\title{
MASTER \\ ACTINIDE PARTITIONING-TRANSMUTATION PROGRAM \\ FINAL REPORT. \\ V. PRECONCEPTUAL DESIGNS AND COSTS OF PARTITIONING FACILITIES AND SHIPPING CASKS (APPENDIX 4)
}

FFP-WTF

COST ESTIMATES

Report prepared by

The Ralph M. Parsons Company

Engineers/Constructors

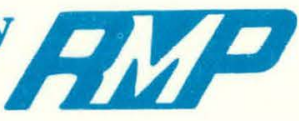

Pasadena, California 91124

for

OAK RIDGE NATIONAL LABORATORY

Oak Ridge, Tennessee 37830 


\section{DISCLAIMER}

This report was prepared as an account of work sponsored by an agency of the United States Government. Neither the United States Government nor any agency Thereof, nor any of their employees, makes any warranty, express or implied, or assumes any legal liability or responsibility for the accuracy, completeness, or usefulness of any information, apparatus, product, or process disclosed, or represents that its use would not infringe privately owned rights. Reference herein to any specific commercial product, process, or service by trade name, trademark, manufacturer, or otherwise does not necessarily constitute or imply its endorsement, recommendation, or favoring by the United States Government or any agency thereof. The views and opinions of authors expressed herein do not necessarily state or reflect those of the United States Government or any agency thereof. 


\section{DISCLAIMER}

Portions of this document may be illegible in electronic image products. Images are produced from the best available original document. 


\section{The following pages are an exact representation of what is in the original document folder.}


This report was prepared as an account of work sponsored by an agency of the United States Government. Neither the United States Government nor any agency thereof, nor any of their employees, makes any warranty, express or implied, or assumes any legal liability or responsibility for the accuracy, completeness, or usefulness of any information, apparatus, product, or process disclosed, or represents that its use would not infringe privately owned rights. Reference herein to any specific commercial product, process, or service by trade name, trademark, manufacturer, or otherwise, does not necessarily constitute or imply its endorsement, recommendation, or favoring by the United States Government or any agency thereof. The views and opinions of authors expressed herein do not necessarily state or reflect those of the United States Government or any agency thereof. 
ACTINIDE PARTITIONING-TRANSMUTATION PROGRAM FINAL REPORT.

V. PRECONCEPTUAL DESIGNS AND COSTS OF PARTITIONING FACILITIES AND SHIPPING CASKS (APPENDIX 4)

\author{
FFP-WTF \\ COST ESTIMATES \\ JUNE 1980 \\ Report prepared by
}

\begin{abstract}
THE RALPH M. PARSONS COMPANY
Pasadena, California 91124

Under Purchase Order No. 85B31056X-01
\end{abstract}

for

OAK RIDGE NATIONAL LABORATORY

Oak Ridge, Tennessee 37830

operated by

UNION CARBIDE CORPORATION

NUCLEAR DIVISION

for the

DEPARTMENT OF ENERGY

Under Contract No. W-7405-eng-26

Job No. 5872-1 


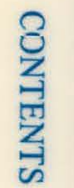




\section{CONTENTS}

Account

PAGE

-- Introduction. ................. i . . . . .

-- Cost Sumaries. . . . . . . . . . . . . . 1

2000 Land Improvements ................ 4

3000 Building

Electrical................ 8

Civil, Architectural, Mechanical ...... 23

Office, Guard House . . . . . . . . . . . . . 44

$5000 \quad$ Other Structures

Genera1. .............. 50

Mock-Up Facility............ 53

6000 Special Facilities

Equipment. ............ 54

Cation Exchange Chromatography (SK-MP-1). . . 55

CMP Extraction (SK-MP-2 \& 3). . . . . . 70

Nitric Acid Recovery (SK-MP-4)....... 102

Salt Waste Treatment (SK-MP-5\&6). . . . . 122

Solid Alpha Waste Treatment (SK-MP-7) . . . 151

Service Utilities (SK-MP-40, 40A, 41) . . . 173

Piping ................ . 223

Instruments. . . . . . . . . . . 224

Computer ............... . 226

Insulation ............... 227

$7000 \quad$ Utilities

Shipping Cask Haulers. . . . . . . . . . 228

Tunnel Piping............... 229

Electrical ............. . . . 230

Tunnels and Railroads. . . . . . . . . . 240

Shipping Cask.............. 243 


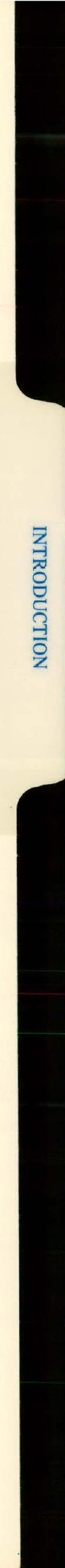




\section{INTRODUCTION}

This Appendix contains cost estimate documents for the Fuels Fabrication Plant Waste Treatment Facility. Plant costs are summarized by Code of Accounts and by Process Function. Costs contributing to each account are detailed.

Process equipment costs are detailed for each Waste Treatment Process. Service utility costs are also summarized and detailed. Shipping cask costs are provided. 
勇 
TABLE 5.5

FFP-WTF COST ESTIMATE BY CODE OF ACCOUNTS

Mid-1979 Dollars

\section{Account}

2000 Land Imp rovement

3000 Building

5000 Other Structures

7000 Utilities

Sub-Tota 1
6000 Special Facilities

\section{Material}

Expense

$1,030,600$

$49,846,400$

$12,357,300$

$36,641,200$

$\frac{7,399,300}{107,274,800}$

Field Indirects and

$S / C$ 'S OH\&P

$$
\text { Sub-Total }
$$

Engineering/Design/Project Management

Conceptual, Tit les I, II, III

$(28 \%)$

Sub-Total

Contingency (35\%)

TOTAL

\begin{tabular}{rc}
\multicolumn{1}{c}{ Labor } & Total \\
945,700 & $1,976,300$ \\
$41,292,600$ & $91,139,000$ \\
$4,684,400$ & $17,041,700$ \\
$14,246,900$ & $50,888,100$ \\
$\frac{11,325,900}{72,495,500}$ & $\frac{18,725,200}{179,770,300}$
\end{tabular}

$72,495,500$

$252,265,800$

$70,634,400$

$322,900,200$

$113,099,800$

$436,000,000$ 
TABLE 5.6

FFP-WTF COST ESTIMATE - BY FUNCTION

Mid-1979 Dollars

Land Improvement

Including Railroad

Material

Expense

Labor

Total

Process Canyon/Mechanical

$1,180,600$

$1,049,200$

$2,229,800$

Support Facilities

Tunnel and Piping

$85,976,100 \quad 55,128,700 \quad 141,104,800$

Support Facilities

$5,790,100 \quad 10,850,100$

$16,640,800$ and Utilities

$\underline{14,378,000} \quad \underline{5,467,500} \quad 19,795,500$

Sub-Tota 1

$107,274,800 \quad 72,495,500$

$179,770,300$

Field Indirects and

$72,495,500$

$S / C$ 's OH\&P

$252,265,800$

Engineering/Design/Project Management

$70,634,400$

Sub-Total

$322,900,200$

Contingency $(35 \%)$

$113,099,800$

TOTAL

$436,000,000$ 


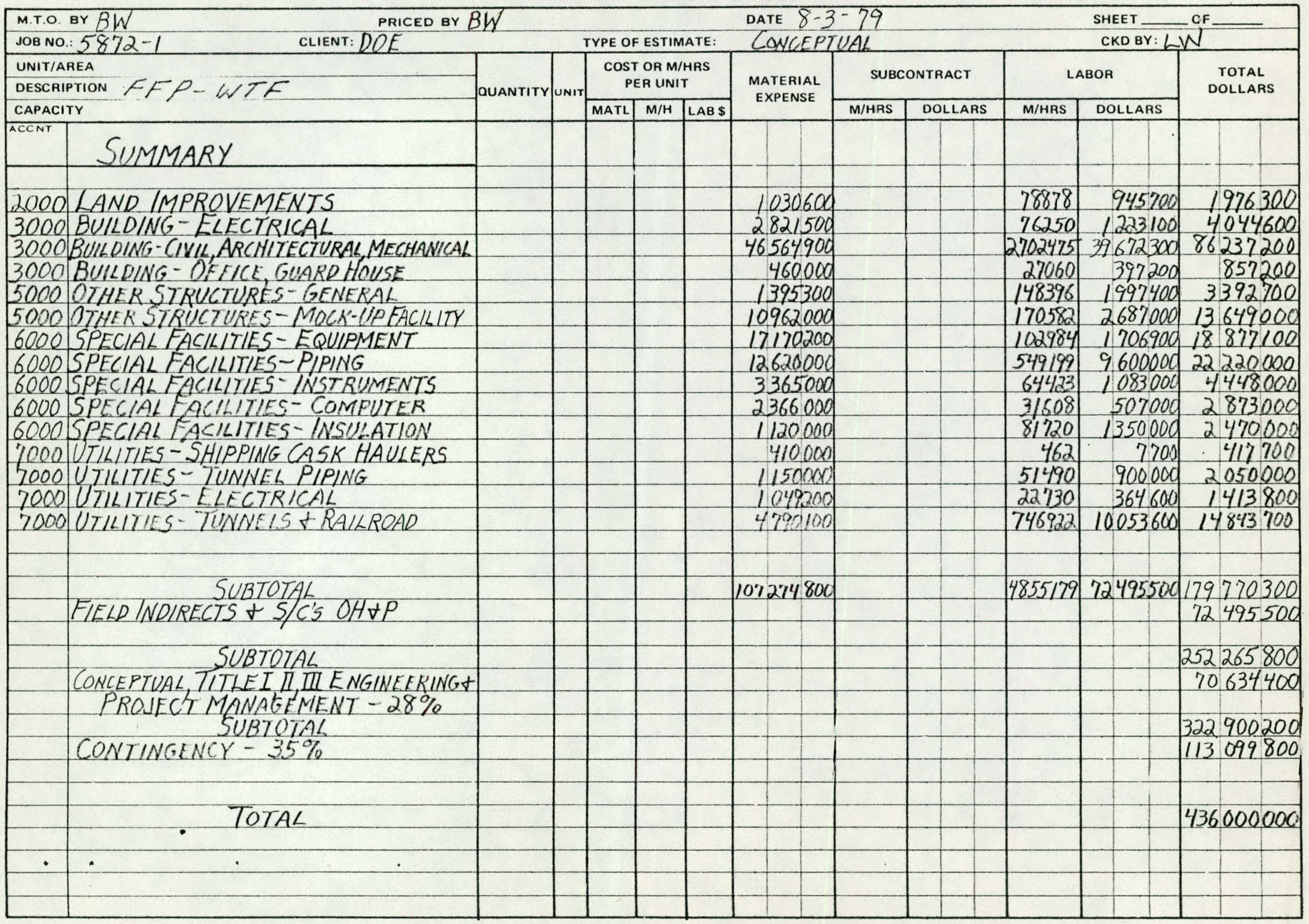


CES $4903-0$ THE RALPH M. PARSONS COMPANY
ESTIMATE WORKSHEET

DATE

SHEET 1 OF 4

MTO: BY

PRICED BY

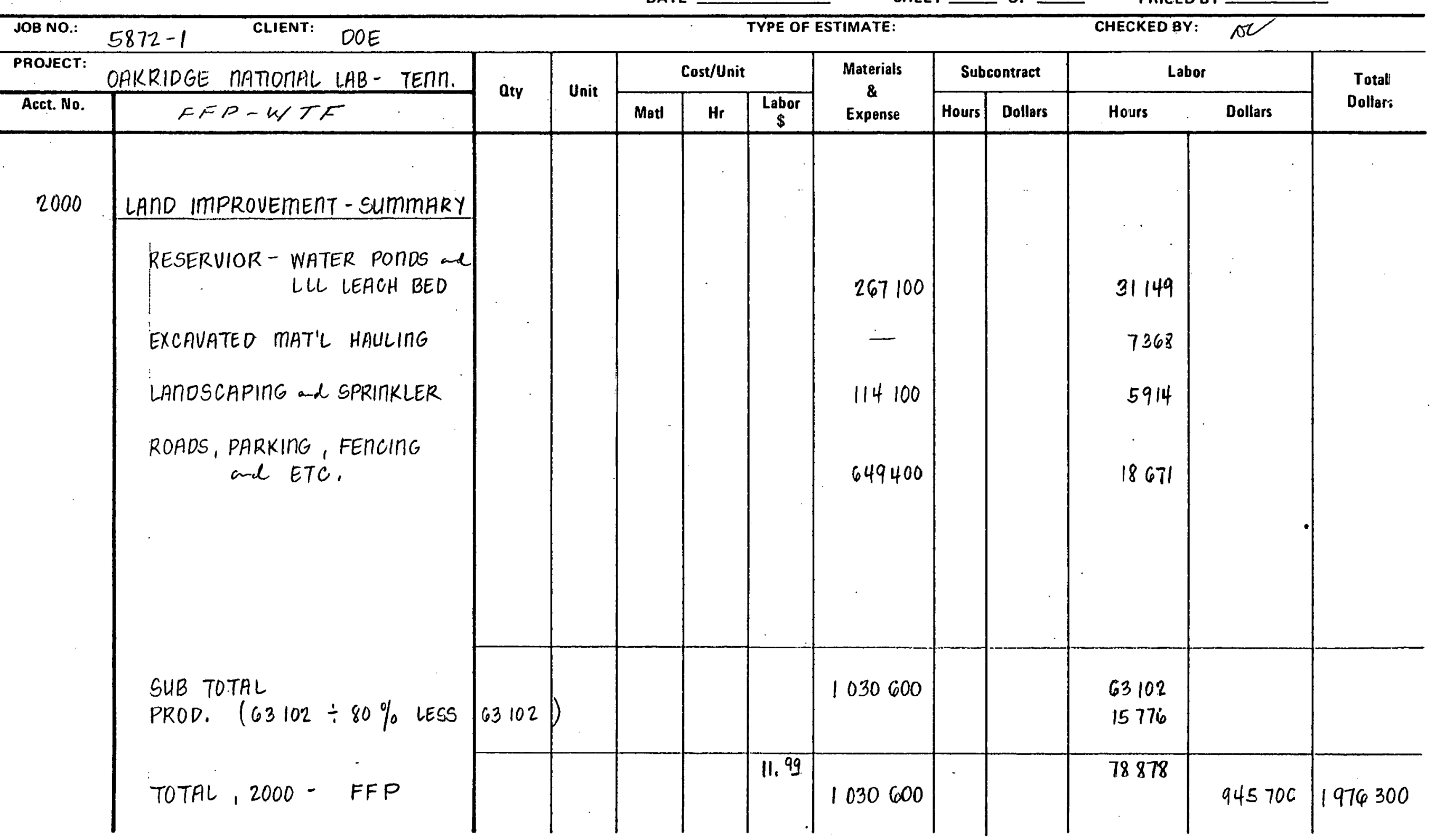


ESTIMATE WORKSHEET

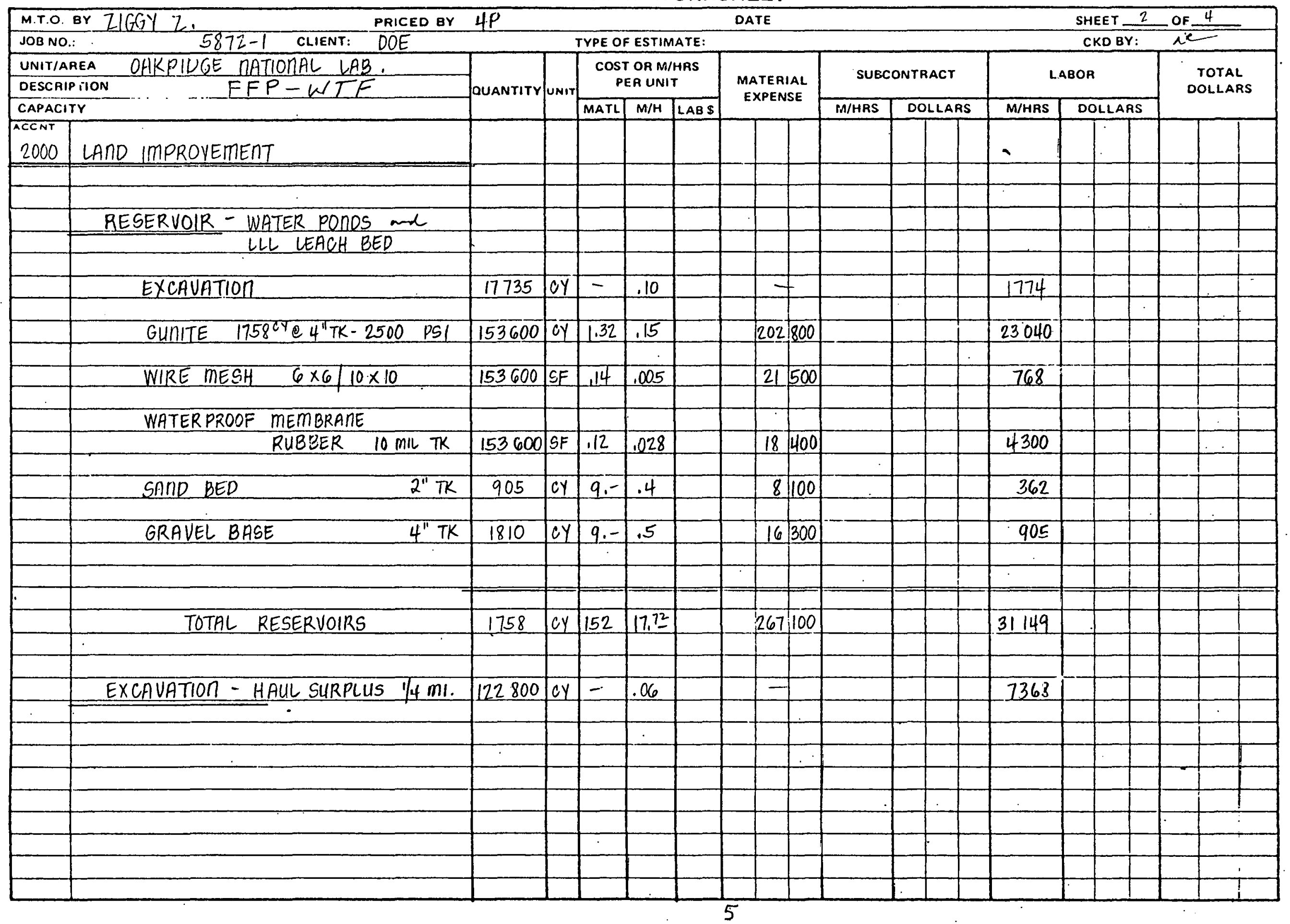


ESTIMATE WORKSHEET

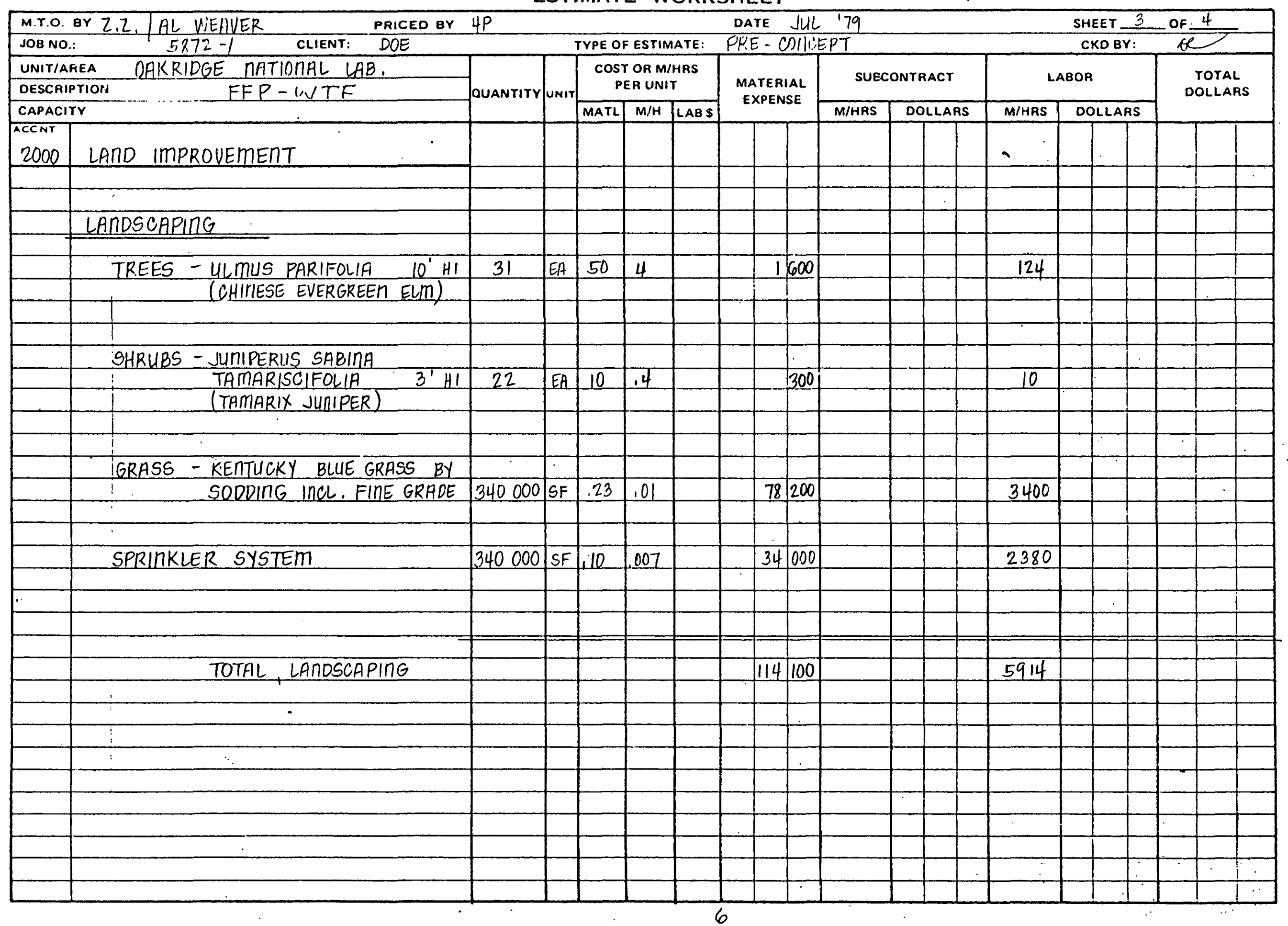


ESTIMATE WORKSHEET

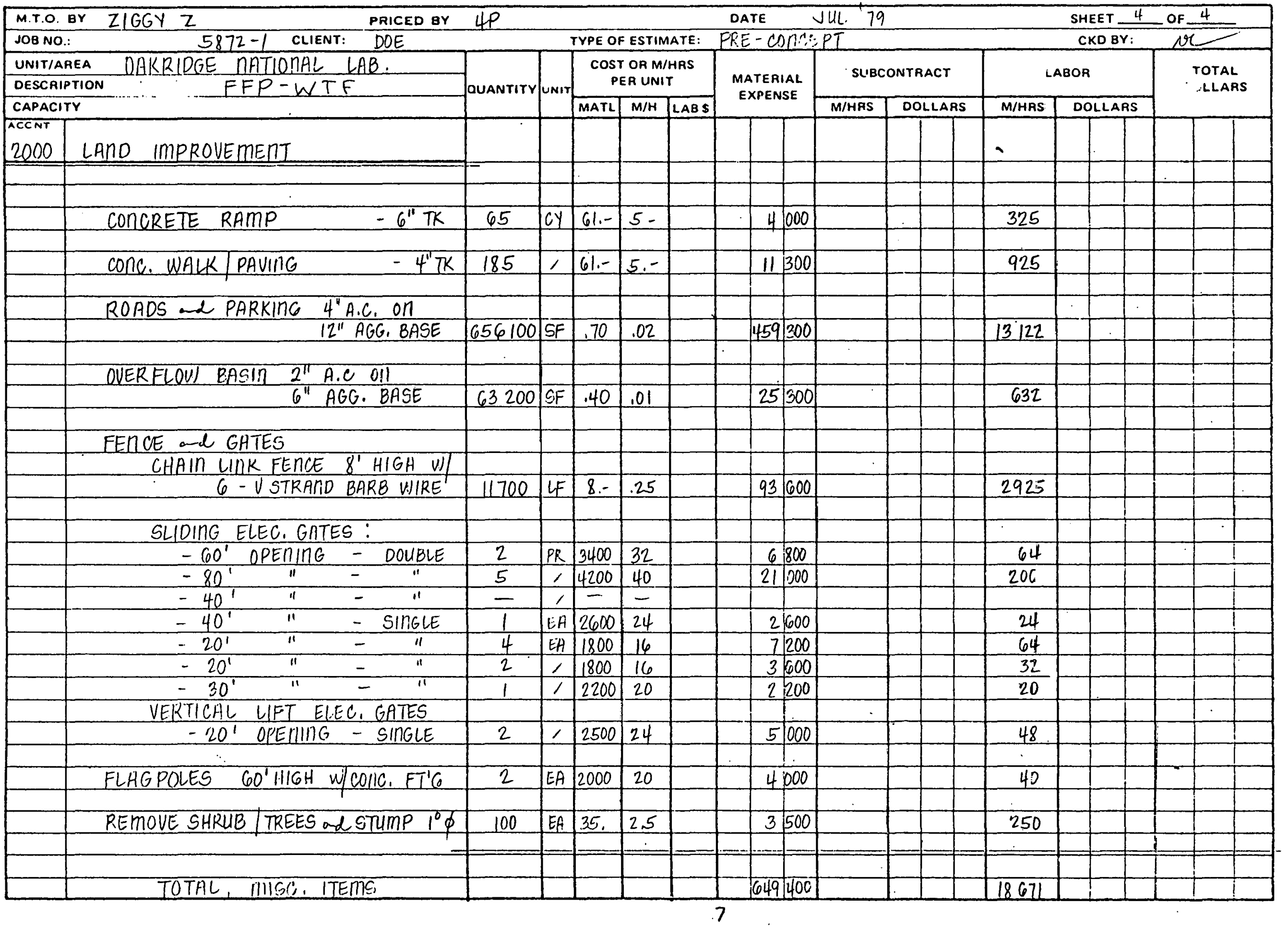


ESTIMATE WORKSHEET

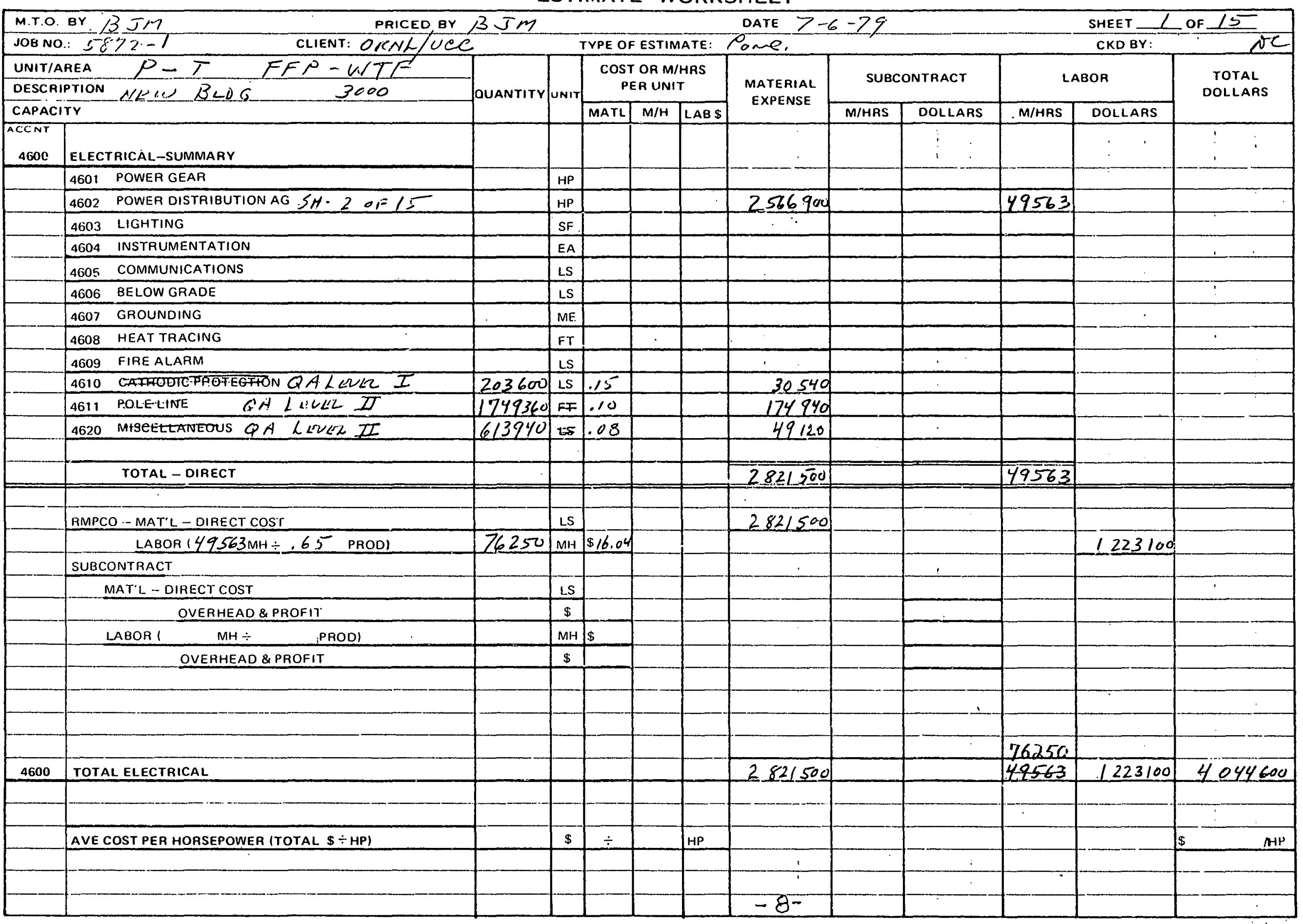




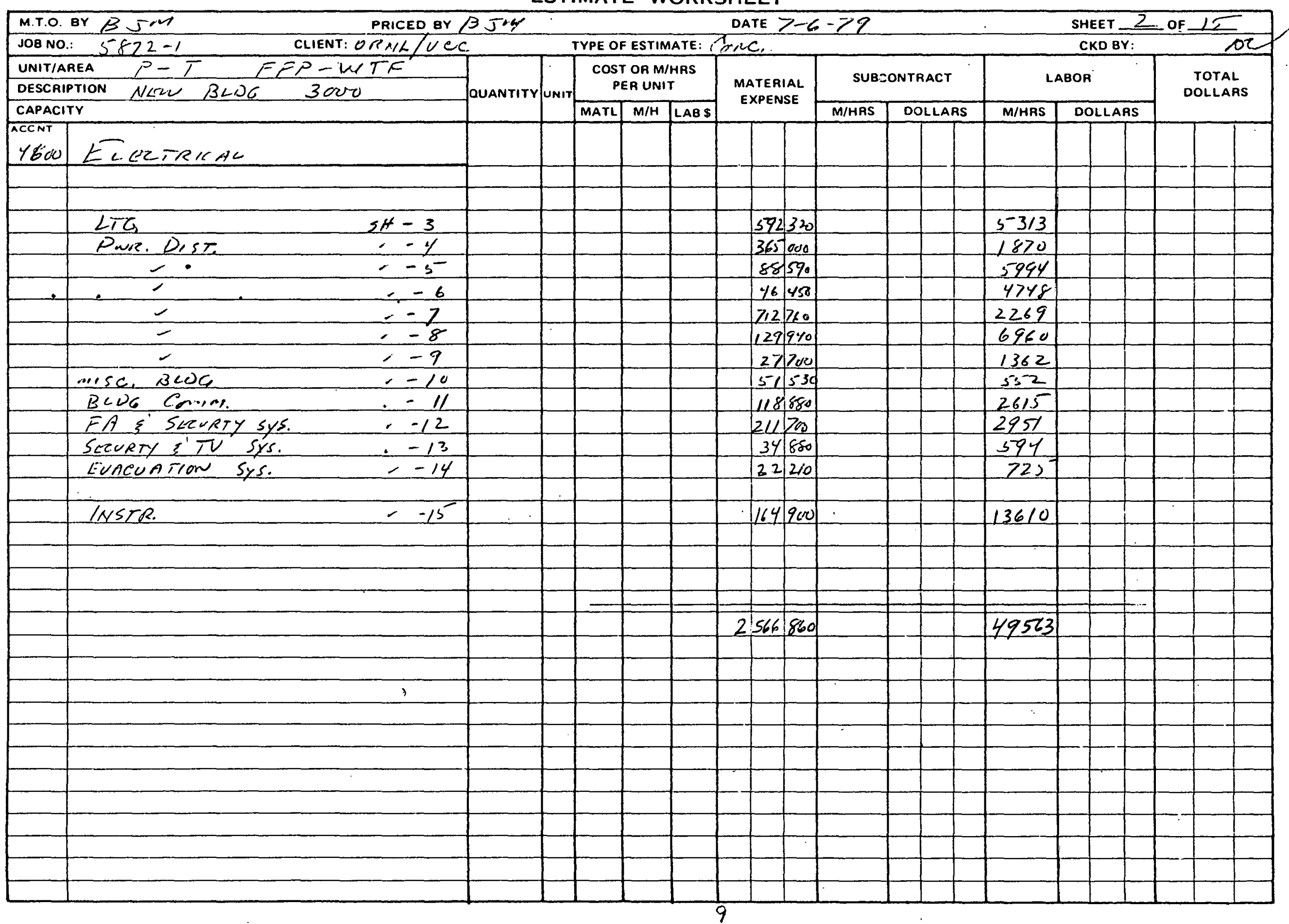


ESTIMATE WORKSHEET

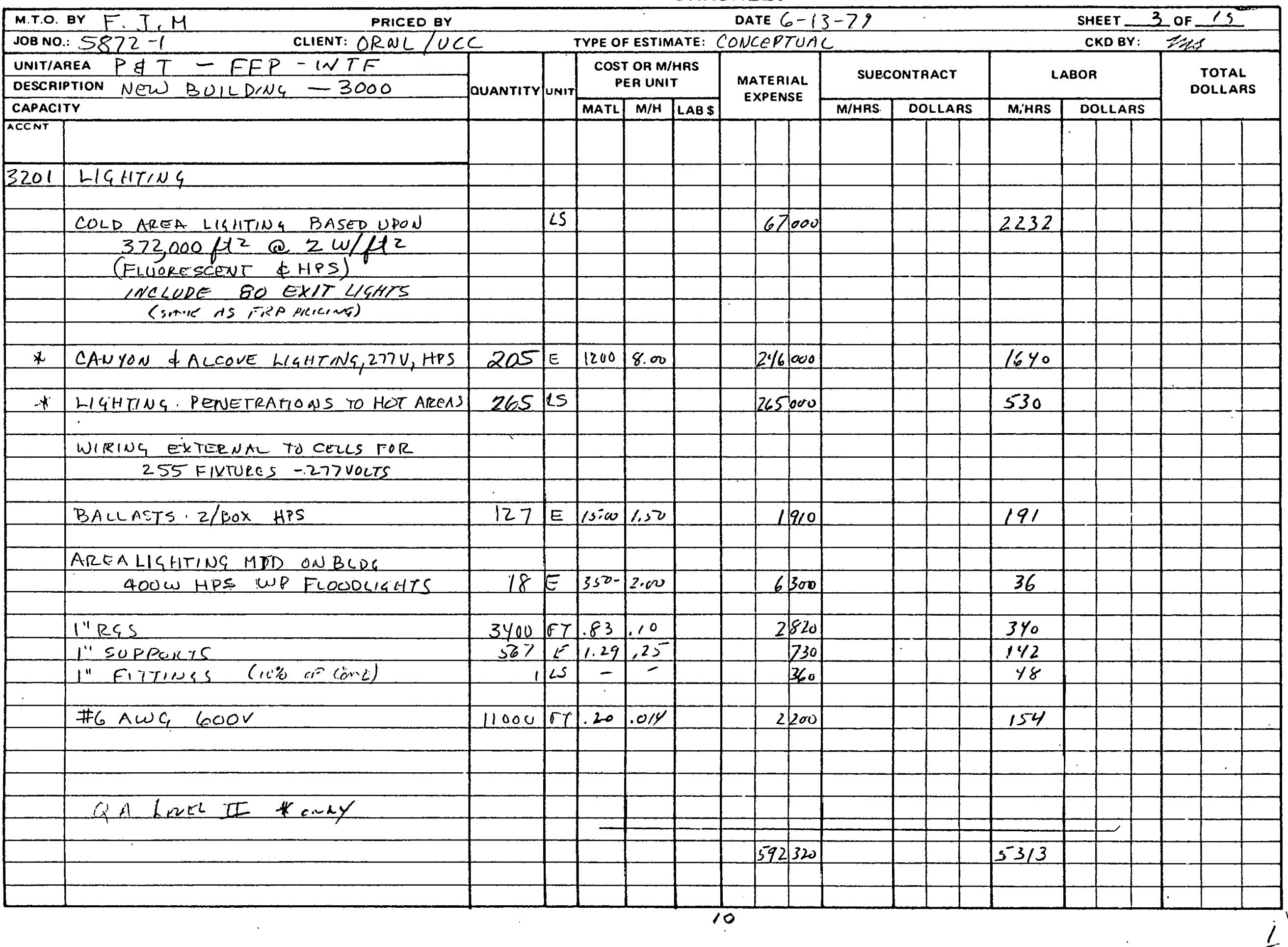


ESTIMATE WORKSHEET

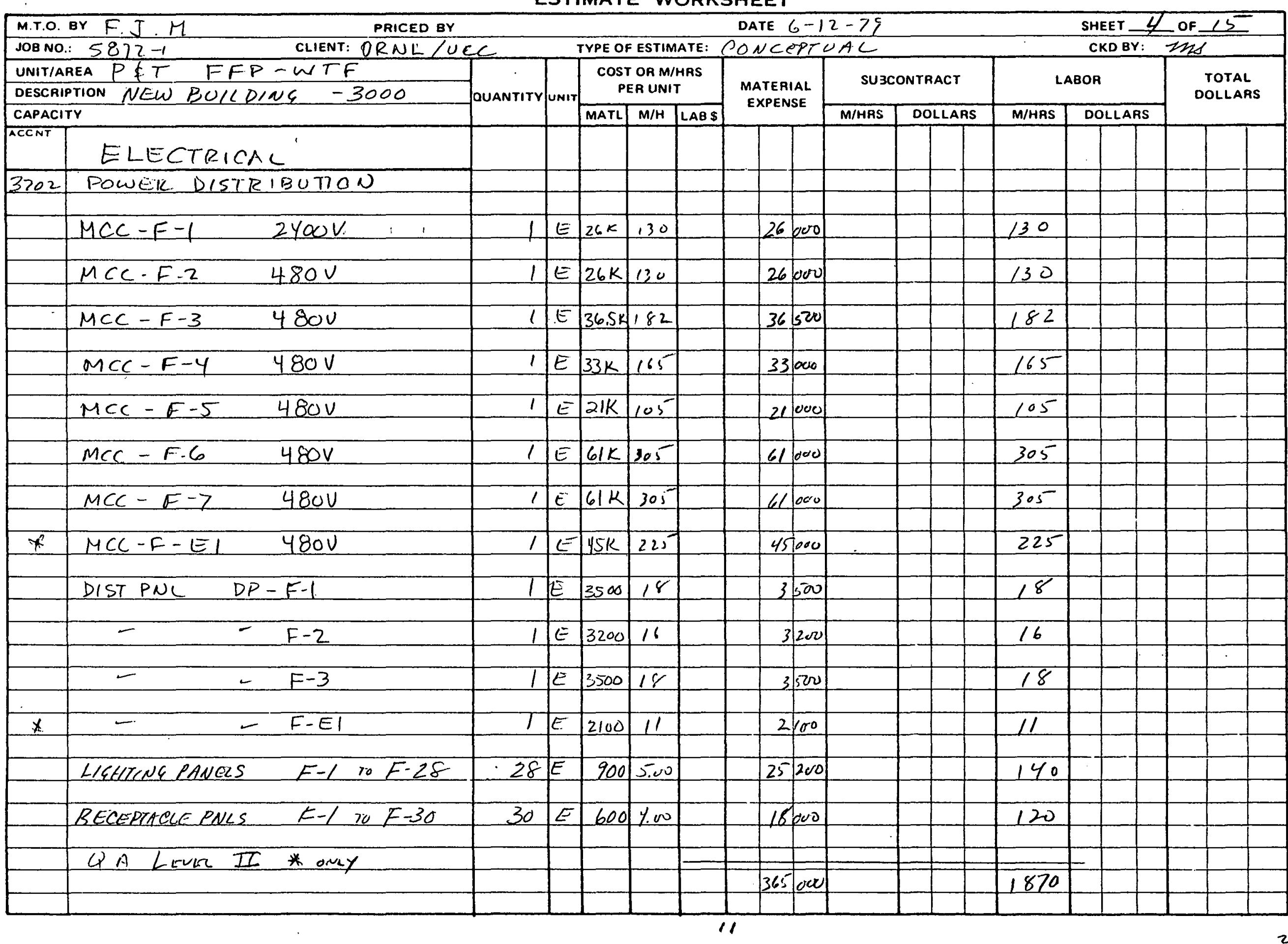


ESTIMATE WORKSHEET

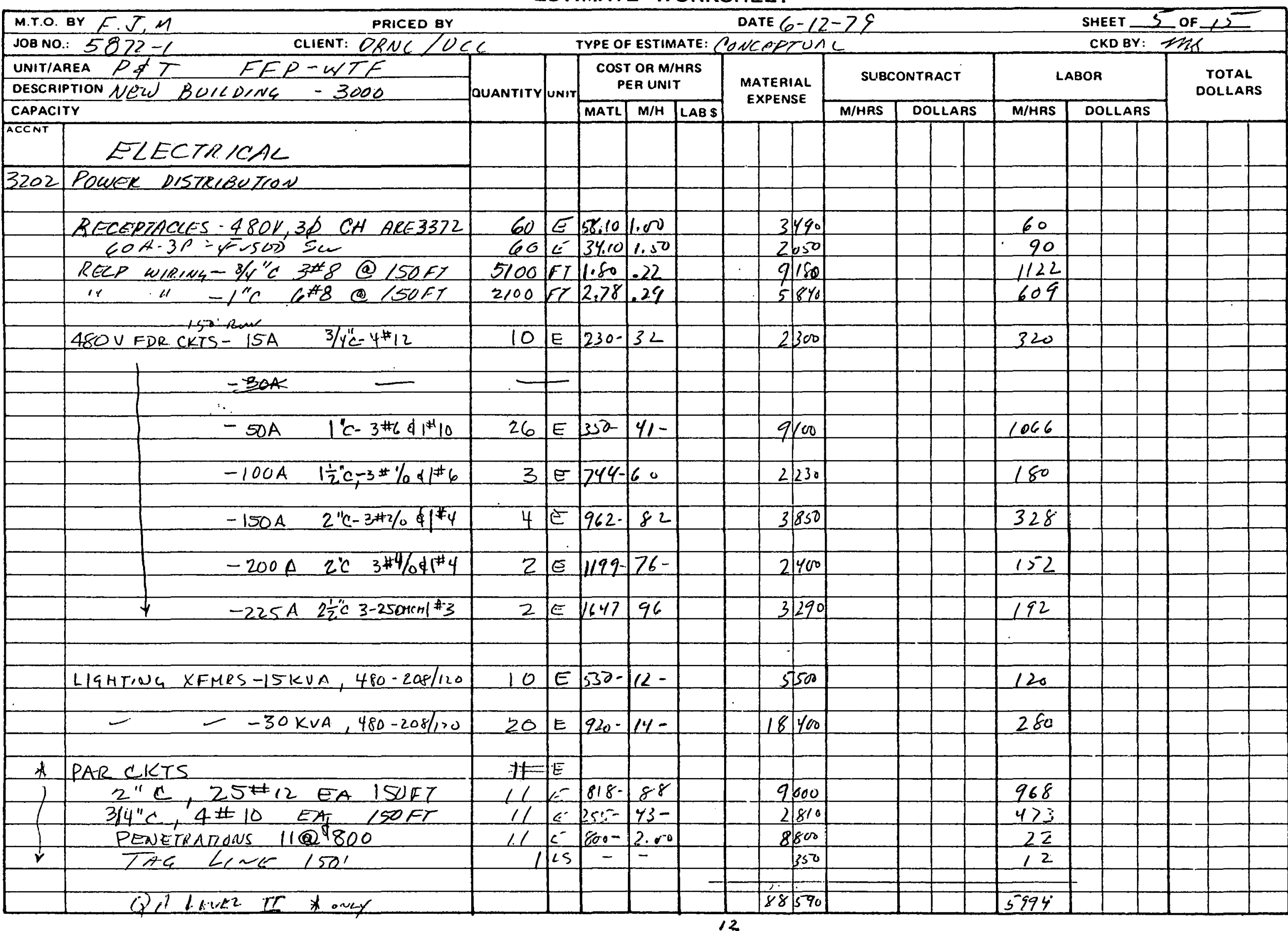


ESTIMATE WORKSHEET

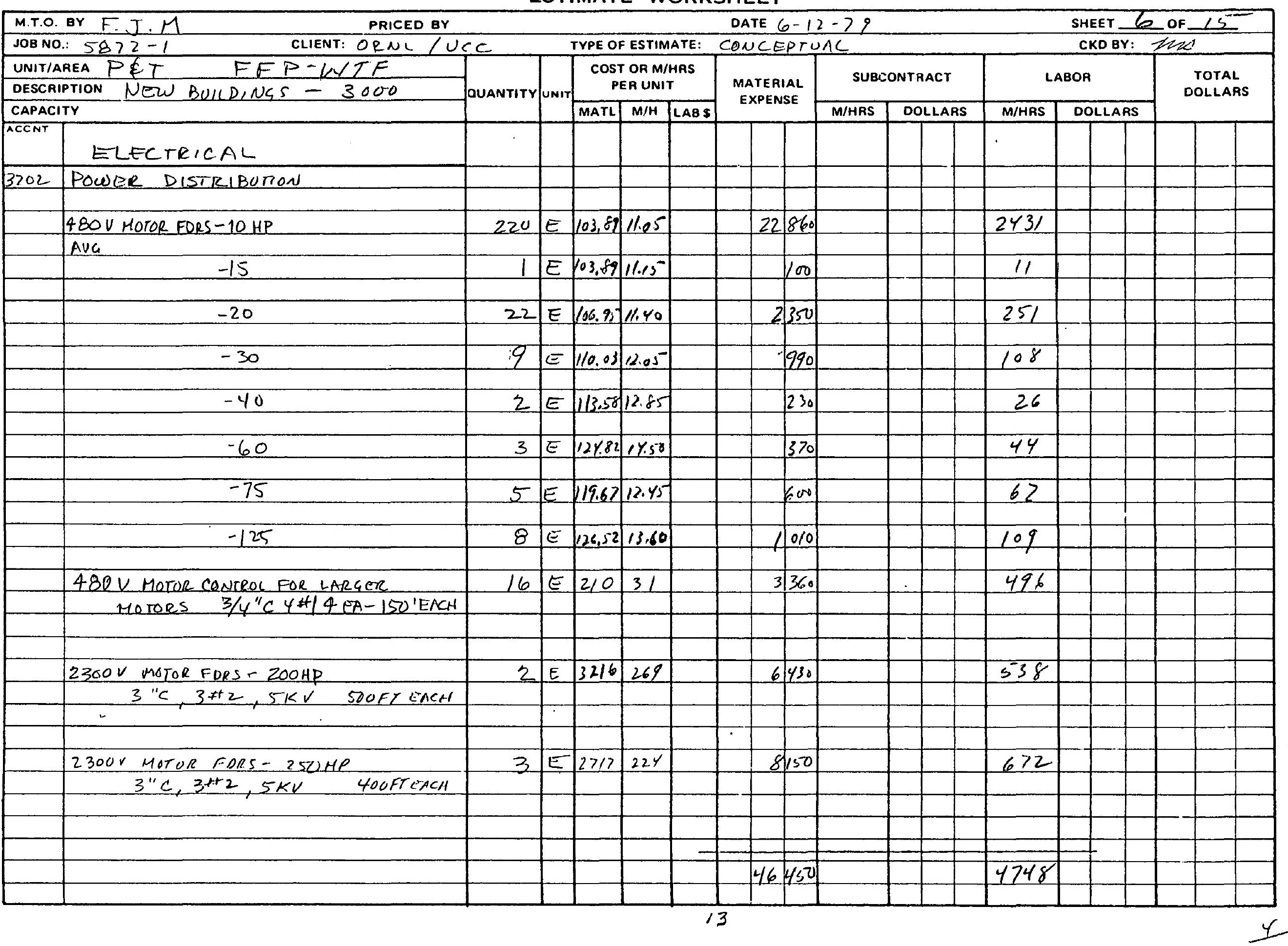


ESTIMATE WORKSHEET

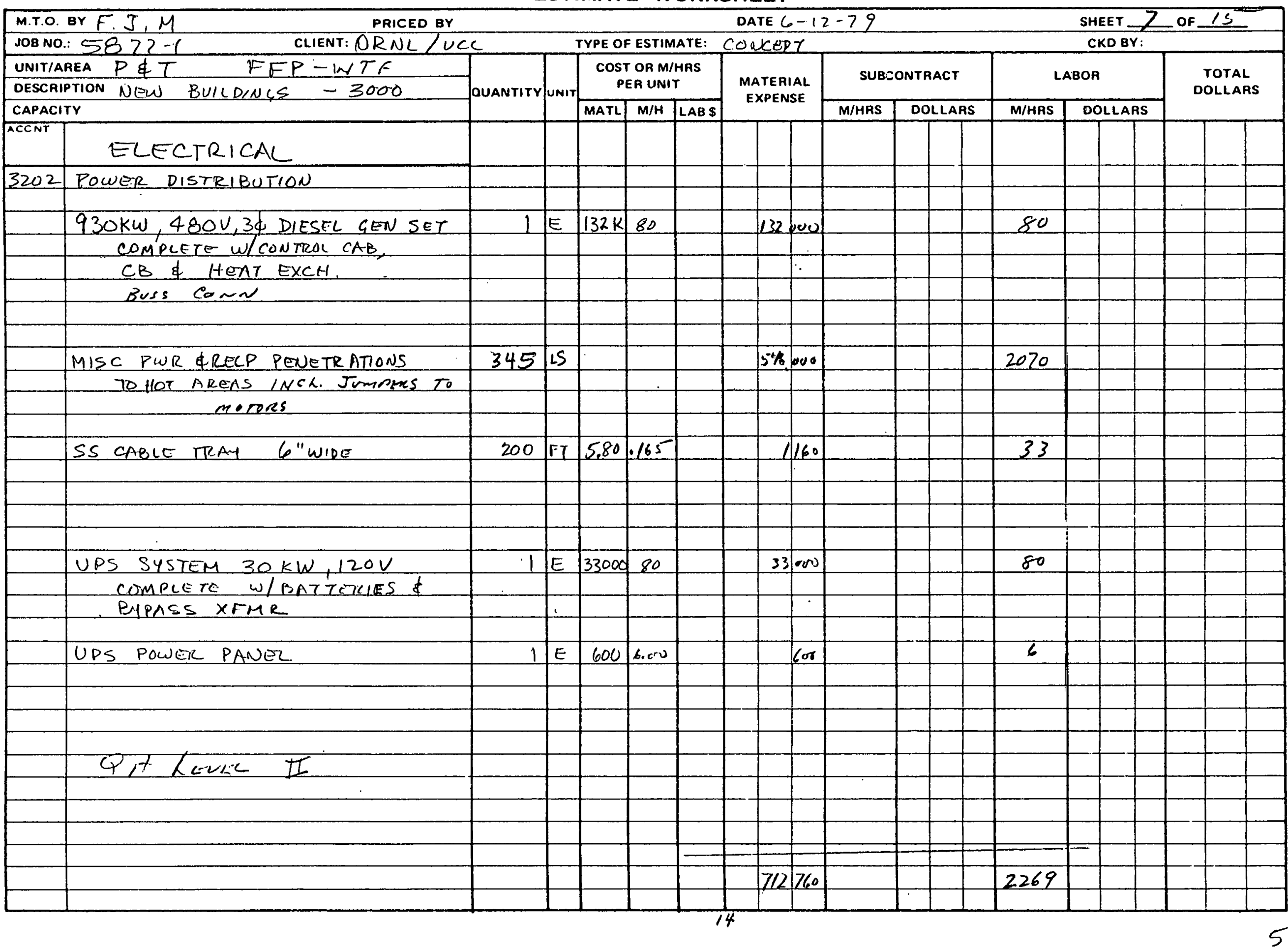


ESTIMATE WORKSHEET

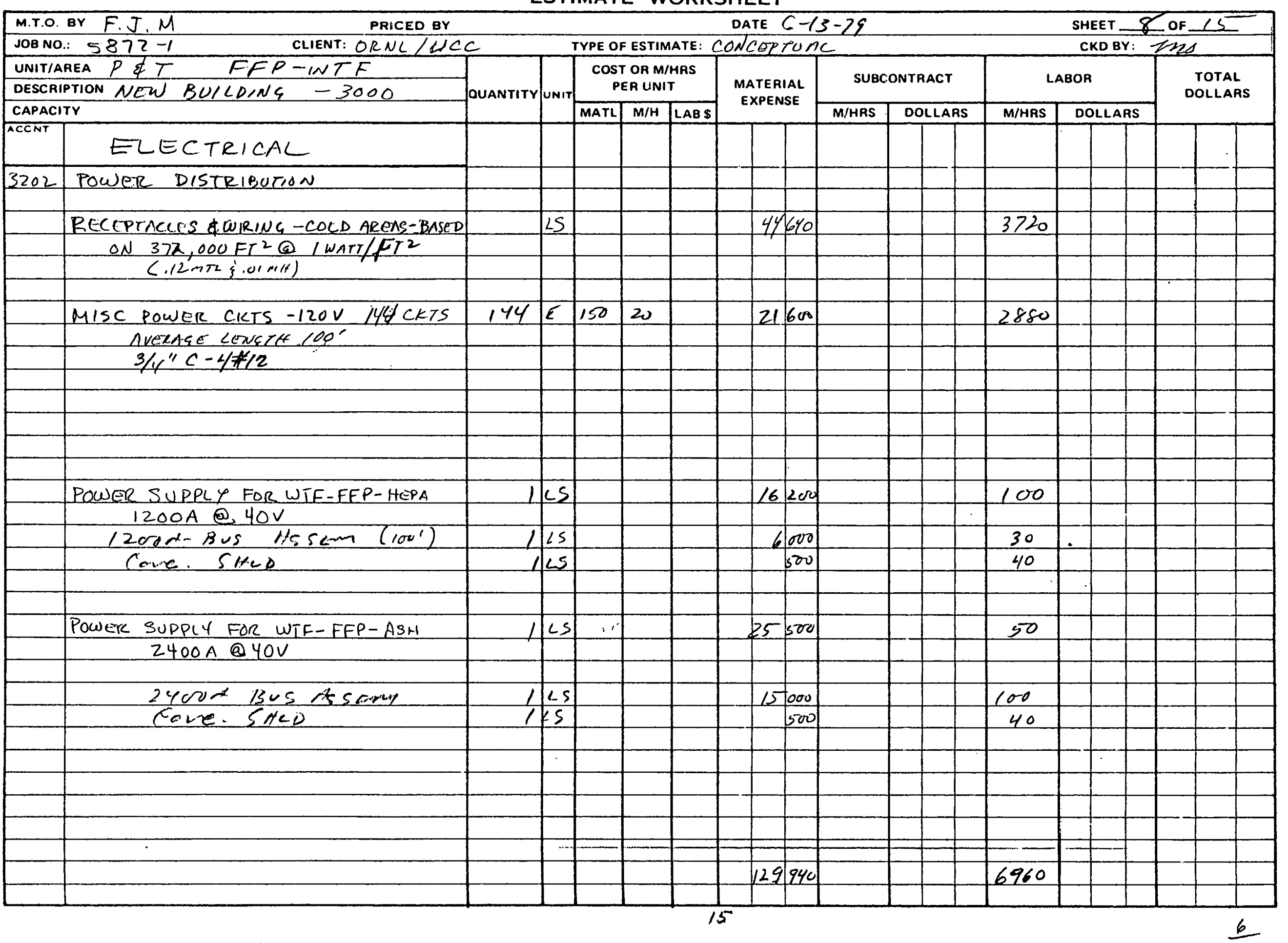


THE RALPH M. PARSONS COMPANY

ESTIMATE WORKSHEET

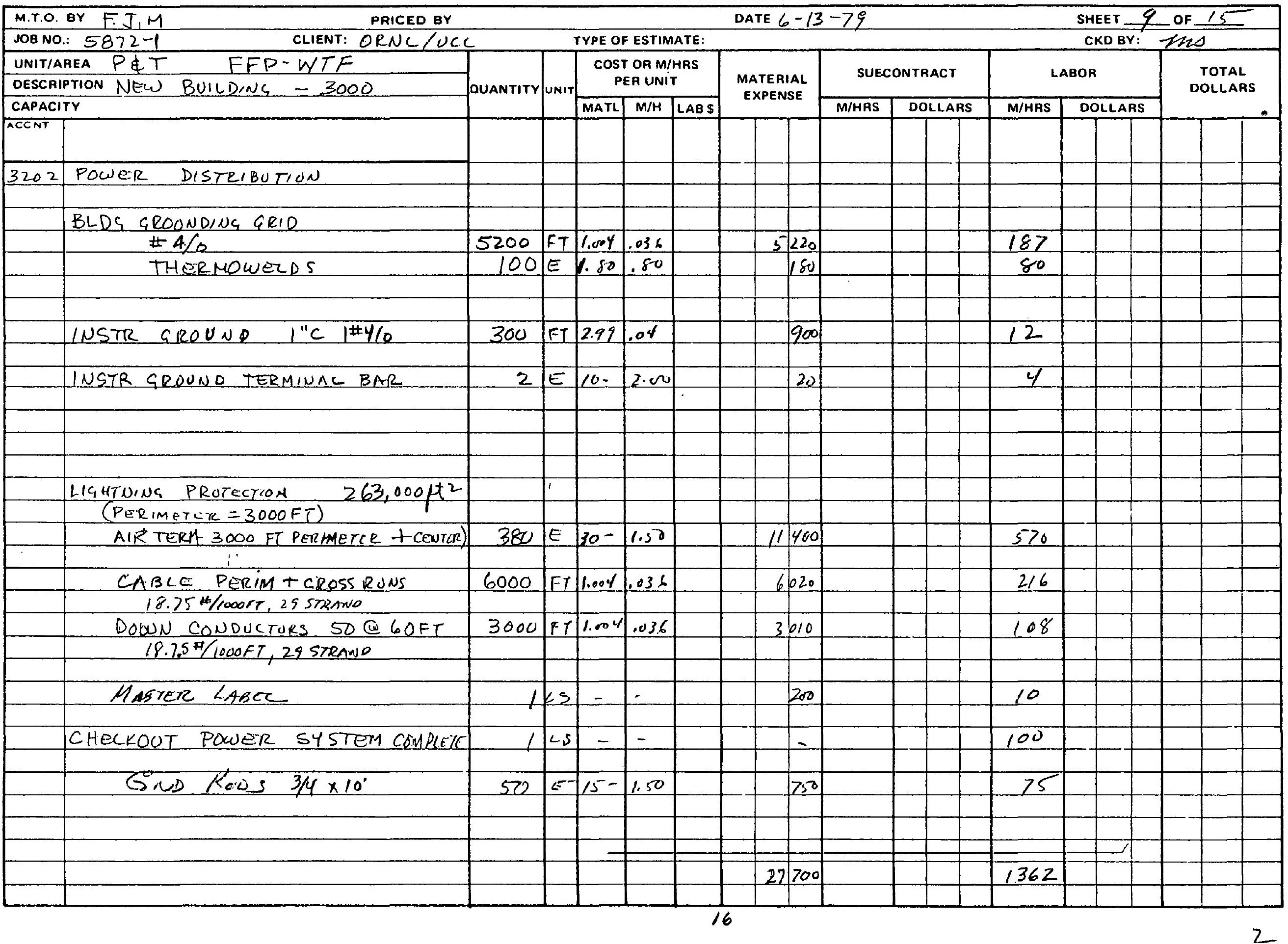


ESTIMATE WORKSHEET

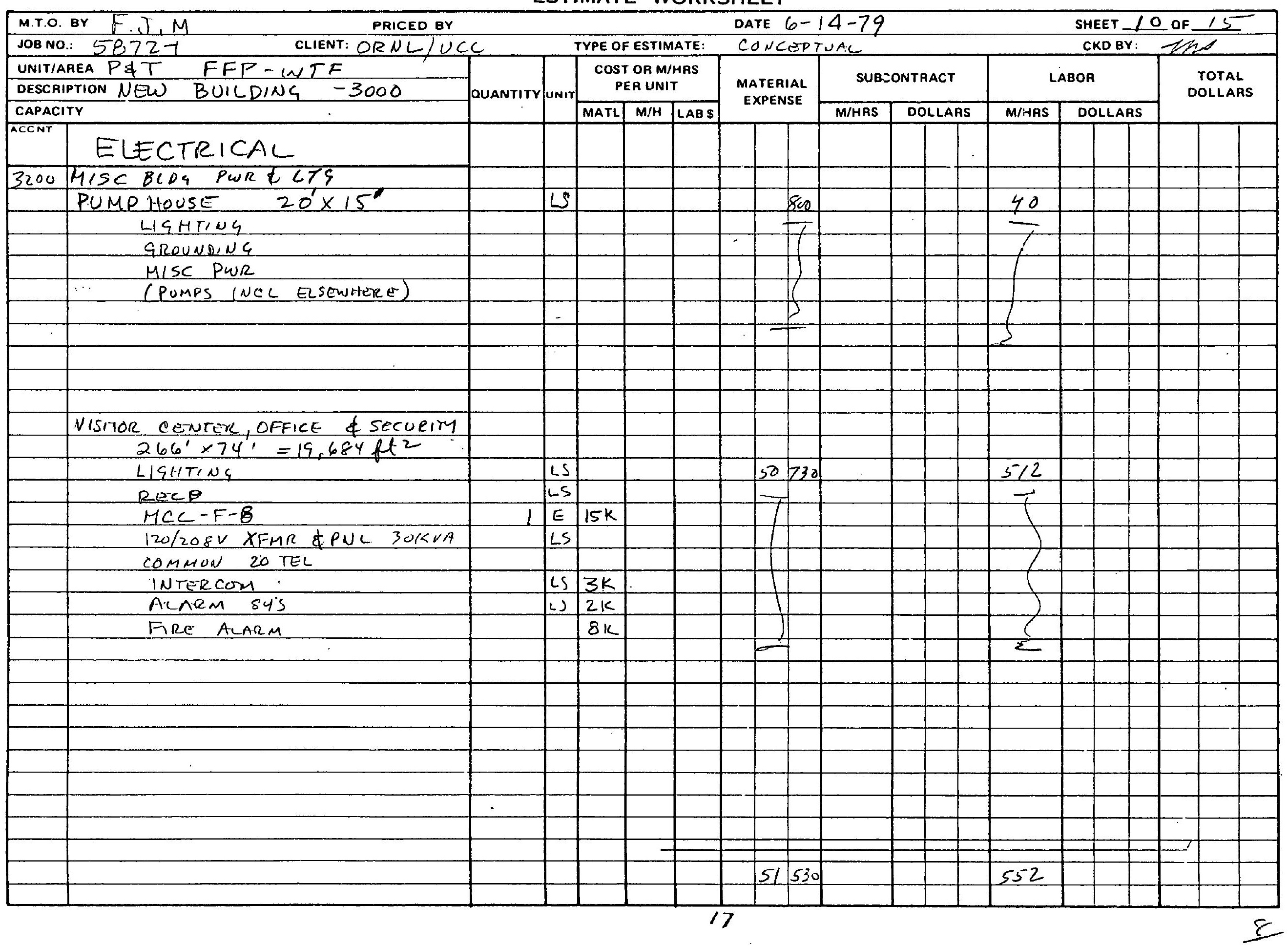


ESTIMATE WORKSHEET

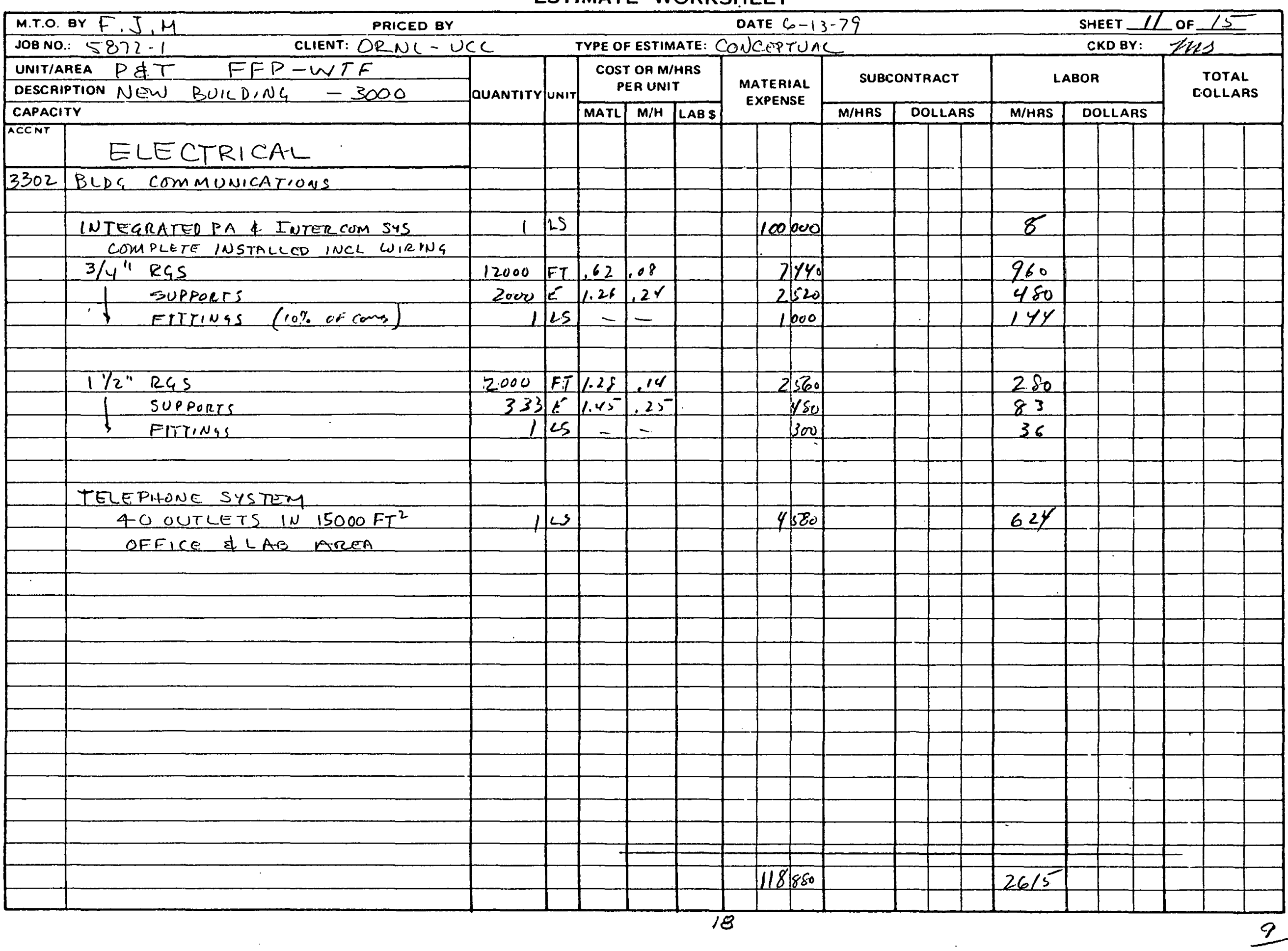


THE RALPH M. PARSONS COMPANY

ESTIMATE WORKSHEET

\begin{tabular}{|c|c|c|c|c|c|c|c|c|c|c|c|c|c|c|c|c|}
\hline M.T.O. & BY F,J,M & & & & & & DATE & $6-$ & $13-79$ & & & & SHEE & 12 & $=0 F 15$ & \\
\hline JOB NO & CLIENT: QRWL/UCO & & & TYPE OF & FESTIM & AATE: $C$ & oncer & PTU & $A C$ & & & & CKD & BY: & tos & \\
\hline UNIT/A & EFP-WTF & & & $\cos T$ & TOR M/I & & & & SUBC & DNTRACT & & & BOR & & TOTAL & \\
\hline DESCAI & PTION NEW BUILDING - 3000 & OUANTITY & 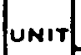 & & & & $\begin{array}{l}\text { MATERI } \\
\text { EXPENS }\end{array}$ & & & & & & & & DOLLAR & \\
\hline CAPACI & & & & MATL & $M / H$ & LABS & & & M/HRS & DOLLAF & & M/HRS & DOL & LARS & & \\
\hline$\overline{A C C N T}$ & ELECTRICAL & & & & & & & & & & & & & & & \\
\hline 3303 & EIRE ALARM \& SECURITI & & & & & & & & & & & & & & & \\
\hline & & & & & & & & & & & & & & & & \\
\hline & WWTEGRATED FA E SECURITM SYS & 1 & LS & $200 \mathrm{k}$ & & & 200 & 000 & & & & 1000 & & & & \\
\hline & operater control colsole with & & & & & & & & & & & & & & & \\
\hline & DISPLAY MODULE I PRINTER. & & & & & & & & & & & & & & & \\
\hline & DOOR Swincries, window mowrtors, & & & & & & & & & & & & & & & \\
\hline & IUFRARED DETECTORS, & & & & & & & & & & & & & & & \\
\hline & MANUAL FA STA, DUCT DETECTORS & & & & & & & & & & & & & & & \\
\hline & IONIZATION DETECTORS \& ALARM BeCLS & & & & & & & & & & & & & & & \\
\hline & S45TEM TO USE MULTIPCEX XMIS5ION. & & & & & & & & & & & & & & & \\
\hline & TOTAL 470 FIELD DEVICES & & & & & & & & & & & & & & & \\
\hline & & & & & & & & & & & & & & & & \\
\hline & & & & & & & & & & & & & & & & \\
\hline & & & & & & & & & & & & & & & & \\
\hline & $3 / 4$ "RGs & 7000 & $\mathrm{Fl}$ & .62 & .08 & & 4. & 340 & & & & 560 & & & & \\
\hline & 11 SUPPOR.75 & 1167 & 5 & 1.26 & .24 & & & $4>0$ & & & & 280 & & & & \\
\hline & "FITIINGS & 1 & 25 & - & - & & & 580 & & & & 84 & & & & \\
\hline & & & & & & & & & & & & & & & & \\
\hline & I"C 245 & 2000 & FT & .83 & .10 & & 1 & 660 & & & & 200 & & & & \\
\hline & "I SUPPOIIS & 333 & E & 1.29 & 25 & & & 430 & & & & 83 & & & & \\
\hline & 11 FITTINCS & & LS & - & - & & & 210 & & & & 28 & & & & \\
\hline & & & & & & & & & & & & & & & & \\
\hline & WIRE $2 / C \# / 8$ & 70,000 & $F 7$ & .043 & .010 & & & 010 & & & & 200 & & & & \\
\hline & & & & & & & & & & & & & & & & \\
\hline & & & & & & & & & & & & 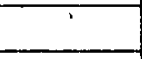 & & & & \\
\hline & & & & & & & & & & & & & & & & \\
\hline & & & & & & & & & & & & & & & & \\
\hline & & & & & & & & & & & & & & & & \\
\hline & CHECKOUT & & 45 & & & & & $E$ & & & & 16 & & & & \\
\hline & & & & & & & & & & & & & & & & \\
\hline & & & & & & Z & $\rightarrow$ & $E$ & & 7 & E & & $-1-$ & & & \\
\hline & & & & & & & 211 & 200 & & & & 2951 & & & & \\
\hline & & & & & & & 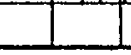 & & & & & & & & & \\
\hline
\end{tabular}


THE RALPH M. PARSONS COMPANY

ESTIMATE WORKSHEET

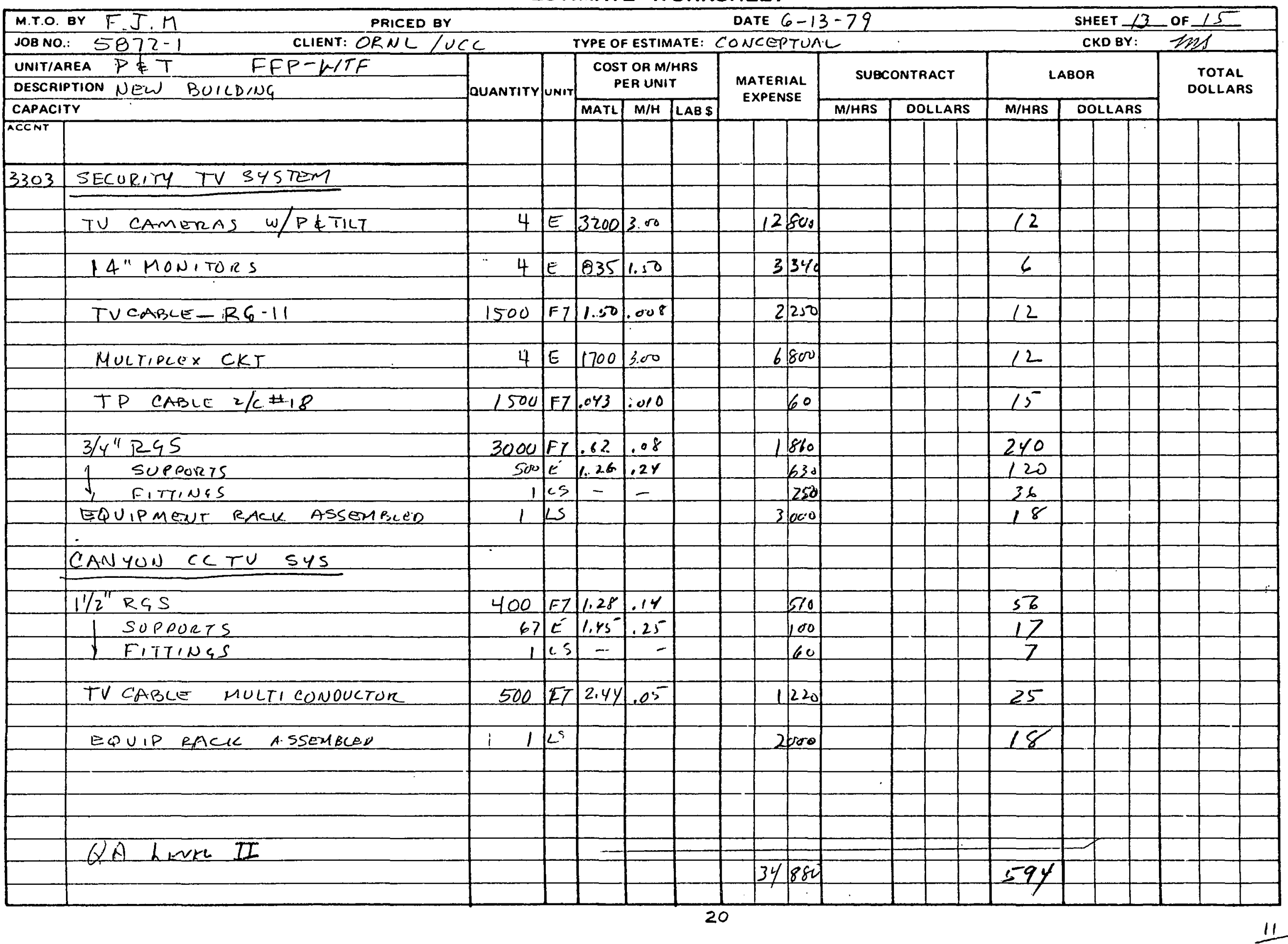


ESTIMATE WORKSHEET

\begin{tabular}{|c|c|c|c|c|c|c|c|c|c|c|c|c|c|c|c|c|c|}
\hline M.T.O. & BY F.J.M & & & & & & DATE 6 & -13 & -79 & & & & & & EET 14 & OF 15 & \\
\hline JOB NC & CLIENT: ORNL/UC & c. & & TYPE OF & ESTIM & IATE: & CONCEF & ETU & Ac & & & & & & KD BY: & zkes & \\
\hline UNIT/A & REA $P \& T \quad F F P-W T F$ & & & $\cos 1$ & OR M/I & & & & suso & ONTR & ACCT & & & BOR & & & \\
\hline DESCA & PTION NEW BUILDING -3000 & QUANTITY & unit & & ER UNIT & & $\begin{array}{l}\text { MATERI } \\
\text { EXPENS }\end{array}$ & & Suso & ONIR & $\mid A C T$ & & & Don & & DOLLAR & \\
\hline$\overline{C A P A C}$ & & & & MATL & $M / H$ & LABS & & & M/HRS & & LLARS & & M/HRS & & LLARS & & \\
\hline$\overline{A C C N T}$ & ELECTRICAL & & & & & & & & & & & & & & & & \\
\hline $330 \%$ & EVACUATION SYSTEM & & & & & & & & & & & & & & & & \\
\hline & & & & & & & & & & & & & & & & & \\
\hline & EUACUATION SUSTEM COMPEETE & 1 & LS & & & & 17 & 800 & & & & & 85 & & & & \\
\hline & INCCUDING G:INITIATING STATIONS & & & & & & & & & & & & & & & & \\
\hline & 8O SIRENS \& COUTROL PANEZ & & & & & & & & & & & & & & & & \\
\hline & & & & & & & & & & & & & & & & & \\
\hline & $3 / 4^{\prime \prime} R S S$ & 2000 & $\mathrm{Ft}$ & .62 & .08 & & 1 & 240 & & & & & 160 & & & & \\
\hline & Supponts & 3.33 & $t^{-}$ & 1.26 & 24 & & & 420 & & & & & 80 & & & & \\
\hline & FitTinss (10\% of condo) & & es & $=$ & - & & & 120 & & & & & 24 & & & & \\
\hline & & & & & & & & & & & & & & & & & \\
\hline & $1^{\prime \prime}$ RSS & 1000 & F7 & .83 & .10 & & & 830 & & & & & 100 & & & & \\
\hline & SUPPORTS & 167 & $L^{\prime}$ & 1.29 & 25 & & & 22 & & & & & 42 & & & & \\
\hline & $(10 \%$ of comp) & 1 & $\angle 5$ & - & - & & & $1 / 10$ & & & & & 14 & & & & \\
\hline & & & & & & & & & & & & & & & & & \\
\hline & $112^{\prime \prime R S S}$ & 500 & $F$ & 1.28 & .14 & & & 640 & & & & & 70 & & & & \\
\hline & supporers & 83 & $L^{\prime}$ & 1.45 & .25 & & & 120 & & & & & 21 & & & & \\
\hline & Fititinss (10\% of comp) & 1 & LS & $=$ & - & & & 80 & & & & & $q$ & & & & \\
\hline & & & & & & & & & & & & & & & & & \\
\hline & & & & & & & & & & & & & & & & & \\
\hline & $\# 10$ AwG & 8000 & $F_{7}$ & .11 .6 & .007 & & & 930 & & & & & 72 & & & & \\
\hline & & & & & & & & & & & & & & & & & \\
\hline & $\pm 1 Y$ AwG & 8000 & $E$ & .056 & .006 & & & 430 & & & & & 48 & & & & \\
\hline & & & & & & & & & & & & & & & & & \\
\hline & & & & & & & & & & & & & & & & & \\
\hline & & & & & & & & & & & & & & & & & \\
\hline & & & & & & & & & & & & & & & & & \\
\hline & & & & & & & & & & & & & & & & & \\
\hline & & & & & & & & & & & & & & & & & \\
\hline & & & & & & & & & & & & & & & & & \\
\hline & & & & & & & & & & & & & & & & & \\
\hline & & & & & - & & & - & & E- & 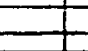 & - & & 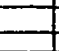 & 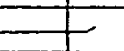 & & \\
\hline & & & & & & & 22 & 210 & & & & & 725 & & & & \\
\hline & & & & & & & & & & & & & & & & & \\
\hline
\end{tabular}




\begin{tabular}{|c|c|c|c|c|c|c|c|c|c|c|c|c|c|c|c|c|c|c|}
\hline M.T.O. & Br $\beta J M$ & $35 x$ & & & & & DATE & $8 \cdot 2$ & $2-79$ & & & & & & EET_ZL & OF. & 15 & \\
\hline JOB NO & CLIENT: ORNL/UCC & & & TYPE OF & ESTIM & AATE: & & & & & & & & & KD BY: & & & \\
\hline UNIT/A & REA WTF $-F F P-W T F$ & & & & $O R \mathrm{M} / \mathrm{H}$ & HAS & & & SU $3 c$ & ONTR & RACT & & & BOR & & & TOTAL & \\
\hline DESCRI & PTION & QUANTITY & |unit & & ER UNIT & & MATERI & IAL & 80 & ש & NAC I & & & 800 & & & DOLLAR & \\
\hline CAPACI & & & & MATL & $M / H$ & LAB \$ & & & M/HRS & & OLLAR & & M/MAS & & LLARS & & & \\
\hline $\begin{array}{l}A C C N T \\
\text { YraO } \\
\end{array}$ & $5<-2+7 x)<A<$ & & & & & & & & & & & & & & & & & \\
\hline & & & & & & & & & & & & & & & & & & \\
\hline 1604 & $\operatorname{LCST3}$ & & & & & & & & & & . & & & & & & & \\
\hline & & & & & & & & & & & & & & & & & & \\
\hline & NSTR (FACTORE) ForOH To8 & 1 & $\angle S$ & & & & 164 & 900 & & & & & 13610 & & & & & \\
\hline & $(586-9313 \times k 614)$ & & & & & & & & & & & & & & & & & \\
\hline & (B/ PROJ. LST.) & & & & & & & & & & & & & & & & & \\
\hline & & & & & & & & & & & & & & & & & & \\
\hline & & & & & & & & & & & & & & & & & & \\
\hline & & & & & & & & & & & & & & & & & & \\
\hline & & & & & & & & & & & & & & & & & & \\
\hline & & & & & & & & & & & & & & & & & $\therefore$ & \\
\hline & & & & & & & & & & & & & & & & & & \\
\hline & & & & & & & & & & & & & & & & & & \\
\hline & & & & & & & & & & & & & & & & & & \\
\hline & & & & & & & & & & & & & & & & & & \\
\hline & & & & & & & & & & & & & & & & & & \\
\hline & & & & & & & & & & & & & & & & & & \\
\hline & & & & & & & & & & & & & & & & & & \\
\hline & & & & & & & & & & & & & & & & & & \\
\hline & & & & & & & & & & & & & & & & & & \\
\hline & & & & & & & & & & & & & & & & & & \\
\hline & & & & & & & & & & & & & & & & & & \\
\hline & & & & & & & & & & & & & & & & & & \\
\hline & & & & & & & & & & & & & & & & & & \\
\hline & & & & & & & & & & & & & & & & & & \\
\hline & & & & & & & & & & & & & & & & & & \\
\hline & & & & & & - & & & & & & & & & & & & \\
\hline & & & & & & & & & & & & & & & & & & \\
\hline & & & & & & & & & & & & & & & & & & \\
\hline & & & & & & & & & & & & & & & & & & \\
\hline & & & & & -4 & & $\square$ & - & & - & & & & 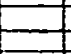 & $\underline{-}$ & & & \\
\hline & QH heVEL TL & & & & & & 164 & 200 & & & & & 13610 & & & & & \\
\hline & & & & & & 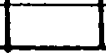 & & & & & & & & & & & & \\
\hline
\end{tabular}




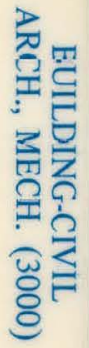





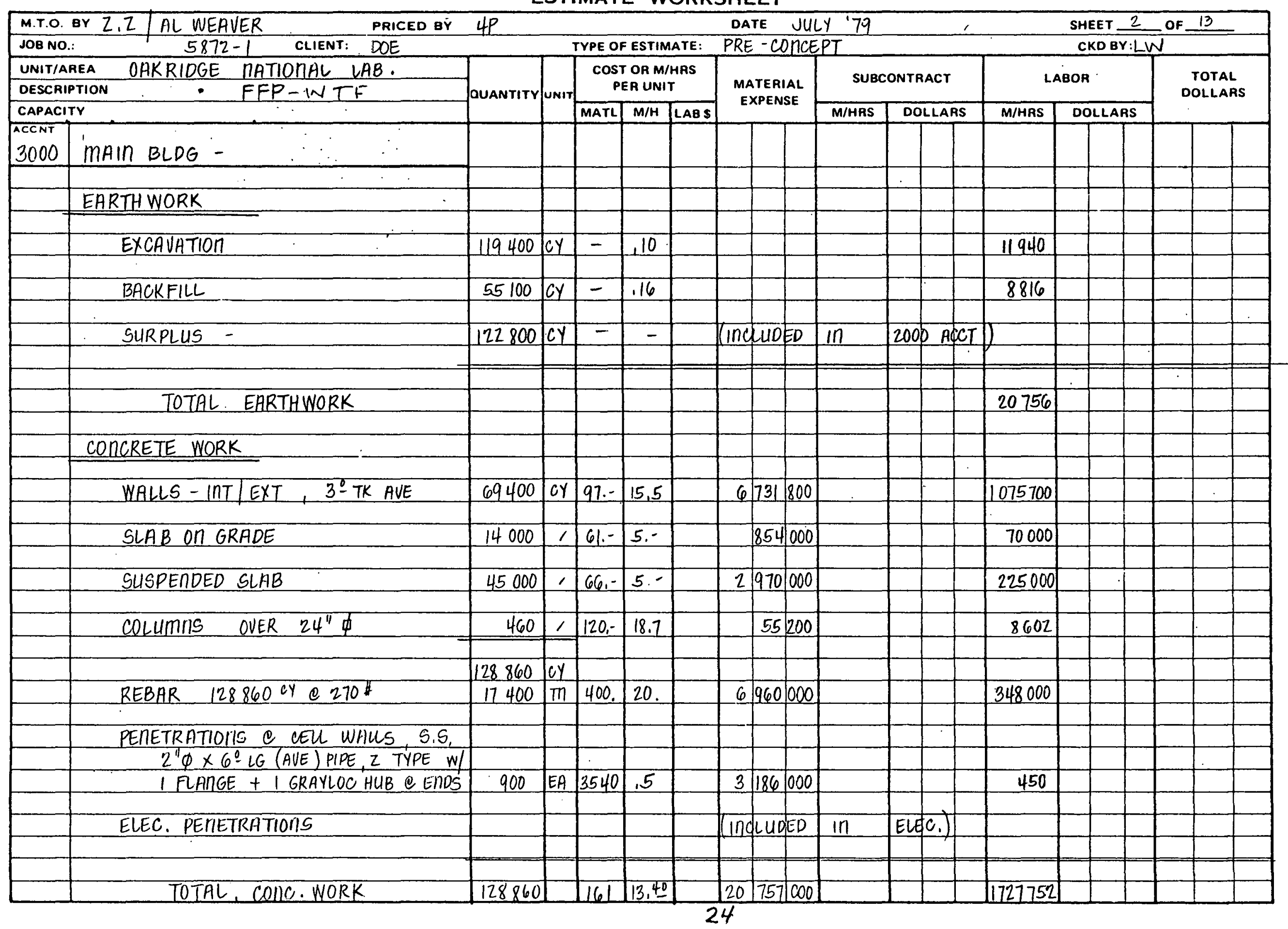




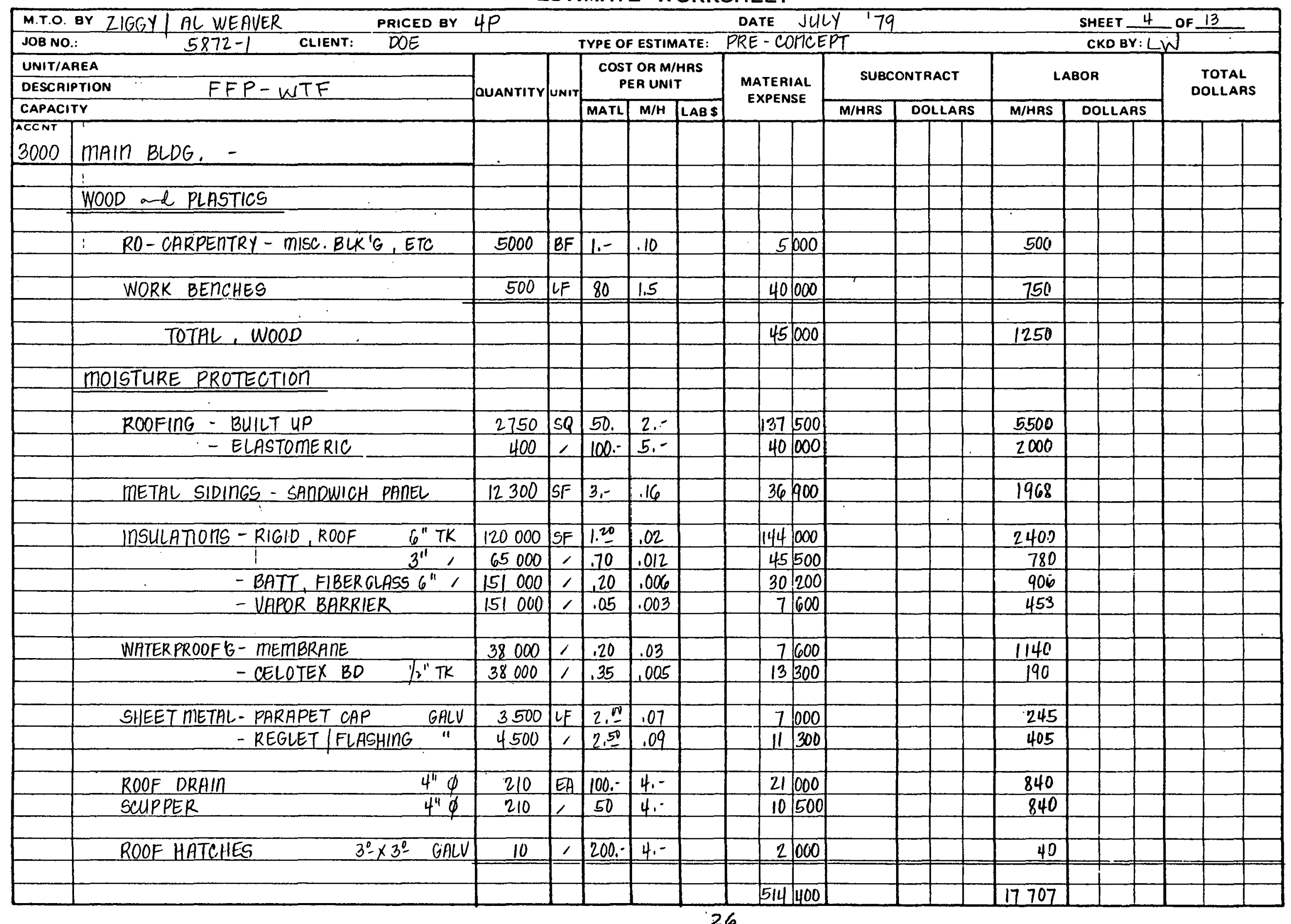


ESTIMATE WORKSHEET

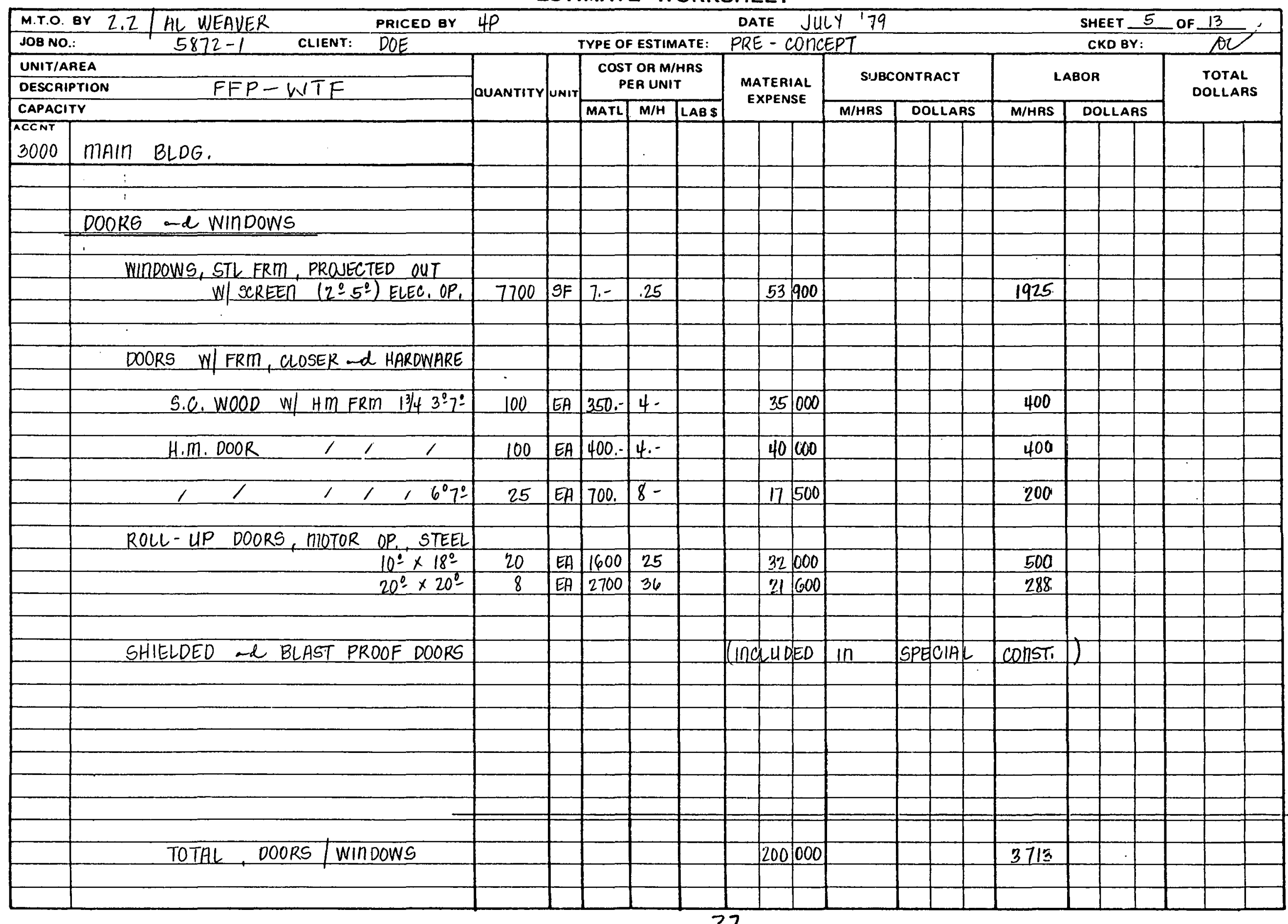


THE RALPH M. PARSONS COMPANY

ESTIMATE WORKSHEET

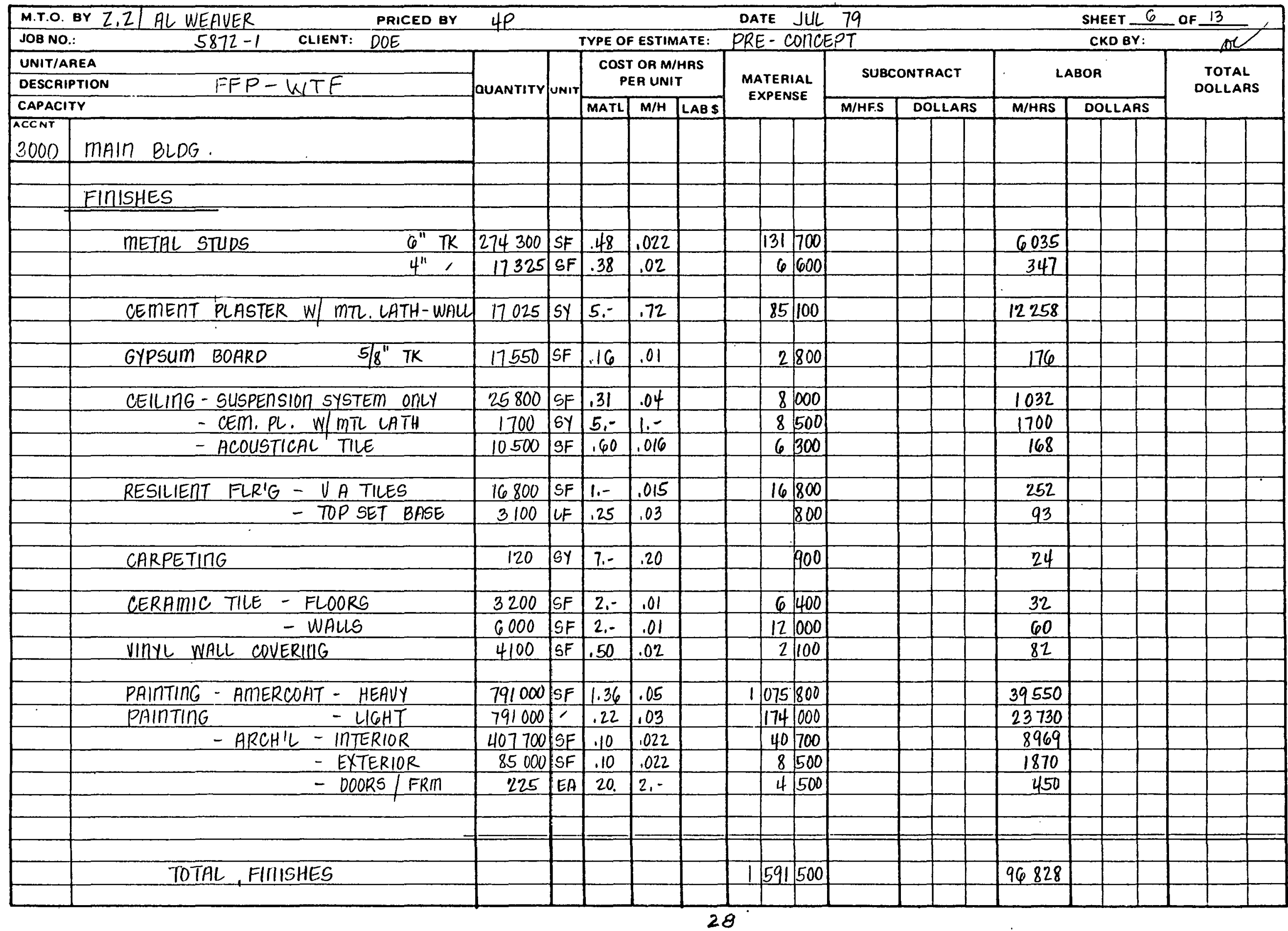


ESTIMATE WORKSHEET

\begin{tabular}{|c|c|c|c|c|c|c|c|c|c|c|c|c|c|c|c|c|}
\hline M.T.O. & BY Z.Z. AL WEAVER & $4 P$ & & & & & DATE & ل & ULY 17 & & & & ET 7 & OF & 13 & \\
\hline JOB NC & $5872-1$ CLIENT: DOE & & & TYPE OI & ESTIN & AATE: & PRE-CO & ONCE & & & & & KD BY: & & 2 & \\
\hline UNIT/A & IEA & & & $\cos 1$ & OR M/ & HRS & & & SLIBC & INTRACT & & BOR & & & & \\
\hline DESCR & $F F P-W T F$ & QUANTITY & | INIT | & & ER UNI & & MATERI & IAL & 3000 & & & & & & JOLLAR & \\
\hline CAPAC & & & & MATL & $M / H$ & LAB \$ & & & M/HRAS & DOLLAR & M/HRS & & LLARS & & & \\
\hline $\begin{array}{l}\text { ACCNT } \\
3000\end{array}$ & MAIn BLDG. - & & & & & & & & & & & & & & & \\
\hline & & & & & & & & & & & & & & & & \\
\hline & & & & & & & & & & & & & & & & \\
\hline & BLDG. SPECIALTIES & & & & & & & & & & & & & & & \\
\hline & & & & & & & & & & & & & & & & \\
\hline & LOCKERS, SIIIGLE TIER $18^{\prime \prime} \times 21^{\prime \prime} \times 72^{\prime \prime} \mathrm{H}$ & 250 & $E A$ & 70.- & .7 & & 17. & 500 & & & 175 & & & & & \\
\hline & & & & & & & & & & & & & & & & \\
\hline & TOILET PARTITIOMS, BAKED EMAMEL & & & & & & & & & & & & & & & \\
\hline & CEILING HUNG METAL & 130 & 1 & $350 .-$ & 3.5 & & 45 & 500 & & & 455 & & & & & \\
\hline & & & & & & & & & & & & & & & & \\
\hline & URIMAL SCREEM - DITTO & 50 & 1 & 120. & 1.6 & & 6 & 000 & & & 80 & & & & & \\
\hline & & & & & & & & & & & & & & & & \\
\hline & TOILET ACCESSORIES & & & & & & & & & & & & & & & \\
\hline & - MIRRORG & 100 & - & 30. & .40 & & 3 & 600 & & & 40 & & & & & \\
\hline & - SOAP DISPENSER & 130 & 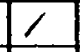 & 25 & .40 & & 3 & 300 & & & 52 & & & & & \\
\hline & - TOILET PAPER HOLDER & 130 & 1 & 10 & .27 & & 1 & 300 & & & 35 & & & & & \\
\hline & - SEAT.COVER DISPETSER & 130 & 1 & 30 & .50 & & 3 & 900 & & & 65 & & & & & \\
\hline & - PAPER TOWEL " and & & & & & & & & & & & & & & & \\
\hline & DISPOSAL & 50 & 1 & 200 & .80 & & 10 & 000 & & & 40 & & & & & \\
\hline & & & & & & & & & & & & & & & & \\
\hline & & & & & & & & & & & & & & & & \\
\hline & & & & & & & & & & & & & & & & \\
\hline & & & & & & & & & & & & & & & & \\
\hline & & & & & & & 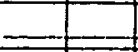 & $\underline{E}$ & & $\underline{\underline{z}}$ & $=$ & $\underline{z}$ & $=$ & & $=$ & \\
\hline & & & & & & & & & & & & & & & & \\
\hline & BLDG. SPECIALTIES & & & & & & 90 & 500 & & & 942 & & & & & \\
\hline & & & & & & & & & & & & & & & & \\
\hline & & & & & & & & & & & & & & & & \\
\hline & & & & & & & & & & & & & & & & \\
\hline & & & & & & & & & & & & & & & & \\
\hline & & & & & & & & & & & & & & & & \\
\hline & & & & & & & & & & & & & & & & \\
\hline & & & & & & & & & & & & & & & & \\
\hline & & & & & & & & & & & & & & & & \\
\hline
\end{tabular}


ESTIMATE WORKSHEET

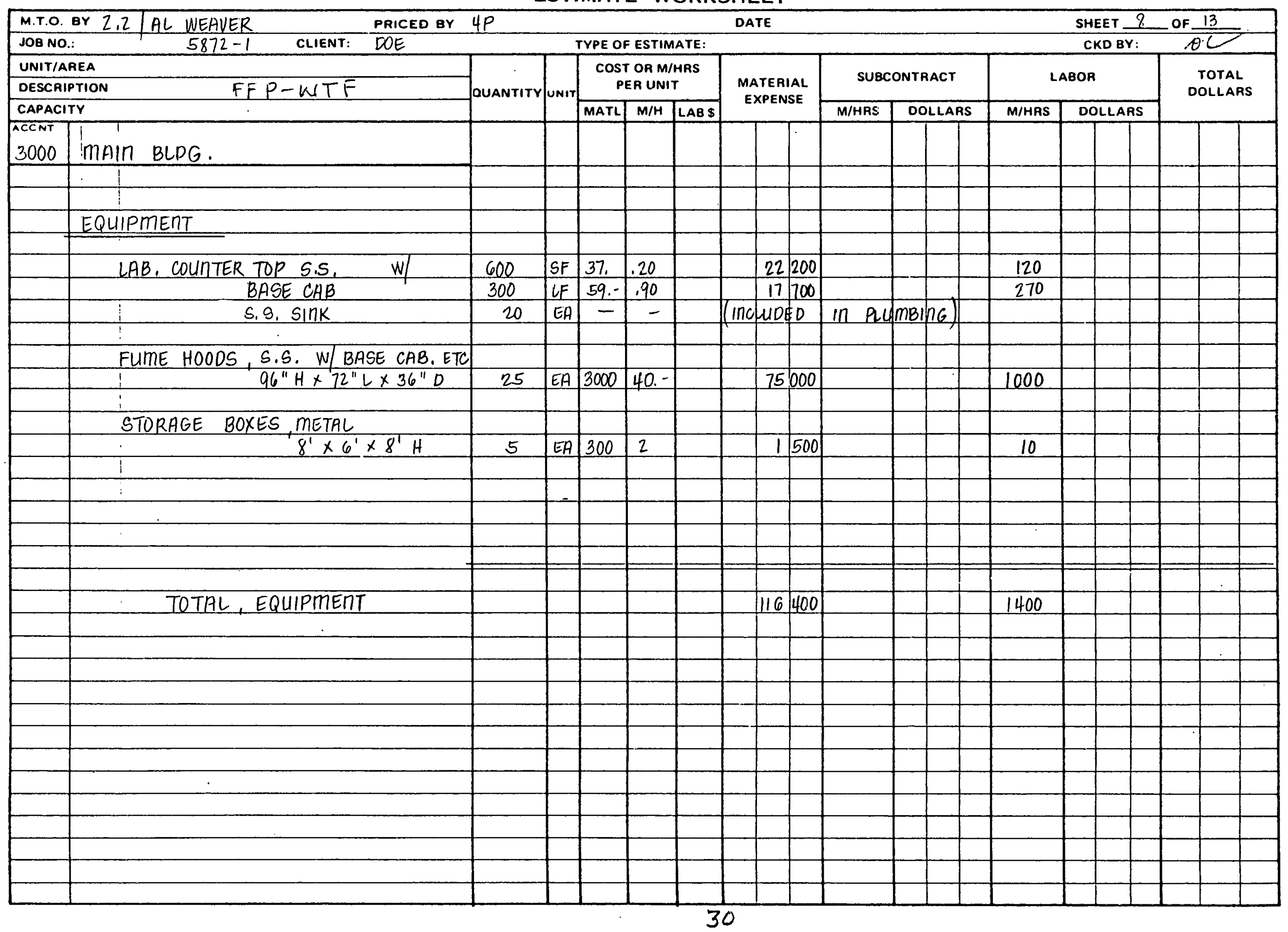


M.T.O. BY DOUGHEPTY

JOB NO.: $5872-1$ CLIENT: POE

UNITIAREA OAKRIDGE MATIOMAL $\angle A B$

OESCRIPTION

CAPACITY

ACCNT

3000

MAIN BUILDING

SPECIAL COIIST (CONT'D)

TRAINSFER CARTS

SHIELDED DOORS, VERTICAUY

TELESCOPING

EQUIPMENT LOHD OUT DOORS

AIR LOCK SEALIIIG DOORS

LIMER PLATE, 14 al $\| G A$, S.S, InOLLIDIIG STUDS

\begin{tabular}{l} 
DOUGHERTY \\
SUANTITV UNIT \\
\hline
\end{tabular}

DATE JULY 79

PRE - CONCEP T

\begin{tabular}{|c|c|c|c|c|c|}
\hline \multirow{2}{*}{\multicolumn{3}{|c|}{ DATE JULY 79}} & \multicolumn{3}{|c|}{ SHEET !) OF 13} \\
\hline & & & & CKD BY: & th \\
\hline \multirow{2}{*}{$\begin{array}{l}\text { MATERIAL } \\
\text { EXPENSE }\end{array}$} & \multicolumn{2}{|c|}{ SUBCONTAACT } & \multicolumn{2}{|c|}{ LABOR } & \multirow{2}{*}{$\begin{array}{l}\text { TOTAL } \\
\text { DOLLARS }\end{array}$} \\
\hline & $M / H R S$ & OOLLARS & M/HRS & DOLLARS & \\
\hline
\end{tabular}

A
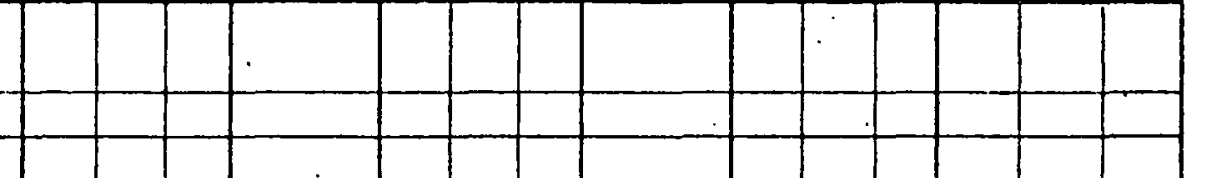

TOTAL, SPECIAL CONST. 


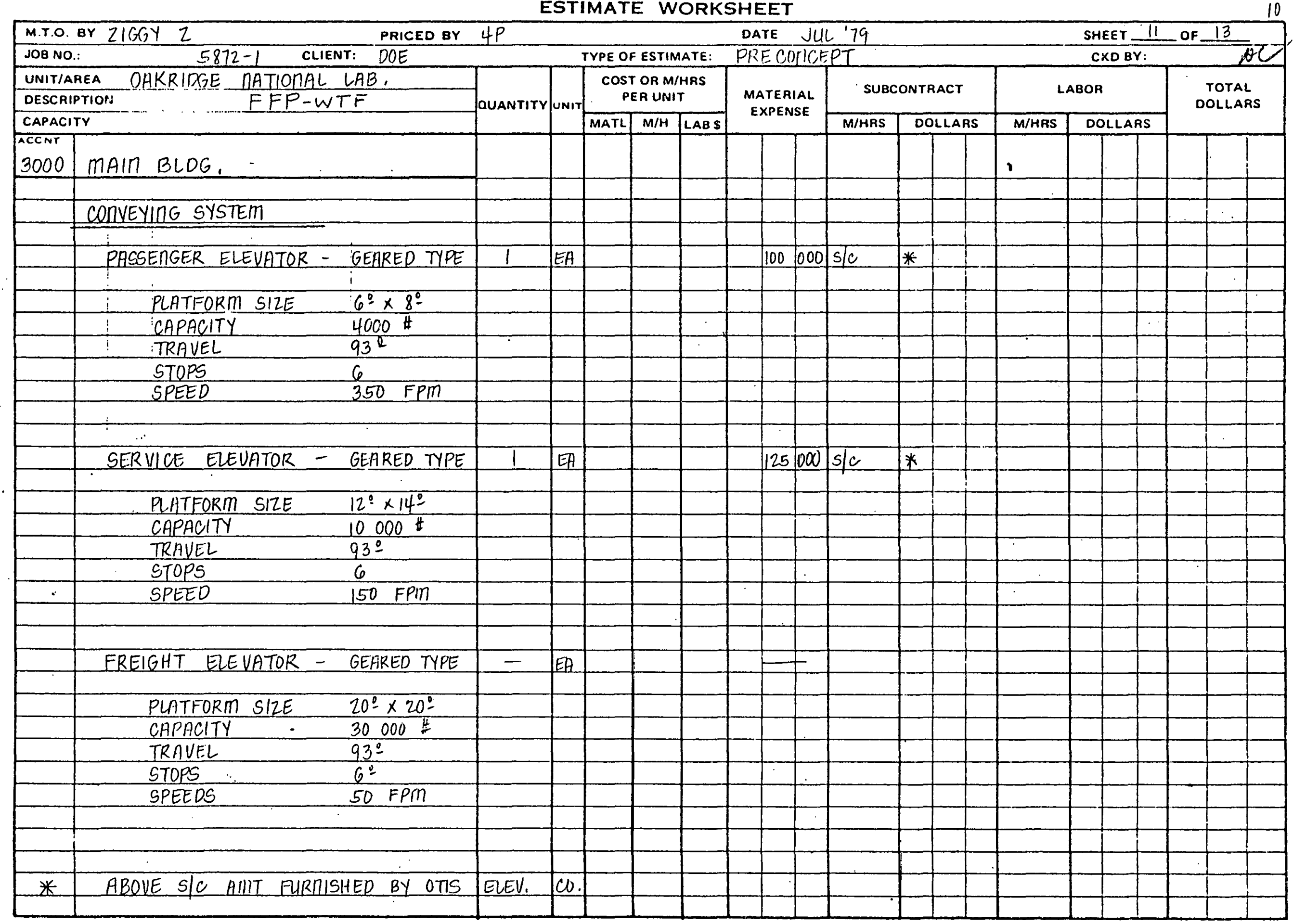




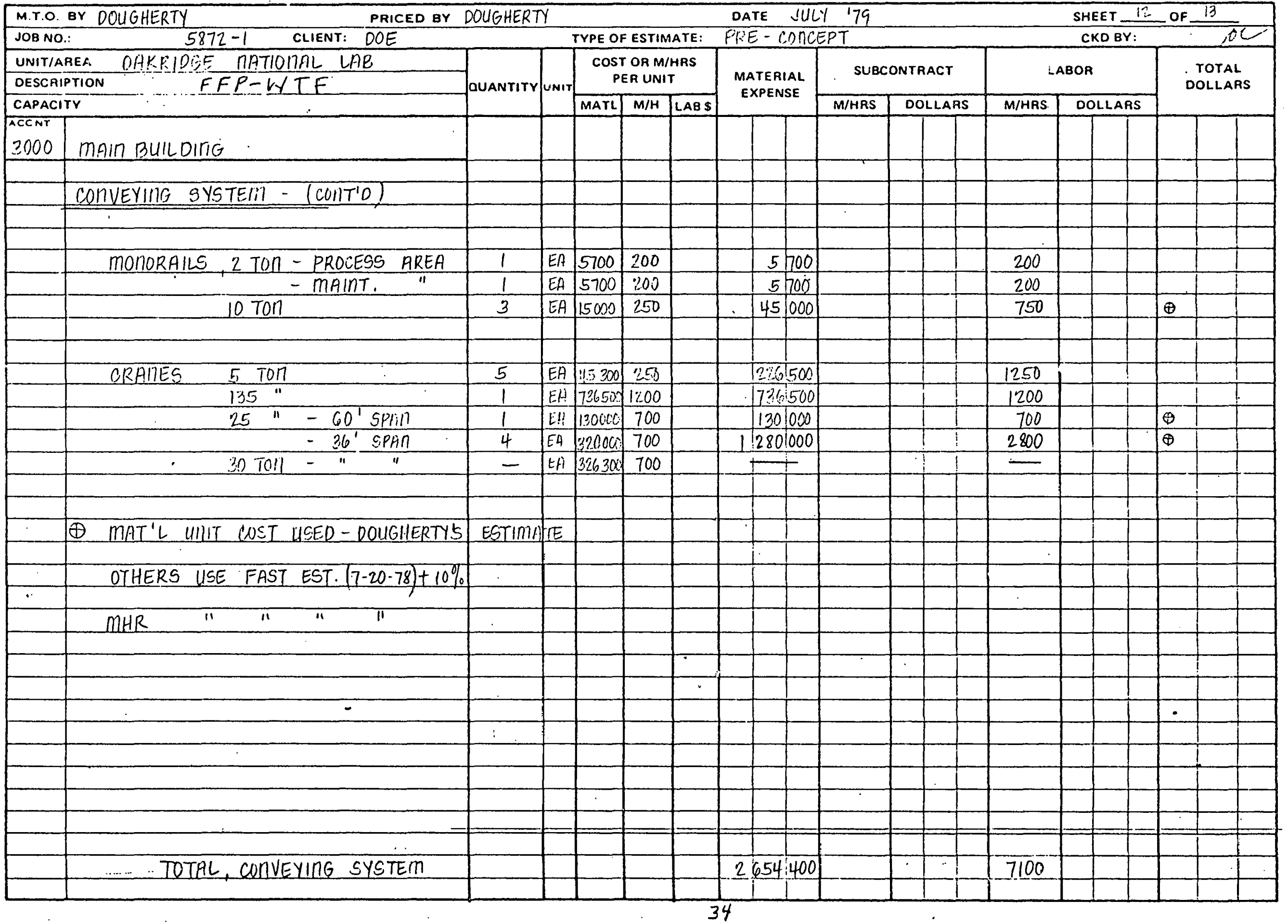


ESTIMATE WORKSHEET

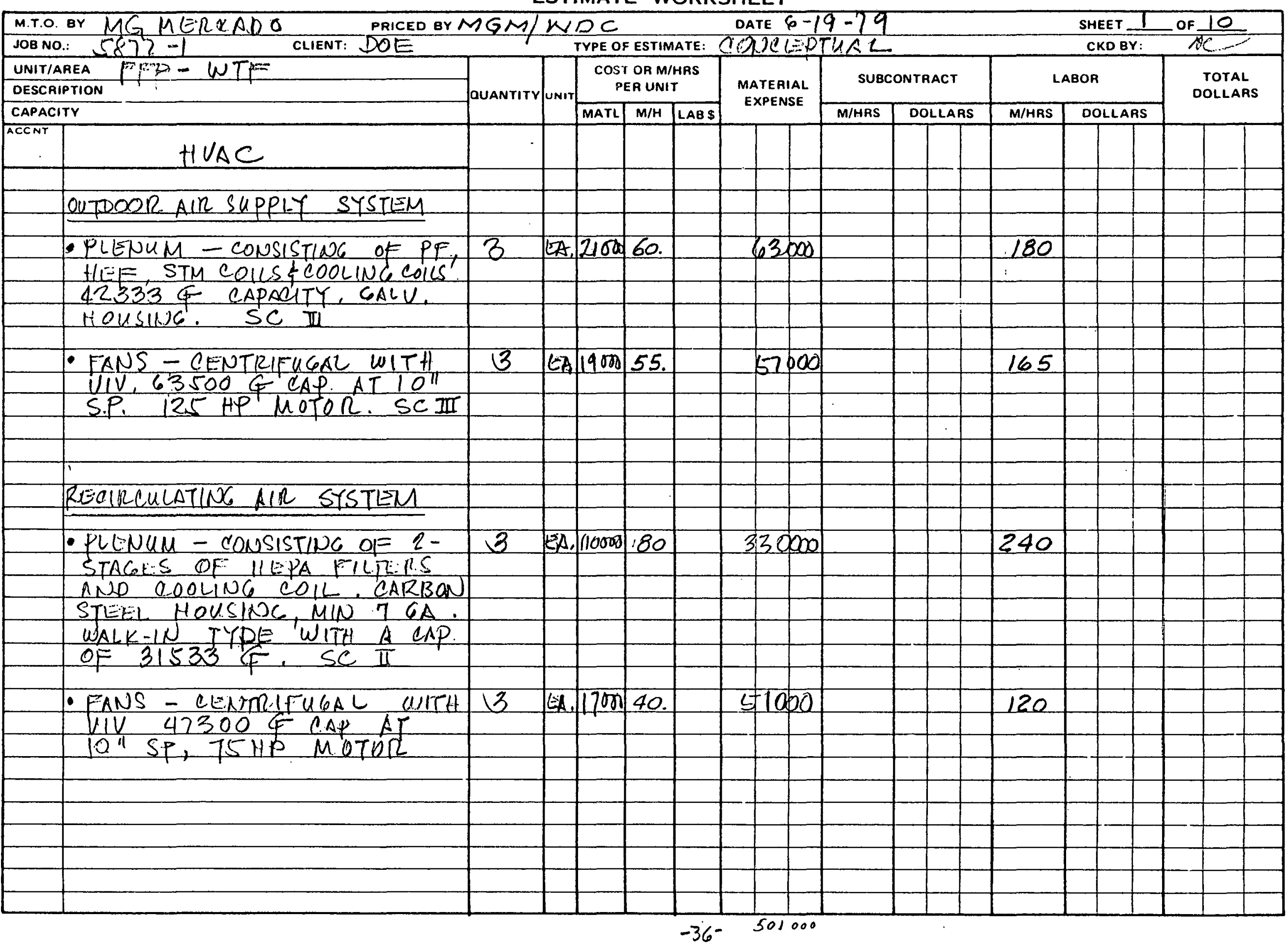


ESTIMATE WORKSHEET

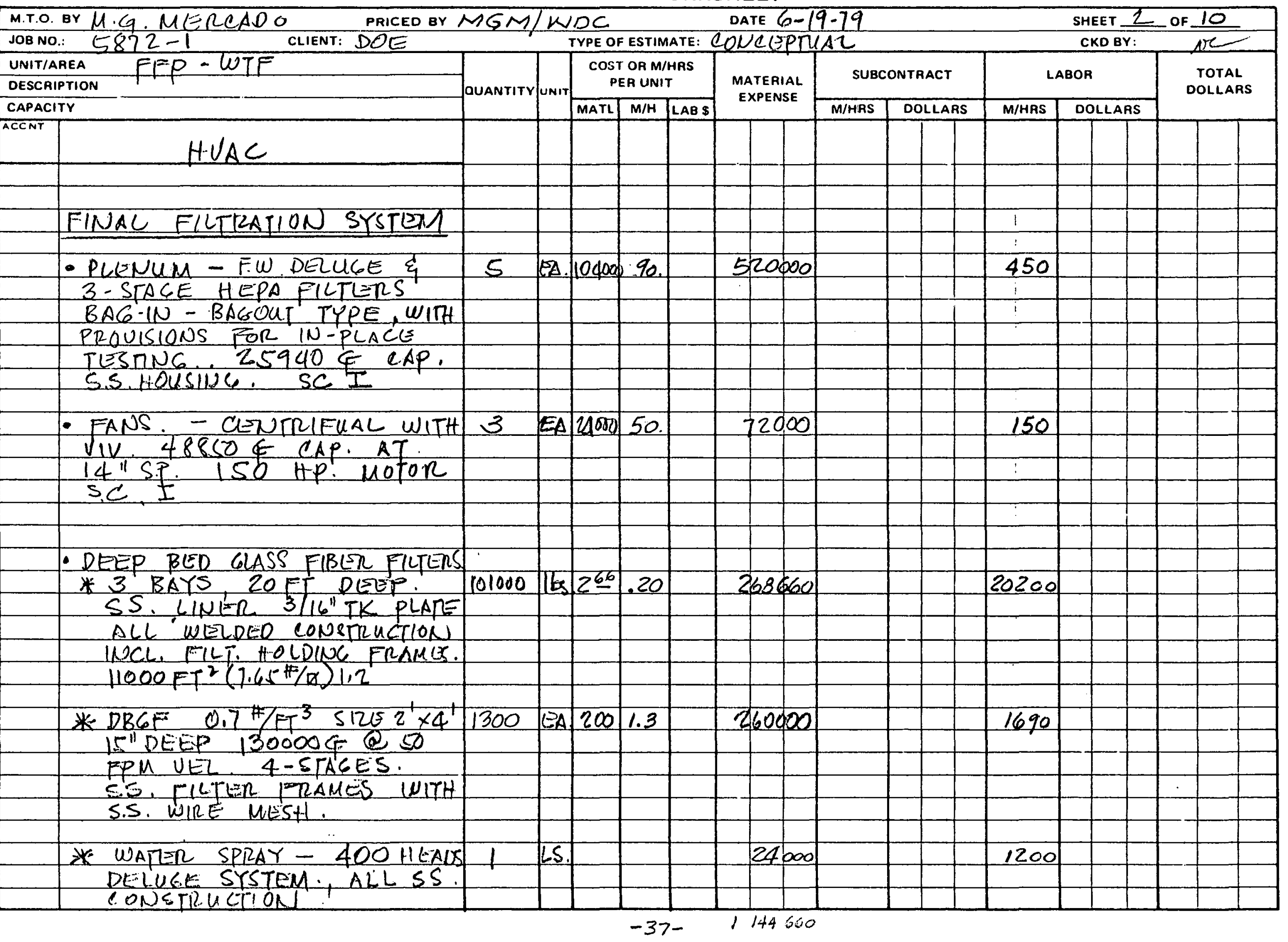


ESTIMATE WORKSHEET

\begin{tabular}{|c|c|c|c|c|c|c|c|c|c|c|c|c|c|}
\hline M.T.O & Br M.G. U ENCADO & MGMI & u & $10 C$ & & & DATE $6-10$ & -79 & & & SHEET D & & 10 \\
\hline JOB N & $\therefore 5872-1 \quad$ CLIENT: DOE & & & TYPE OF & ESTIM & IATE: & COLIQLP & TULL & & & CKD BY: & & 2 \\
\hline UNIT/I & AEA TP - WTF & & & & $O R M /$ & & & SUBC & NTRACT & & BOR & & \\
\hline DESCR & Prion & GUANTITY & UNIT & & ER UNIT & & $\begin{array}{l}\text { MATERIAL } \\
\text { EXPENSE }\end{array}$ & & & & & & OLLARS \\
\hline CAPAC & & & & MATL & $M / H$ & LAB S & & M/HAS & DOLLAR & M/HAS & DOLLARS & & \\
\hline ACCNT & $H \cup A C$. & & & & & & & & & & & & \\
\hline & & & & & & & & & & & & & \\
\hline & & & & & & & & & & & & & \\
\hline & IN-CELL PRE-FILTERATION) - & 62 & las. & 300 & 2. & & 18600 & & & 124 & & & \\
\hline & S.S, WIRE CRIO OR MUSH, & & & & & & & & & & & & \\
\hline & WEDGE TYPE FILTLRS FOR & & & & & & & & & & & & \\
\hline & REMOTE SERUKE BI MANI & & & & & & & & & & & & \\
\hline & pulator filter dap. \& zo00G & & & & & & & & & & & & \\
\hline & $(51800+72000=1238004)$ & & & & & & & & & & & & \\
\hline & & & & & & & & & & & & & \\
\hline & & & & & & & & & & & & & \\
\hline & COUTROL DAMPERS WITH AIR & 40 & exs. & 3500 & 30. & & 400,00 & & & 1200 & & & \\
\hline & OPERATORS AUERAGE OF & & & & & & & & & & & & \\
\hline & $30 \pi$ & & & & & & & - & & & & & \\
\hline & & & & & & & & & & & & & \\
\hline & & & & & & & & & & & & & \\
\hline & HUAC CONTROLS - & $\perp$ & LS. & - & - & & 150000 & $j$ & & 2000 & & & \\
\hline & PNEUMATC \& OLLCTRL CONIRA & & & & & & & & & & & & \\
\hline & I0) $\quad N C L U D E$ & & & & & & & & & & & & \\
\hline & - FlOW MONITORS & & & & & & & & & & & & \\
\hline & - PRESS 0.0NTROLlens & & & & & & & & & & & & \\
\hline & - Temp controlcens & & & & & & & & & & & & \\
\hline & - PNEUUMATIC TUBING. $\left(C_{a}\right)$ & & & & & & & & & & & & \\
\hline & - TLSTING & & & & & & & & & & & & \\
\hline & & & & & & & & & & & & & \\
\hline & & & & & & & & & & $i$ & & & \\
\hline & WATIR COOOLNO COLS - & 10 & EA. & 1800 & 20. & & 18000 & & & 200 & & & : \\
\hline & 6 ROWS EACH AppNOX. & & & & & & & & & 1 & & & \\
\hline & $24 F T^{2}$ & & & & & & & & & & & & \\
\hline & & & & & & & & & & & & & \\
\hline & STM. RUHEAT COILS - & & & & & & & & & & & & \\
\hline & 4 ROUS WACH APPROX & 15 & EA. & 500 & 15. & & 226500 & & & 225 & & & \\
\hline & $20 \mathrm{FT}$ ? & & & & & & & & & & & & \\
\hline & & & & & & & & & & & & & \\
\hline
\end{tabular}


ESTIMATE WORKSHEET

\begin{tabular}{|c|c|c|c|c|c|c|c|c|c|c|c|c|c|}
\hline M.T.O. & BY M.G.MENCAOU & MGA1 & $W C$ & Qc & & & DATE $6-19$ & -79 & & & SHEET 4 & of 10 & \\
\hline JOB NO. & $: 5872-1 \quad$ CLIENT: DOE & & & TYPE OF & FESTIM & IATE: & andecpl & UAL & & & CKD BY: & DC & \\
\hline UNIT/A & REA $F F P-W_{T E}$ & & & $\cos 1$ & TOR M/r & HRS & & SUBC & ONTRACT & & BOR & & \\
\hline DESCRI & PTION & DUANTITY & | & & ER UNIT & & $\begin{array}{l}\text { MATERIAL } \\
\text { EXPENSE }\end{array}$ & & & & & DOLLARS & \\
\hline CAPACI & & & & MATL & $\mathrm{M} / \mathrm{H}$ & LABS & & M/HRS & DOLLARS & M/HAS & DOLLARS & & \\
\hline ACCNT & $H \cup A C$ & & & & & & & & & & & & \\
\hline & & & & & & & & & & & & & \\
\hline & & & & & & & & & & & & & \\
\hline & DUCTURRK & & & & & & & & & & & & \\
\hline & - GALV STEEL SHEETMETAL & 192000 & 16s. & .97 & .07 & & 186240 & & & 13440 & & & \\
\hline & $160000 \& Q 1 \# / G(1,2)$ & & & & & & & & & & & & \\
\hline & & & & & & & & & & & & & \\
\hline & - S.S. DUCTWORK ALL WELDED & 80073 & |bs. & 3,20 & .10 & & 256000 & & & 8000 & & & \\
\hline & consTrucTION $1660.41 N$. & & & & & & & & & & & & \\
\hline & $130,000 q 0151 / \%(1,2)(1 / 3)$ & & & & & & & & & & & & \\
\hline & & & & & & & & & & & & & \\
\hline & QPUANTITY ABOVE HOLUDES & & & & & & & & & & & & \\
\hline & BOD'S VOL. DAMPLES, \& SCILAP & & & & & & & & & & & & \\
\hline & t & & & & & & & & & & & & \\
\hline & & & & & & & & & & & & & \\
\hline & - DUCT iNSULAT7ON - R.1610 & 40000 & $S E$ & 1.33 & $.0 \times 3$ & & B3200 & & & 2520 & & & \\
\hline & TYPE MIN, 1T/2 THKK & & & & & & & & & & & & \\
\hline & PROVIDE IN SUPPLY ONLY & & & & & & & & & & & & \\
\hline & & & & & & & & & & & & & \\
\hline & & & & & & & & & & & & & \\
\hline & & & & & & & & & & & & & \\
\hline & EILTERS - & & & & & & & & & & & & \\
\hline & & & & & & & & & & & & & \\
\hline & - ZONE RETURN FLTERS & 90 & EN. & 100 & .50 & & 000 & & & 45 & & & \\
\hline & G"TK MED EFFRIUNCY & & & & & & & & & & & & \\
\hline & 17000 FAPACIT GACH & & & & & & & & & & & & \\
\hline & & & & & & & & & & & & & \\
\hline & & & & & & & & & & & & & \\
\hline & - INITIAL TEST AHA REPLACE-T & & & & & & & & & & & & \\
\hline & MLNT F16 TLnS. & & & & & & & & & & & & \\
\hline & $P F$ & 165 & EA. & 50 & .50 & & 8250 & & & & & & \\
\hline & $F(E)$ & 75 & $E A$ & 150 & .50 & & 112.10 & & & 408 & & & \\
\hline & $H E P A$ & 250 & EA. & 2.00 & .50 & & 50000 & & & & & & \\
\hline & $D B G=$ & 325 & $k: \Delta$, & 200 & .50 & & 67000 & & & & & & \\
\hline
\end{tabular}


ESTIMATE WORKSHEET

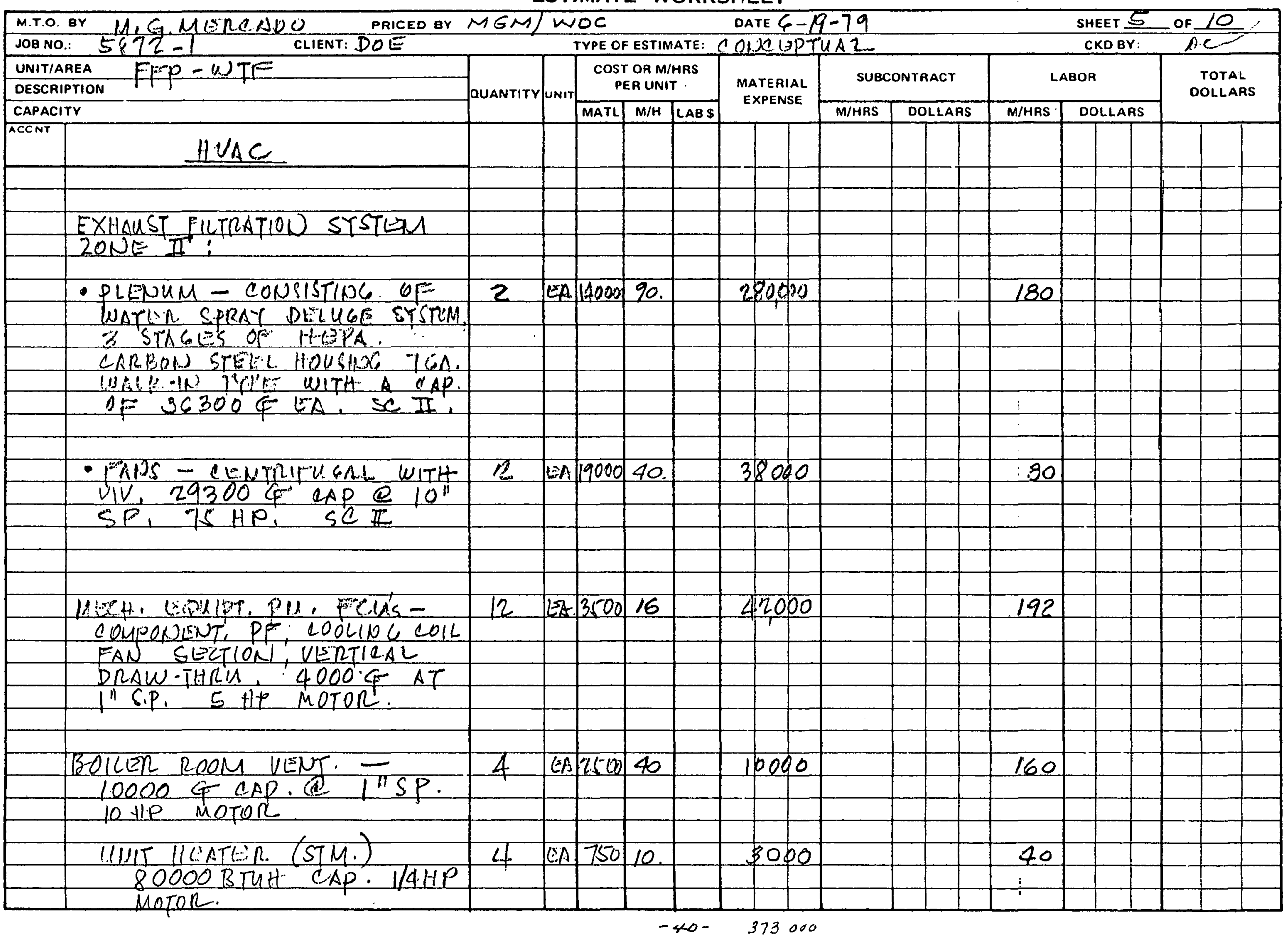


ESTIMATE WORKSHEET

\begin{tabular}{|c|c|c|c|c|c|c|c|c|c|c|c|c|c|c|c|}
\hline M.T.O. & Br $M, G \cdot M E R C A D O$ & MGMI & Ko & $\overline{D C}$ & & & DATE $6-1 /$ & -79 & & & & SHEET & 6 & of 10 & \\
\hline JOB NO & $\therefore \quad 5872-1$ CLIENT: DOE & & & TYPE OF & FESTIM & MATE: & CONCEP & UnL & & & & CKD BY & & $A C$ & \\
\hline UNIT/A & REA FEP-UTF & & & & TOR MI & & & SUBC & NTPACT & & & BOR & & & \\
\hline DESCAI & Prion & QUANTITY & |UNIT| & & & & $\begin{array}{l}\text { MATERIAL } \\
\text { EXPENSE }\end{array}$ & & TVIHACT & & & & & DOLLARS & \\
\hline CAPACI & & & & MATL & $M / H$ & LAB \$ & & M/HRS & DOLLAR & & M/HRS & DOLLAR & & & \\
\hline$\overline{A C C N T}$ & HVAC. & & & & & & & & & & & & & & \\
\hline & & & & & & & & & & & & & & & \\
\hline & & & & & & & & & & & & & & & \\
\hline & BAT. RM UPS LXNA, FAN - & & EA, & 200 & 12 & & 2.000 & & & & 12 & & & & \\
\hline & & & $-\infty$ & $\ldots$ & & & & & & & & & & & \\
\hline & $4000 \mathrm{G} 1.51 \mathrm{s.P}$. & & & & & & & & & & & & & & \\
\hline & $3 \mathrm{HP}$ MOTOR & & - & & & & & & & & & & & & \\
\hline & & & & & & & & & & & & & & & \\
\hline & & & & & & & & & & & & & & & \\
\hline & & & & & & & & & & & & & & & \\
\hline & UTILITY EQUIP. ROQH & 1 & EA. & 12000 & 40. & & 12000 & & & & 40 & & & & \\
\hline & - HEATHUC \& VETNTL WNIT & & & & & & & & & & & & & & \\
\hline & WITH. PF \& STM. HEATINC & & & & & & & & & & & & & & \\
\hline & 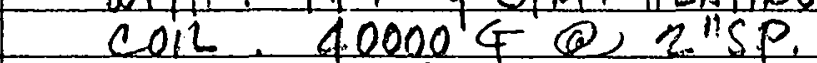 & & & & & & & & & & & & & & \\
\hline & 3-0 te woton sc-T & & & & & & & & & & & & & & \\
\hline & - & & & & & & & & & & & & & & \\
\hline & - ODA PURGE UNIT WITH & & ED & 500 & 30. & & 5000 & & & & 30 & & & & \\
\hline & PF, HEATINC coll $(20 \mathrm{k \omega})$ & & & & & & & & & & & & & & \\
\hline & 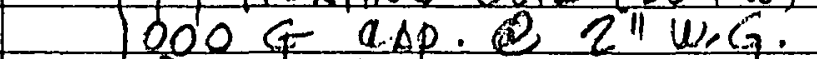 & & & & & & & & & & & & & & \\
\hline & SP. SC. & & & & & & & & & & & & & & \\
\hline & & & & & & & & & & & & & & & \\
\hline & & & & & & & & & & & & & & & \\
\hline & HLAT RISLOVERY STSTEM - & , & & & & & & & & & & & & & \\
\hline & & & & & & & & & & & & & & & \\
\hline & - PUMP \& MOTOR - 100 GPM & 1 & $\ln$ & 2500 & 15 & & 2500 & & & & 15 & & & & \\
\hline & (Q) TOFT HLAD $10 \mathrm{He}$ & & & & & & & & & & & & & & \\
\hline & & & & & & & & & & & & & & & \\
\hline & - HEAT RECOVERY cOILS & $\eta$ & LA. & 3000 & 40 . & & 2000 & & & & 80 & & & & \\
\hline & APPROX. $60 \mathrm{~B}$ & & & & & & & & & & & & & & \\
\hline & & & & & & & & & & & & & & & \\
\hline & & & & & & & & & & & & & & . & \\
\hline & STM. UNIT HEATER - & & & & & & & & & & & & & & \\
\hline & C.0000BTnH. W/LHP POTOR & 8 & $E A$. & 600 & 10 & & 1800 & & & & 80 & & & & \\
\hline & & & & & & & & & & & & & & & \\
\hline
\end{tabular}


ESTIMATE WORKSHEET

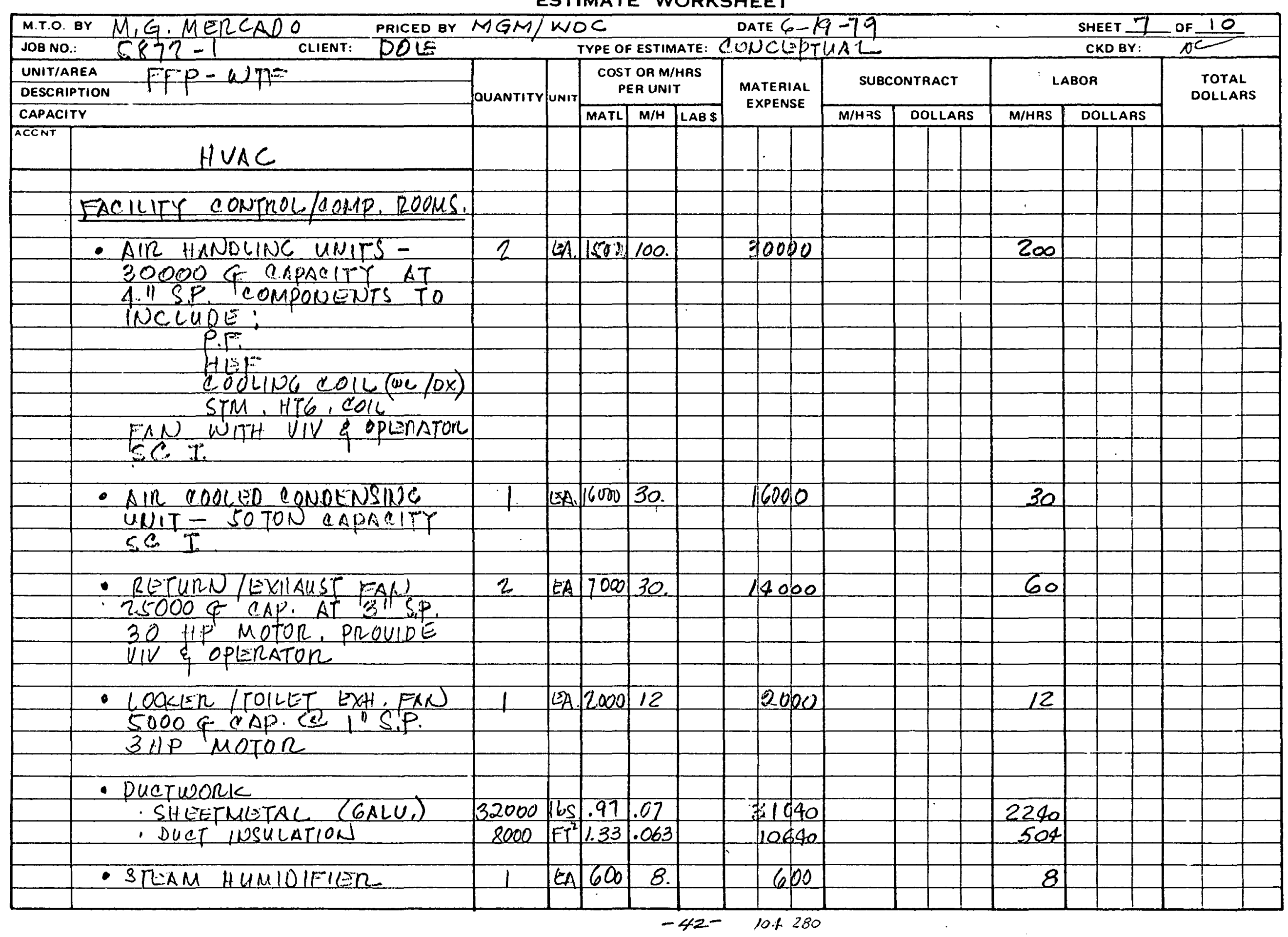


ESTIMATE WORKSHEET

\begin{tabular}{|c|c|c|c|c|c|c|c|c|c|c|c|c|c|c|c|c|}
\hline M.T.O & BY M.G.MERCADO & $M G M / 1$ & WO & & & & & ATE $6-19$ & -79 & & & SHEET_ & 8 & OF & 10 & \\
\hline $\operatorname{JOBN}$ & $\therefore 5872-1$ CLIENT: DOE & & & TYPEO & FESTIN & AATE: & COR & र्टिय & $A L$ & & & CKOBY & 4 & +20 & & \\
\hline UNIT/I & REA EFP-WTE & & & & T OR M & & & & SUBC & ONTRACT & & $\triangle B O B$ & & & & \\
\hline DESCR & PTION & GUANTITY & UNIT & & ER UNI & & & $\begin{array}{l}\text { ATERIAL } \\
\text { XPENSEE }\end{array}$ & & & & & & & DOLLAR & \\
\hline CAPAC & & & & MATL & $\mathrm{M} / \mathrm{H}$ & LAB \$ & & & M/tRS & DOLLAR & M/HAS & DOLLAA & & & & \\
\hline$\overline{A C C N T}$ & $H V_{A C}$ & & & & & & & & & & & & & & & \\
\hline & $=$ & & & & & & & & & & & & & & & \\
\hline & - electric rltieat colls & 10 & LA. & 1500 & 8. & & & 15000 & & & 80 & & & & & \\
\hline & WITH CONTACTORS SCR & & & & & & & & & & & & & & & \\
\hline & \& LOCDL CONTROL PANE? & & & & & & & & & & & & & & & \\
\hline & $10 \mathrm{k \omega}$ & & & & & & & & & & & & & & & \\
\hline & & & & & & & & & & & & & & & & \\
\hline & & & & & & & & & & & & & & & & \\
\hline & BALANCINC \& TESTINX OE & & LS & - & - & & & - & & & 400 & & & & & \\
\hline & HUAC SYSTEM FOR EFP. & & & & & & & & & & & & & & & \\
\hline & & & & & & & & & & & & & & & & \\
\hline & & & & & & & & & & & & & & & & \\
\hline & HVAC PIPING: & & & & & & & & & & & & & & & \\
\hline & CHILLED WATER \& STEAM & 1 & LS & & & & & 50000 & & & 1600 & & & & & \\
\hline & & & & & & & & & & & & & & & & \\
\hline & & & & & & & & & & & & & & & & \\
\hline & & & & & & & & & & & & & & & & \\
\hline & & & & & & & & & & & & & & & & \\
\hline & & & & & & & & & & & & & & & & \\
\hline & & & & & & & & & & & & & & & & \\
\hline & & & & & & & & & & & & & & & & \\
\hline & & & & & & & & & & & & & & & i & \\
\hline & & & & & & & & & & & & & & & & \\
\hline & . & & & & & & & & & & & & & & & \\
\hline & & & & & & & & & & & & & & & & \\
\hline & & & & & & & & & & & & & & & & \\
\hline & & & & & & & & & & & & & & & & \\
\hline & & & & & & & & . & & & & & & & & \\
\hline & & & & & & & & • & & & & & & & & \\
\hline & & & & & & & & & & & & & & & & \\
\hline & SUB TOTAL & & & & & & 3 & 253280 & & & 58600 & & & & & \\
\hline & PRoDactivity a $65 \%$ & & $\ldots$ & & & & & & & & 31554 & & & & & \\
\hline & & & & & & & & & & & & & & & & \\
\hline & TOTAL HVAC & & - & & & & 3 & 253300 & & & $90 / 54$ & & & & & \\
\hline
\end{tabular}




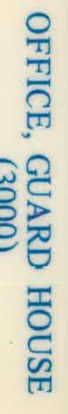



ESTIMATE WORKSHEET

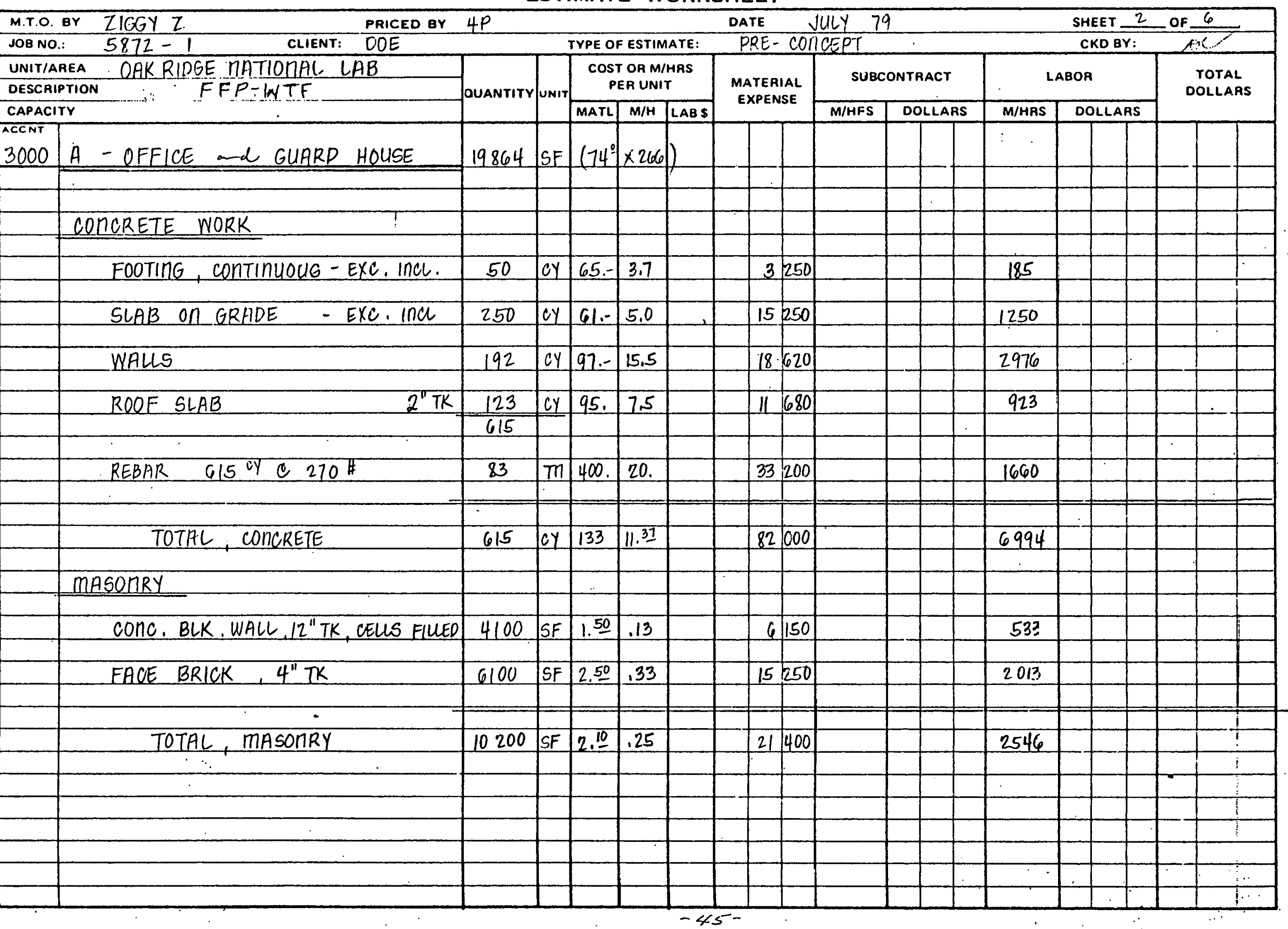


ESTIMATE WORKSHEET

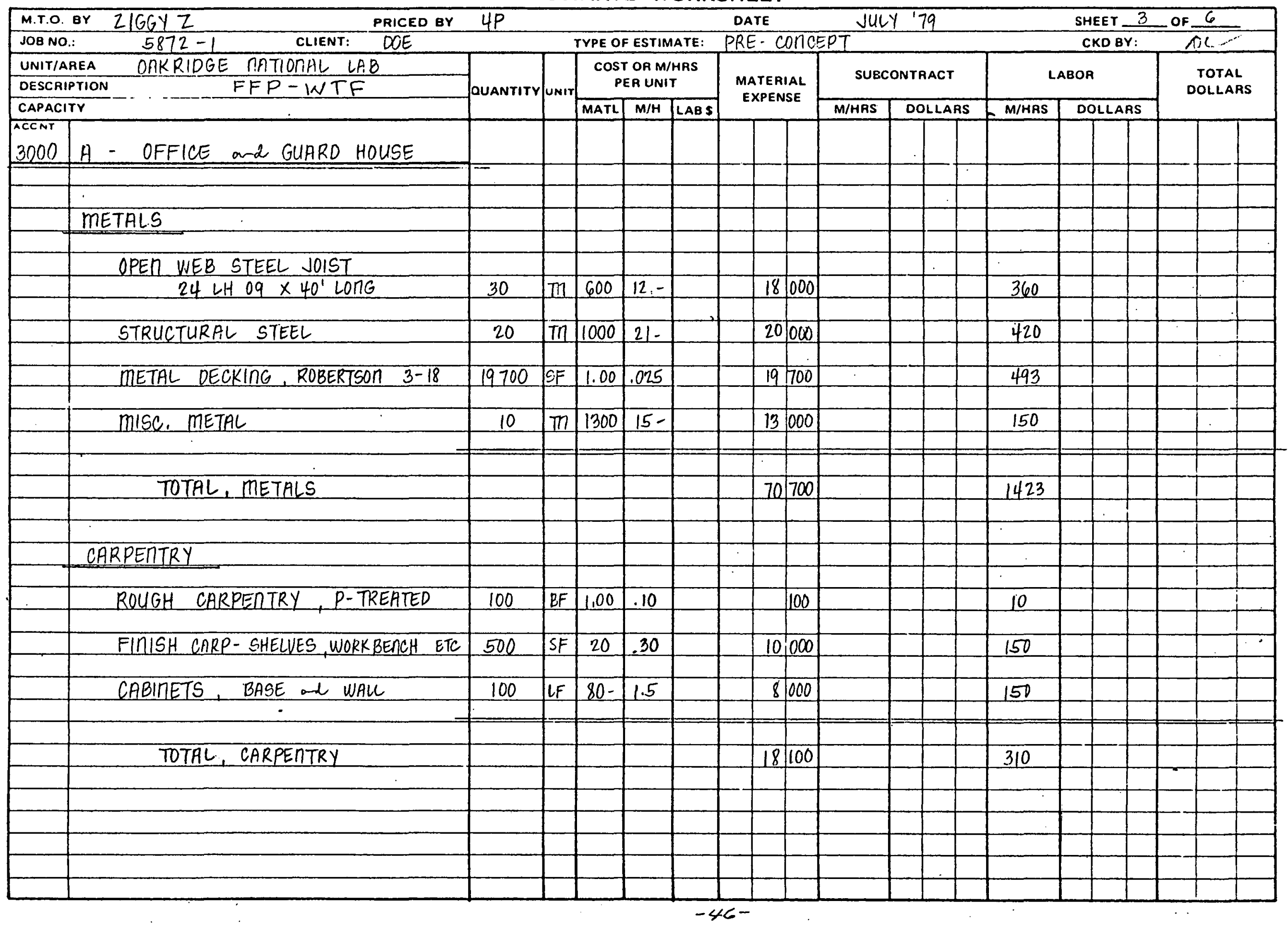


ESTIMATE WORKSHEET

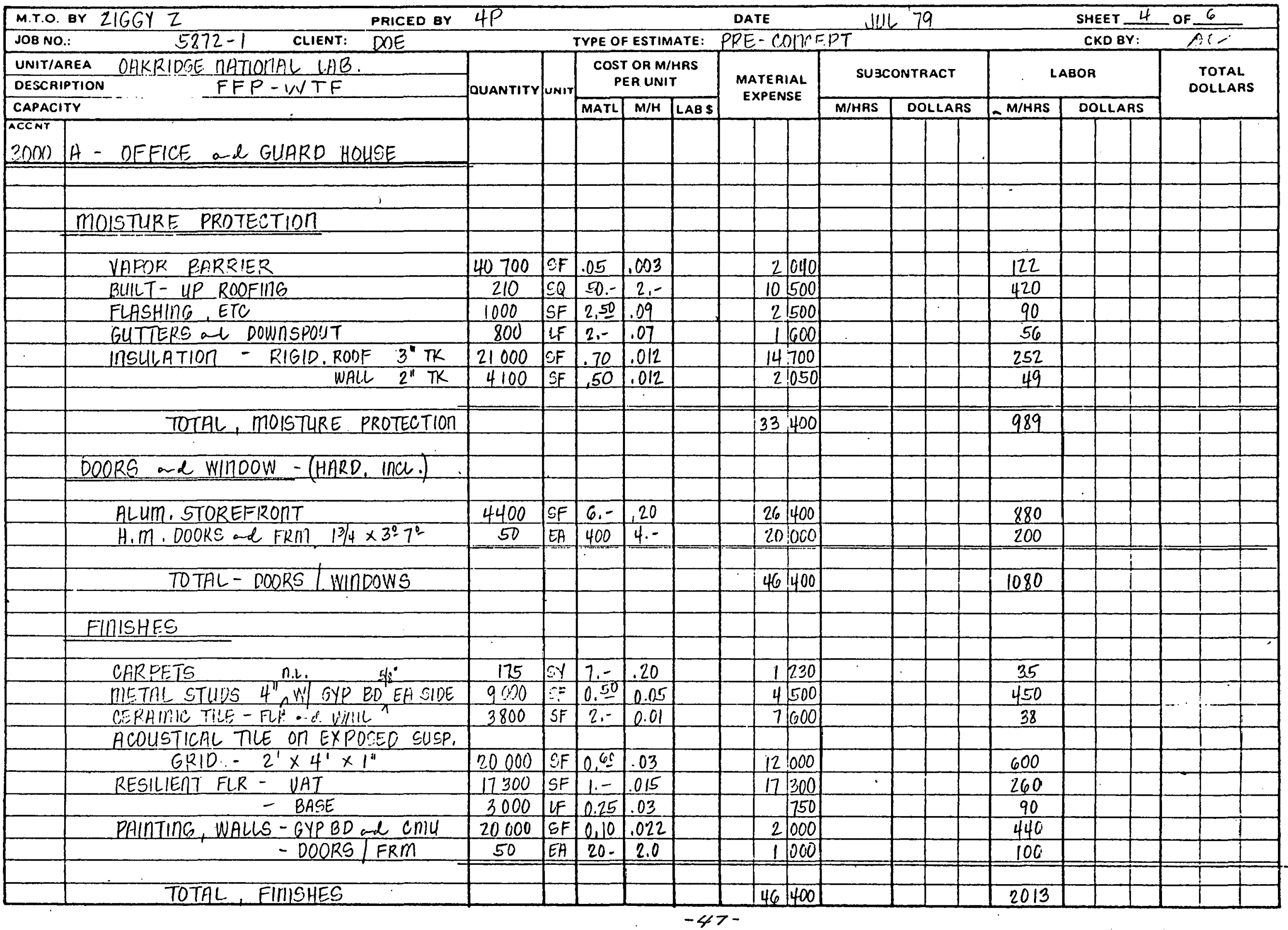


ESTIMATE WORKSHEET

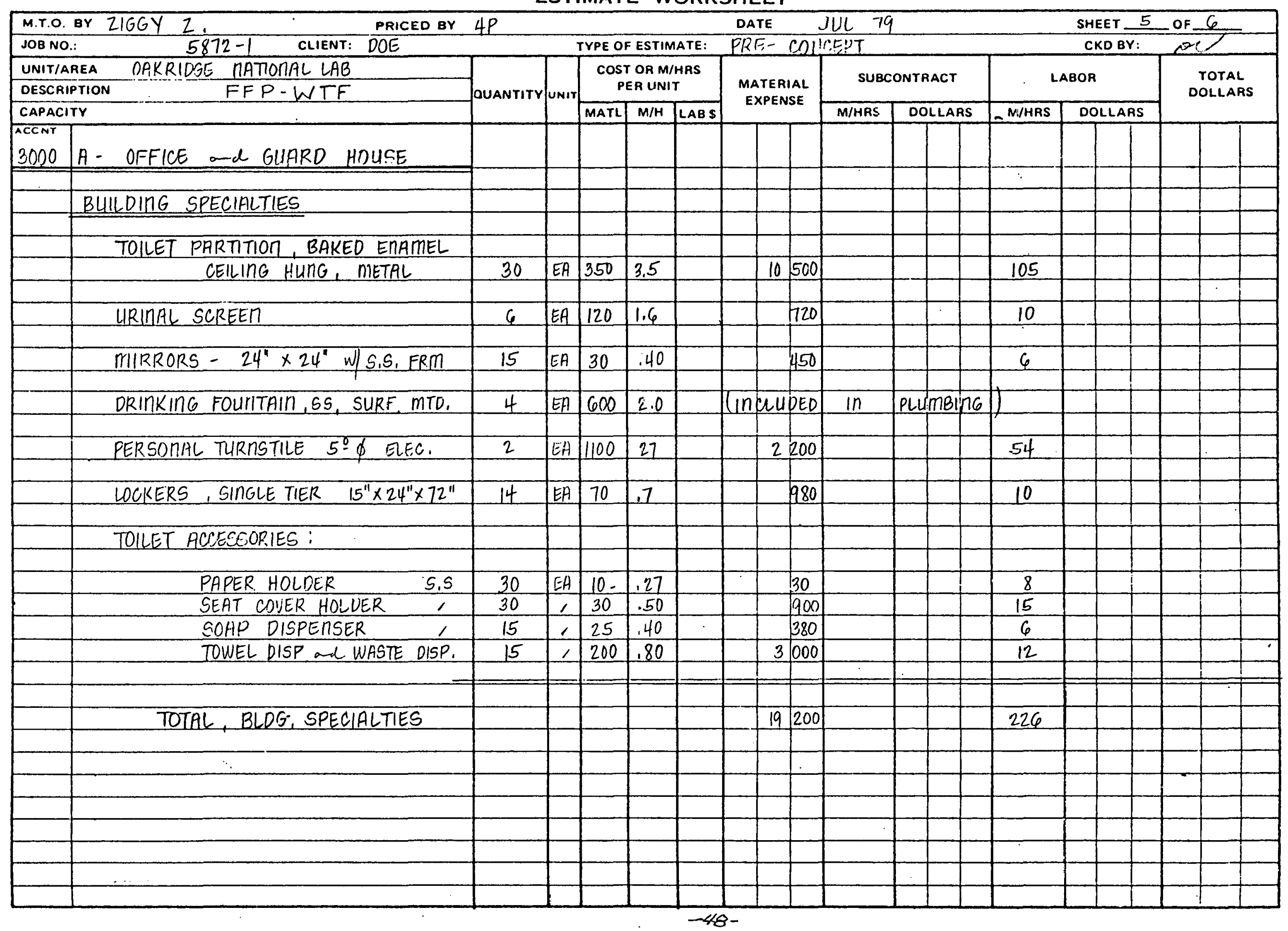


ESTIMATE WORKSHEET

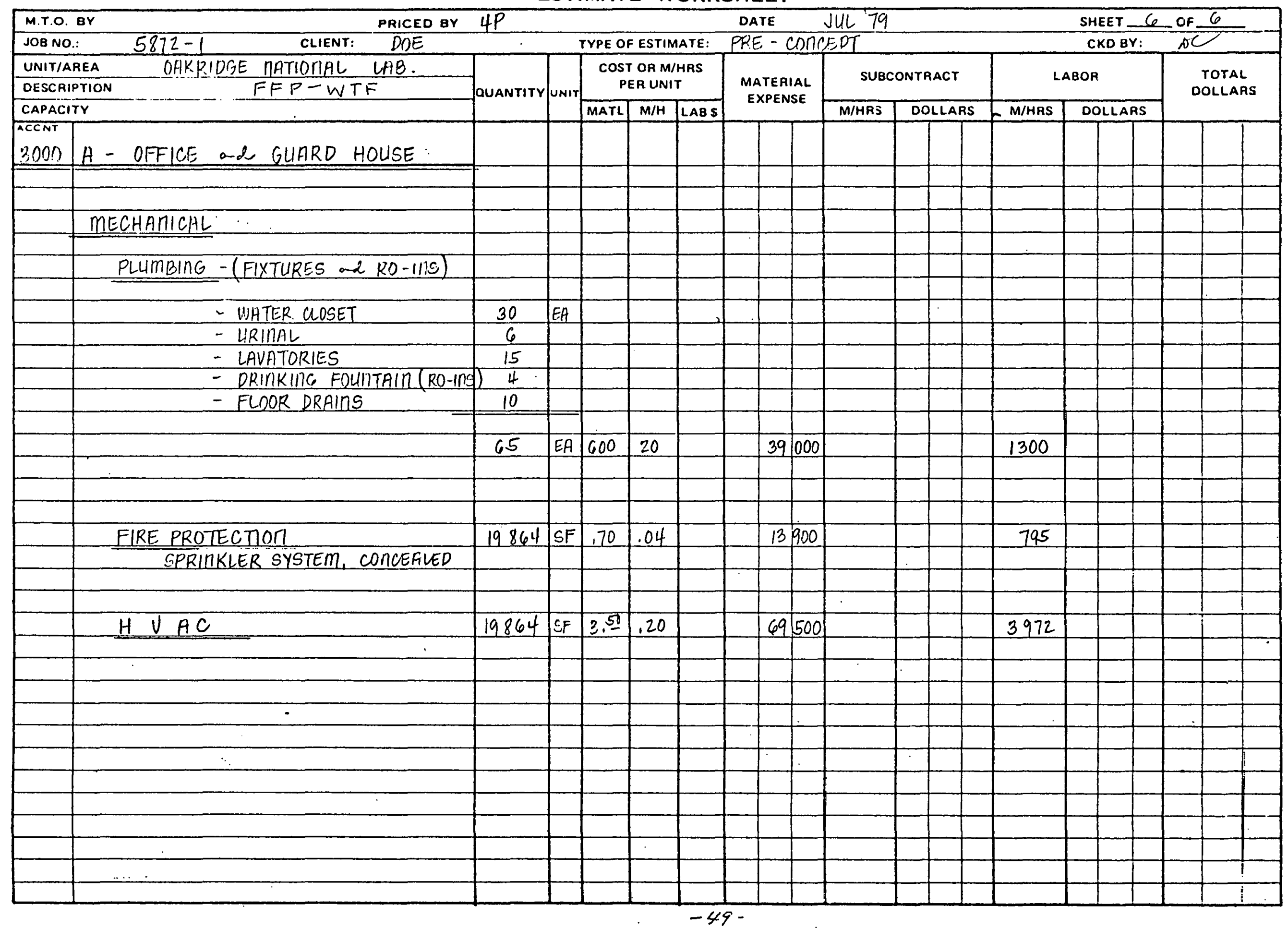




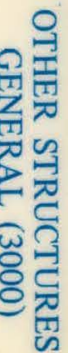




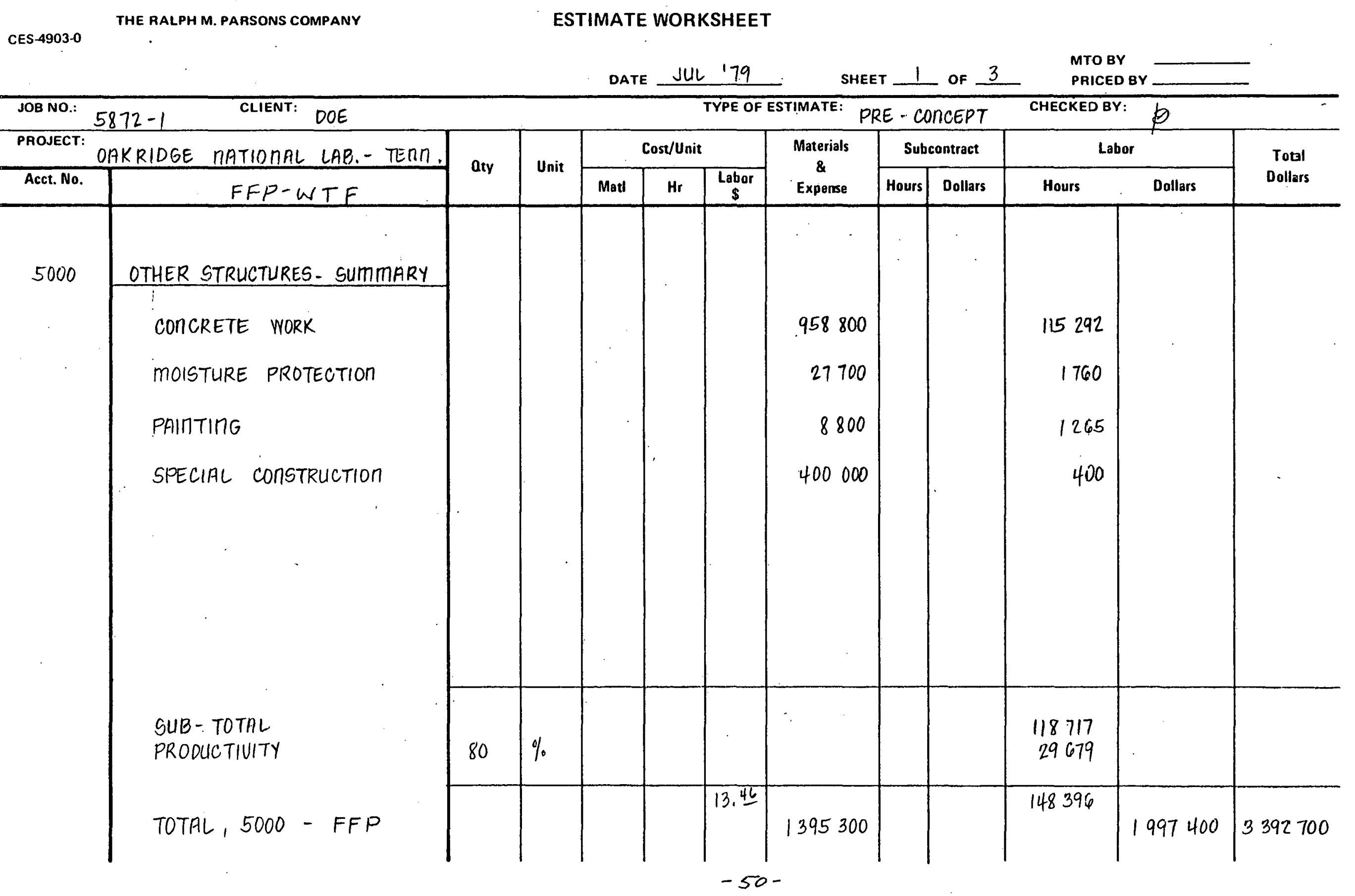


ESTIMATE WORKSHEET

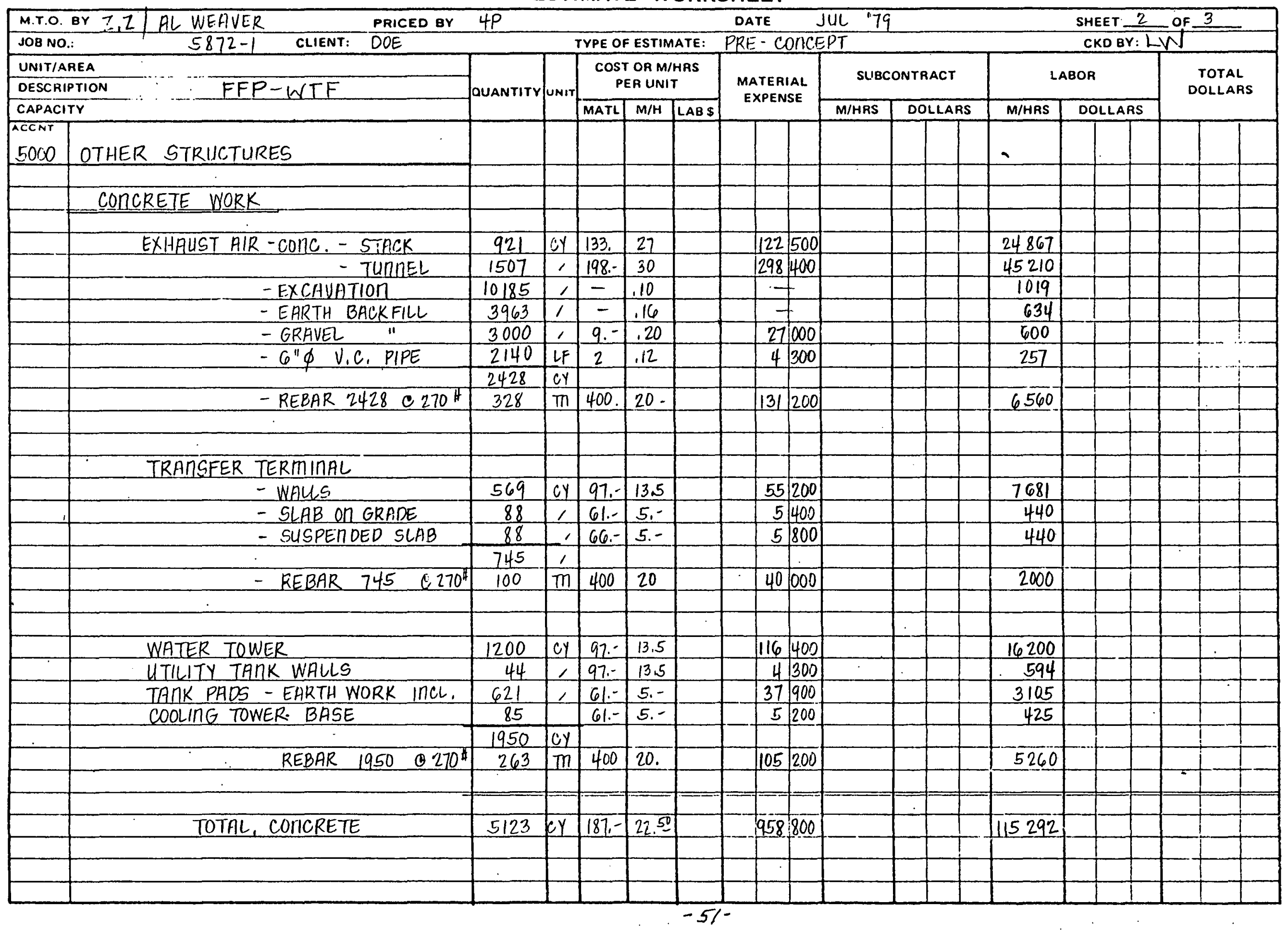


ESTIMATE WORKSHEET

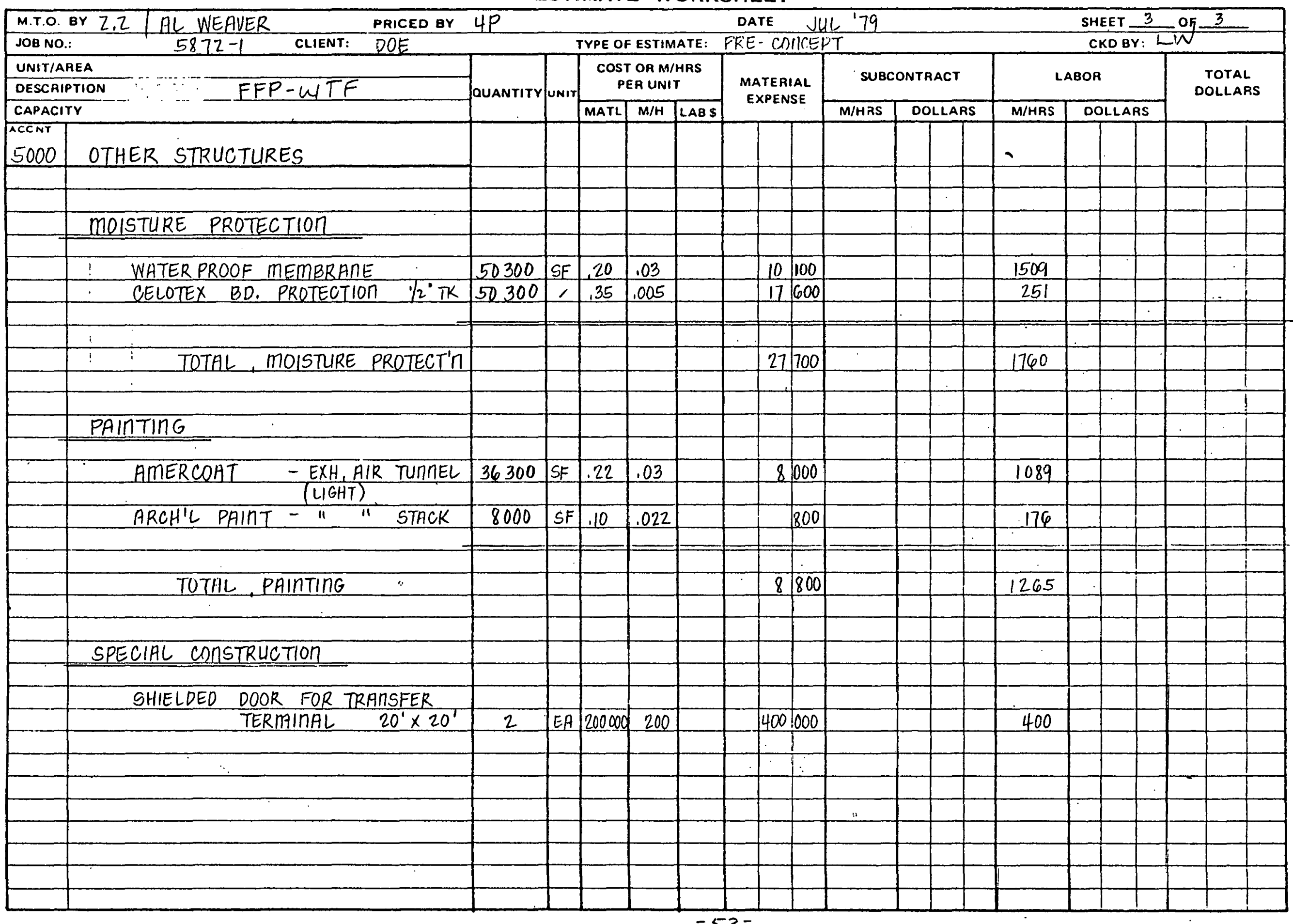




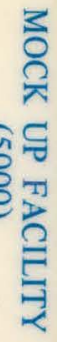


ESTIMATE WORKSHEET

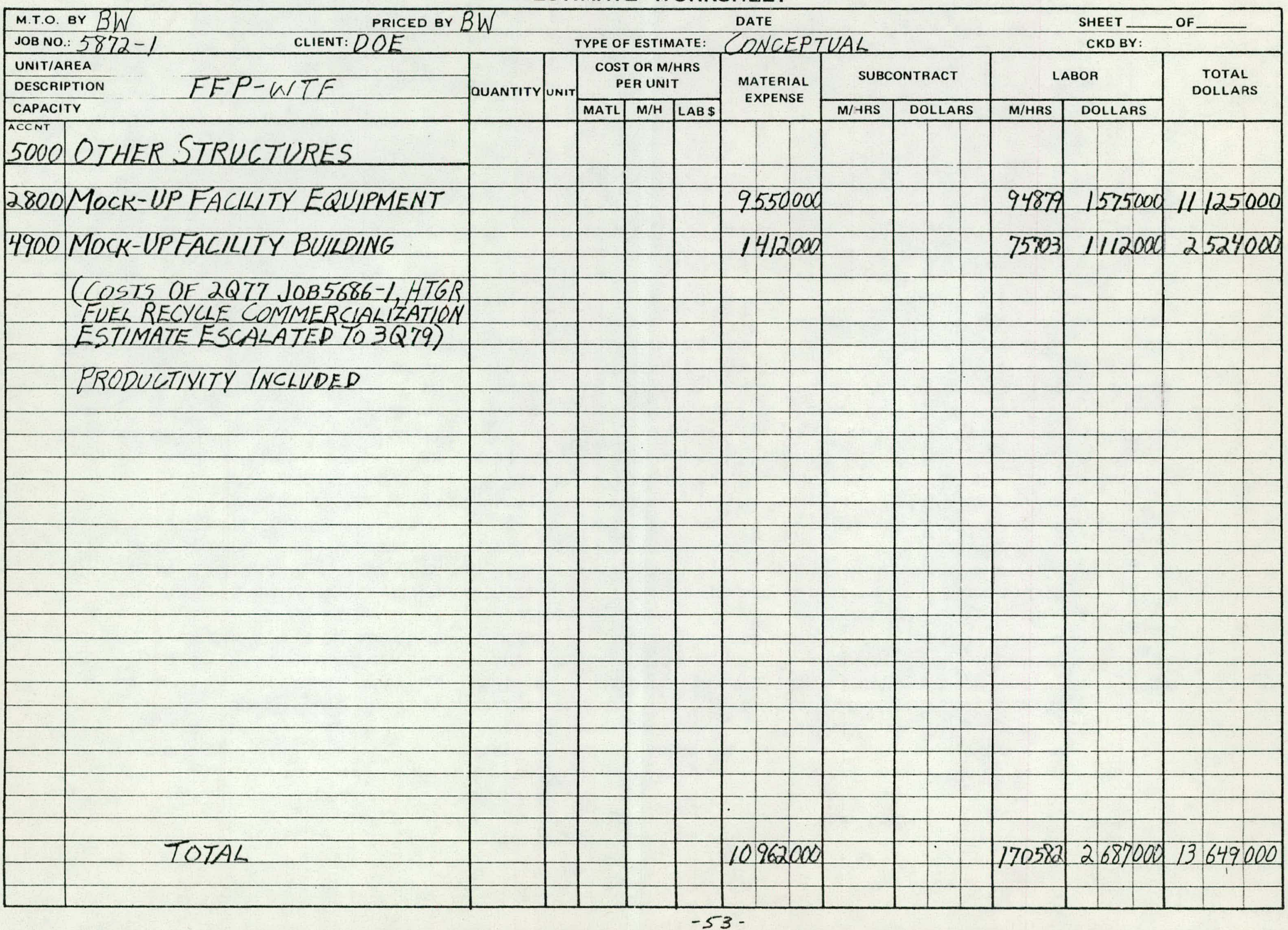


ESTIMATE WORKSHEET

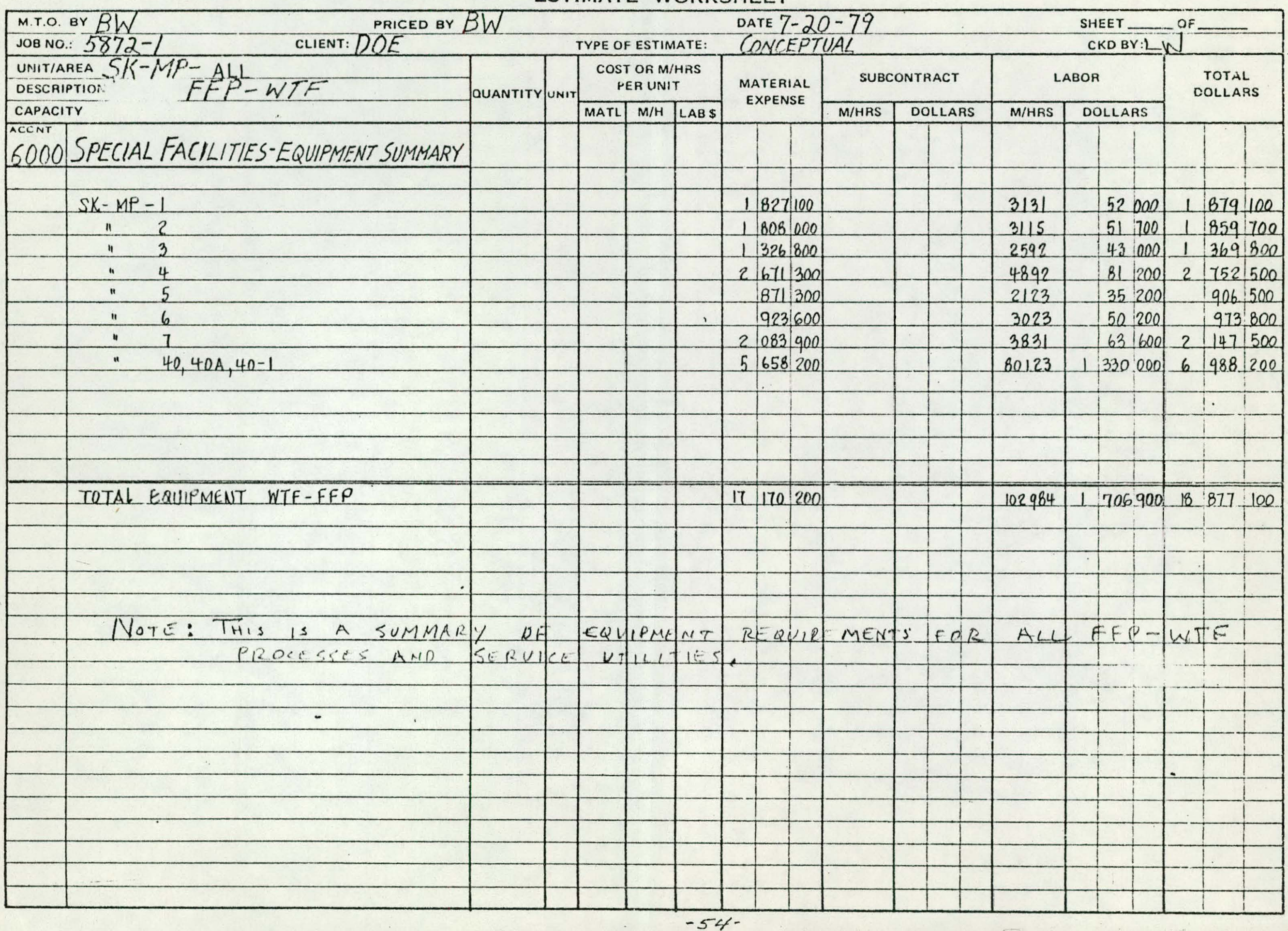




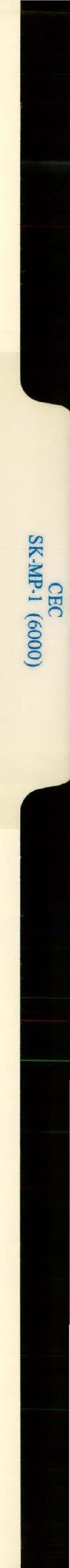


ESTIMATE WORKSHEET

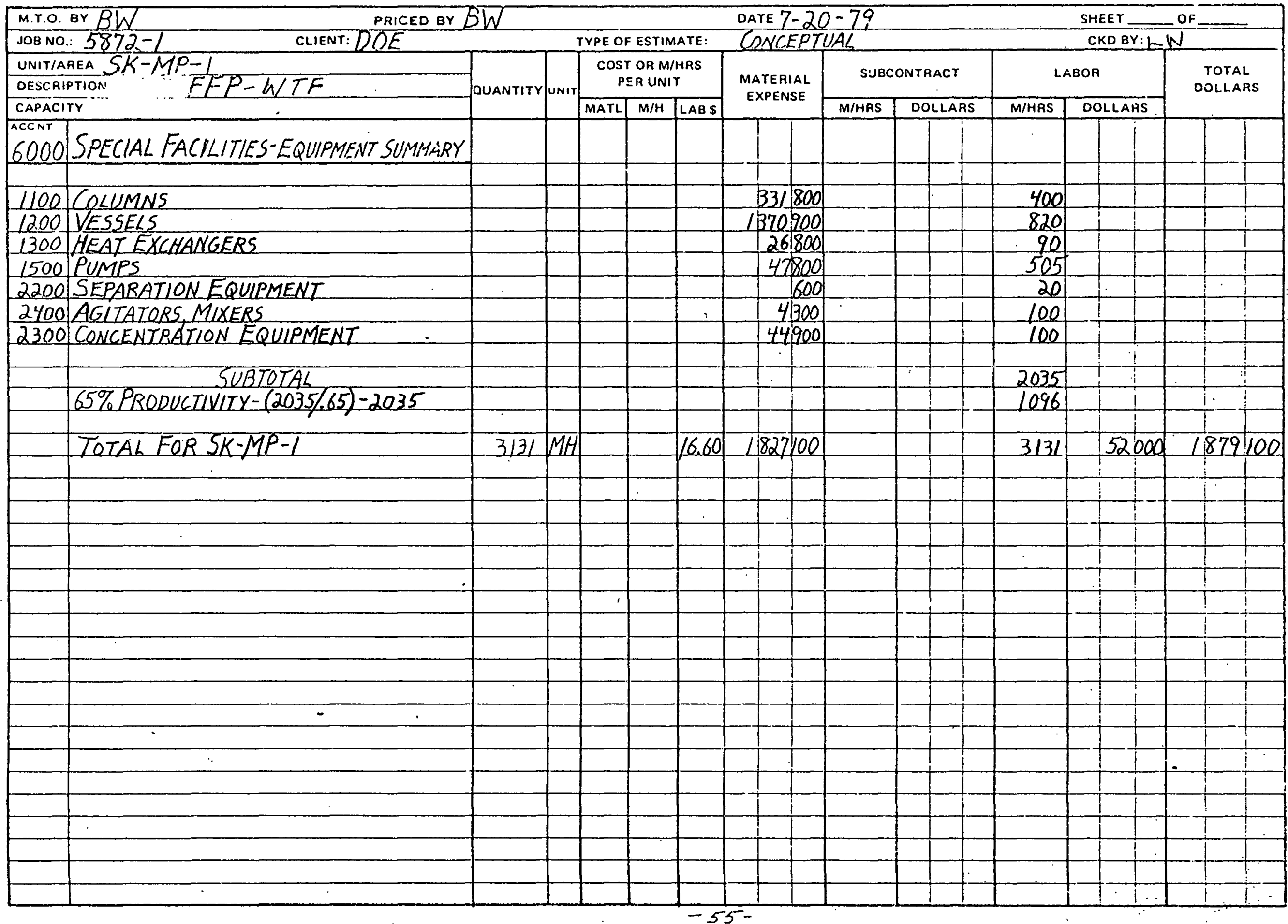


ESTIMATE WORKSHEET

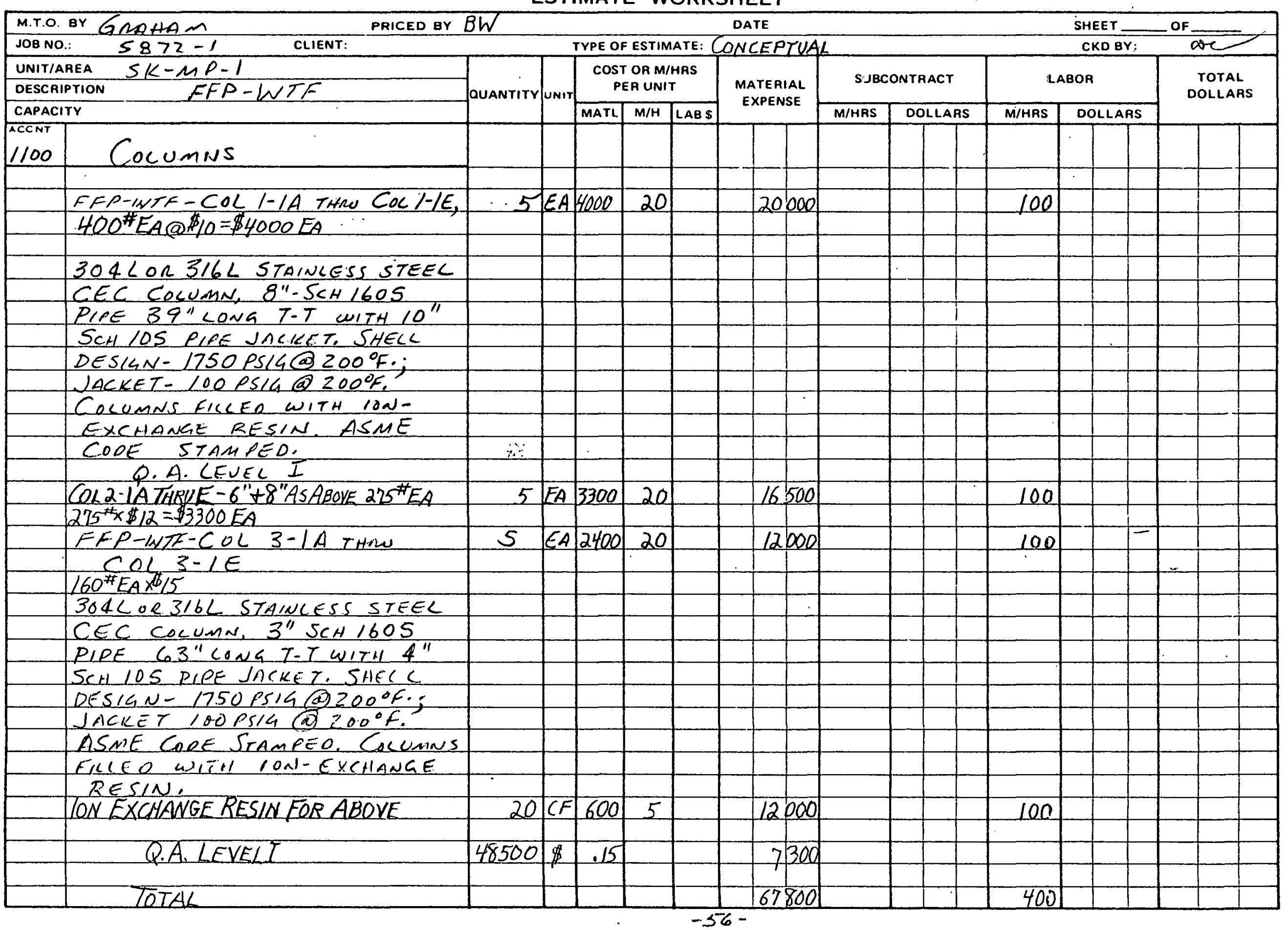


THE RALPH M. PARSONS COMPANY

ESTIMATE WORKSंHEET

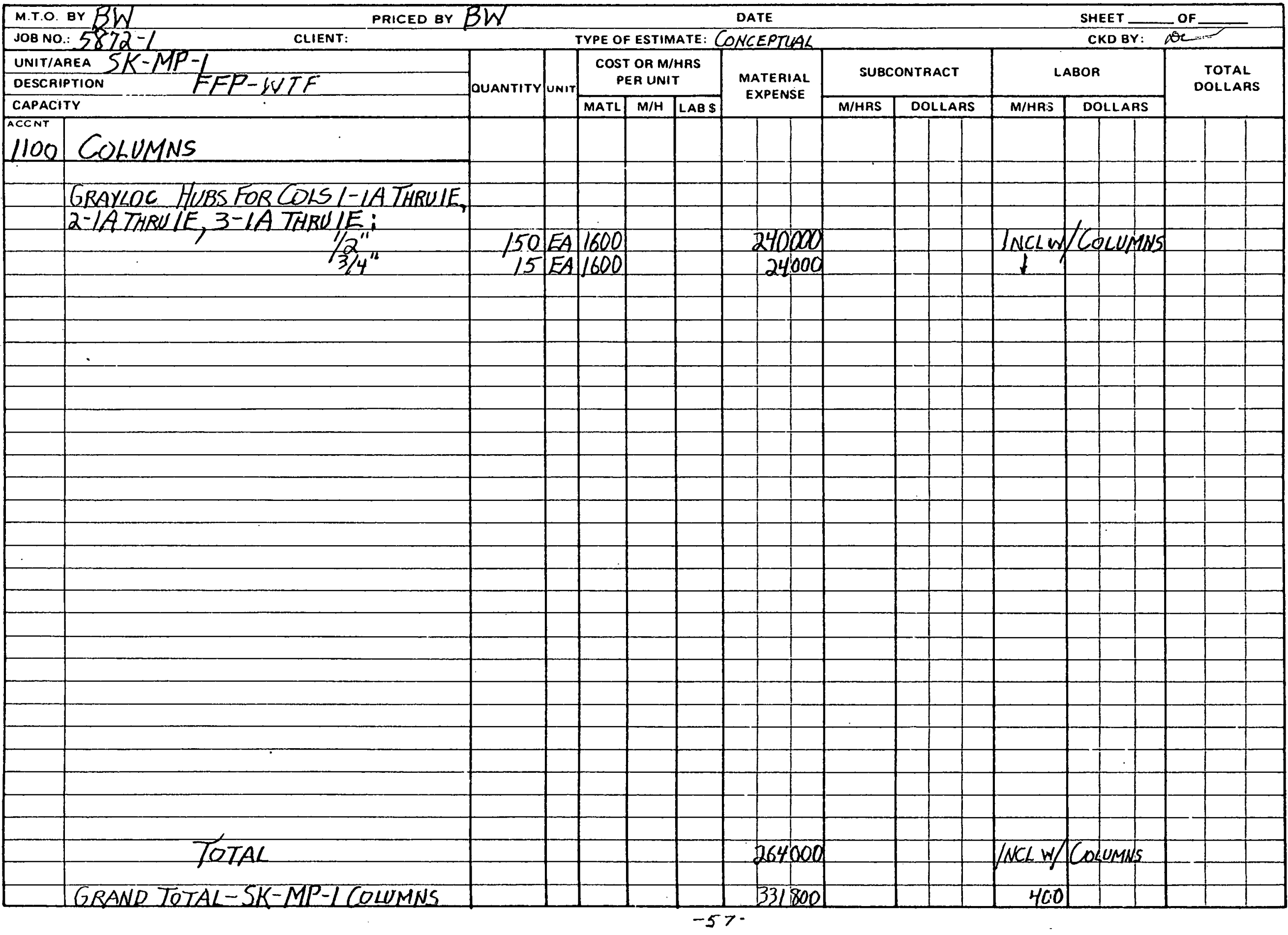


ESTIMATE WORKSHEET

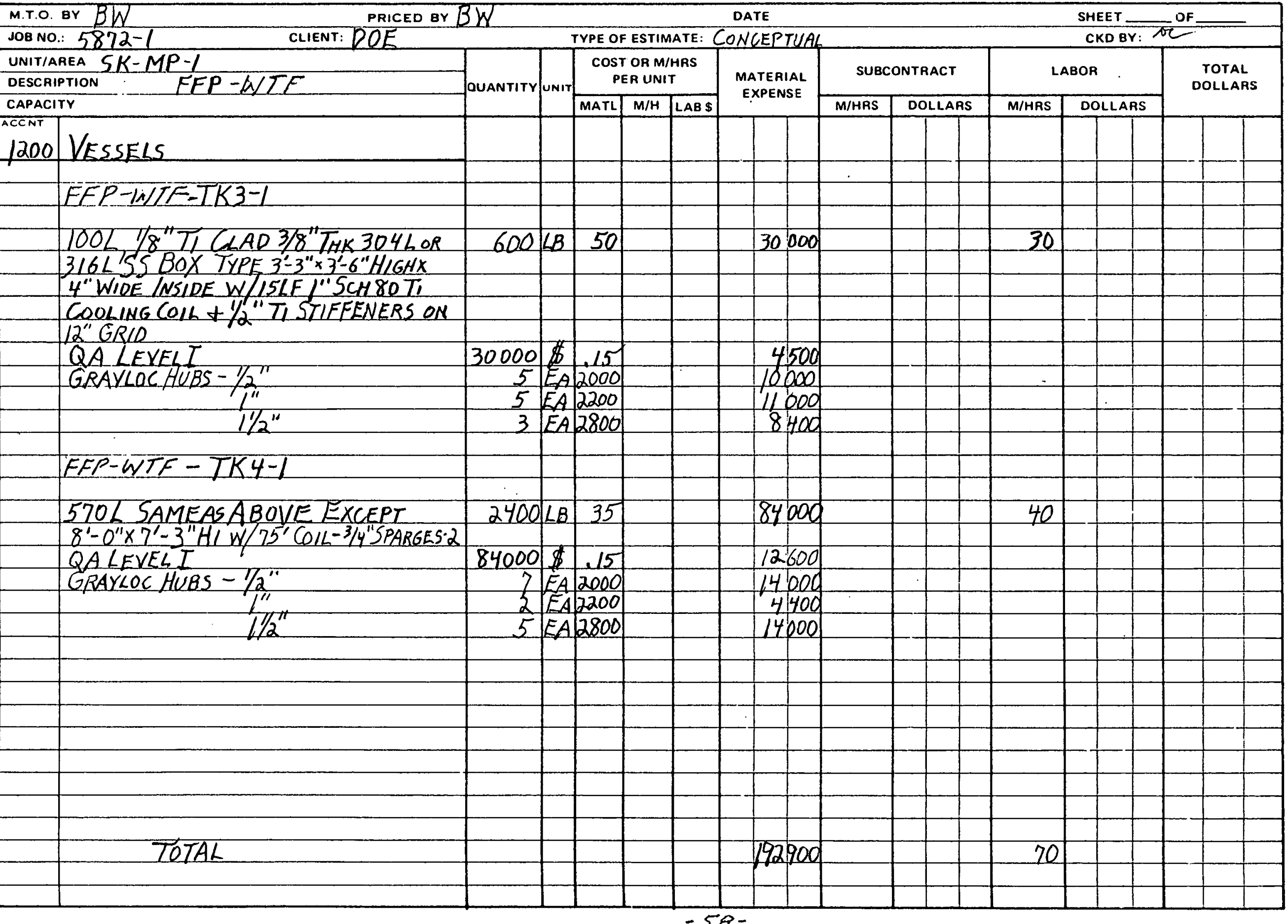


ESTIMATE WORKSHEET

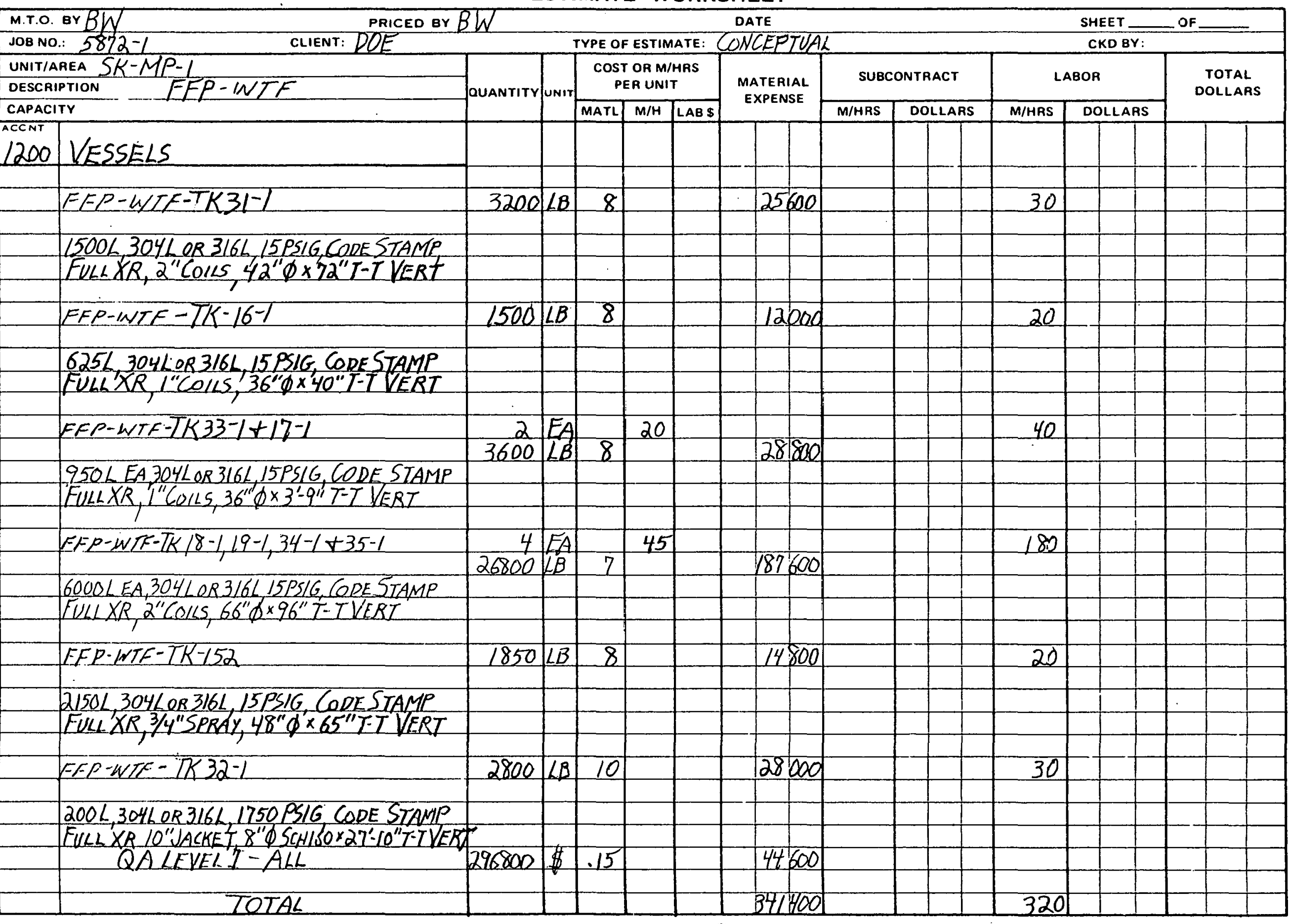


ESTIMATE WORKSHEET

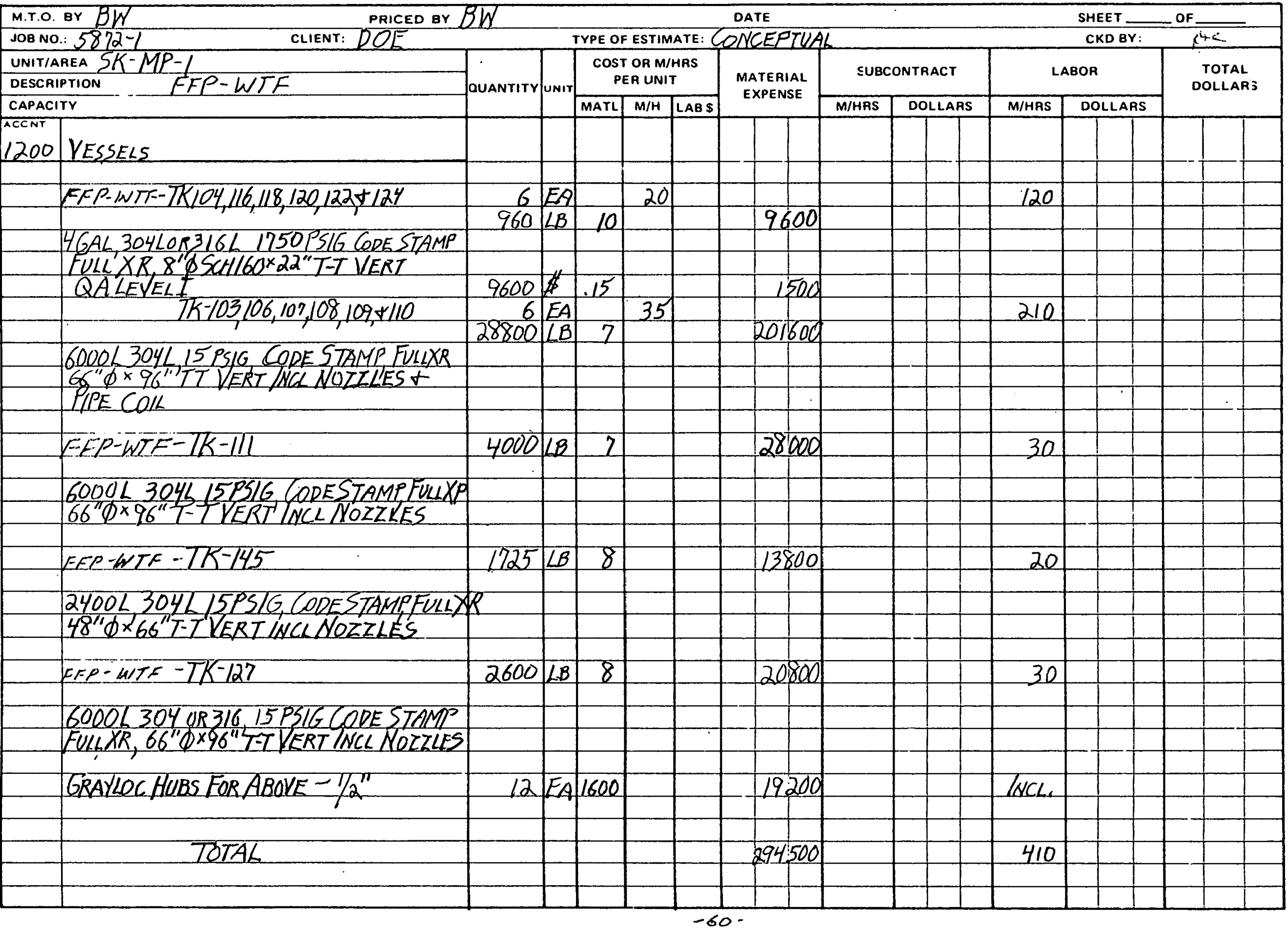


ESTIMATE WORKSHEET

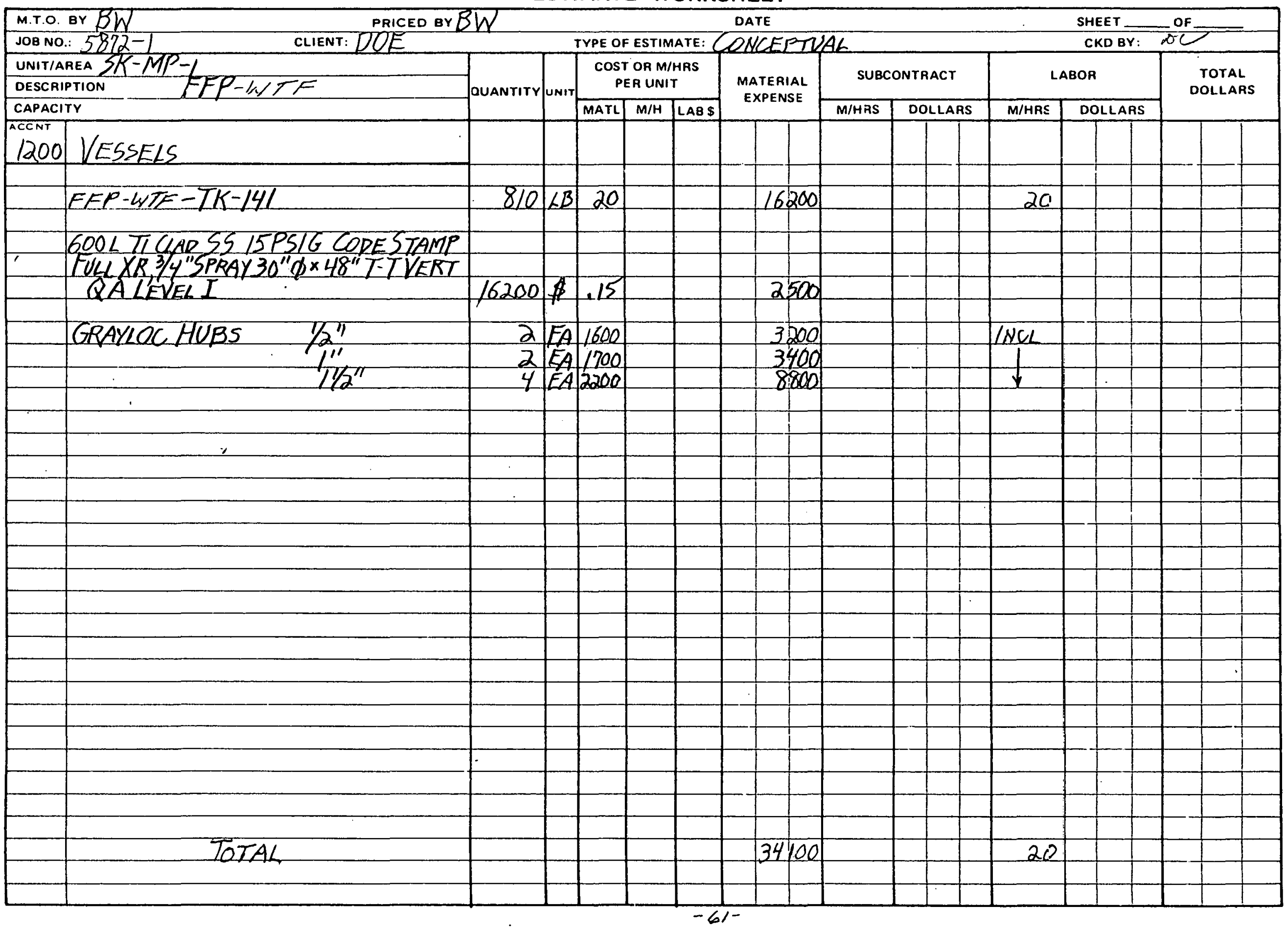


THE RALPH M. PARSONS COMPANY

ESTIMATE WORKSHEET

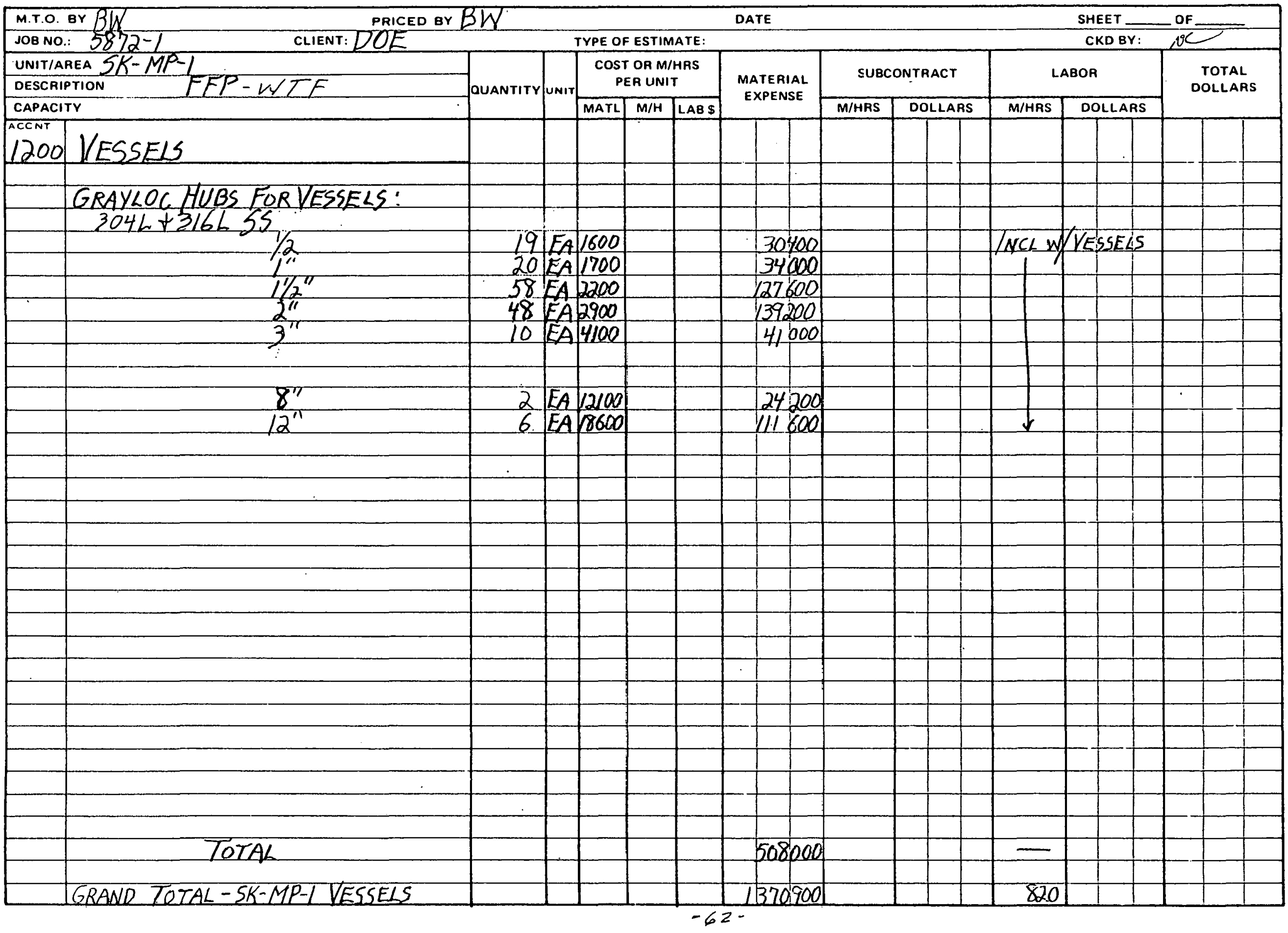


THE RALPH M. PARSONS COMPANY

ESTIMATE WORKSHEET




ESTIMATE WORKSHEET

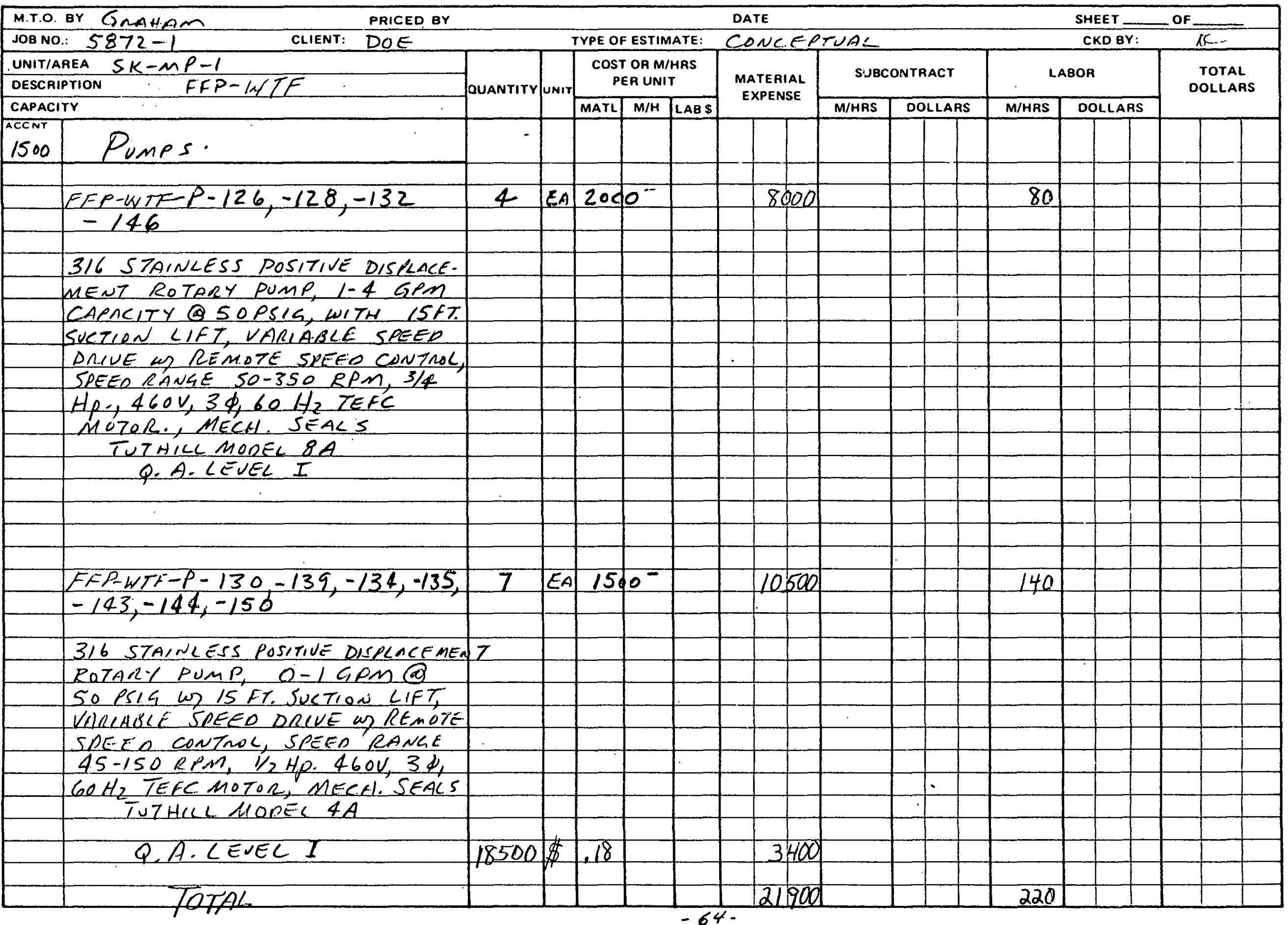


ESTIMATE WORKSHEET

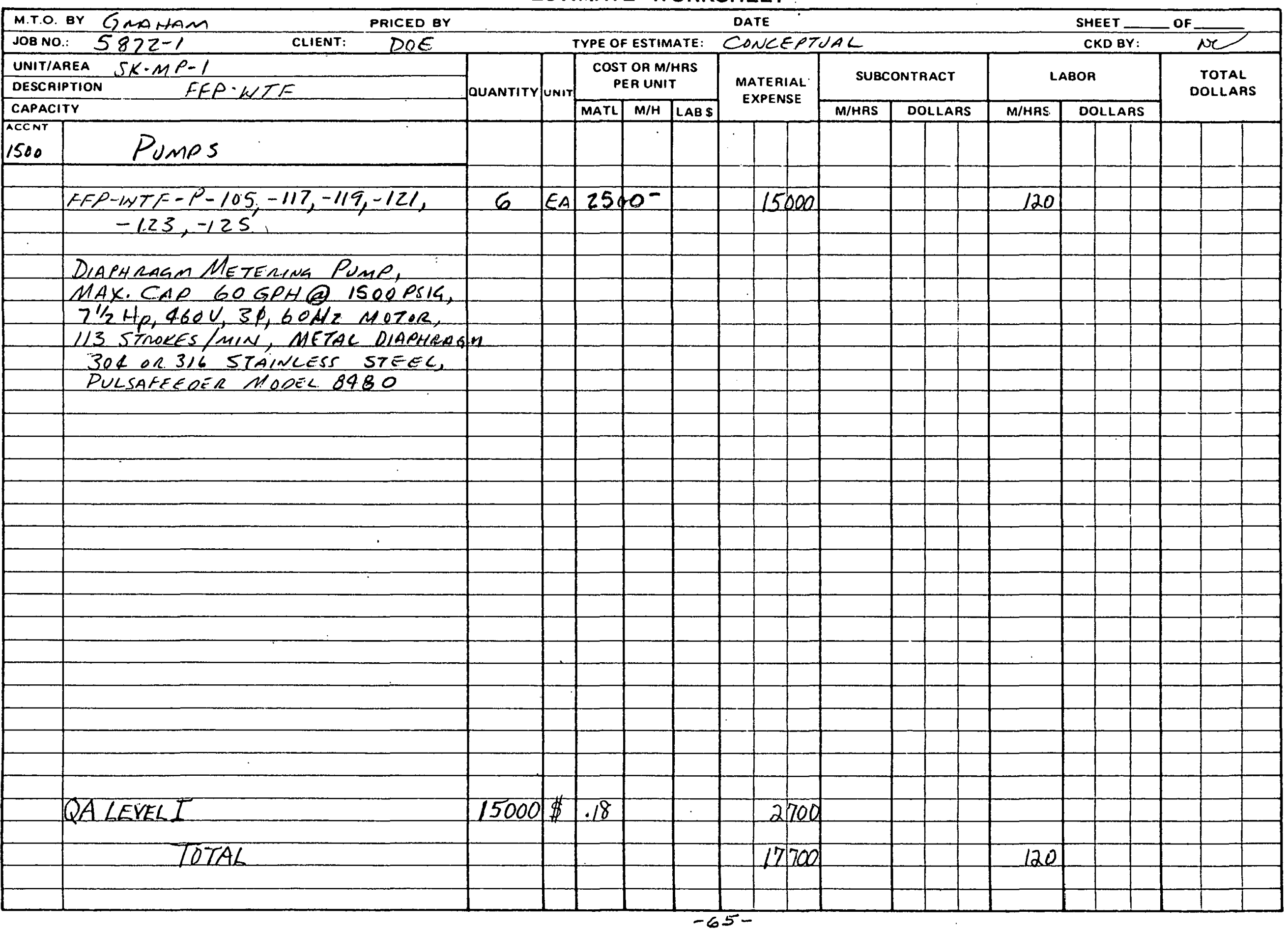


ESTIMATE WORKSHEET

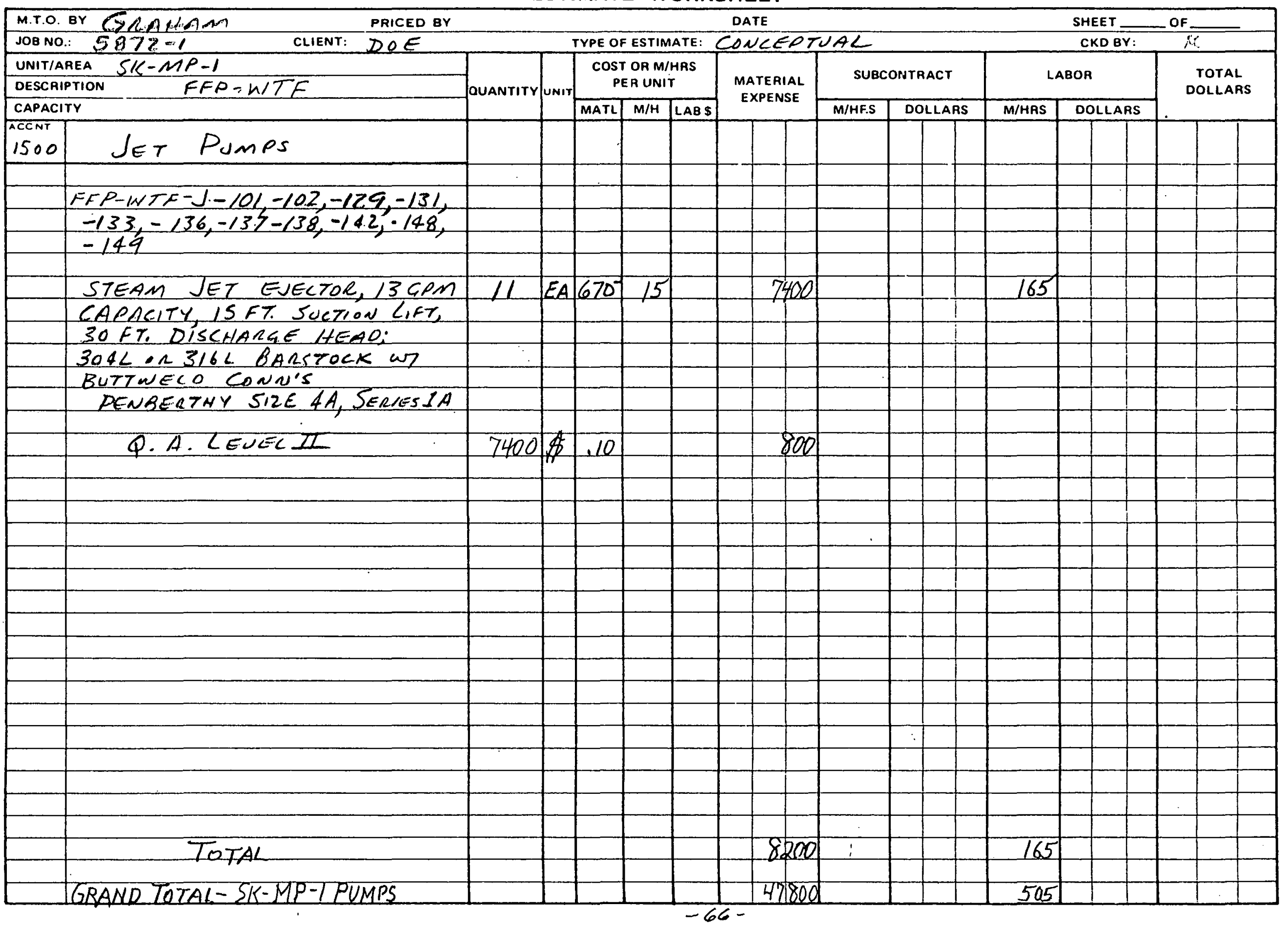


ESTIMATE WORKSHEET

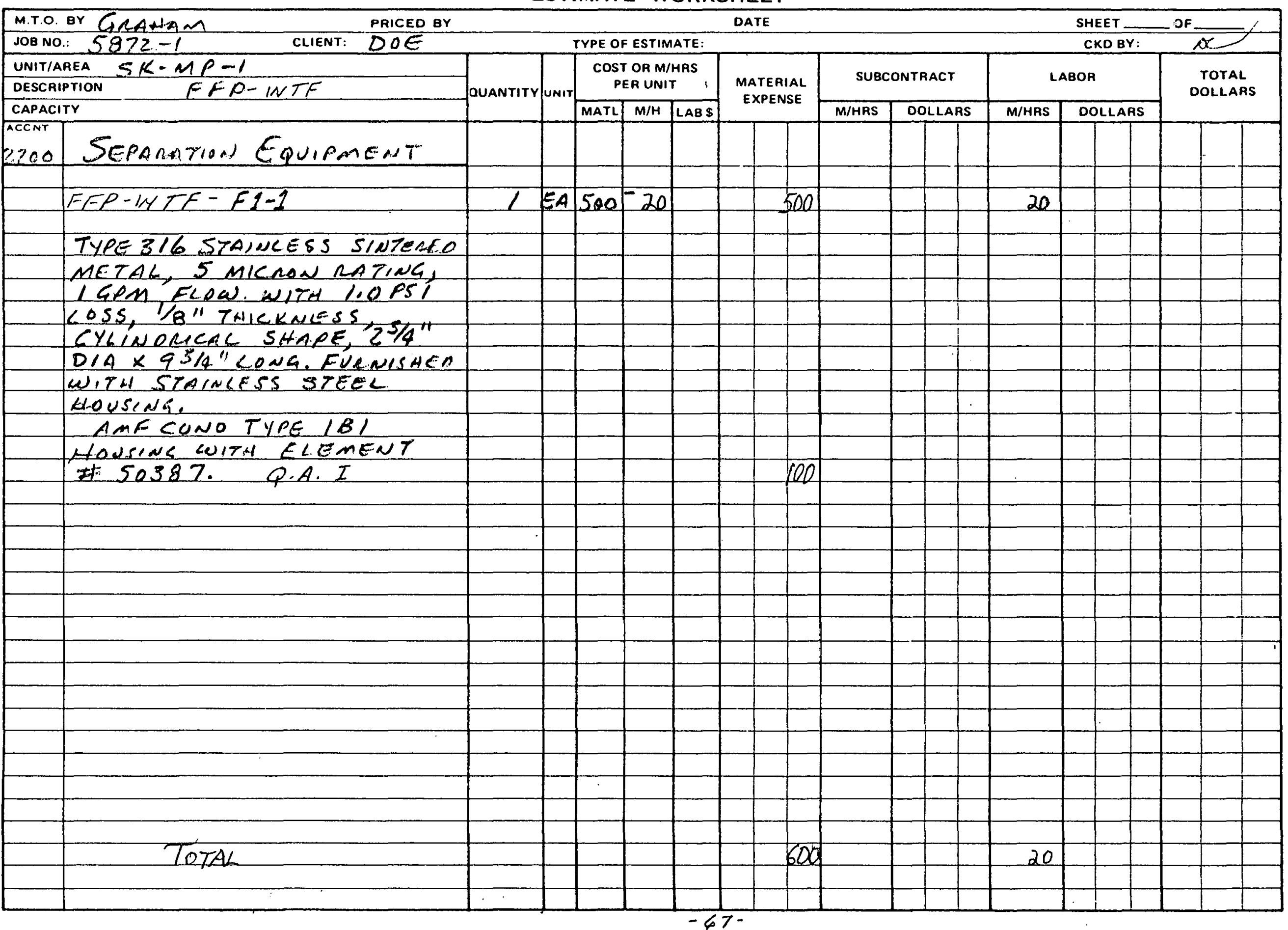


ESTIMATE WORKSHEET

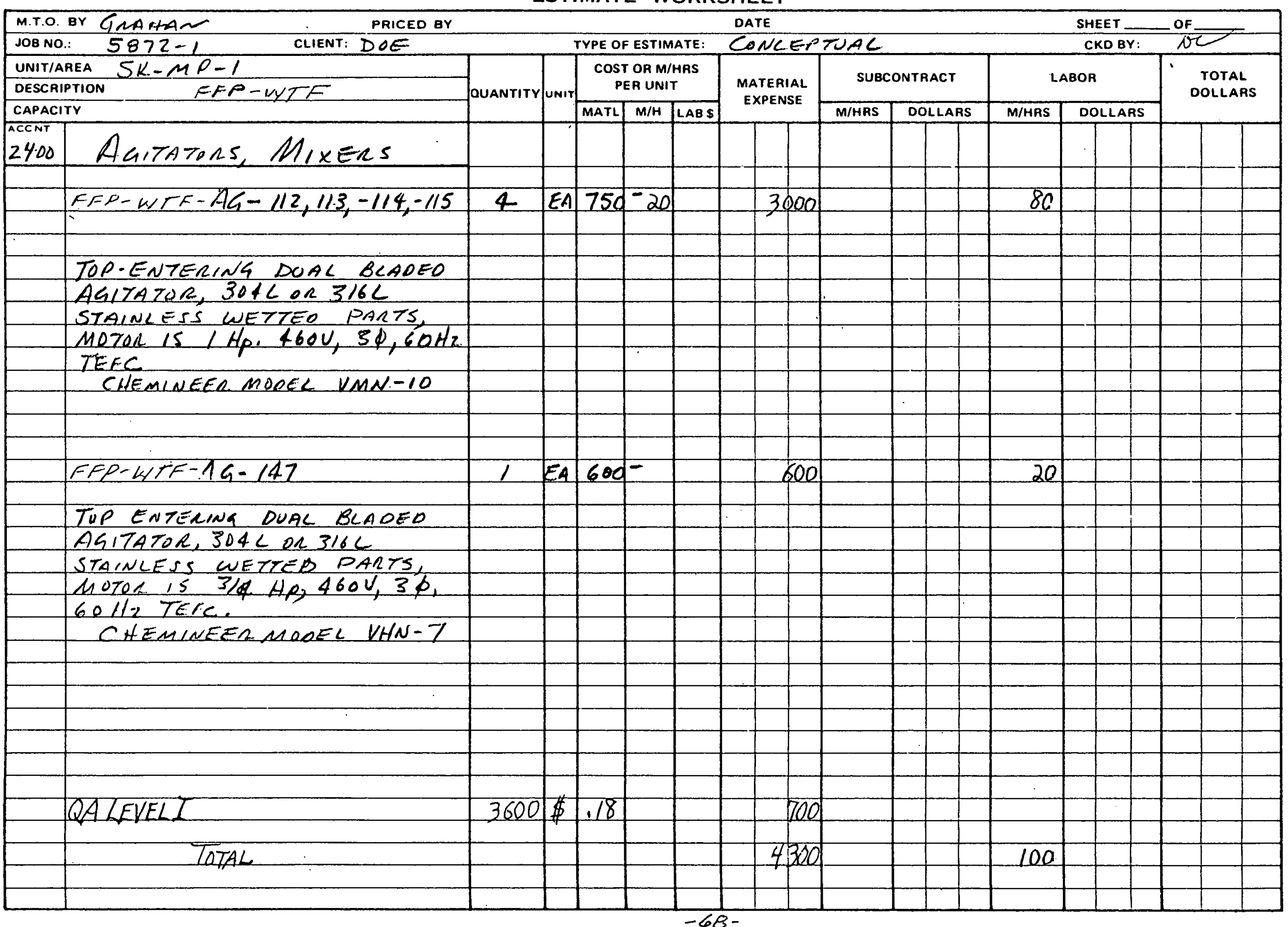


THE RALPH M. PARSONS COMPANY

ESTIMATE WORKSHEET

\begin{tabular}{|c|c|c|c|c|c|c|c|c|c|c|c|c|c|c|c|}
\hline M.T.O. & GRANAM & & & & & & DATE & & & & & & SHEET & & OF \\
\hline JOB NO & $\therefore 5872-1$ & & & TYPE OF & ESTIM & ATE: & & & & & & & CKD BY & & $a C$ \\
\hline UNIT/A & REA $S K-M P-1$ & & & $\cos T$ & & & & & SJUBC & ONTRACT & & & BOR & & \\
\hline DESCRI & PTION FFP-WTF & DUANTITY & |unit & & R UNI & & $\begin{array}{l}\text { MATERI } \\
\text { EXPENS }\end{array}$ & $\begin{array}{ll}\text { RIAL } \\
\text { NSE }\end{array}$ & Sube & ONIKACI & & & 80 & & DOLLARS \\
\hline CAPACI & & & & MATL & $M / H$ & LAB \$ & & & M/HRS & DOLLAR & & M/HRS & DOLLAF & & \\
\hline 2300 & Concentration Equip't. & & & & & & & & & & & & & & \\
\hline & & & & & & & & & & & & & & & \\
\hline & FFP-USTF-CONC $1-1$ & $L$ & $E A$ & 2506 & 0 & & & 5000 & & & & 50 & & & \\
\hline & & & & & & & & & & & & & & & \\
\hline & Titanum CLAO 316 STANuless & & & & & & & & & & & & & & \\
\hline & STEEL CONCENTRATOR, 6" & & & & & & & & & & & & & & \\
\hline & DIA $\times 54^{\prime \prime}$ COLUMN WITH & & & & & & & & & & & & & & \\
\hline & DISENGAGINA SECTION, REGOILER. & & & & & & & & & & & & & & \\
\hline & Q.A.LEVELI & 25000 & \$\$ & .15 & & & & 8800 & & & & & & & \\
\hline & & & & & & & & & & & & & & & \\
\hline & $F F P-W T F-\operatorname{CoNC} 9-1$ & 1 & FA & 14,04 & 0 & & & 1000 & & & & 50 & & & \\
\hline & VESSEL WT $800 \angle B ; H X W T 400 \angle B$ & 1200 & $\angle B$ & $1 / 667$ & & & & & & & & & & & \\
\hline & 316 STAINLESS STEEL CONCENTEATOR & & & & & & & & & & & & & & \\
\hline & 8"PIPE COLUMN 108 "T-T WITH & & & & & & & & & & & & & & \\
\hline & $12 " D \angle A \times 24 "$ DISENGAGING & & & & & & & & & & & & & & \\
\hline & SECTION REBOILER WITH & & & & & & & & & & & & & & \\
\hline & IS SQ.FT. SURAACE. DESIGN & & & & & & & & & & & & & & \\
\hline & FOR 100 PS1G@3S0 Of. & & & & & & & & & & & & & & \\
\hline & Q.A.LEVELI & 14000 & \$5 & .15 & & & 2 & 100 & & & & & & & \\
\hline & & & $\pi$ & & & & & & & & & & & & \\
\hline & & & & & & & & & & & & & & & \\
\hline & & & & & & & & & & & & & & & \\
\hline & & & & & & & & & & & & & & & \\
\hline & & & & & & & & & & & & & & & \\
\hline & & & & & & & & & & & & & & & \\
\hline & & & & & & & & & & & & & & & \\
\hline & & & & & & & & & & & & & & & \\
\hline & & & & & & & & & & & & & & & \\
\hline & & & & & & & & & & & & & & & \\
\hline & 1 & & & & & & & & & & & & & & \\
\hline & & & & & & & & & & & & & & & \\
\hline & TOTAK & & & & & & 44 & 1900 & & & & 100 & & & \\
\hline & & & & & & & & & & & & & & & \\
\hline & & & & & & & & & & & & & & & \\
\hline
\end{tabular}


ESTIMATE WORKSHEET

\begin{tabular}{|c|c|c|c|c|c|c|c|c|c|c|c|c|c|c|c|c|c|c|}
\hline \multicolumn{7}{|c|}{ M.T.O. BY $B W$ PRICED BY $B W$} & \multicolumn{5}{|c|}{ DATE $7-20-79$} & \multicolumn{7}{|c|}{ SHEET } \\
\hline \multicolumn{3}{|c|}{ JOB NO.: $5872-1 \quad$ CLIENT: DOE } & \multicolumn{4}{|c|}{ TYPE OF ESTIMATE: } & \multicolumn{5}{|c|}{ CONCEPTUAL } & \multicolumn{7}{|c|}{ CKOBY: $\mathrm{N}$} \\
\hline \multicolumn{2}{|c|}{ UNIT/AREA $S K-M P-2$} & \multirow{3}{*}{ DUanriry } & \multirow{3}{*}{ UNIT } & \multirow{2}{*}{\multicolumn{3}{|c|}{$\begin{array}{l}\text { COST OR M/HRS } \\
\text { PER UNIT }\end{array}$}} & \multirow{3}{*}{\multicolumn{3}{|c|}{$\begin{array}{c}\text { MATERIAL } \\
\text { EXPENSE }\end{array}$}} & \multirow{2}{*}{\multicolumn{2}{|c|}{ SUBCONTRACT }} & \multirow{2}{*}{\multicolumn{4}{|c|}{ LABOR }} & \multirow{3}{*}{\multicolumn{3}{|c|}{$\begin{array}{c}\text { TOTAL } \\
\text { DOLLARS }\end{array}$}} \\
\hline OESCAIP & TFE: FE-INTE & & & & & & & & & & & & & & & & & \\
\hline \multicolumn{2}{|c|}{ CAPACITY } & & & \multirow[t]{2}{*}{ MATL } & \multirow[t]{2}{*}{$M / H$} & \multirow[t]{2}{*}{ LAB\$ } & & & & \multirow[t]{2}{*}{ M/HAS } & DOLLAA & M/HRS & & OLLAF & & & & \\
\hline 6000 & SPECIAL FACILITIES-EQUIPMENT SUMMARY & & & & & & & & & & & & & & & & & \\
\hline & & & & & & & & & & & & & & & & & & \\
\hline 1100 & COLIMNS & & & & & & & 221 & 600 & & & 150 & & & & & & \\
\hline 1200 & VESSELS & & & & & & 1 & 343 & 800 & & & 680 & & & 1 & & & \\
\hline 1300 & HEAT EXCHANGERS & & & & & & & 48 & 300 & & & 170 & & & $i$ & & & \\
\hline 1.500 & PUMPS & & & & & & & 34 & 100 & & & 425 & & & & & & \\
\hline 2300 & CONCENTRATION EQUIPUENT & & & & & & & 140 & 500 & & & 160 & & & & & & \\
\hline 2400 & AGITATORS MIXERS & & & & & 1 & & 9 & BeO & & & 320 & & & & & & \\
\hline 2800 & OTHER MATOR EQUIPMENT & & & & & & & $q$ & 900 & & & 120 & & & & & & \\
\hline & & & & & & & & 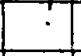 & & & & & & & 1 & & & \\
\hline & & & & & & & &. & & & & & & & + & & & \\
\hline & SUE TOTAL & & & & & & 1 & 808 & eee & & & 2025 & & & $T$ & &.. & \\
\hline & & & & & & & & & & & & & & & & & & \\
\hline & $65 \%$ PROQUCTIVITY $(2025 \div .65)-2025$ & & & & & & & & & & & 1090 & & & & & & \\
\hline & & & & & & & & & & & & 3115 & & & & & I & \\
\hline & & 3115 & $\mathrm{MH}$ & & & 16.60 & & & & & & & & 51 & 700 & & & \\
\hline & & & & & & & & & & & & & & & & & q & \\
\hline & & & & & & & & & & & & & & & T & & i & \\
\hline & TOTAL FOR SK-MP-2 & & & & & & 1 & 808 & 000 & & & 3115 & & 51 & 1700 & 1 & 859 & 700 \\
\hline & & & & & & & & & & & & & & & & & & \\
\hline & & & & & & & & & & & & & & & & & & \\
\hline & & & & & & & & & & & & & & & & & & \\
\hline & & & & & & & & & & & & & & & & & & \\
\hline & & & & & & & & & & & & & & & & & & \\
\hline & & & & & & & & & & & & & & & & & & \\
\hline & - & & & & & & & & & & & & & & & & & \\
\hline & & & & & & & & & & & & & & & & & & \\
\hline & & & & & & & & & & & & & & & $i$ & & & \\
\hline & & & & & & & & & & & & & & & & & & \\
\hline & & & & & & & & & & & & & & & & & & \\
\hline & & & & & & & & & & & & & & & & & & \\
\hline & & & & & & & & & & & & & & & & & & \\
\hline & & & & & & & & & & & & & & & & & & \\
\hline & & & & & & & & & & & & & & . & & & I & \\
\hline
\end{tabular}


ESTIMATE WORKSHEET

\begin{tabular}{|c|c|c|c|c|c|c|c|c|c|c|c|c|c|c|c|}
\hline M.T.O. & BY GRANAM & & & & & & DATE & & & & & SHEET - & $\underline{\underline{z}}$ & & \\
\hline JOB NC & CLIENT: DOE/CKC- & NO & & TYPE OF & FESTIM & ATE: & CONCEATU & & & & & CKD BY: & & or & \\
\hline UNIT/A & REA (SK-MP-2) CMP EXTRACTION & & & & TOR M/ & & & SUBC & NTRACT & & & $B O R$ & & TOTAL & \\
\hline DESCR & IPTION FFP-UITE & QUANTITY & UNIT & & ER UNI & & $\begin{array}{l}\text { MATERIAL } \\
\text { EXPENSE }\end{array}$ & SUBC & NTRAC & & & toun & & DOLLAR: & \\
\hline CAPAC & & & & MATL & $M / \mathbf{H}$ & LAB \$ & & M/HAS & DOLLAR & & M/HAS & DOLLAR & & & \\
\hline $\begin{array}{l}\text { ACCNT } \\
1100\end{array}$ & CoLUMNS & & & & & & & & & & & & & & \\
\hline & & & & & & & & & & & & & & & \\
\hline & $F F P-W T F F-C O L 6-2$ & $\angle$ & $E A$ & 25 & sto & & 25000 & & & & 50 & & & & \\
\hline & $304 L$ OR $316 L$ STAINLESS COLUMN, & & & का & & & & & & & & & & & \\
\hline & $24 " \Delta \angle A \times 30^{\prime \prime}$ TOP VESSEL, 18"DIA & & & & & & & & & & & & & & \\
\hline & $\times 24 "$ BOTTOM JESSEL, 6"SLH 40 & & & & & & & & & & & & & & \\
\hline & PIPE COLUMal WI INTERNAL & & & & & & & & & & & & & & \\
\hline & NOZZLE PLATES, O.A. HEIAHT = & & & & & & & & & & & & & & \\
\hline & $27^{\prime}$ D. DESIAN PRESS. 30P5IS. & & & & & & & & & & & & & & \\
\hline & Q.A.LEUEL I & 25000 & $\$$ & .15 & & & 3800 & & & & & & & & \\
\hline & & & & & & & & & & & & & & & \\
\hline & & & & & & & & & & & & & & & \\
\hline & $F F P^{2}-W T F-\operatorname{COL} 7-2$ & $L$ & $E A$ & 50 & 600 & & 50000 & & & & 50 & & & & \\
\hline & HASTELLOY "E" STRP COLUMN, & & & & & & & & & & & & & & \\
\hline & 24"DIAX 30" TOP VESSEL, 18 "DIA & & & & & & & & & & & & & & \\
\hline & $\times 24$ " BOTTOM VESSEL, 6 "SCH.4O & & & & & & & & & & & & & & \\
\hline & PIPE COLUMN WI INTENNAL NOZZLE & & & & & & & & & & & & & & \\
\hline & PLATES, O.A. HEILHT- ZT'I & & & & & & & & & & & & & & \\
\hline & DESIGN PRESSURE- 30 PSIG, 1400 LD & & & & & & & & & & & & & & \\
\hline & Q.A.LEVELI & 50000 & $\$$ & .15 & & & 7500 & & & & & & & & \\
\hline & & & & & & & & & & & & & & & \\
\hline & & & & & & & & & & & & & & & \\
\hline & $E E P-M / T F-.015-2$ & I & $E A$ & 30,0 & 200 & & 30000 & & & & 50 & & & & \\
\hline & 314L OR 3/6L EXTRACTION COLUMN & & & & & & & & & & & & & & \\
\hline & 24 "OIA X30" TOP VESSEL, 18 "DIA & & & & & & & & & & & & & & \\
\hline & X24"BOTTOM UESSEC 6 "SCM 40 & & & & & & & & & & & & & & \\
\hline & PIRE Cocuma as INTERNAL NOZZLE & & & & & & & & & & & & & & \\
\hline & PLATES, O.A HEIGHT- 35'I & & & & & & & & & & & & & & \\
\hline & DESIGN PRESSURE - 30 PSIG & & & & & & & & & & & & & & \\
\hline & QA.LEKEC I & 30000 & 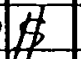 & .15 & & & 4500 & & & & & & & & \\
\hline & & & & & & & & & & & & & & & \\
\hline & TOTAK & & & & & & 120800 & & & & 150 & & & & \\
\hline & & & & & & & & & & & & & & & \\
\hline & & & & & & & & & & & & & & & \\
\hline
\end{tabular}




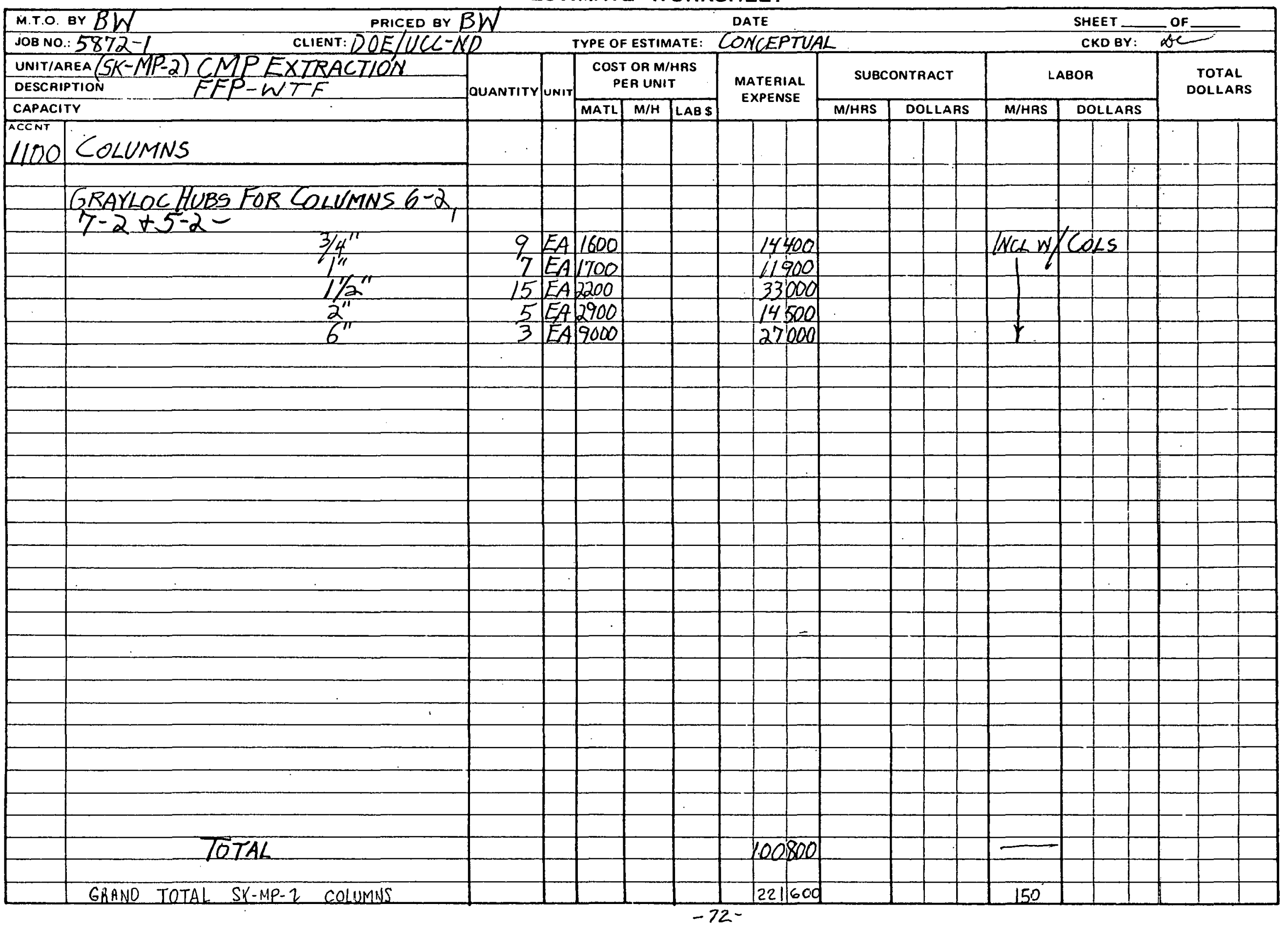


ESTIMATE WORKSHEET

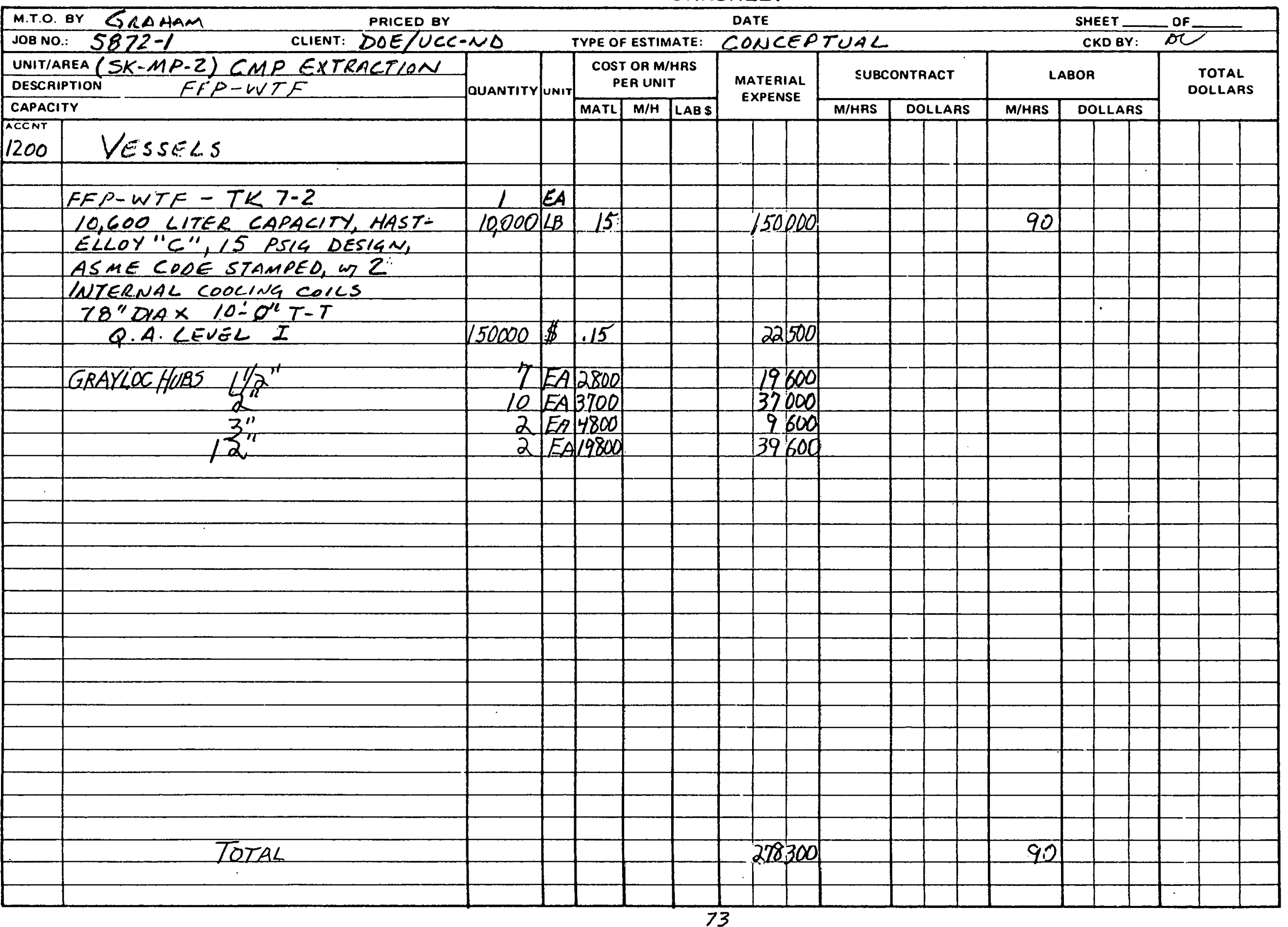


ESTIMATE WORKSHEET

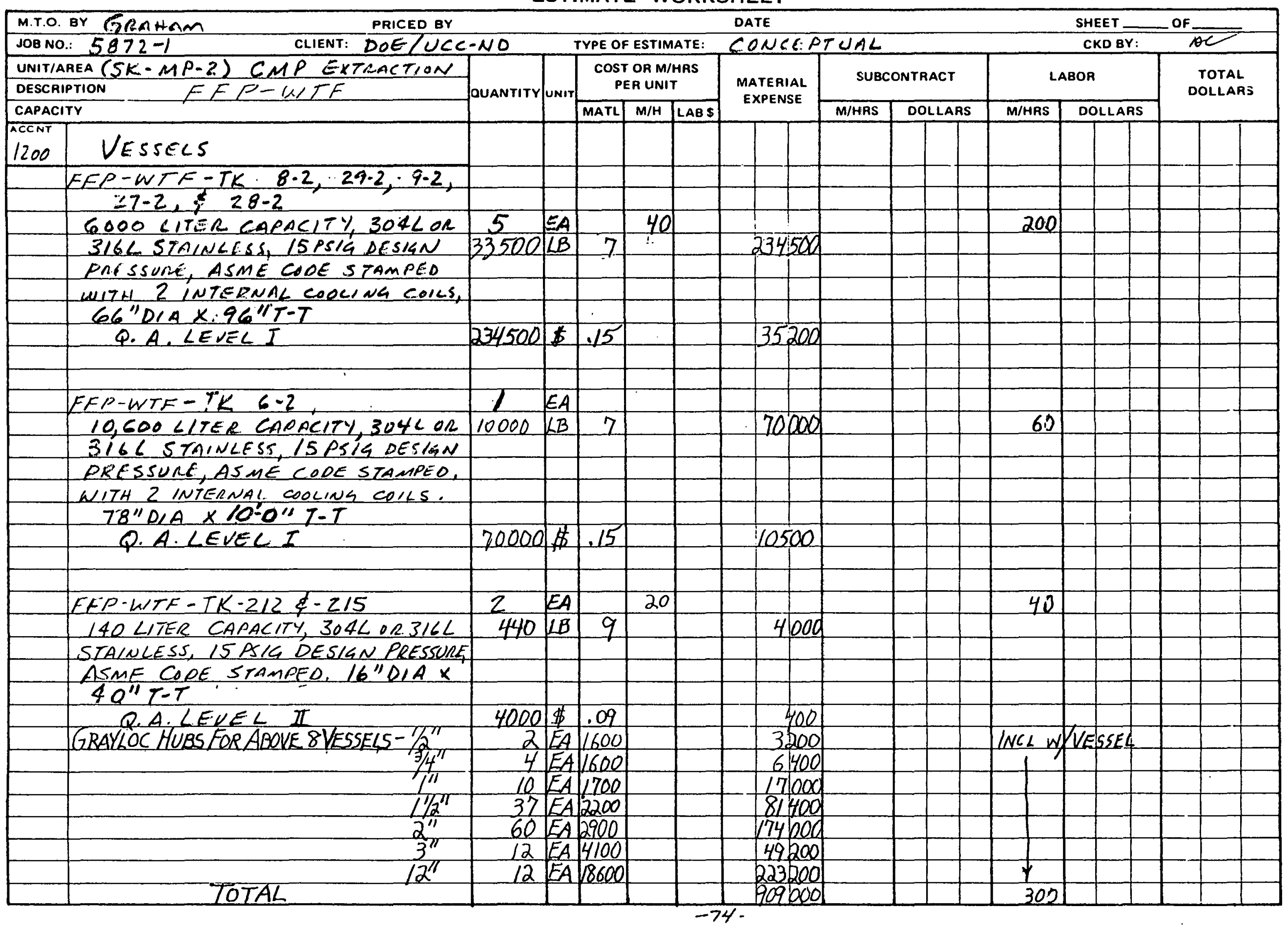


ESTIMATE WORKSHEET

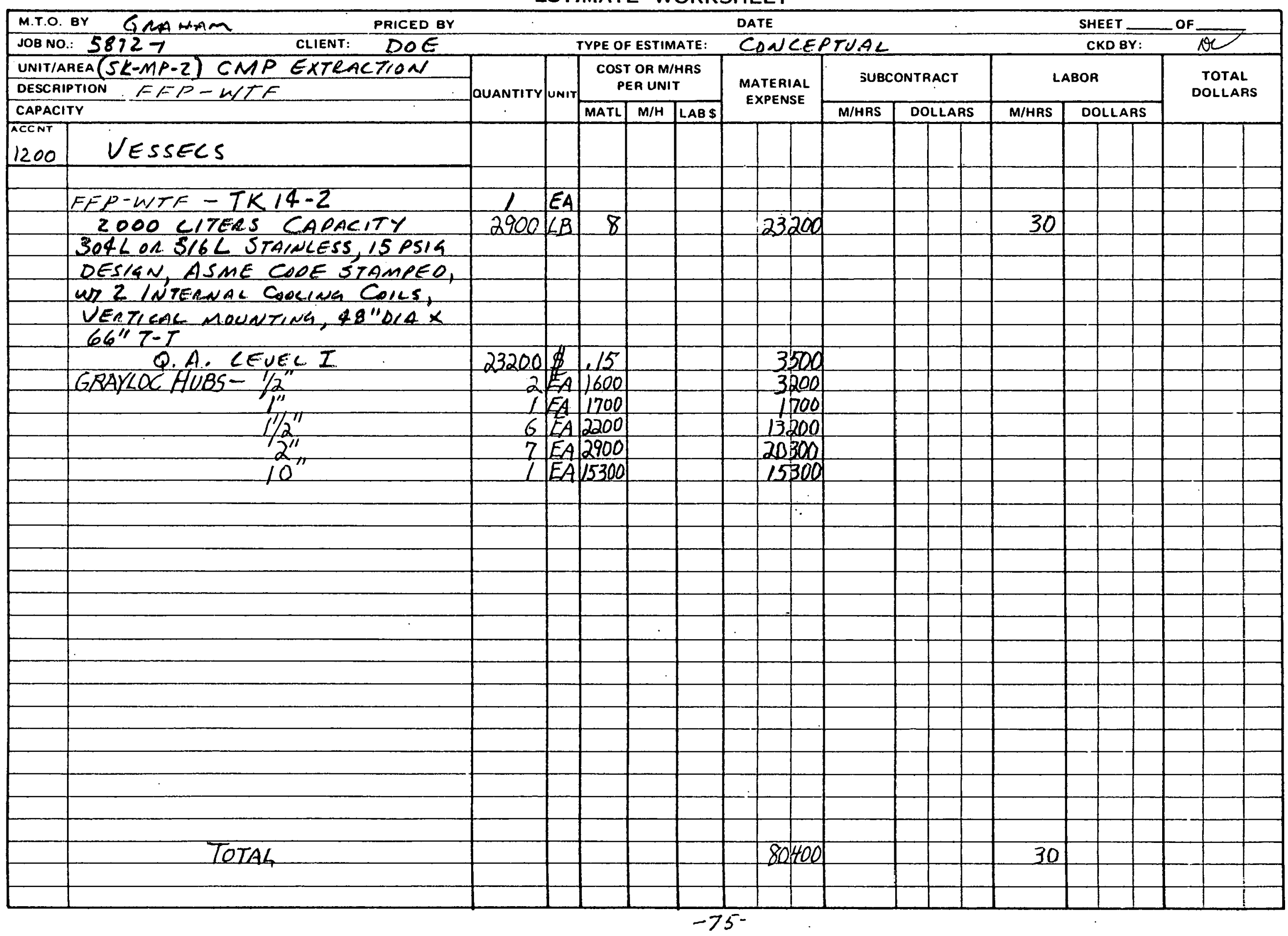


ESTIMATE WORKSHEET

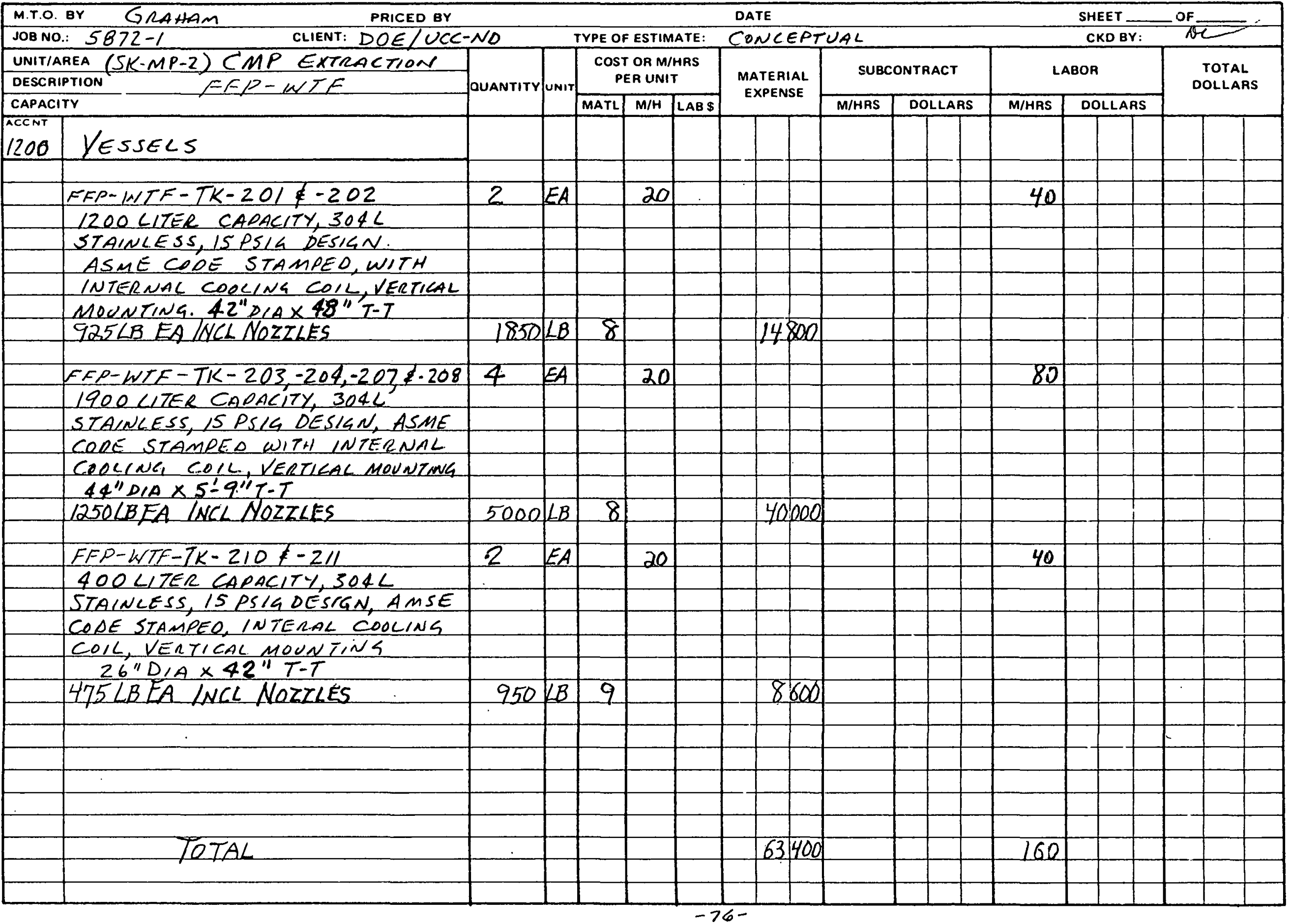


ESTIMATE WORKSHEET

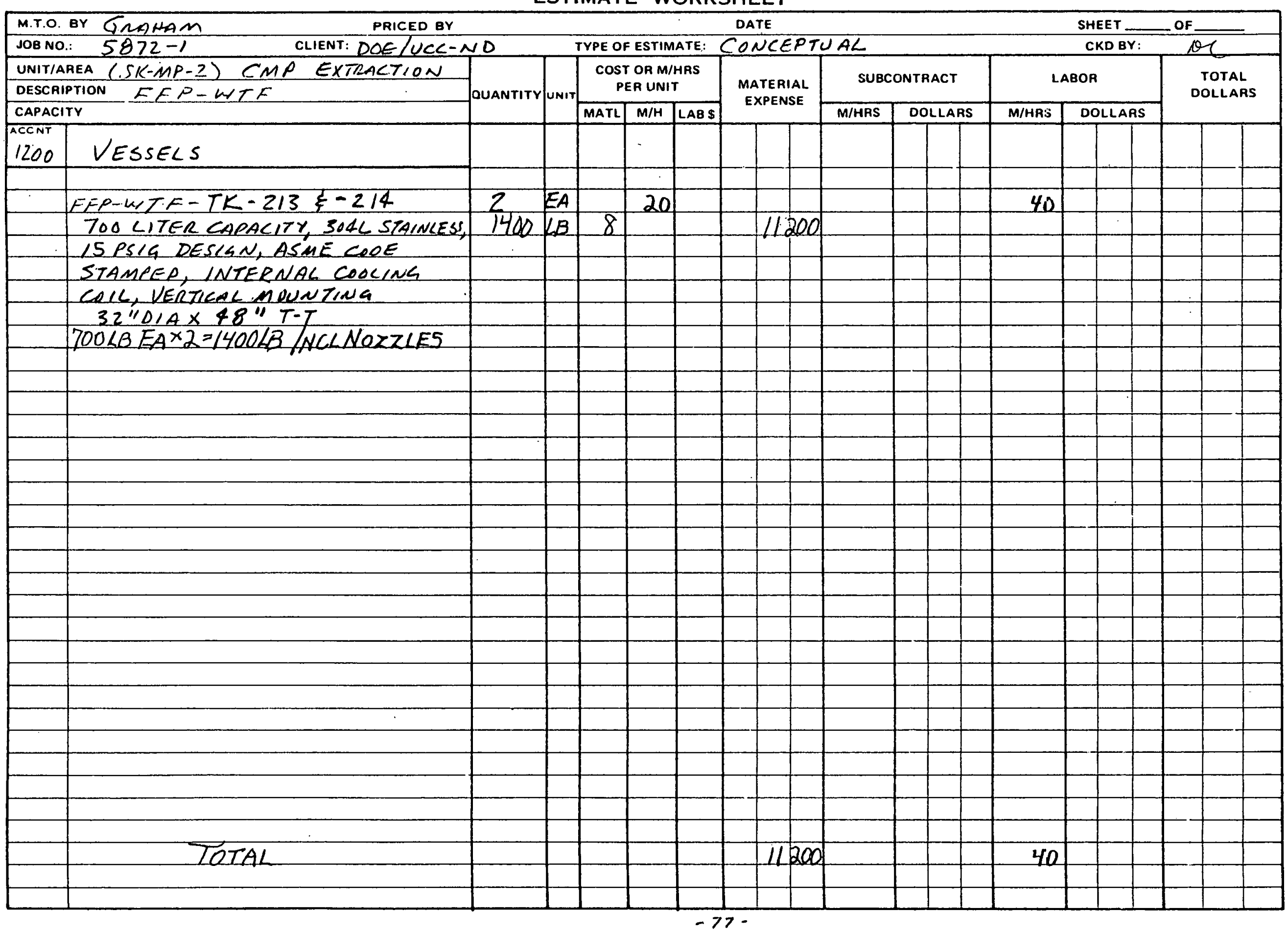


ESTIMATE WORKSHEET

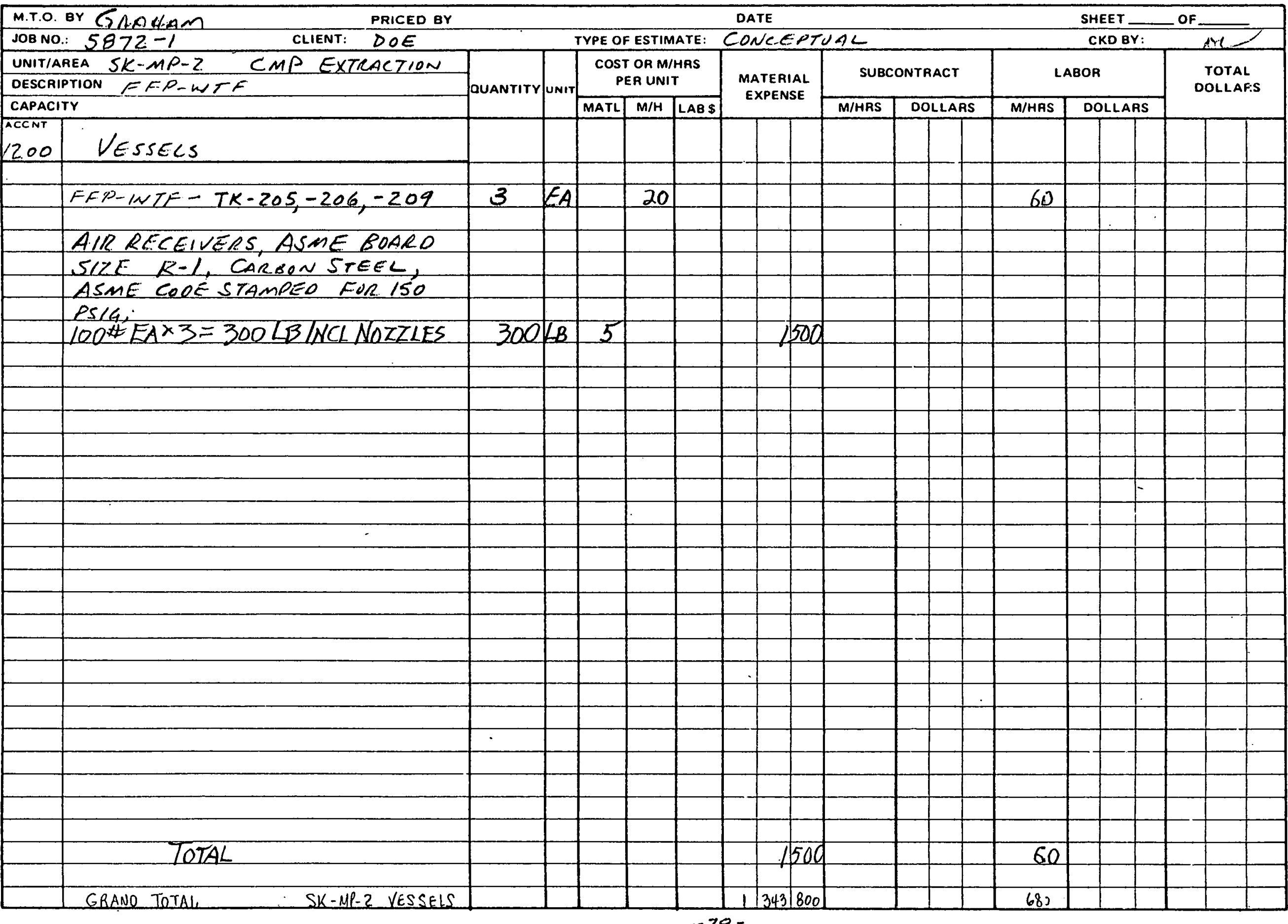


ESTIMATE WORKSHEET

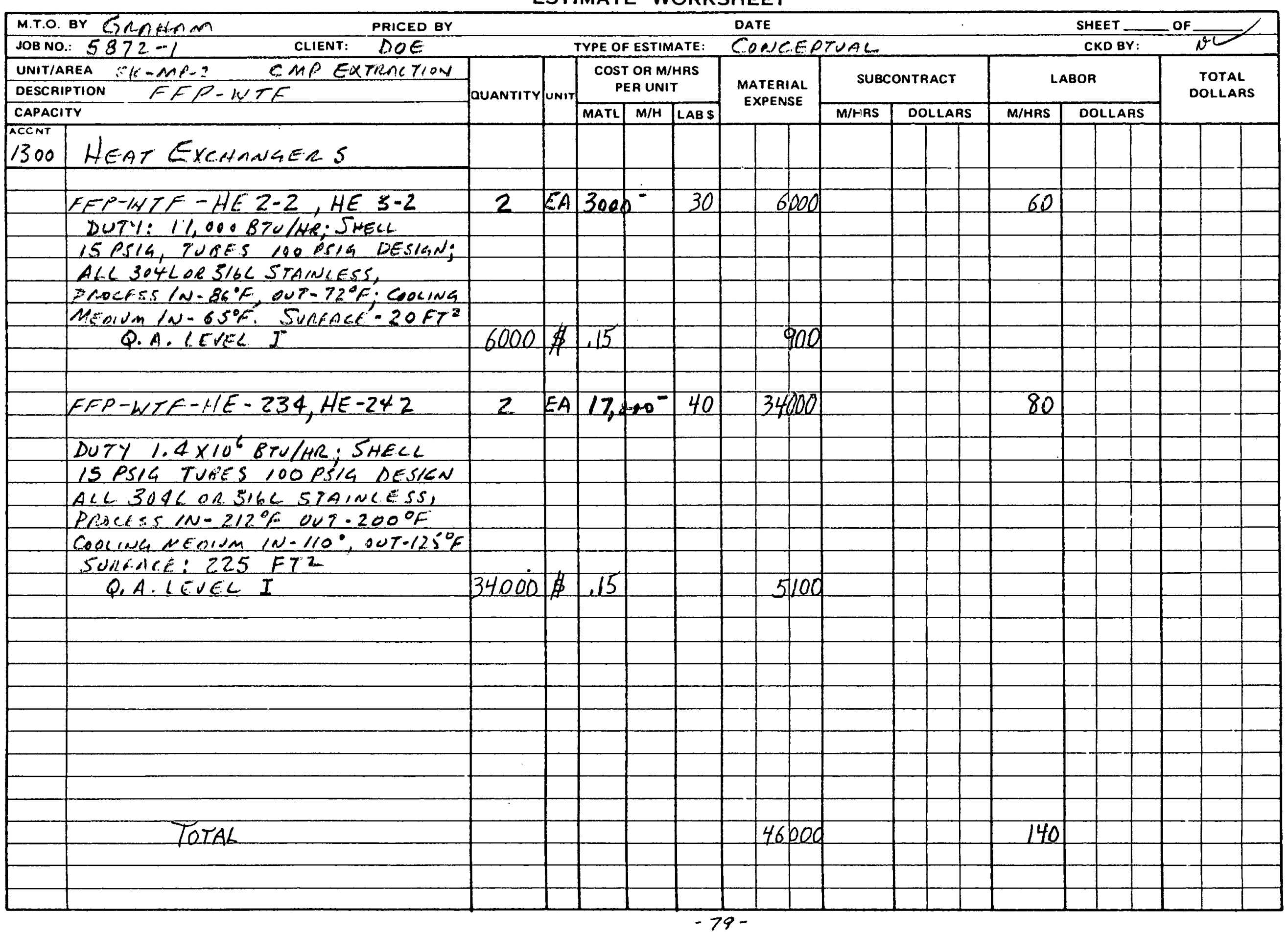


ESTIMATE WORKSHEET

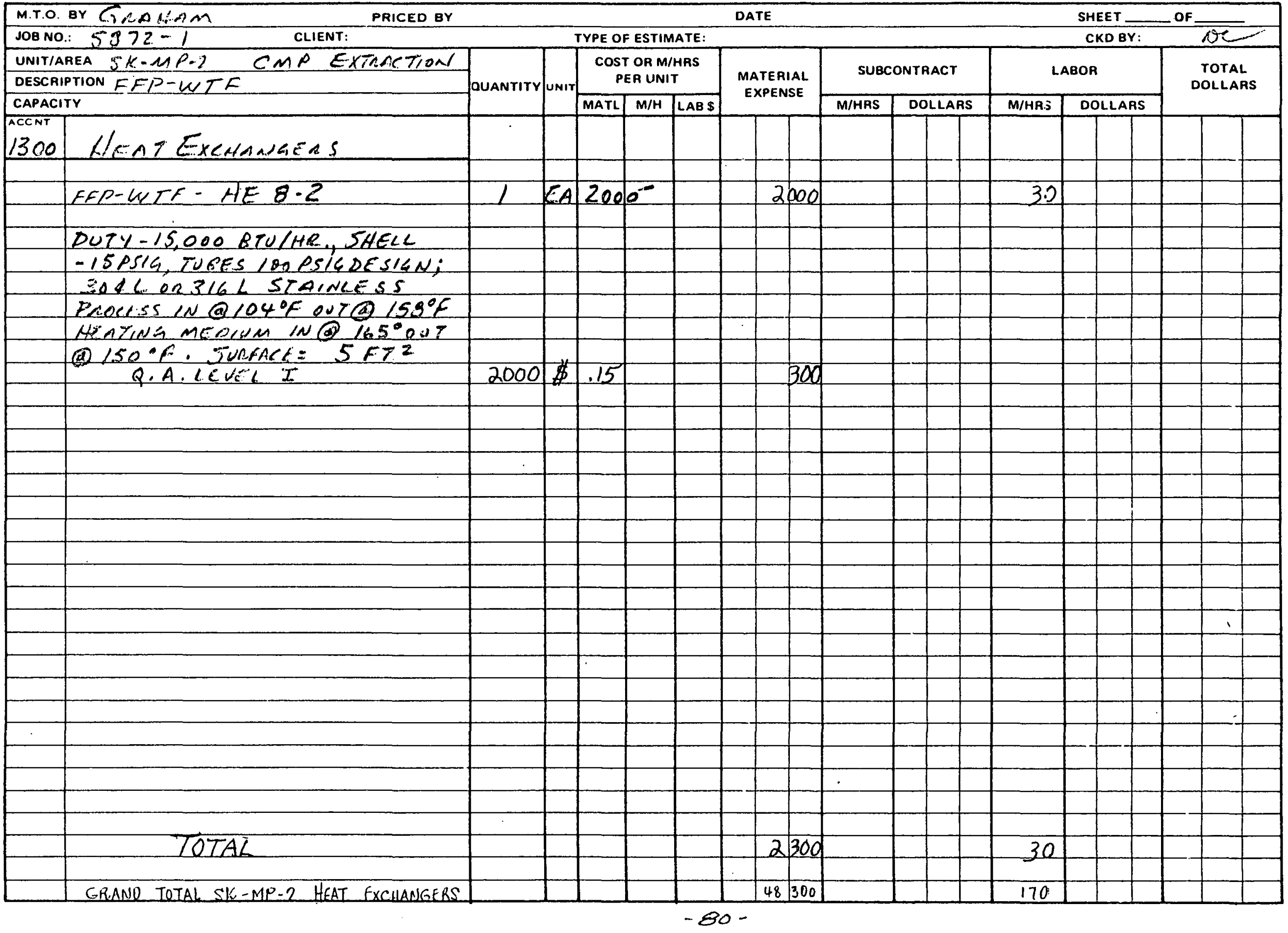


ESTIMATE WORKSHEET

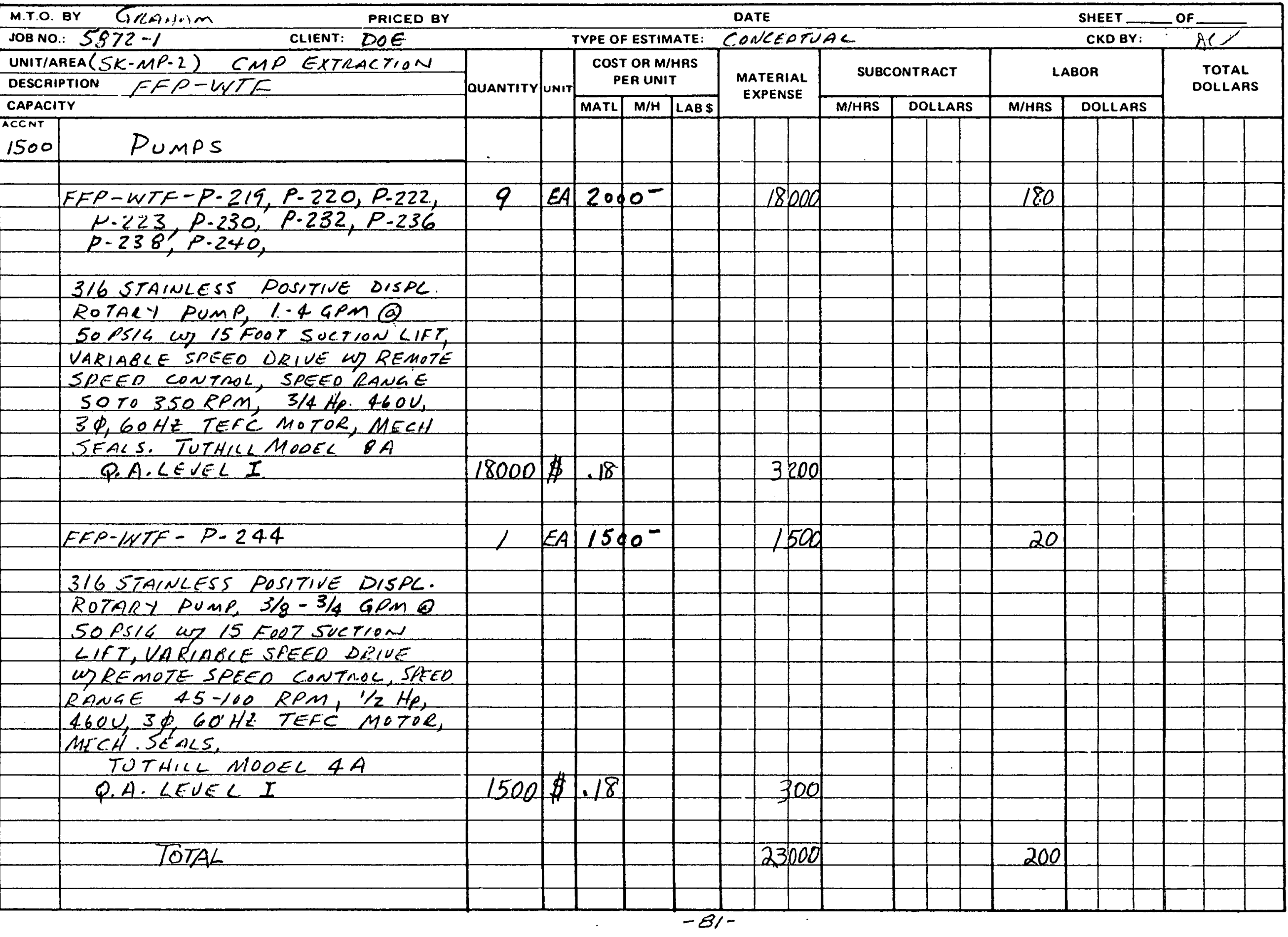


THE RALPH M. PARSONS COMPANY

ESTIMATE WORKSHEET

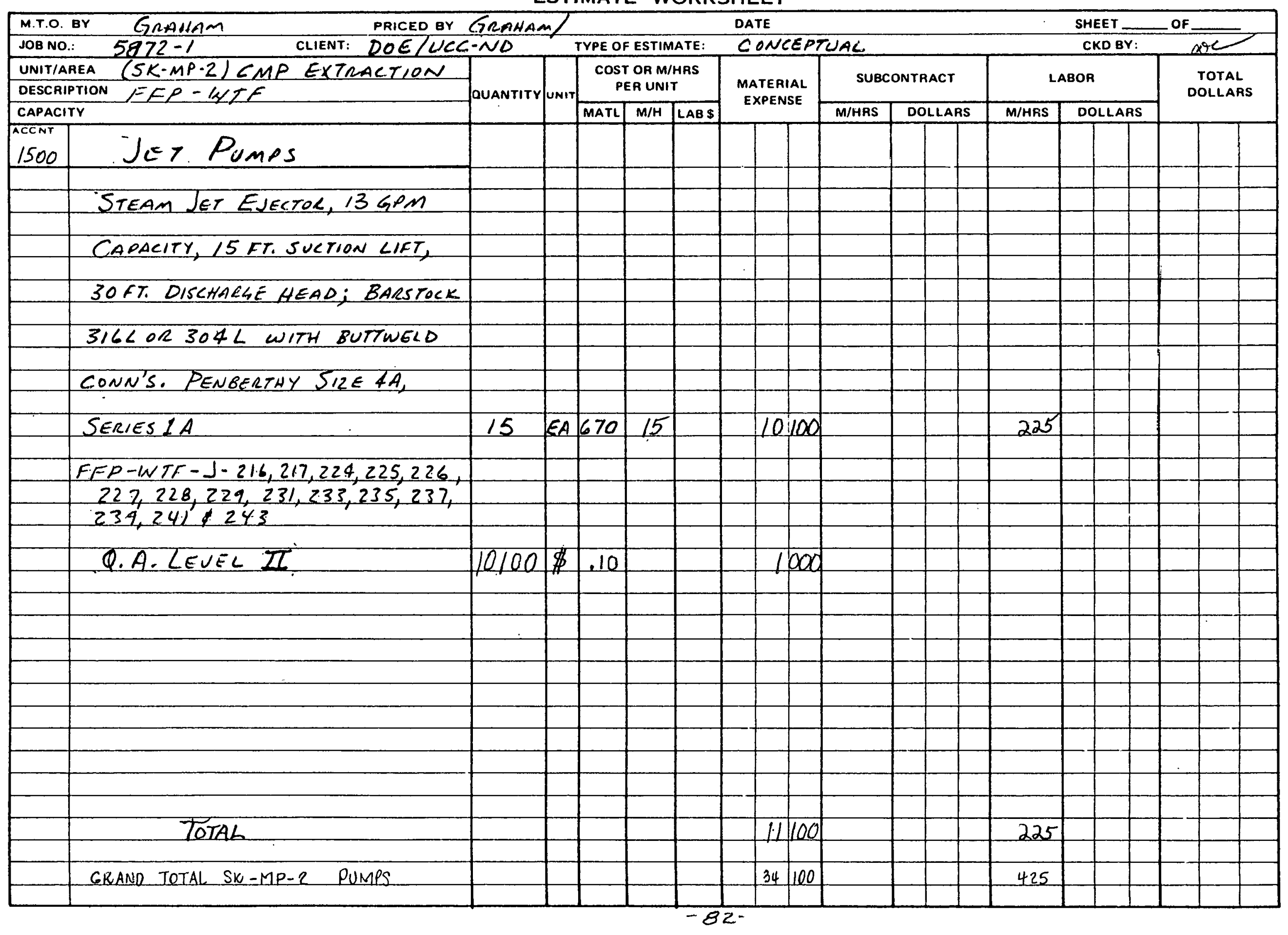


C-2000-0

THE RALPH M. PARSONS COMPANY

ESTIMATE WORKSHEET

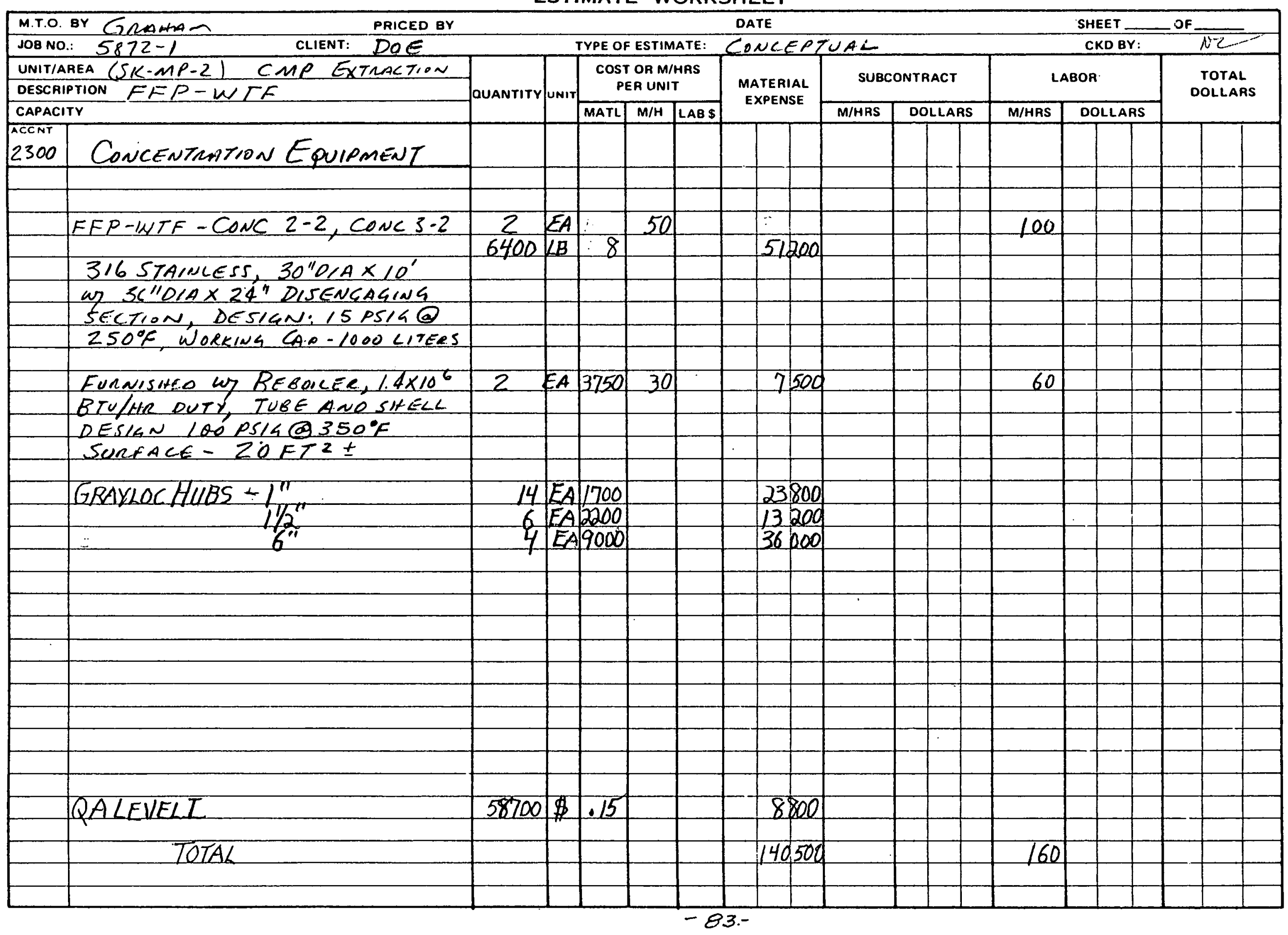


ESTIMATE WORKSHEET

\begin{tabular}{|c|c|c|c|c|c|c|c|c|c|c|c|c|c|c|c|c|}
\hline \multirow{2}{*}{\multicolumn{2}{|c|}{$\begin{array}{ll}\text { M.T.O. BY GRANAM } & \\
\text { JOB NO.: } S \& 72 \% & \text { PLICENT: }\end{array}$}} & & \multirow{2}{*}{\multicolumn{4}{|c|}{ TYPE OF ESTIMATE: }} & \multicolumn{5}{|l|}{ DATE } & \multicolumn{5}{|c|}{ SHEET__ OF } \\
\hline & & & & & & & & & & & & \multicolumn{3}{|c|}{ CKD BY: } & \multirow{3}{*}{\multicolumn{2}{|c|}{ 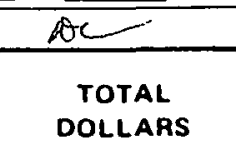 }} \\
\hline UNIT/A & REA SK:MP-2 CMP EXTRACTIOA & \multirow{3}{*}{ QUANTITY } & \multirow{3}{*}{ UNIT } & \multirow{2}{*}{\multicolumn{3}{|c|}{$\begin{array}{c}\text { COST OR M/HRS } \\
\text { PER UNIT }\end{array}$}} & \multirow{3}{*}{\multicolumn{2}{|c|}{$\begin{array}{l}\text { MATERIAL } \\
\text { EXPENSE }\end{array}$}} & \multirow{2}{*}{\multicolumn{3}{|c|}{ SUBCONTRACT }} & \multirow{2}{*}{\multicolumn{3}{|c|}{ LABOR }} & & \\
\hline \multicolumn{2}{|c|}{ DESCAIPTION FEP-WTE } & & & & & & & & & & & & & & & \\
\hline \multicolumn{2}{|c|}{ CAPACITY } & & & MATL & $M / H$ & LAB\$ & & & M/HF.S & \multicolumn{2}{|c|}{ DOLLARS } & \multirow[t]{2}{*}{ M/HRS } & \multicolumn{2}{|c|}{ DOLLARS } & 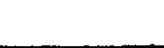 & \\
\hline $\begin{array}{l}\text { ACCNT } \\
24.00\end{array}$ & AlitATORS, MIXERS & & & & & & & & & & & & & & & \\
\hline & & & & & & & & & & & & & & & & \\
\hline & FFP-IN TF AG-245,-24G,-247, & 5 & $E A$ & 750 & -20 & & & 800 & & & & 100 & & & & \\
\hline & $-248-249$ & & & & & & & & & & & & & & & \\
\hline & $-2,1$ & & & & & & & & & & & & & & & \\
\hline & TOP ENTERING DUAL BLADEO & & & & & & & & & & & & & & & \\
\hline & AGITATOR, $304 L$ OR 3166 STAINLESS & & & & & & & & & & & & & & & \\
\hline & WETTEO PARTS, MOTOR IS / HP. & & & & & & & & & & & & & & & \\
\hline & $460 \mathrm{~V}, 3 \phi, 60 \mathrm{~Hz}$ TEFC & & & & & & & & & & & & & & & \\
\hline & CHEMINEFR MODEL VMN-10 & & & & & & & & & & & & & & & \\
\hline & Q.A.LEVEL II & & & & & & & & & & & & & & & \\
\hline & & & & & & & & & & & & & & & & \\
\hline & $F F P U T F-16-250$ & & & & & & & & & & & & & & & \\
\hline & & & & & & & & & & & & & & & & \\
\hline & TOP ENTEANG DUAL BLAOED & T & $E A$ & $500 t$ & $E$ & & & 500 & & & & 20 & & & & \\
\hline & AGITATOR SOHL OR 316L STAINLESS & & & & & & & & & & & & & & & \\
\hline & WETTEO PARTS, MOTUR IS $1 / 2 \mathrm{HP}$. & & & & & & & & & & & & & & & \\
\hline & $460 \mathrm{~V}, 3 \phi, 60 \mathrm{~Hz}$ TEFC & & & & & & & & & & & & & & & \\
\hline & CHE MINEER MODEL VHN-S & & & & & & & & & & & & & & & \\
\hline & Q.A.LEVEL II & & & & & & & & & & & & & & & \\
\hline & & & & & & & & & & & & & & & & \\
\hline & & & & & & & & & & & & & & & & \\
\hline & & & & & & & & & & & & & & & & \\
\hline & & & & & & & & & & & & & & & & \\
\hline & & & & & & & & & & & & & & & & \\
\hline & & & & & & & & & & & & & & & & \\
\hline & & & & & & & & & & & & & & & & \\
\hline & & & & & & & & & & & & & & & & \\
\hline & & & & & & & & & & & & & & & & \\
\hline & QA LEVELII & $430 d$ & & .10 & & & & 500 & & & & & & & & \\
\hline & & & & & & & & & & & & & & & & \\
\hline & TOTAL & & & & & & & 800 & & & & 120 & & & & \\
\hline & & & & & & & & & & & & & & & & \\
\hline & & & & & & & & & & & & & & & & \\
\hline
\end{tabular}


ESTIMATE WORKSHEET

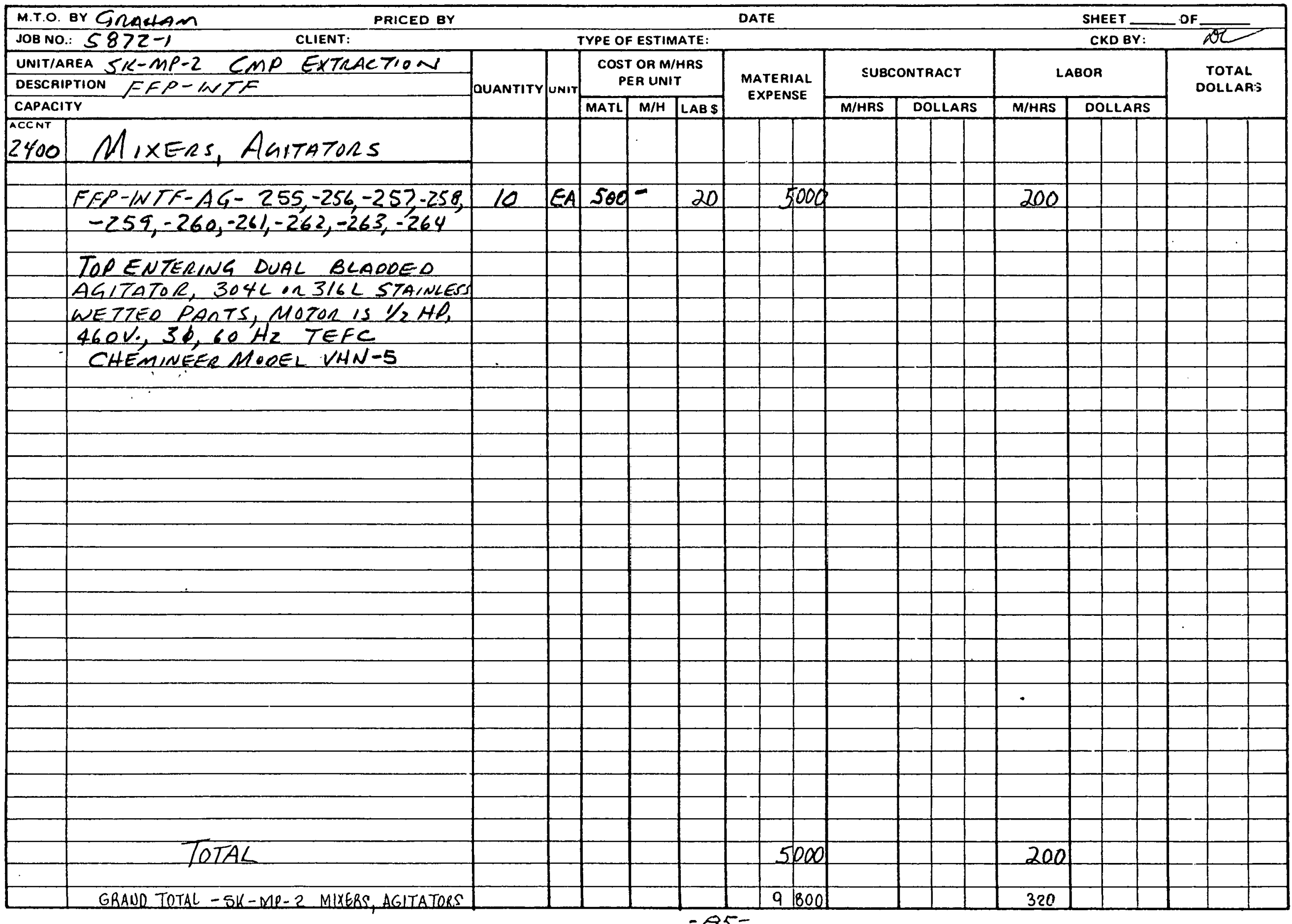


ESTIMATE WORKSHEET

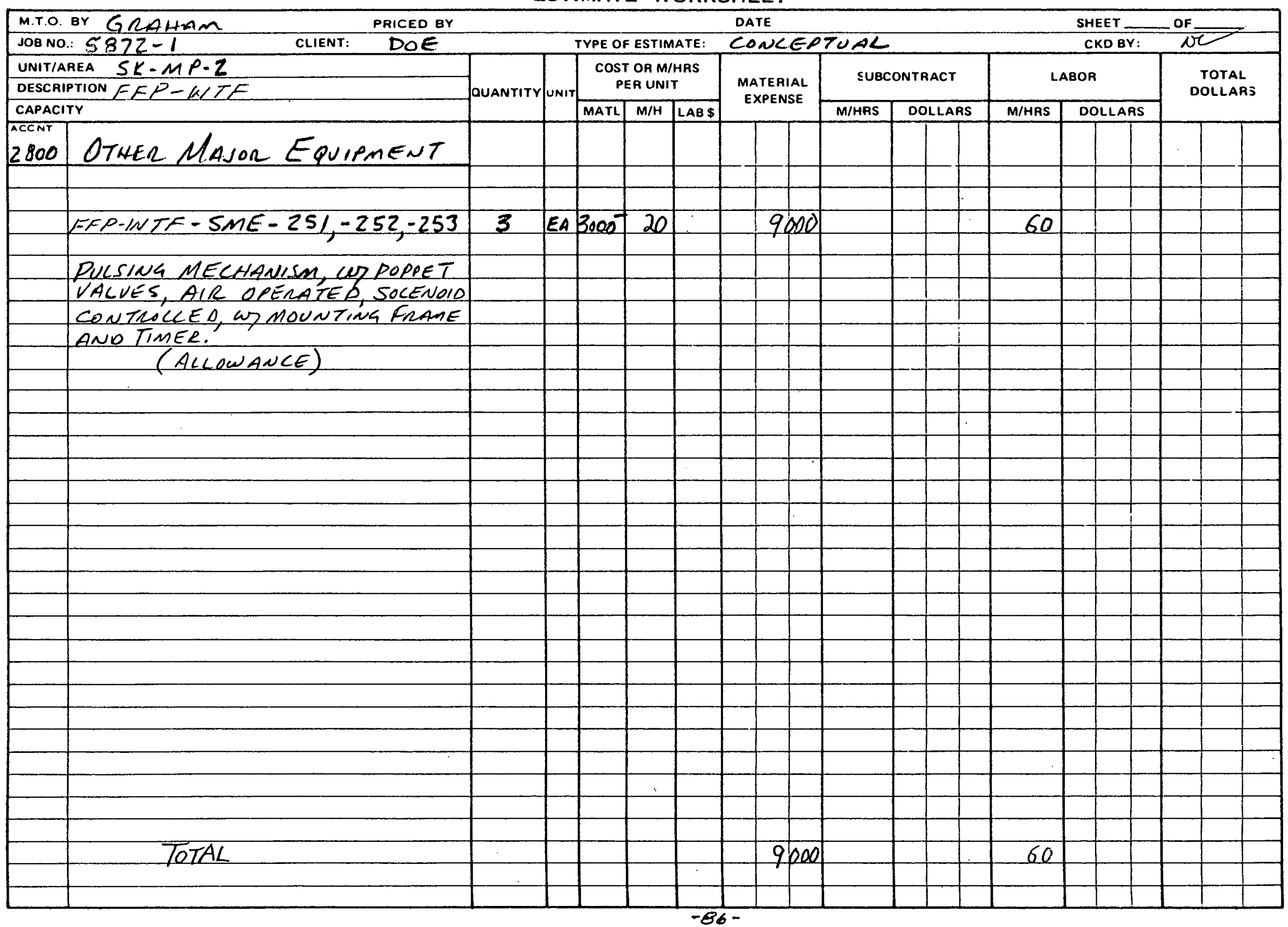


ESTIMATE WORKSHEET

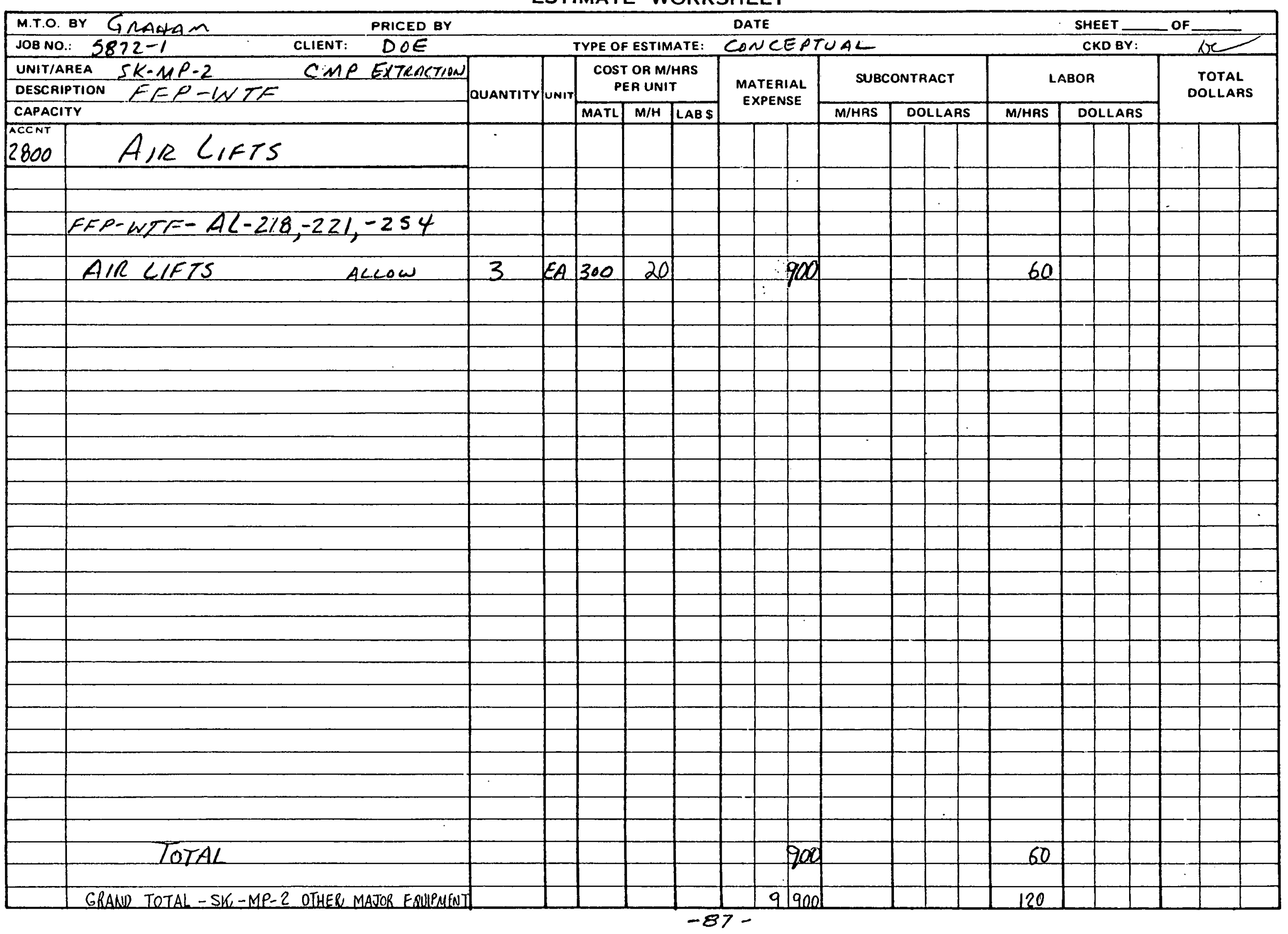




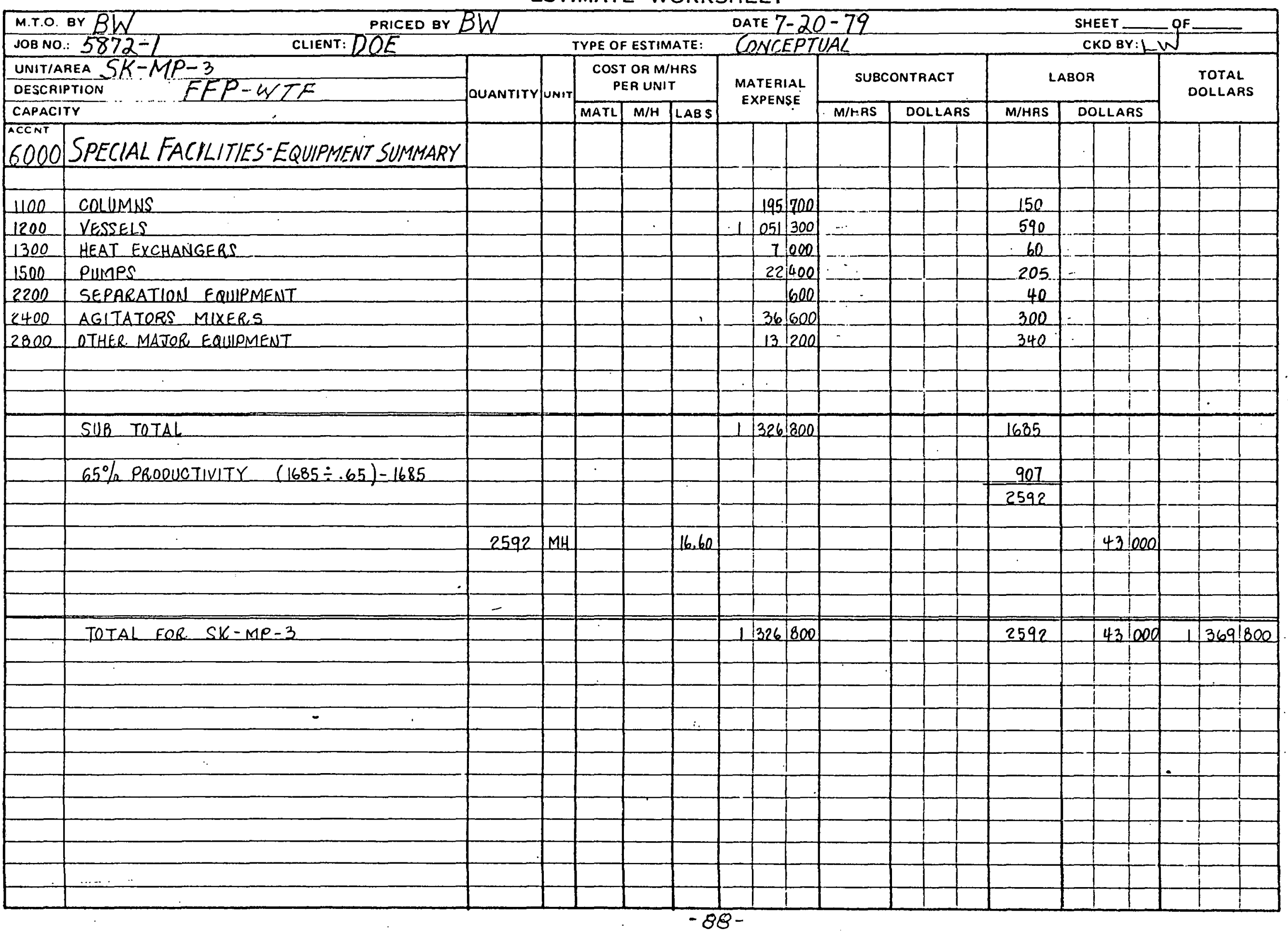


ESTIMATE WORKSHEET

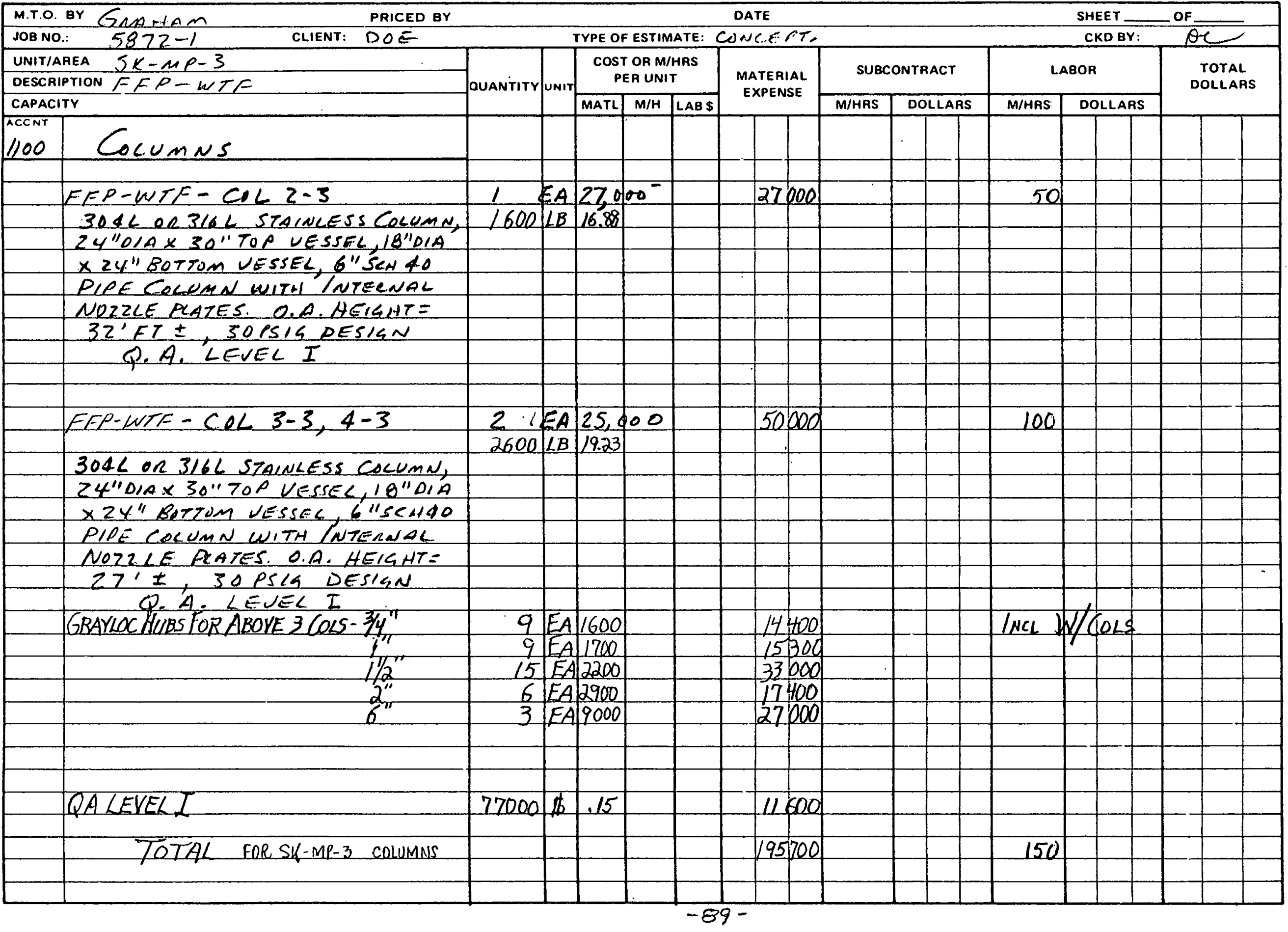


THE RALPH M. PARSONS COMPANY

ESTIMATE WORKSHEET

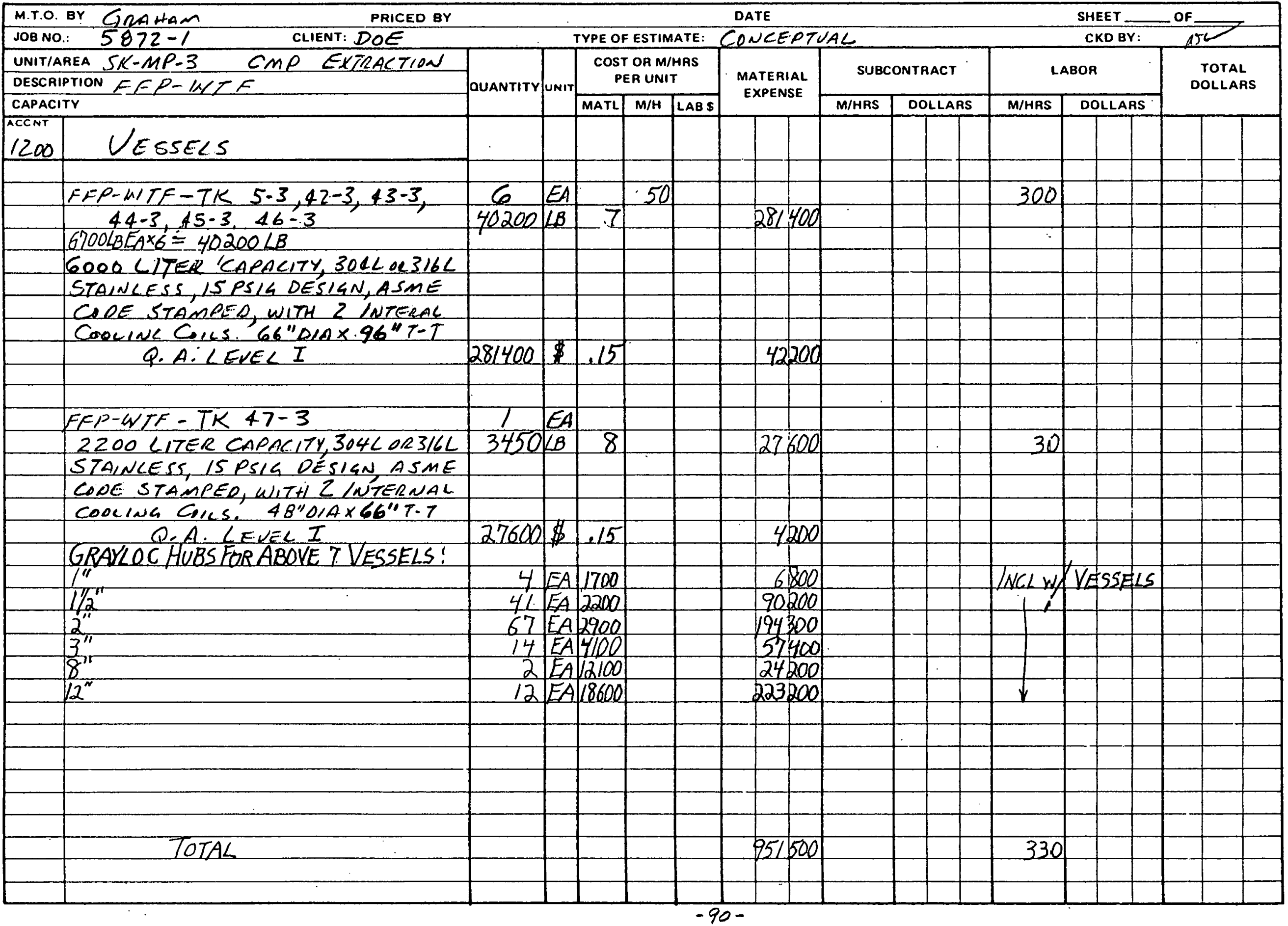


ESTIMATE WORKSHEET

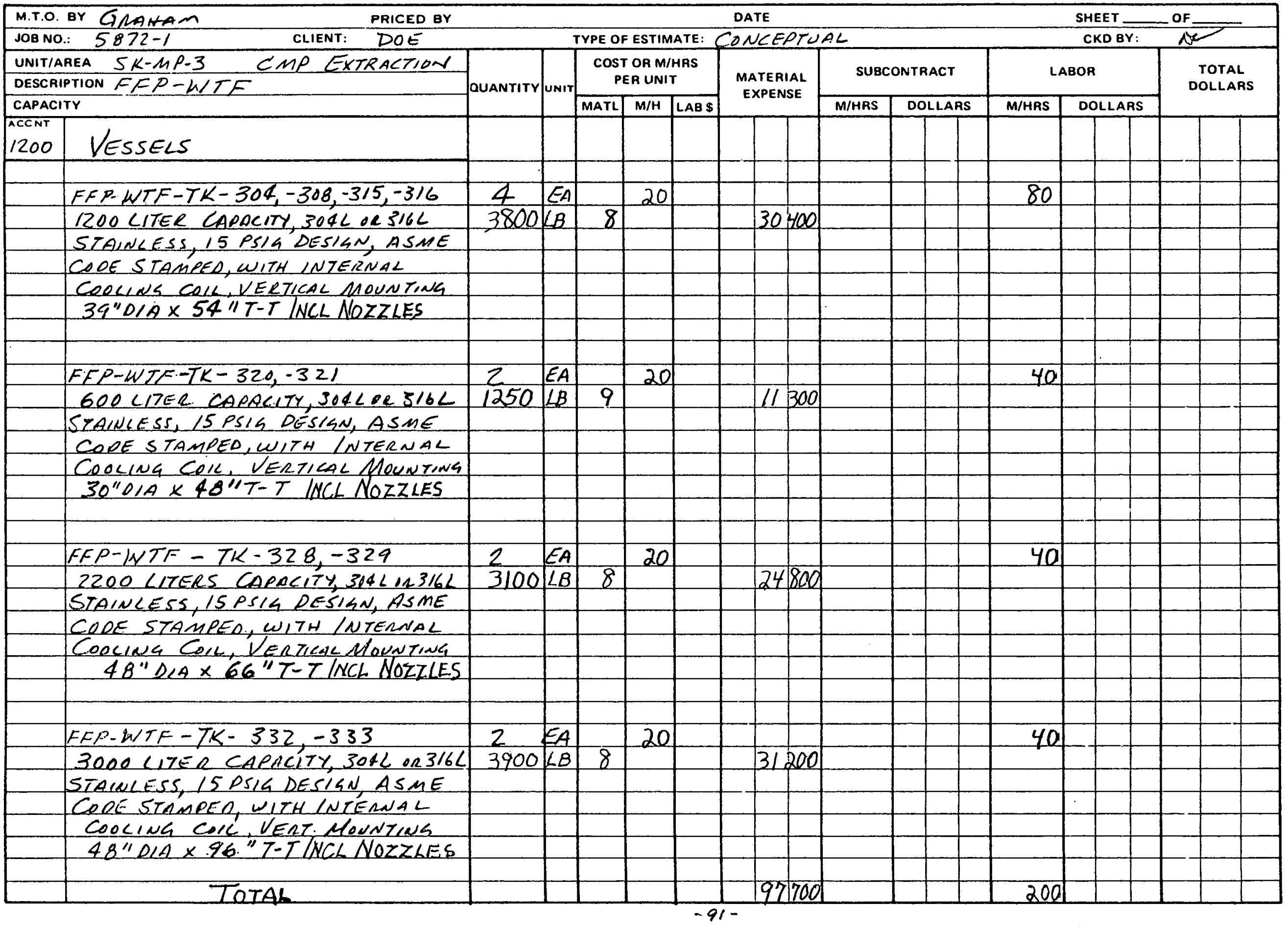


ESTIMATE WORKSHEET

\begin{tabular}{|c|c|c|c|c|c|c|c|c|c|c|c|c|c|c|c|c|c|c|}
\hline M.T.O. & BY GRANAM & & & & & & DA & & & & & & & & & EET & OF & \\
\hline JOB NC & $\therefore 5872-1$ & & & TYPE OI & ESTIM & IATE: & $\operatorname{Eon}$ & ICE & $P T$ & $\triangle A L$ & & & & & & KD BY: & $a$ & \\
\hline UNIT/A & CMP EXTRACTION & & & $\overline{\cos 1}$ & OR M/ & HRS & & & & suBC & ONTR & ACT & & & BOR & & & \\
\hline DESCR & PTION FFD-UVTF & DUANTITY & unit & & :R UNI & & & TERI & & & & & & & & & DOLLARS & \\
\hline CAPAC & & & & MATL & $M / H$ & LAB \$ & & & & M/HAS & & LLAR & & M/HRS & & DLLARS & & \\
\hline $\begin{array}{l}\overline{A C C N T} \\
1200\end{array}$ & VESSELS & & & & & & & & & & & & & & & & & \\
\hline & & & & & & & & & & & & & & & & & & \\
\hline & FEP-11TR-TK-335-337-339 & 3 & $E A$ & & 20 & & & & & & & & & 60 & & & & \\
\hline & & 300 & $\angle B$ & 7 & & & & & 100 & & & & & & & & & \\
\hline & AIR RECEIVER ASME BOARD & & & & & & & & & & & & & & & & & \\
\hline & SIRE R.L CARBON STEEL, & & & & & & & & & & & & & & & & & \\
\hline & ASME CDQE STAMPEO FOR 150 & & & & & & & & & & & & & & & & & \\
\hline & $P S / G, 100 \angle B \times 3=300 \angle B / N C L$ NOZZLES & & & & & & & & & & & & & & & & & \\
\hline & & & & & & & & & & & & & & & & & & \\
\hline & & & & & & & & & & & & & & & & & & \\
\hline & & & & & & & & & & & & & & & & & & \\
\hline & $\therefore$ & & & & & & & & & & & & & & & & & \\
\hline & $\therefore$ & & & & & & & & & & & & & & & & & \\
\hline & & & & & & & & & & & & & & & & & & \\
\hline & & . & & & & & & & & & & & & & & & & \\
\hline & & & & & & & & & & & & & & & & & & \\
\hline & $\therefore$ & & & & & & & & & & & & & & & & & \\
\hline & & & & & & & & & & & & & & & & & & \\
\hline & & & & & & & & & & & & & & & & & & \\
\hline & & & & & & & & & & & & & & & & & & \\
\hline & & & & & & & & & & & & & & & & & & \\
\hline & & & & & & & & & & & & & & & & & & \\
\hline & & & & & & & & & & & & & & & & & & \\
\hline & & & & & & & & & & & & & & & & & & \\
\hline & & & & & & & & & & & & & & & & & & \\
\hline & & & & & & & & & & & & & & & & & & \\
\hline & & & & & & & & & & & & & & & & & & \\
\hline & & & & & & & & & & & & & & & & & & \\
\hline & & & & & & & & & & & & & & & & & & \\
\hline & & & & & & & & & & & & & & & & & & \\
\hline & & & & & & & & & & & & & & & & & & \\
\hline & TOTAL & & & & & & & & 100 & & & & & 60 & & & & \\
\hline & & & & & & & & & & & & & & & & & & \\
\hline & GRAND TOTAL SIL-MP-3 VESSEIS & & & & & & $\perp$ & 051 & 300 & & & & & 59i) & & & & \\
\hline
\end{tabular}


ESTIMATE WORKSHEET

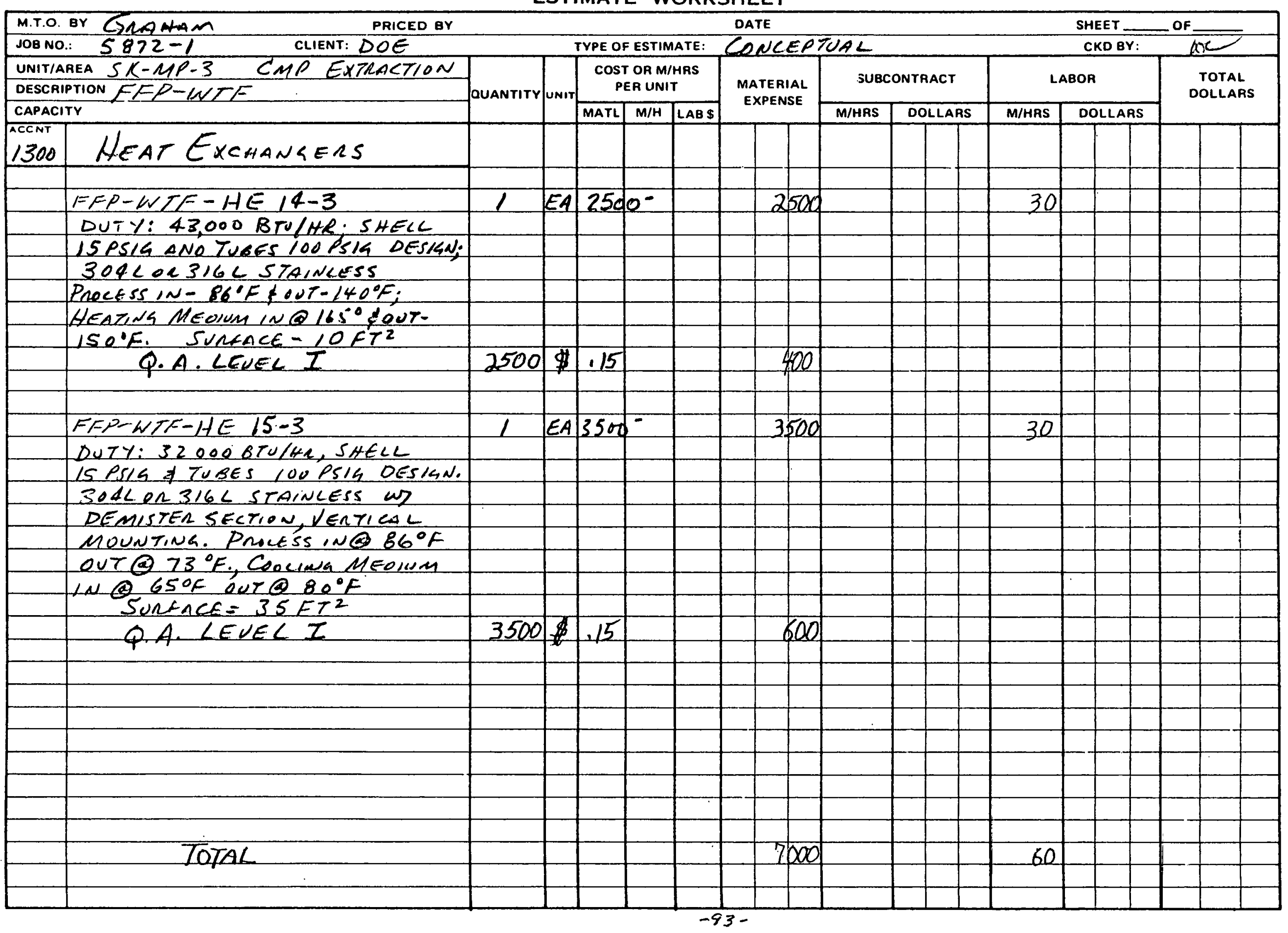


ESTIMATE WORKSHEET

\begin{tabular}{|c|c|c|c|c|c|c|c|c|c|c|c|c|c|c|c|}
\hline M.T.O. & BY. GMANA & & & & & & DATE & & & & & SHEET & 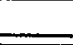 & of & \\
\hline JOB NO & $\therefore \quad 5872-1$ & & & TYPE OF & FESTIM & ATE: & CONCEPTUA & & & & & CKD BY: & & 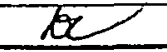 & \\
\hline UNIT/A & AEA $5 K-M P-3$ CMP EXTRACTION & & & & OR M & & & SUBC & NTRACT & & & BOR & & & \\
\hline DESCAI & PTION FEP-WTE & QUANTITY & 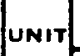 & & ER UNI & & $\begin{array}{l}\text { MATERIAL } \\
\text { EXPENSE }\end{array}$ & & & & & & & DOLLARS & \\
\hline CAPACI & & & & MATL & $\mathbf{M} / \mathbf{H}$ & LABS & & M/T.RS & DOLLAA & & M/HAS & DOLLAAS & & & \\
\hline $\begin{array}{l}\text { ACCNT } \\
1500\end{array}$ & Pumps & & & & & & & & & & & & & & \\
\hline & & & & & & & & & & & & & & & \\
\hline & & & & & & & & & & & & & & & \\
\hline & FFP-WTF $-P-302,-303$ & 2 & $E A$ & 250 & 0 & & 5000 & & & & 40 & & & & \\
\hline & & & & & & & & & & & & & & & \\
\hline & & & & & & & & & & & & & & & \\
\hline & 316 STAINLESS POSITIVE DISAKACE. & & & & & & & & & & & & & & \\
\hline & MENT ROTARY PUMP, 6-14 GPM & & & & & & & & & & & & & & \\
\hline & Q8 50 PSIG WITH 15 FOST SU $\angle T I O N$ & & & & & & & & & & & & & & \\
\hline & LIFT, VARAABCE SPEEO DRIVE UP & & & & & & & & & & & & & & \\
\hline & REMOTE SPEEO CONTNOL, SPEED & & & & & & & & & & & & & & \\
\hline & RANGE-230TS500RPM, / HP, & & & & & & & & & & & & & & \\
\hline & $460 \mathrm{~V}, 3 \& 60 \mathrm{HL}$ TEFC MOTOR & & & & & & & & & & & & & & \\
\hline & MECAI. SEALS & & & & & & & & & & & & & & \\
\hline & TUTHICL MOPEL $15 \mathrm{~A}$ & & & & & & & $\dot{5}$ & & & & & & & \\
\hline & Q.A.LEVELI I & 5000 & $\$$ & 18 & & & 900 & & & & & & & & \\
\hline & & & & & & & & & & & & & & & \\
\hline & & & & & & & & & & & & & & & \\
\hline & EFP-NTF-P-312,-318-319-324 & 4 & EA & 200 & $0^{-}$ & & 8000 & & & & 80 & & & & \\
\hline & , & & & & & & & & & & & & & & \\
\hline & 316 STAINLESS POSITIVE DISPLACEMENT & & & & & & & & & & & & & & \\
\hline & ROTARY PUMP $1-4$ GPM@ 50 & & & & & & & & & & & & & & \\
\hline & PSIG WITH 15 FOOT SUCTION LIFT, & & & & & & & & & & & & & & \\
\hline & VARIARCE SPEEO DRIVE US RENATE & & & & & & & & & & & & & & \\
\hline & SNEED CONTROL SPEEO RANGE & & & & & & & & & & & & & & \\
\hline & so ro $350 \mathrm{RPM}, 3 / 4 \mathrm{He}, 460 \mathrm{~V}, 3 \mathrm{C}_{1}$ & & & & & & & & & & & & & & \\
\hline & GOHL TEFC MOTOR MECH. & & & & & & & & & & & & & & \\
\hline & SEALS. & & & & & & & & & & & & & & \\
\hline & TuTHILL MOOEL BA & & & & & & & & & & & & & & \\
\hline & Q.A.LEVELI & 8000 & $\not$ & 18 & & & 1500 & & & & & & & & \\
\hline & & & & & & & & & & & & & & & \\
\hline & Torah & & & & & & 15400 & & & & 120 & & & & \\
\hline & & & & & & & & - & & & & & & & \\
\hline & & & & & & & & & & & & & & & \\
\hline
\end{tabular}


ESTIMATE WORKSHEET

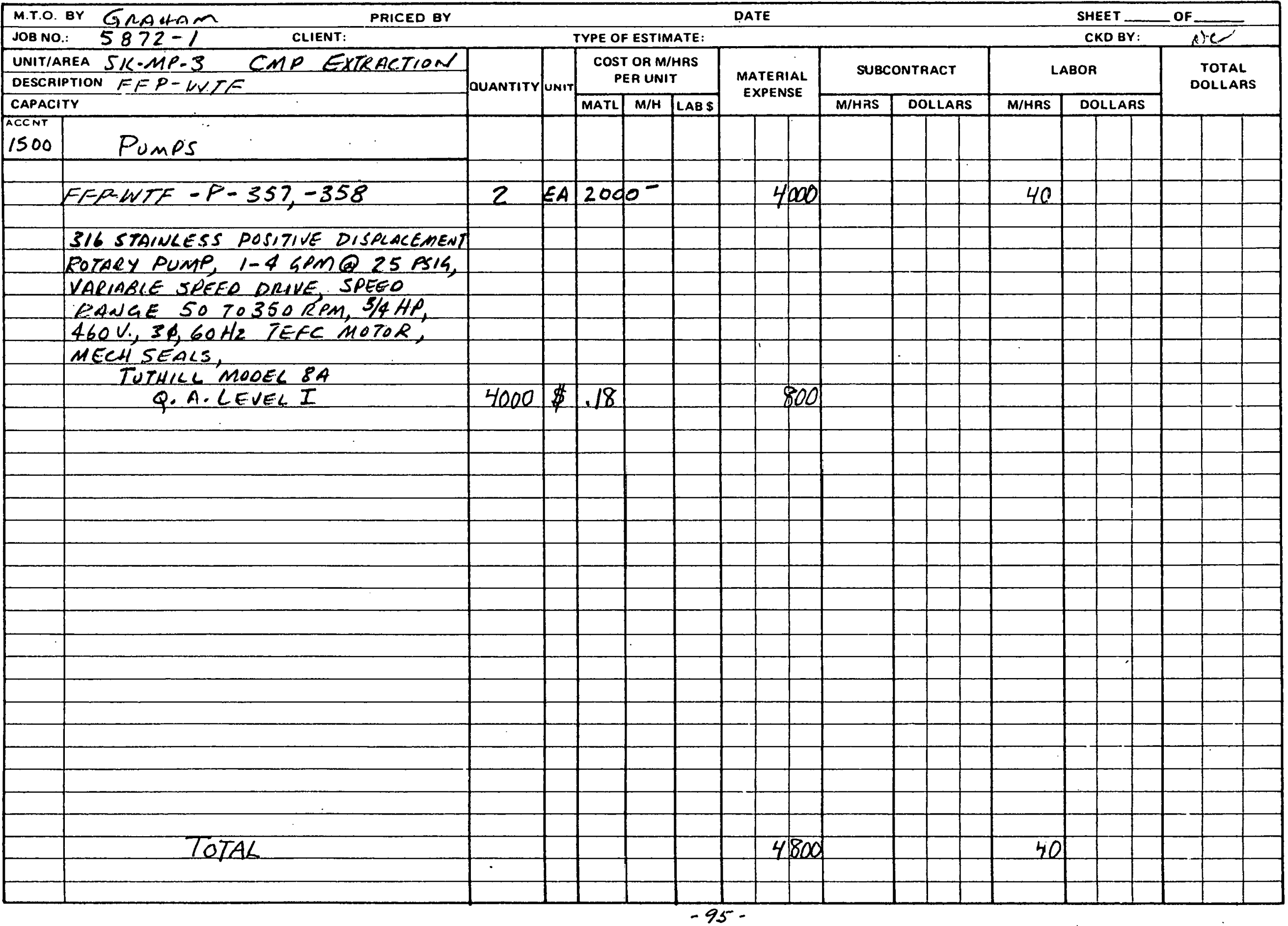


ESTIMATE WORKSHEET

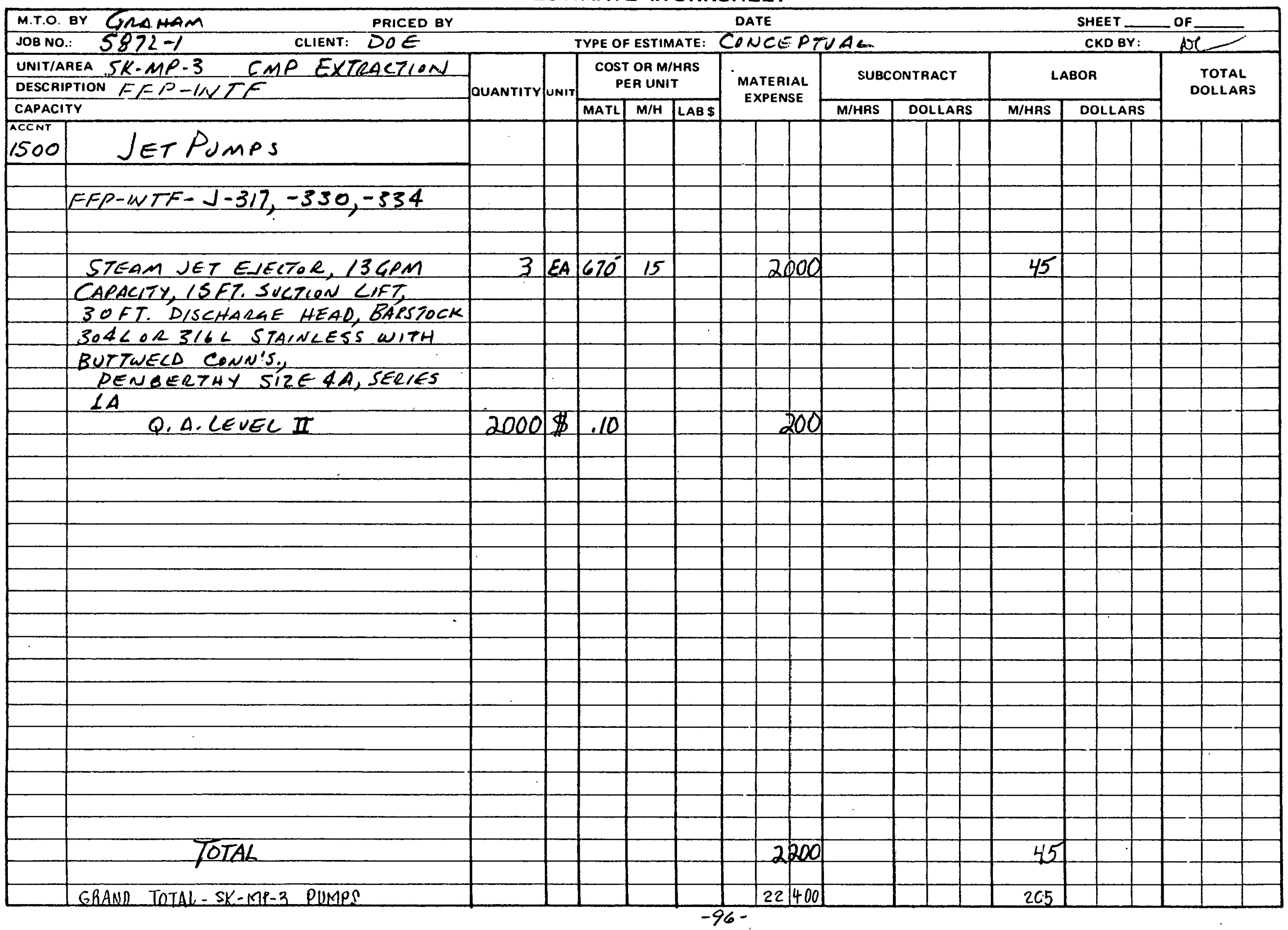


THE RALPH M. PARSONS COMPANY

ESTIMATE WORKSHEET

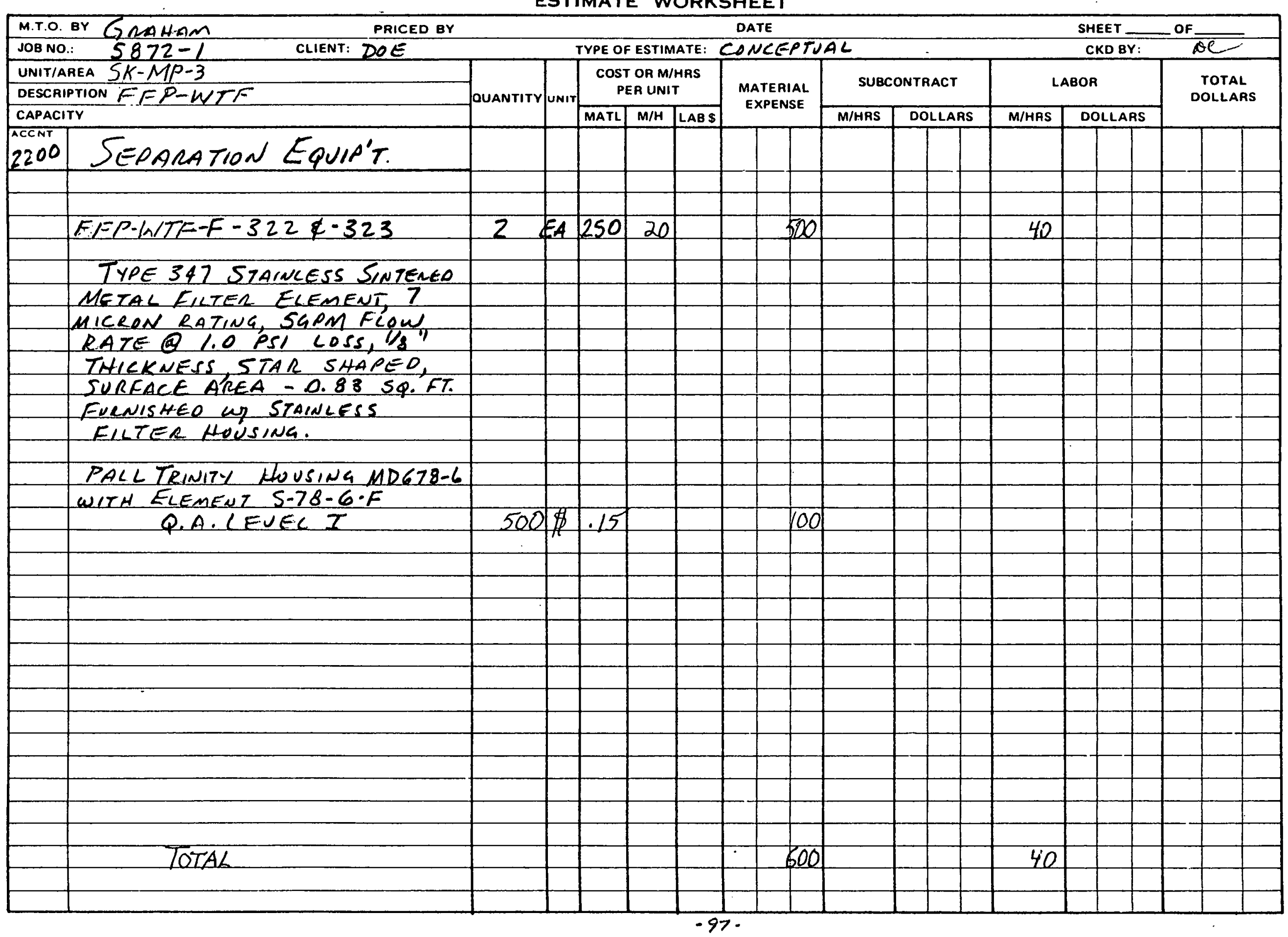


THE RALPH M. PARSONS COMPANY

ESTIMATE WORKSHEET

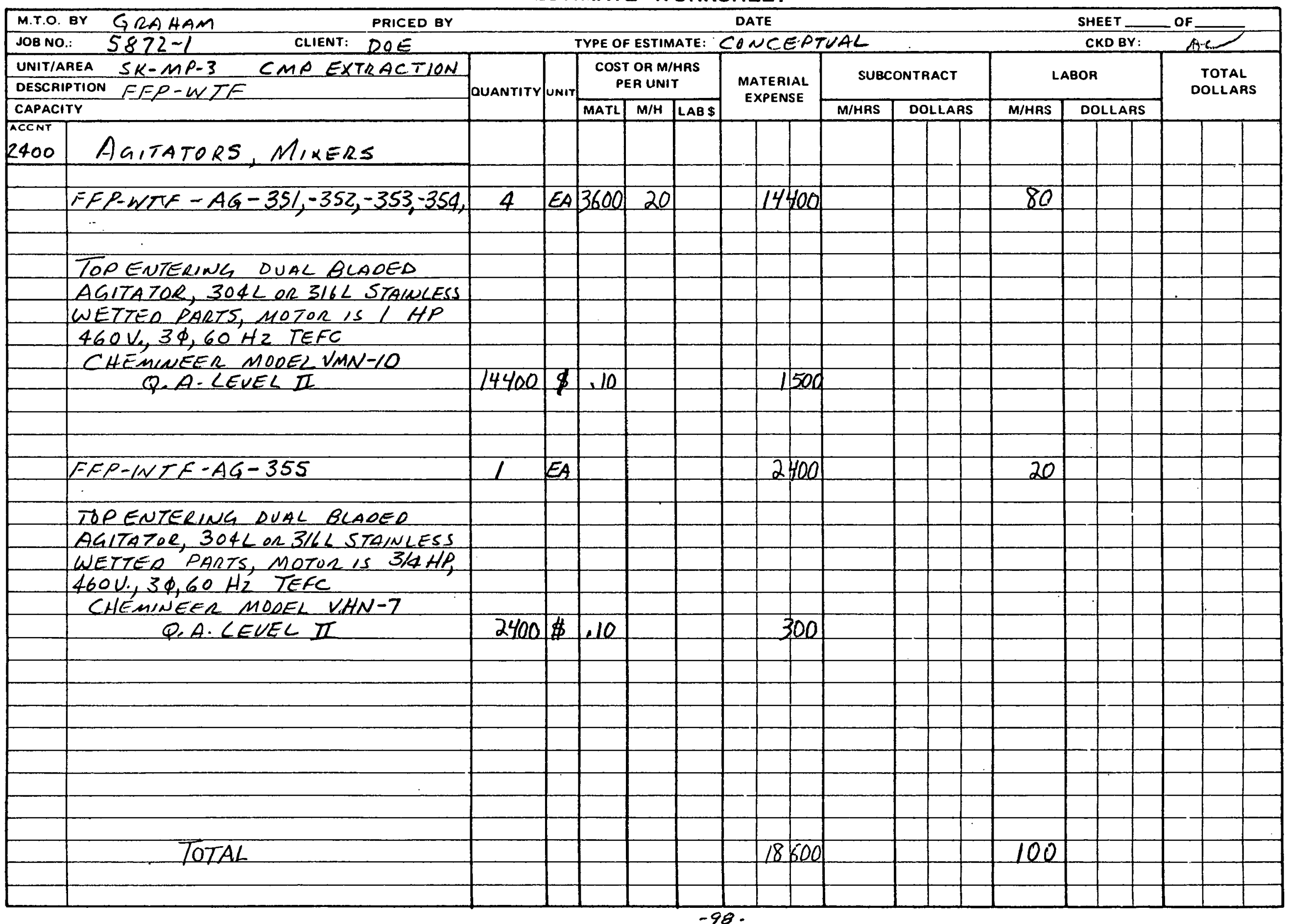


ESTIMATE WORKSHEET

\begin{tabular}{|c|c|c|c|c|c|c|c|c|c|c|c|c|c|c|c|c|}
\hline \multicolumn{3}{|c|}{ M.T.O. BY GNAWAM $\quad$ PRICED BY } & \multicolumn{9}{|c|}{ DATE } & \multicolumn{4}{|c|}{  } & \\
\hline \multicolumn{3}{|c|}{ JOBNO:: $5872-1 \quad$ CLIENT: DOE } & \multicolumn{9}{|c|}{ TYPE OF ESTIMATE: CONCEPTUAL } & \multicolumn{3}{|c|}{ CKD BY: } & \multirow{4}{*}{\multicolumn{2}{|c|}{$\begin{array}{l}\text { TOTAL } \\
\text { DOLLARS }\end{array}$}} \\
\hline UNIT/A & CMP EXTRACTION & \multirow{4}{*}{ QuANTITY } & \multirow{4}{*}{ UNIT } & \multirow{2}{*}{\multicolumn{3}{|c|}{$\begin{array}{l}\text { COST OR M/HAS } \\
\text { PER UNIT }\end{array}$}} & \multirow{3}{*}{\multicolumn{2}{|c|}{$\begin{array}{l}\text { MATERIAL } \\
\text { EXPENSE }\end{array}$}} & \multirow{2}{*}{\multicolumn{3}{|c|}{ SUBCONTAACT }} & \multirow{2}{*}{\multicolumn{3}{|c|}{ LABOR }} & & \\
\hline \multirow{2}{*}{\multicolumn{2}{|c|}{$\begin{array}{l}\text { DESCRIPTION FFP-WTF } \\
\text { CAPACITY }\end{array}$}} & & & & & & & & & & & & & & & \\
\hline & & & & \multirow[t]{2}{*}{ MATL } & \multirow[t]{2}{*}{$\mathrm{M} / \mathrm{H}$} & \multirow[t]{2}{*}{ LAB \$ } & & & \multirow[t]{2}{*}{ M/HRS } & \multicolumn{2}{|c|}{ DOLLARS } & \multirow[t]{2}{*}{ M/HFS } & \multicolumn{2}{|c|}{ DOLLARS } & & \\
\hline $\begin{array}{l}\text { ACCNT } \\
2400\end{array}$ & AGitatons Mixers & & & & & & & & & & & & & & & \\
\hline & & & & & & & & & & & & & & & & \\
\hline & $F F P-W T F-A G-341,-342,-343$ & 4 & $E A$ & 1500 & 20 & & & 000 & & & & 80 & & & & \\
\hline & -344 & & & & & & & & & & & & & & & \\
\hline & & & & & & & & & & & & & & & & \\
\hline & DUAL BLARED & & & & & & & & & & & & & & & \\
\hline & AGITATOR, SOLLOR $316 C$ STAINLESS & & & & & & & & & & & & & & & \\
\hline & WETTED PARTS, MOTOR IS $1 / 2 \mathrm{HP}$, & & & & & & & & & & & & & & & \\
\hline & $460 \mathrm{~V}, 3 \phi, 60 \mathrm{~Hz}$ TEFC & & & & & & & & & & & & & & & \\
\hline & CHEMINEER MOOEL VHN-5 & & & & & & & & & & & & & & & \\
\hline & & & & & & & & & & & & & & & & \\
\hline & & & & & & & & & & & & & & & & \\
\hline & & & & & & & & & & & & & & & & \\
\hline & & & & & & & & & & & & & & & & \\
\hline & $E F P-4 / T E-A G-345,-346$ & 2 & $E A$ & 1200 & 20 & & & 400 & & & & 40 & & & & \\
\hline & & & & & & & & & & & & & & & & \\
\hline & & & & & & & & & & & & & & & & \\
\hline & TOPENTERINA DUAL BLAREO & & & & & & & & & & & & & & & \\
\hline & AGITATOR, $304 L O R$ B/6L STAINLESS & & & & & & & & & & & & & & & \\
\hline & WETTEO PARTS, MOTOR IS $1 / 4 \mathrm{HP}$. & & & & & & & & & & & & & & & \\
\hline & $110 \mathrm{~V}, 1 \phi, 60 \mathrm{~Hz}$ TEFC & & & & & & & & & & & & & & & \\
\hline & CAEMINEER MODEL VHN-2 & & & & & & & & & & & & & & & \\
\hline & & & & & & & & & & & & & & & & \\
\hline & & & & & & & & & & & & & & & & \\
\hline & $E F P-W T F-A G-347,-348,-349$ & 4 & $E A$ & 2400 & 20 & & & 600 & & & & 80 & & & & \\
\hline & -550 & & & & & & & & & & & & & & & \\
\hline & & & & & & & & & & & & & & & & \\
\hline & TOPENTERING DUAL BLAPED & & & & & & & & & & & & & & & \\
\hline & AGITATOR, $304 C$ R $316 L$ STARLESS & & & & & & & & & & & & & & & \\
\hline & WETTEO PARTS MOTOR IS $3 / 4 \mathrm{HW}$. & & & & & & & & & & & & & & & \\
\hline & $4600,3 \phi, 60 H_{2}$ TEFC & & & & & & & & & & & & & & & \\
\hline & CHE MUNETI MOOEL VHN-7 & & & & & & & & & & & & & & & \\
\hline & TOTAL & & & & & & IB & $\log$ & & & & 200 & & & & \\
\hline & I GRANO TOTAL SK-MP-3 AGITATOBS MIXERS & & & & & & 36 & $1600 \mid$ & & & & 300 & & & & \\
\hline
\end{tabular}


ESTIMATE WORKSHEET

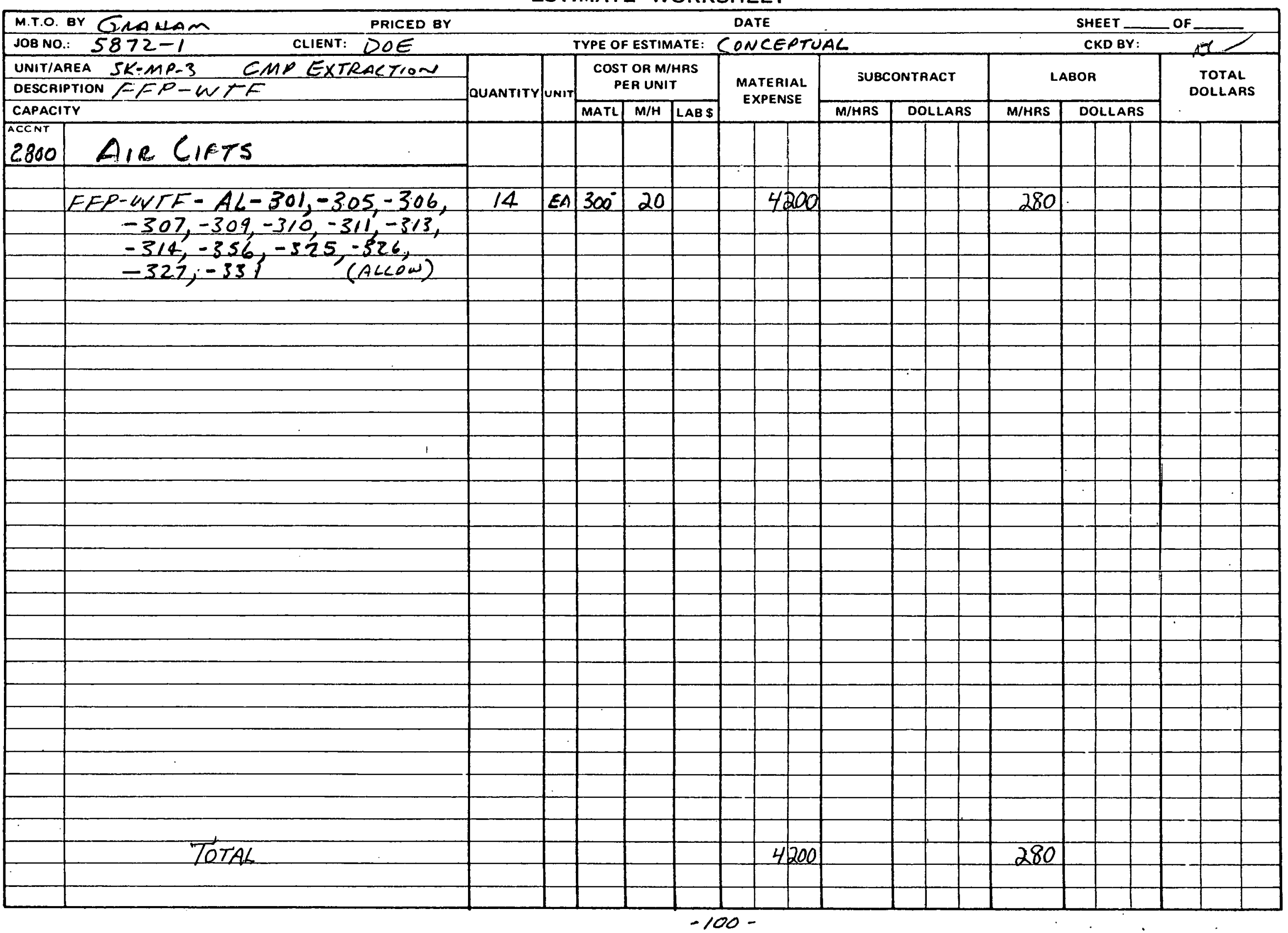


THE RALPH M. PARSONS COMPANY

ESTIMATE WORKSHEET

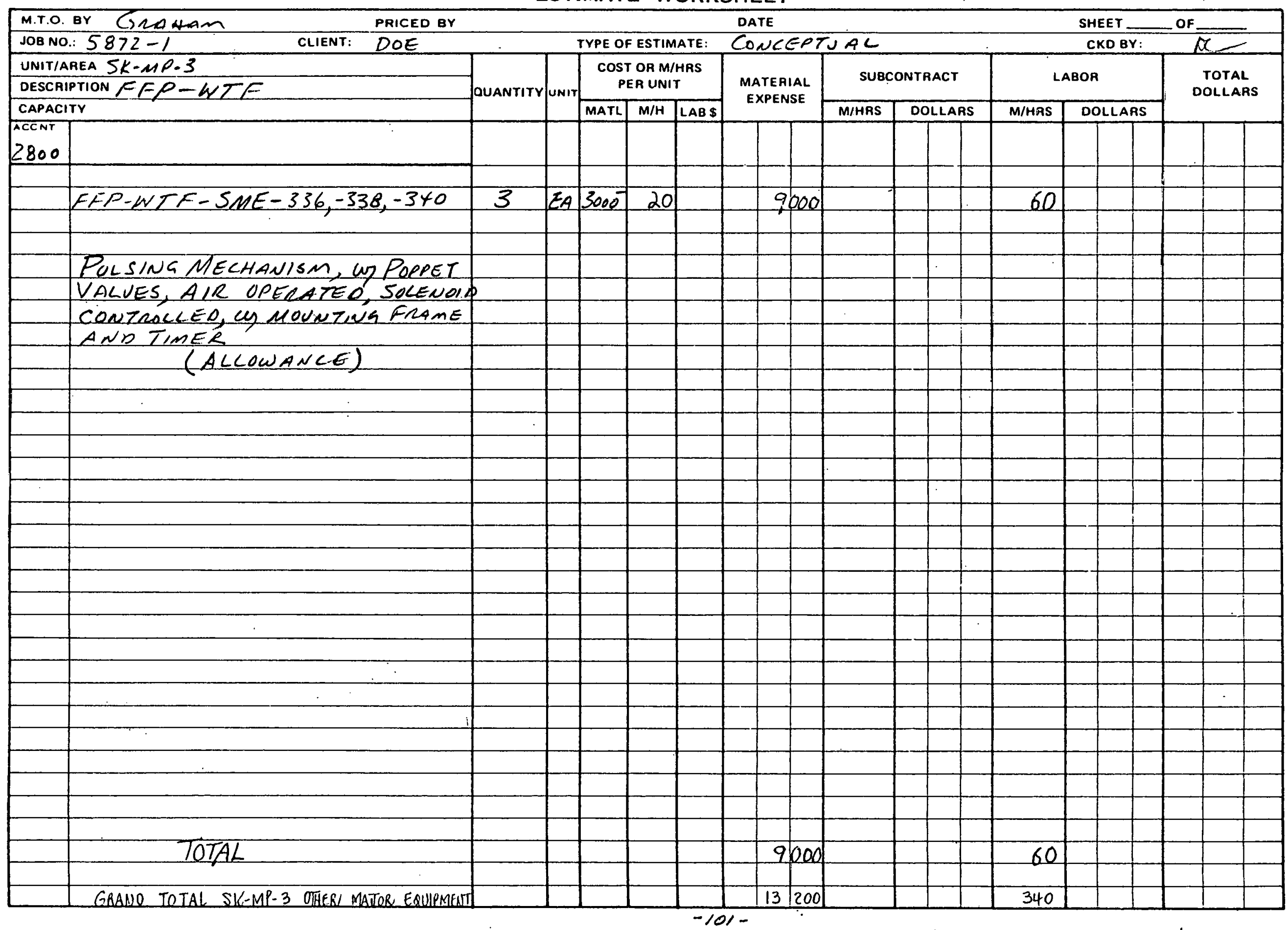


n

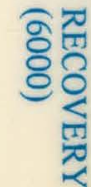


ESTIMATE WORKSHEET

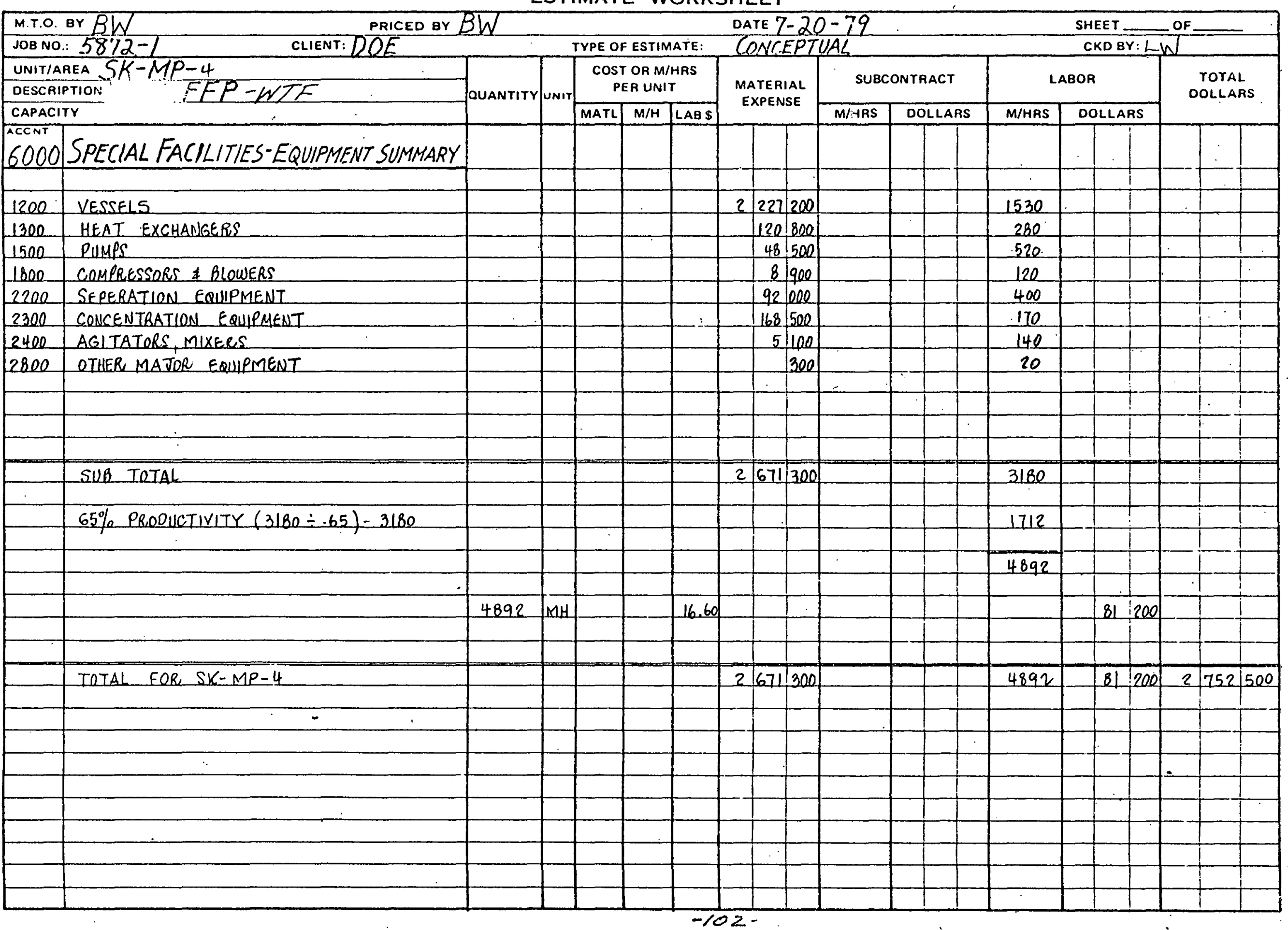


ESTIMATE WORKSHEET

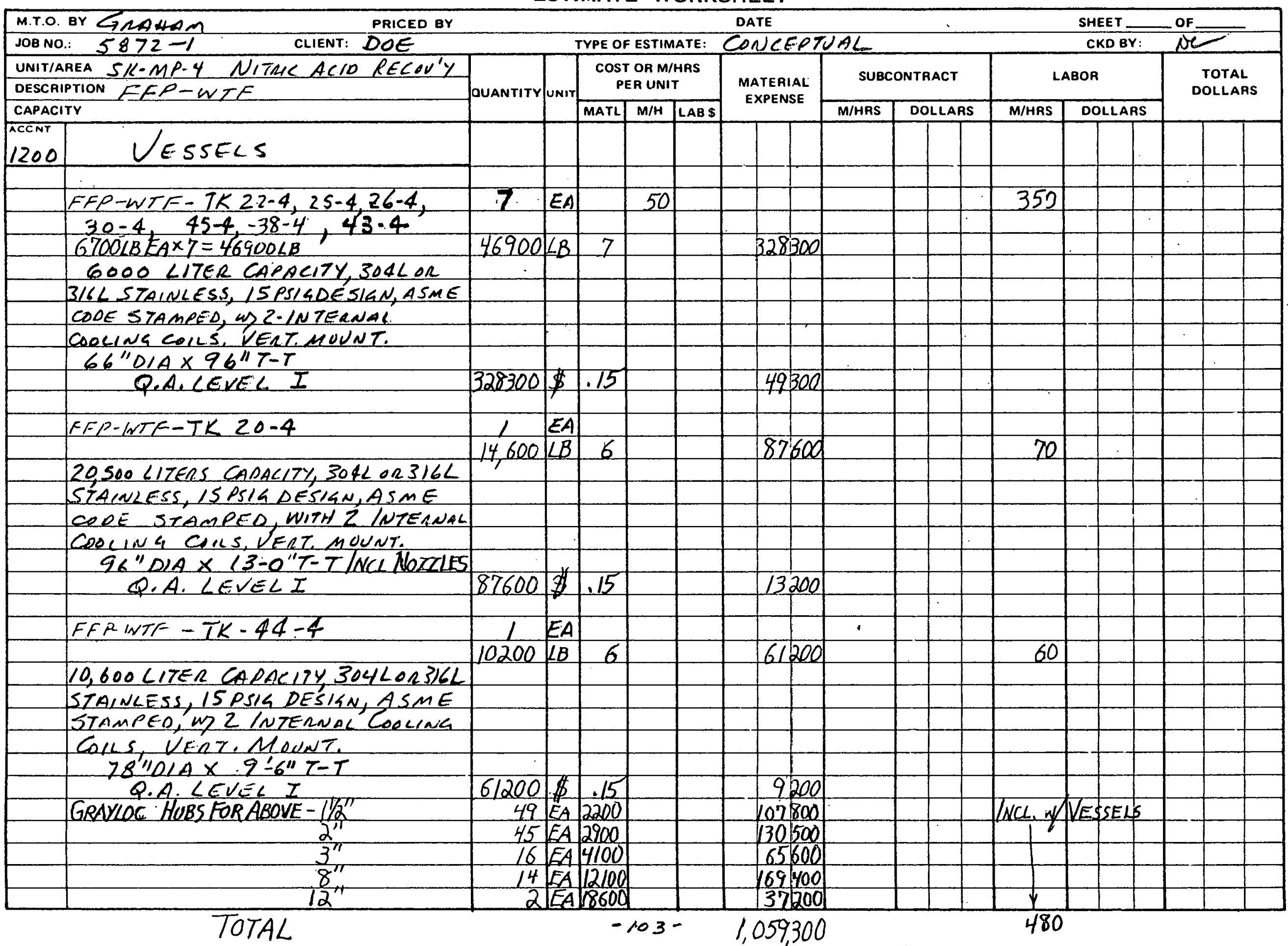


ESTIMATE WORKSHEET

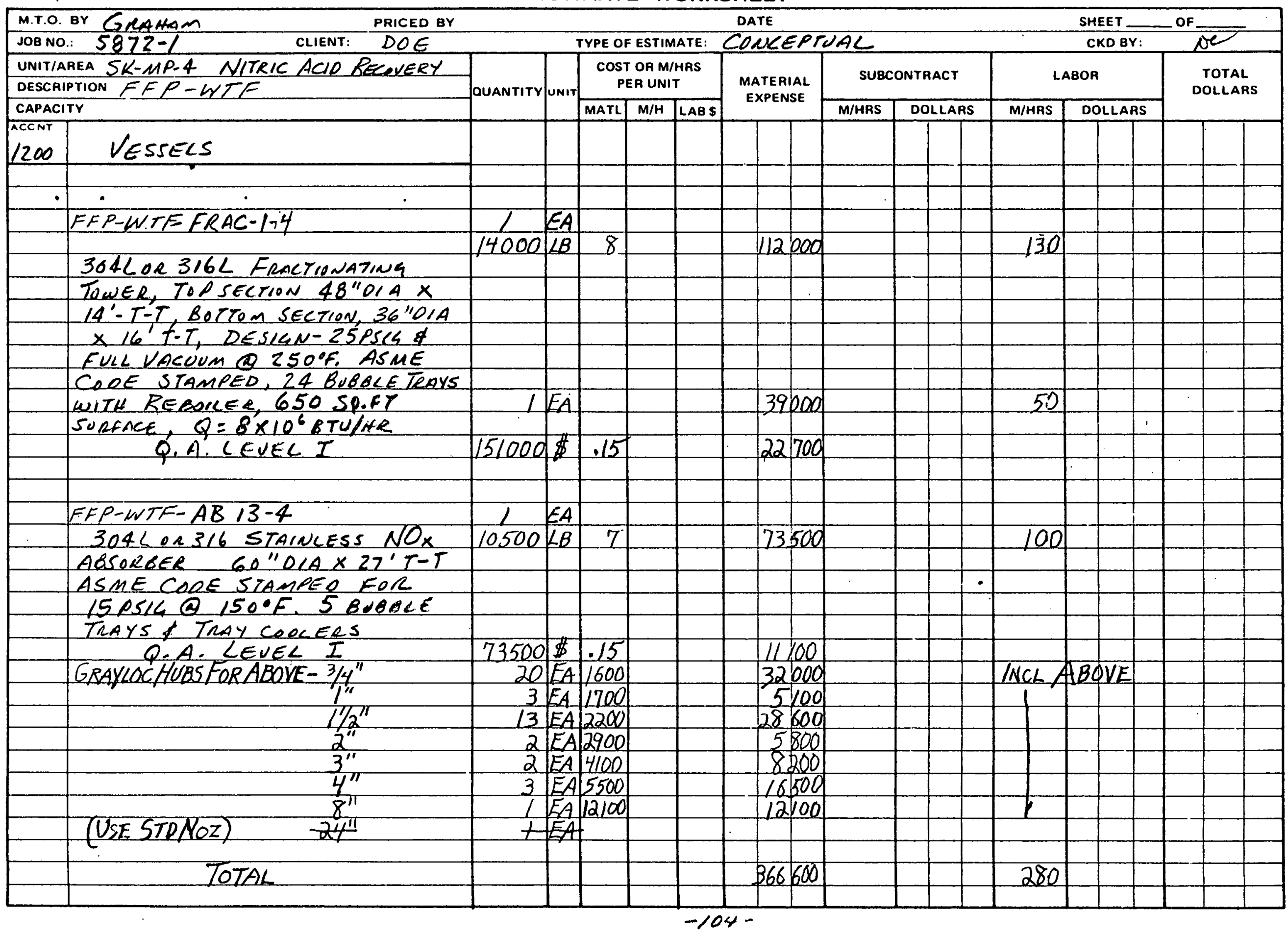


ESTIMATE WORKSHEET

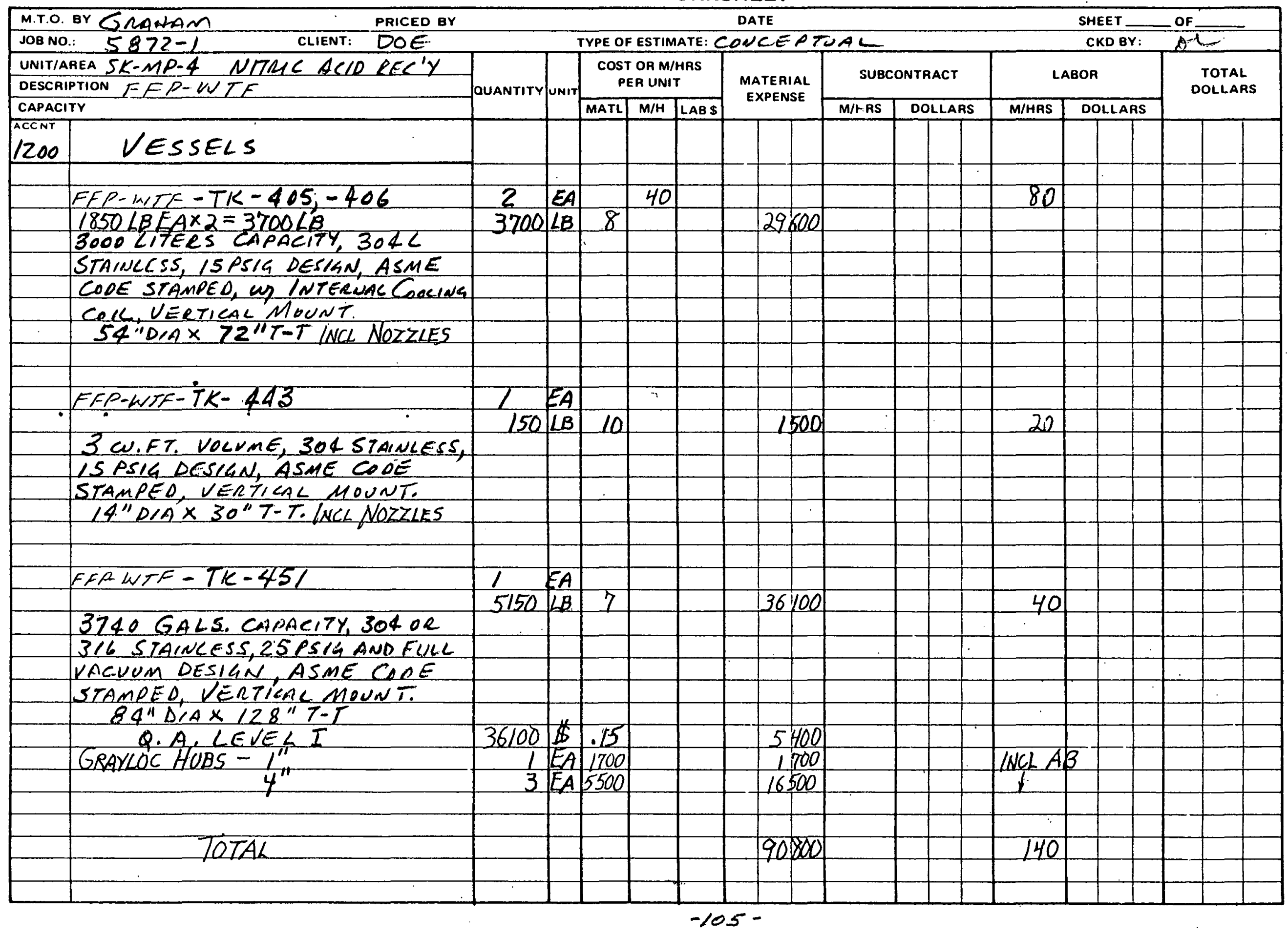


ESTIMATE WORKSHEET

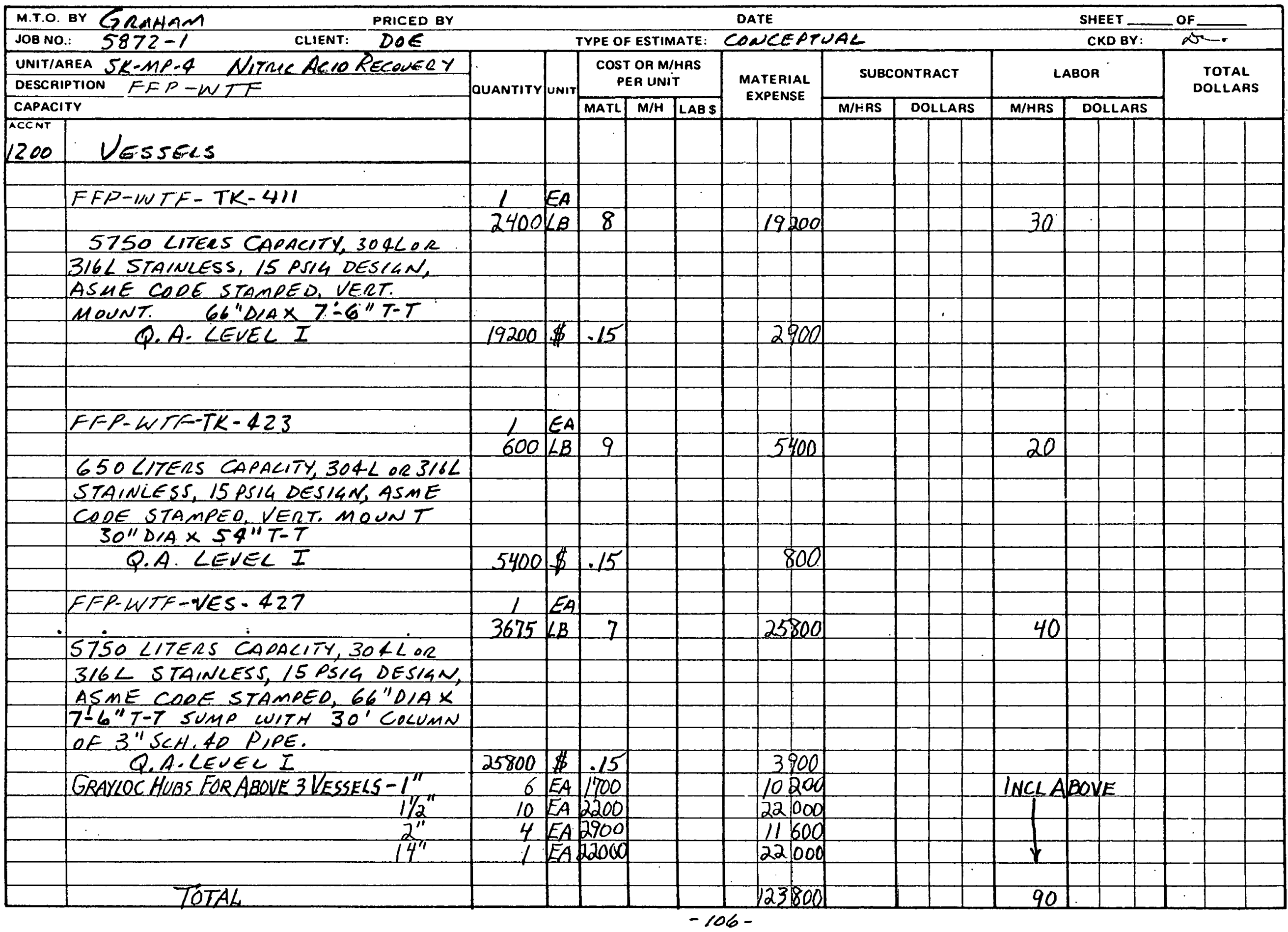


ESTIMATE WORKSHEET

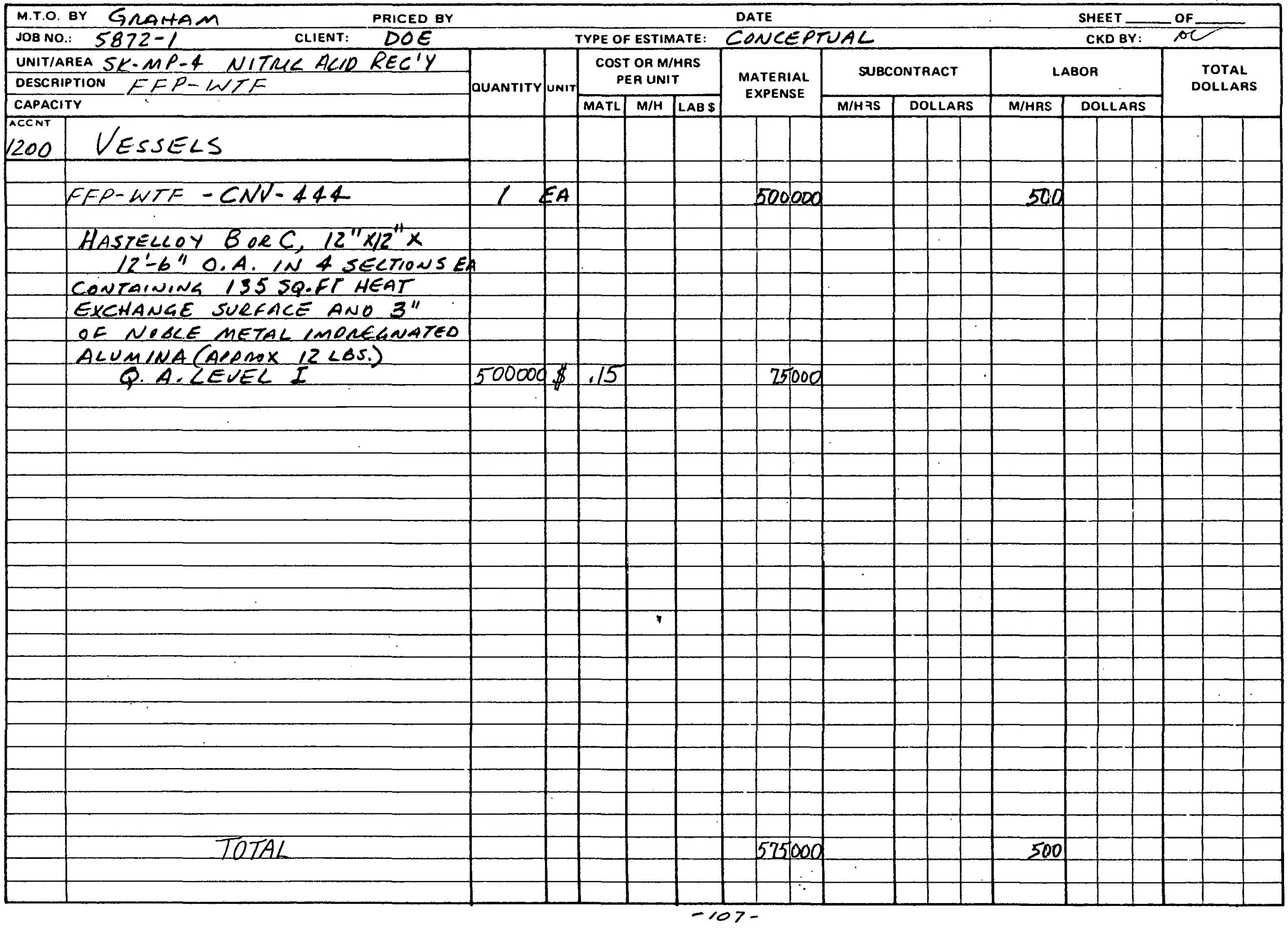


ESTIMATE WORKSHEET

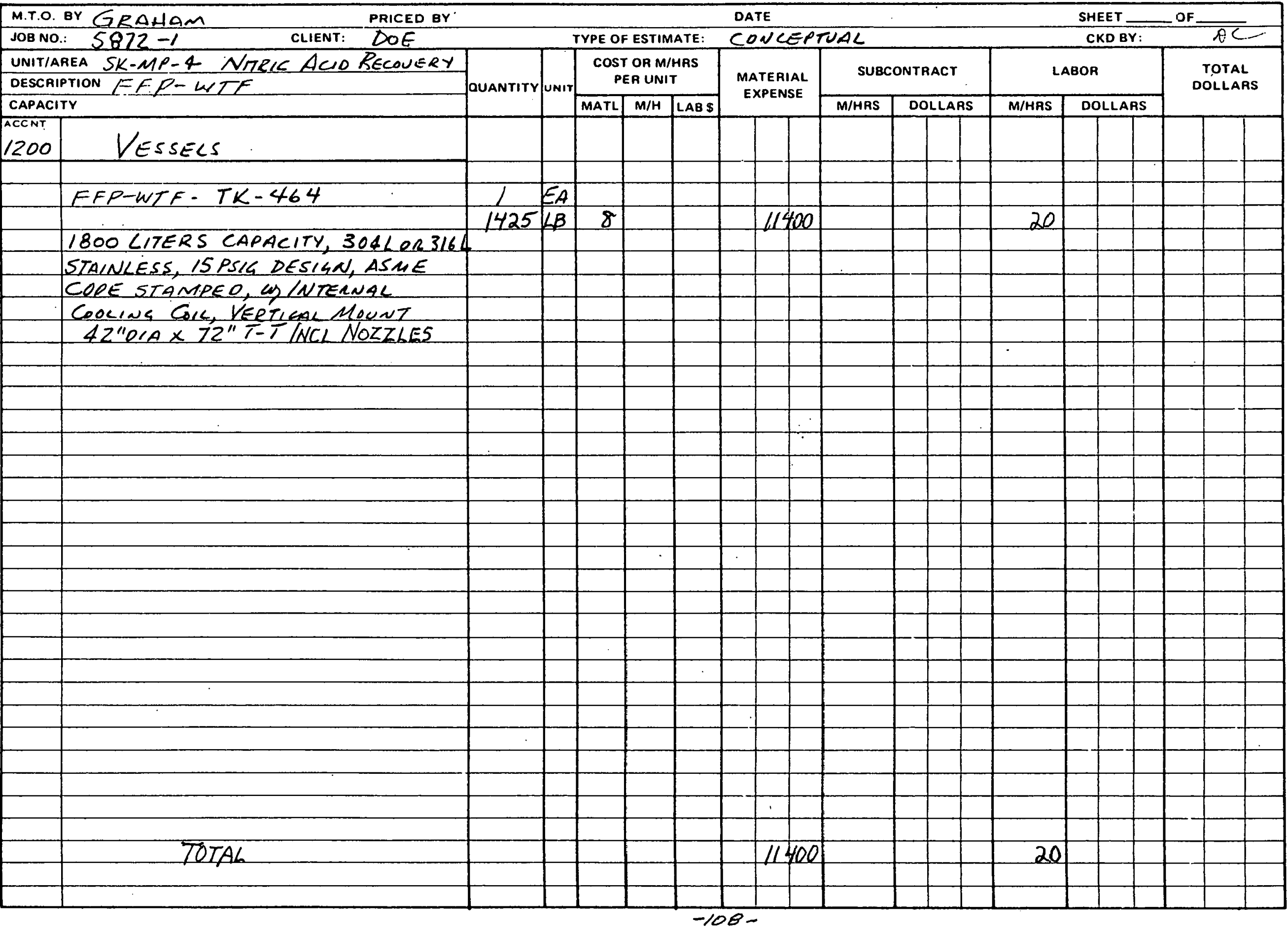


ESTIMATE WORKSHEET

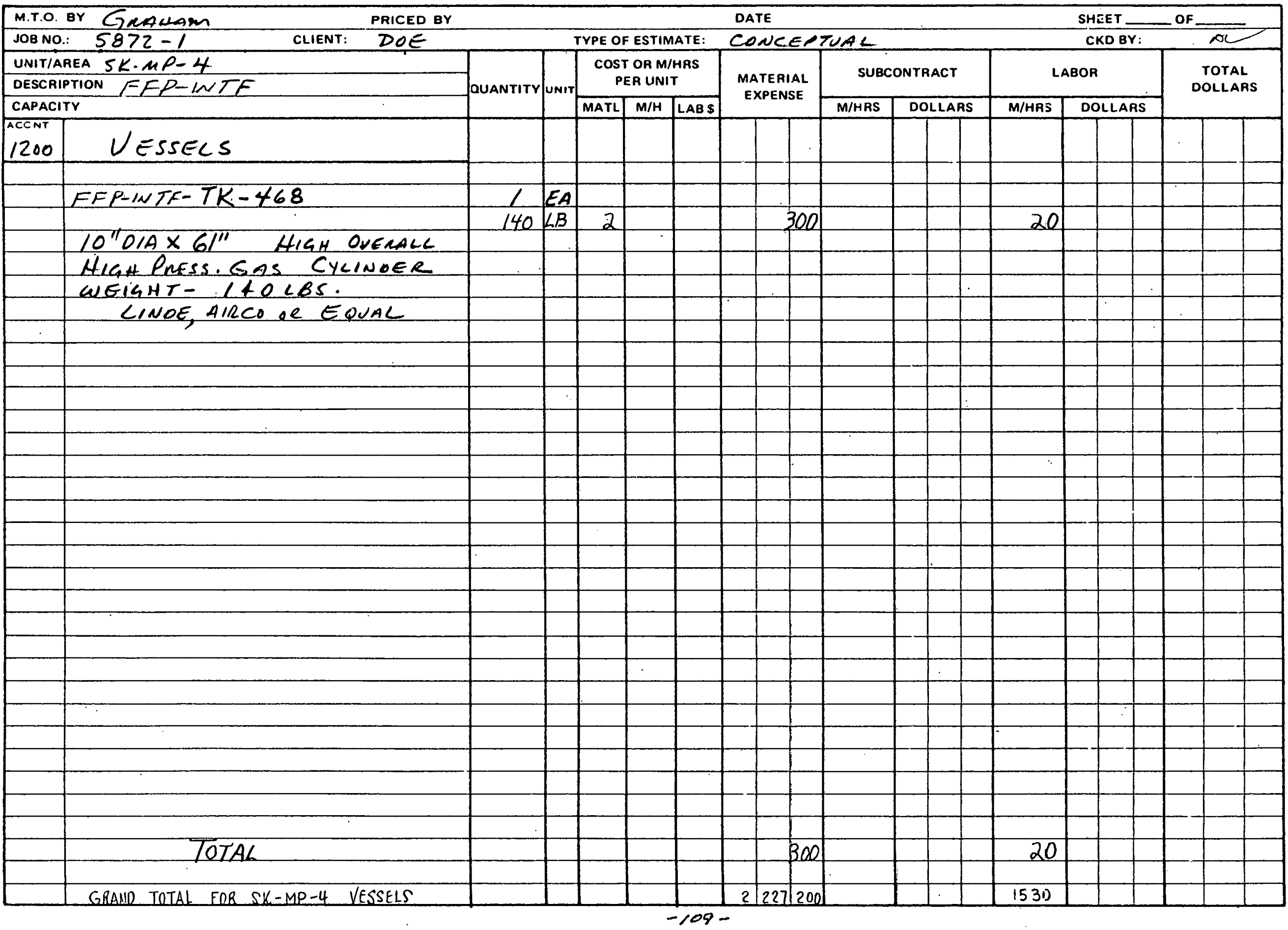


ESTIMATE WORKSHEET

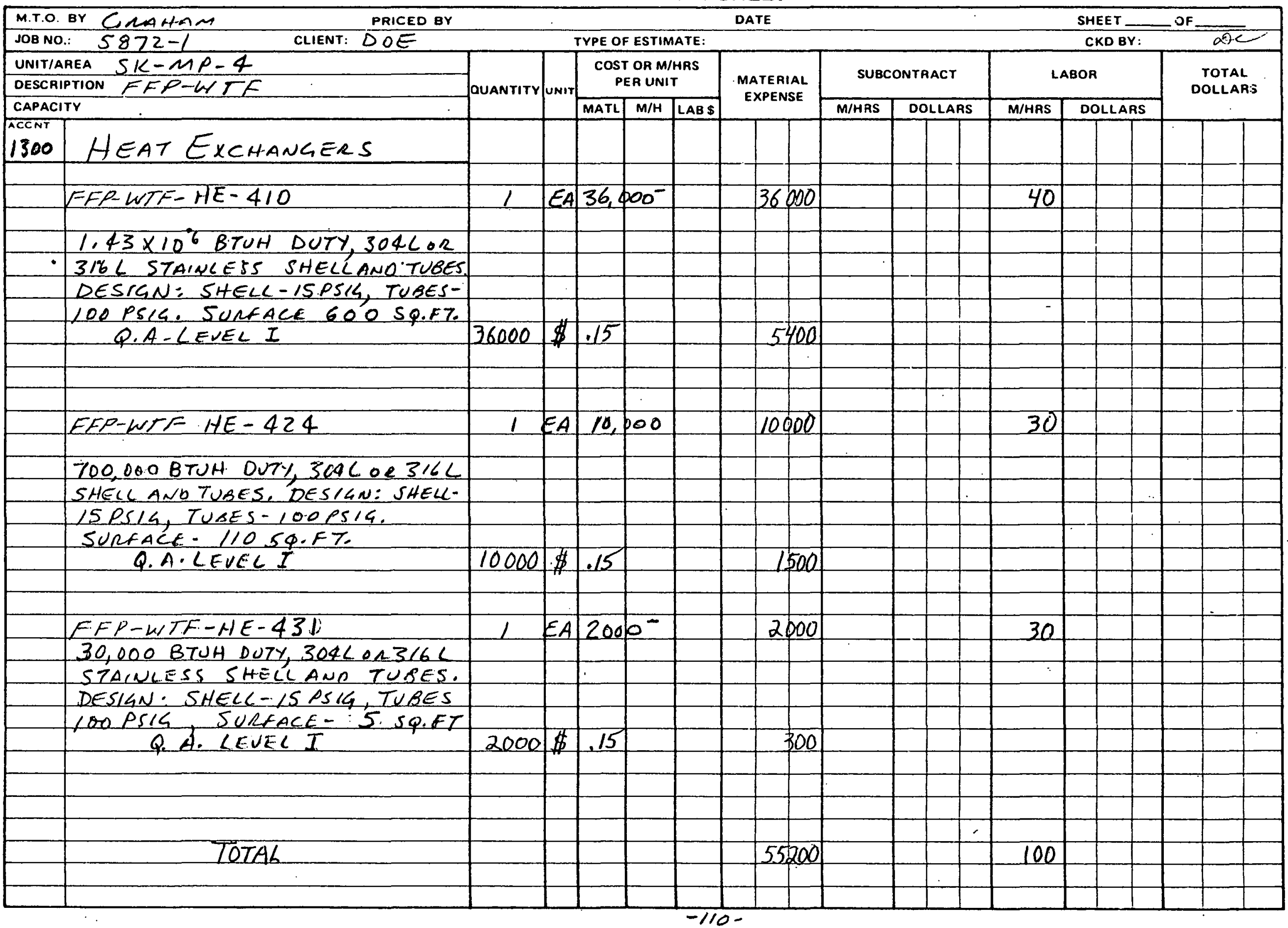


ESTIMATE WORKSHEET

\begin{tabular}{|c|c|c|c|c|c|c|c|c|c|c|c|c|c|}
\hline M.T.O. & BY GRANAMM & & & & & & & TE & & & & SHEET & \\
\hline JOB NO & $\therefore \quad 5972-1$ & & & TYPE OF & ESTIM & ATE: & & & & & & CKD BY: & $D^{C}$ \\
\hline UNIT/A & AEA $S K-M P-4$ & & & & OR M/I & & & & SUBC & NTRACT & & BOR & \\
\hline DESCAI & PTION FFP M MTE & OUANTITY & UNIT & & ER UNIT & & & $\begin{array}{l}\text { QTERIAL } \\
\text { XPENSE }\end{array}$ & $30 \mathrm{BC}$ & NIRACT & & & DOLLARS \\
\hline CAPACI & & & & MATL & $M / H$ & LAB \$ & & & M/HRS & DOLLARS & M/HF.S & DOLLARS & \\
\hline $\begin{array}{l}\text { ACCNT } \\
1300\end{array}$ & H. Ex ExaRS & & & & & & & & & & & & \\
\hline & & & & & & & & & & & & & \\
\hline & FFP-WYFF-HE-445 (Q.A.-I) & L & $E A$ & 16,4 & 00 & & & 16000 & & & 30 & & \\
\hline & & & & & & & & & & & & & \\
\hline & 10,000 BTUH DUTY, $304 L$ OR $316 \mathrm{~L}$ & & & & & & & & & & & & \\
\hline & STAINLESS SHELL ANO TUBES. & & & & & & & & & & & & \\
\hline & DESIGN: SHECL 15 PSIS, TUBES- & & & & & & & & & & & & \\
\hline & 100 PSIL. SURFACE 20050.57. & & & & & & & & & & & & \\
\hline & . & & & & & & & & & & & & \\
\hline & FFP-WTF - HE - 446 & 18 & $E A$ & $40 d$ & $0^{-}$ & & & 4000 & & & 30 & & \\
\hline & & & & & & & & & & & & & \\
\hline & 20,000 BTUH DUTY,304LOR316L & & & & & & & & & & & & \\
\hline & STAINLESS STEEL SHELC \& TUBES. & & & & & & & & & & & & \\
\hline & DESIGN: SHELC - 15 PSIG TUOES - & & & & & & & & & & & & \\
\hline & LOOPSIS. SURFACE - $4054 . F T$. & & & & & & & & & & & & \\
\hline & & & & & & & & & & & & & \\
\hline & & & & & & & & $\therefore$ & & & & & \\
\hline & FFRMTF-HE-463-465.(Q.A.7) & 2 & $E A$ & $16, d$ & $00^{-}$ & & & 32000 & & & 60 & & \\
\hline & & & & & & & & & & & & & \\
\hline & 220,000 BTUH DU7\% 304L 0 3 3/6L & & & & & & & & & & & & \\
\hline & STAINLESS SHELL AND TUBES. & & & & & & & & & & & & \\
\hline & DESIGN: SHECL-15 PSIL, TUBES- & & & & & & & & & & & & \\
\hline & LOO.PSIG. SURFACE- 200 SQ.FT. & & & & & & & & & & & & \\
\hline & & & & & & & & & & & & & \\
\hline & & & & & & & & & & & & & \\
\hline & $F F P-4 T F-H E-442(Q, A .-I$ & $L$ & $E A$ & $30 \emptyset$ & $20^{-}$ & & & 3000 & & & 30 & & \\
\hline & & & & & & & & & & & & & \\
\hline & ZOKW ELELTRKACLY AEATED & & & & & & & & & & & & \\
\hline & GASS HEATER; 304 ON 316 & & & & & & & & & & & & \\
\hline & STAINLESS HOUSING & & & & & & & & & & & & \\
\hline & & & & & & & & & & & & & \\
\hline & QALEVEL I FOR AGL ABOVE & 55000 & $\$$ & .15 & & & & 8300 & & & & & \\
\hline & & & & & & & & & & & & & \\
\hline & TorAl & & & & & & & 63300 & & & 150 & & \\
\hline
\end{tabular}


ESTIMATE WORKSHEET

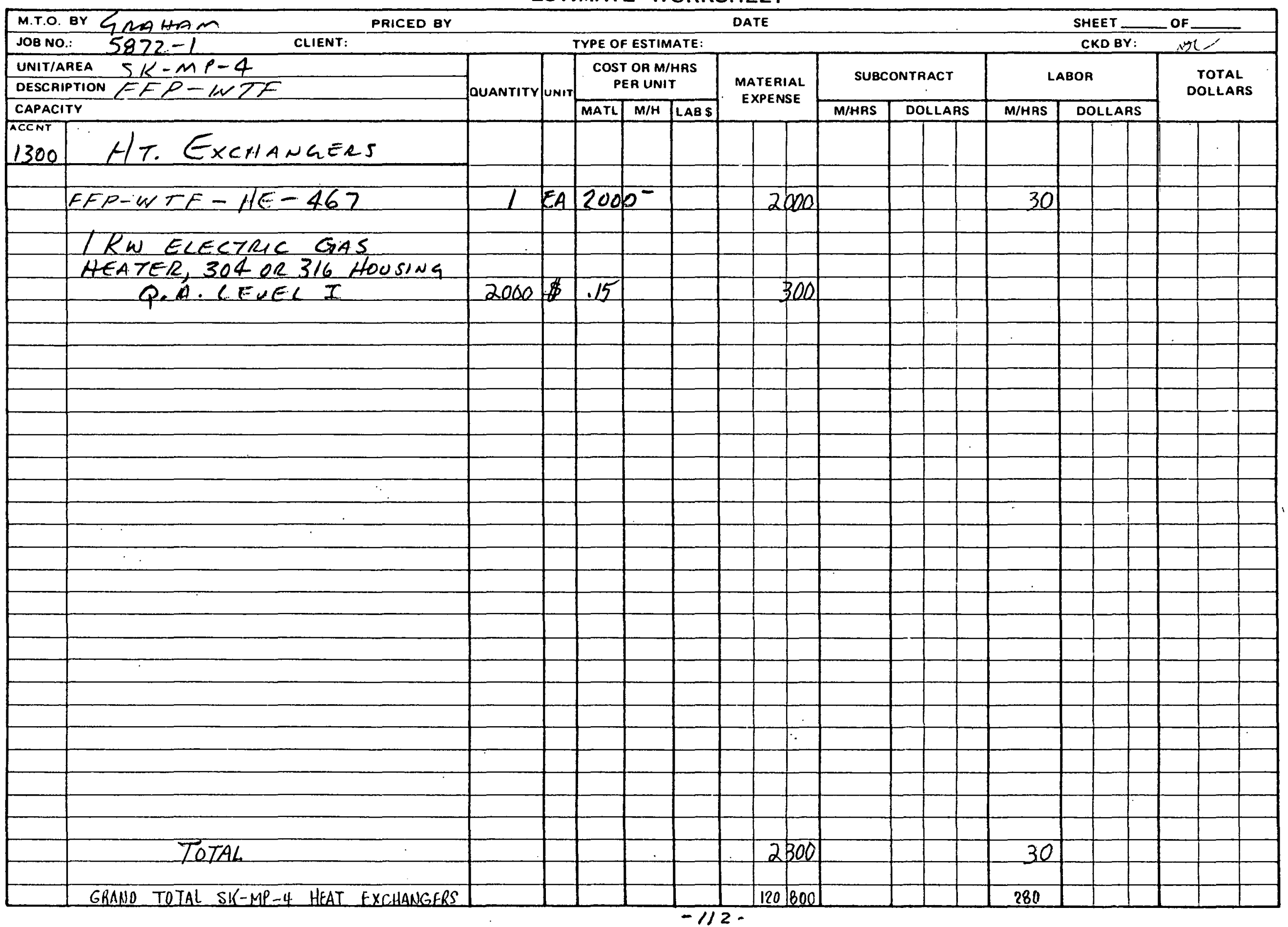


THE RALPH M. PARSONS COMPANY

ESTIMATE WORKSHEET

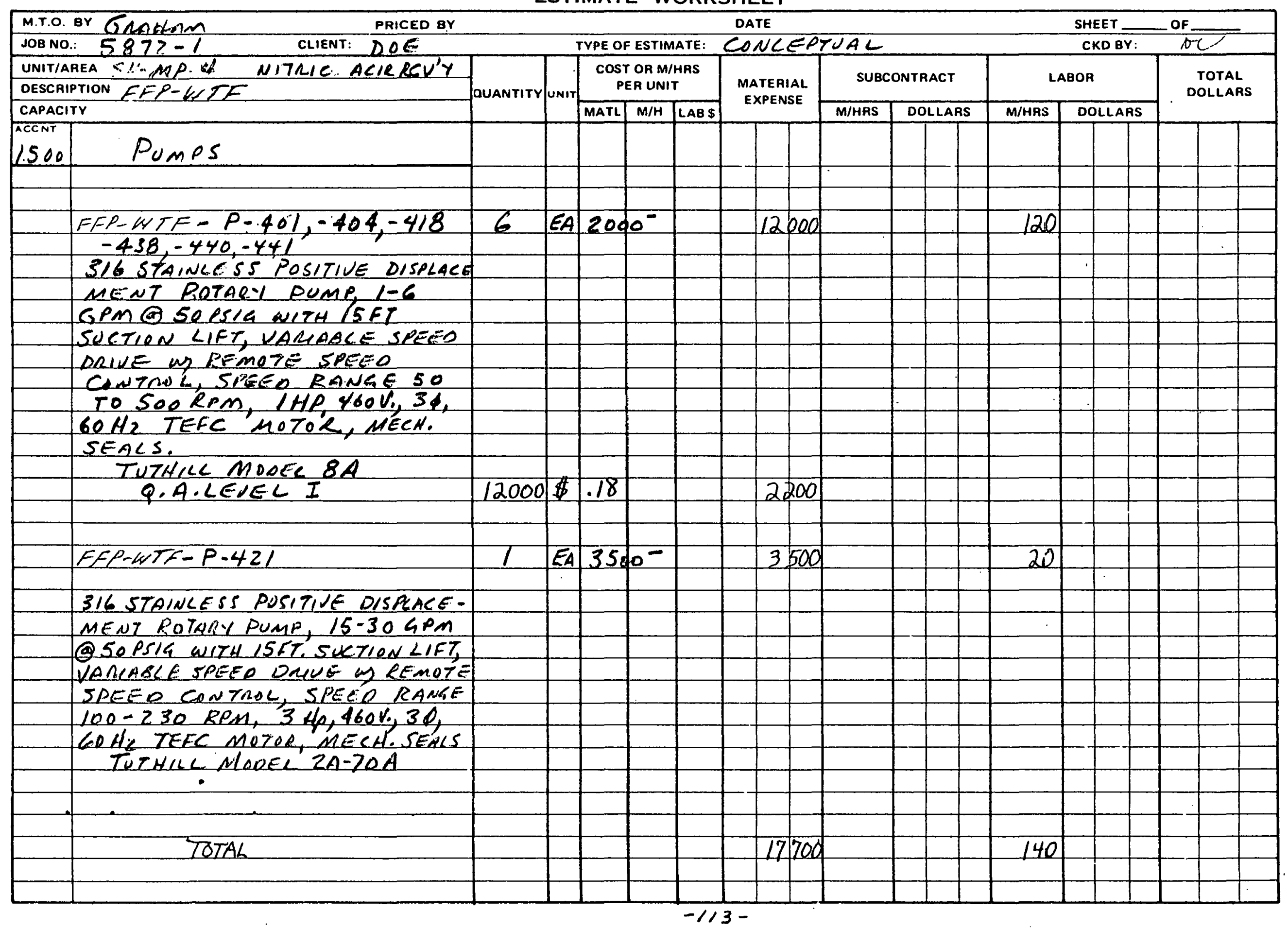


ESTIMATE WORKSHEET

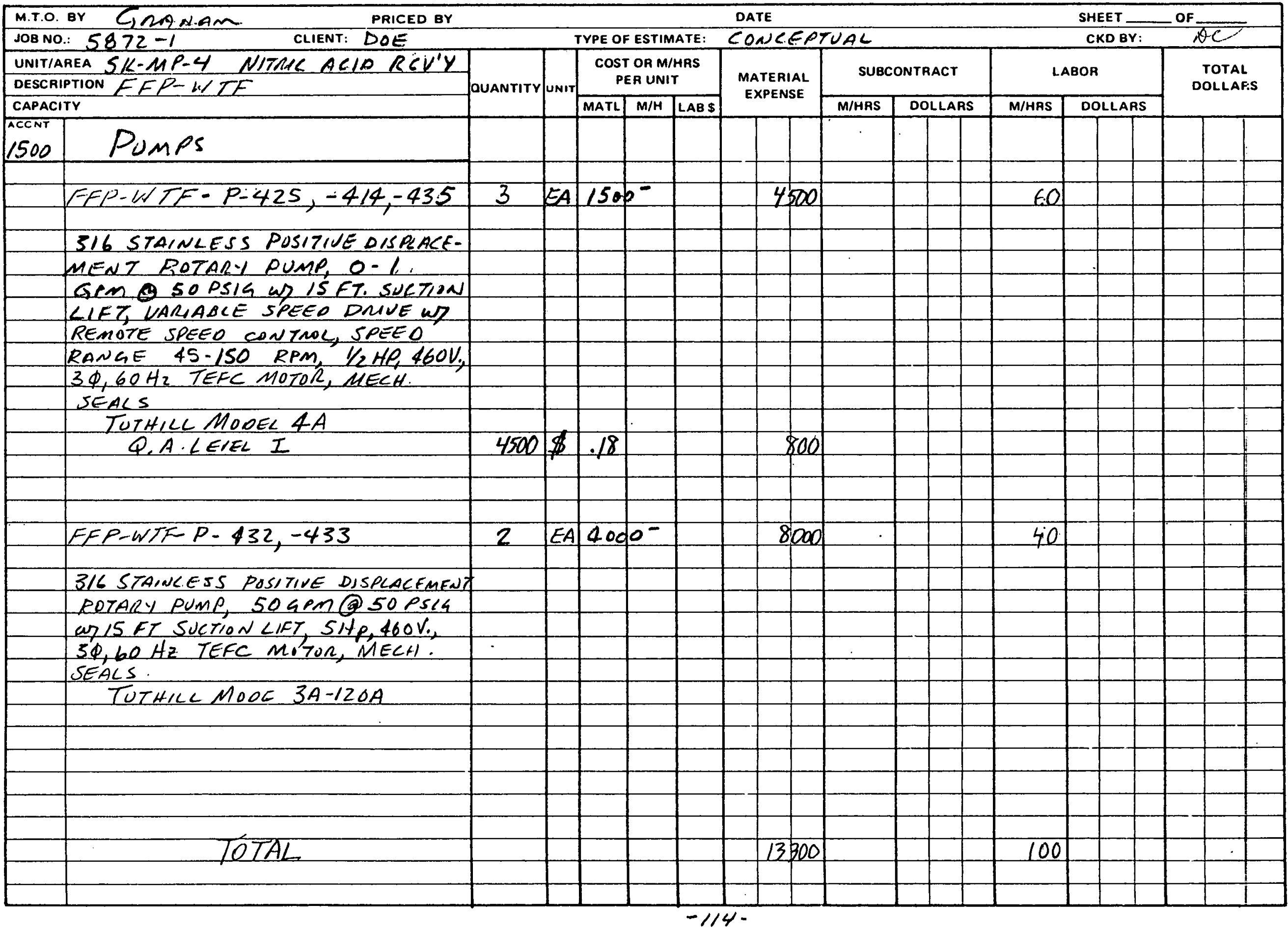


ESTIMATE WORKSHEET

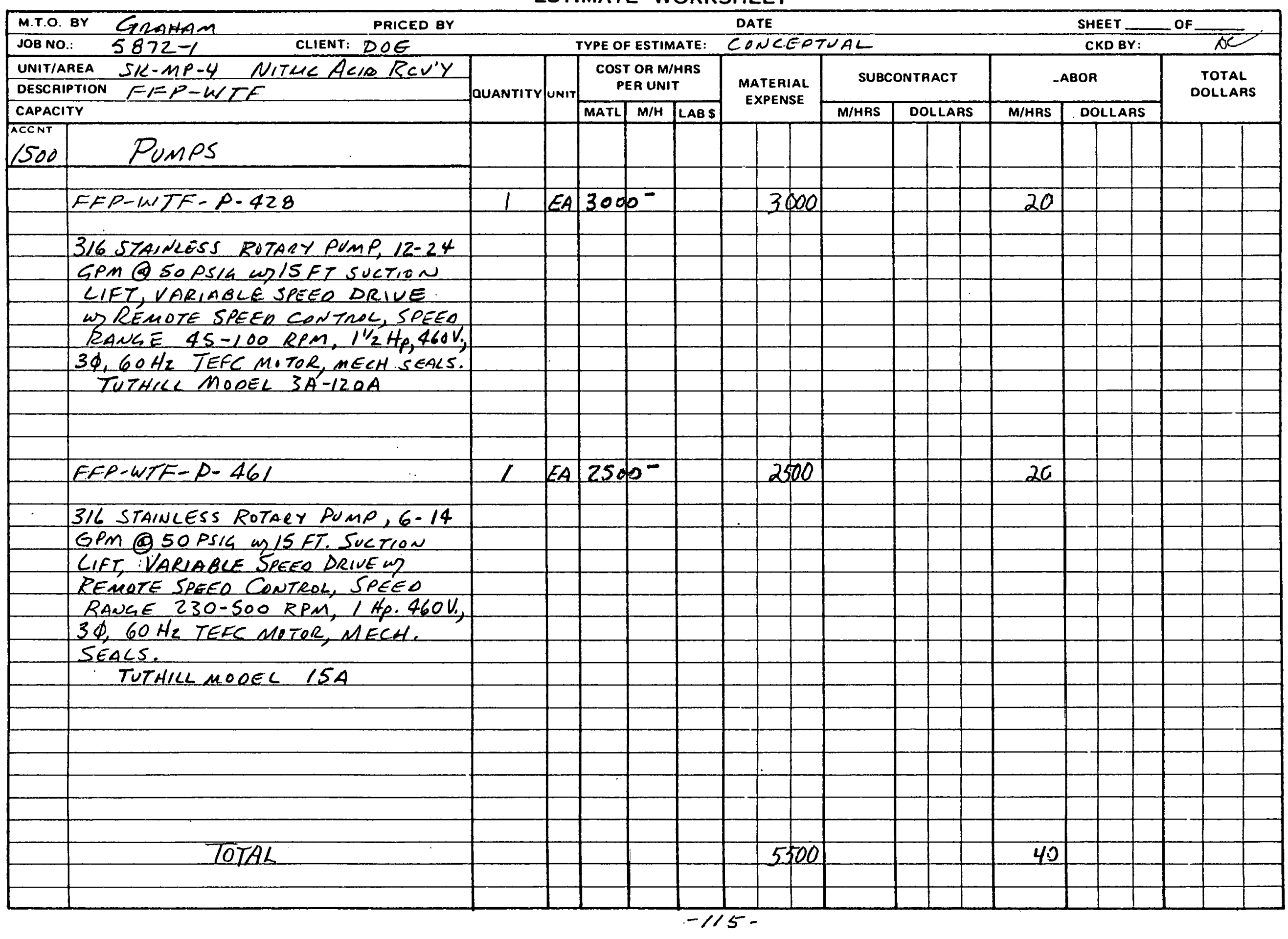


ESTIMATE WORKSHEET

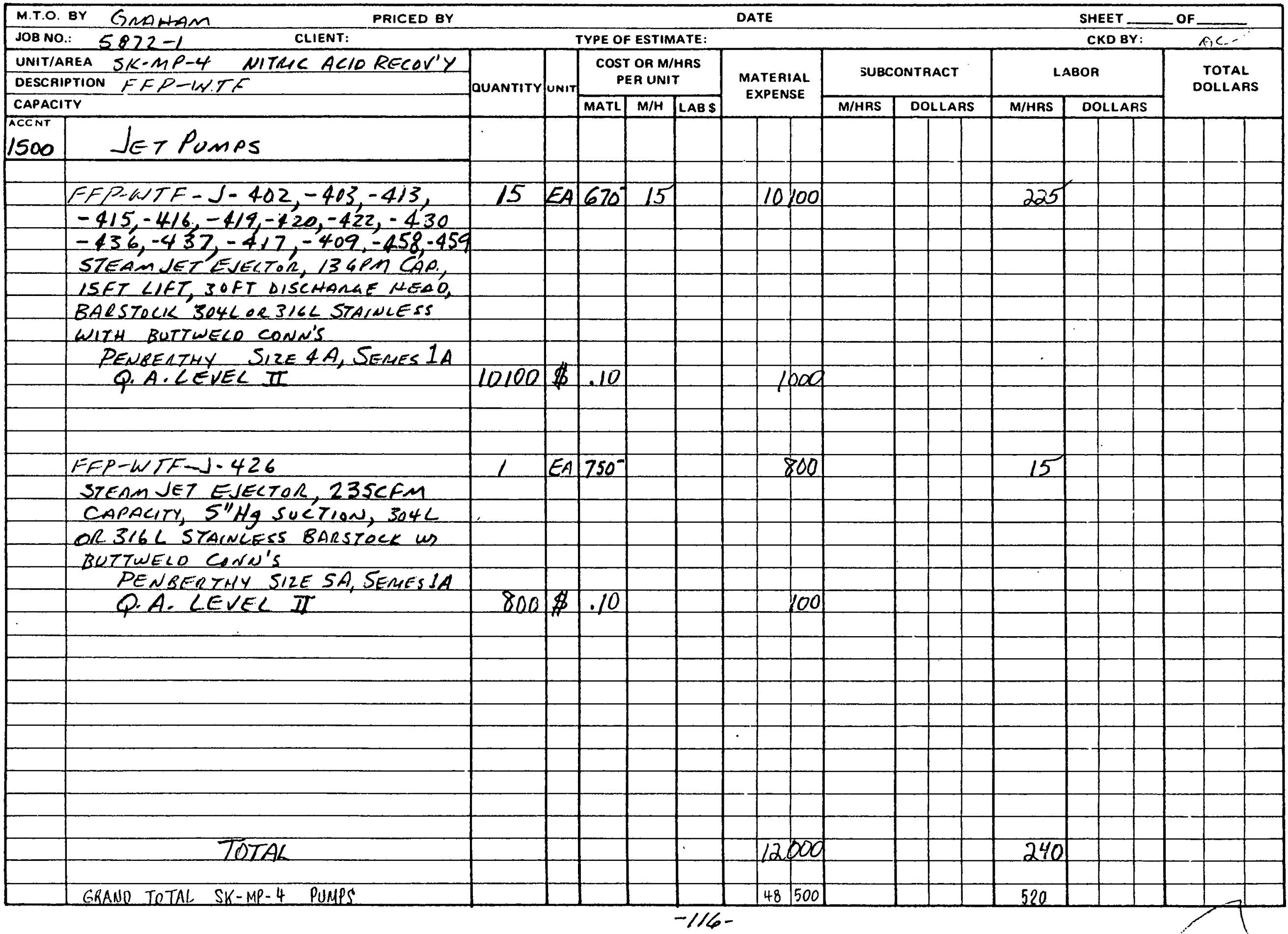


ESTIMATE WORKSHEET

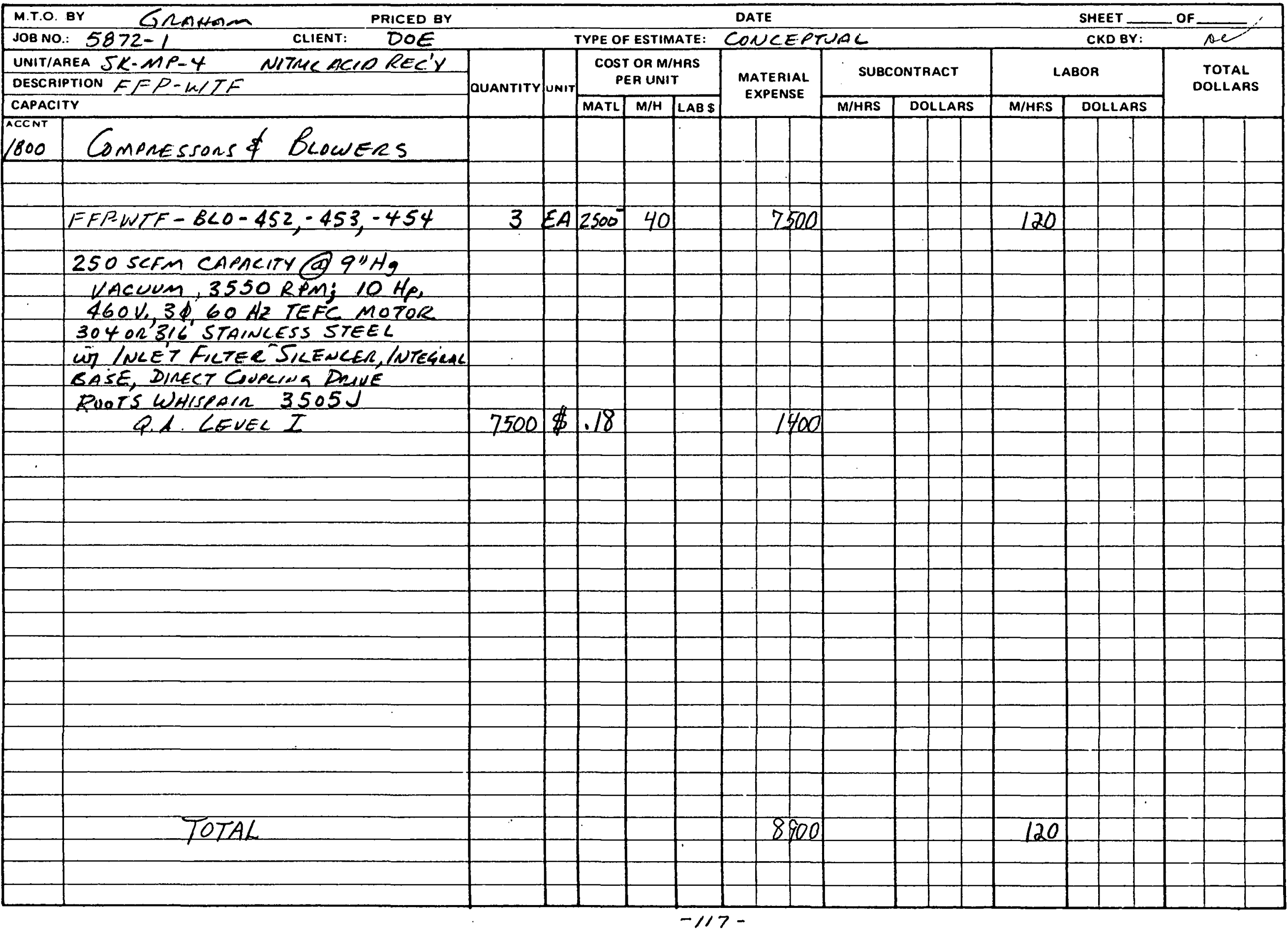


ESTIMATE WORKSHEET

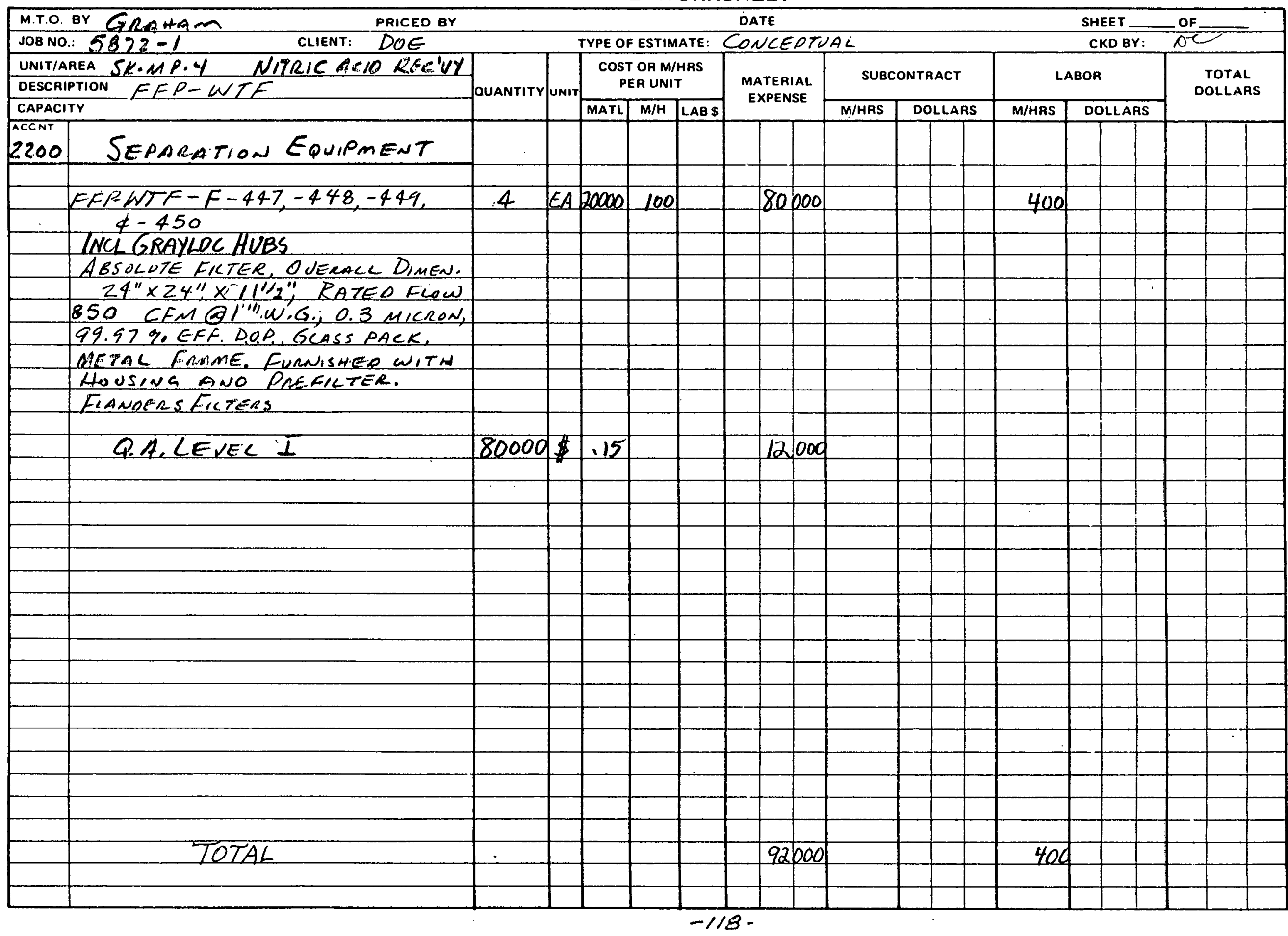


ESTIMATE WORKSHEET

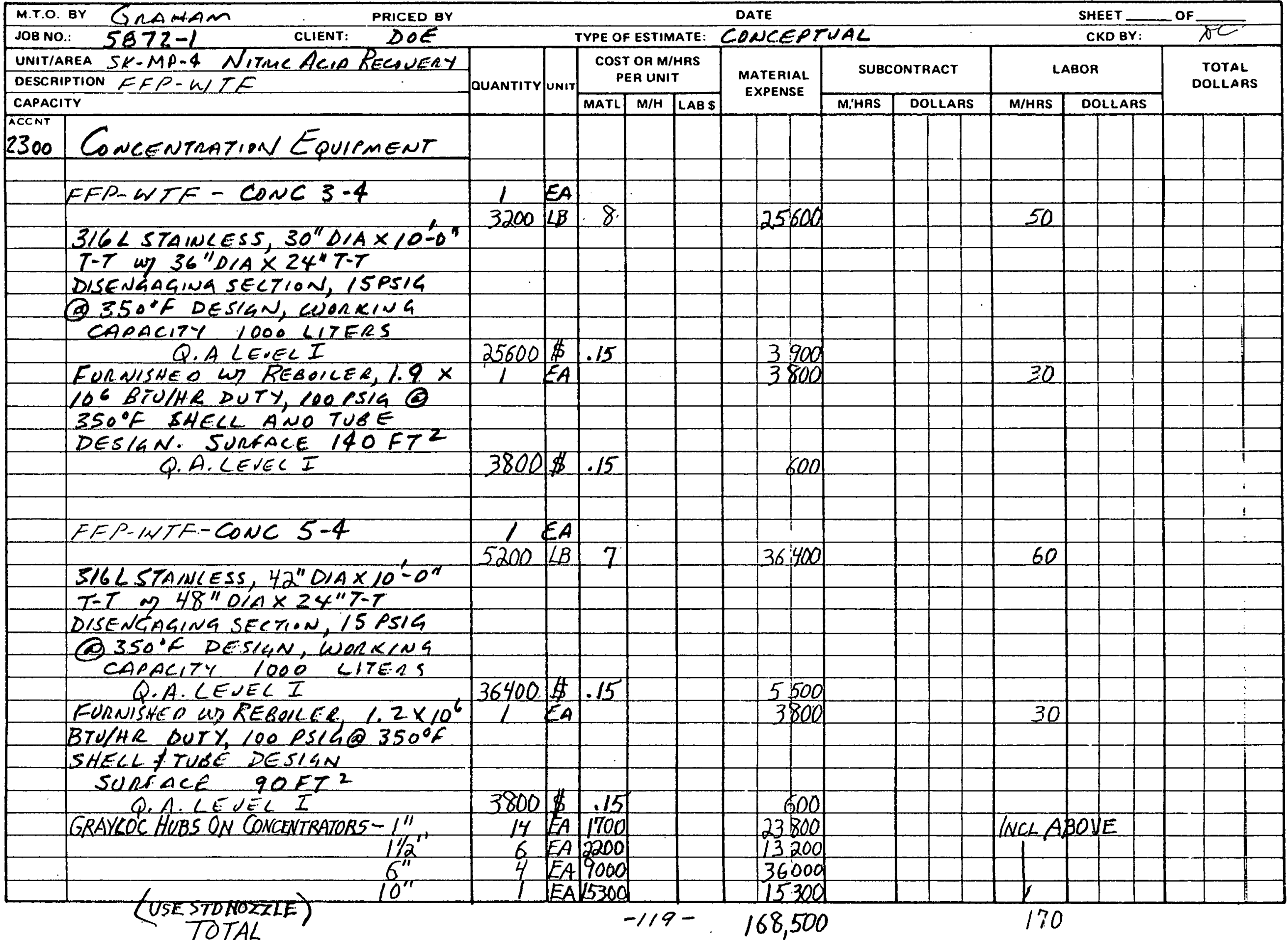


ESTIMATE WORKSHEET

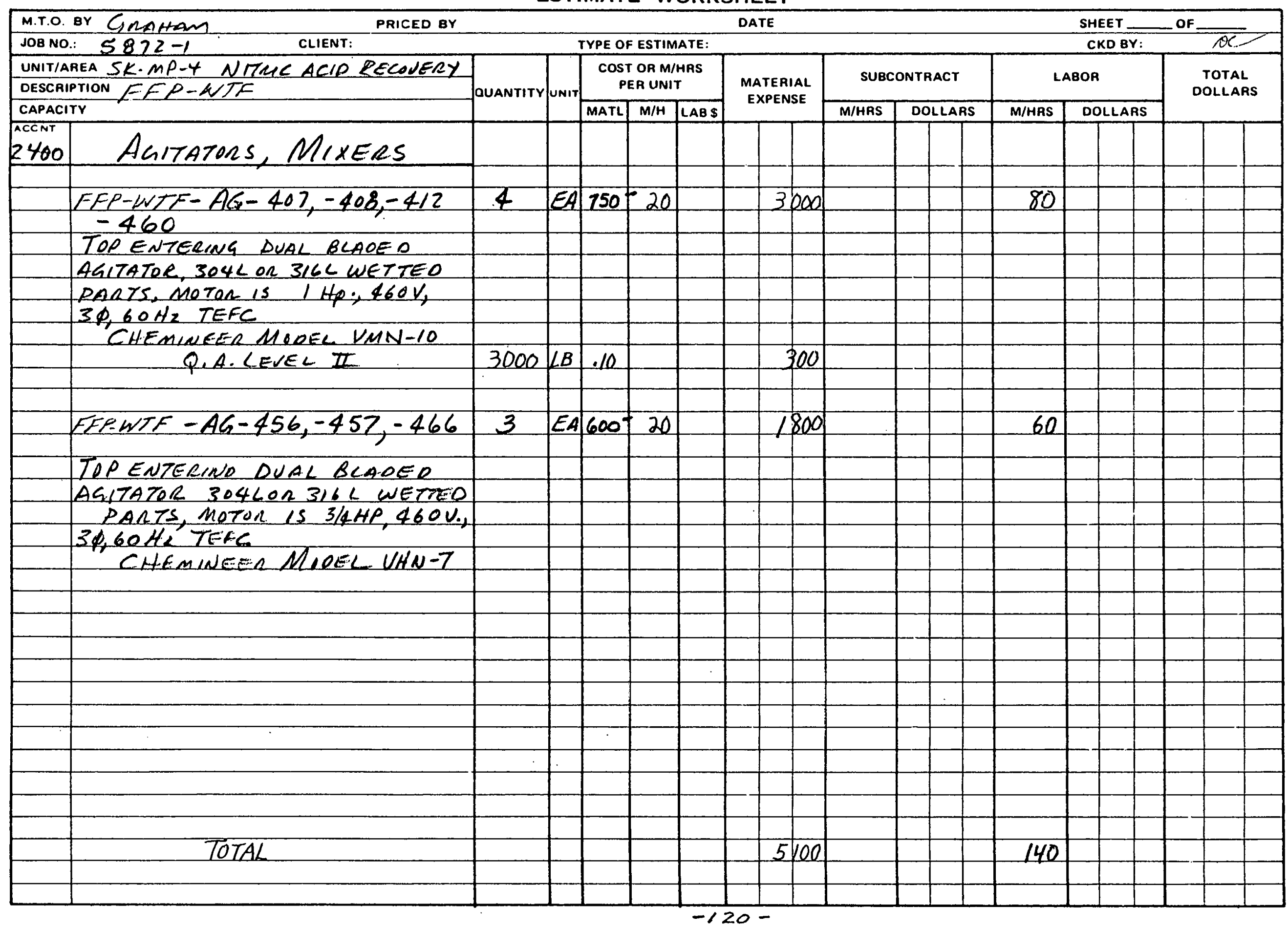


THE RALPH M. PARSONS COMPANY

ESTIMATE WORKSHEET

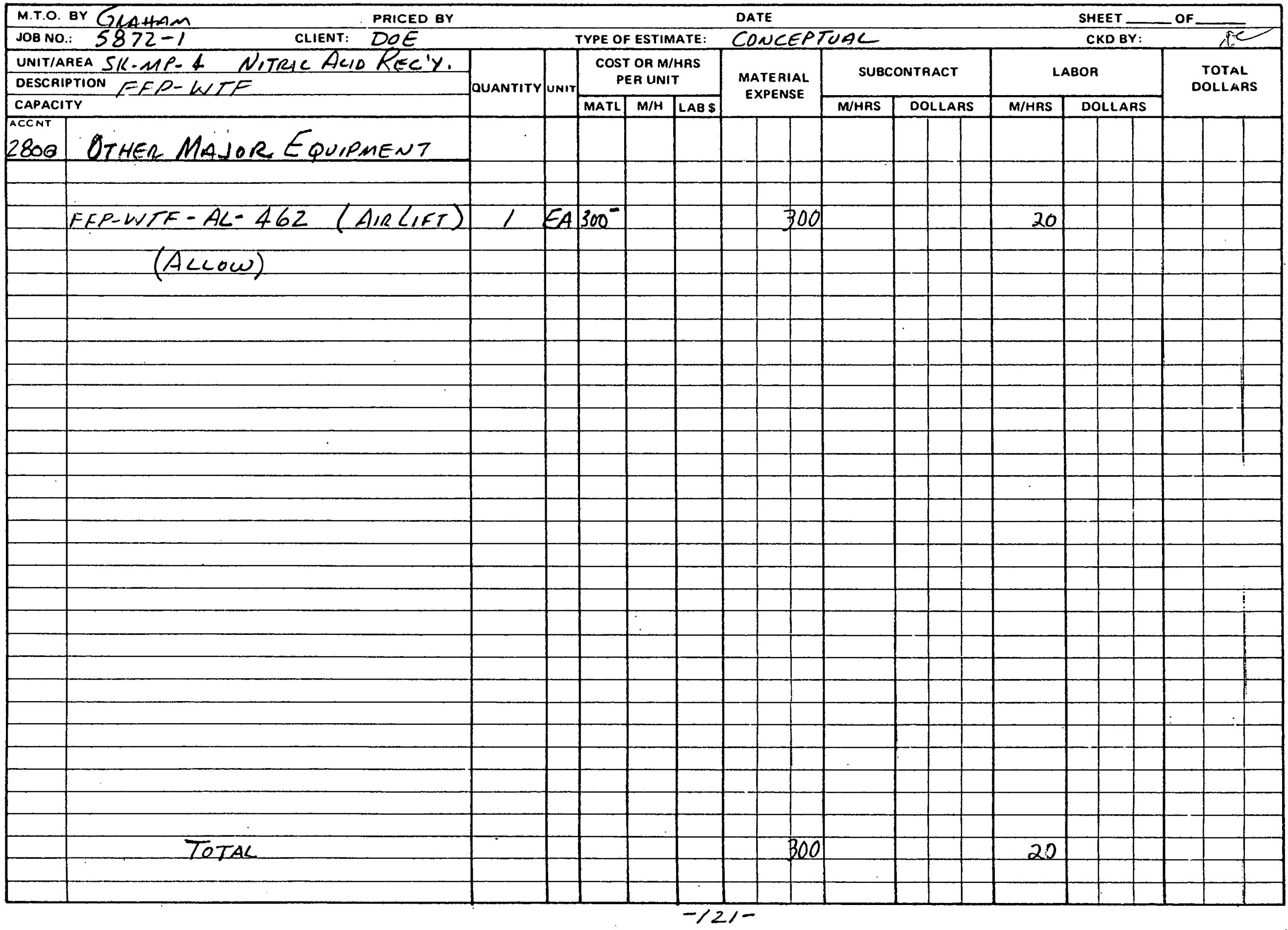


ESTIMATE WORKSHEET

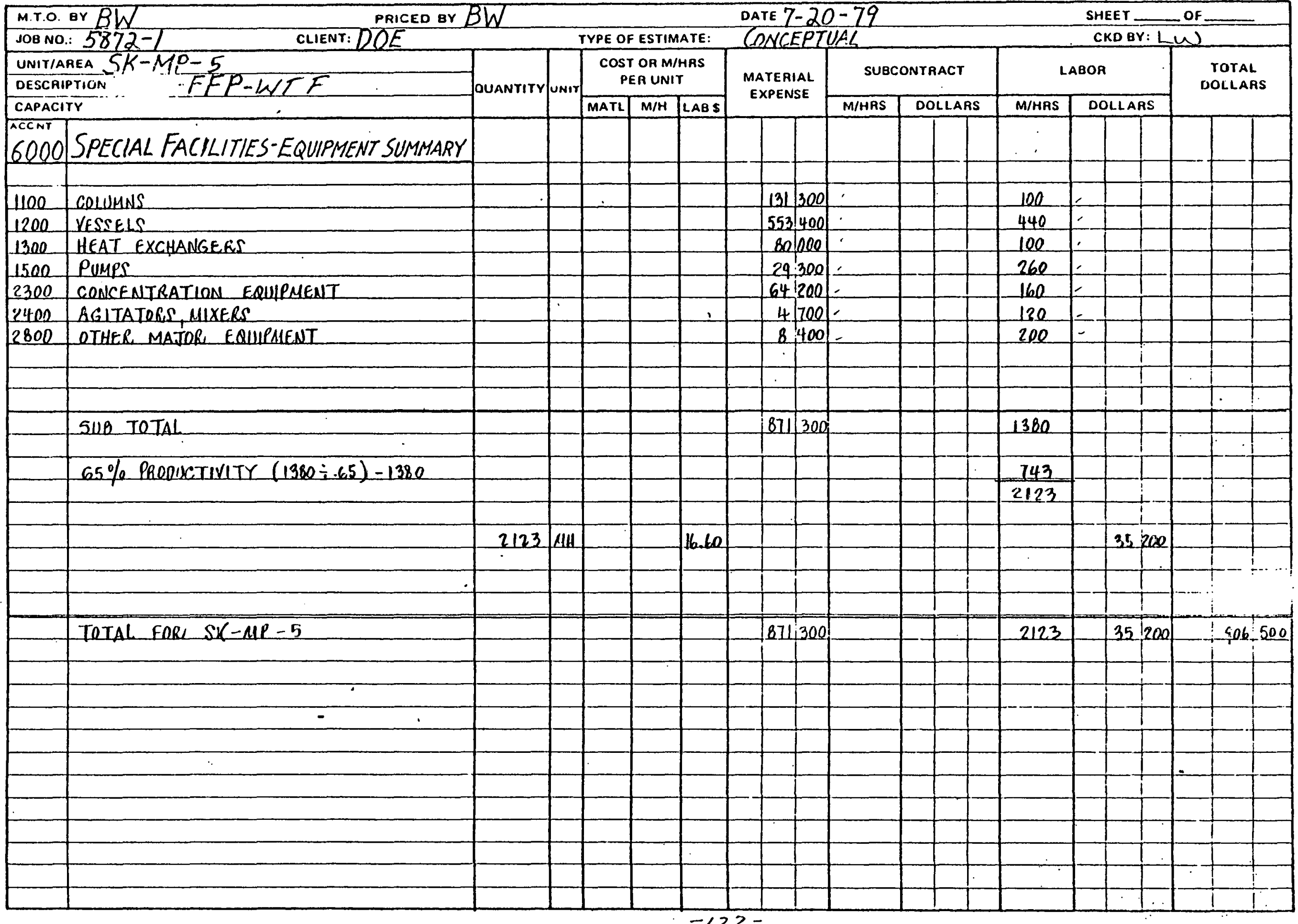


ESTIMATE WORKSHEET

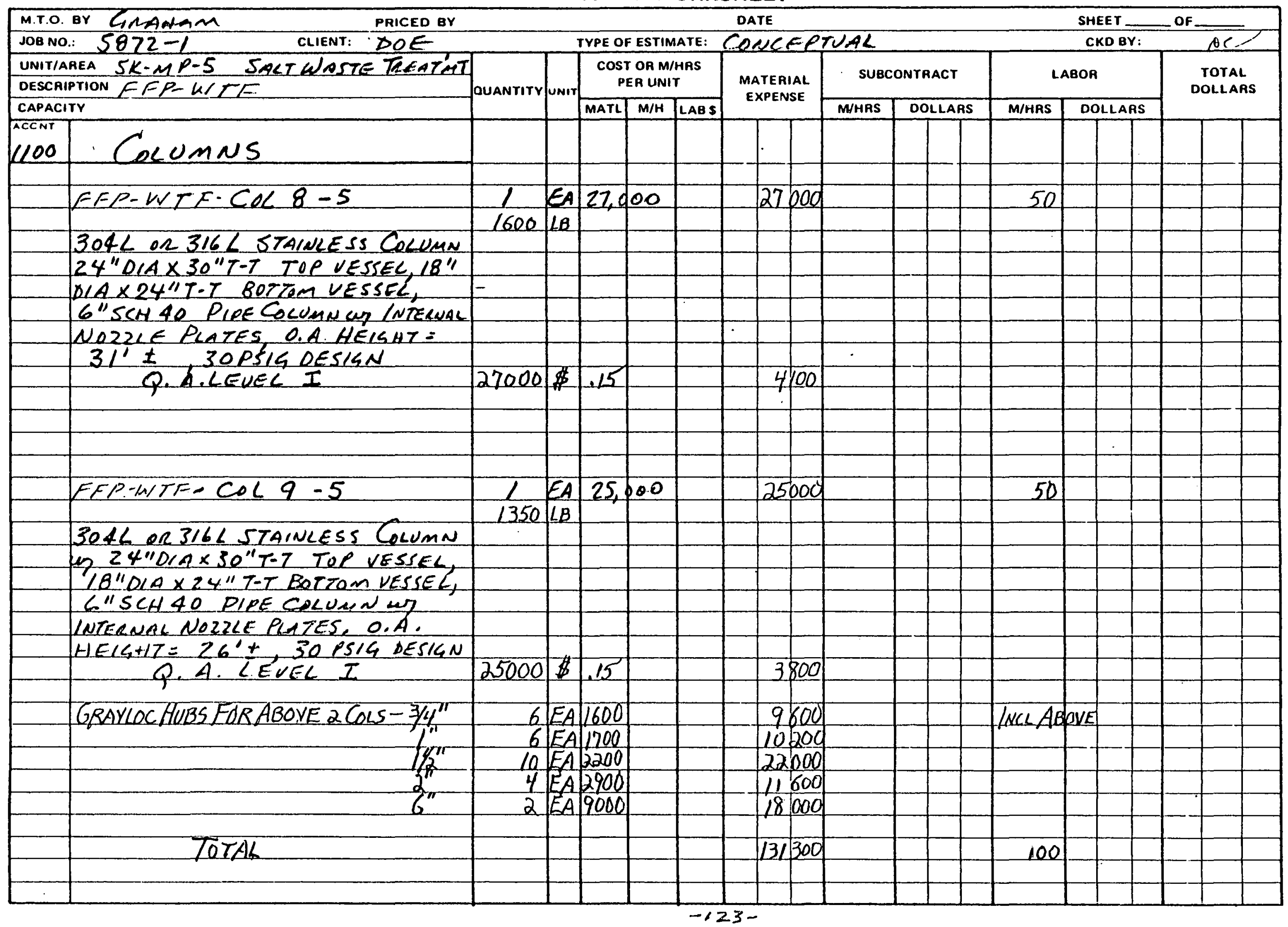


ESTIMATE WORKSHEET

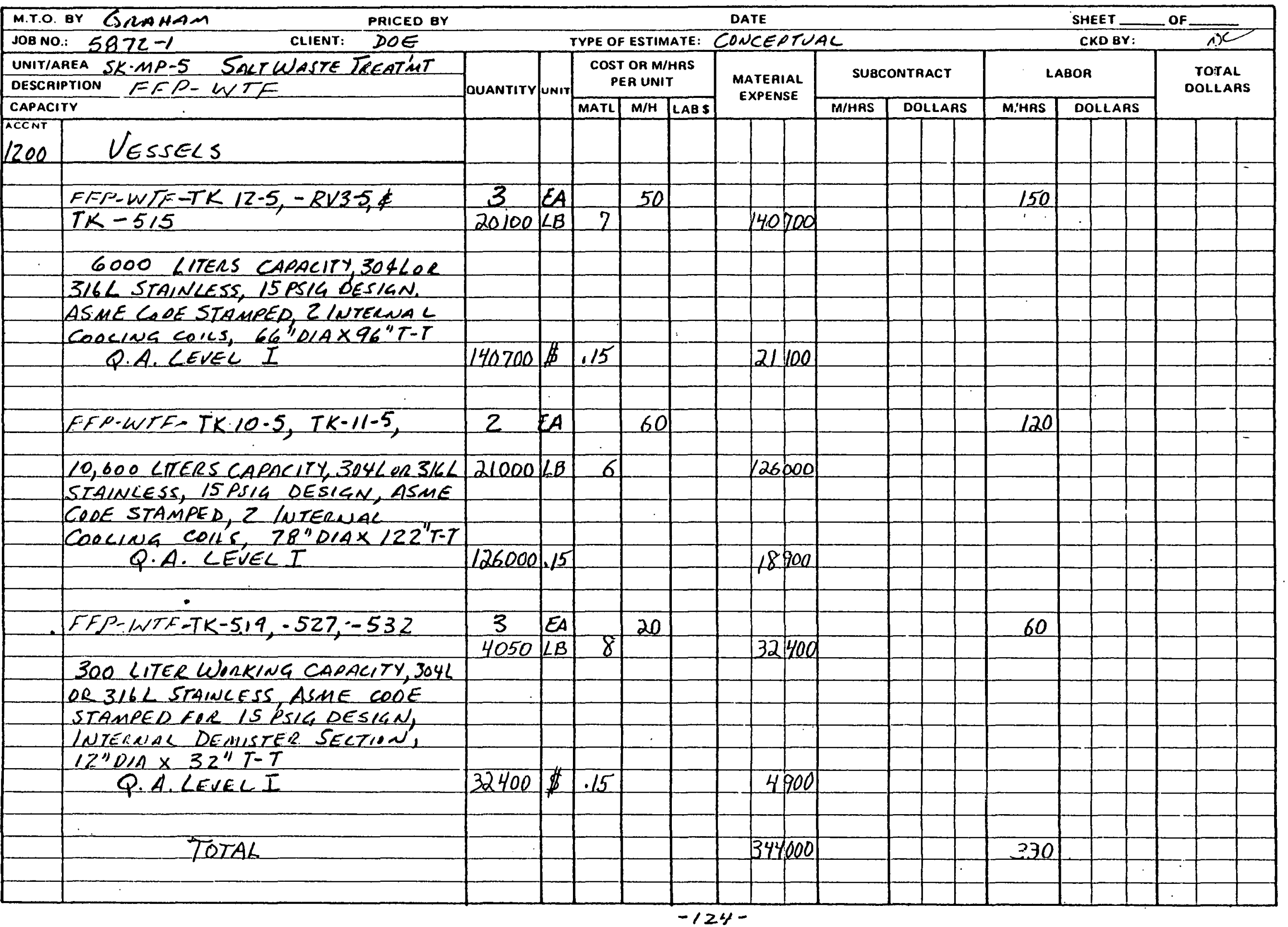


ESTIMATE WORKSHEET

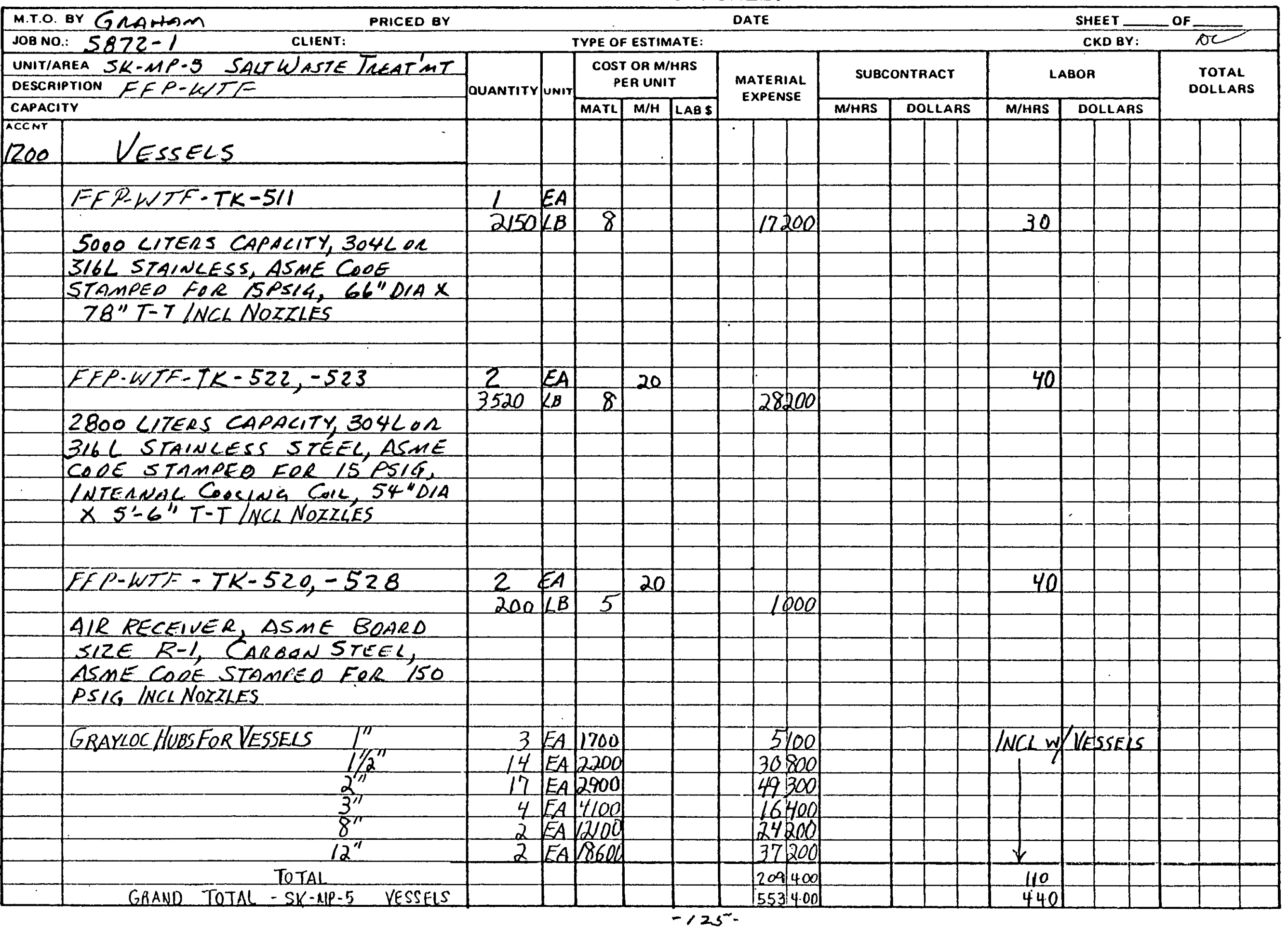


ESTIMATE WORKSHEET

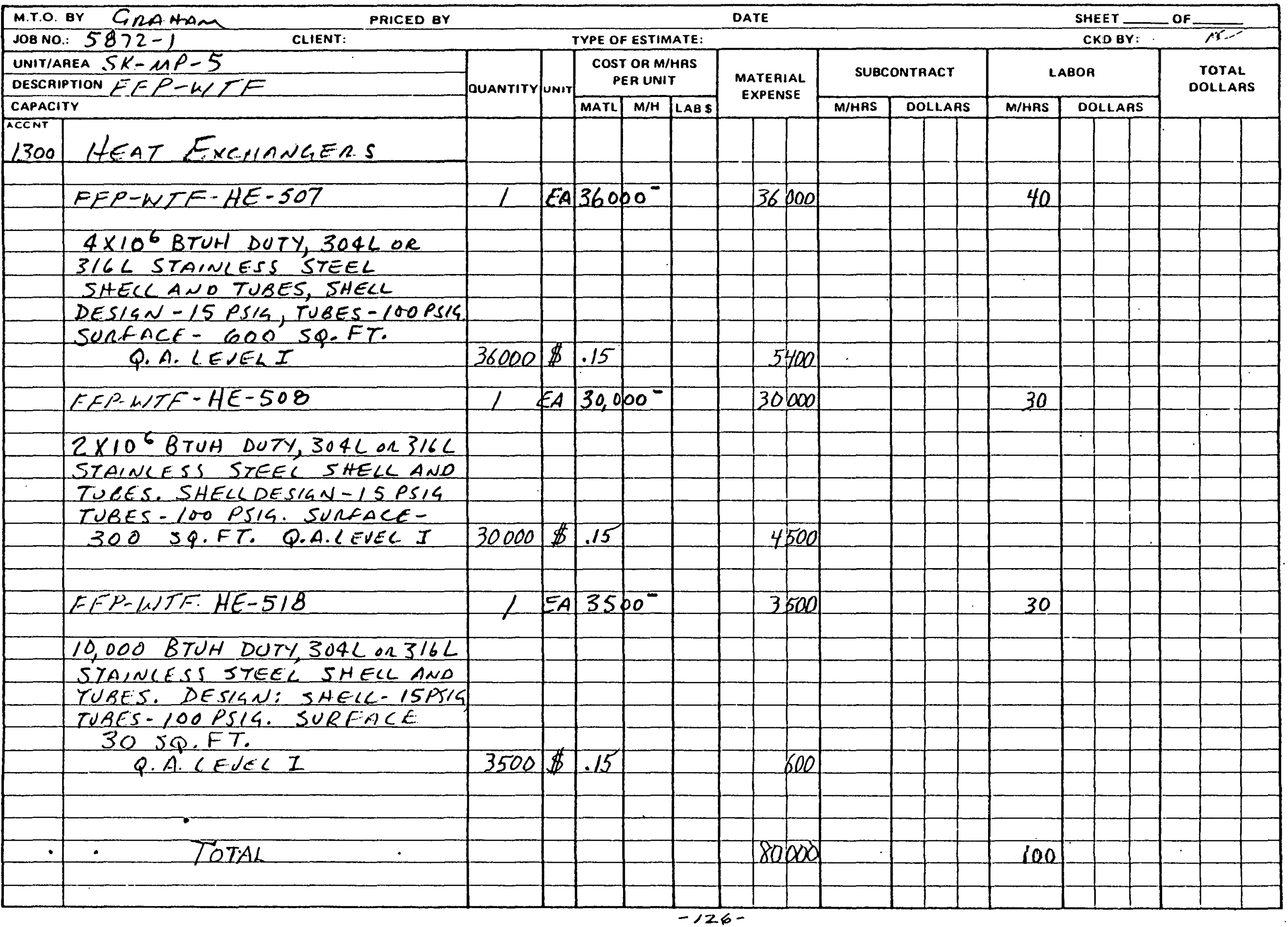


ESTIMATE WORKSHEET

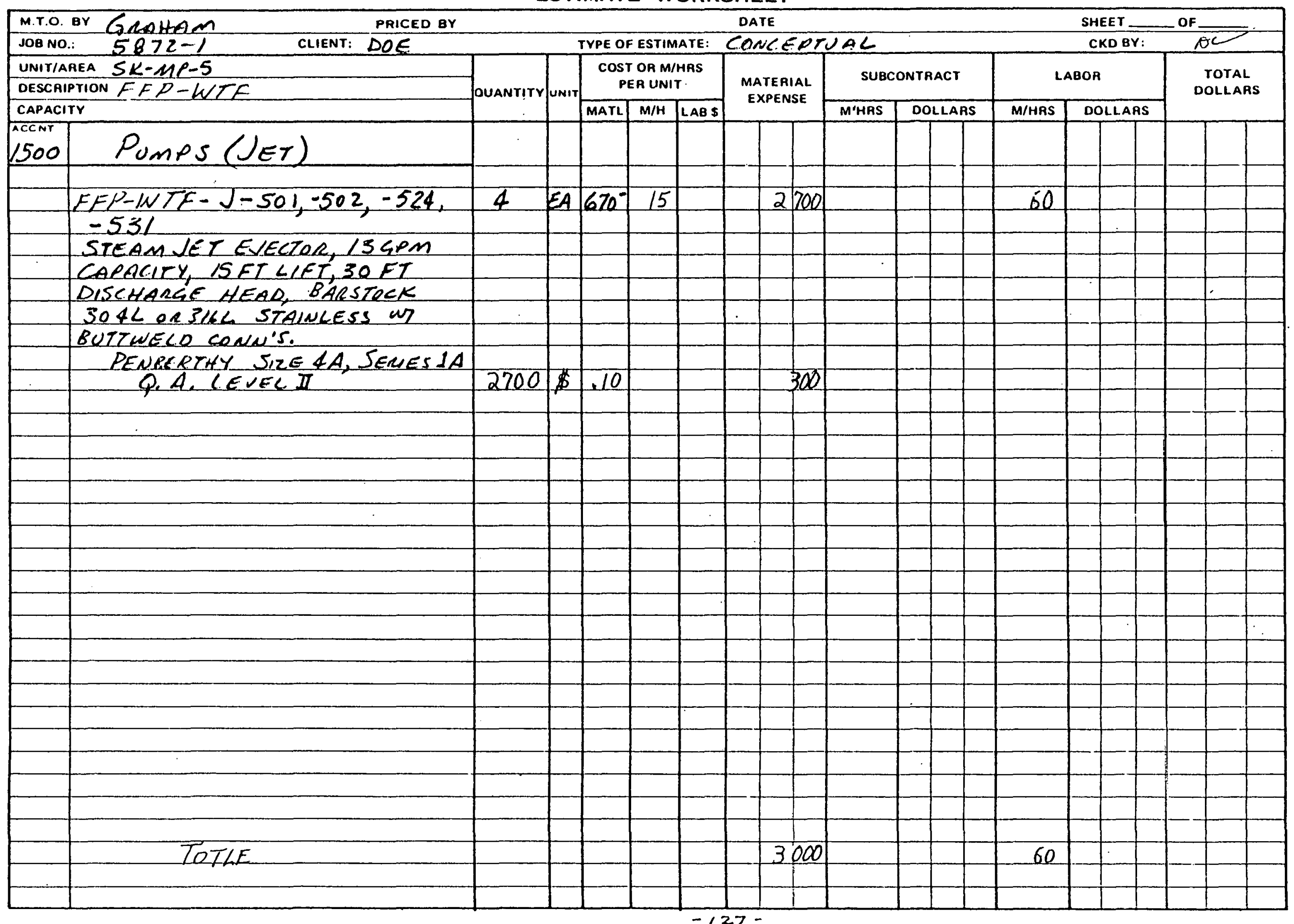


ESTIMATE WORKSHEET

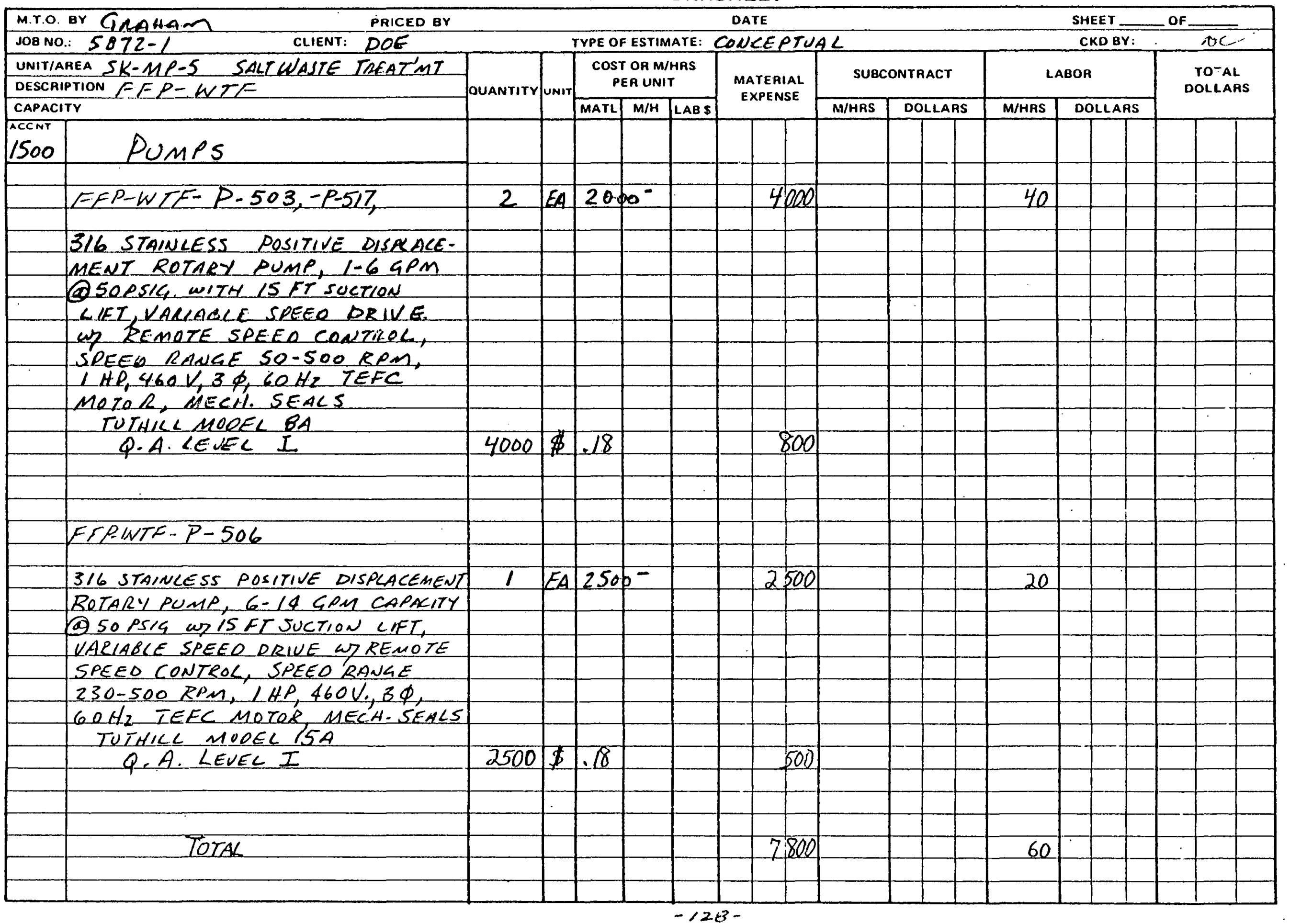


ESTIMATE WORKSHEET

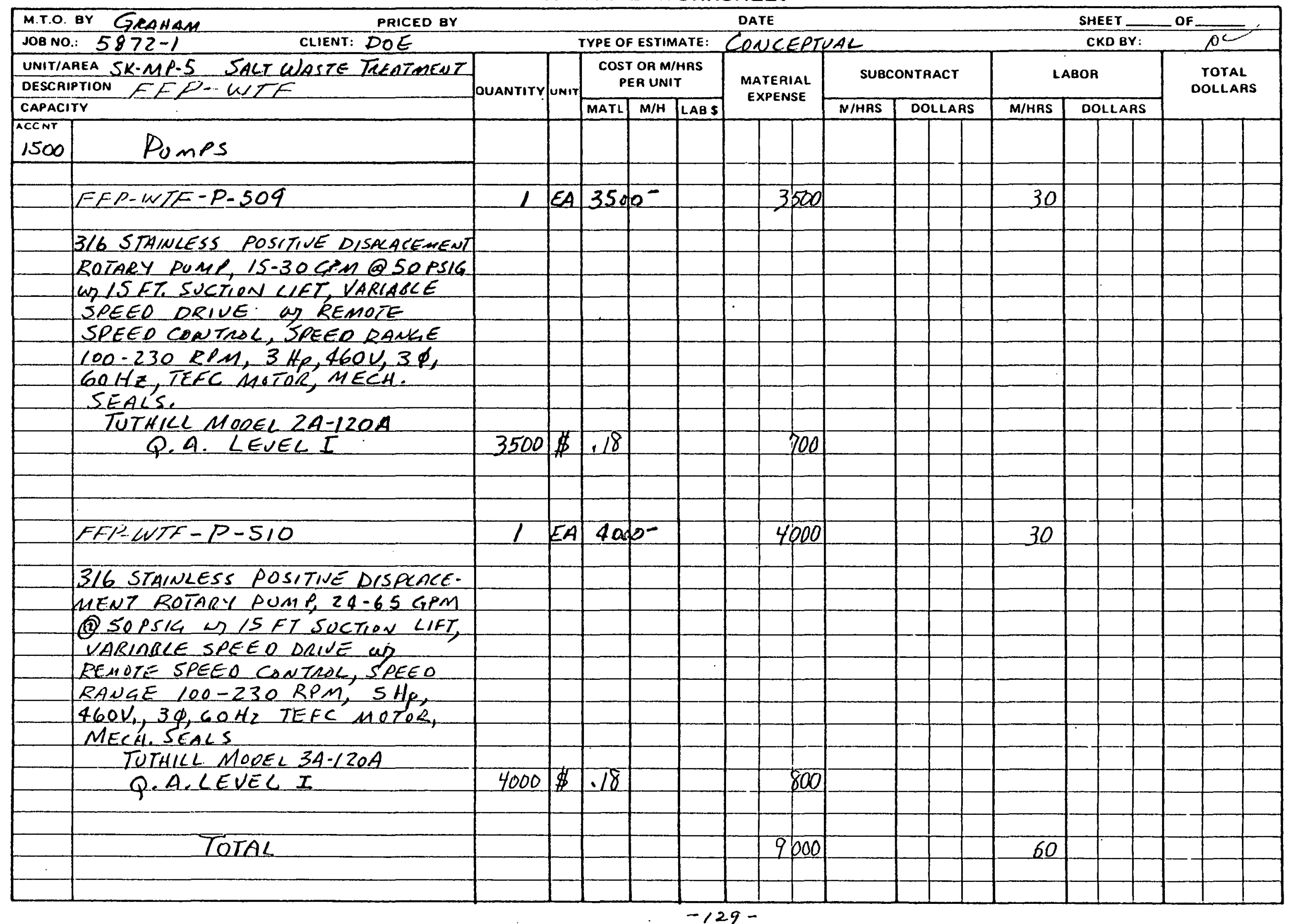


ESTIMATE WORKSHEET




ESTIMATE WORKSHEET

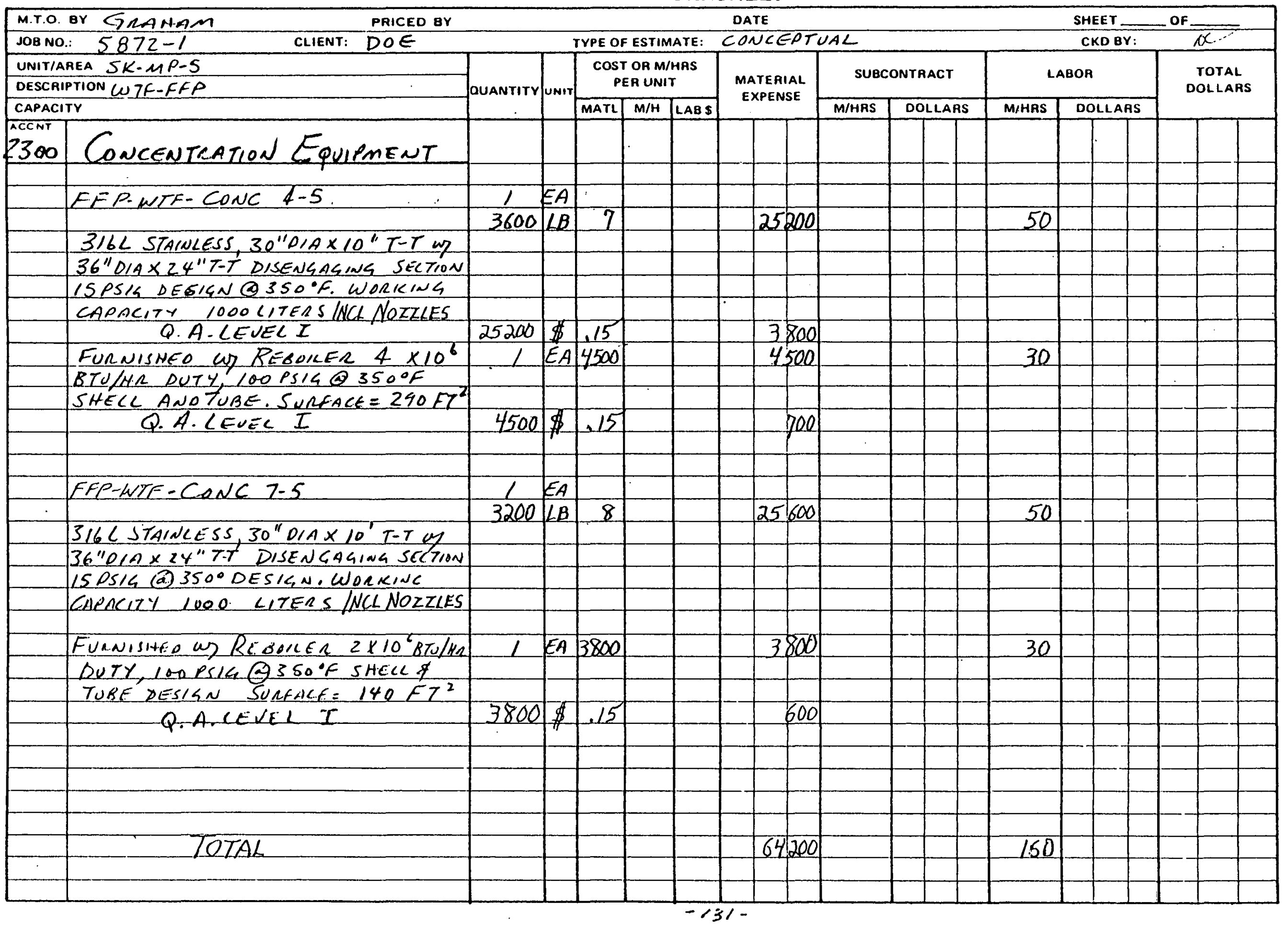


ESTIMATE WORKSHEET

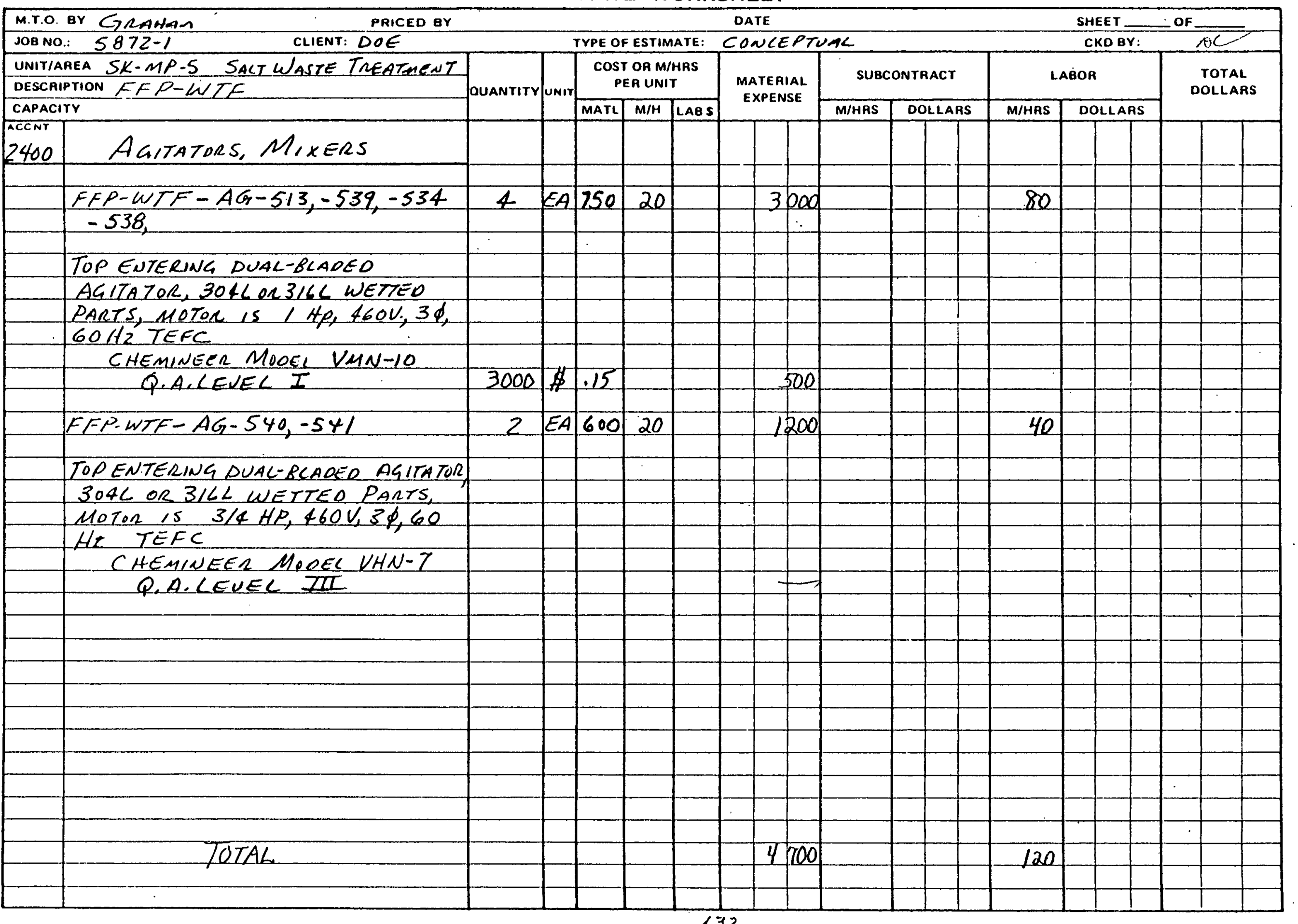


THE RALPHM. PARSONS COMPANY

ESTIMATE WORKSHEET




THE RALPH M. PARSONS COMPANY

ESTIMATE WORKSHEET

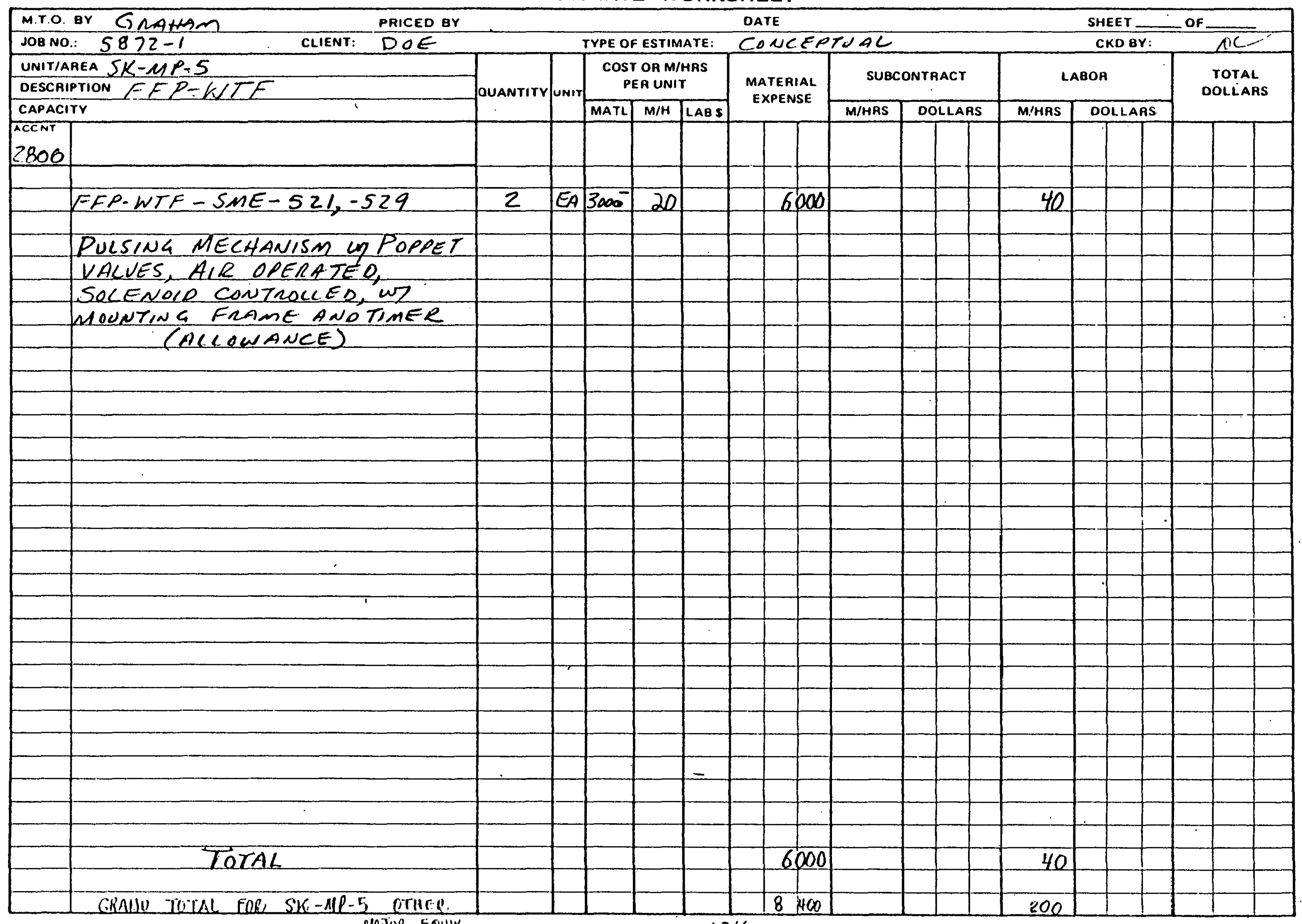

ARDOR EQUIT 


\begin{tabular}{|c|c|c|c|c|c|c|c|c|c|c|c|c|c|c|c|c|c|c|c|}
\hline \multirow{2}{*}{\multicolumn{4}{|c|}{$\begin{array}{l}\text { M.T.O. BY BW } \\
\text { JOB NO.: } 5872-1 \\
\text { PRICED OY BWW }\end{array}$}} & \multirow{2}{*}{\multicolumn{3}{|c|}{ TYPE OF ESTIMATE: }} & \multirow{2}{*}{\multicolumn{6}{|c|}{$\begin{array}{l}\text { DATE } 7-20-79 \\
\text { CONCEPTUAL }\end{array}$}} & \multirow{2}{*}{\multicolumn{7}{|c|}{$\frac{\text { SHEET }}{\text { CKD BY:LW }}$}} \\
\hline & & & & & & & & & & & & & & & & & & & \\
\hline \multirow{2}{*}{\multicolumn{2}{|c|}{ 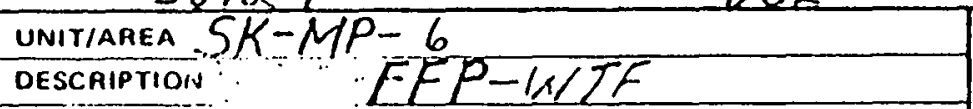 }} & \multirow{4}{*}{ QUANTITY } & \multirow{4}{*}{ Unir } & \multirow{2}{*}{\multicolumn{3}{|c|}{$\begin{array}{c}\text { COST OR M/HRS } \\
\text { PER UNIT }\end{array}$}} & \multirow{3}{*}{\multicolumn{2}{|c|}{$\begin{array}{l}\text { MATERIAL } \\
\text { EXPENSE }\end{array}$}} & \multirow{2}{*}{\multicolumn{3}{|c|}{ SUBCONTRACT }} & & \multirow{2}{*}{\multicolumn{4}{|c|}{ LABOR }} & \multirow{3}{*}{\multicolumn{3}{|c|}{$\begin{array}{l}\text { TOTAL } \\
\text { DOLLARS }\end{array}$}} \\
\hline & & & & & & & & & & & & & & & & & & & \\
\hline \multicolumn{2}{|c|}{$\begin{array}{l}\text { CAPACITY } \\
\text { ACCNT }\end{array}$} & & & \multirow[t]{2}{*}{ MATL } & \multirow[t]{2}{*}{$\mathrm{M} / \mathrm{H}$} & \multirow[t]{2}{*}{ LAB \$ } & & & \multirow[t]{2}{*}{ M/HRS } & \multicolumn{3}{|c|}{ DOLLARS } & \multirow[t]{2}{*}{ M/HAS } & \multicolumn{3}{|c|}{ DOLLARS } & & & \\
\hline 6000 & SPECIAL FACILITIES-EQUIPMENT SUMMARY & & & & & & & & & & & & & & & & & & \\
\hline & & & & & & & & & & & & & & & & & & & \\
\hline 1100 & collumiss & & & & & & $117 \mathrm{~s}$ & 400 & & & & & 200 & & & & & & \\
\hline 1200 & VESSELS & & & & & & 646 & 100 & & 7 & & & 720 & & & & & & \\
\hline 1300 & HEAT EXCHANGERS & & & & & & 38 & 100 & & & & & .90 & & & & & & \\
\hline 1500 & PuMPS & & & & & & 31 & 100 & & $\therefore$ & & & 355 & & & & & & \\
\hline 2300 & SEPE RATION EQUIPAIENT & & & & & & 36.0 & gee & & & & & 100 & & & & & & \\
\hline 2300 & COUCENTRATION EQUUPMENT & & & & & 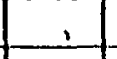 & 33 il & 400 & & & & & 80 & & & & & & \\
\hline 2400 & AGIIATORS MIXERS & & & & & & 6 & 800 & & & & & 220 & & & & & & \\
\hline 2800 & OTHER MATOR EQUIPMENT & & & & & & $13]$ & 800 & & & & & 200 & & & & & & \\
\hline & & & & & & & & & & & & & & & & & & & \\
\hline & & & & & & & & & & & & & & & & 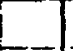 & & i. & \\
\hline & & & & & & & & & & & & & & & & & & & \\
\hline & SUB TOTAL & & & & & & 9235 & 600 & & & & & 1965 & & & & & & \\
\hline & & & & & & & & & & & & & & & & & & & \\
\hline & $65 \%$ provuctivitr $(1965 \div-65)-1965$ & & & & & & & & & & & & 1058 & & & & & & \\
\hline & & & & & & & & & & & & & 3023 & & & & & & \\
\hline & & & & & & & & & & & & & & & & & & & \\
\hline & & 3023 & 114 & & & 16.60 & & & & & & & $\because$ & & & 200 & & & \\
\hline & & & & & & & & & & & & & & & & & & & \\
\hline & & & & & & & & & & & & & & & & I & & & \\
\hline & TQTAL FOR SK-MP-6 & & & & & & & & & & & & 3023 & & & 200 & & 973 & 800 \\
\hline & & & & & & & & & & & & & & & & & & & \\
\hline & & & & & & & & & & & & & & & & & & & \\
\hline & & & & & & & & & & & & & & & & 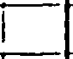 & & & \\
\hline & - & & & & & & & & & & & & & & & & & $i$ & \\
\hline & & & & & & 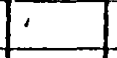 & & & & & & & & & & & & & \\
\hline & & & & & & & & & & & & & & & & & $=$ & & \\
\hline & & & & & & & & & & & & & & & & & & & \\
\hline & & & & & & & & & & & & & & & & & & & \\
\hline & & & & & & & & & & & & & & & & & & & \\
\hline & & & & & & & & & & & & & & & & & & & \\
\hline
\end{tabular}


Les 20000

THE RALPH M. PARSONS COMPANY

ESTIMATE WORKSHEET

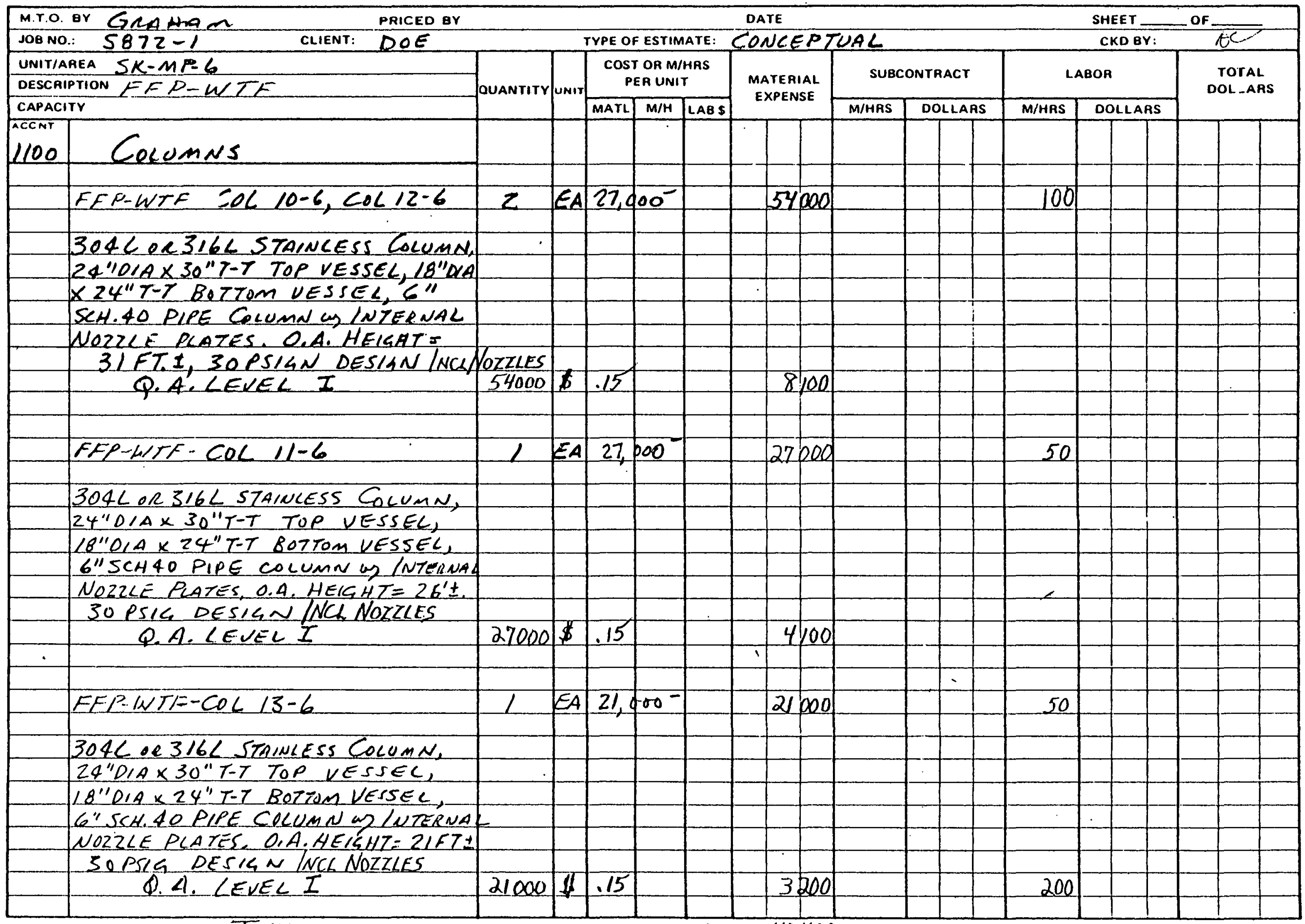


CtS 20000

THE RALPH M. PARSONS COMPANY

ESTIMATE WORKSHEET

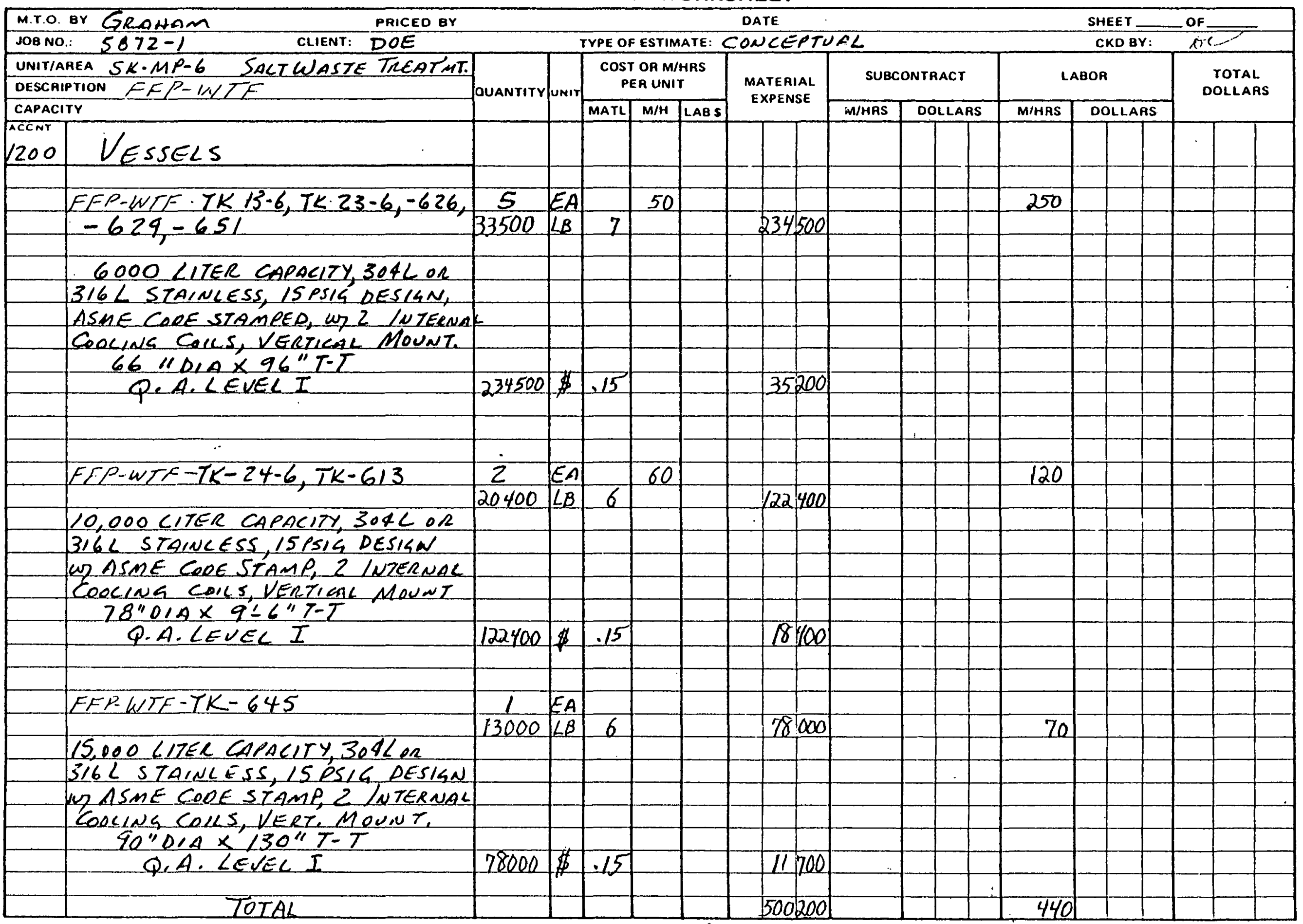


ESTIMATE WORKSHEET

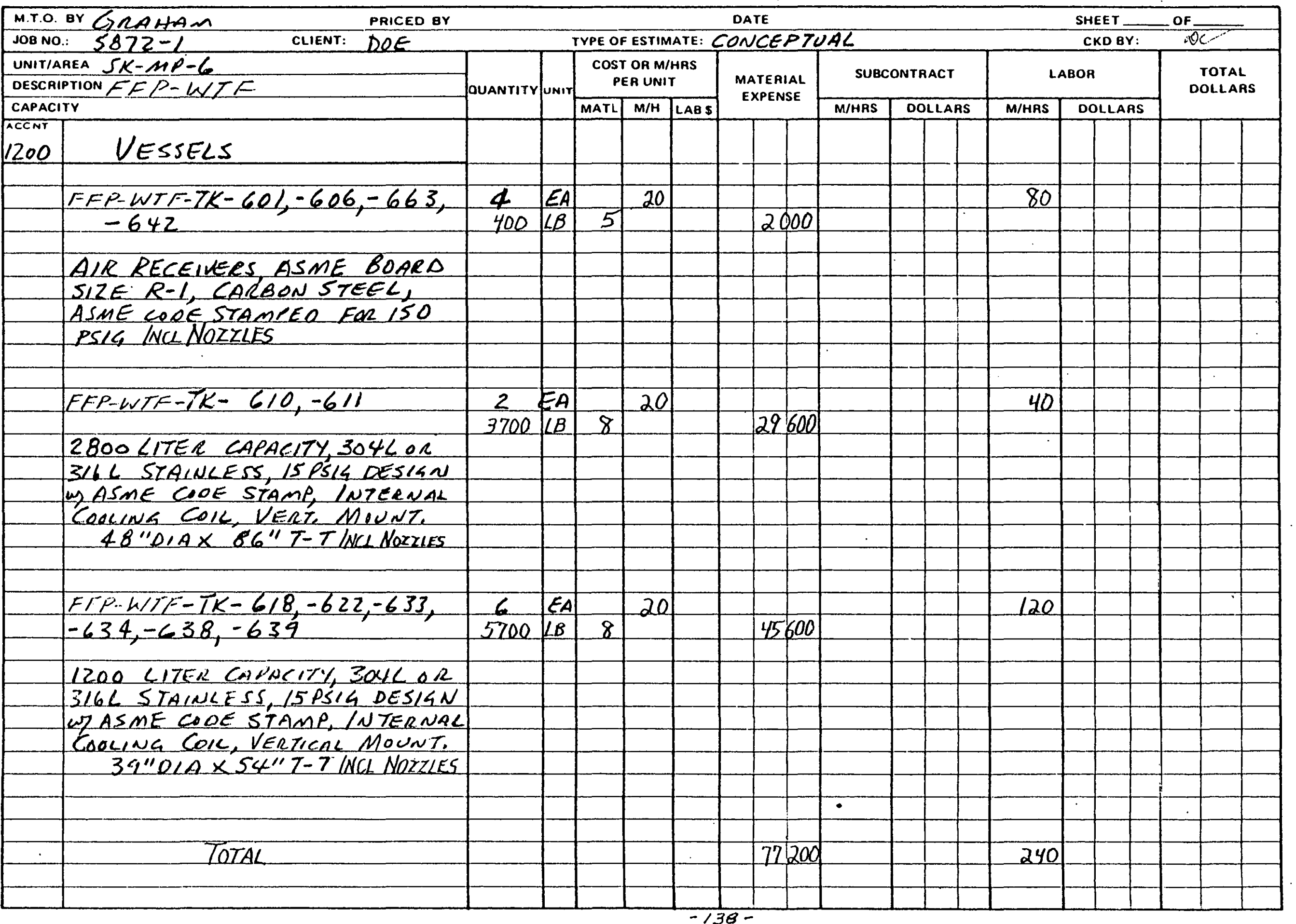


THE RALPH M. PARSONS COMPANY

M.T.O. BY GRAHAN
JOBNO.: $582 Z=1$
UNITIAREA $5 K-M P=6$

Description EFP-WTE

CAPACITY

ACCNT

1200 VESSELS

$$
\text { FFP-WTF-TK-662 }
$$

10,000 LITER CAPACITY $304 L$ OR 3lGL STAINLESS, 15 PSIL DESLGN WI ASME COOE STAMP VERTICAL MUUNT. 78"OLAX G'G"T-T Q. A. LEVEL I GRAYLOC HUBS - I" CLENT: DOE

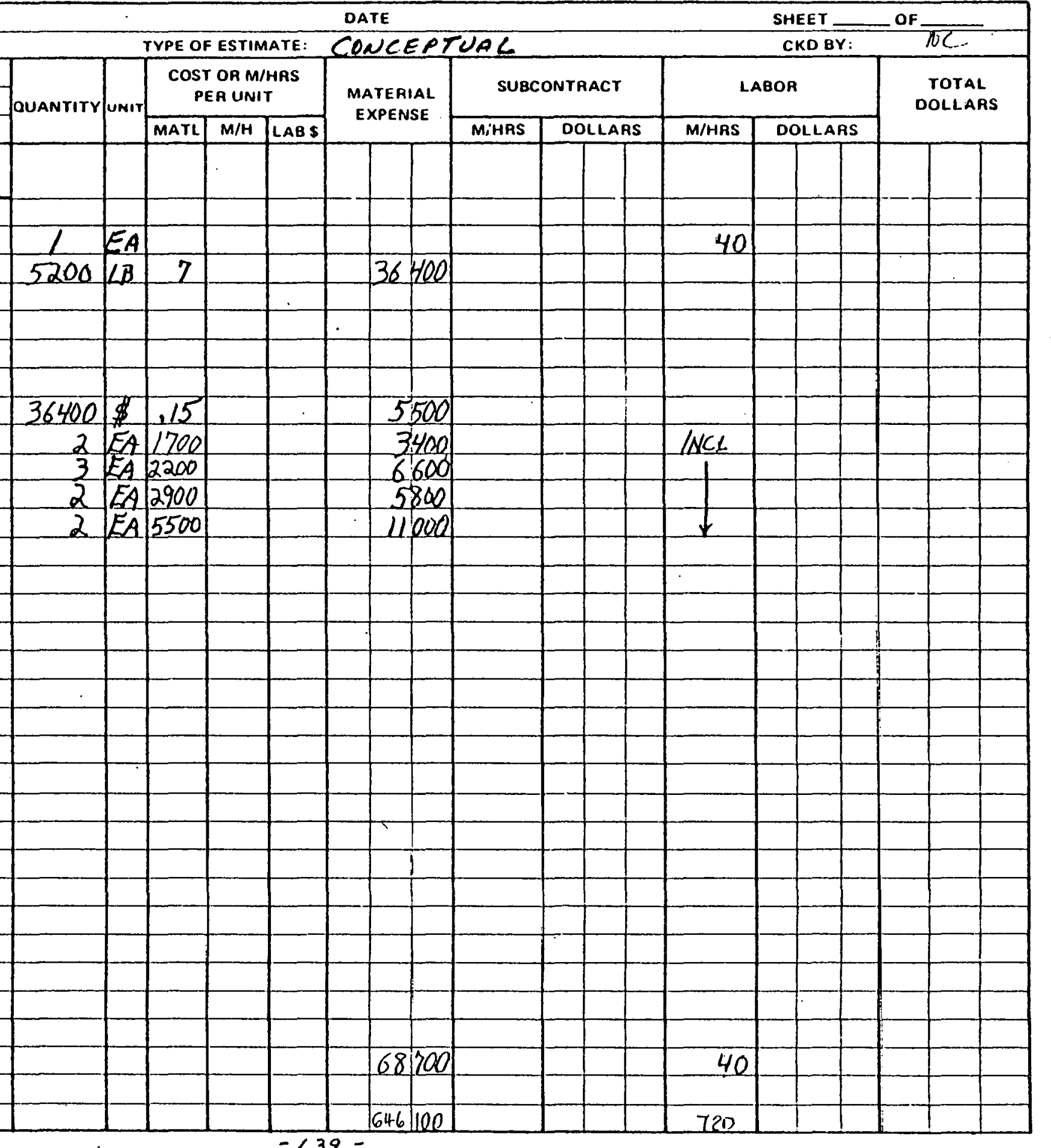


ESTIMATE WORKSHEET

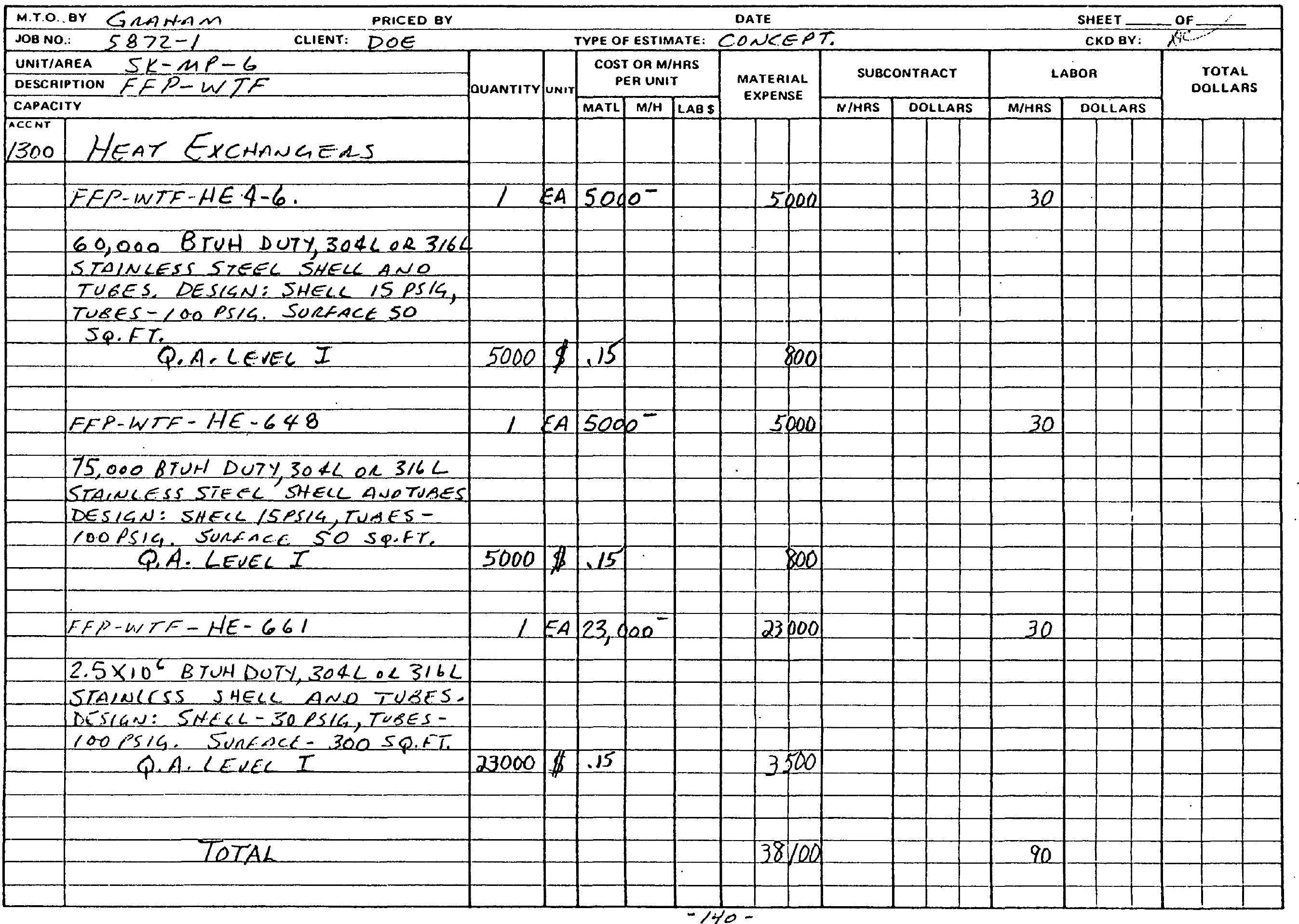


LS $3000-0$

THE RALPH M. PARSONS COMPANY

ESTIMATE WORKSHEET

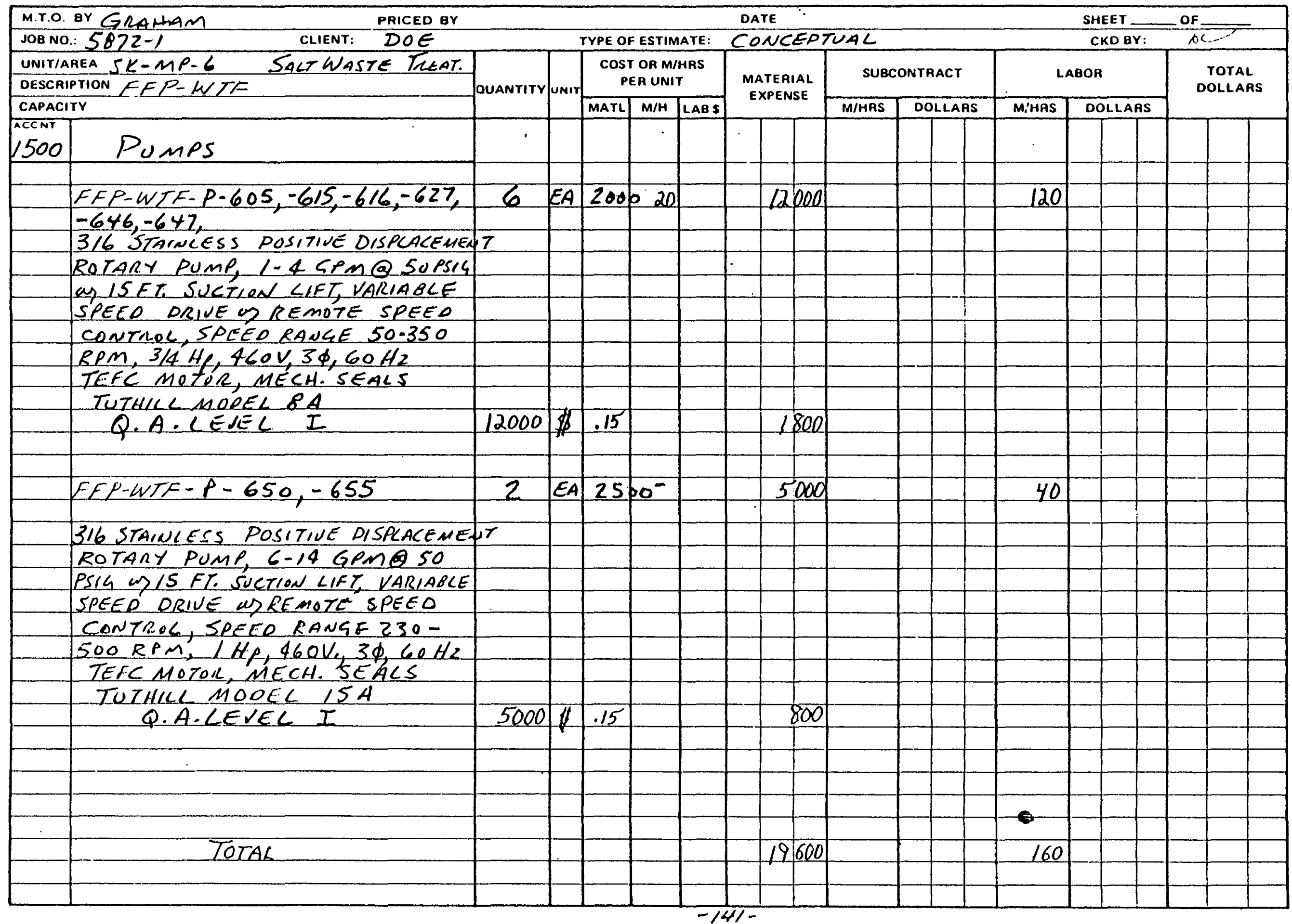


ESTIMATE WORKSHEET

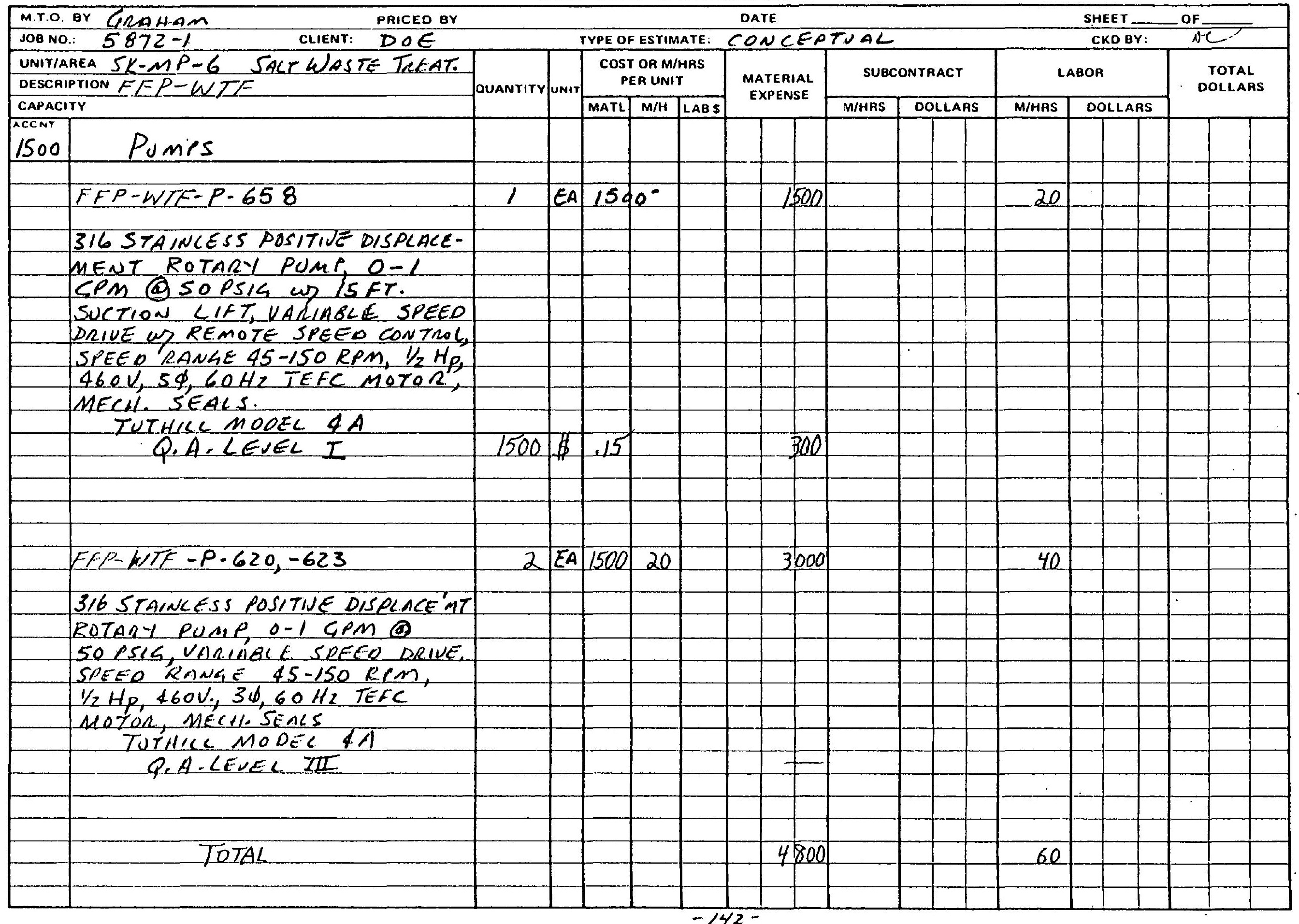


ESTIMATE WORKSHEET

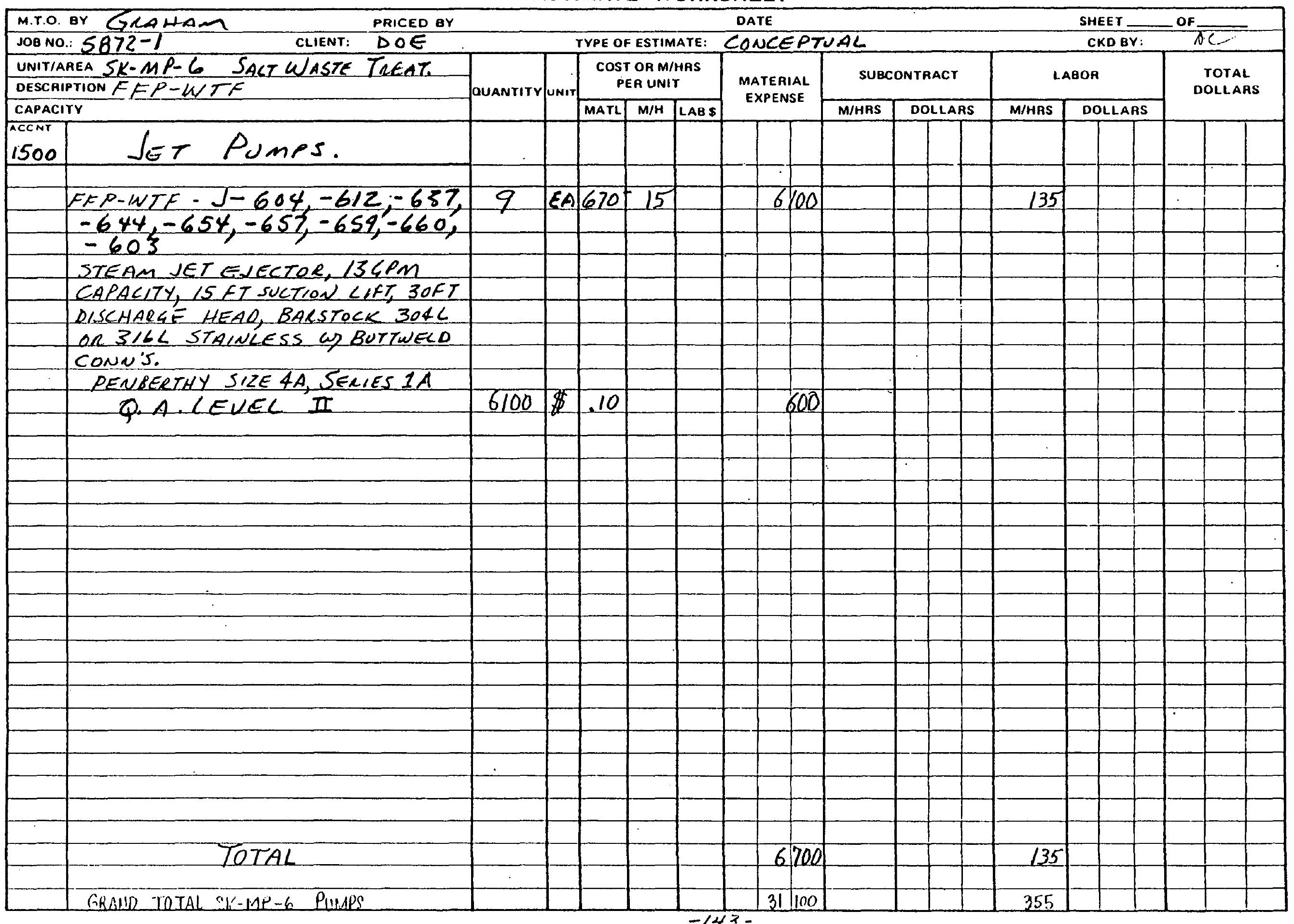


LES 20000

THE RALPH M. PARSONS COMPANY

ESTIMATE WORKSHEET

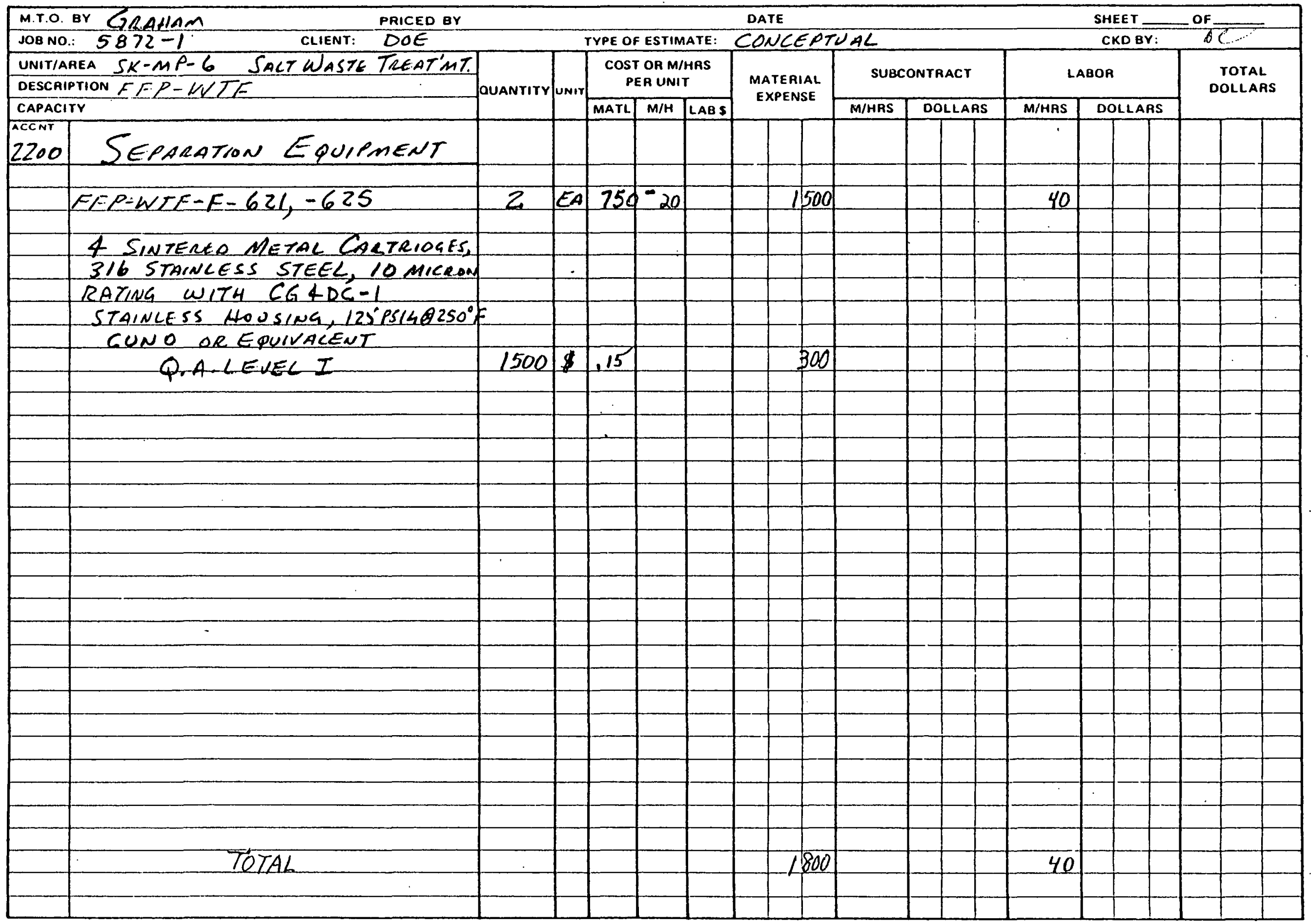


Les 20000 ;

THE RALPH M. PARSONS COMPANY

ESTIMATE WORKSHEET

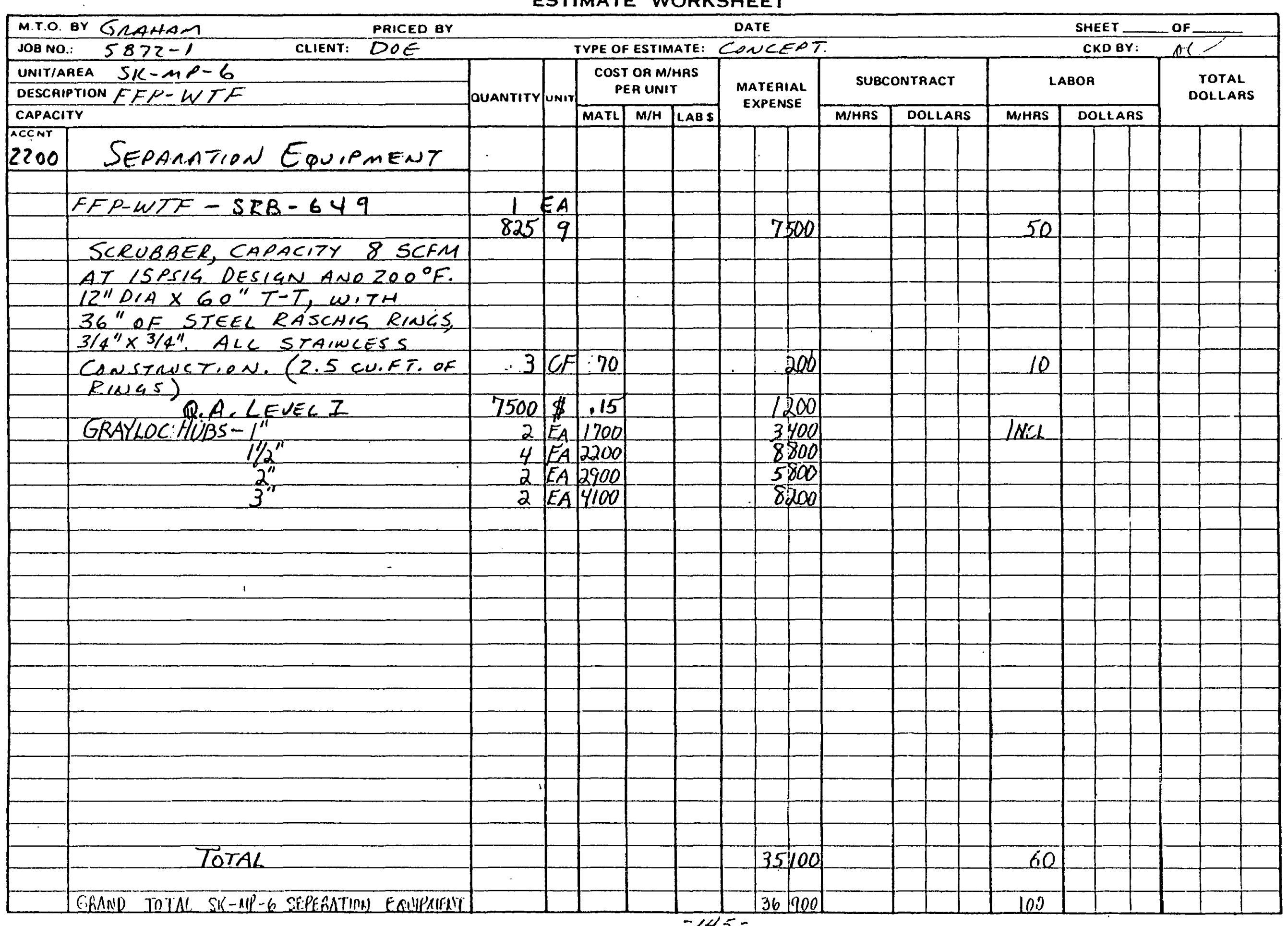


ESTIMATE WORKSHEET

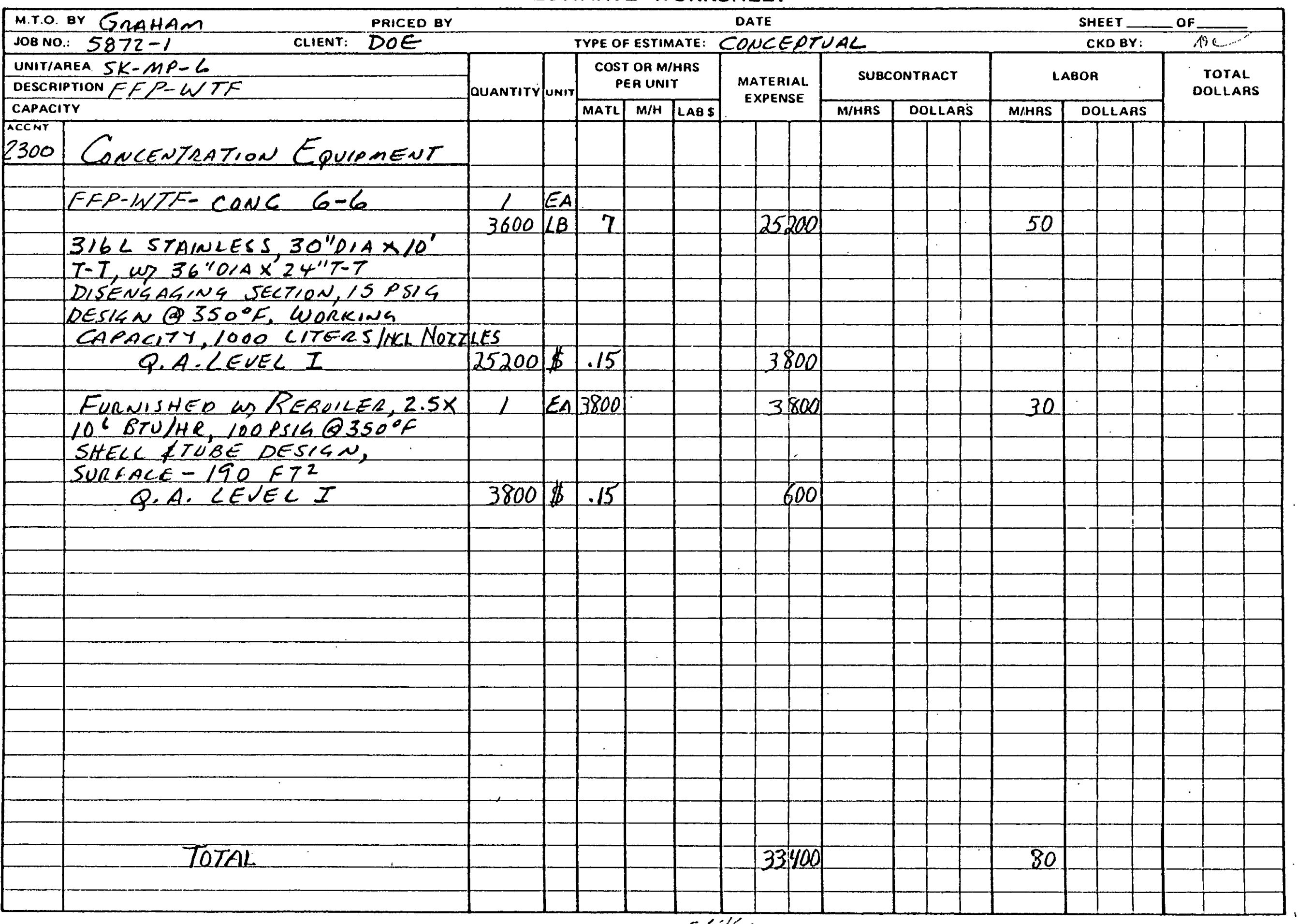


ESTIMATE WÓRKSHEET

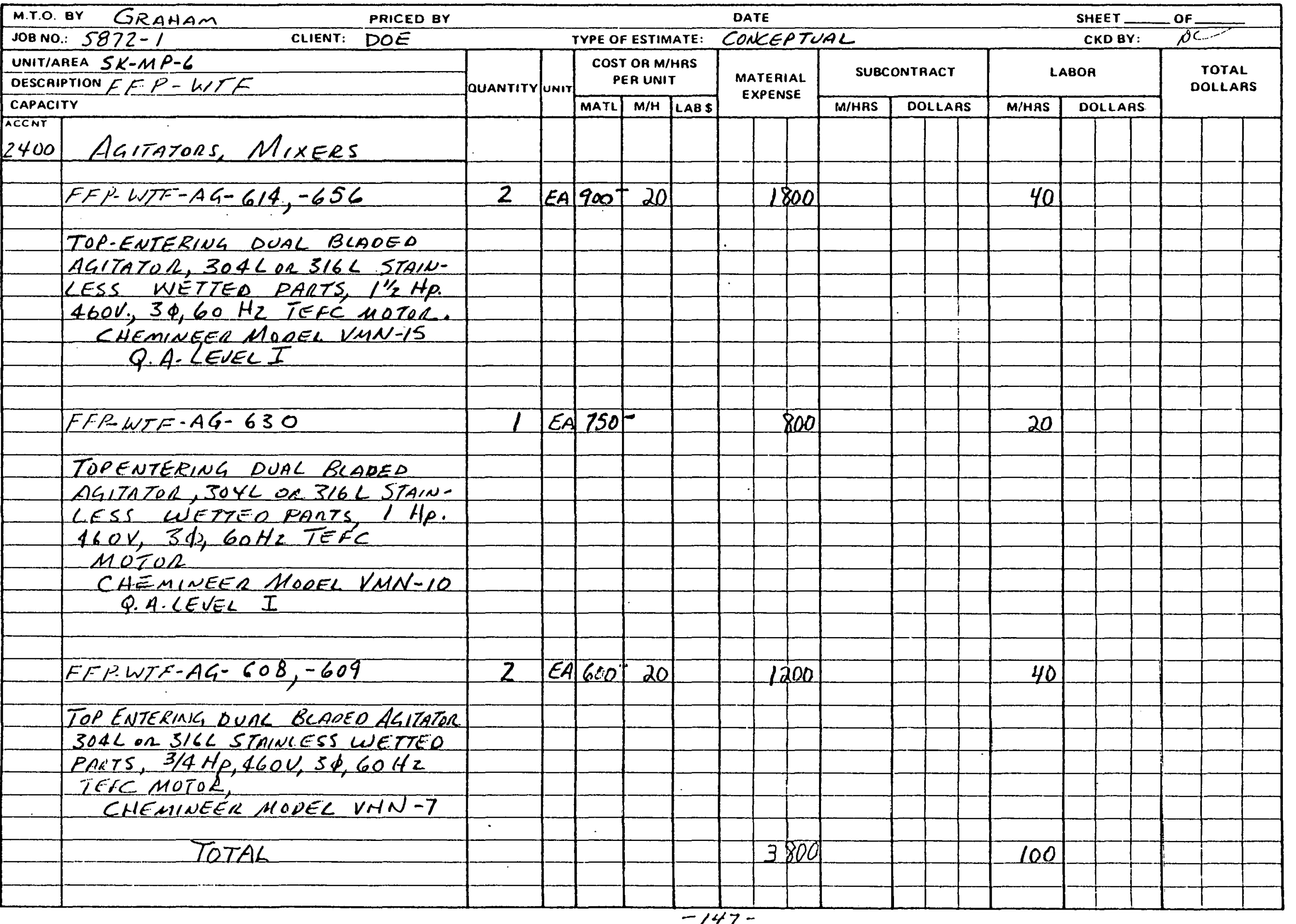


ESTIMATE WORKSHEET

PRICED BY

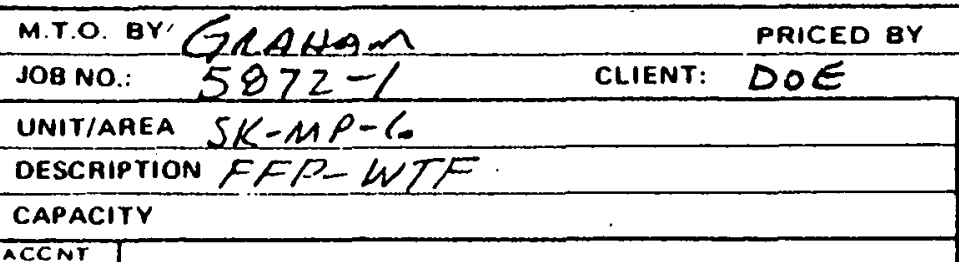

ztoo Agitatons, Mexens

FFP-WFF -AG $-619,-624,-635$,

$-656,-640,-641$

Top Entering Dual Bladeo Aaitatde,

$304 L$ on $316 L$ STACNCESS WETTEO

RACOS, $1 / 2 \mathrm{HP}, 460 \mathrm{~V}, 3 \mathrm{~B}, 60 \mathrm{H}_{2}$ TEFE

MOTOR.

CHEMINEER MODEL VHN-5

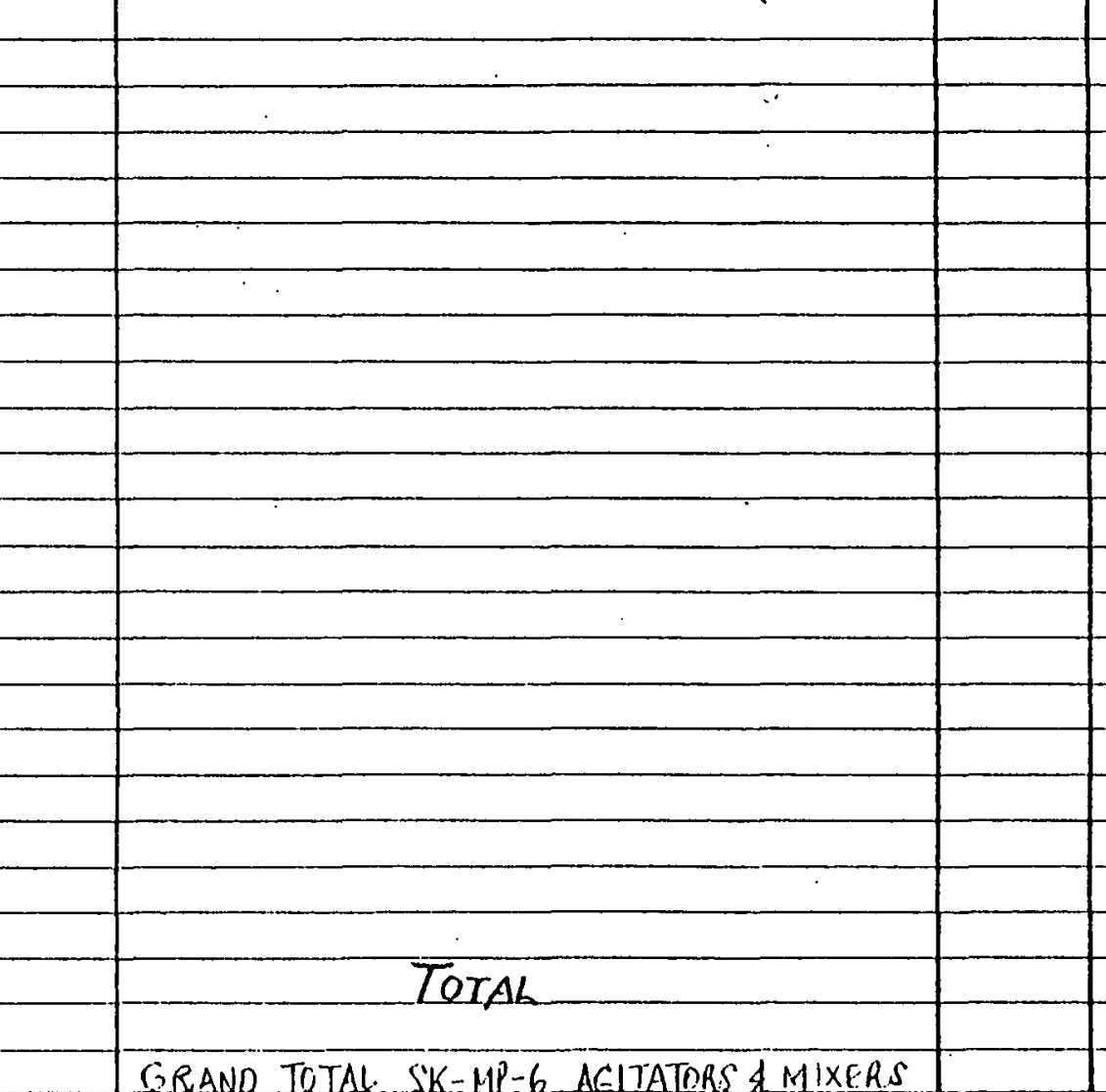

GRAND TOTAL SK-MP-6 AELTATORS A MIXERS
DATE

TYPE OF ESTIMATE: CON CENTUAL

COST OA M/HRS

PER UNIT

\begin{tabular}{|l|l|l|}
\hline MATL & M/H & LABS \\
\hline
\end{tabular}

EXPENSE

EA $500^{-}-20$

3000

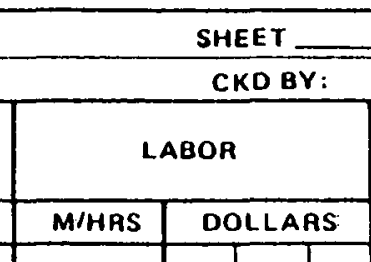

120 
THE RALPH M. PARSONS COMPANY

ESTIMATE WORKSHEET

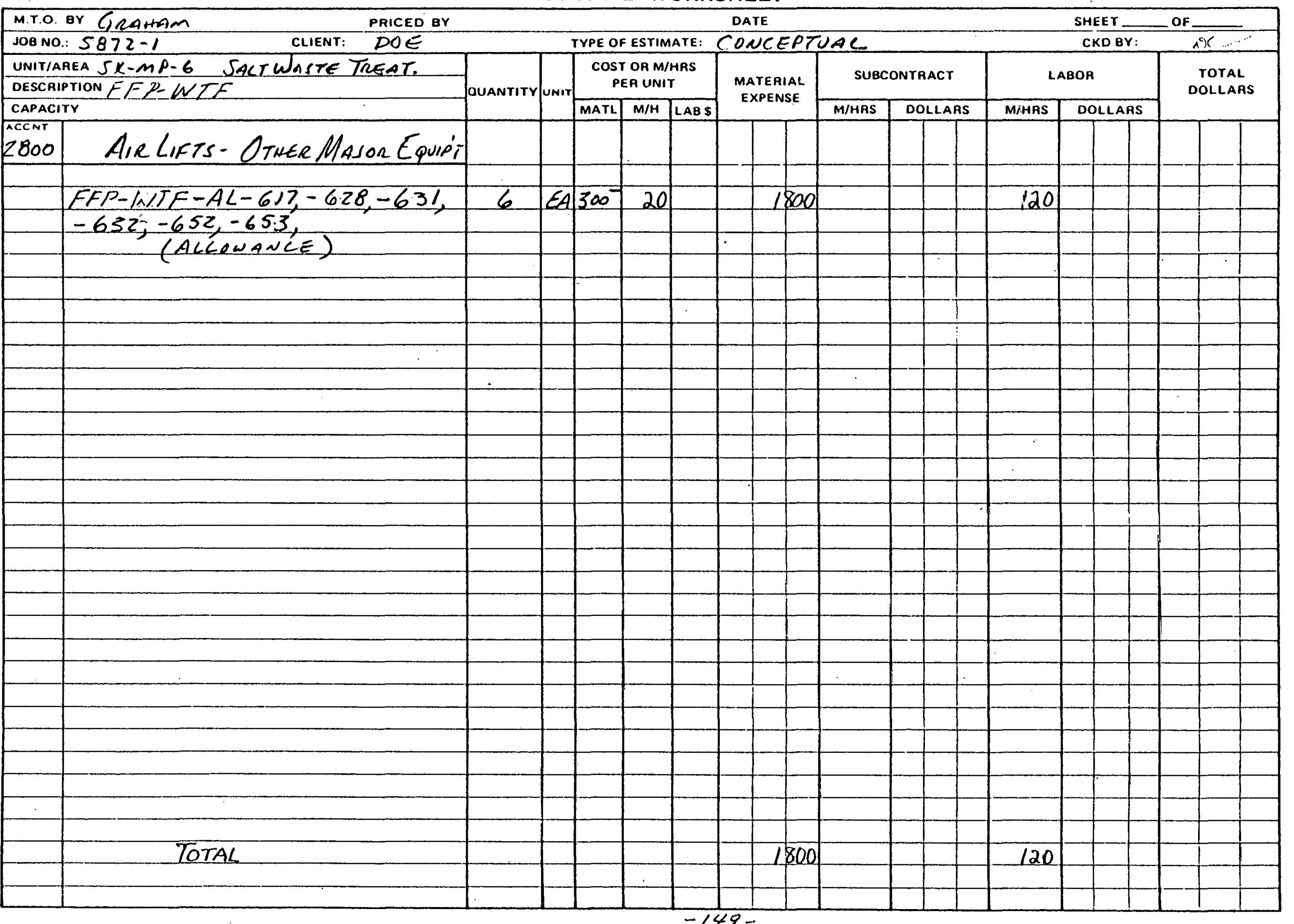


THE RALPH M. PARSONS COMPANY

ESTIMATE WORKSHEET

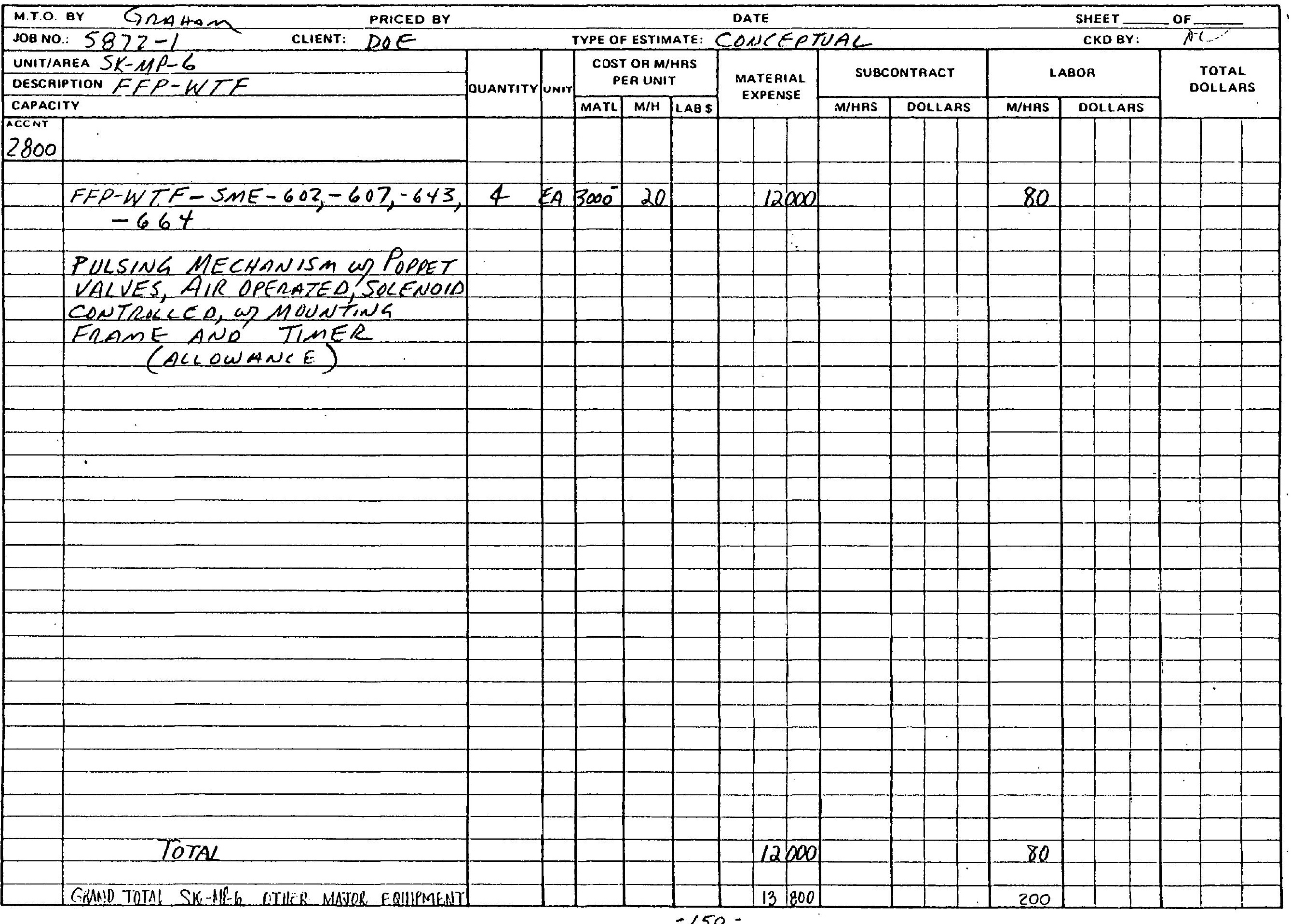




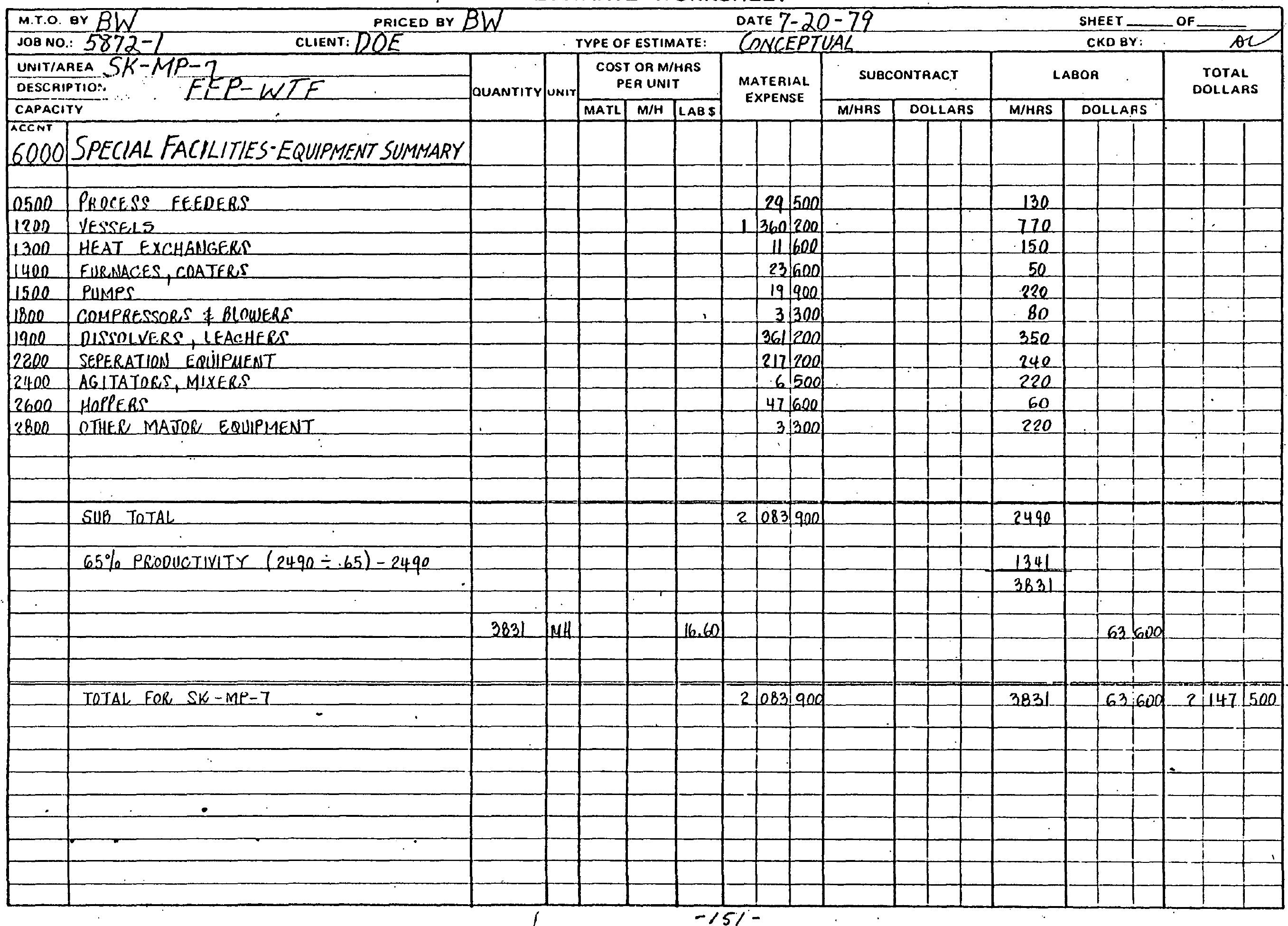


ESTIMATE WORKSHEET

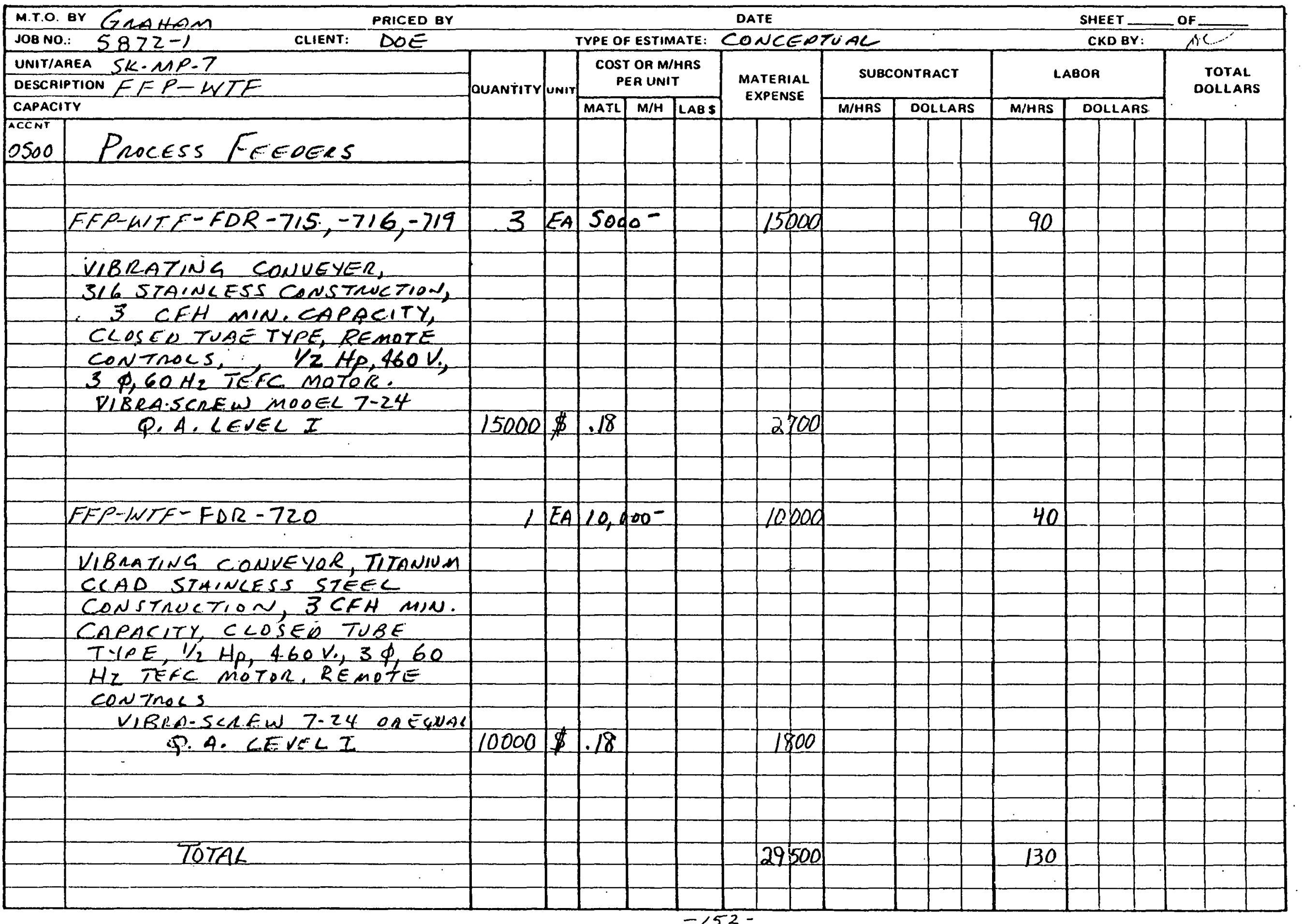


ESTIMATE WORKSHEET

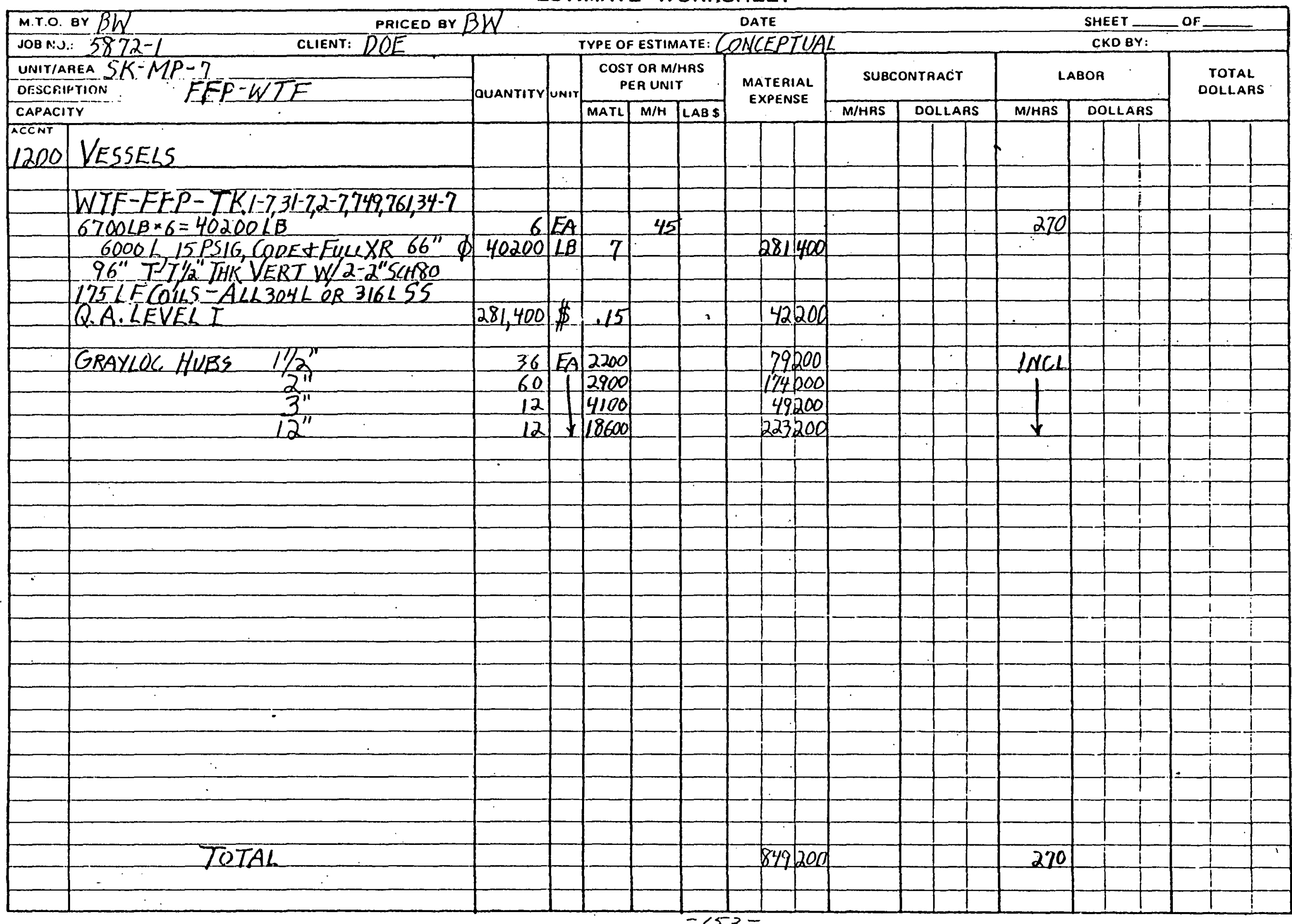


ESTIMATE WORKSHEET PRICEO BY $B W$ CLIENT: DOE

DATE

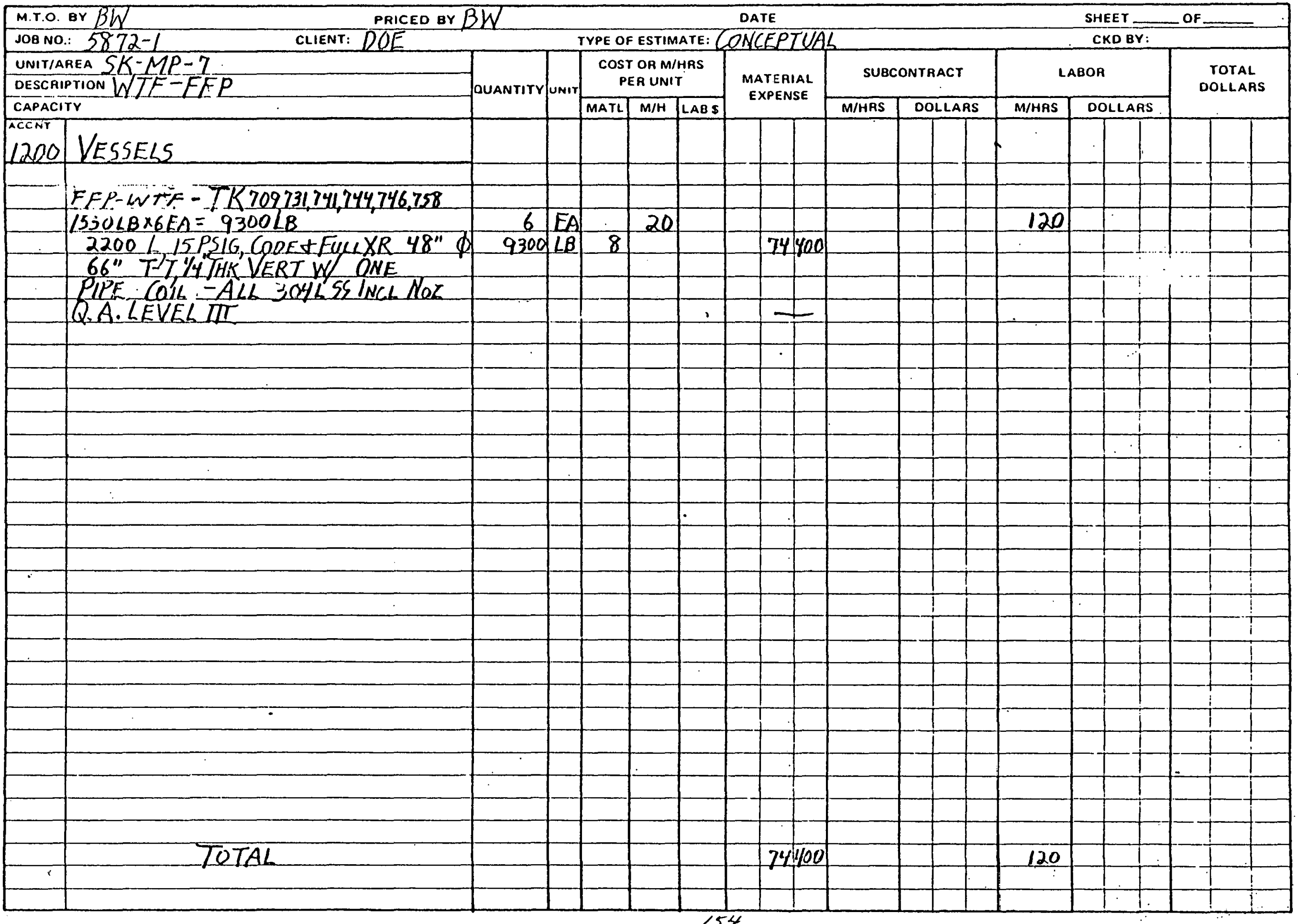


c $2000.0 /$

THE RALPH M. PARSONS COMPANY

ESTIMATE WORKSHEET

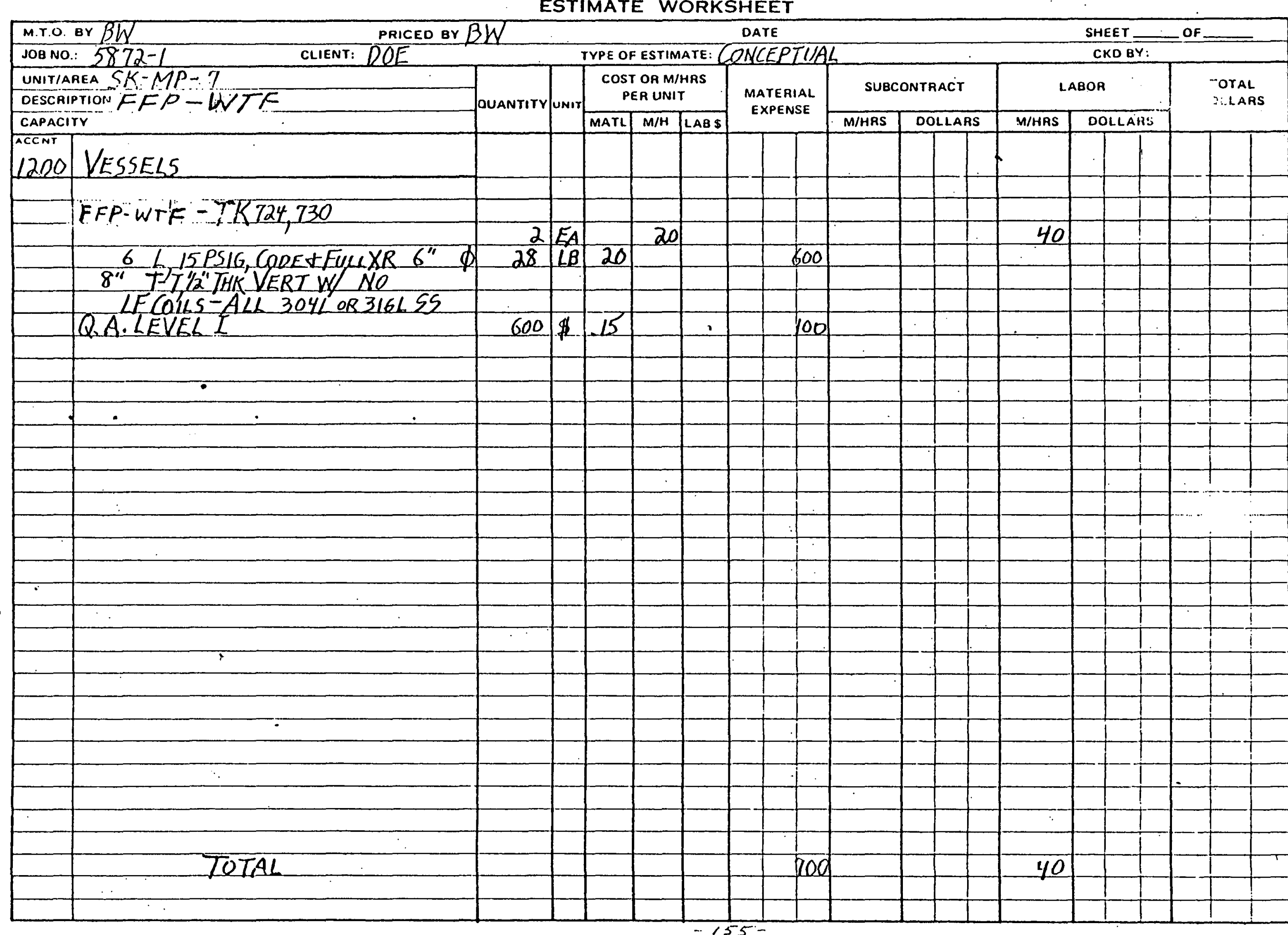




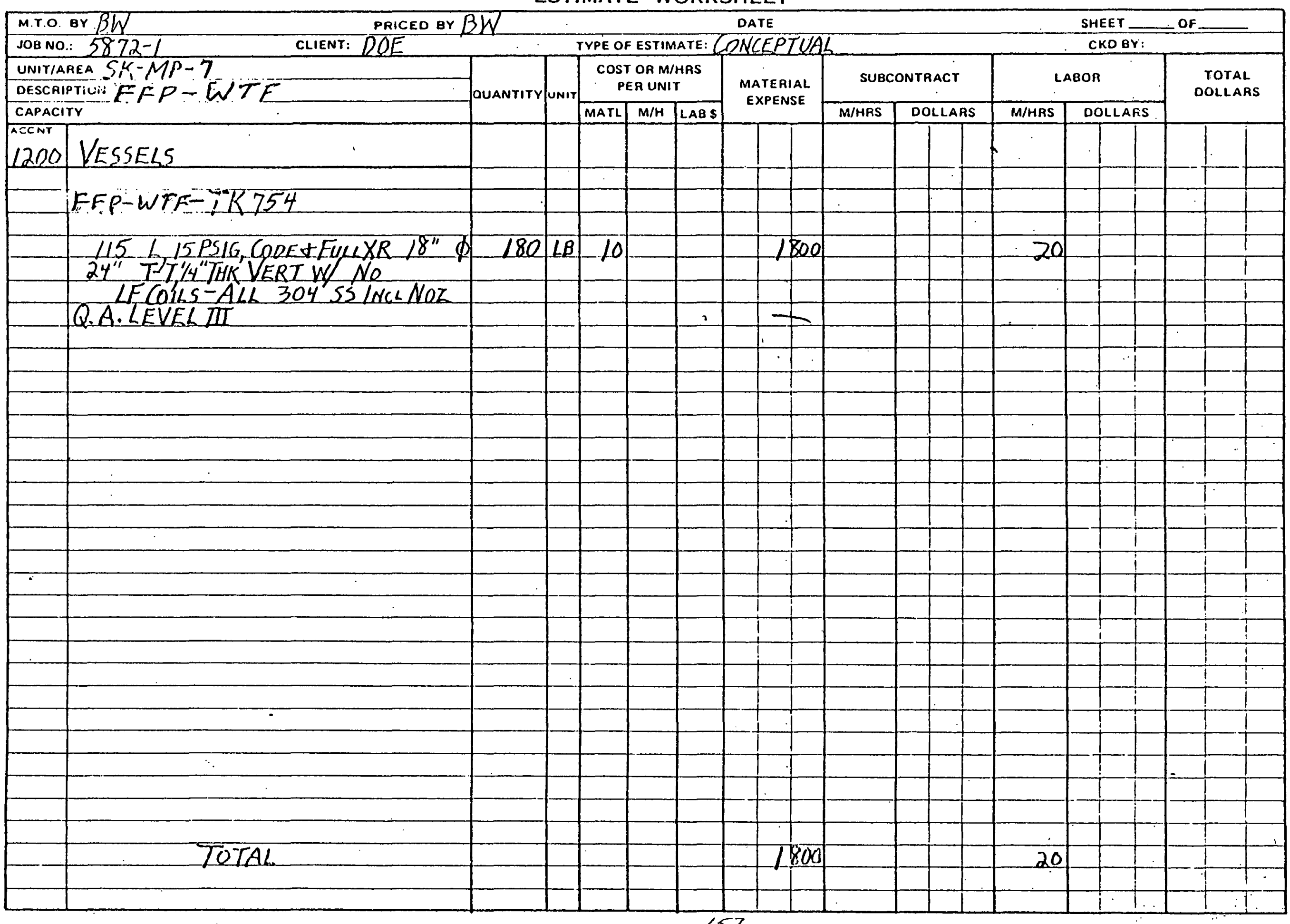


ESTIMATE WORKSHEET

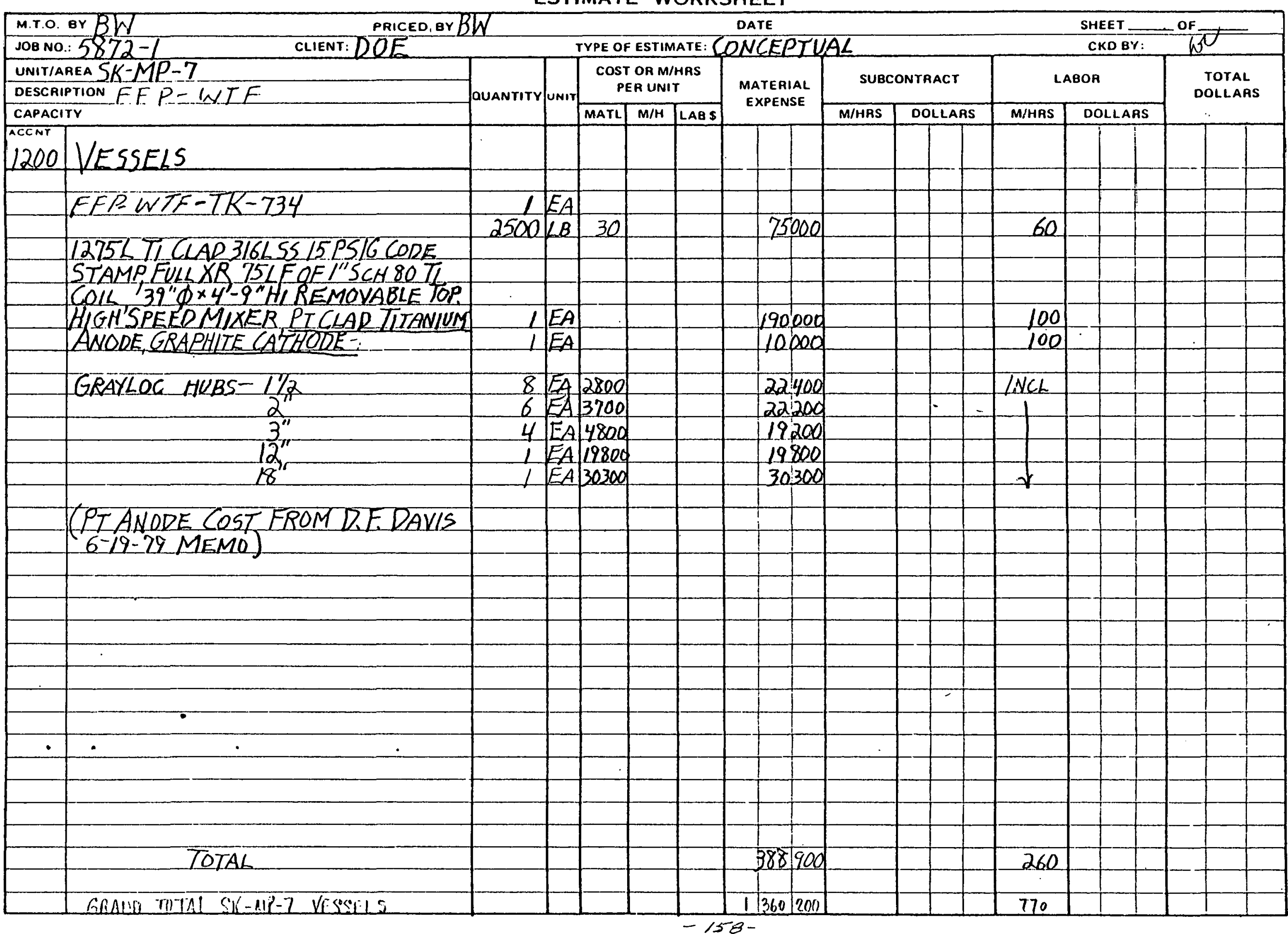


ESTIMATE WORKSHEET

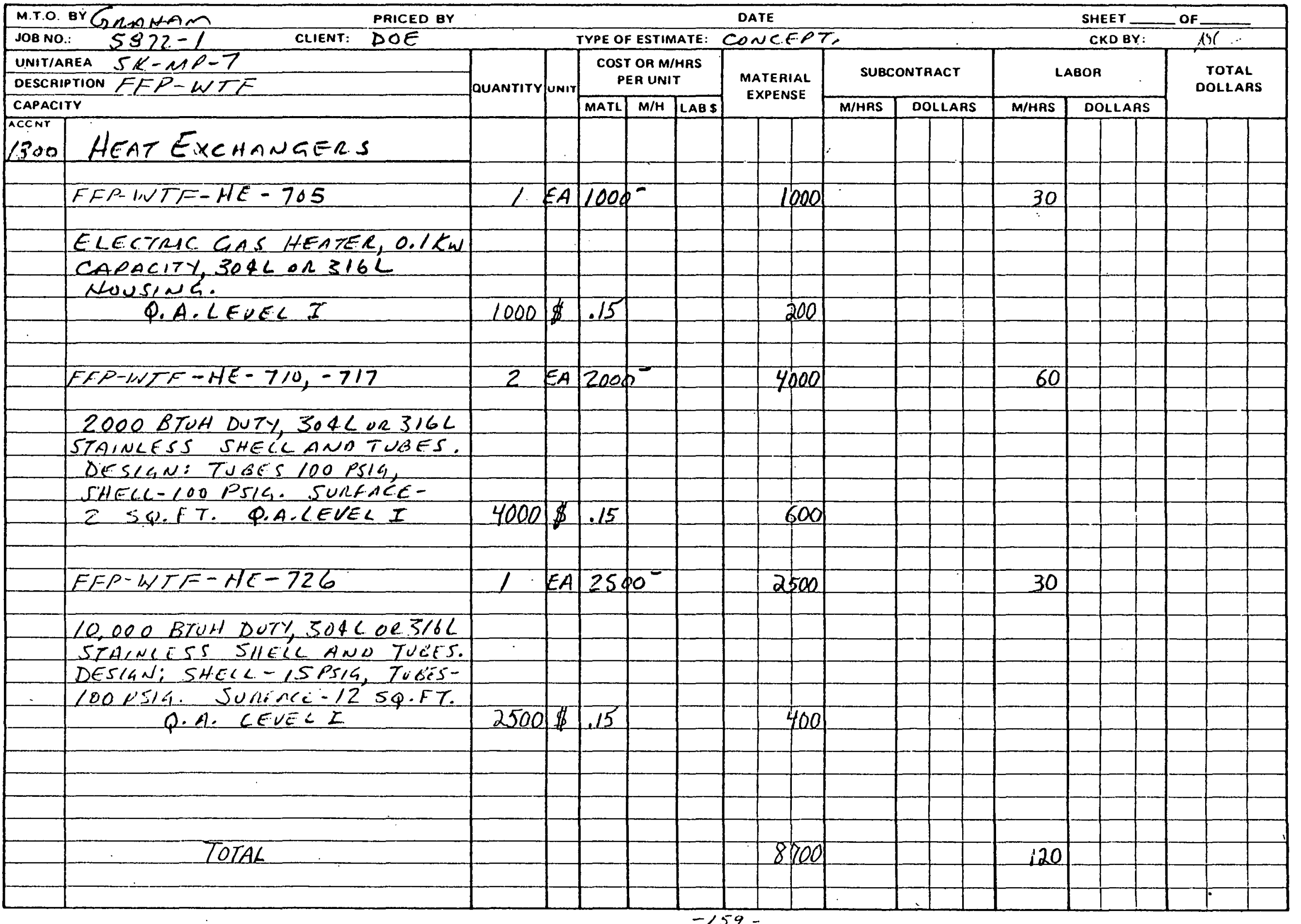


ESTIMATE WORKSHEET

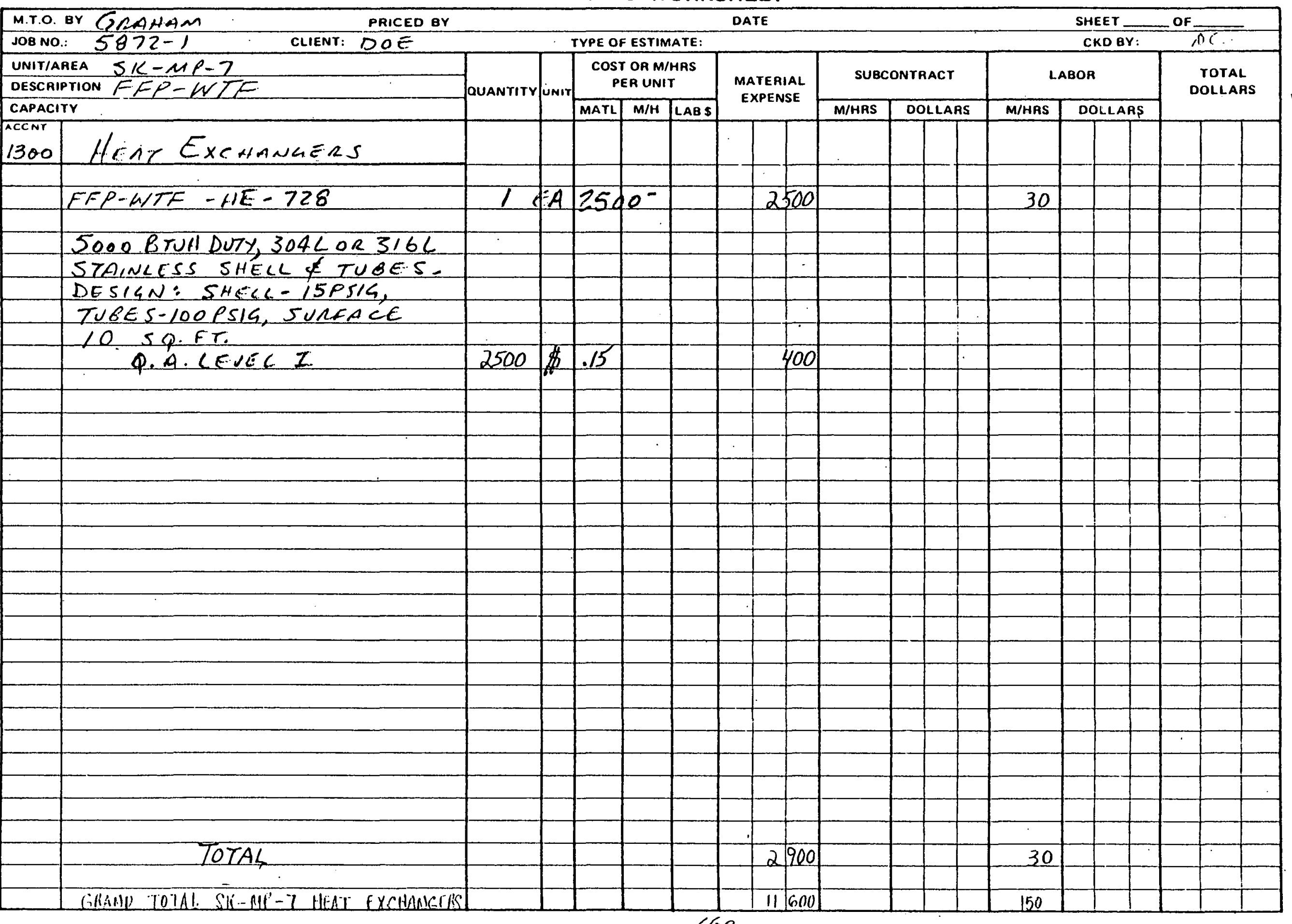


ESTIMATE WORKSHEET

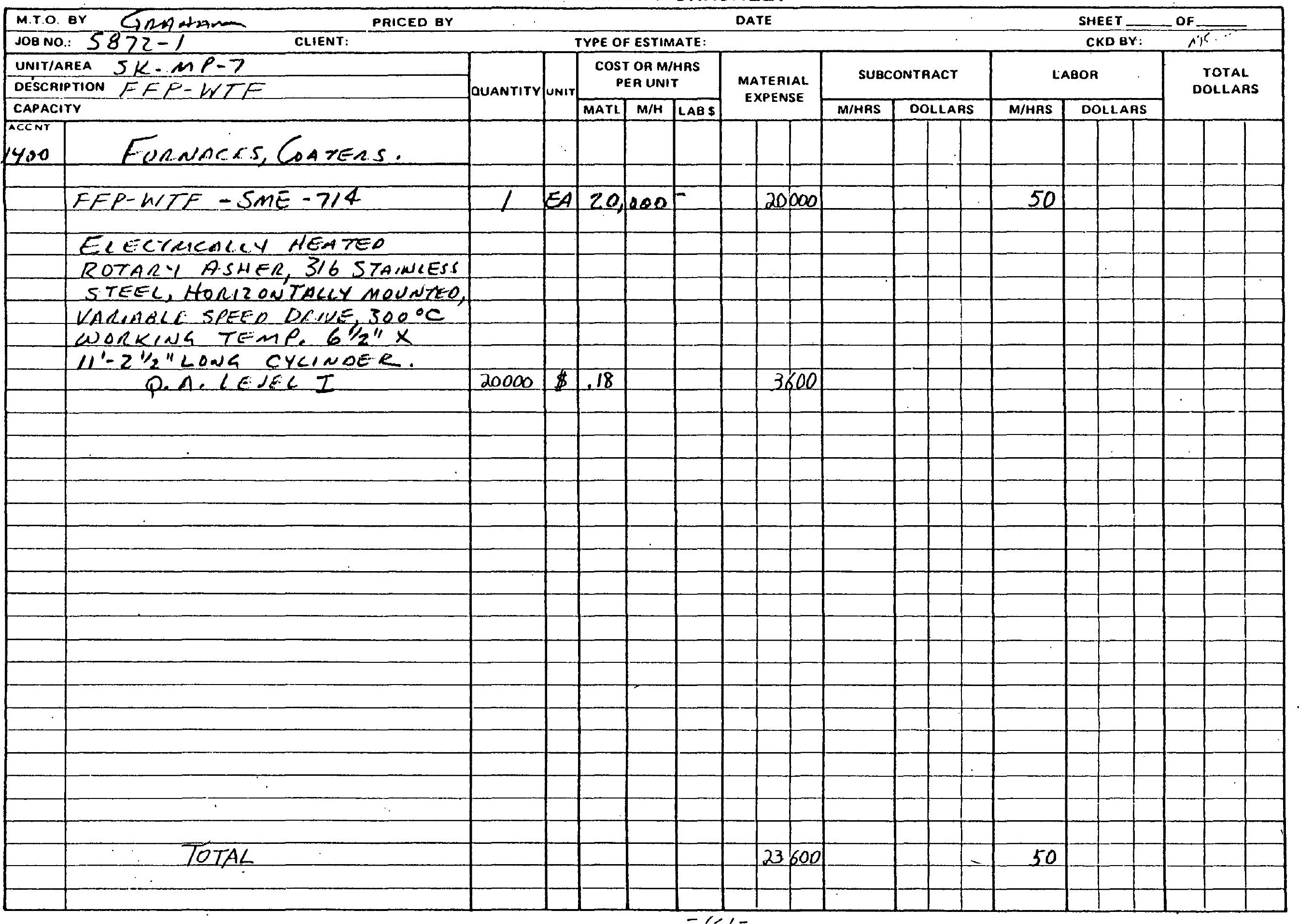


ESTIMATE WORKSHEET

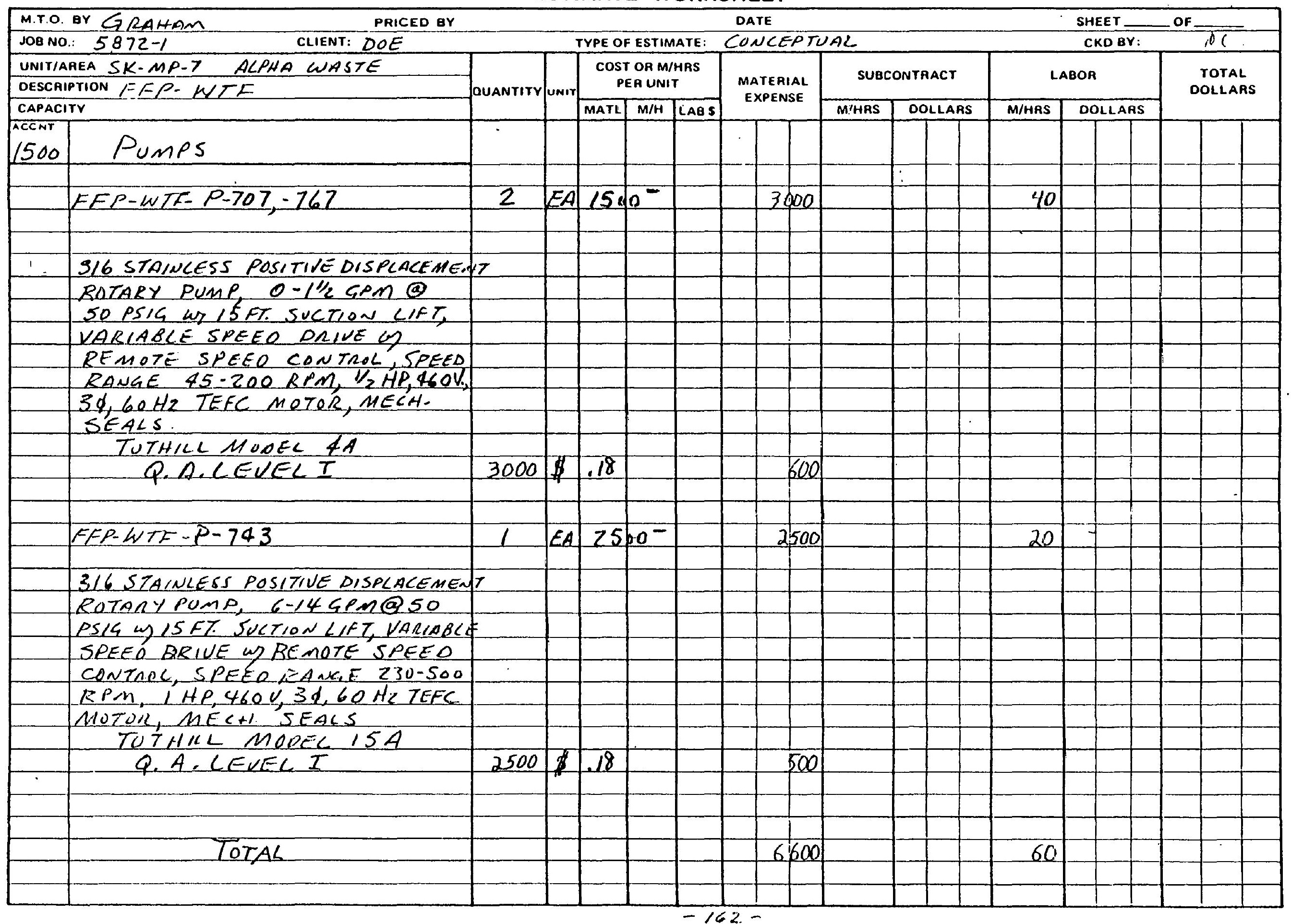


ESTIMATE WORKSHEET

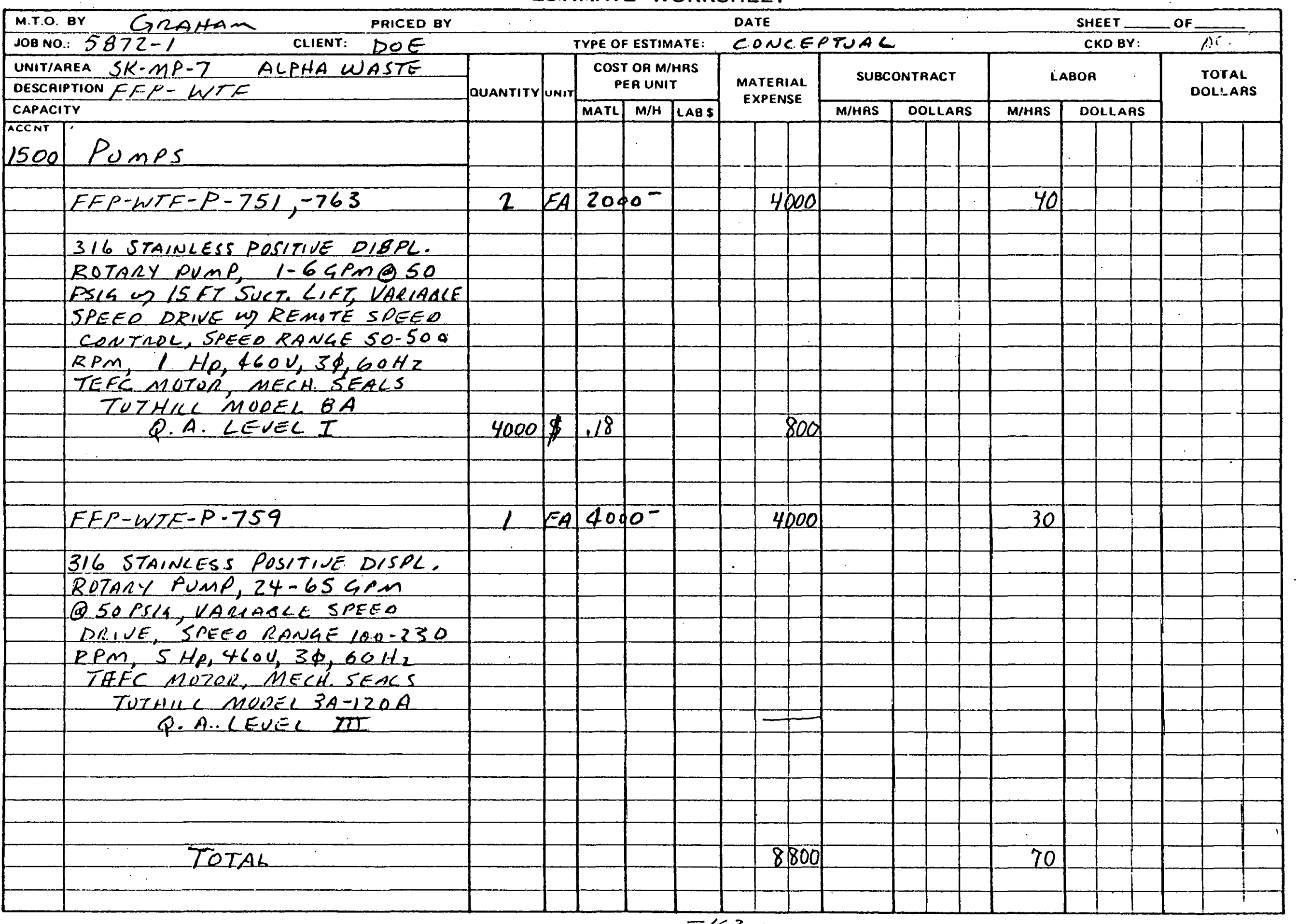


THE RALPH M. PARSONS COMPANY

THE RALPM M
ESTIMATE WORKSHEET

DATE

TYPE OF ESTIMATE: CONLEDTUAL

\begin{tabular}{|c|c|c|c|c|c|c|c|c|c|c|}
\hline & & & & & & & & & & \\
\hline & & TYPE OF & $\widehat{E S T I I}$ & AATE: & CONCED & VAL & & & CKD BY: & .06 \\
\hline GuANTITY & lunit & $\cos 1$ & $\begin{array}{l}\text { OR M } \\
R \text { UN }\end{array}$ & $\begin{array}{l}\text { HAS } \\
T\end{array}$ & MATERIAL & SUBC & UTRACT & & DOA & $\begin{array}{l}\text { TCIAL } \\
\text { DOLLARS }\end{array}$ \\
\hline & & MATL & $\mathbf{M} / \mathbf{H}$ & LAB \$ & & M/HAS & DOLLARS & M/HAS & DOLLARS & \\
\hline
\end{tabular}

M.r.O. BY GROANA PRICED B

JOB NO.: $5872-1$ CLIENT: DOE

UNITIAREA SK-MP-7 ALPHA NASTE CAPACITY

ACCNT

1500

FFP-WTF-J-722,-736, -737,
$-739,-756,-75 \zeta$

STEAMVET EJELTOR $13 \mathrm{GPM}$

CAPACITY, 15 FT. SUCTION LIFT,

$30 \mathrm{FT}$. DISCHARGE HEAO,

30IL BARSTOCK W BUTTWECD

CONN'S.

DENBERTHY SIZE 4A, SERES 1A Q.A. LEVEL II

TOTAL

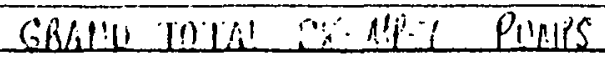

EA $670^{\circ} 15$

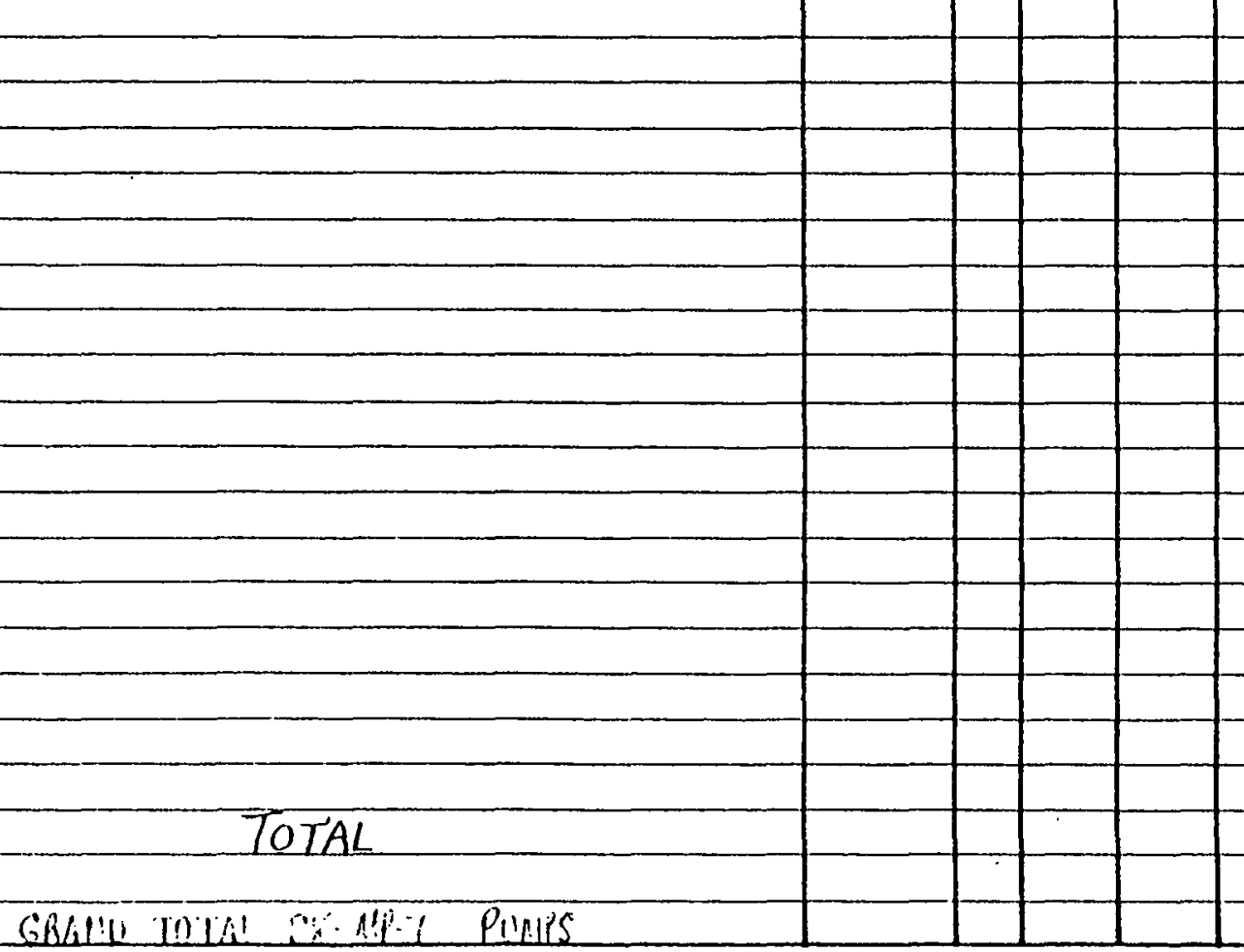


ESTIMATE WORKSHEET

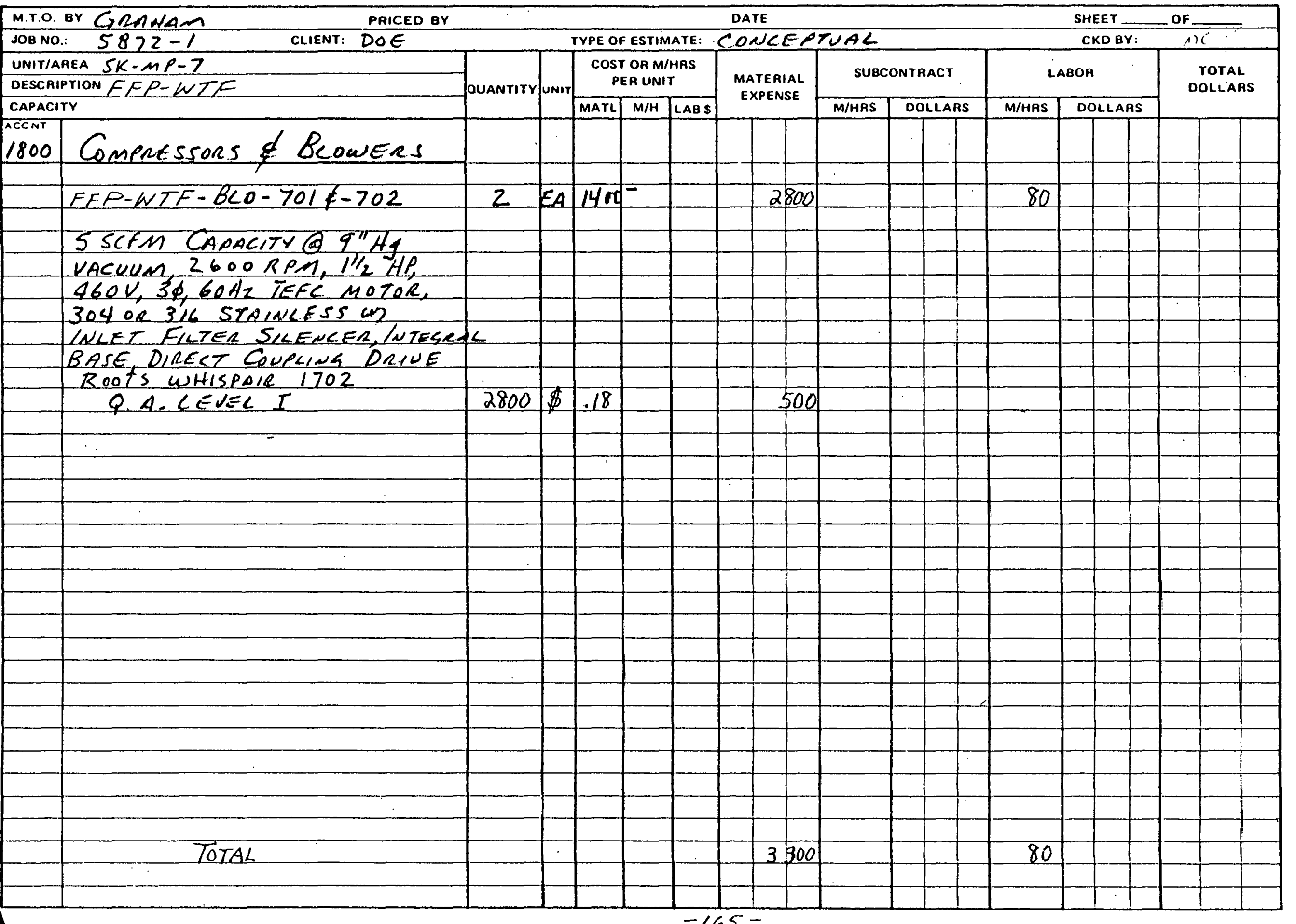


ESTIMATE WORKSHEET

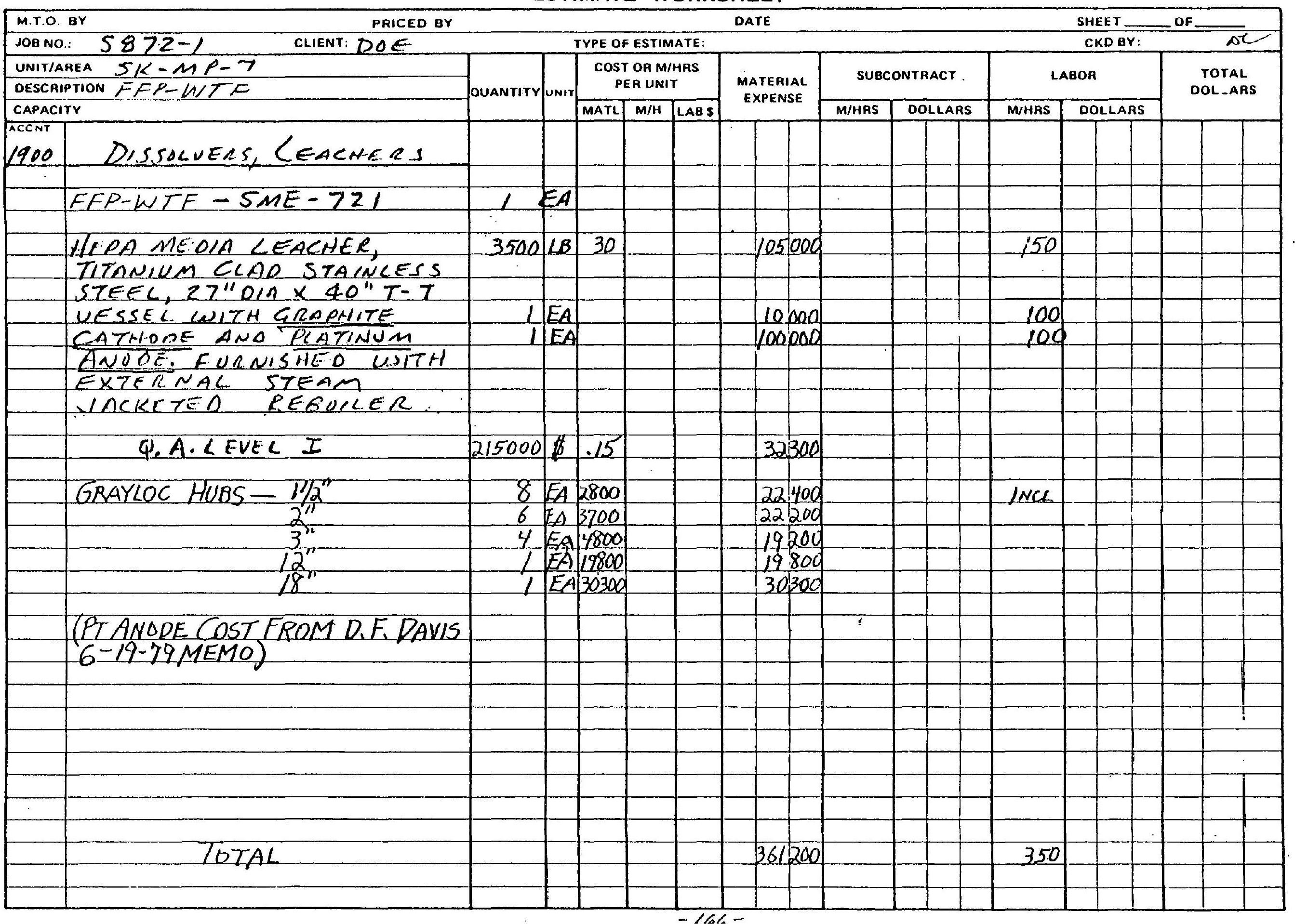


THE RALPH M. PARSONS COMPANY

ESTIMATE WORKSHEET

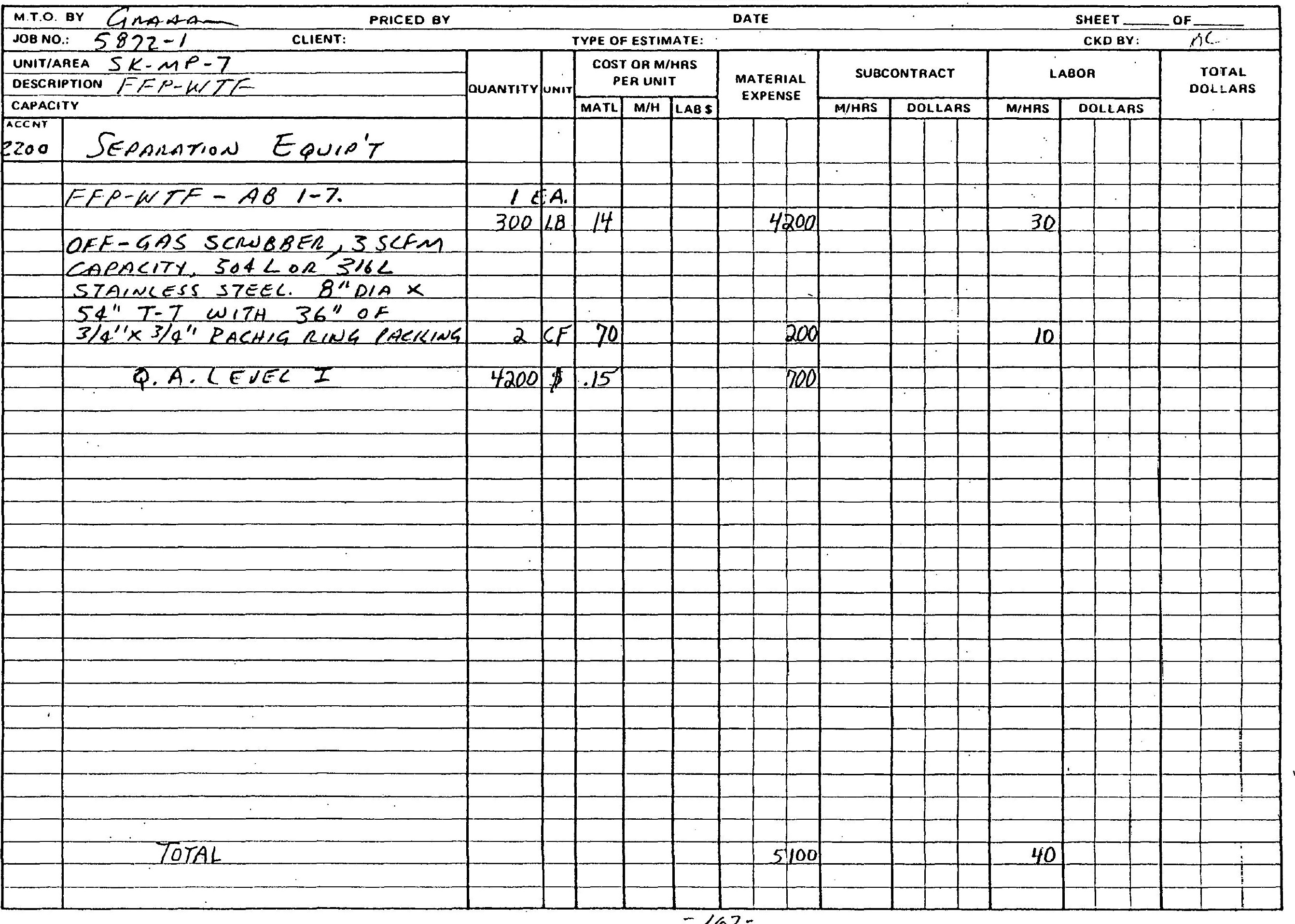


CIS 20000

THE RALPH M. PARSONS COMPANY

ESTIMATE WORKSHEET

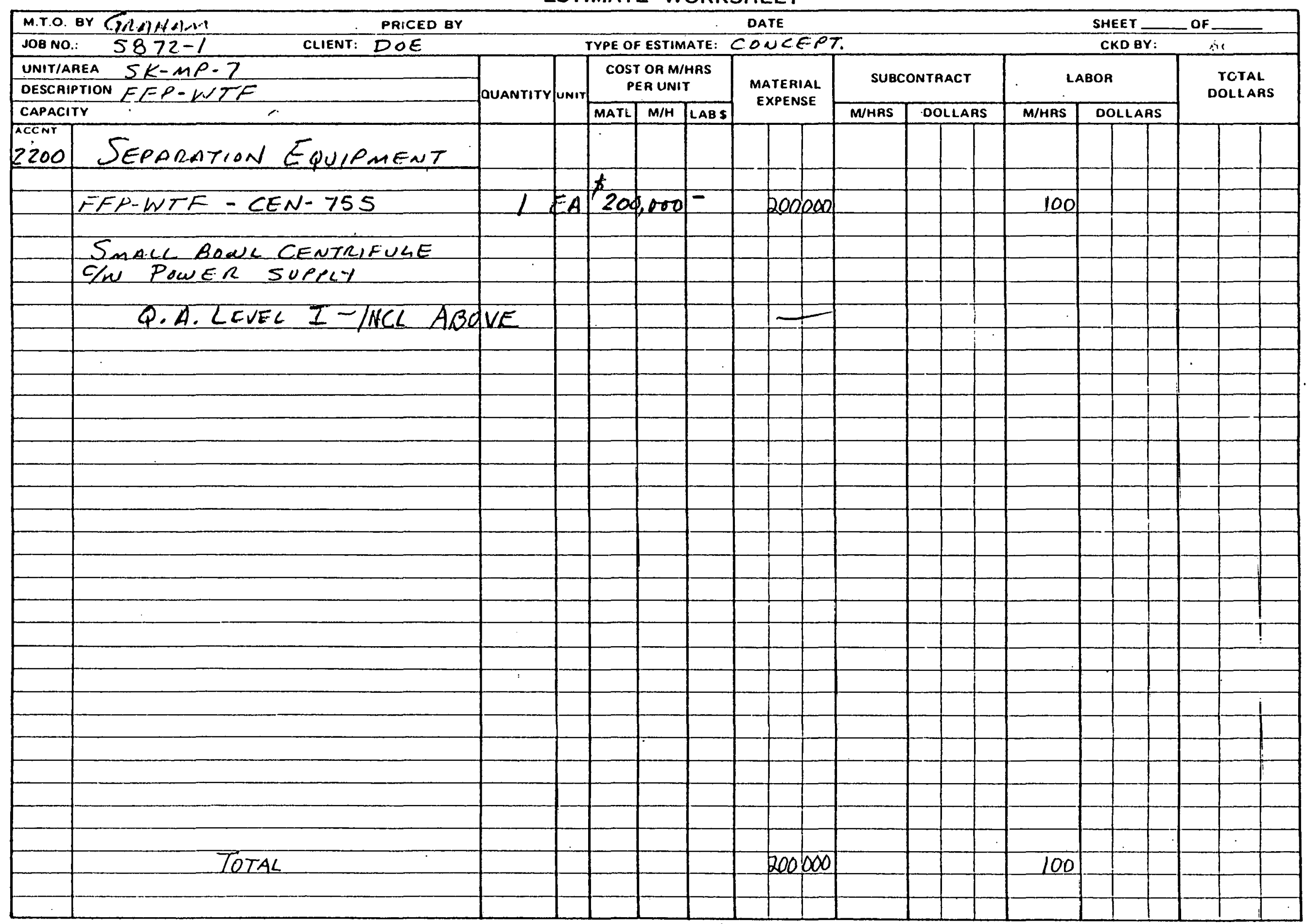


$\cos 20000$

THE RALPH M. PARSONS COMPANY

ESTIMATE WORKSHEET

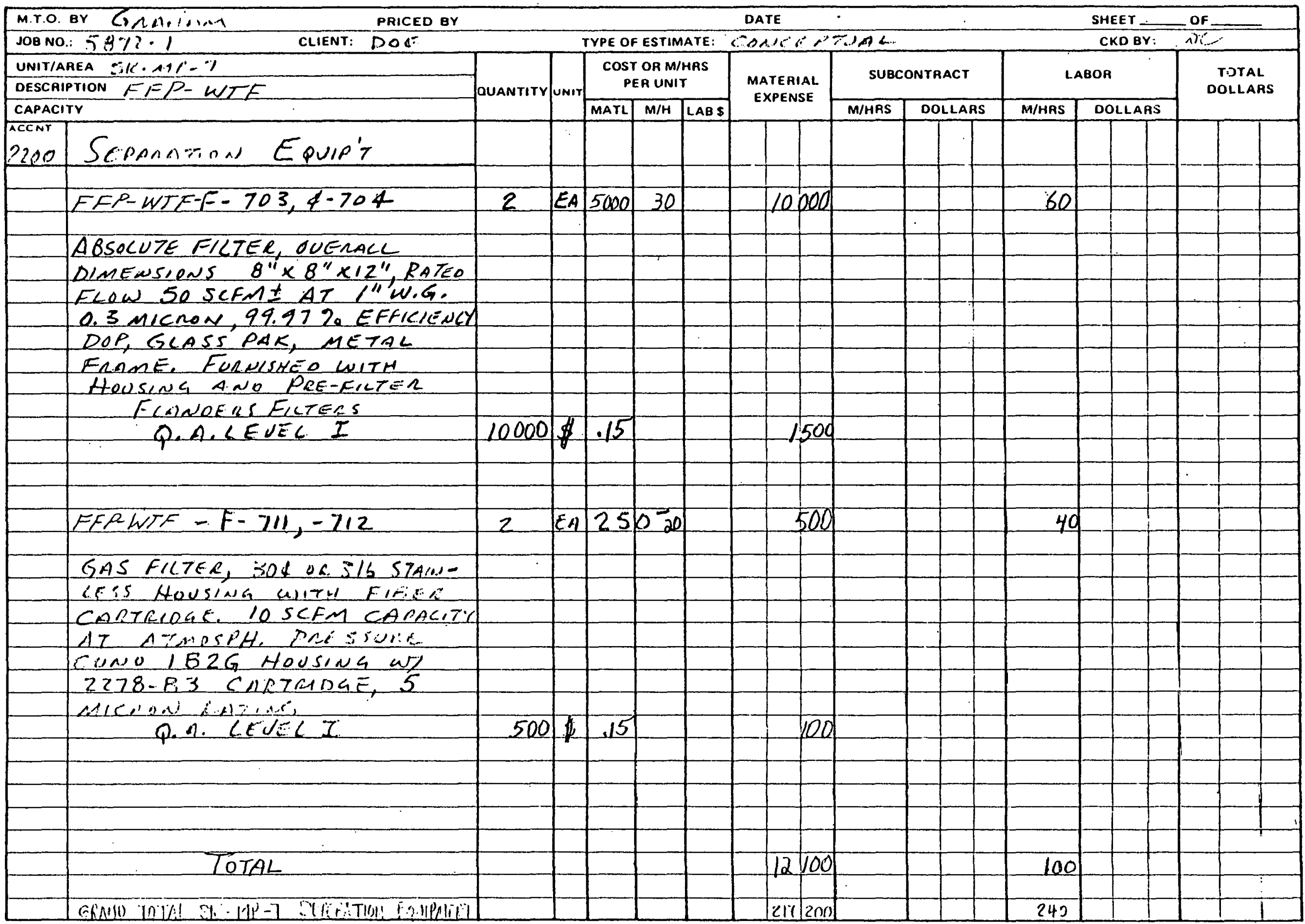


ESTIMATE WORKSHEET

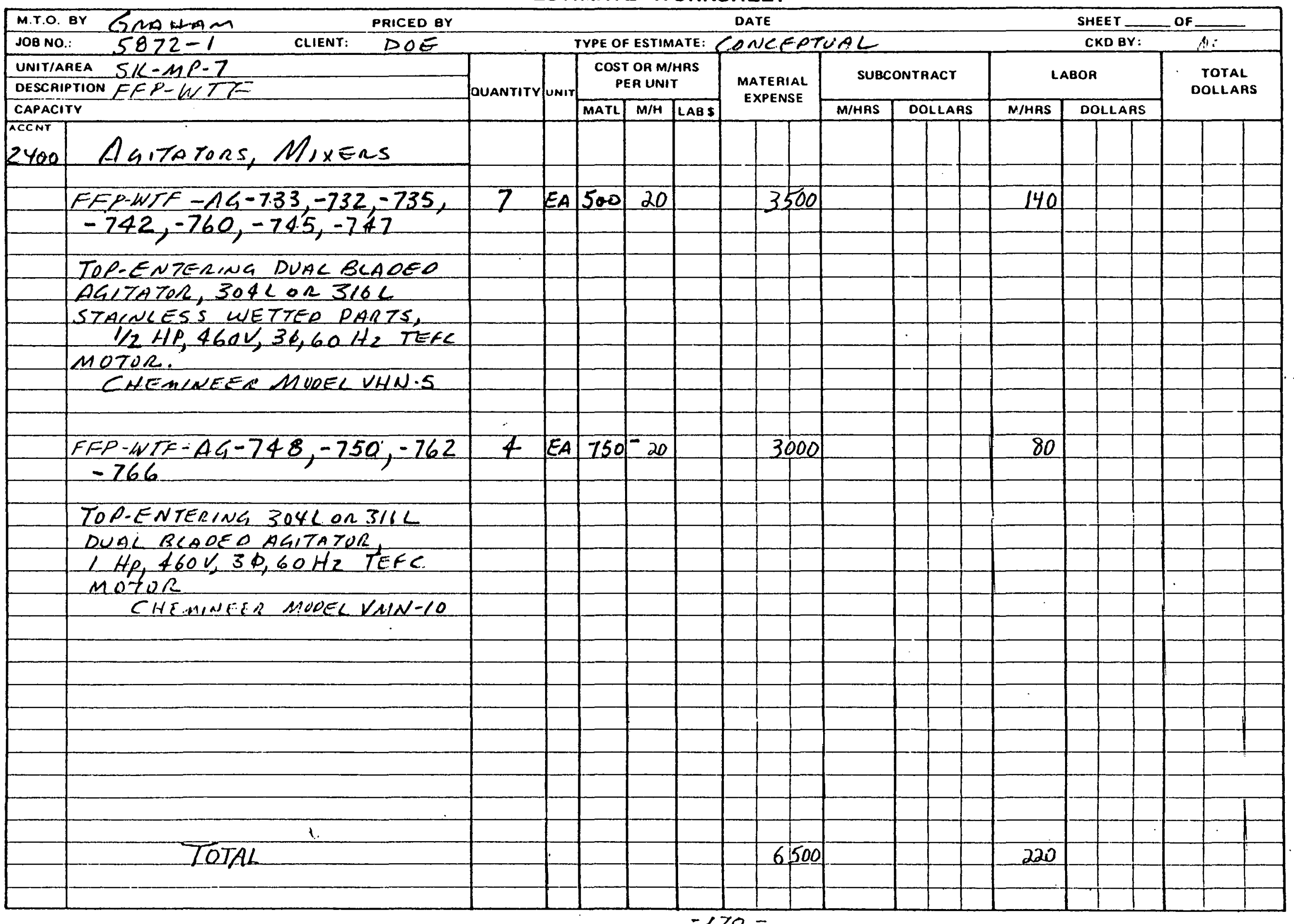


ESTIMATE WORKSHEET

\begin{tabular}{|c|c|c|c|c|c|c|c|c|c|c|c|c|c|c|c|c|c|c|}
\hline M.T.O. & PRICED BY & & & & & & DATE & & & & & & & SHEET: & 5 & -OF & & \\
\hline JOB NO & $\therefore 872-1$ & & & TYPE OP & ESTIM & IATE: & & & & & & & & CKD BY & & & & \\
\hline UNIT/A & AEA $S K-M P-7$ & & & $\overline{\cos r}$ & OR M/I & & & & & & & & & & & & & \\
\hline OESCAI & Prion FEP-WTE & aUANTITY & UNit & & EA UNIT & & $\begin{array}{l}\text { MATERIA } \\
\text { EXPENS }\end{array}$ & & SUBC & N & & & & $80 \mathrm{H}$ & & & OLLAR & \\
\hline CAPACI & & & & MATL & $M / H$ & LABS & & & PA/HAS & & $\angle$ LAA & & MIHAS & DOLLAF & & & & \\
\hline $\begin{array}{l}\text { ACCNT } \\
2600\end{array}$ & Ho.ppers & & & & & & & & & & & & & & & & & \\
\hline . & & & & & & & & & & & & & & & & & & \\
\hline & EFP-WTF - $10-713$ & $L$ & $E A$ & & & & & & & & & & & & & & & \\
\hline & & 950 & $\angle B$ & 8 & & & & 600 & & & & & 20 & & & & & \\
\hline & FEED HOPPER, $304 \mathrm{LOR} 316 \mathrm{~L}$ & & & & & & & & & & & & & & & & & \\
\hline & STAINLESS STEEL 35 W.FT & & & & & & & & & & & & & & & & & \\
\hline & CAPACITY WITH AIR -OPEMATED & & & & & & & & & & & & & & & & & \\
\hline & SEAC PCATE 36"DIAX & & & & & & & & & & $i$ & & & & & & & \\
\hline & 4G" WITH CONE BOTTOM. & & & & & & & & & & & & & & & & & \\
\hline & Q.A.LEVEL I & 2600 & $\$$ & .15 & & & & 200 & & & & & & & & & & \\
\hline & & & & & & & & & & & & & & & & $\therefore$ & & \\
\hline & & & & & & & & & & & & & & & & & & \\
\hline & FEP-LVTF - $40-718, H 0-770$ & 2 & $E A$ & & 20 & & & & & & & & 40 & & & . & & \\
\hline & STANCESS STEWL, TO CU.FT. & & & & & & & & & & & & & & & & & \\
\hline & Colp, $17 \% \quad 24^{\prime \prime} 017 \times 53^{\prime \prime}$ & & & & & & & & & & & & & & & & & \\
\hline & Lelcewens a cone Butrom & & & & & & & & & & & & & & & & & \\
\hline & Q.A.IE $/ E C I$ & 5500 & If & .15 & & & & 900 & & & & & & & & & & \\
\hline & & & th & & & & & & & & & & & & & & & \\
\hline & GRAYLOC ALUBS: $1^{\prime \prime}$ & 7 & EA & 1700 & & & & 900 & & & & & $I N C L$ & & & & & \\
\hline & $3^{n}$ & 5 & $E A$ & 4100 & & & 20.5 & 500 & & & & & $\downarrow$ & & & & & \\
\hline & . & & & & & & & & & & & & & & & & & \\
\hline & & & & & & & & & & & & & & & & & & \\
\hline & & & & & & & & & & & & & & & & & & \\
\hline & & & & & & & & & & & & & & & & & & \\
\hline & & & & & & & & & & & & & & & & & & \\
\hline & & & & & & & & & & & & & & & & & & \\
\hline & & & & & & & & & & & & & & & & & & \\
\hline & & & & & & & & & & & & & & & & & & \\
\hline & & & & & & & & & & & & & & & & & & , \\
\hline & & & & & & & & & & & & & & & & & & \\
\hline
\end{tabular}


ESTIMATE WORKSHEET

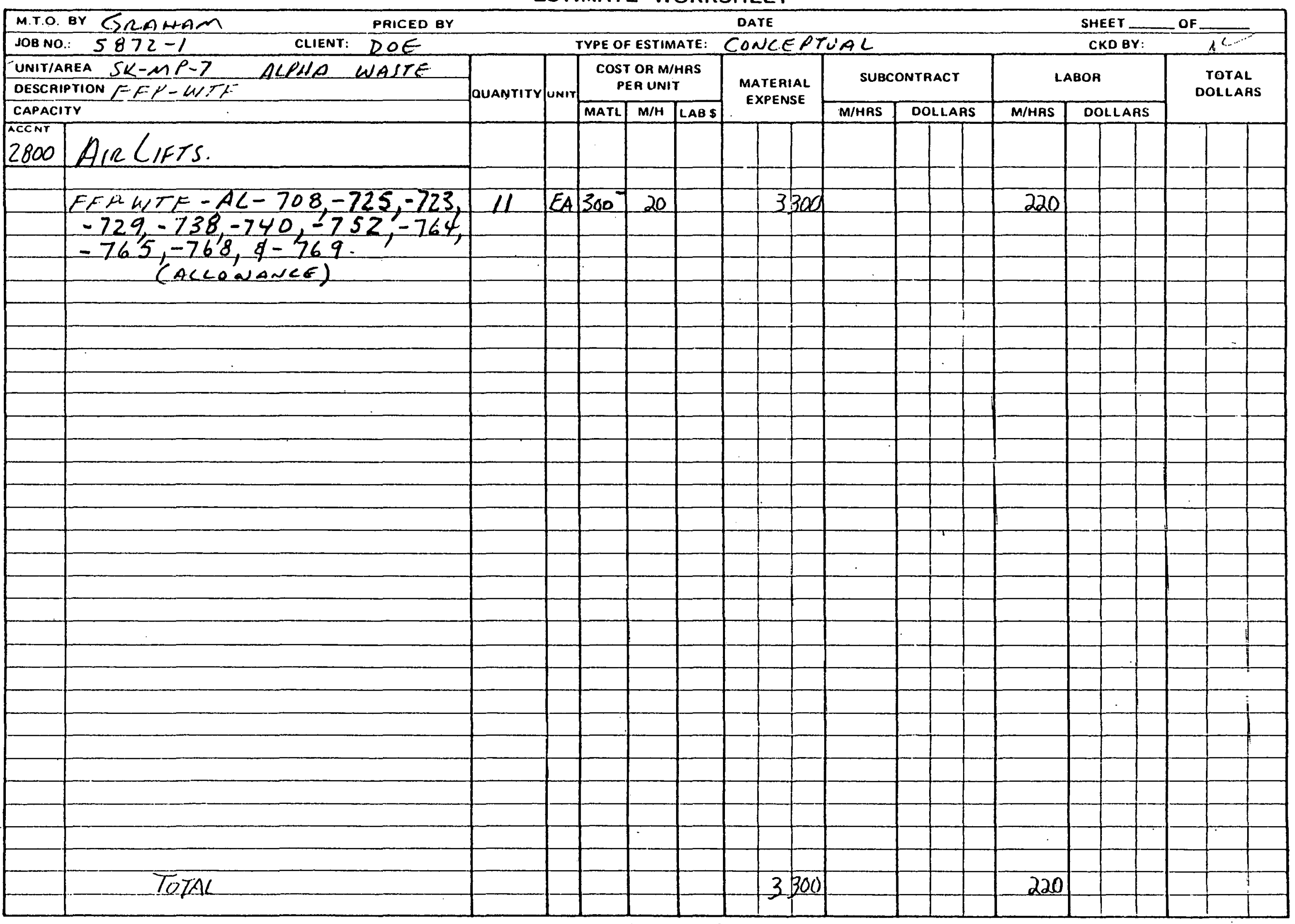




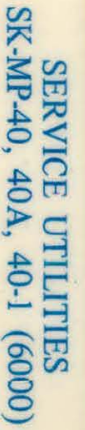


ESTIMATE WORKSHEET

\begin{tabular}{|c|c|c|c|c|c|c|c|c|c|c|c|c|c|c|c|c|c|c|c|}
\hline \multirow{2}{*}{\multicolumn{4}{|c|}{$\begin{array}{ll}\text { M.T.O. BY BW } & \text { PRICED \&Y BW } \\
\text { JOB NO.: } 5872-1 & \text { CLIENT: } D O C E\end{array}$}} & \multirow{2}{*}{\multicolumn{3}{|c|}{ TYPE OF ESTIMATE: }} & \multirow{2}{*}{\multicolumn{7}{|c|}{$\begin{array}{l}\text { DATE } 7-20-79 \\
\text { CONCEPTLAL }\end{array}$}} & \multicolumn{6}{|c|}{ SHEET $=$ OF } \\
\hline & & & & & & & & & & & & & & & & CD BY: & & te. & \\
\hline \multirow{2}{*}{\multicolumn{2}{|c|}{ UNITIAAEA $S K-M P-40,40 A, 40-1$}} & \multirow{4}{*}{ OUANTITY } & \multirow{4}{*}{ Unit } & \multirow{2}{*}{\multicolumn{3}{|c|}{$\begin{array}{l}\text { COST OR M/HAS } \\
\text { PER UNIT }\end{array}$}} & \multirow{3}{*}{\multicolumn{3}{|c|}{$\begin{array}{l}\text { MATERIAL } \\
\text { EXPENSE }\end{array}$}} & \multirow{2}{*}{\multicolumn{3}{|c|}{ SUBCONTRACT }} & \multirow{2}{*}{\multicolumn{4}{|c|}{ LABOR }} & \multirow{3}{*}{\multicolumn{3}{|c|}{$\begin{array}{l}\text { YOTAL } \\
\text { DOLLARS }\end{array}$}} \\
\hline & & & & & & & & & & & & & & & & & & & \\
\hline \multicolumn{2}{|c|}{\begin{tabular}{|l|l|} 
CAPACITY \\
ACCNT \\
\end{tabular}} & & & \multirow{2}{*}{ MATL } & \multirow[t]{2}{*}{$M / H$} & \multirow[t]{2}{*}{ LABS } & & & & \multirow[t]{2}{*}{ M/HRS } & \multicolumn{2}{|c|}{ DOLLARS } & & \multirow[t]{2}{*}{ M/HRS } & \multicolumn{2}{|c|}{ DOLLARS } & & & \\
\hline 6000 & SPECIAL FACILITIES-EQUIPMENT SUMMARY & & & & & & & & & & & & & & & & & & \\
\hline & & & & & & & & & & & & & & & & & & & \\
\hline 1200 & VESSELS & & & & & & & 539 & 900 & & & & & 1530 & & & & & \\
\hline 1500 & PUMPS & & & & & & & 237 & 400 & & & & & 2485 & & & & & \\
\hline 1600 & BOILERS & & & & & & & 360 & 000 & & & & & 7770 & & & & & \\
\hline 1700 & COOLING TOLEERS & & & & & & & 52 & 500 & & & & & 1750 & & & & & \\
\hline 1800 & COMPRESSORS \& BLOWERS & & & & & 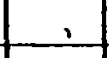 & & 53 & 000 & & & & & 330 & & & & & \\
\hline-1900 & TANKS & & & & & & 1 & 638 & 900 & & & & & 27115 & & & & & \\
\hline 2200 & SEPERATION EQUUPMENT & & & & & & & 200 & een & & & & & 960 & & & & & \\
\hline 2400 & AGITATORS & & & & & & & 1 & 200 & & & & & 40 & & & & & \\
\hline 2800 & OTHER MATOR EQUPMENT & & & & & & 1 & 033 & 500 & & & & & 15190 & & & & & \\
\hline & & & & & & & & & & & & & & & & & & & \\
\hline & SUB TOTAL & & & & & & 5 & 658 & 200 & & & & & 52080 & & & & 1 & \\
\hline & & & & & & & & & & & & & & & & & & & \\
\hline & $65 \%$ PROOUCTIVITY $(52080 \div .65)-52080$ & & & & & & & & & & & & & $2 B 043$ & & & & & \\
\hline & & & & & & & & & & & & & & 80123 & & & & & \\
\hline & & 80123 & $\mathrm{MH}$ & & & 16.62 & & & & & & & & & 1 & $330: 000$ & & & \\
\hline & & & & & & & & & & & & & & & & $i$ & & & i \\
\hline & & & ' & & & & & & & & & & & & & & & & \\
\hline & TOTAL FOR SK-MP-40, $40 A, 40-1$ & & & & & & 5 & 658 & 200 & & & & & 80123 & 1 & 330.000 & 6 & 988 & 200 \\
\hline & & & & & & & & & & & & & & & & i & & & 1 \\
\hline & & & & & & & & & & & & & & & & & & & $i$ \\
\hline & - & & & & & & & & & & & & & & & 1 & & & 7 \\
\hline & & & & & & & & & & & & & & & & $i$ & & & \\
\hline & $\because$ & & & & & & & & & & & & & & & $T$ & & 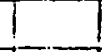 & \\
\hline & & & & & & & & & & & & & & & & $i$ & & & \\
\hline & & & & & & & & & & & & & & & & & & & \\
\hline & & & & & & & & & & & & & & & & & & & \\
\hline & & & & & & & & & & & & & & & & $!$ & & & \\
\hline
\end{tabular}


THE RALPH M. PARSONS COMPANY

ESTIMATE WORKSHEET

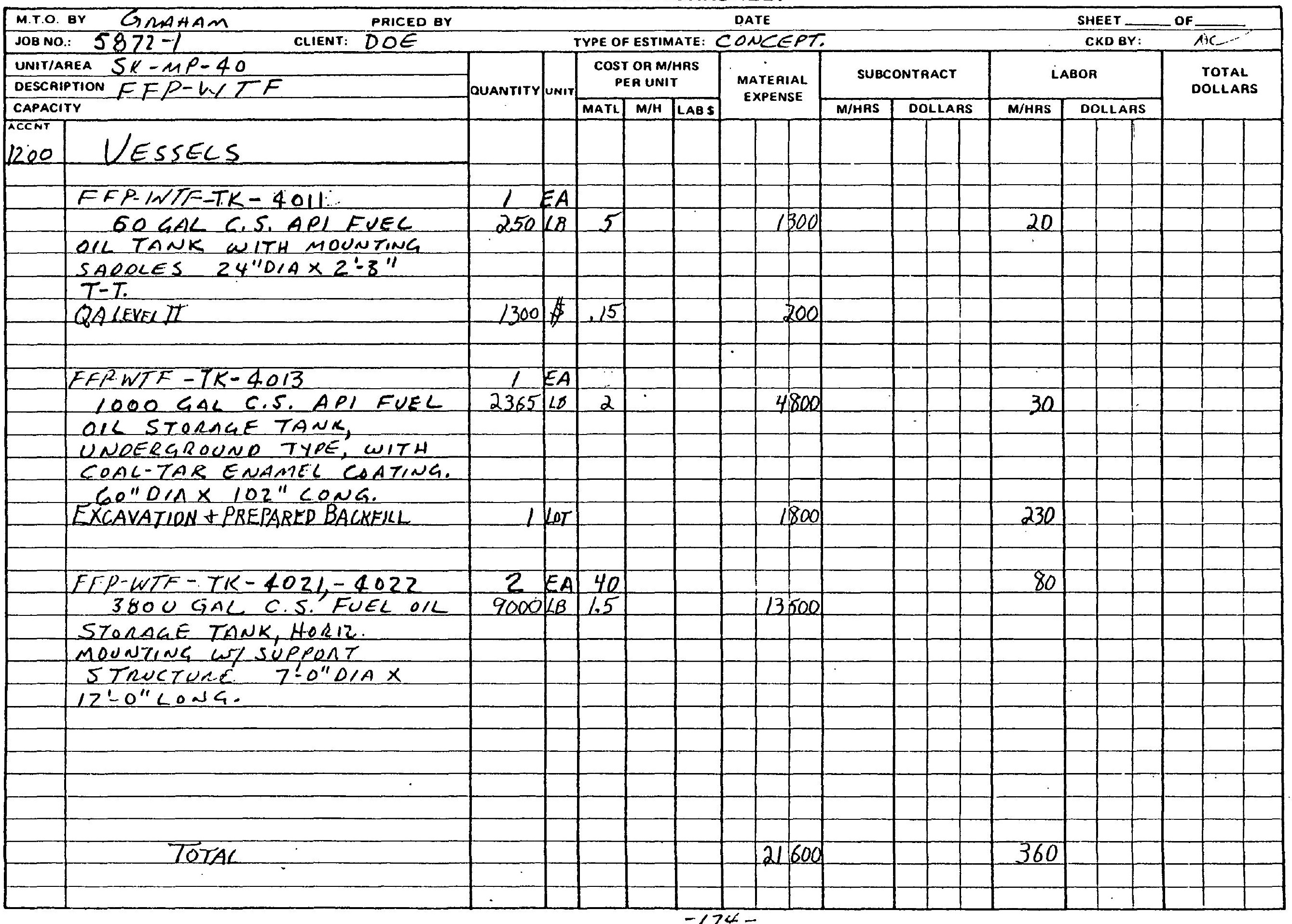


ESTIMATE WORKSHEET

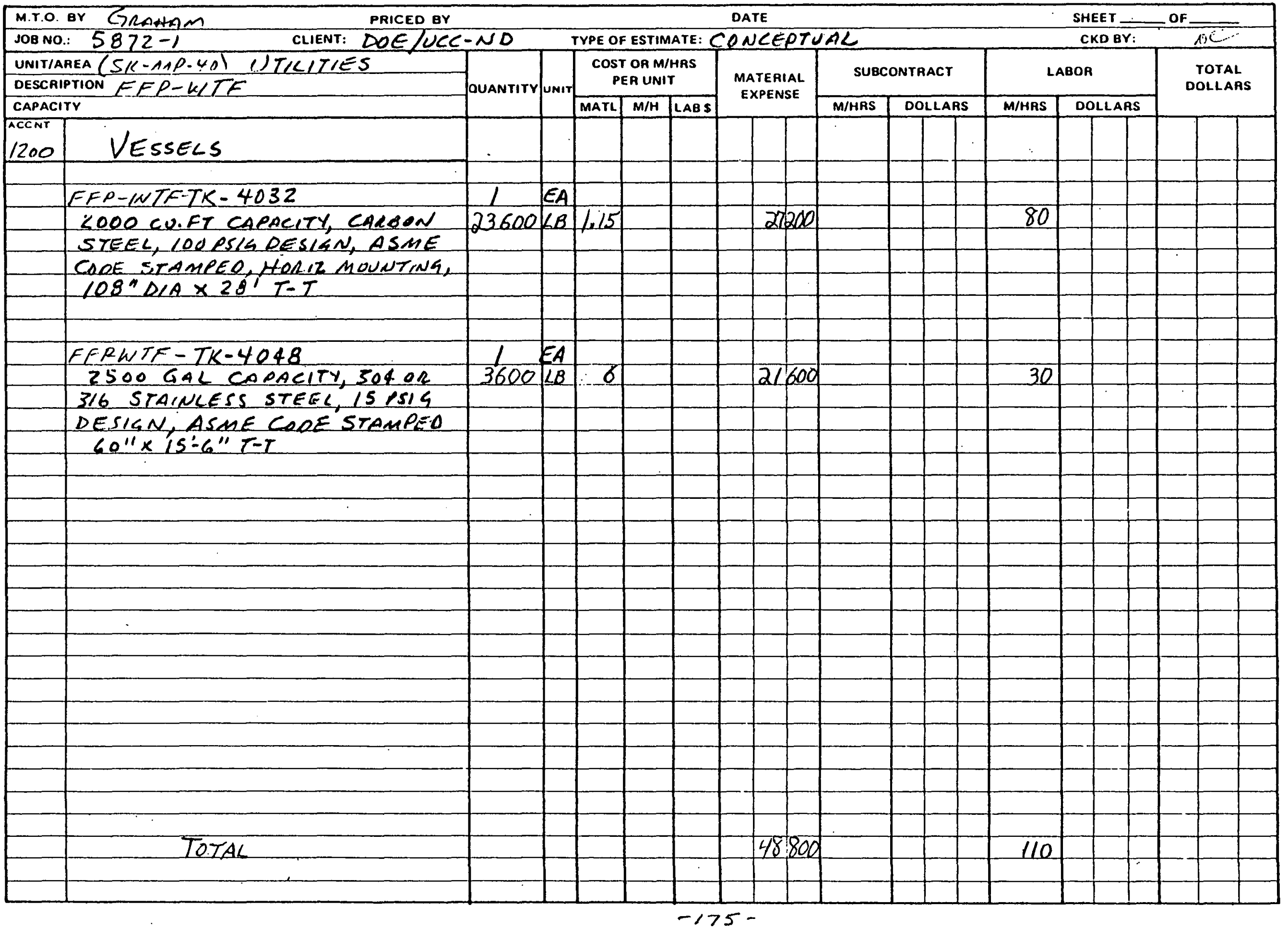


ESTIMATE WORKSHEET

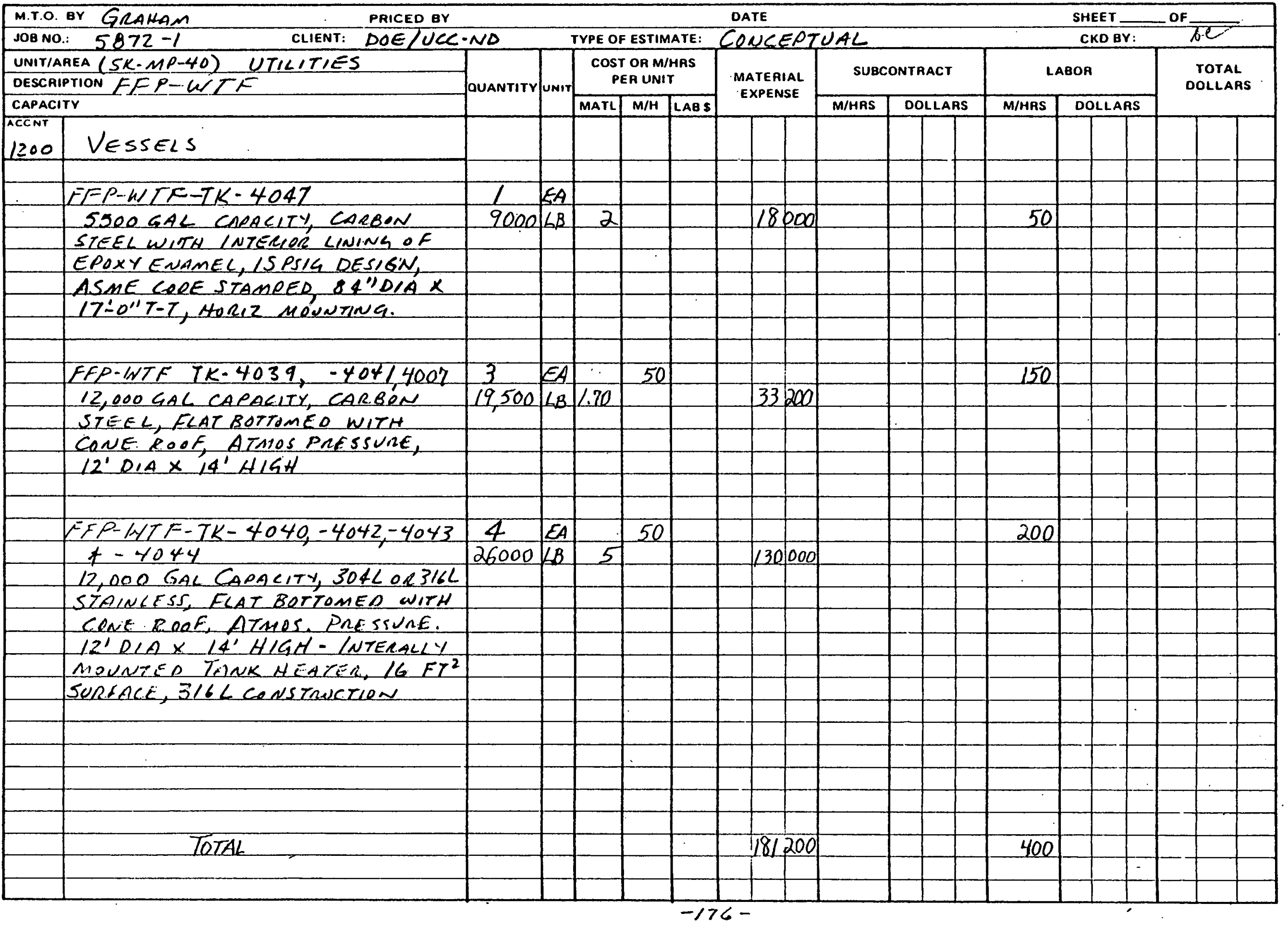


ESTIMATE WORKSHEET

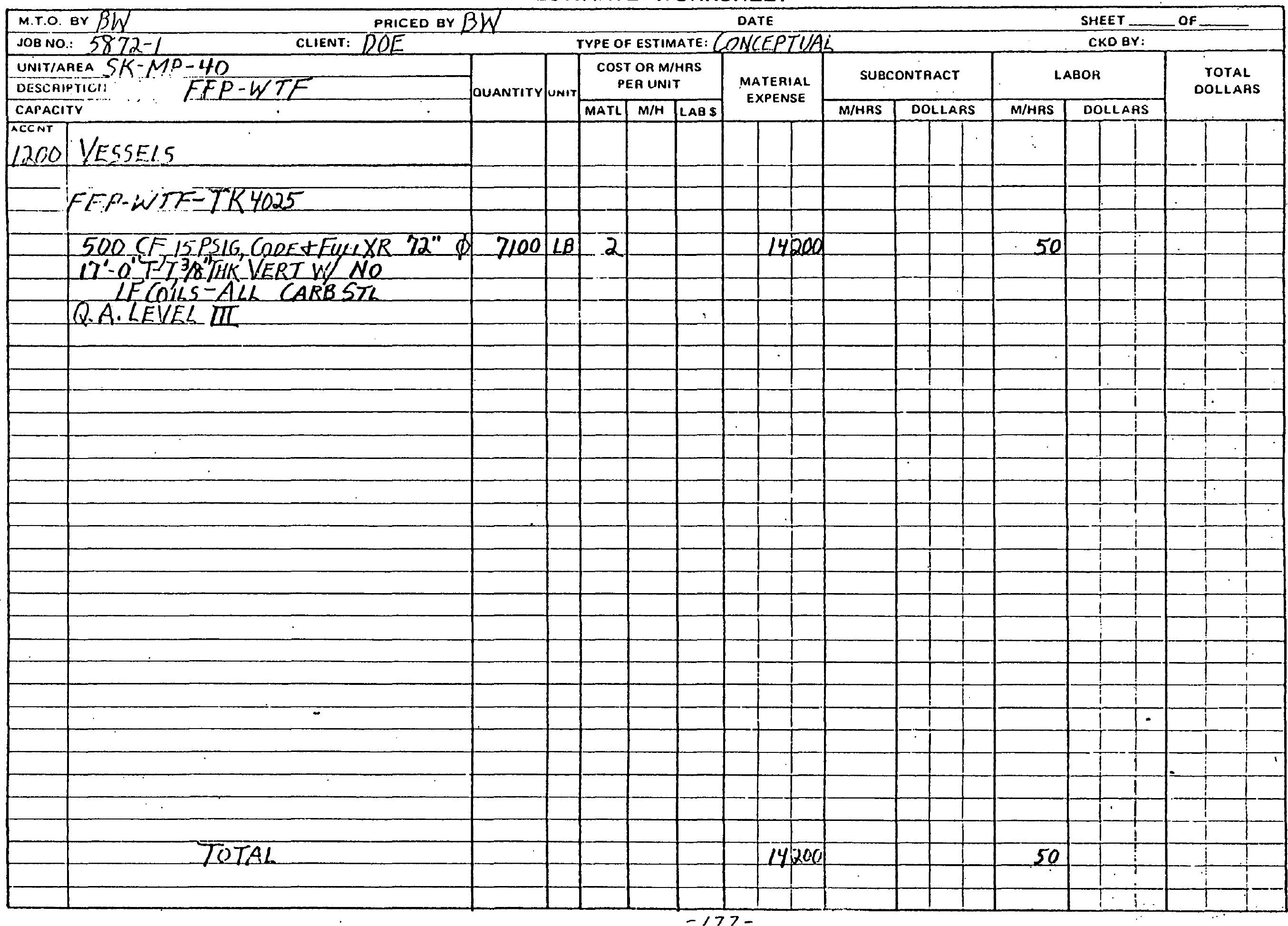


ESTIMATE WORKSHEET

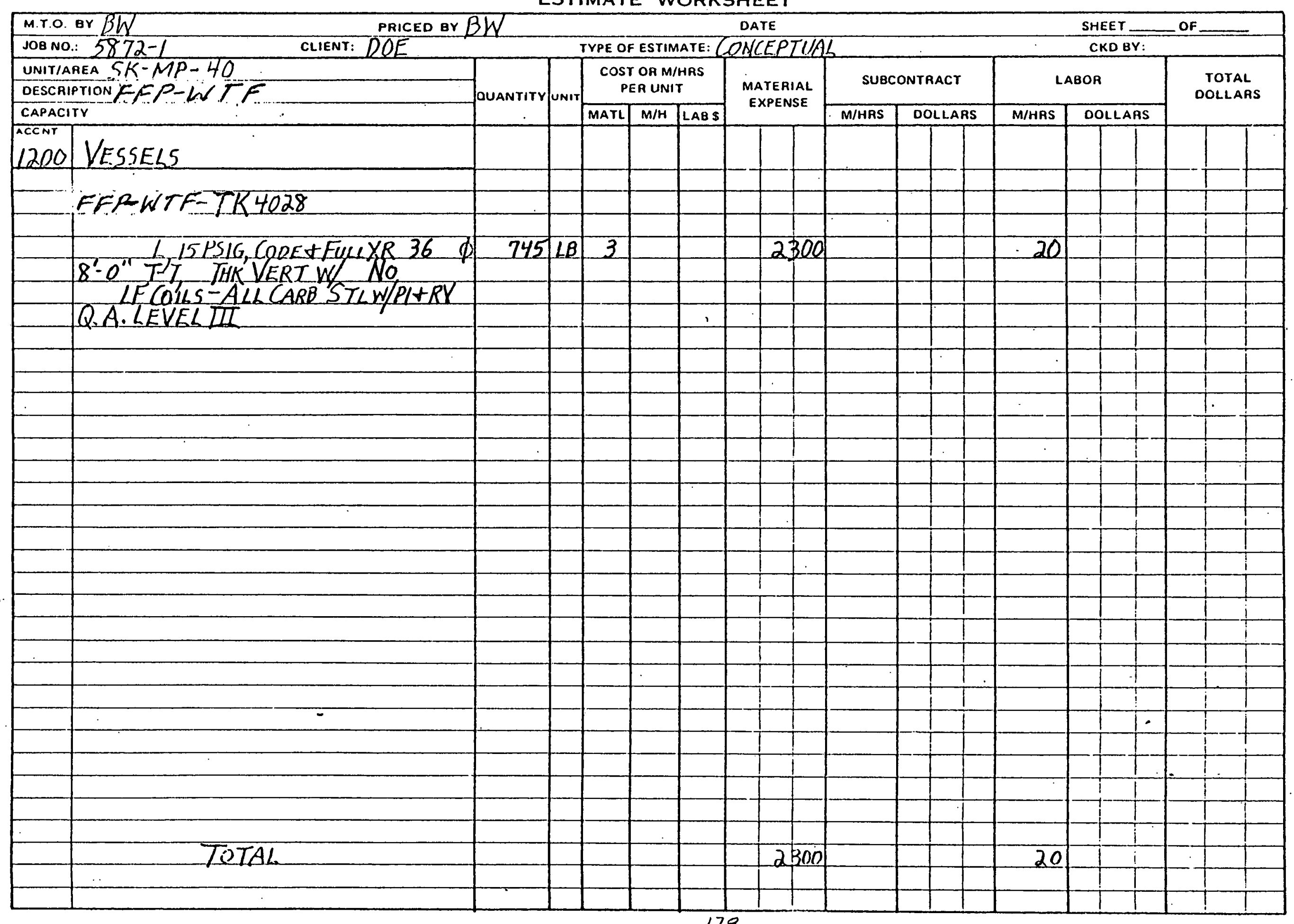


ESTIMATE WORKSHEET

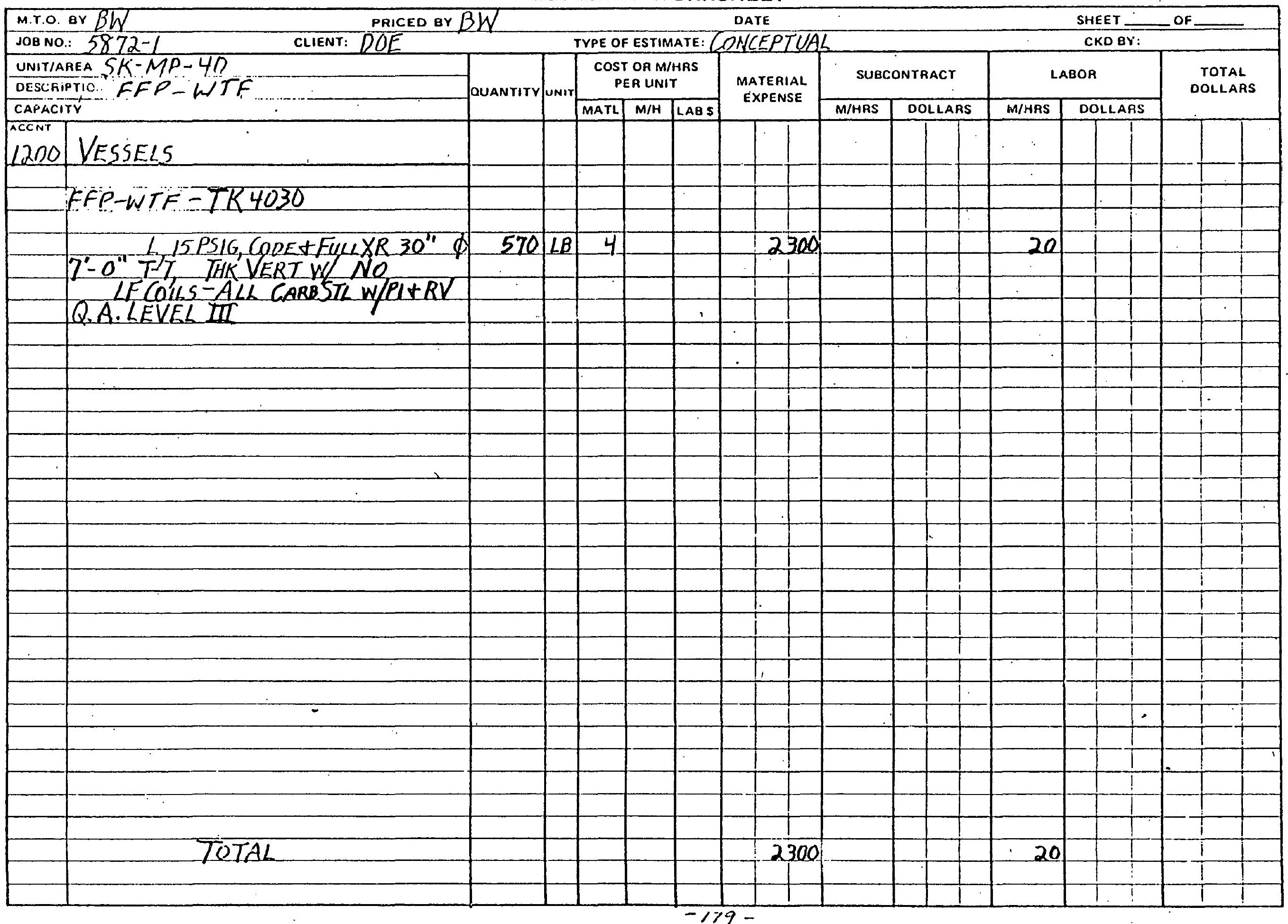


ESTIMATE WORKSHEET

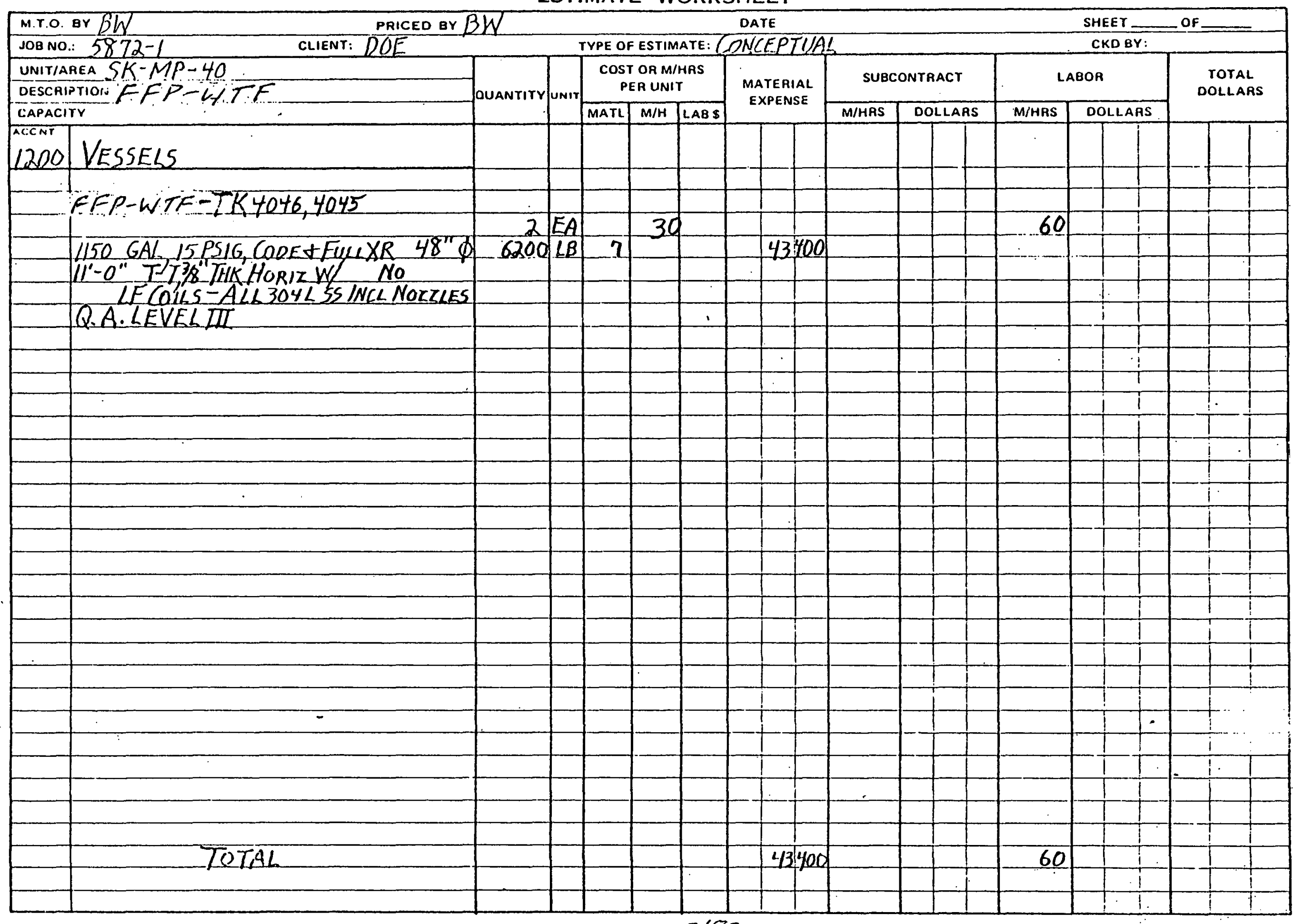


ESTIMATE WORKSHEET

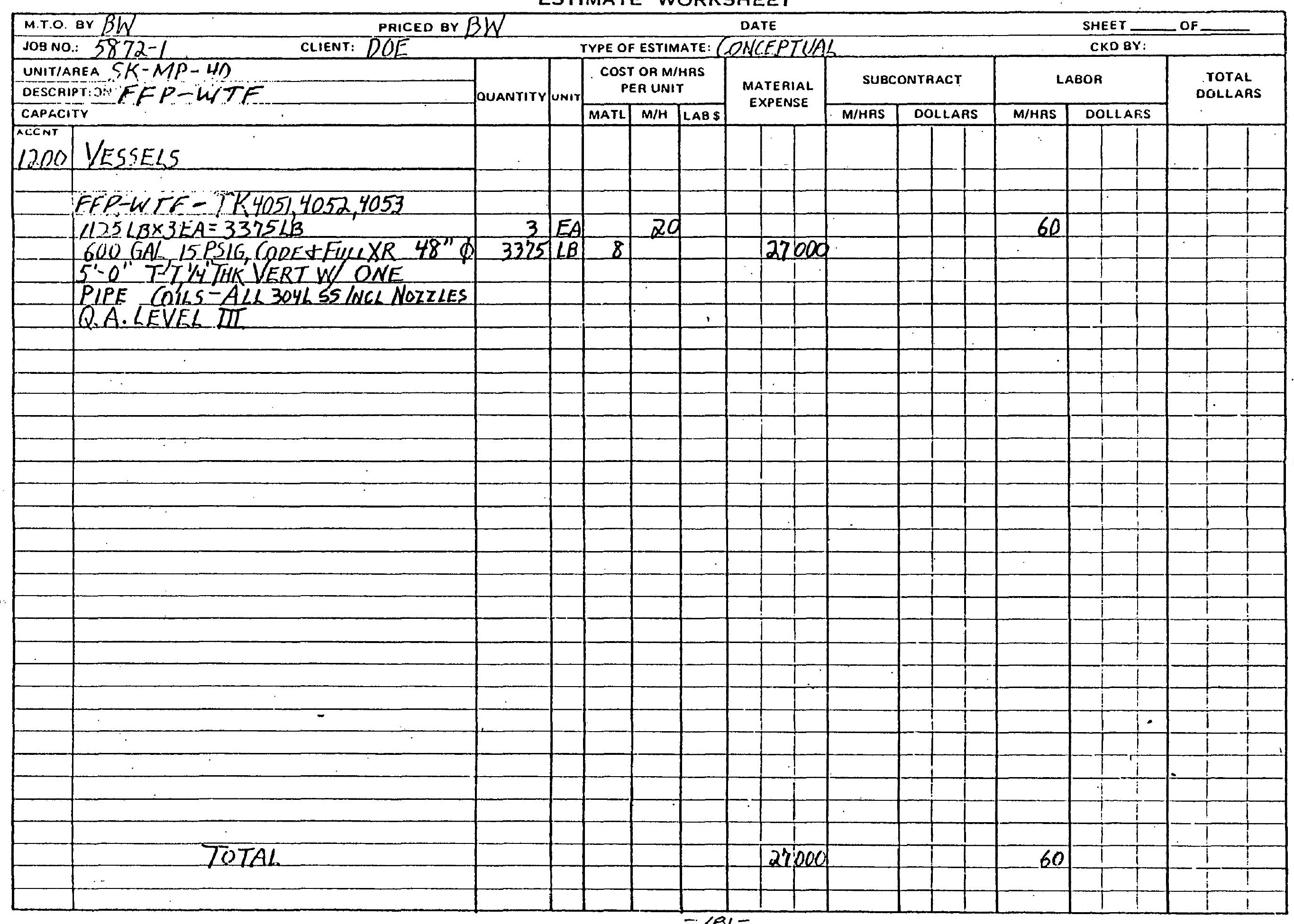


ESTIMATE WORKSHEET

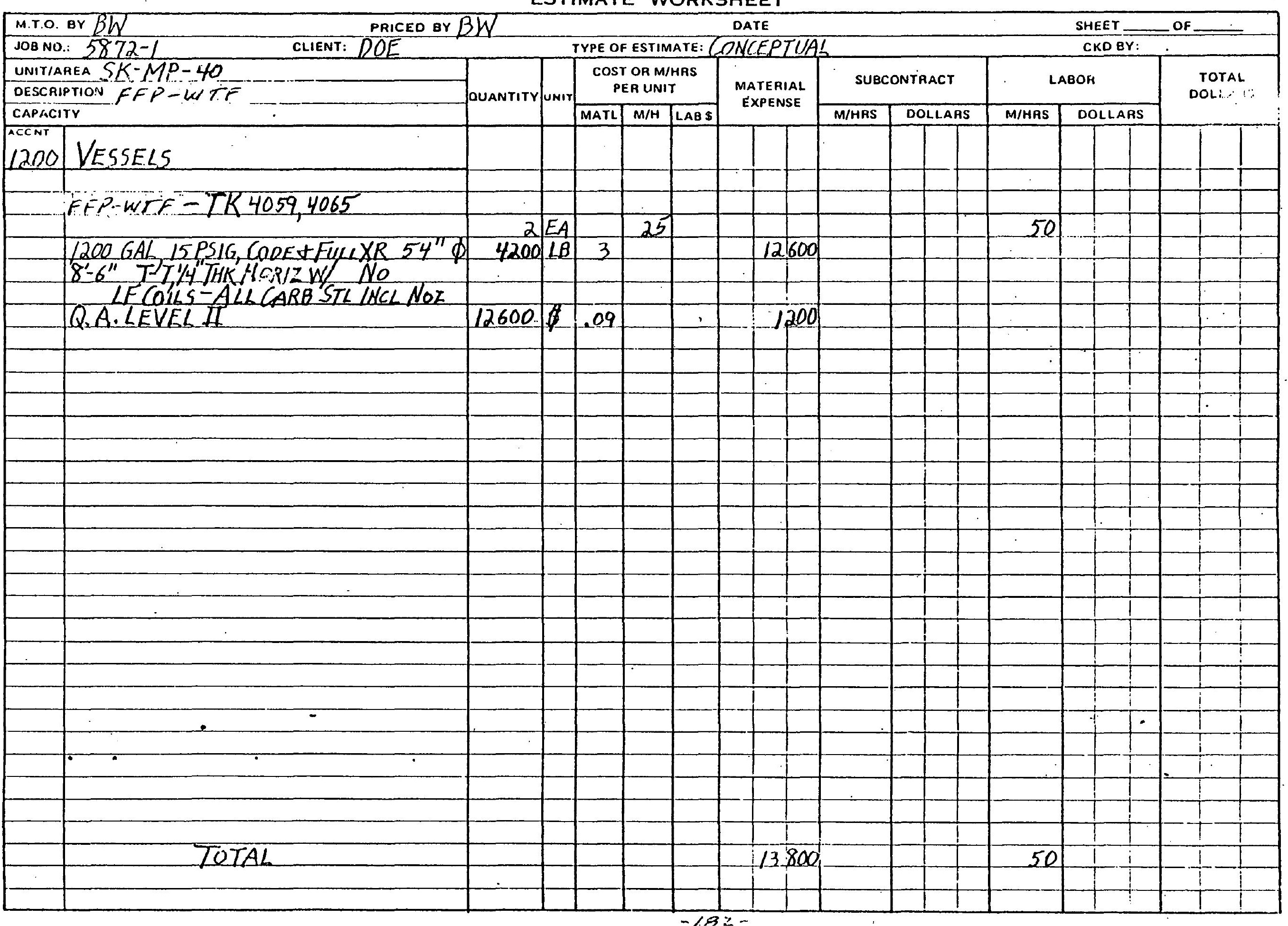


ESTIMATE WORKSHEET

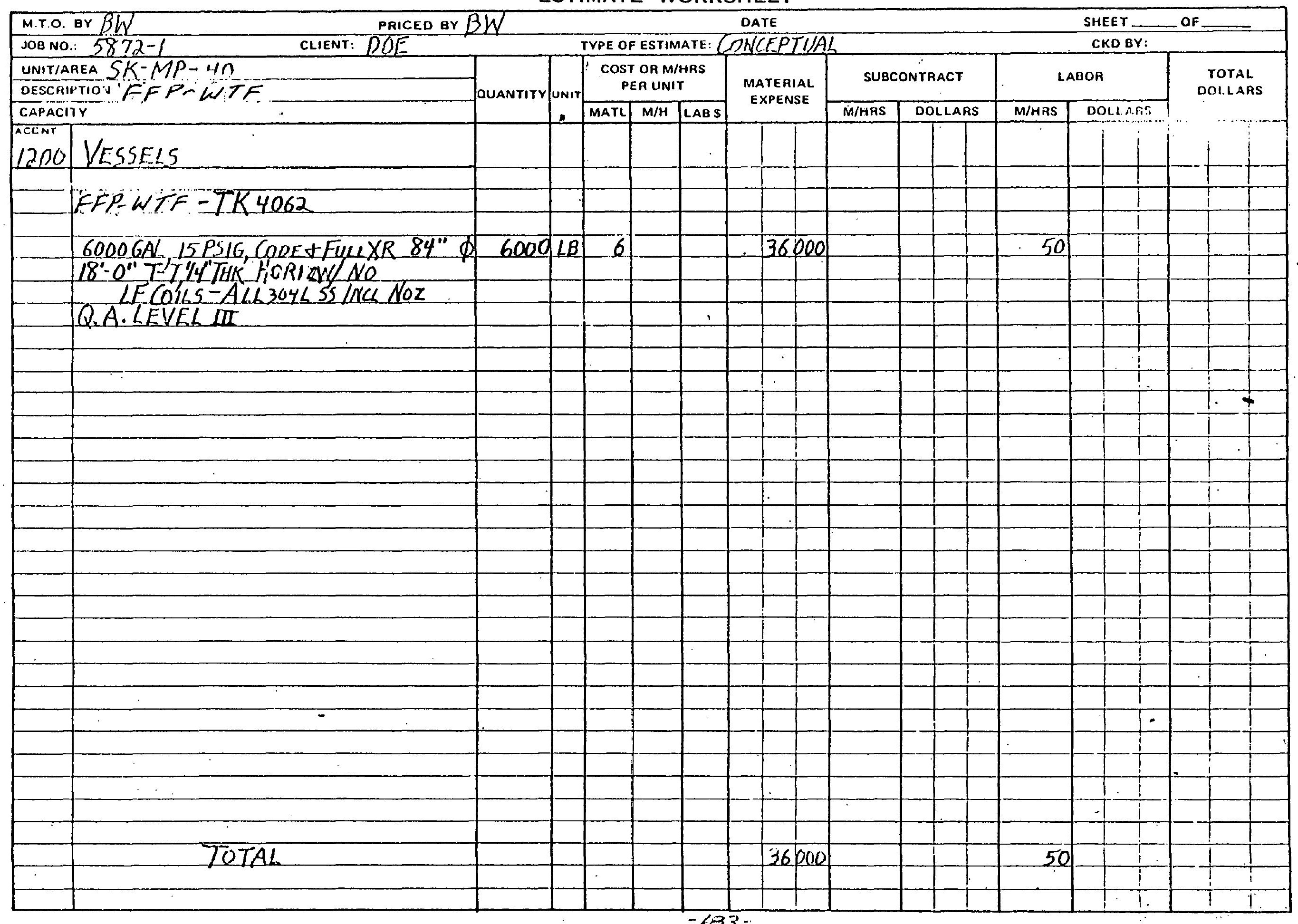


ESTIMATE WORKSHEET

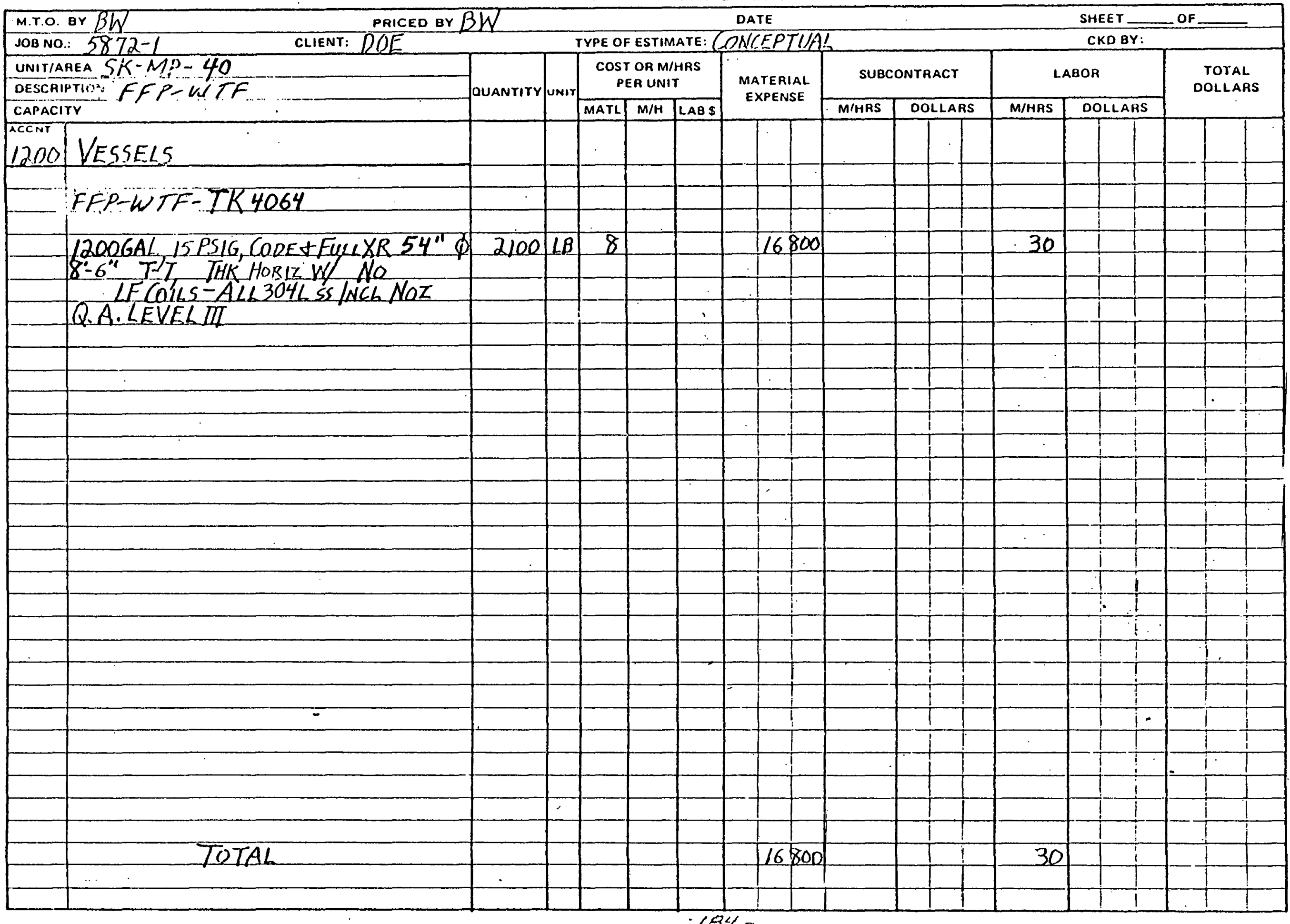


ESTIMATE WORKSHEET

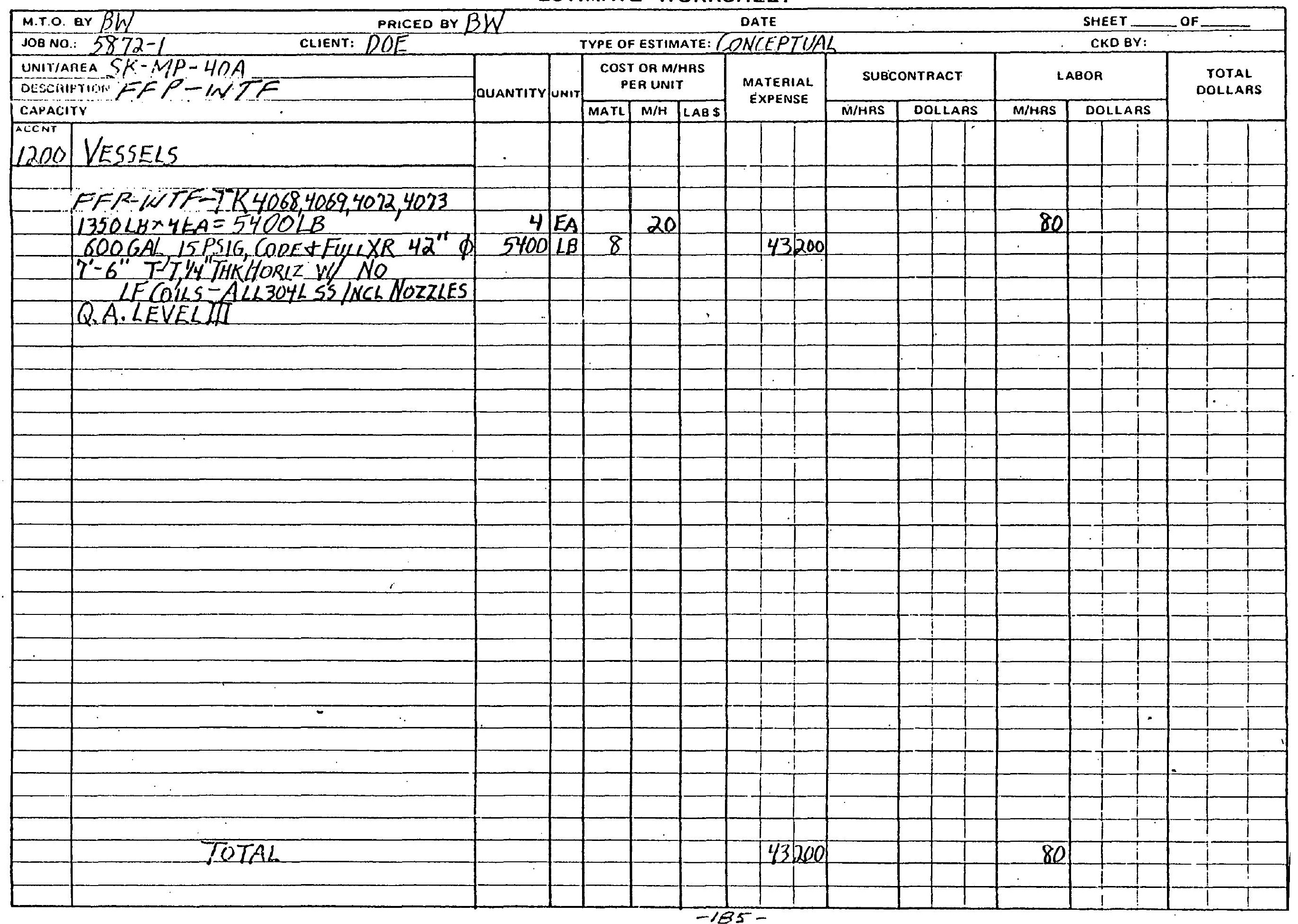


ESTIMATE WORKSHEET

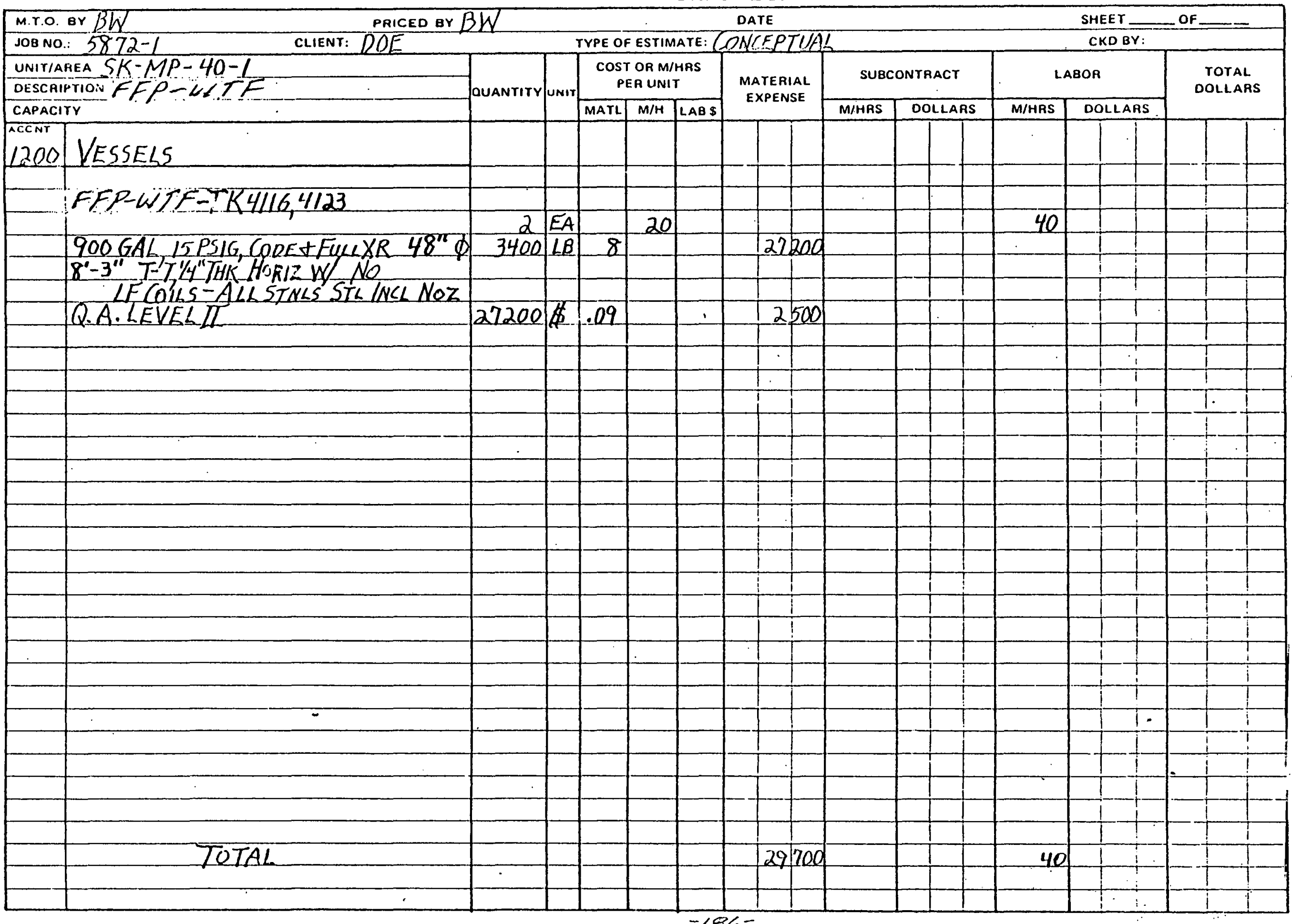


ESTIMATE WORKSHEET

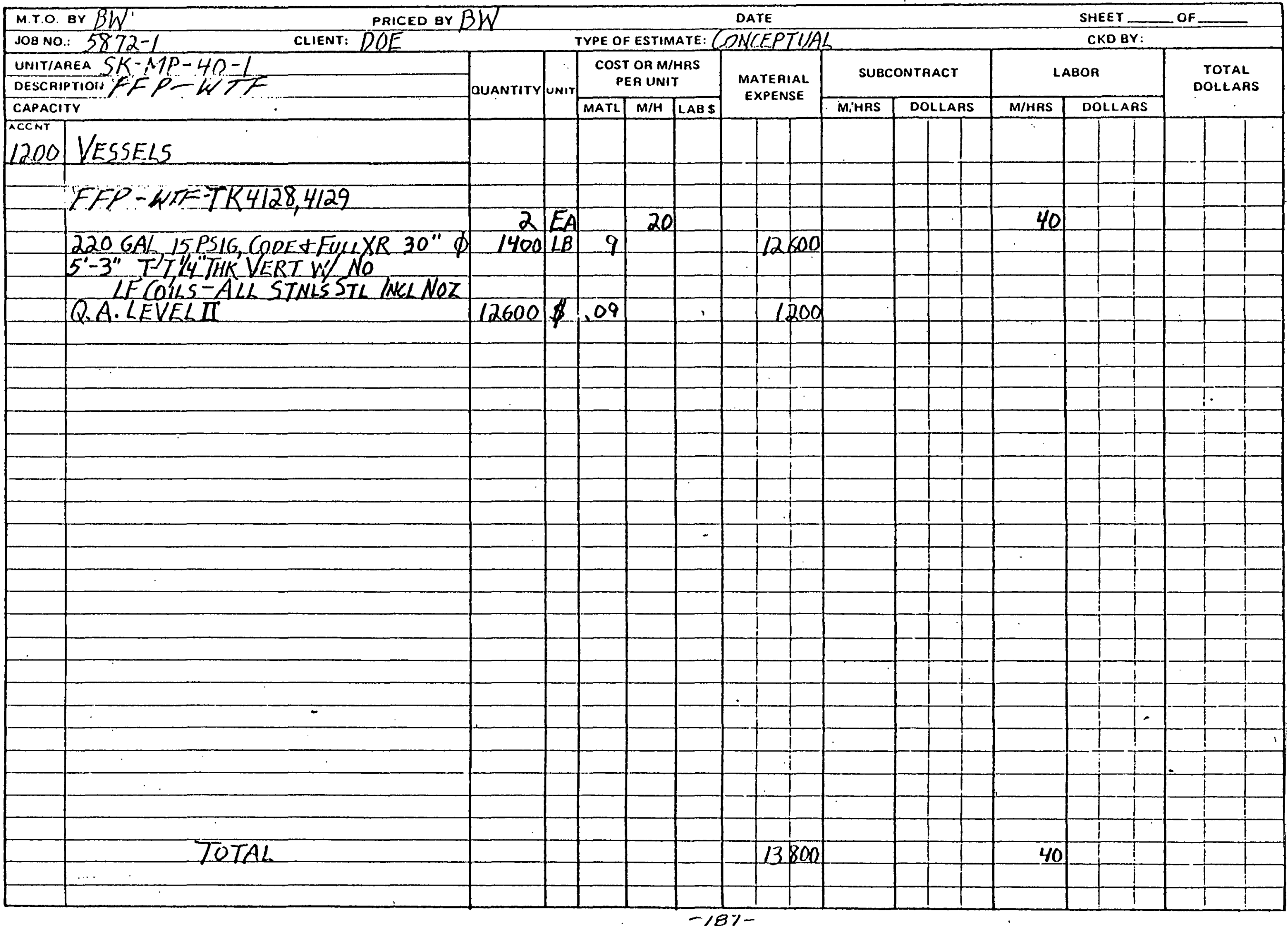




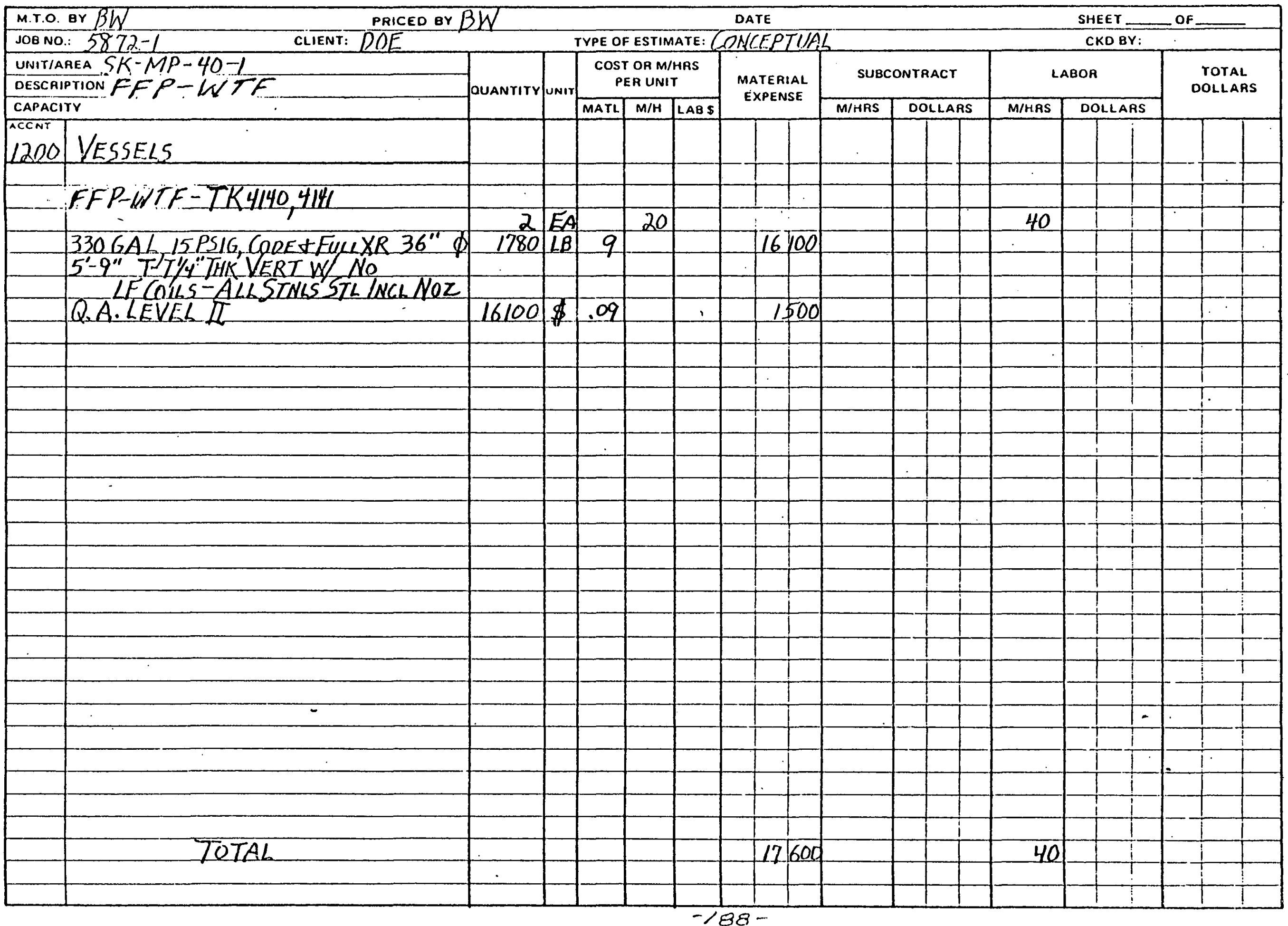




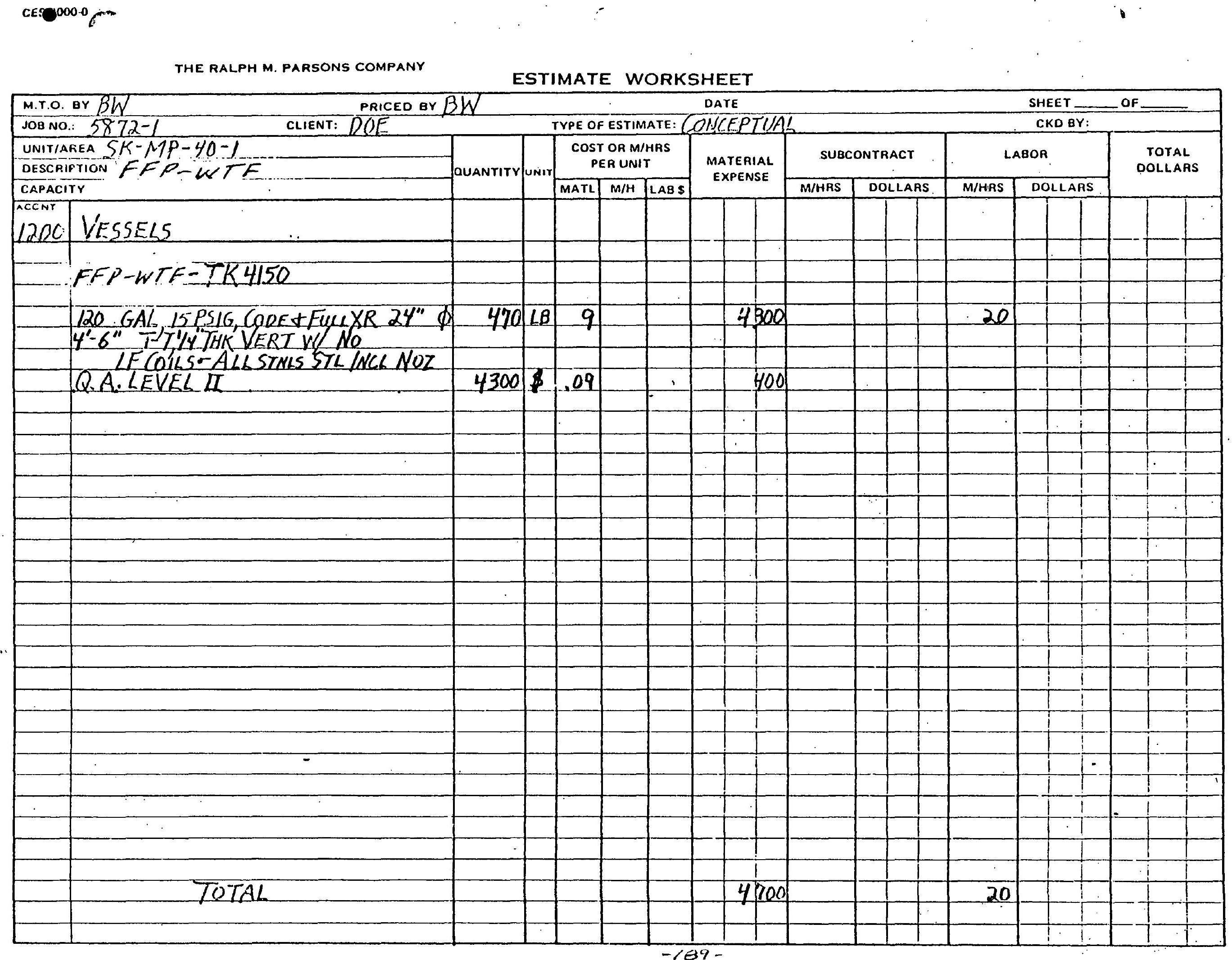


ESTIMATE WORKSHEET

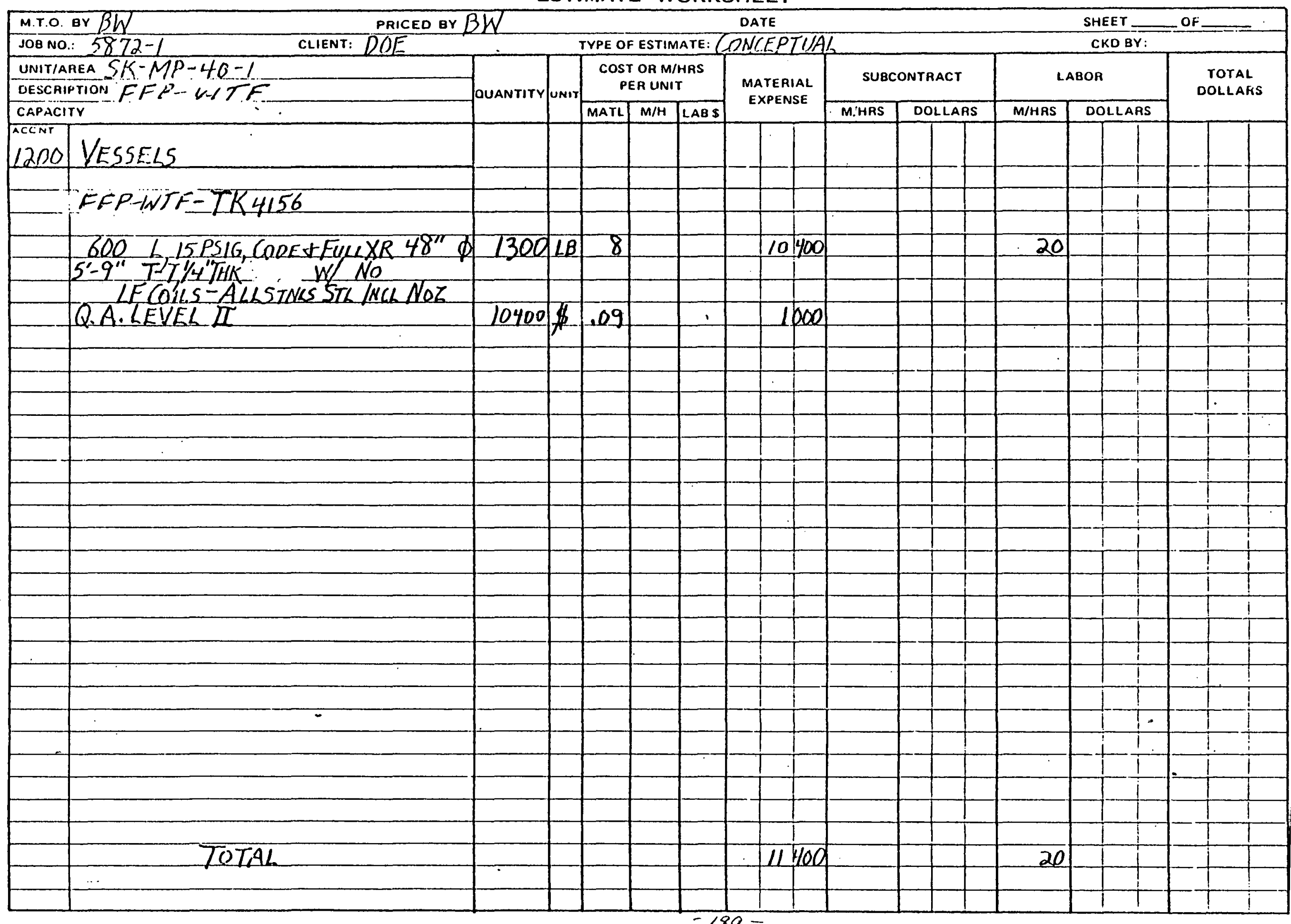


ESTIMATE WORKSHEET

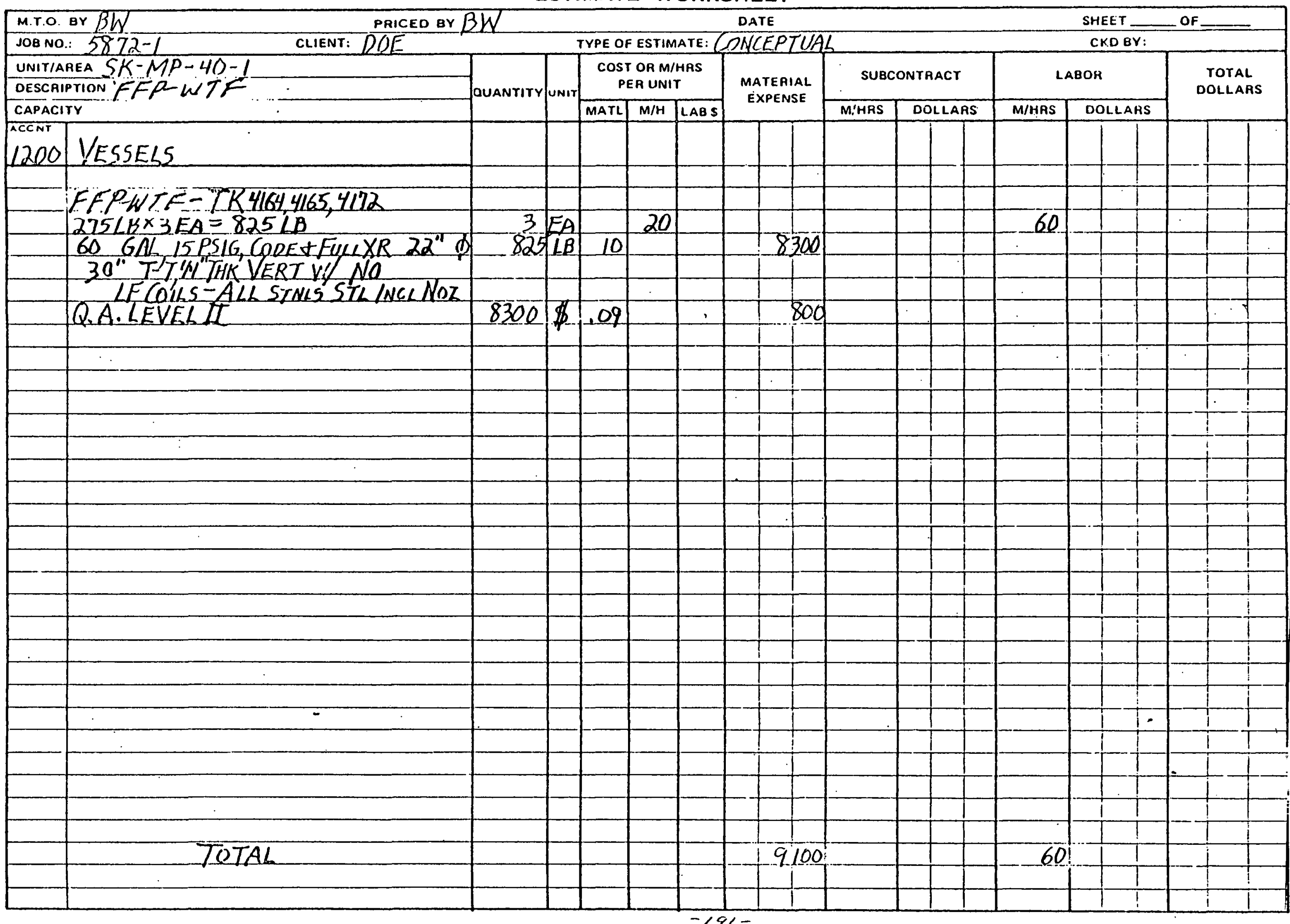


ESTIMATE WORKSHEET

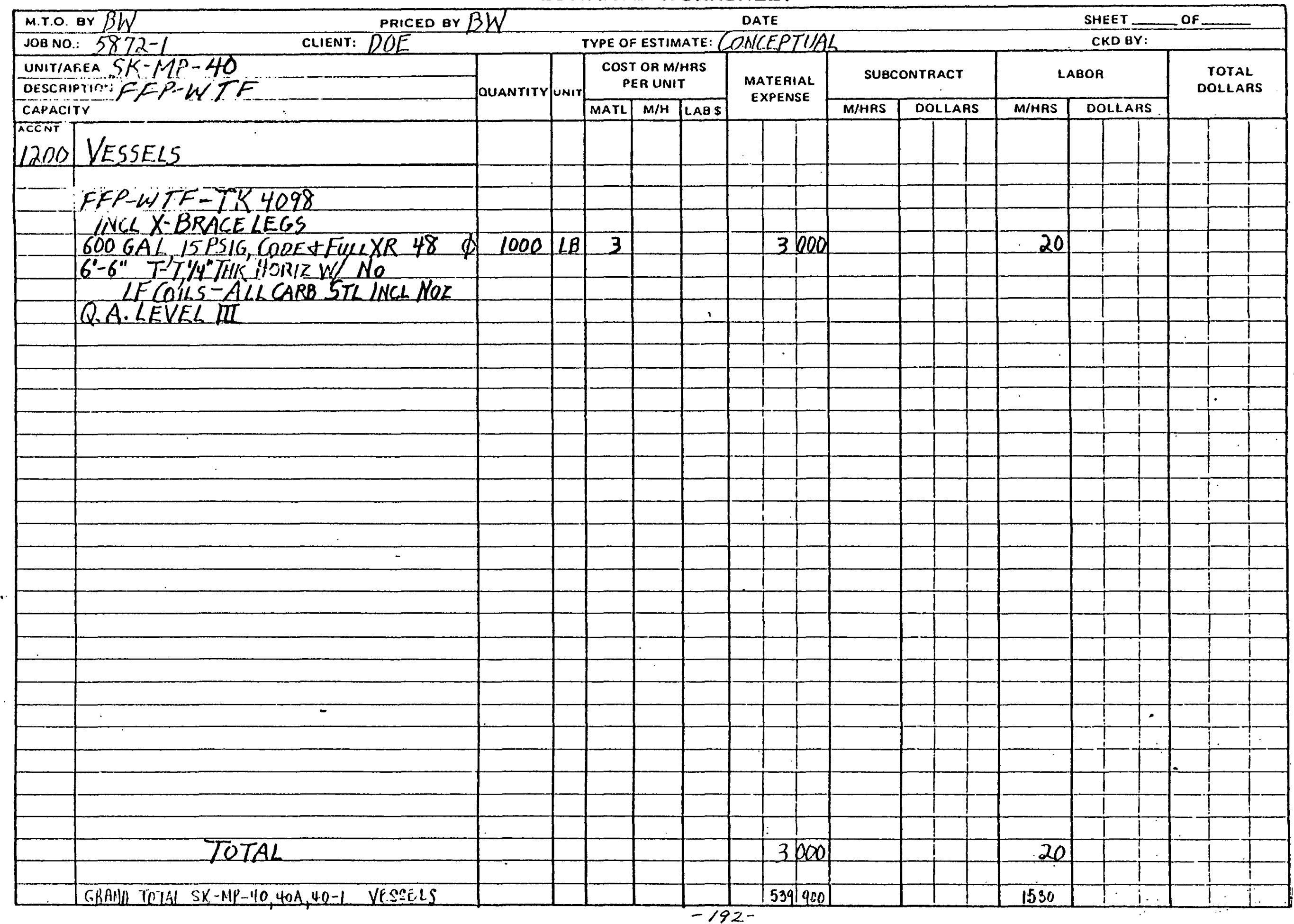


ESTIMATE WORKSHEET

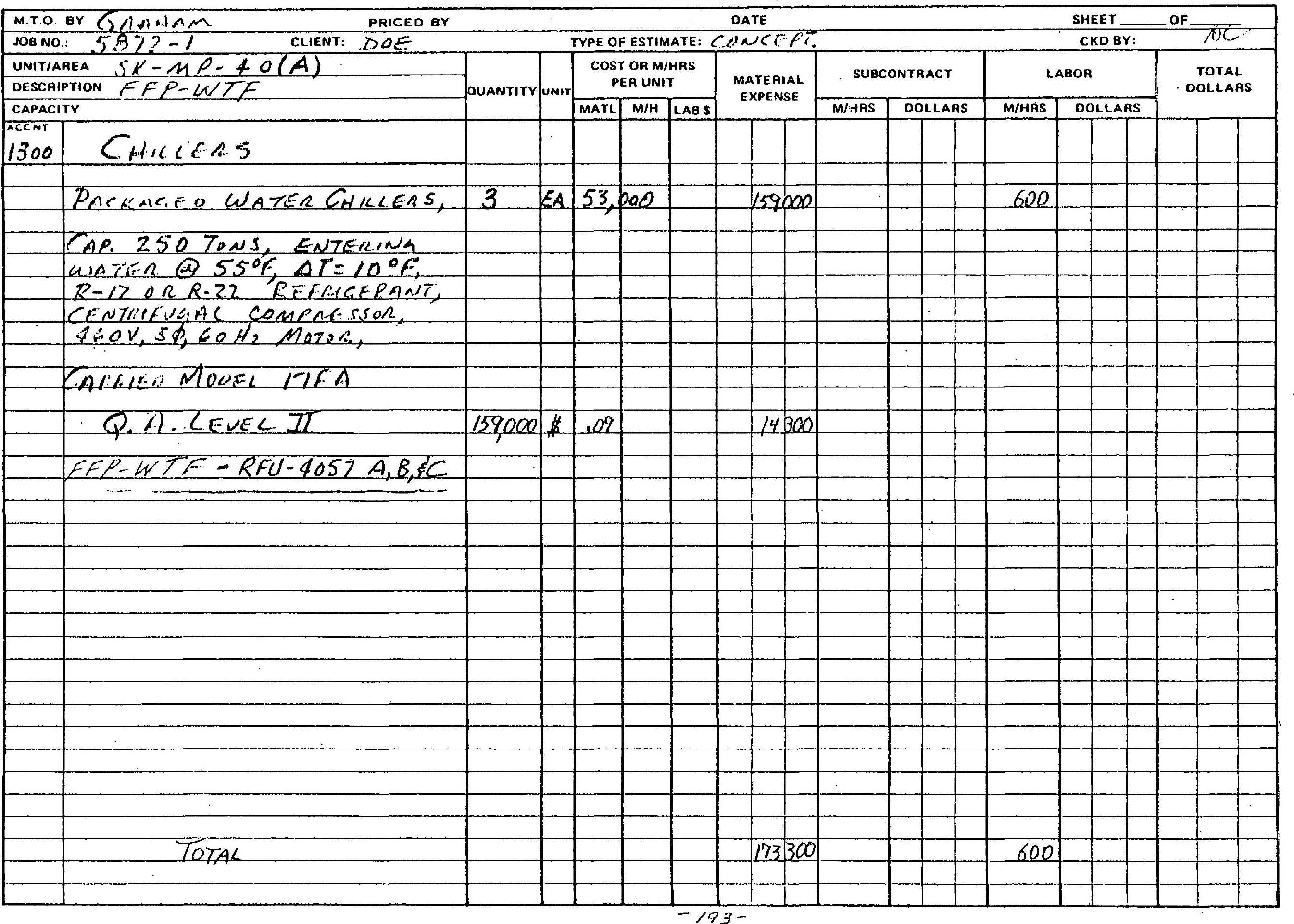


CES 20000

THE RALPH M. PARSONS COMPANY

ESTIMATE WORKSHEET

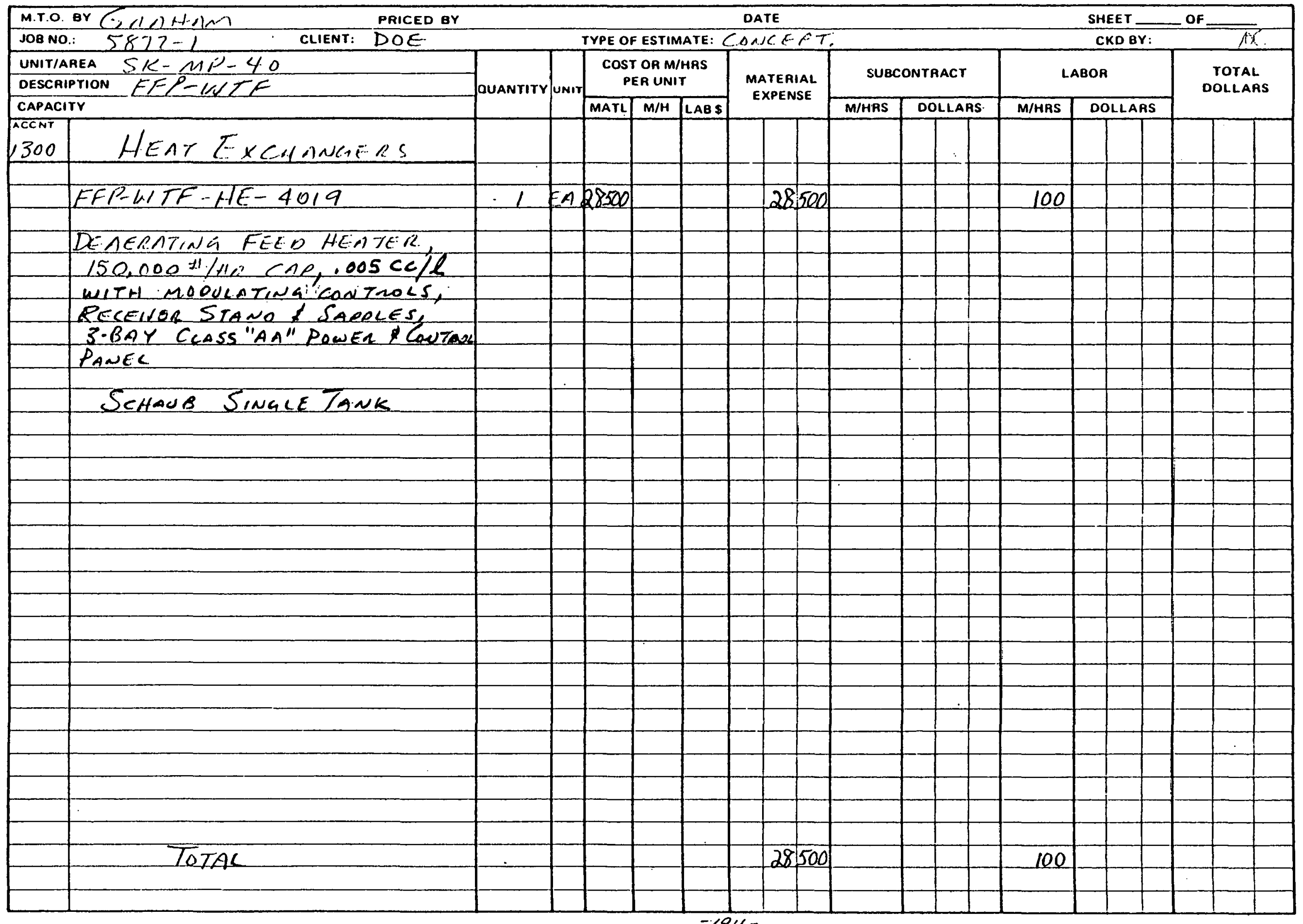


ESTIMATE WORKSHEET

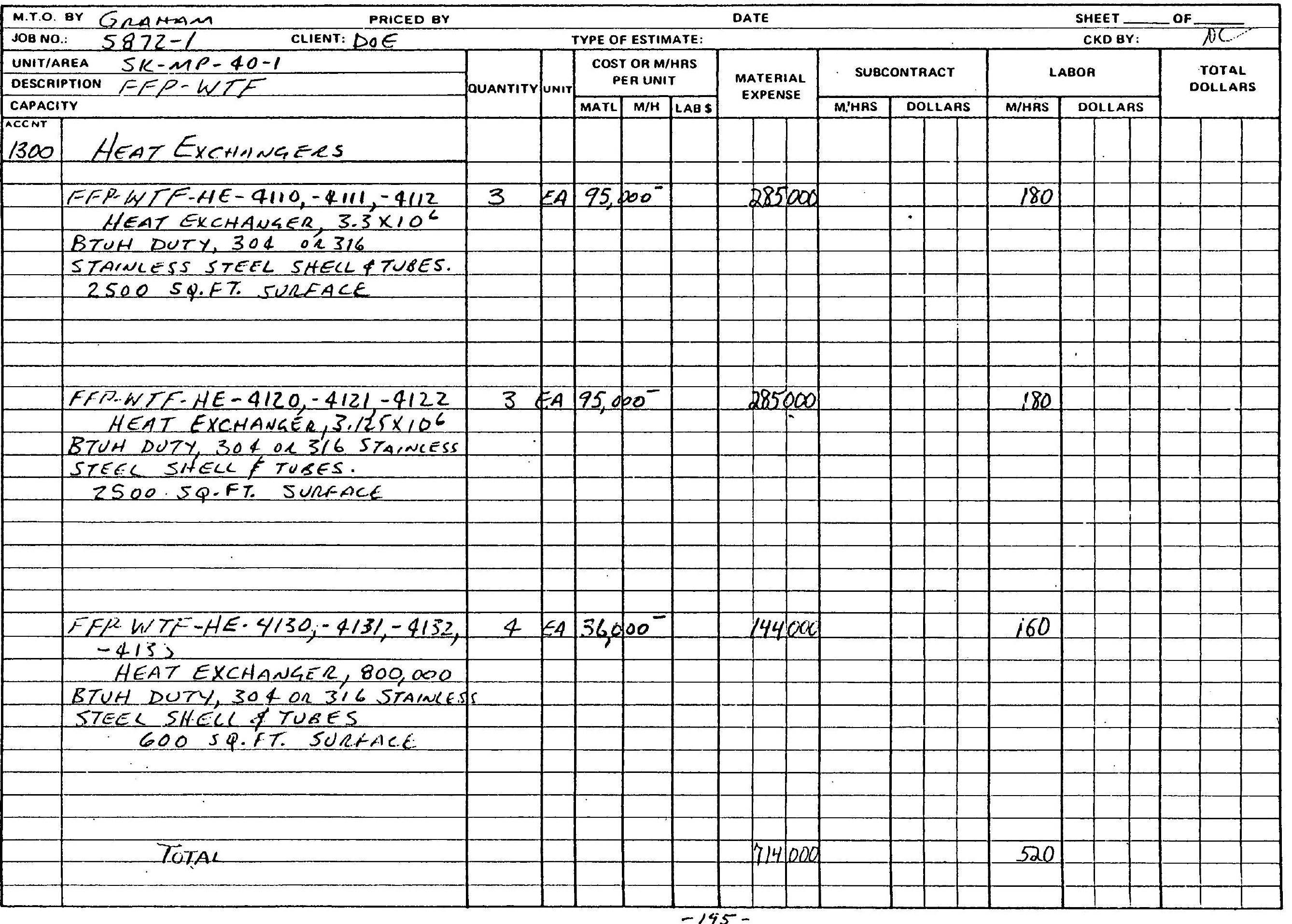


ESTIMATE, WORKSHEET

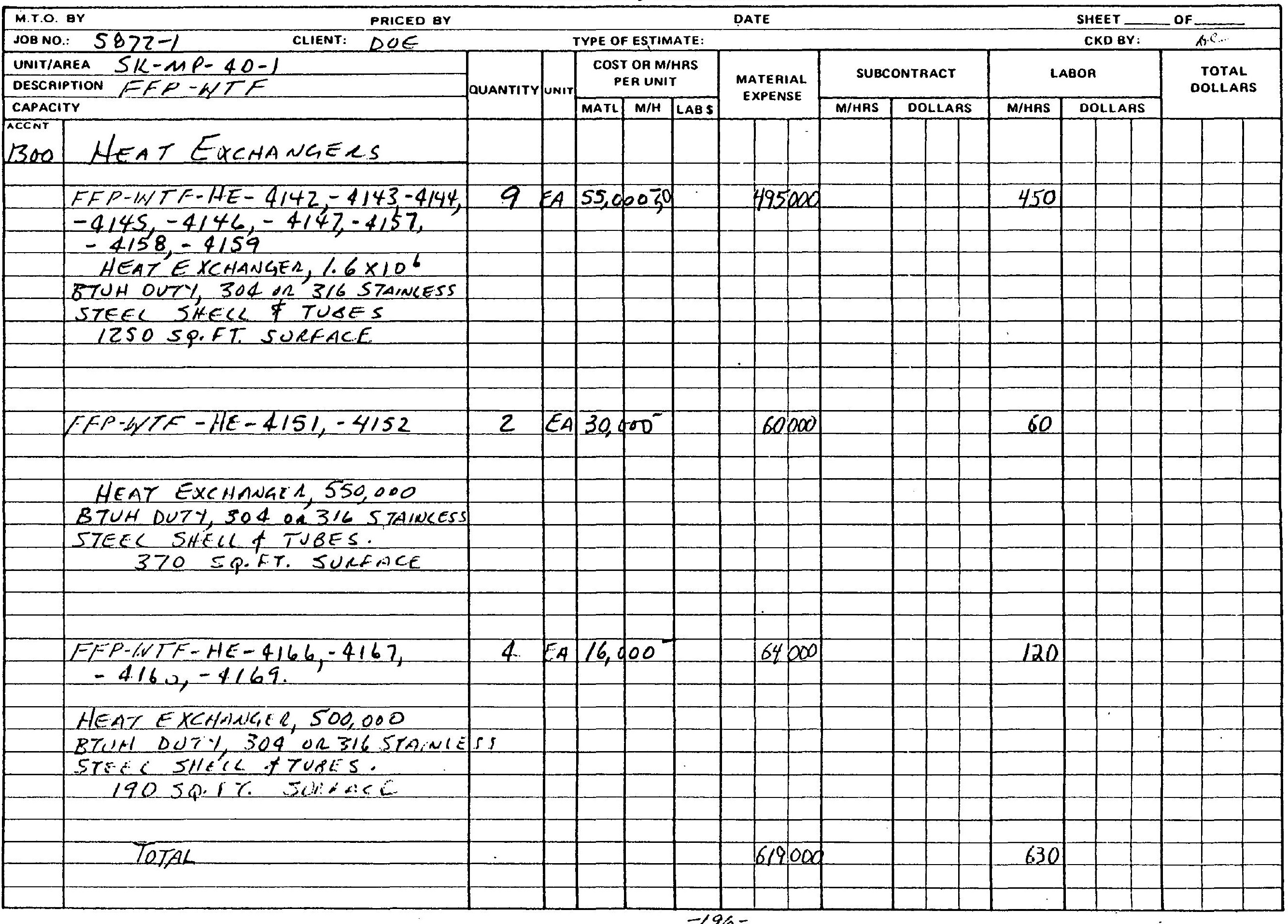


ESTIMATE WORKSHEET

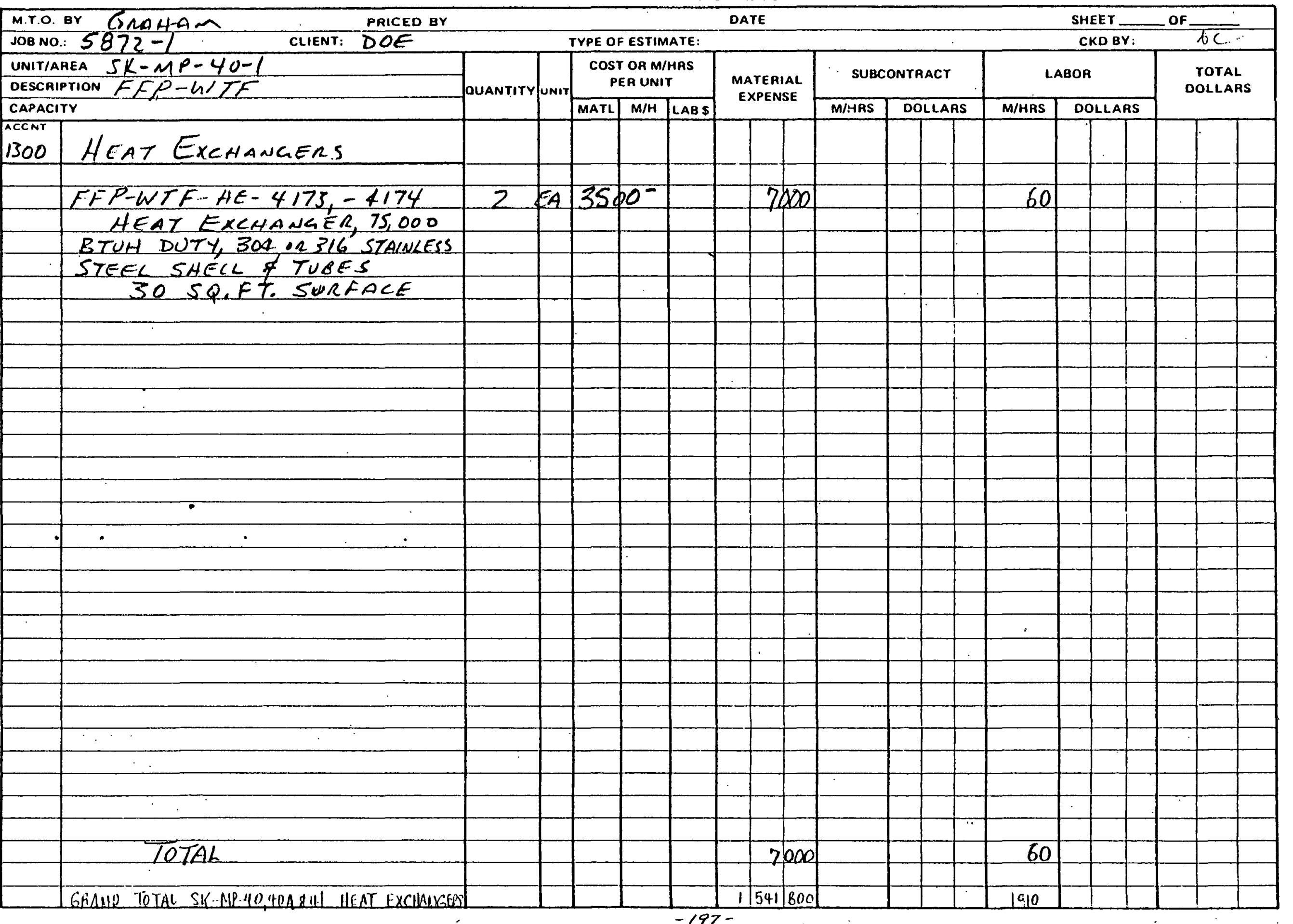


ESTIMATE WORKSHEET

M.T.O. OY GMA NAM PRICED QY

JOB NO.: $5877-1$

UNITIAREA SK-MP- 40

Deschiption E EP-MIT

CAPACITY

\begin{tabular}{l|l}
\hline ASCNT & PUMP \\
\hline
\end{tabular}

EFP-INTE-P-4001 $A \div B$

1000 SPM@100 Cs/ w7

$600 \mathrm{FT}$ suctiod LIFT.

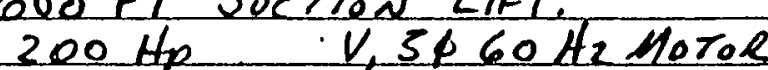

BROUZE LMPELLERS, ENAMELED Bowes
EA

1350090

27000

180 
Cts $2000-0$

THE RALPH M. PARSONS COMPANY

ESTIMATE WORKSHEET

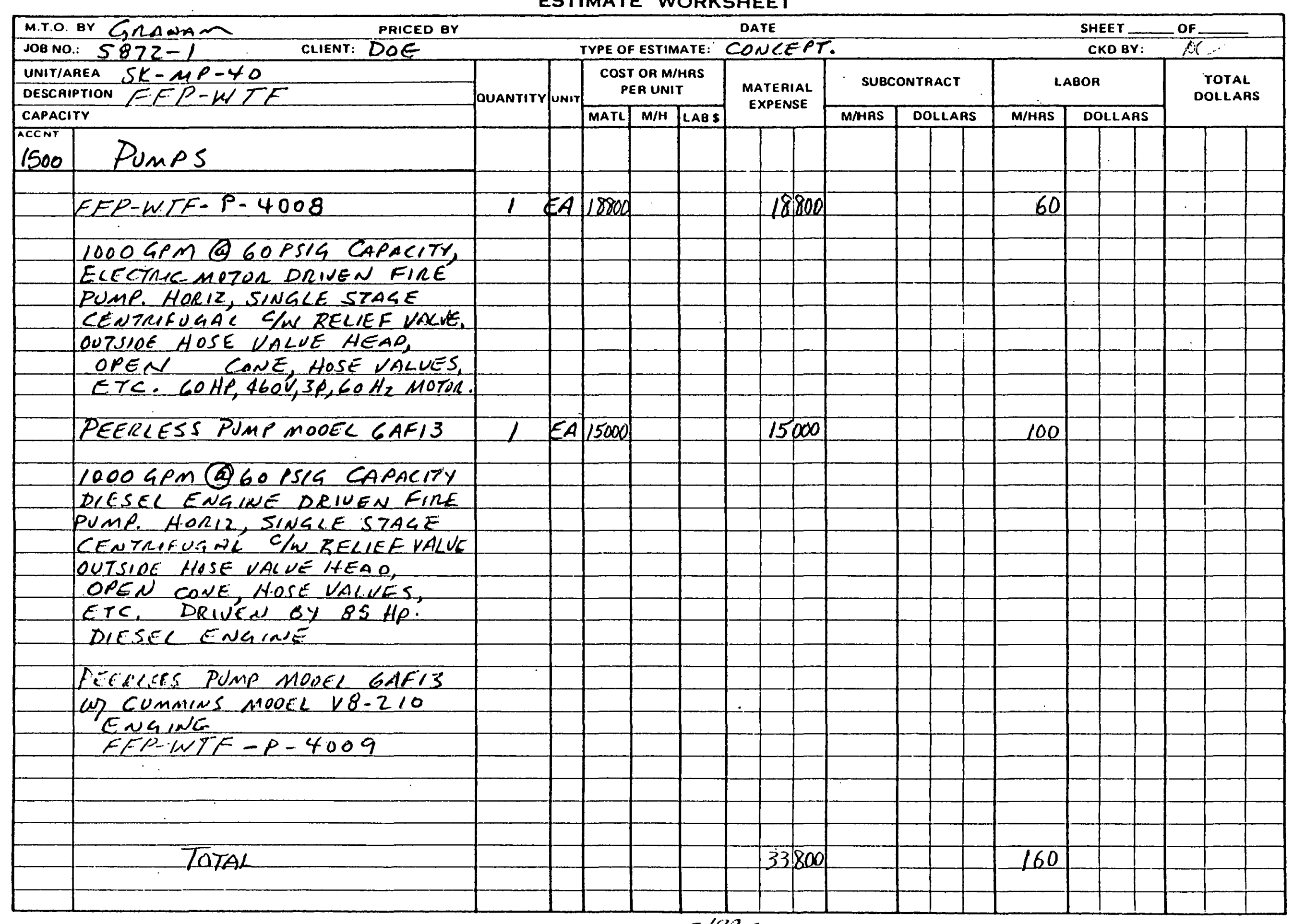


ES $2000-$

THE RALPH M. PARSONS COMPANY

ESTIMATE WORKSHEET

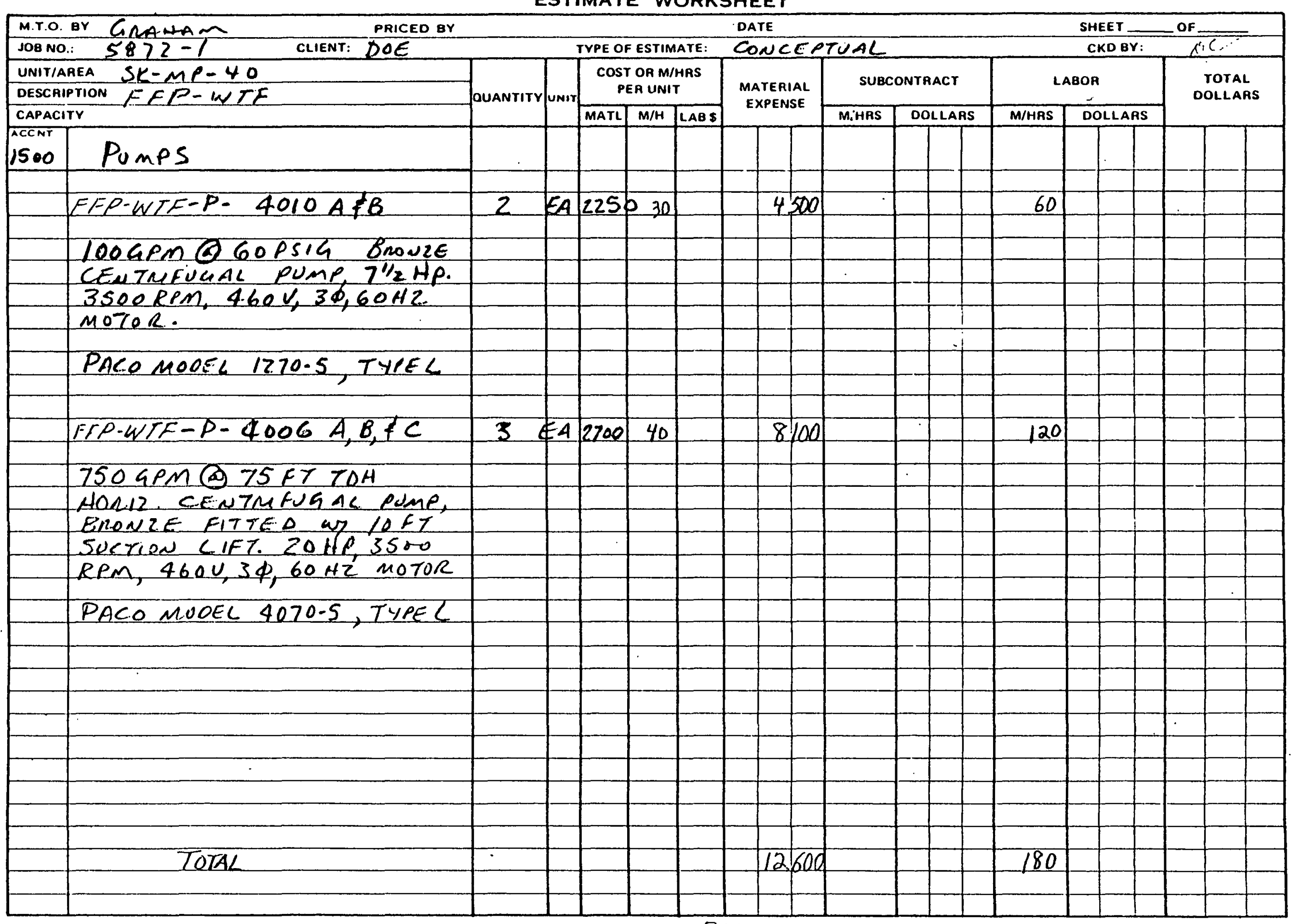


CES 2000-0

THE RALPH M. PARSONS COMPANY

ESTIMATE WORKSHEET

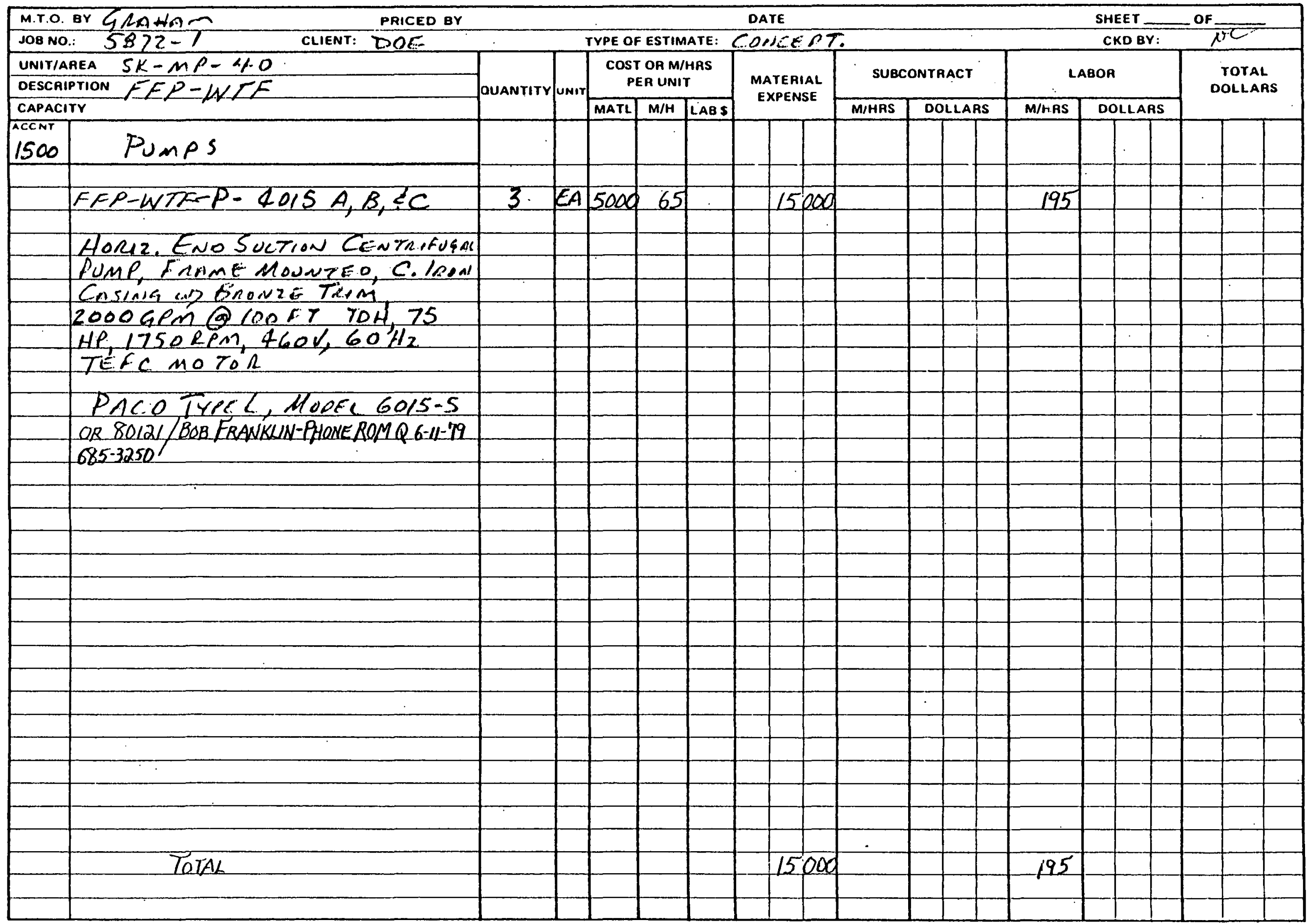


LES $2000-0$

THE RALPH M. PARSONS COMPANY

ESTIMATE WORKSHEET

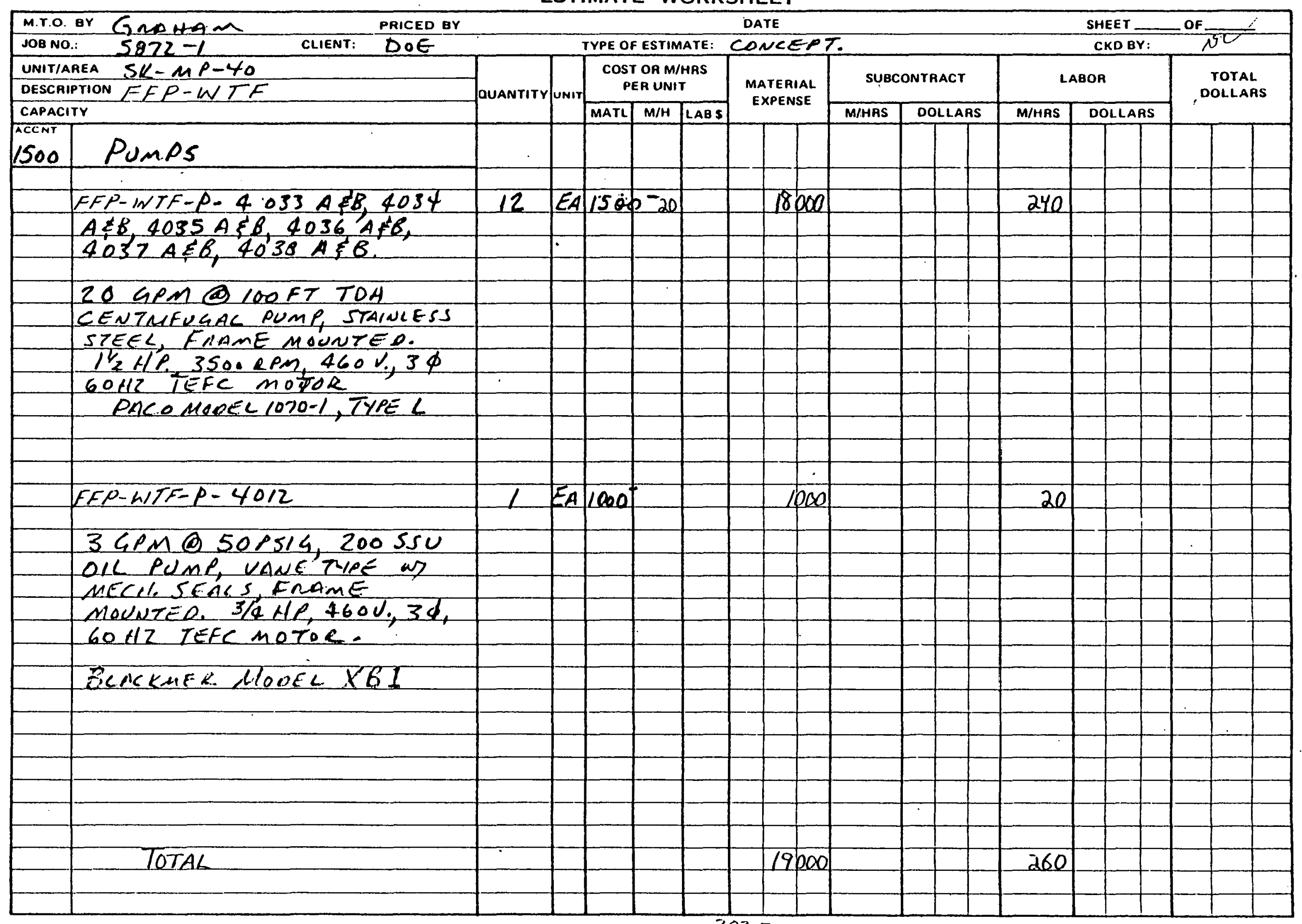


ESTIMATE WORKSHEET

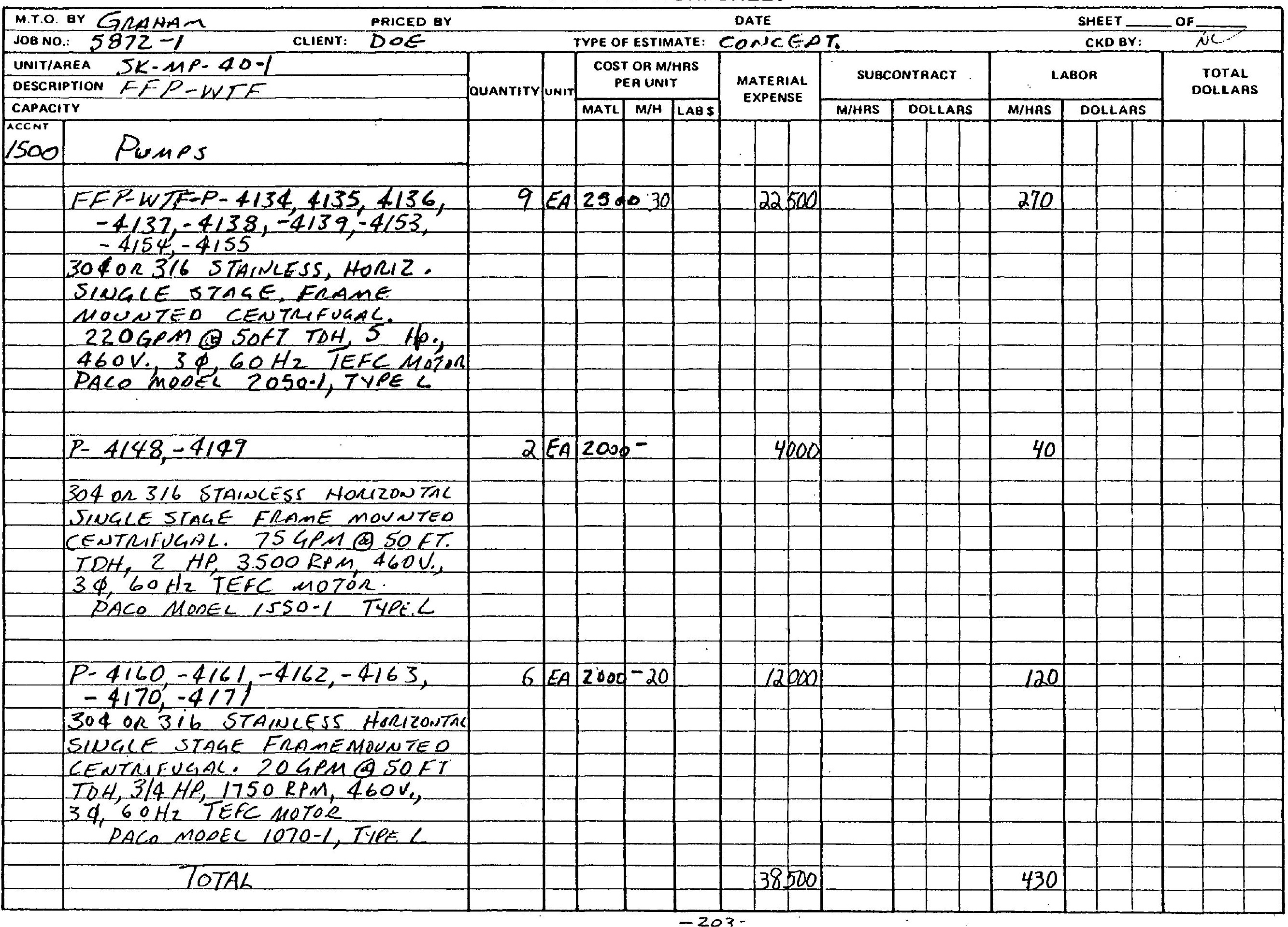


ESTIMATE WORKSHEET

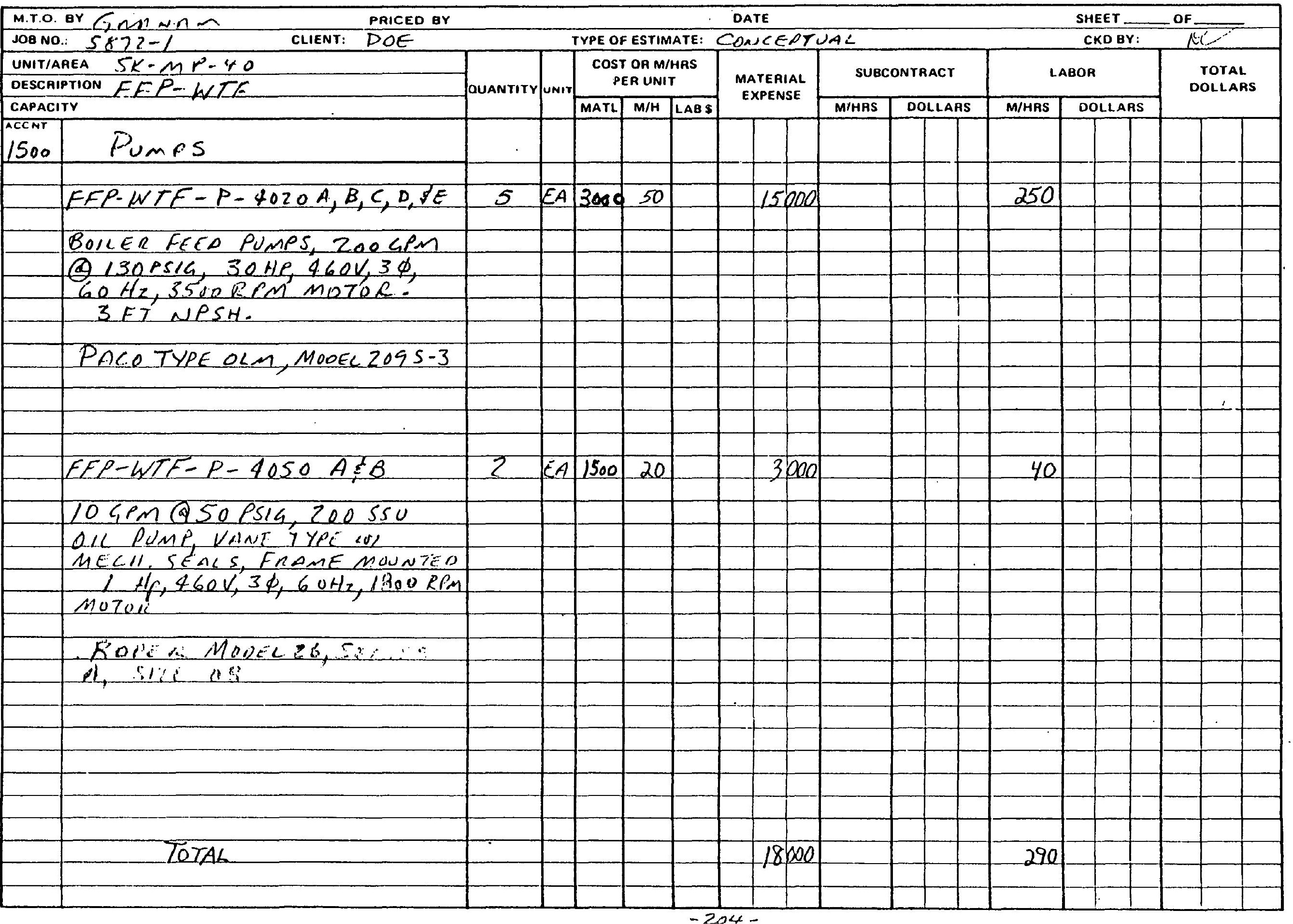


ESTIMATE WORKSHEET

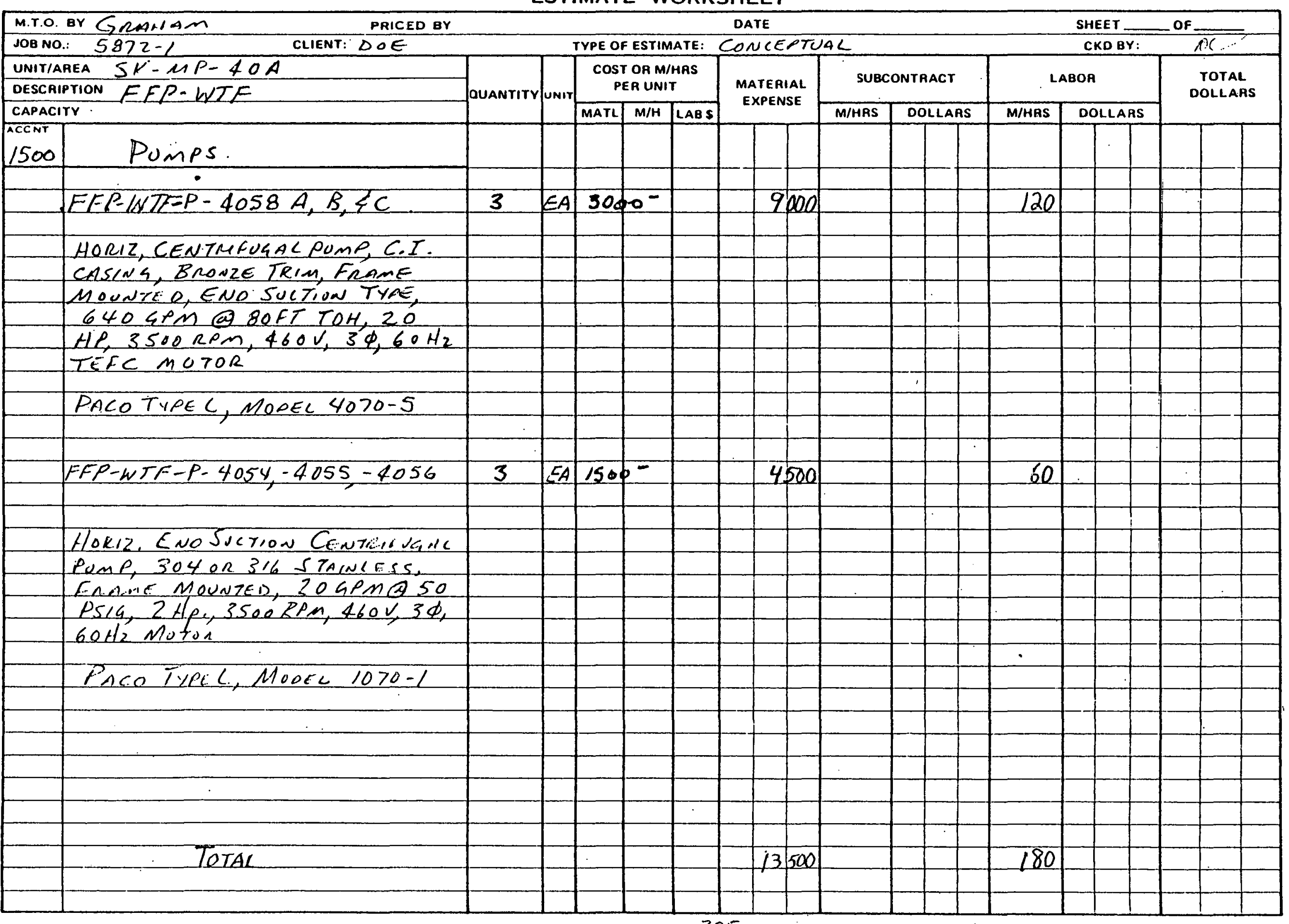


LES $2000-0$

THE RALPH M. PARSONS COMPANY

ESTIMATE WORKSHEET

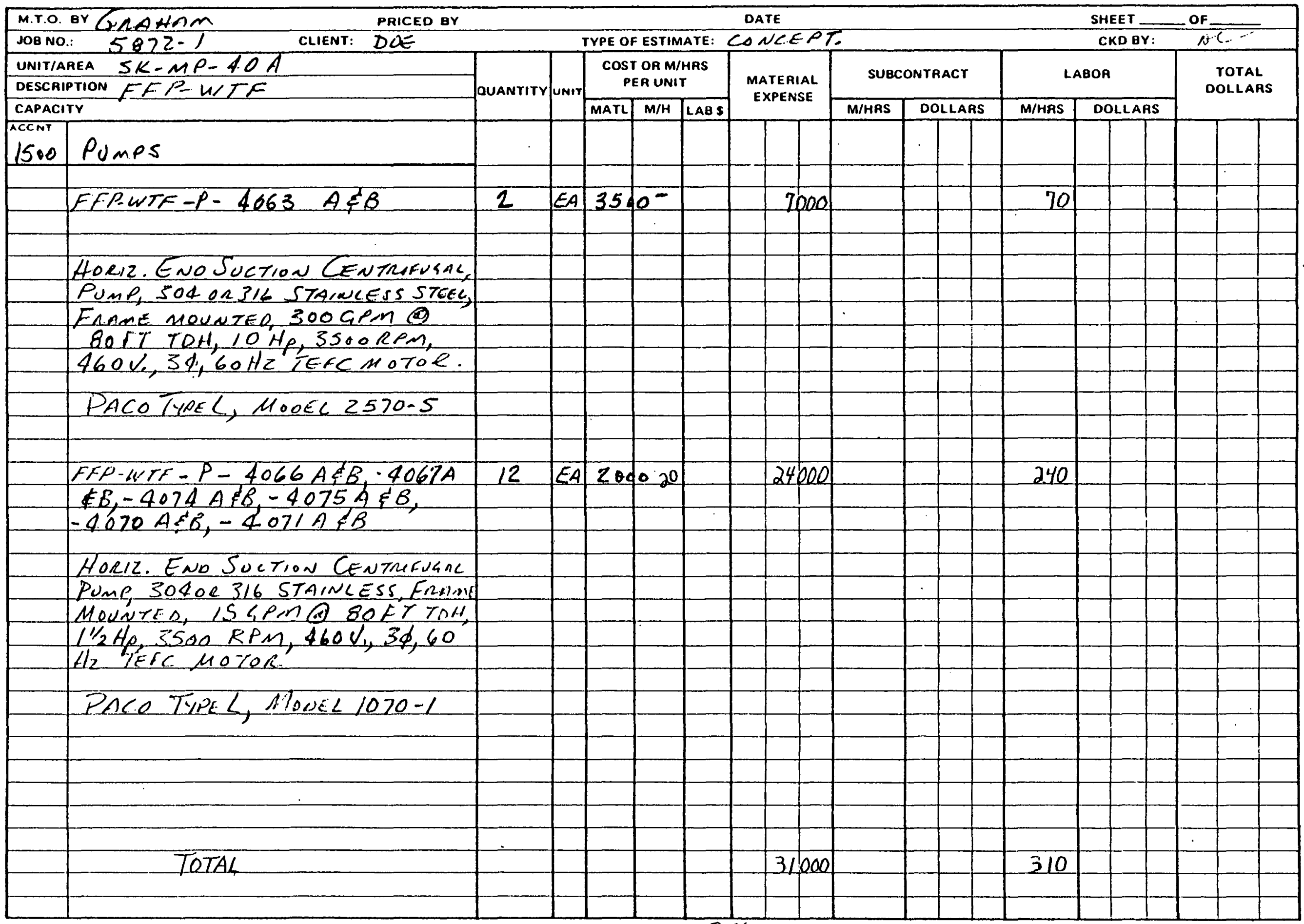


ES 20000

THE RALPH M. PARSONS COMPANY

ESTIMATE WORKSHEET

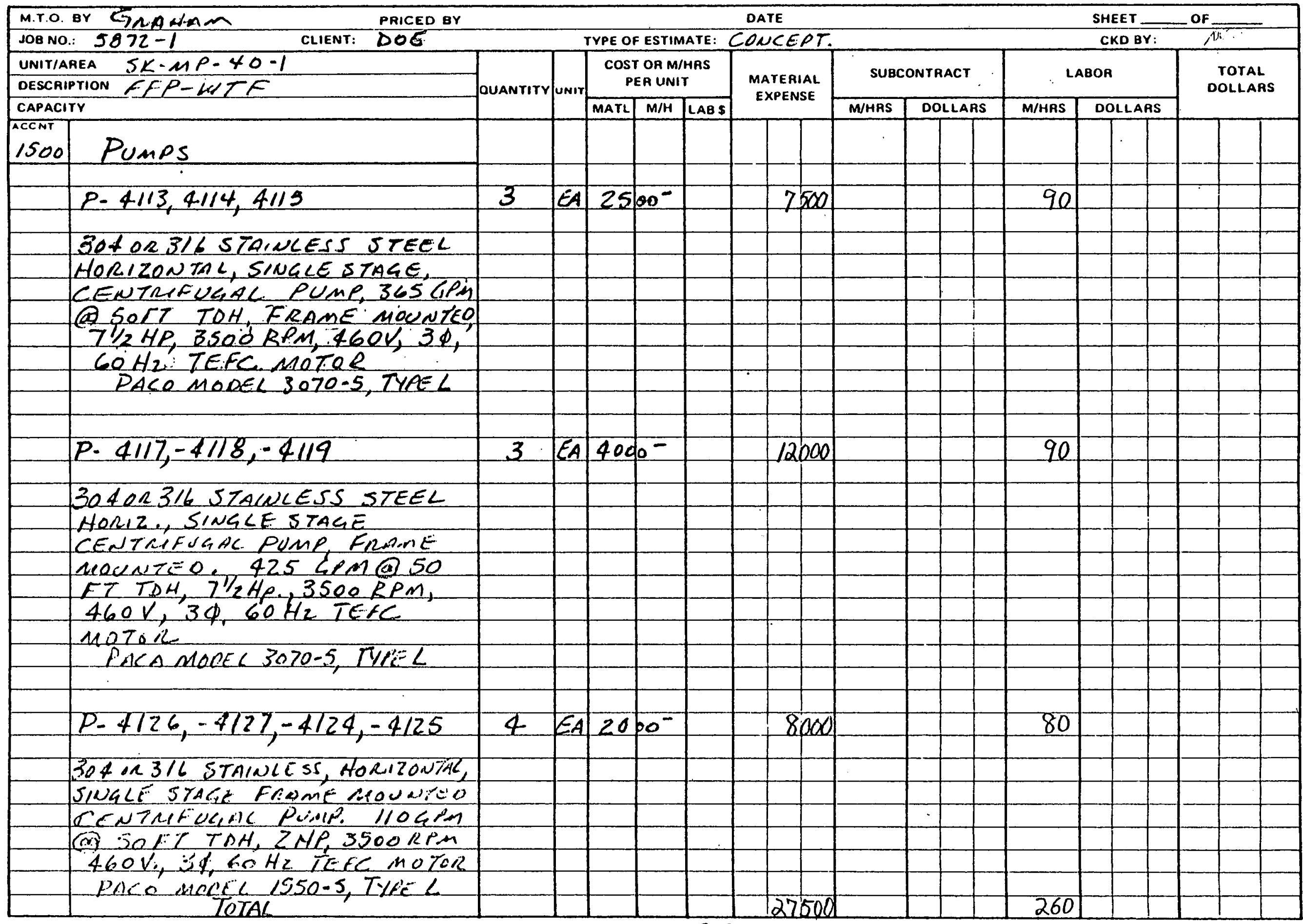


ESTIMATE WORKSHEET

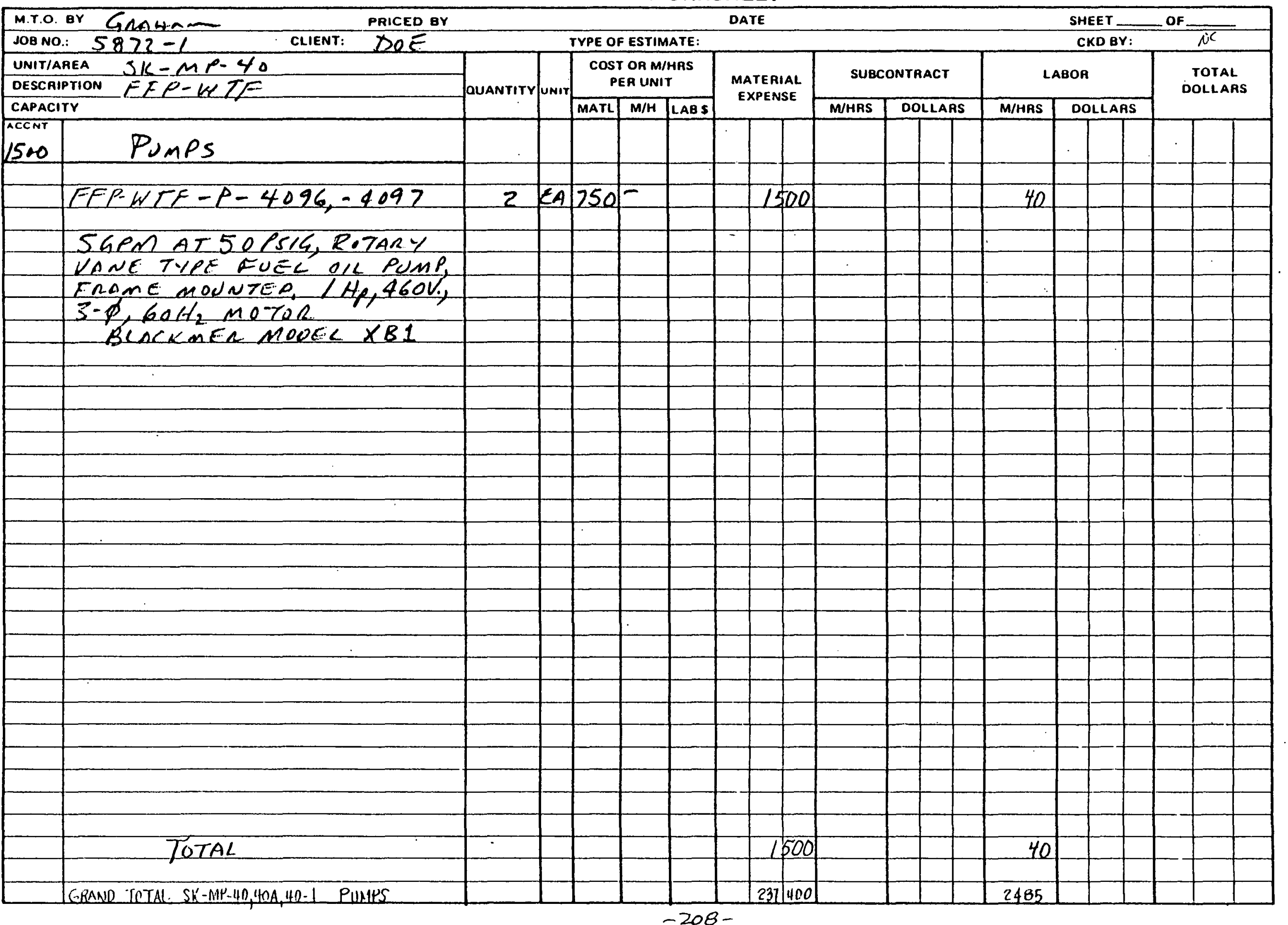


ESTIMATE WORKSHEET

\begin{tabular}{|c|c|c|c|c|c|c|c|c|c|c|c|c|c|c|c|c|c|c|}
\hline M.T.O & or Gancsin & & & & & & DATE & & & & & & & SHE & EET & -OF. & & \\
\hline$J 0 B N$ & $\therefore 5822-1$ & & & TYPE OF & ESTIM & IATE: & $600 / 6$ & Eas & & & & & & & COQY: & & 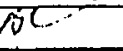 & \\
\hline UNITII & REA $S K-M P-40$ & & & $\cos T$ & $O R M / 1$ & & & & SUB & ONTR & RACY & & & BOA & & & & \\
\hline DESCR & PrION FER-WLF & JoUantitY & UNir & & ER UNIT & & $\begin{array}{l}\text { MATERI } \\
\text { EXPENS }\end{array}$ & & & & & & & & & & DOLLARS & \\
\hline CAPAC & & & & MATL & $M / H$ & LAB S & & & M/HAS & & OLLAR & & M/HAS & & LLARS & & & \\
\hline 1600 & BOILERS & & & & & & & & & & & & & & & & & \\
\hline & & & & & & & & & & & & & & & & & & \\
\hline & FFP-WTF. $5-4017 S-4018$ & 2 & $E A$ & 1756 & 20a & & 3500 & 200 & & & & & 700 & & & & & \\
\hline & 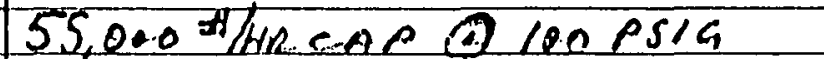 & & & & & & & & & & & & & & & & & \\
\hline & DRY If SAT., PACNAGED UNIT & & & & & & & & & & & & & & & & & \\
\hline & ar) conesere centrons s, fosceo & & & & & & & & & & & & & & & & & \\
\hline & DESEZ QL FIREA FULCY & & & & & & & & & & & & & & & & & \\
\hline & MOCULNTED, LUNTHA TSCE TYRE & & & & & & & & & & & & & & & & & \\
\hline & & & & & & & & & & & & & & & & & & \\
\hline & ClFAUEA linoeE'S AlORE & & & & & & & & & & & & & & & & & \\
\hline & $D<-14$ & & & & & & & & & & & & & & & & & \\
\hline & & & & & & & & & & & & & & & & & & \\
\hline & & & & & & & & & & & & & & & & & & \\
\hline & ACCDWNACE FOR STACE & I & $E A$ & 10,0 & 00 & & 10 & 100 & & & & & 70 & & & & & \\
\hline & & & & & & & & & & & & & & & & & & \\
\hline & & & & & & & & & & & & & & & & & & \\
\hline & & & & & & & & & & & & & & & & & & \\
\hline & & & & & & & & & & & & & & & & & & \\
\hline & & & & & & & & & & & & & & & & & & \\
\hline & & & & & & & & & & & & & & & & & & \\
\hline & & & & & & & & & & & & & & & & & & \\
\hline & & & & & & & & & & & & & & & & & & \\
\hline & & & & & & & & & & & & & & & 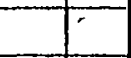 & & & \\
\hline & & & & & & & & & & & & & & & & & & \\
\hline & & & & & & & & & & & & & & & & & & \\
\hline & & & & & & & & & & & & & & & & & & \\
\hline & & & & & & & & & & & & & & & & & & \\
\hline & & & & & & & & & & & & & & & & & & \\
\hline & & & & & & & & & & & & & & & & & & \\
\hline
\end{tabular}


ESTIMATE WORKSHEET

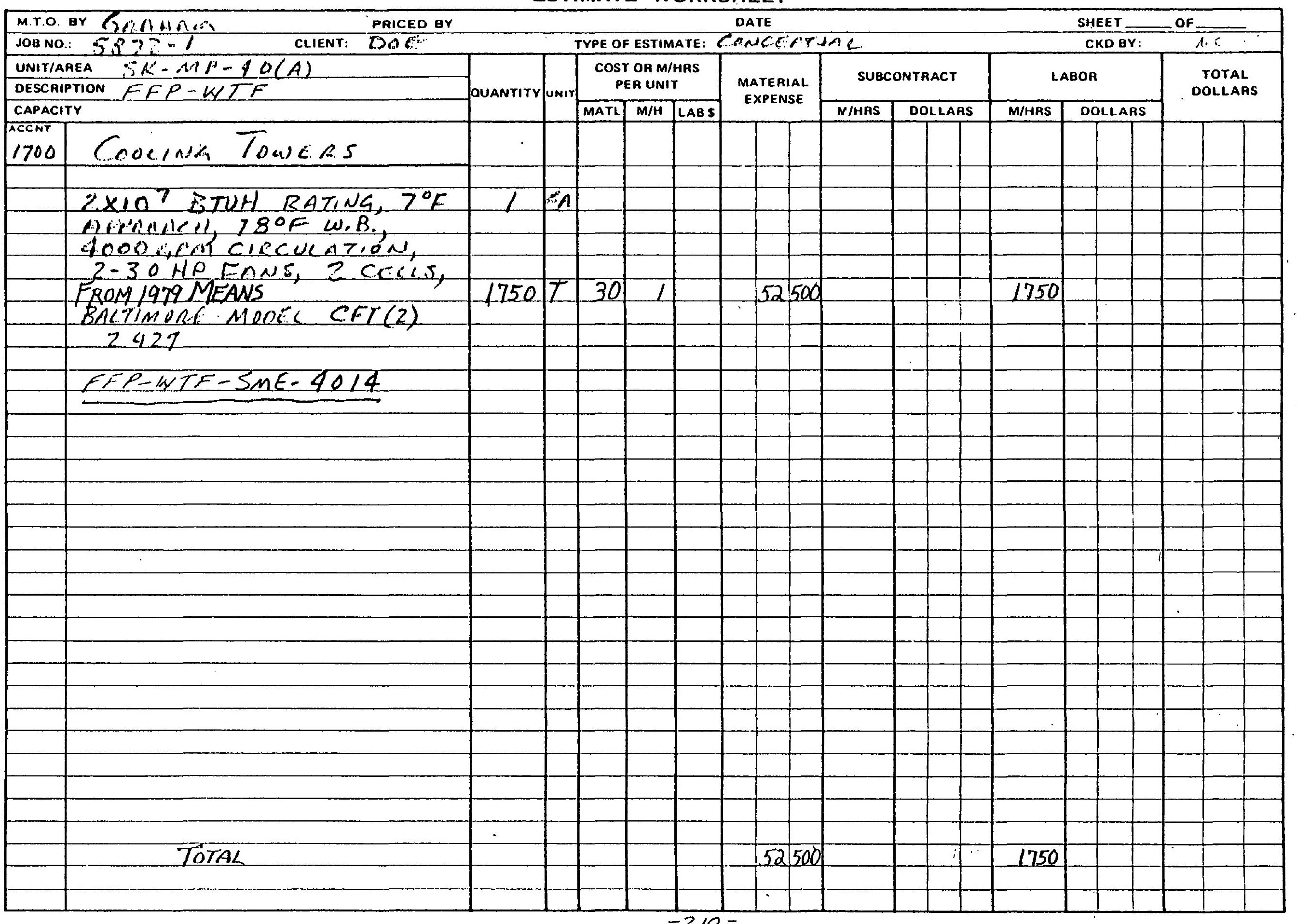




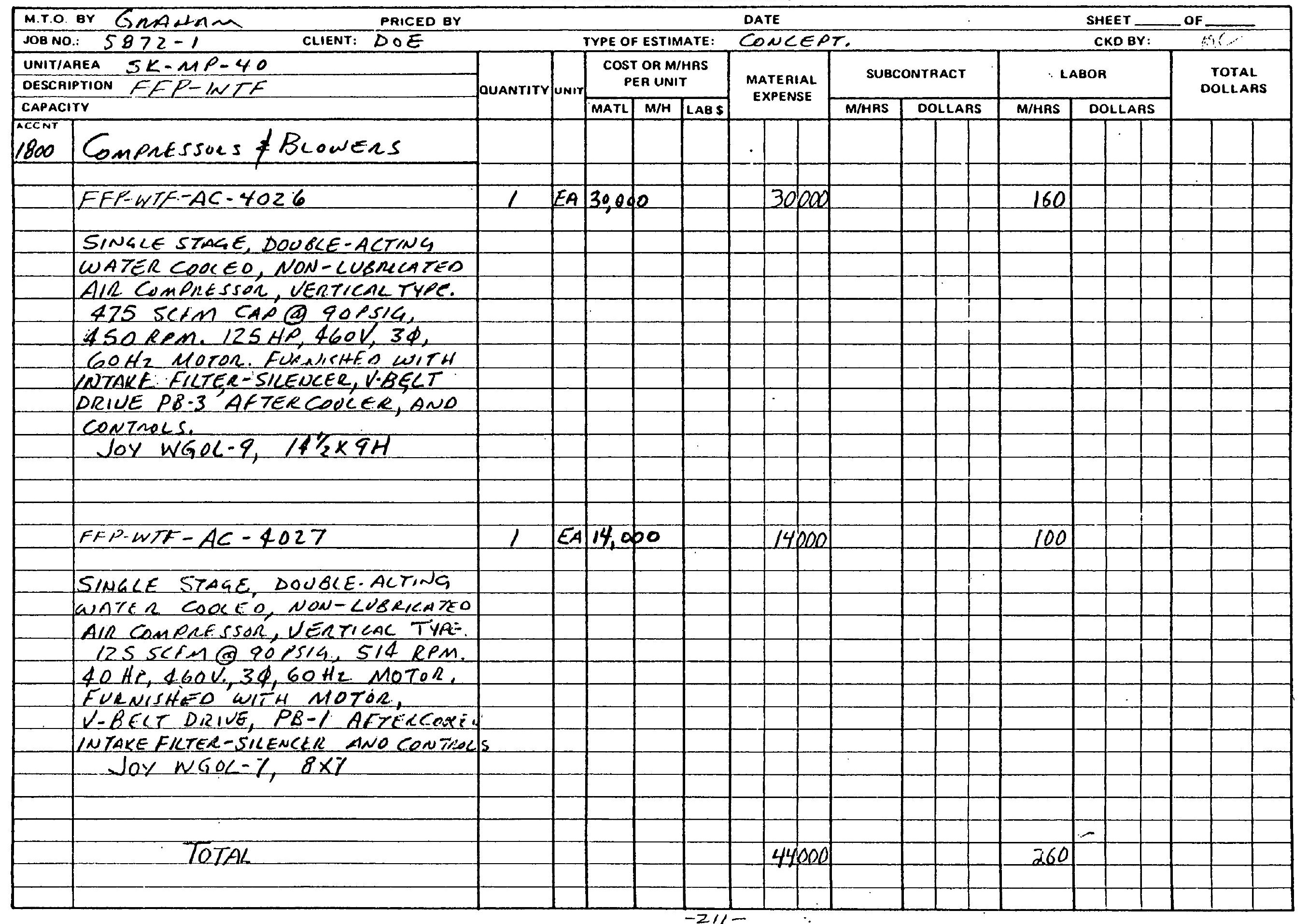


$C_{50} 20000$.

THE RALPH M. PARSONS COMPANY

ESTIMATE WORKSHEET

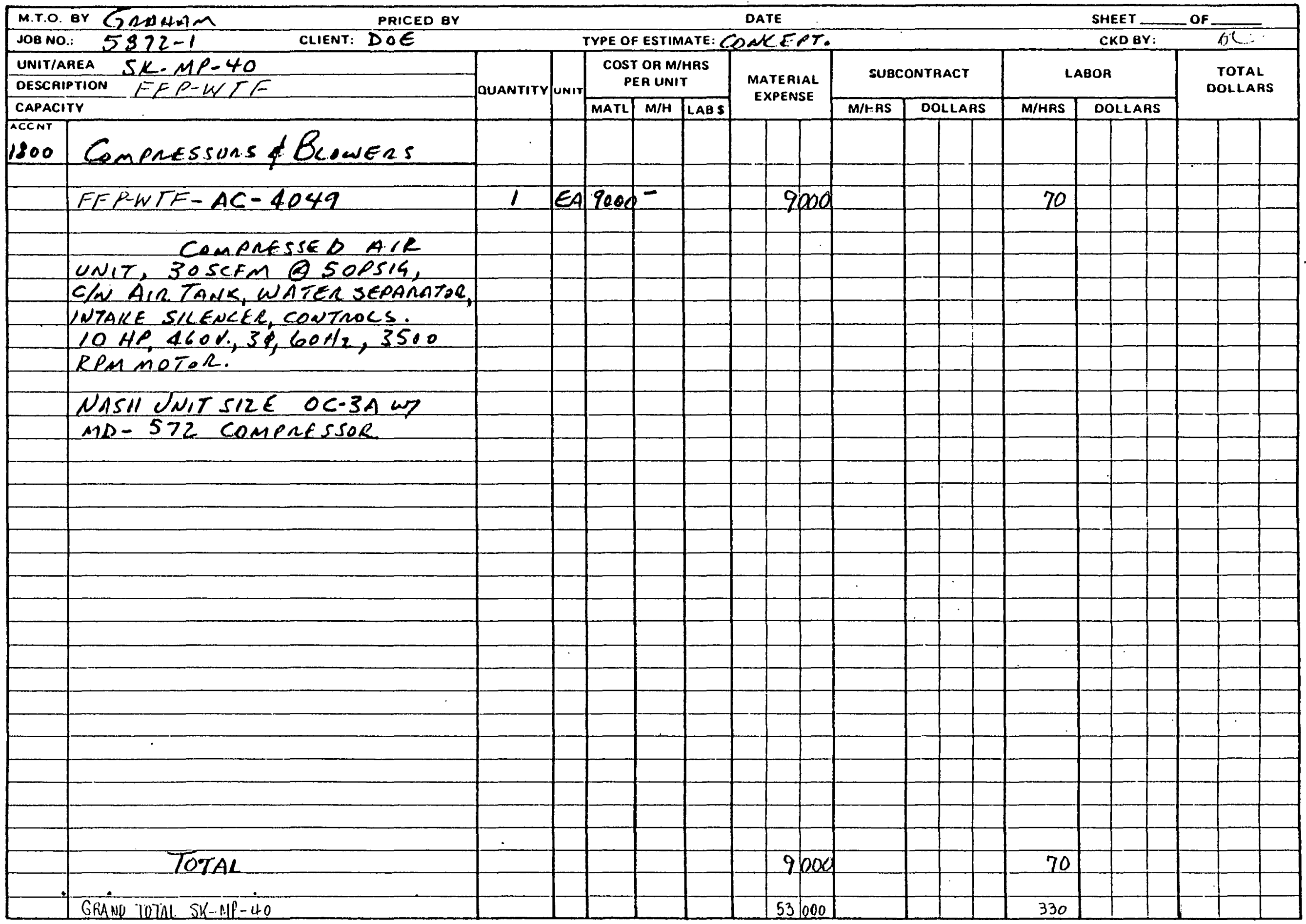


ESTIMATE WORKSHEET

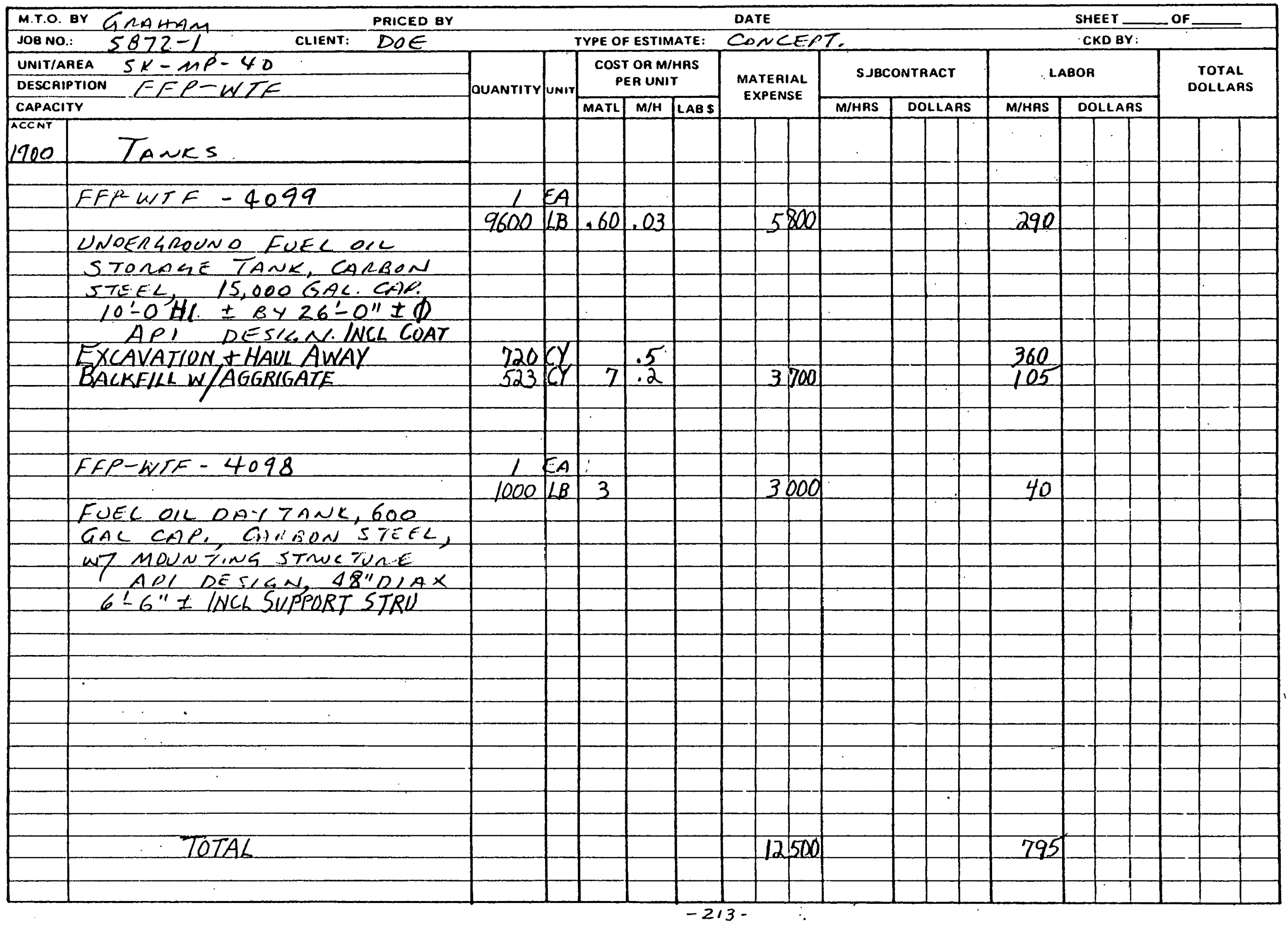


THE RALPH M. PARSONS COMPANY

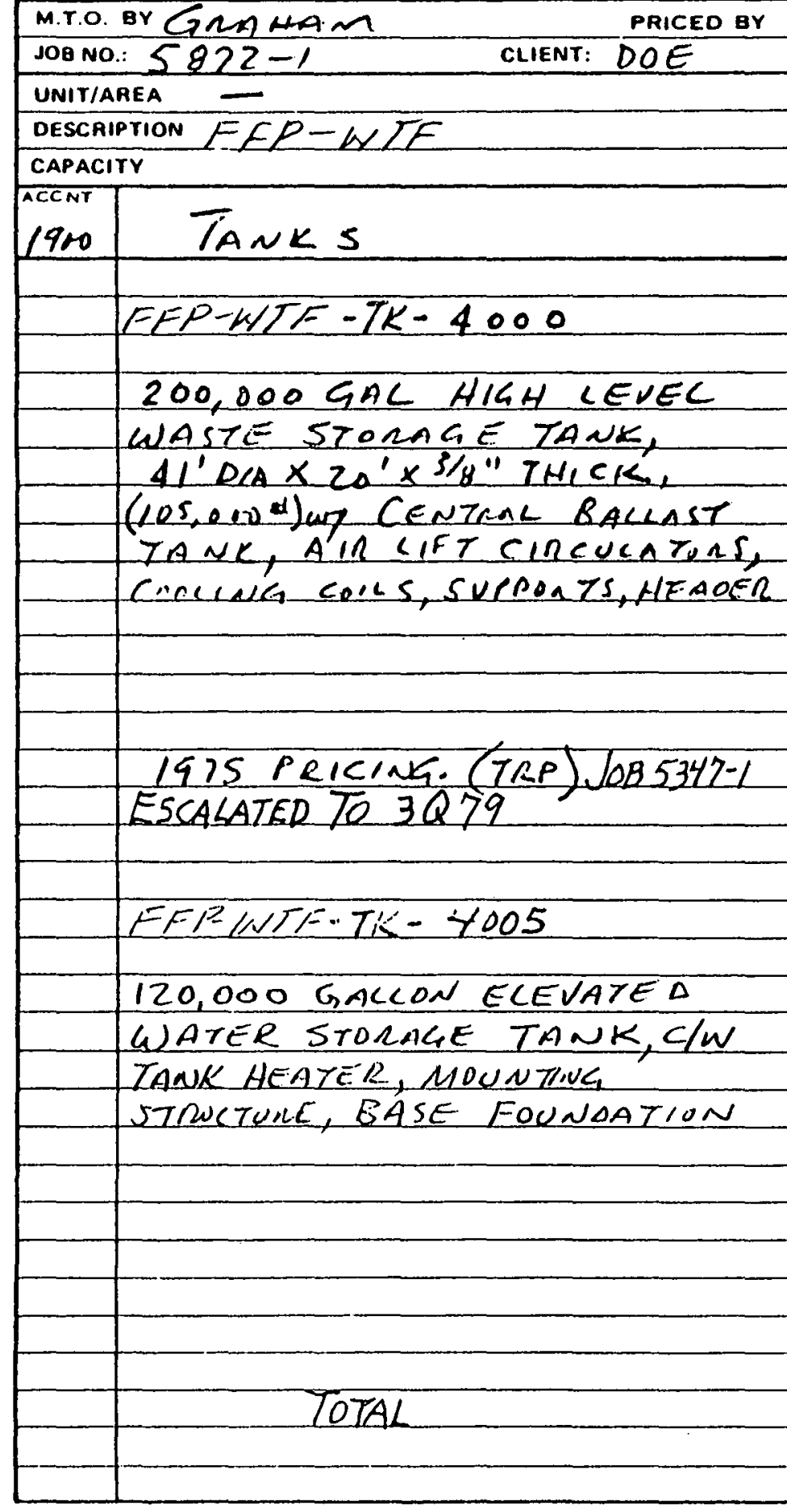

ESTIMATE WORKSHEET

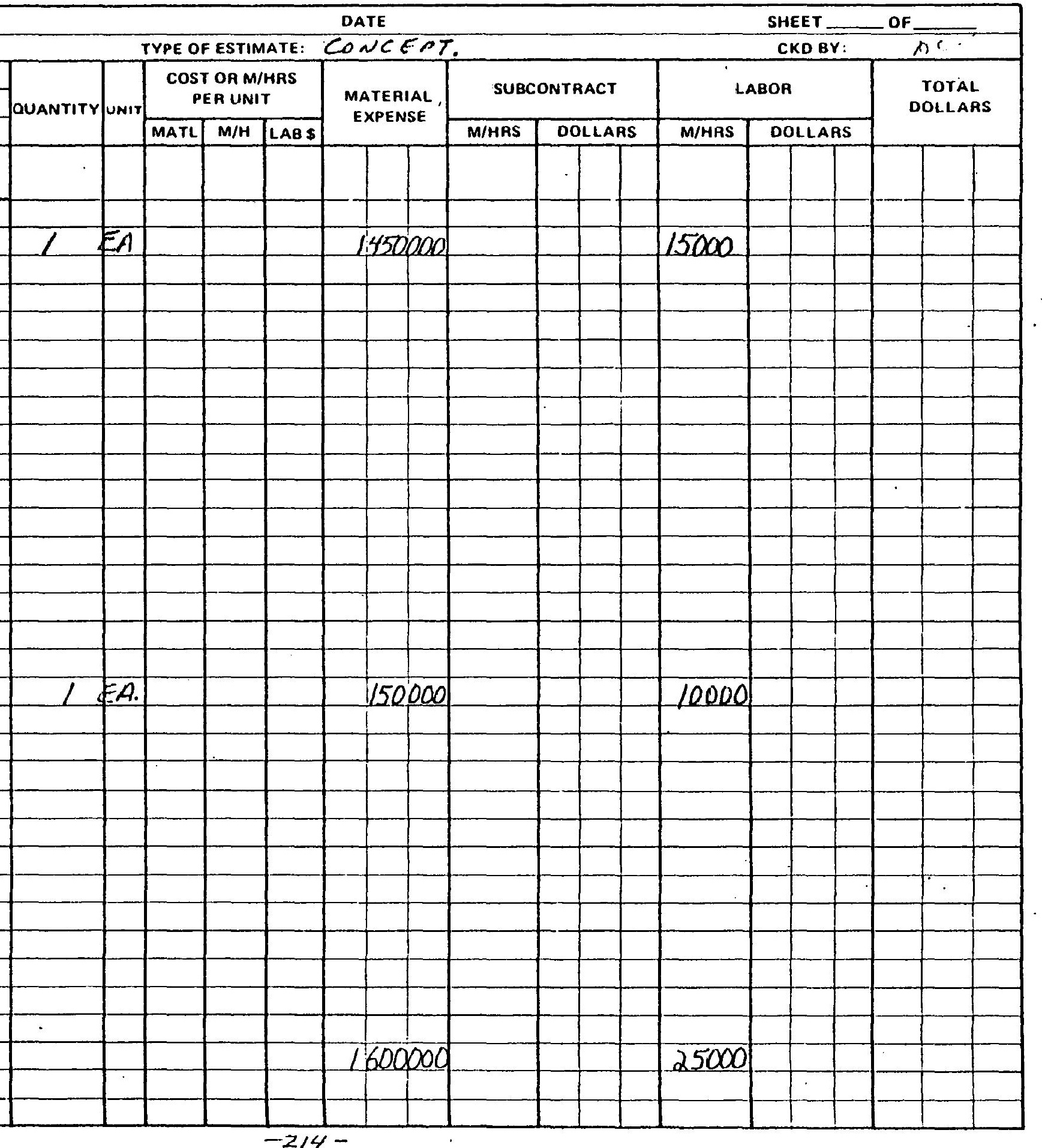




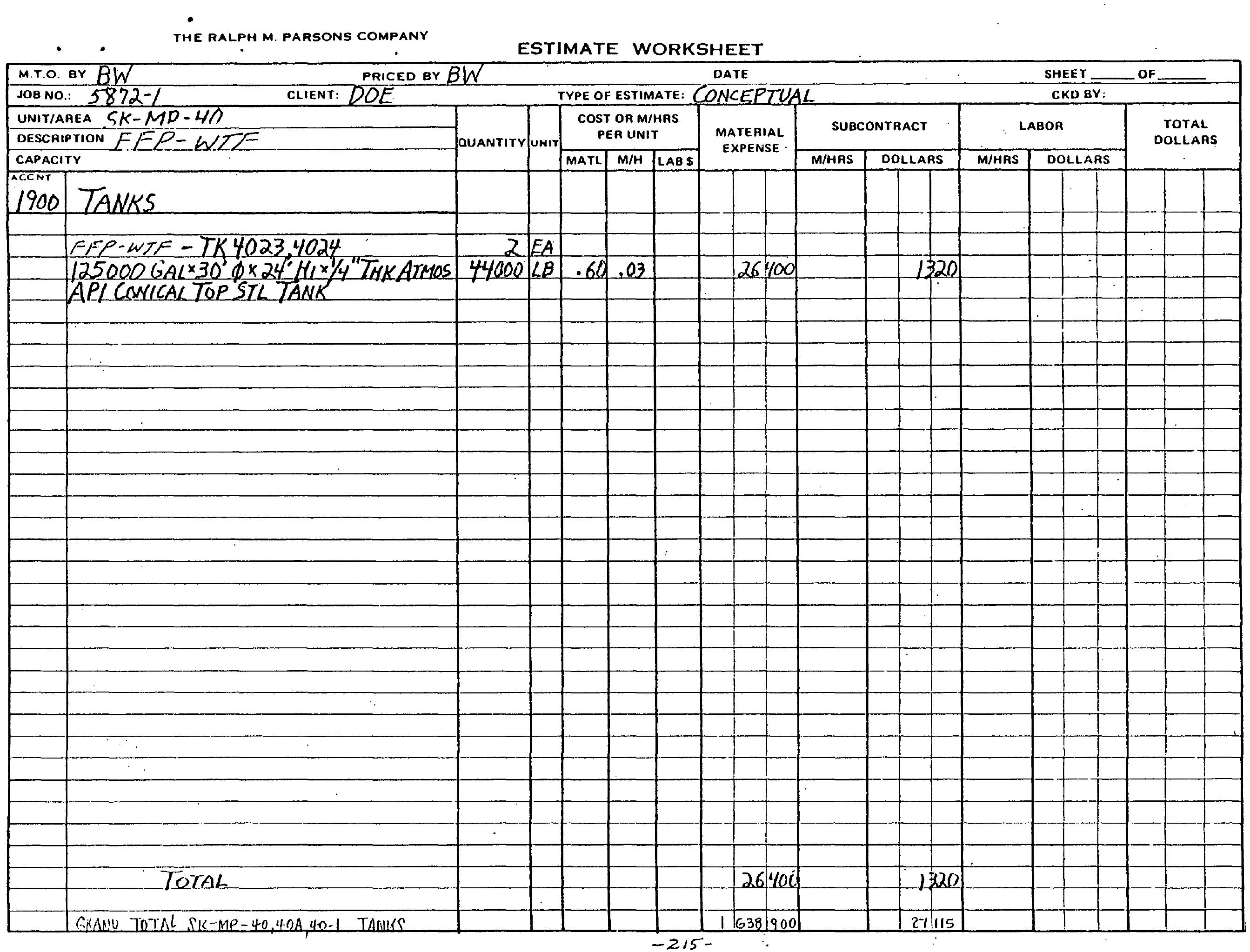


ESTIMATE WORKSHEET

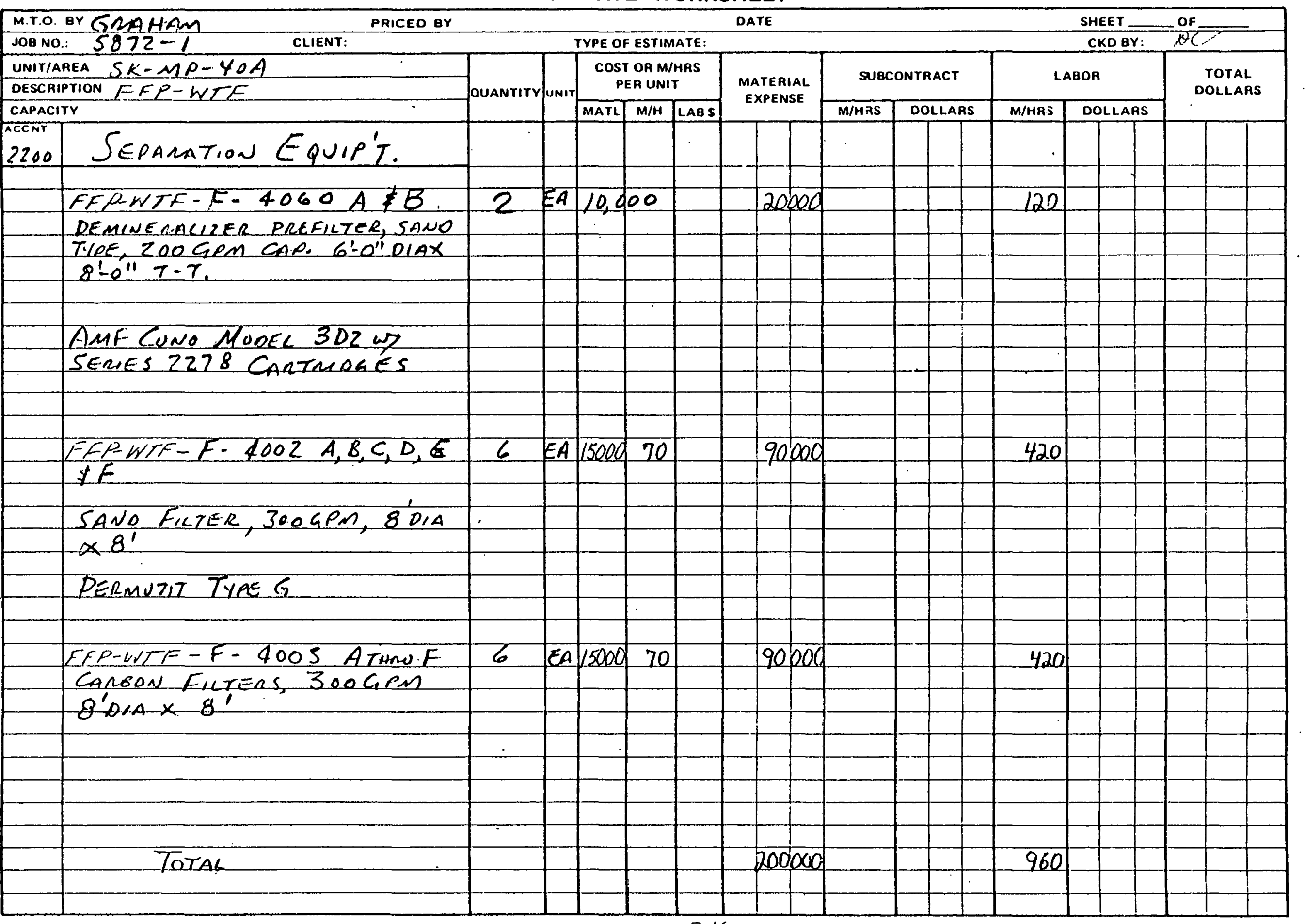


ESTIMATE WORKSHEET

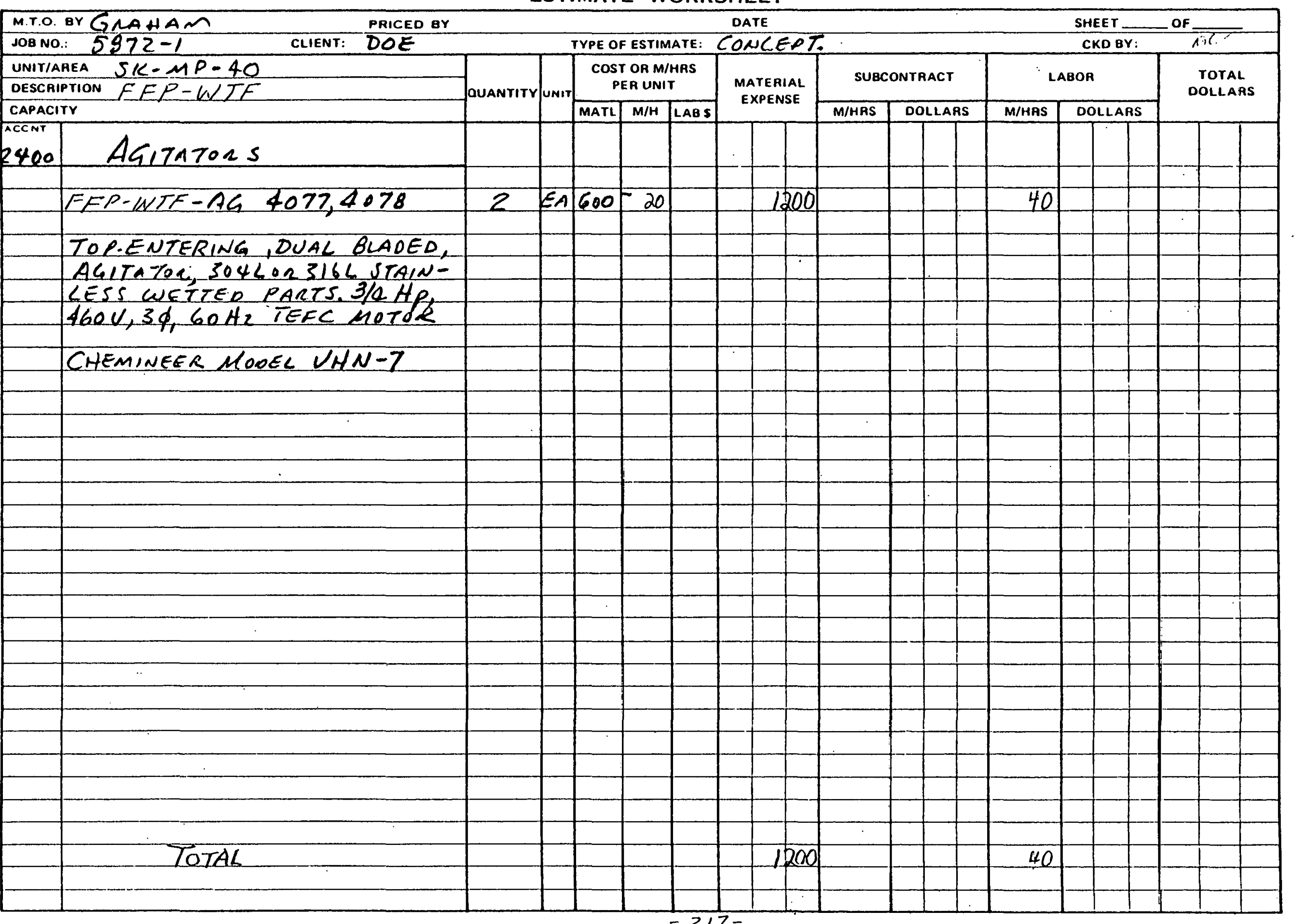


ESTIMATE WORKSHEET

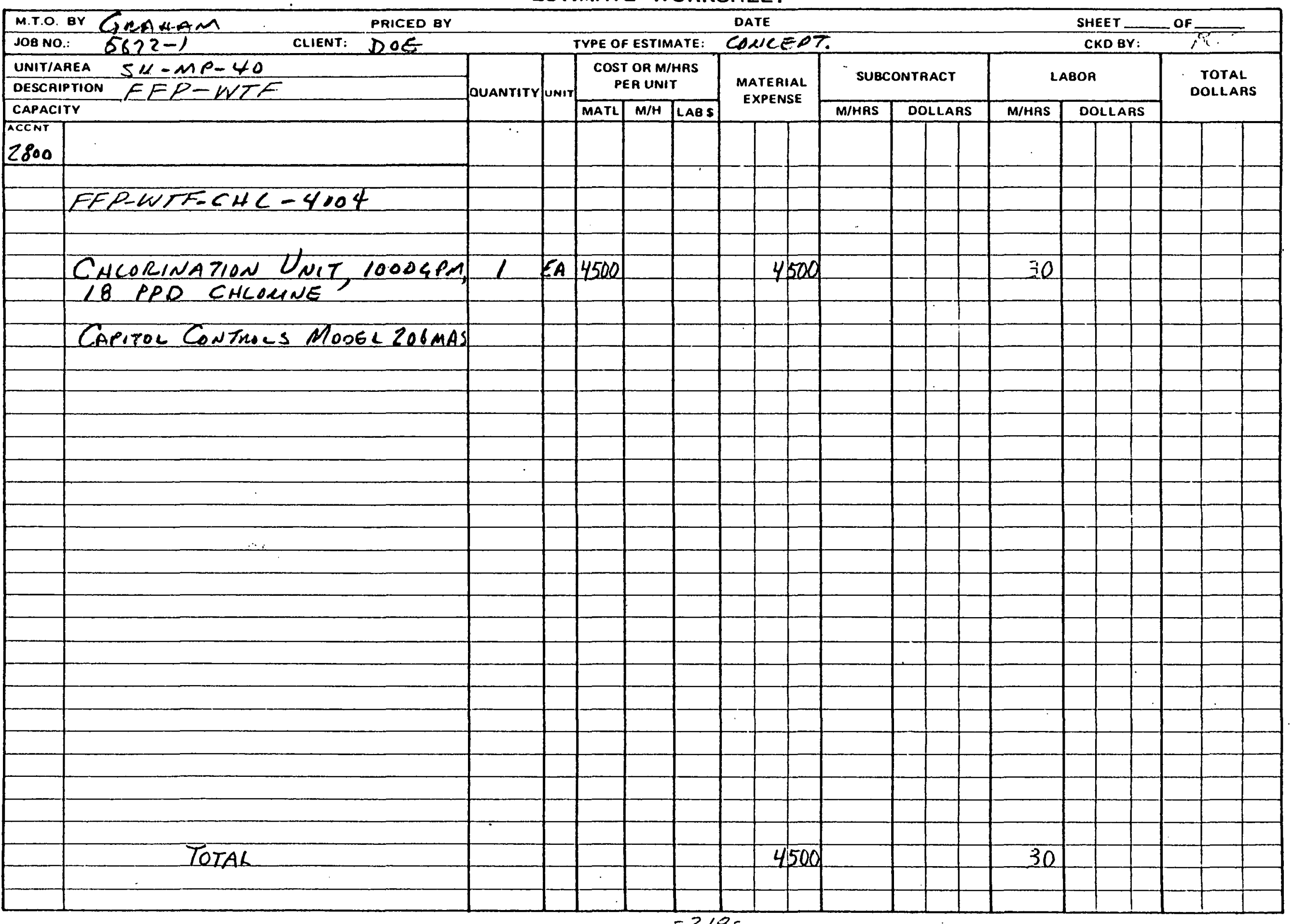


ESTIMATE WORKSHEET

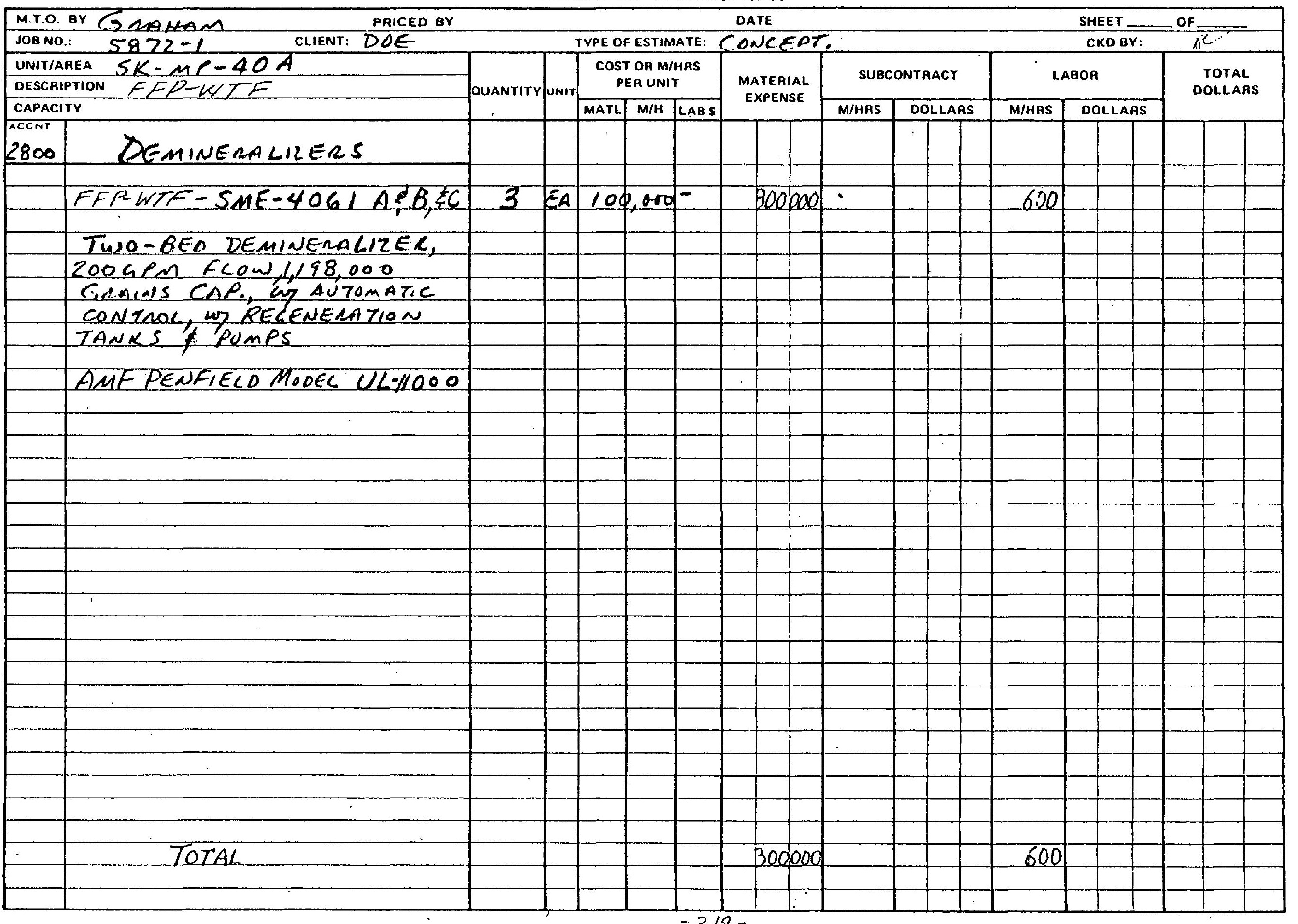


ESTIMATE WORKSHEET

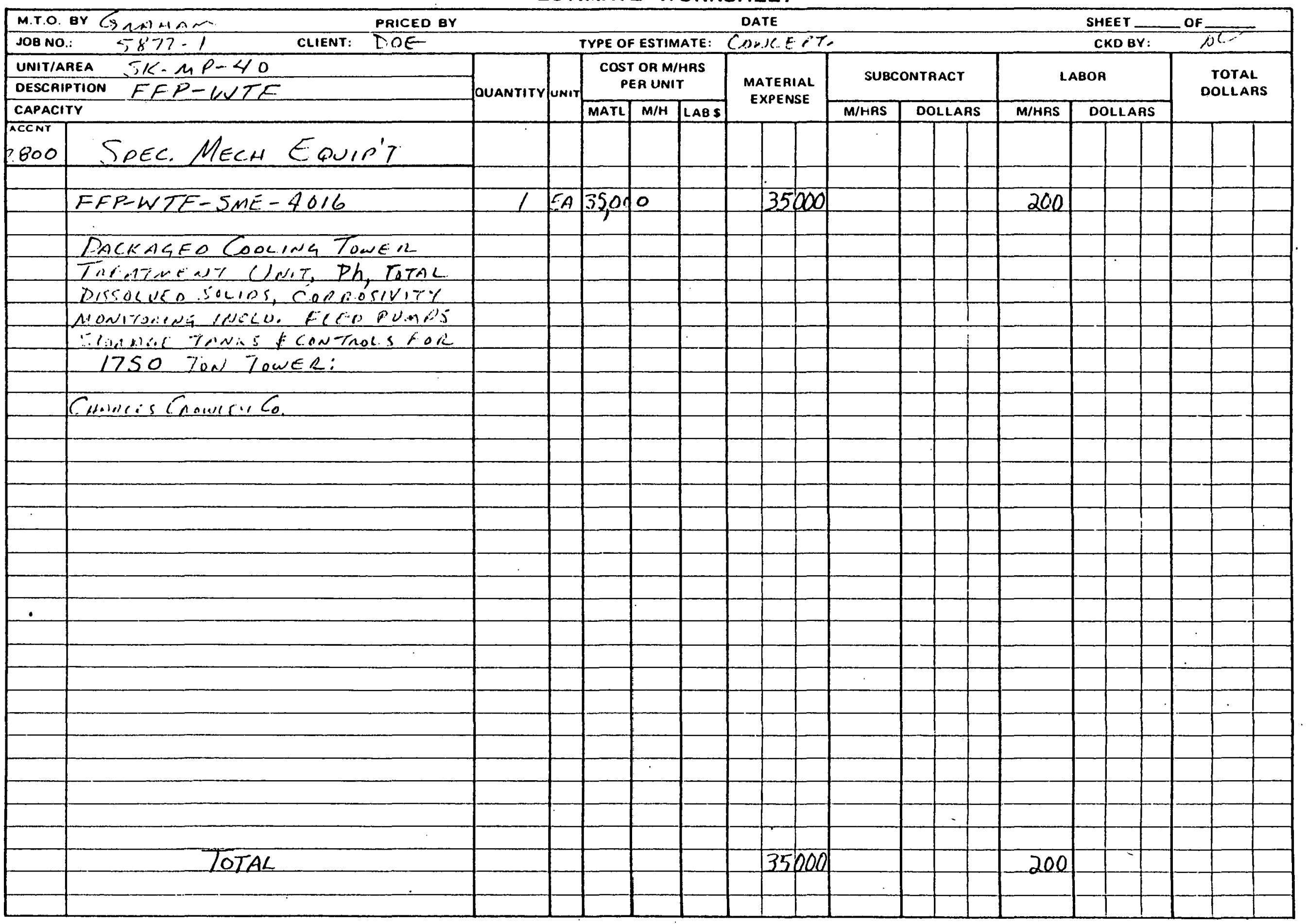


ESTIMATE WORKSHEET

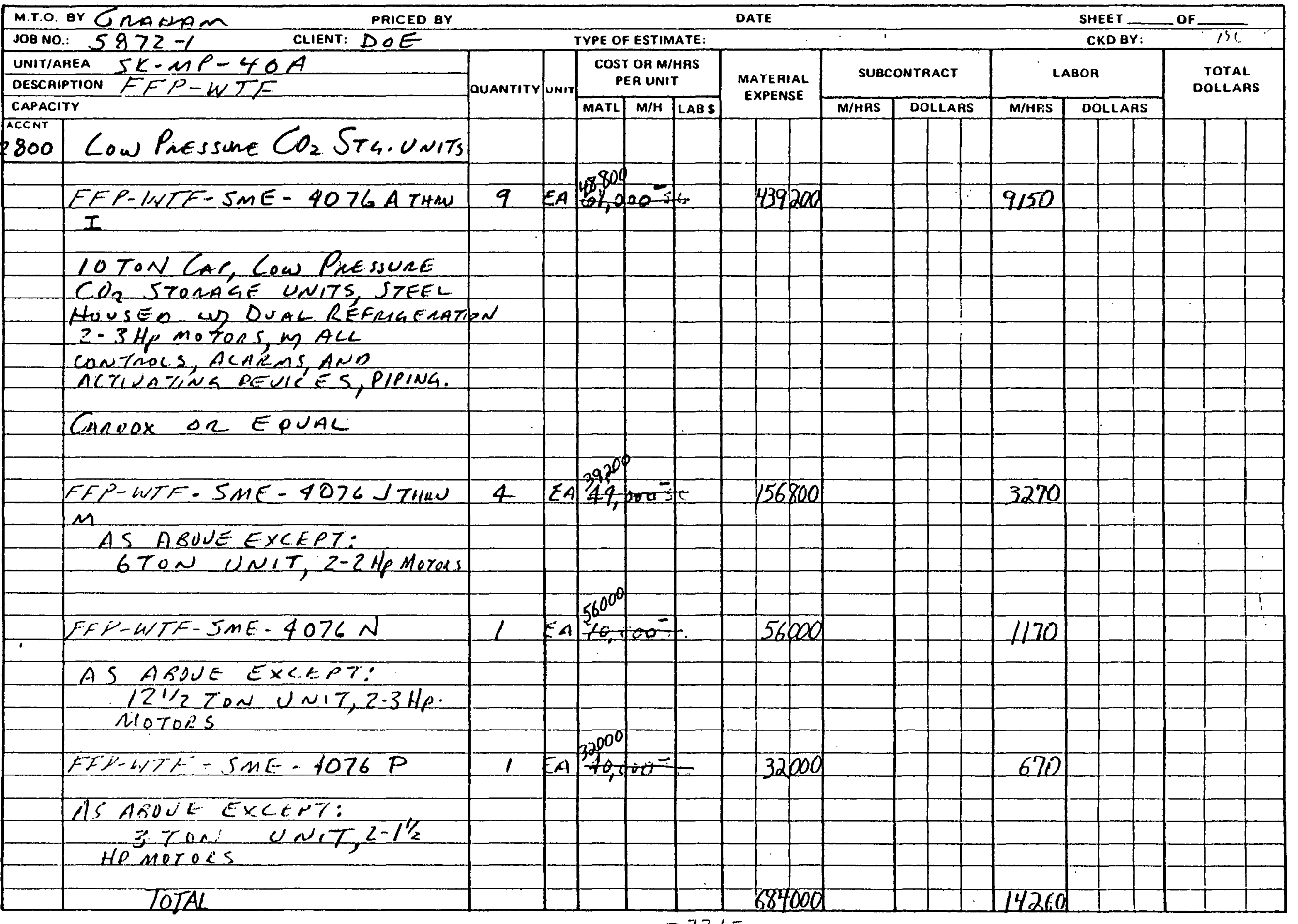


ES 20000

THE RALPH M. PARSONS COMPANY

ESTIMATE WORKSHEET

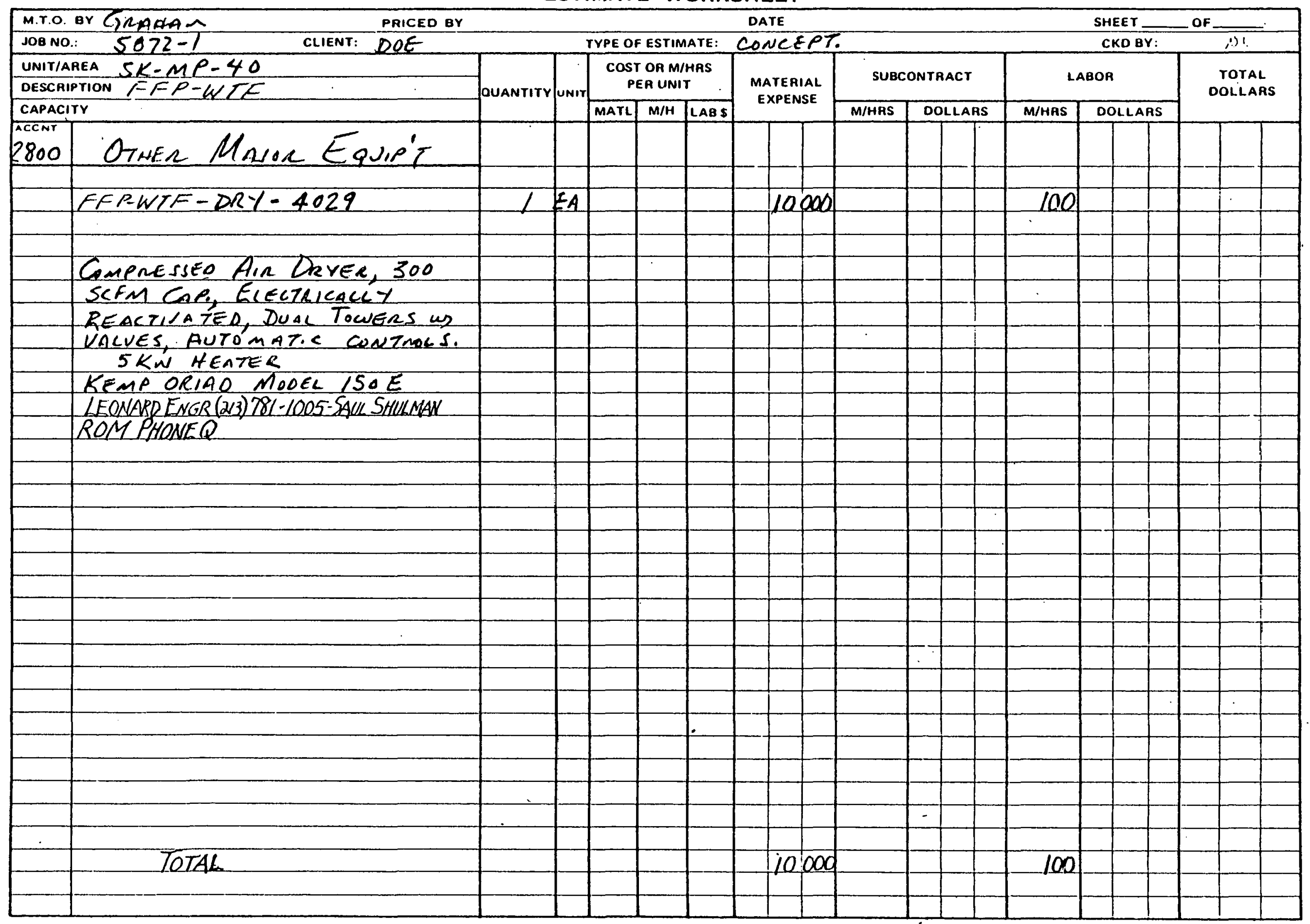


o्ర̃ 
ESTIMATE WORKSHEET

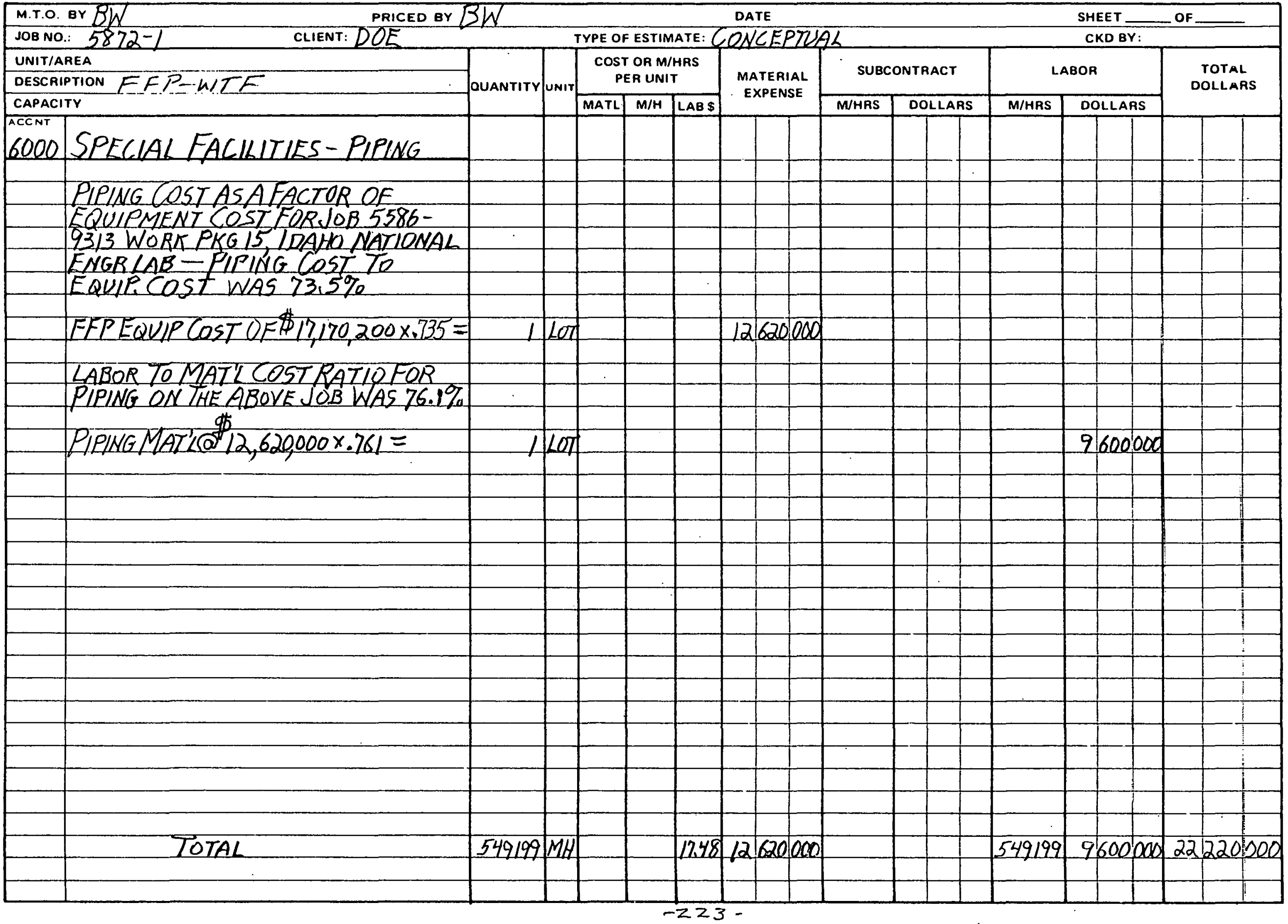


ESTIMATE WORKSHEET

\begin{tabular}{|c|c|c|c|c|c|c|c|c|c|c|c|c|c|c|c|}
\hline M.T.O. & BH $/ \mathrm{SWH}$ & & & & & & DATE & & $7 / 6$ & 179 & & SHEET & 1 & OF 2 & \\
\hline JOBNO & CLIENT: DOE & & & TYPE OF & FESTIM & TATE: & & Ros & & & & CKD B & & $A C$ & \\
\hline UNIT/A & EEP-WTE & & & $\cos$ & OR $\mathrm{M} / \mathrm{I}$ & & & & & & & & & & \\
\hline DESCAI & TION SPECIAL FACILITIES-ACCT 6.000 & GUANTITY & UNIT & & ER UNIT & & $\begin{array}{l}\text { MATER } \\
\text { EXPEN }\end{array}$ & RIAL & SUBC & NTRACT & & BOR & & DOLLA & \\
\hline CAPACI & & & & MATL & $\mathbf{M} / \mathbf{H}$ & LAB \$ & & & M/HRS & DOLLAR & M/HAS & DOLLA & & & \\
\hline $\begin{array}{l}A C C N T \\
4400\end{array}$ & INSTRUMENTS AND CONTROLS SUMMARY & & & & & & & & & & & & & & \\
\hline & & & & & & & & & & & & & & & \\
\hline 4402 & Lab 4200 Control Valves & 548 & 54 & 505 & 1.7 & & 276 & 740 & & & 932 & & & & \\
\hline 4403 & Lab 4200 Press Relief Valves & 138 & EA & 1043 & 2 & & 143 & 930 & & & 276 & & & & \\
\hline 4404 & Lab 4491 Level Instr. & 184 & $\underline{E A}$ & 2743 & $1 /$ & & 504 & 710 & & & 2024 & & & & \\
\hline 4405 & Lab 4491 Gauge Glasses & & & & & & & & & & & & & & \\
\hline 4406 & Lab 4491 Flow and D/P Instr. & 395 & $E A$ & 736 & 8.5 & & 290 & 330 & & & 3358 & & & & \\
\hline 4407 & Lab 4491 Pressure Instr. & 166 & $E A$ & 904 & 8 & & 150 & 060 & & & 1328 & & & & \\
\hline 4408 & Lab 4491 Temperature Instr. & 453 & $\leq 4$ & 207 & 3.9 & & 93 & 770 & & & 1767 & & & & \\
\hline 4409 & Lab $4491 \mathrm{~T} / \mathrm{C}$ and Wells & 164 & $\angle A$ & 997 & 3.3 & & 163 & 510 & & & $54 i$ & & & & \\
\hline 4410 & Lab 4491 Thermometers/We11s & & & & & & & & & & & & & & \\
\hline 4411 & Lab 4491 Electrical Switches & 184 & $E A$ & 123 & 2.6 & & 22 & 630 & & & 478 & & & & \\
\hline 4412 & Lab 4491 Pressure Gauges & 129 & $E A$ & 53 & 2 & & 6 & 840 & & & 258 & & & & \\
\hline 4413 & Lab 4491 Analyzers Systems & 4 & EA & 19732 & 86 & & 786 & 920 & & & 344 & & & & \\
\hline 4414 & Lab 4491 Miscel laneous & 209 & $E A$ & 3862 & 16.4 & & 807 & 160 & & & 3428 & & & & \\
\hline 4415 & Lab 4491 Orif. Plts/Meterruns & & & & & & & & & & & & & & \\
\hline 4416 & Lab 4492 Panel Mounted Instr. & 1750 & EA & 2,3 & 2 & & 372 & 750 & & & 3500 & & & & \\
\hline 4417 & Lab 4492 Control Panels & 1 & $\angle T$ & & & & 208 & 750 & & & 701 & & & & \\
\hline 4418 & Lab 4491 Computers/Data Log. & & & & & & & & & & & & & & \\
\hline 4419 & Lab 4491 Multiplex Sys. & & & & & & & & & & & & & & \\
\hline 4420 & Furnished Installed By Other & & & & & & & & & & & & & & \\
\hline 4421 & Lab 4491 Furnished Loose By Other & & & & & & & & & & & & & & \\
\hline 4422 & & & & & & & & & & & & & & & \\
\hline 4423 & & & & & & & & & & & & & & & \\
\hline 4424 & & & & & & & & & & & & & & & \\
\hline 4425 & Lab 4491 Sub. Hdr. Pipe. Mult. Spt. & 7 & . & & & & & & & & & & & & \\
\hline 4426 & Lab 4491 Instr. Housings & \} & $L T$ & & & & $16 Z$ & 500 & & & $160 ; 0$ & & & & \\
\hline 4427 & Lab 4491 Instr. Insta1. Mat'1. & $S$ & & & & & & & & & & & & & \\
\hline & & & & & & & & & & & & & & & \\
\hline & & & & & & & & & & & & & & & \\
\hline & $C / F$ & 4324 & & & & & 3282 & 600 & & & 34949 & & & & \\
\hline & & & & & & & & & & & & & & & \\
\hline & & & & & & & & & & & & & & & \\
\hline & & & & & & & & & & & & & & & \\
\hline
\end{tabular}




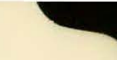

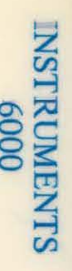


THE RALPH M. PARSONS COMPANY

ESTIMATE WORKSHEET

\begin{tabular}{|c|c|c|c|c|c|c|c|c|c|c|c|c|c|c|c|c|}
\hline M.T.O. BY & BH $/$ SWH & & & & & & \multicolumn{2}{|l|}{ DATE } & & 779 & \multicolumn{6}{|c|}{ SHEET 2 OF 2} \\
\hline JOB NO.: & CLIENT: DOE & & & \multicolumn{3}{|c|}{ TYPE OF ESTIMATE: } & & KOM & & & \multicolumn{3}{|c|}{ CKD BY: } & \multicolumn{3}{|c|}{$x$} \\
\hline \multirow{3}{*}{\multicolumn{2}{|c|}{$\begin{array}{l}\text { UNITIAREA FFP- WT F } \\
\text { DESCRIPTION SPECIAL TACILITIES-ACC'T } 6000 \\
\text { CAPACITY }\end{array}$}} & \multirow{3}{*}{ DUANTITY } & \multirow{3}{*}{ UNIT } & \multirow{2}{*}{\multicolumn{3}{|c|}{$\begin{array}{l}\text { COST OR M/HRS } \\
\text { PER UNIT }\end{array}$}} & \multirow{3}{*}{\multicolumn{2}{|c|}{$\begin{array}{c}\text { MATERIAL } \\
\text { EXPENSE }\end{array}$}} & \multirow{2}{*}{\multicolumn{2}{|c|}{ SUBCONTRACT }} & \multirow{2}{*}{\multicolumn{3}{|c|}{ LABOR }} & \multirow{3}{*}{\multicolumn{3}{|c|}{$\begin{array}{c}\text { TOTAL } \\
\text { DOLLARS }\end{array}$}} \\
\hline & & & & & & & & & & & & & & & & \\
\hline & & & & MATL & $M / H$ & LAB \$ & & & M/HRS & DOLLARS & M/HRS & DOLLA & ARS & & & \\
\hline $\begin{array}{r}\text { ACCNT } \\
4400 \\
\end{array}$ & INSTRUMENTS AND CONTROLS SUMMARY & & & & & & & & & & & & & & & \\
\hline & $3 / 1=$ & 4324 & $E A$ & & & & 3282 & $6 \times 0$ & & & 34945 & & & & & \\
\hline 4481 & Ereight & 1 & UT & & & & 82 & 100 & & & & & & & & \\
\hline 4499 & Calibration & 1 & LOT & & & & & & & & 6930 & & & & & \\
\hline & & & & & & & & & & & & & & & & \\
\hline & & & & & & & & & & & & & & & & \\
\hline & Subtotal & 4324 & $E A$ & & & & 3364 & 700 & & & 41875 & & & & & \\
\hline & Productivity $41875 \mathrm{MH} \div .65$ & 64423 & $\mathrm{MH}$ & & & 16.81 & & & & & 64423 & 082 & 950 & & & \\
\hline & & & & & & & & & & & & & & & & \\
\hline & & & & & & & & & & & & & & & & \\
\hline & Total & 4324 & $E A$ & & & & 3365 & 200 & & & 64423 & 083 & 000 & 4 & 4481 & 000 \\
\hline & & & & & & & & & & & & & & & & \\
\hline & & & & & & & & & & & & & & & & \\
\hline & & & & & & & & & & & & & & & & \\
\hline & - & & & & & & & & & & & & & & & \\
\hline & & & & & & & & & & & & & & & & \\
\hline & & & & & & & & & & & & & & & & \\
\hline & & & & & & & & & & & & & & & & \\
\hline & & & & & & & & & & & & & & & & \\
\hline & & & & & & & & & & & & & & & & \\
\hline & & & & & & & & & & & & & & & & \\
\hline & & & & & & & & & & & & & & & & \\
\hline & & & & & & & & & & & & & & & & \\
\hline & & & & & & & & & & & & & & & & \\
\hline & & & & & & & & & & & & & & & & \\
\hline & & & & & & & & & & & & & & & & \\
\hline & & & & & & & & & & & & & & & & \\
\hline & & & & & & & & & & & & & & & & \\
\hline & & & & & & & & & & & & & & & & \\
\hline & & & & & & & & & & & & & & & & \\
\hline & & & & & & & & & & & & & & & & \\
\hline & & & & & & & & & & & & & & & & \\
\hline & & & & & & & & & & & . & & & & & \\
\hline & . & & & & & & & & & & & & & & & \\
\hline
\end{tabular}


ESTIMATE WORKSHEET

\begin{tabular}{|c|c|c|c|c|c|c|c|c|c|c|c|c|c|c|}
\hline M.T.O. E & PRICED BY & $3 \mathrm{~W}$ & & & & & DATE & & & & & IEET . & OF & \\
\hline JOB NO.: & $5872-1 \quad$ CLIENT: DOE & & & TYPE OF & ESTIM & MATE: & DNCEPTUE & & & & & KD BY: & & \\
\hline UNIT/AR & REA & & & $\cos T$ & OR M/ & /HRS & & SUBC & DNTRACT & & $B C$ & & TOTAL & \\
\hline DESCRIP & TION FFP-WTF & OUANTITY & Unit & & R UNI & & $\begin{array}{l}\text { MATERIAL } \\
\text { EXPENSE }\end{array}$ & 3000 & TIVACI & & & & DOLLAF & \\
\hline CAPACIT & & & & MATL & $M / H$ & LAB \$ & & $\mathrm{M} / \mathrm{HRS}$ & DOLLARS & M/HRS & & OLLARS & & \\
\hline $\begin{array}{l}\mathrm{ACCNT} \\
6000\end{array}$ & SPECIAL FACILITIES-COMPUTER & & & & & & & & & & & & & \\
\hline & & & & & & & & & & & & & & \\
\hline 0600 & COMPUTER & & & & & & 2057000 & & & & & & 2057 & 1000 \\
\hline & & & & & & & & & & & & & & \\
\hline & $2 / 3$ COST OF 2Q77 JOB 5687-1 HTGR & & & & & & & & & & & & & \\
\hline & FUEL RECYCLE COMMERCIALIZATION & & & & & & & & & & & & & \\
\hline & ESTIMATE ESCALATED TO $3 Q 79)$ & & & & & & & & & & & & & \\
\hline & & & & & & & & & & & & & & \\
\hline & PRODUCTIVITY INCLUDED & & & & & & & & & & & & & \\
\hline & & & & & & & & & & & & & & \\
\hline & & & & & & & & & & & & & & \\
\hline & & & & & & & & & & & & & & \\
\hline & & & & & & & & & & & & & & \\
\hline & & & & & & & & & & & & & & \\
\hline & & & & & & & & & & & & & & \\
\hline & & & & & & & & & & & & & & \\
\hline & & & & & & & & & & & & & & \\
\hline & & & & & & & & & & & & & & \\
\hline & & & & & & & & & & & & & & \\
\hline & & & & & & & & & & & & & & \\
\hline & & & & & & & & & & & & & & \\
\hline & & & & & & & & & & & & & & \\
\hline & & & & & & & & & & & & & & \\
\hline & & & & & & & & & & & & & & \\
\hline & & & & & & & & & & & & & & \\
\hline & & & & & & & & & & & & & & \\
\hline & & & & & & & & & & & & & & \\
\hline & & & & & & & & & & & & & & \\
\hline & TOTAK & & & & & & 2366000 & & & $3 / 608$ & & 507000 & 2873 & 000 \\
\hline
\end{tabular}



ESTIMATE WORKSIUEET






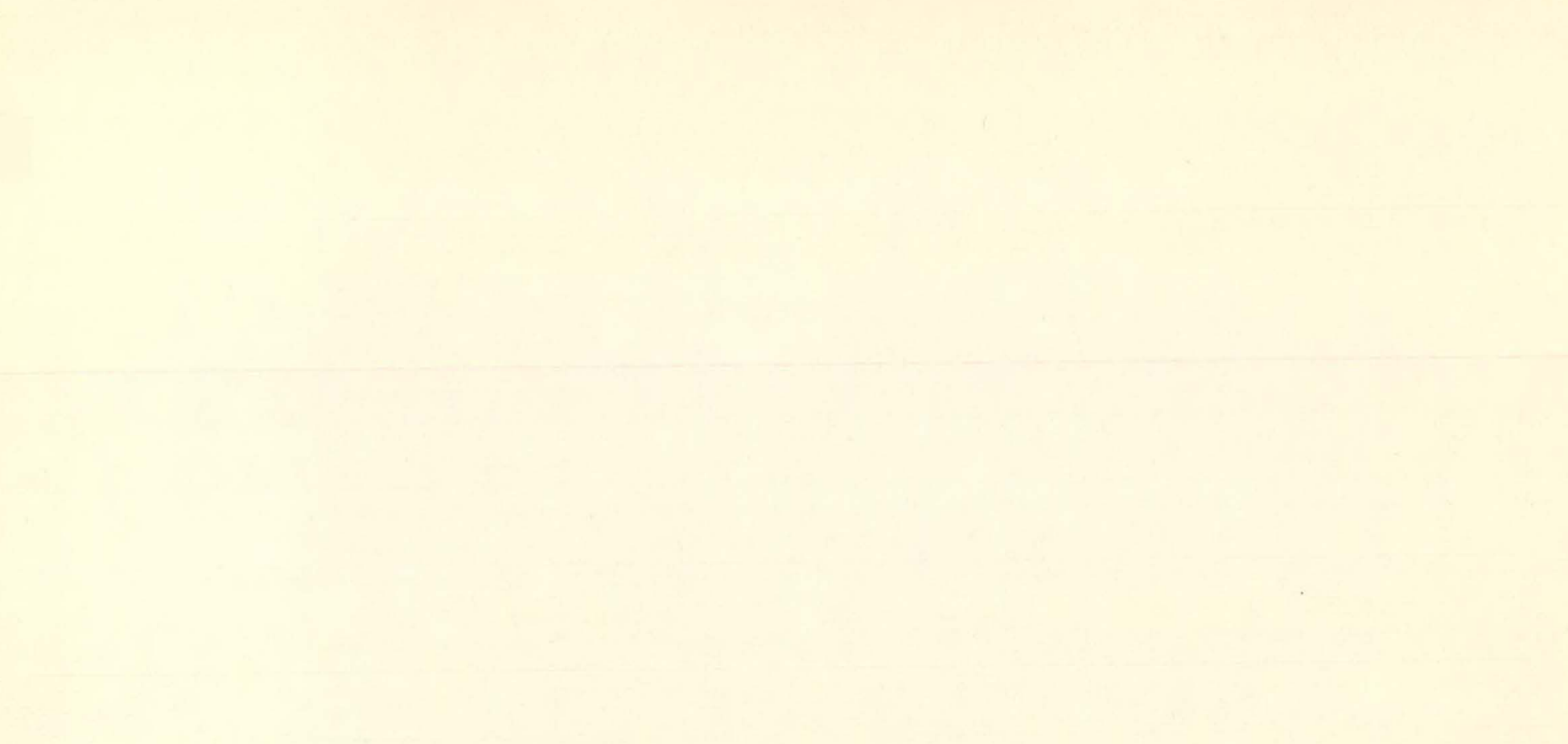

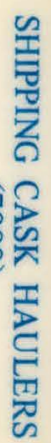




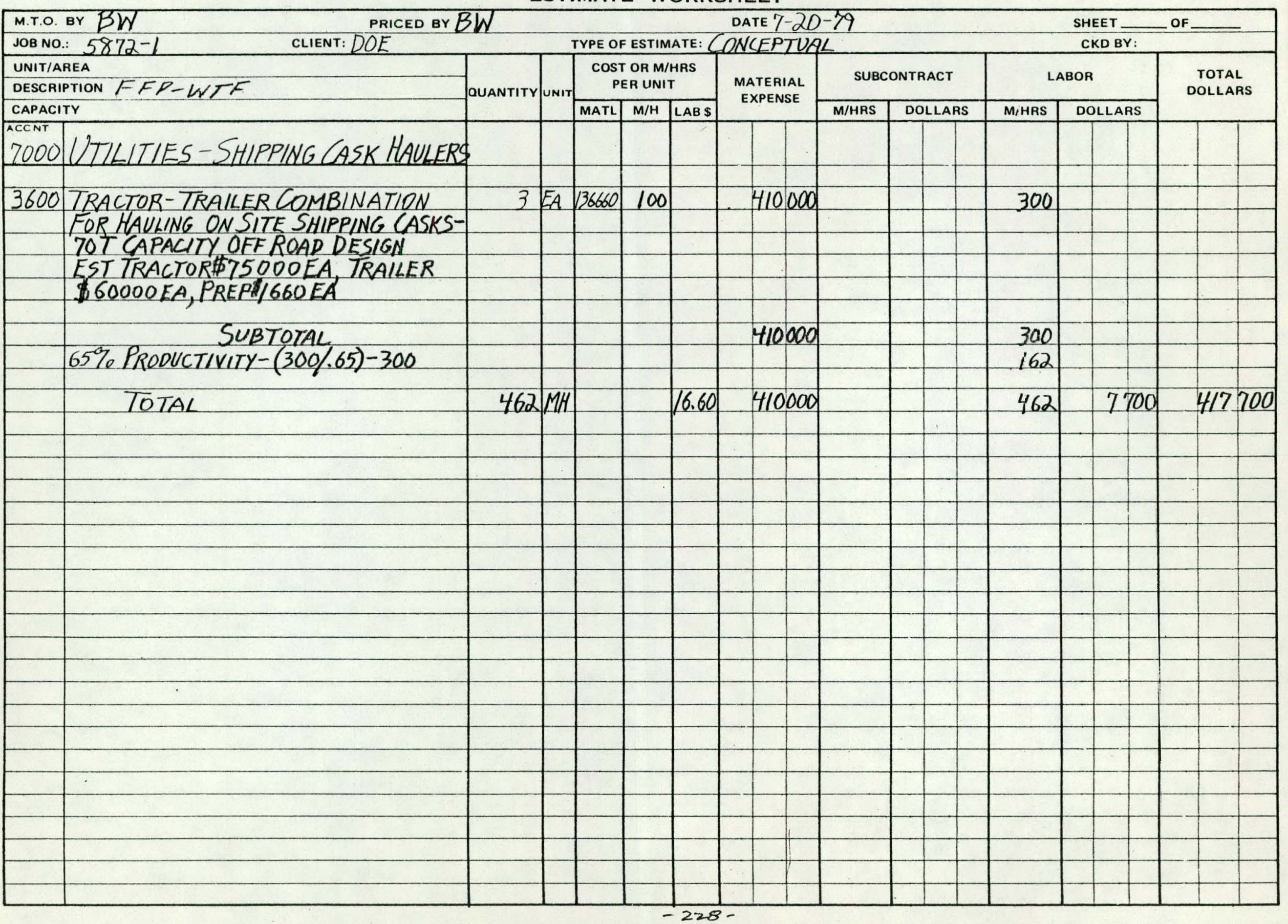


(1) 
LEd 2000-0 ,

THE RALPH M. PARSONS COMPANY

ESTIMATE WORKSHEET

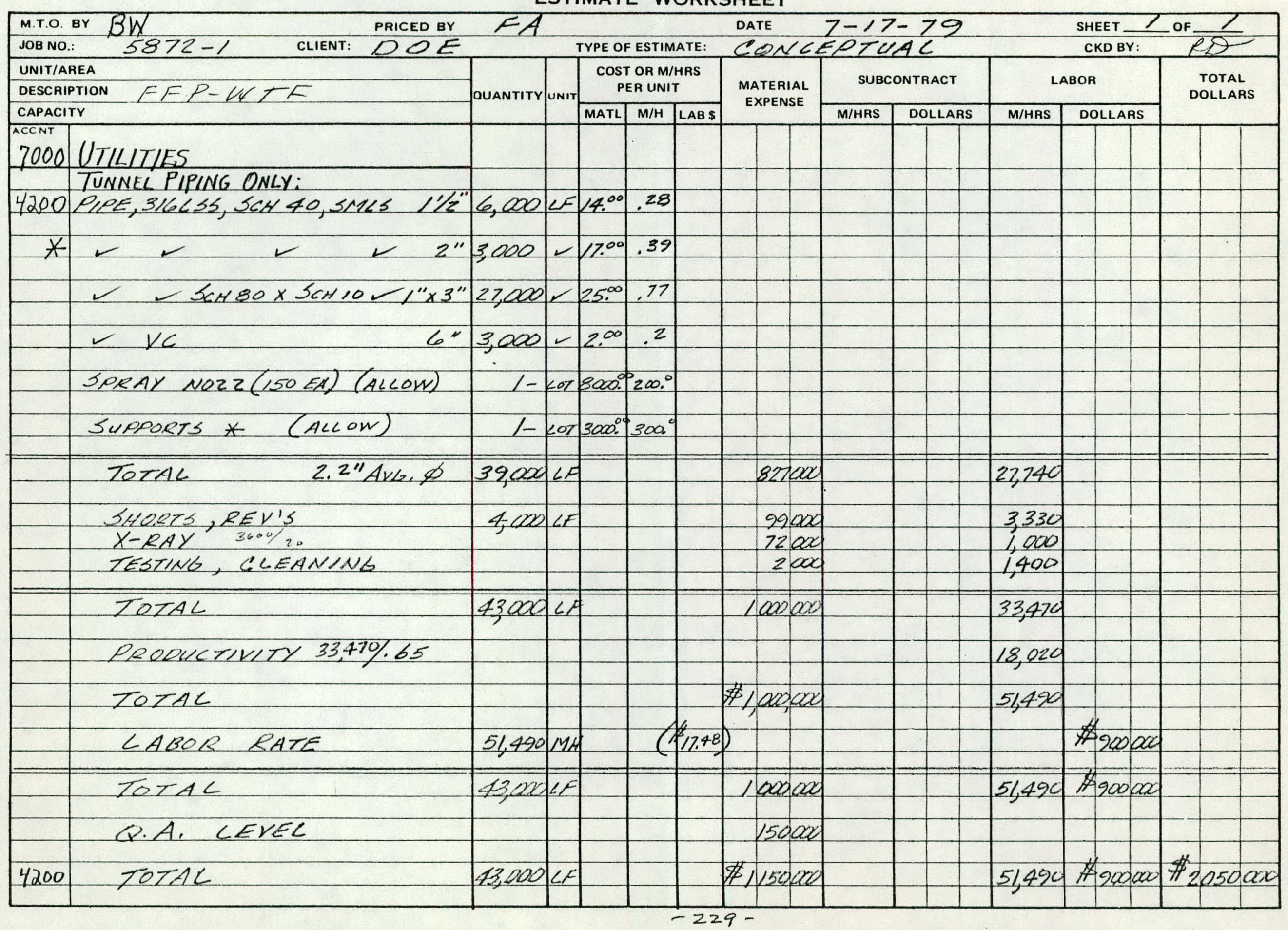


2.

顽: 
ESTIMATE WORKSHEET

\begin{tabular}{|c|c|c|c|c|c|c|c|c|c|c|c|c|c|}
\hline \multicolumn{4}{|c|}{ M.т.о. ву $B 5 M$} & \multicolumn{7}{|c|}{ DATE $7-6-79$} & \multicolumn{3}{|c|}{ SHEET $\angle$ OF $\angle O$} \\
\hline \multicolumn{3}{|c|}{ JOB NO.: $: 822-1$} & & \multicolumn{7}{|c|}{ TYPE Of estimate: Pone. } & \multicolumn{2}{|r|}{ CKD BY: } & \multirow{4}{*}{ 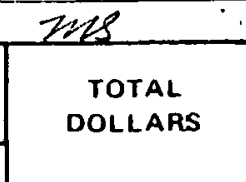 } \\
\hline UNIT/A & REA $\quad P-T F P-V V T F$ & \multirow{3}{*}{ QUANTITY } & \multirow{3}{*}{ UNIT } & \multirow{2}{*}{\multicolumn{3}{|c|}{$\begin{array}{c}\text { COST OR M/HAS } \\
\text { PER UNIT }\end{array}$}} & \multirow{3}{*}{$\begin{array}{l}\text { MATERIAL } \\
\text { EXPENSE }\end{array}$} & \multirow{2}{*}{\multicolumn{3}{|c|}{ SUBCONTRACT }} & \multirow{2}{*}{\multicolumn{2}{|c|}{ LABOR }} & \\
\hline DESCRI & $U_{T, L I T Y}$ & & & & & & & & & & & & \\
\hline \multicolumn{2}{|c|}{ CAPACITY } & & & MATL & $\mathrm{M} / \mathrm{H}$ & LAB \$ & & \multirow[t]{2}{*}{ M/HRS } & \multicolumn{2}{|c|}{ DOLLARS } & \multirow[t]{2}{*}{ M/HRS } & DOLLARS & \\
\hline $\begin{array}{r}A C C N T \\
4600\end{array}$ & ELECTRICAL-SUMMARY & & & · & & & $\begin{array}{l}1 \\
\vdots \\
1\end{array}$ & & $i$ & & & 1 & i \\
\hline & 4601 POWER GEAR & & $\mathrm{HP}$ & & & & $i$ & & $!$ & 1 & & $\therefore$ & \\
\hline & 4602 POWER DISTRIBUTION AG $S H \cdot 2$ of 10 & & HP & & & & 1027800 & & & 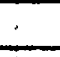 & 14775 & & \\
\hline & 4603 LIGHTING & & SF & & & & $!$ & & ; & 1 & & & 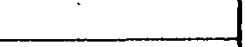 \\
\hline & 4604 INSTRUMENTATION & & EA & & & & & & $i$ & & & $\vdots$ & \\
\hline & 4605 COMMUNICATIONS & & LS & & & & & & 1 & $!$ & & $\mathrm{i}$ & $i$ \\
\hline & 4606 BELOW GRADE & & LS & & & & & & $i$ & 1. & & . & $\therefore$ \\
\hline & 4607 GROUNDING & & ME. & & & & $\because$ & & $\therefore$ & 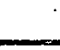 & & 1 & \\
\hline & 4608 HEAT TRACING & & FT & & & & $\therefore$ & & 1 & $\vdots$ & & & \\
\hline & 4609 FIRE ALARM & & LS & & & & $:$ & & 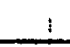 & . & & $\therefore$ & : \\
\hline & 4610 CATHODIC PPOTECTION & & LS & & & & $\therefore$ & & $\vdots$ & + & & & \\
\hline & 4611 POLE LINE & & FT & & & & $!$ & & 1 & $i$ & & . & \\
\hline & 4620 MiseEtanteoUs QA LEVEL IL & 267930 & $E \delta$ & .08 & & & 21400 & & 1 & 1 & &. & \\
\hline & & & & & & & i & & . & $\vdots$ & & $\dot{1}$ & \\
\hline & TOTAL - DIRECT & . & & & & & 1049200 & & $\vdots$ & & 14775 & $\vdots$ & \\
\hline & & & & & & & & & $i$ & 1 & & & \\
\hline & RMPCO - MAT'L - DIRECT COST & & LS & & & & 1049200 & & & 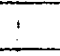 & & 1 & \\
\hline & LABOR $(14775 \mathrm{MH} \div .65$ PROD & 22730 & MH & $\$ 16.04$ & & & & & $\therefore$ & 1 & & 364600 & \\
\hline & SUBCONTRACT & & & & & & $\vdots$ & & 1 & 1 & & & \\
\hline & MAT'L - DIRECT COST & & LS & & & & & & 1 & i & & & ' \\
\hline & OVERHEAD \& PROFIT & & $\$$ & & & & & & $\dot{5}$ & & & 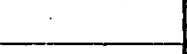 & \\
\hline & LABOR $1 \quad \mathrm{MH} \div \quad \mathrm{PRODI}$ & & $\mathrm{MH}$ & $\$$ & & & & & & & & & 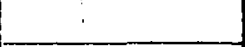 \\
\hline & OVERHEAD \& PROFIT & & $\$$ & & & & 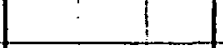 & & $\therefore$ & & & & \\
\hline & & & & & & & i & & & & & 1 & \\
\hline & & & & & & & . & & $\therefore$ & 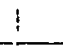 & & 1 & 1 \\
\hline & & & & & & & 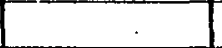 & & $i$ & $!$ & & $i$ & $\therefore 1$ \\
\hline & 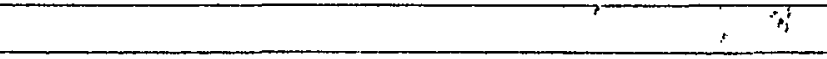 & & & & & & 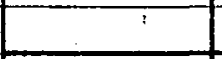 & & $i$ & $i$ & & ! & \\
\hline 4600 & TOTAL ELECTRICAL & & & & & & 1249200 & & 1 & 1 & 22730 & 364600 & 1413.00 \\
\hline & & & & & & & $!$ & & 1 & 1 & & 1 & \\
\hline & & & & & & & ; & & $\vdots$ & $\vdots$ & & $i$ & \\
\hline & AVE COST PER HORSEPOWER (TOTAL $\$ \div \mathrm{HP}$ ) & & $\$$ & $\div$ & & HP & 1 & & 1 & $i$ & & & FHP \\
\hline & . & & & & & & $i$ & & 1 & $i$ & & $!$ & \\
\hline & & & & & & & $?$ & & 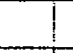 & 1 & & $i$ & . \\
\hline & & & & & & -2 & $130-1$ & & 1 & 1 & & $\vdots$ & ' \\
\hline
\end{tabular}




\begin{tabular}{|c|c|c|c|c|c|c|c|c|c|c|c|c|c|c|c|c|c|c|}
\hline M.T.O. & PRICED BY & $3 \pi$ & & & & & DATE 7 & $7-6$ & $-7 \varepsilon$ & & & & & EET - & 3 & OF & 10 & \\
\hline JOB NO. & CLIENT: ORNALUC & e & & TYPE OF & ESTIM & ATE: $C$ & ane & & & & & & & KD BY & & ins & & \\
\hline UNIT/AI & $F F P-W T E^{\prime}$ & & & $\cos 1$ & OR M/ & HRS & & & & & & & & & & & & \\
\hline DESCAII & UTL. & OUANTITY & 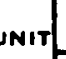 & & R UNI & & $\begin{array}{l}\text { MATERIA } \\
\text { EXPENS }\end{array}$ & & $5 \sqrt{16}$ & NIRACI & & & HOH & & & & OLLAR & \\
\hline CAPACI & & & & MATL & $M / H$ & LAB \$ & & & M/HRS & DOLLAF & & M/HRS & & $\overline{L L A F}$ & & & & \\
\hline $\begin{array}{l}A C C N T \\
4 / 600\end{array}$ & ELectrican & & & & & & & & & & & & & & & & & \\
\hline & & & & & & & & & & & & & & & & & & \\
\hline & & & & & & & & & & & & & & & & & & \\
\hline & APRA LTE & & & & & & 224 & 450 & & & & 588 & & & & & & \\
\hline & $\checkmark-$ Scrurety & & & & & & 550 & 010 & & & & 2088 & & & & & & \\
\hline & Pan, D, sT & & & & & & 5325 & 970 & & & & 1077 & & & & & & \\
\hline & -6 & & & & & & 238 & $8 \%$ & & & & 2366 & & & & & & \\
\hline & -7 & & & & & & 124 & Gsa & & & & 4318 & & & & & & \\
\hline & emmo\% Ser sys. & & & & & & 55 & 920 & & & & 1794 & & & & & & \\
\hline & FENCE" lecpiensione sys. & & & & & & 43 & 260 & & & & 879 & & & & & & \\
\hline & AREA secure ITY TV sys. & & & & & & 1696 & 680 & & & & 1665 & & & & & & \\
\hline$\cdot$ & 7 & & & & & & & & & & & & & & & & & \\
\hline 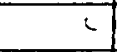 & & & & & & & & & & & & & & & & & & \\
\hline & & & & & & & & & & & & & & & & & & \\
\hline & & & & & & & & & & & & & & & & & & \\
\hline & & & & & & & & & & & & & & & & & & \\
\hline & & & & & & & & & & & & & & & & & & \\
\hline & & & & 7 & & & & & & - & E- & & $=$ & & $=$ & & & \\
\hline & & & & & & & 1027 & 780 & & & & 14775 & & & & & & \\
\hline & & & & & & & & & & & & & & & & & & \\
\hline & & & & & & & & & & & & & & & & . & & \\
\hline & & & & & & & & & & & & & & & & & & \\
\hline & & & & & & & & & & & & & & & & & & \\
\hline & & & & & & & & & & & & & & & & & & \\
\hline & & & & & & & & & & & & & & & & & & \\
\hline & & & & & & & & & & & & & & & & & & \\
\hline & & & & & & & & & & & & & & & & & & \\
\hline & & & & & & & & & & & & & & & & & & \\
\hline & & & & & & & & & & & & & & & & & & \\
\hline & & & & & & & & & & & & & & & & & & \\
\hline & & & & & & & & & & & & & & & & & & \\
\hline & & & & & & & & & & & & & & & & & & \\
\hline & & & & & & & & & & & & & & & & & & \\
\hline & . & & & & & & & & & & & & & & & & & \\
\hline
\end{tabular}


THE RALPH M. PARSONS COMPANY

ESTIMATE WORKSHEET

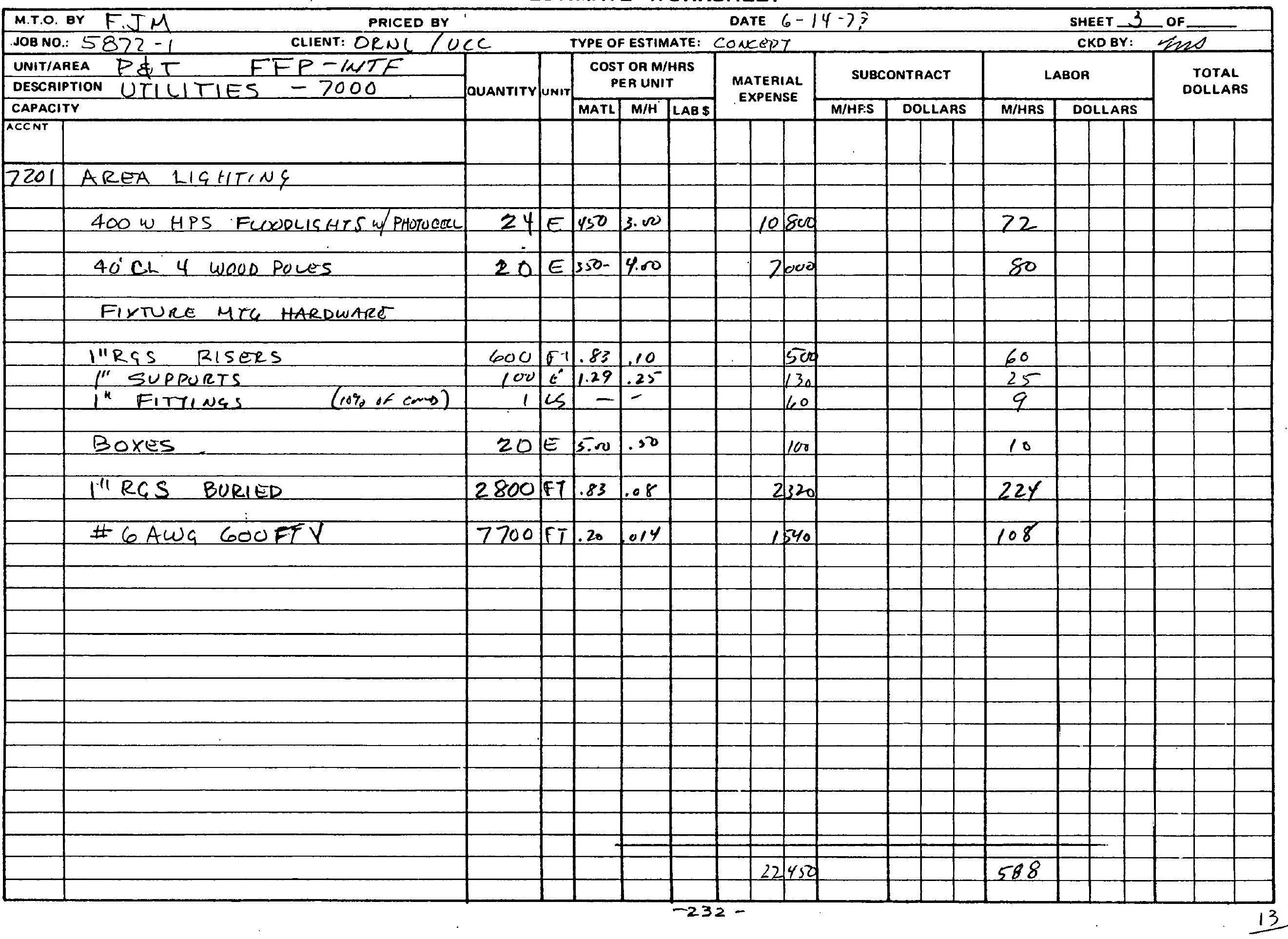


ESTIMATE WORKSHEET

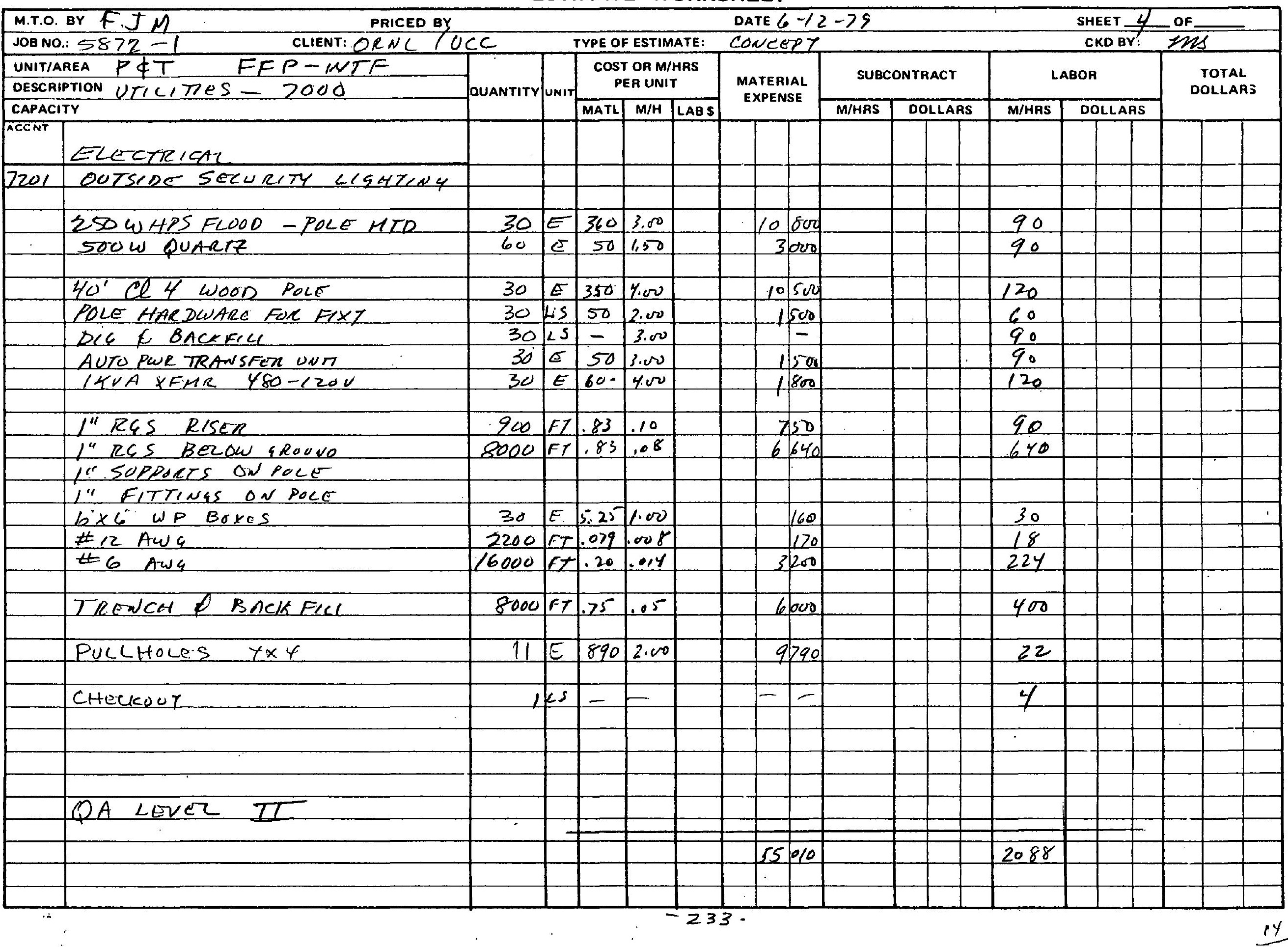


ESTIMATE WORKSHEET

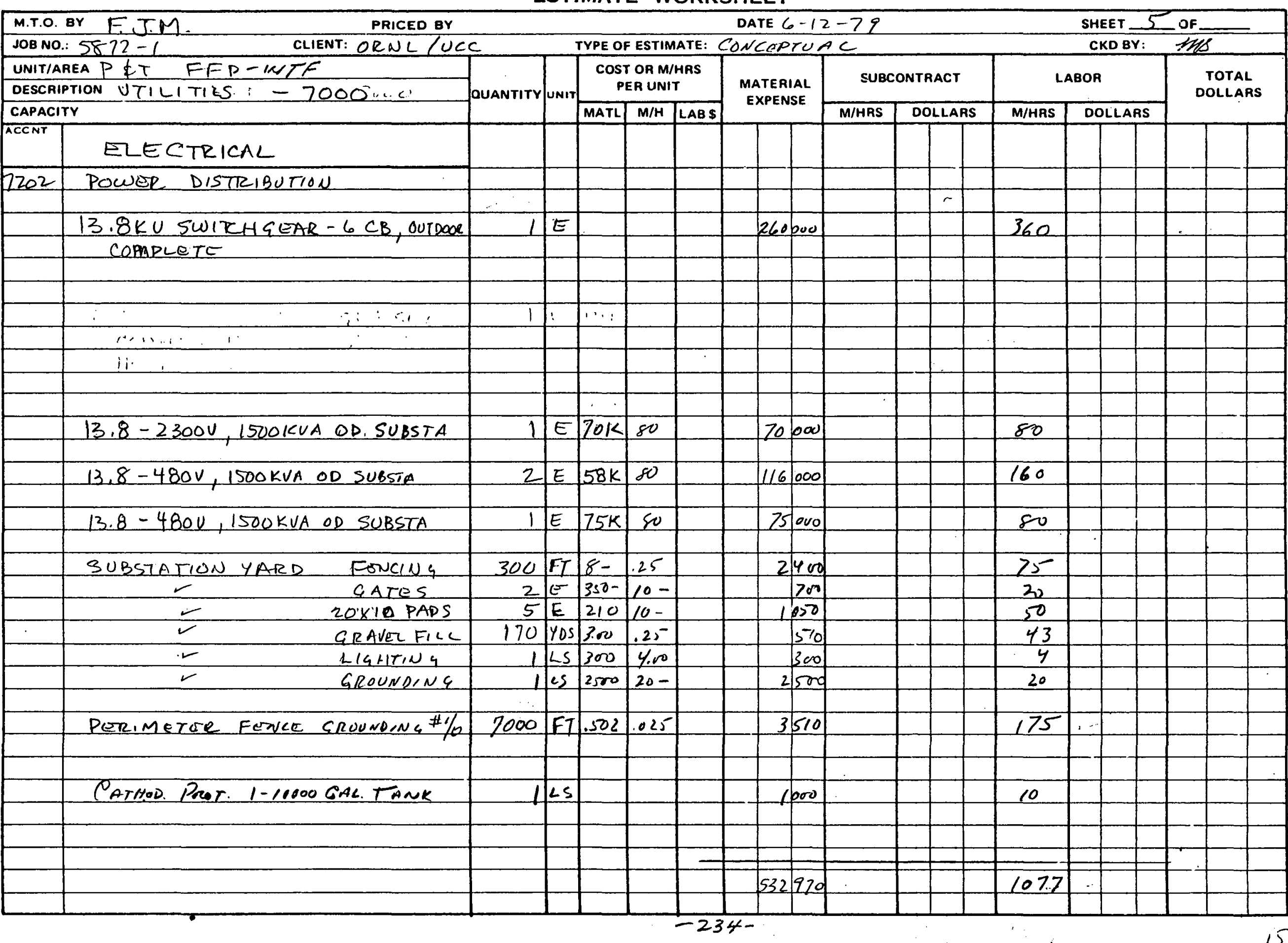


ESTIMATE WORKSHEET

$\begin{array}{ll}\text { M.T.O. BY F J.M } & \text { PRICED BY } \\ \text { JOB NO.: } 5877-1 & \text { CLIENT: ORNL/UCC }\end{array}$

UNIT/AREA P\&T FEP-NTF

DESCAIPTION UTILITIES - 7000

CAPACITY

\begin{tabular}{l|l} 
ACCNT \\
ELECTRICAL
\end{tabular}

\begin{tabular}{l}
\hline 202 \\
\hline $20 W E R$ DISTRIBUTIOU
\end{tabular}

DUCT BANK $4 \times 2^{11}$ CONCENC, PVC

$200 \mathrm{HP}, 2.4 \mathrm{KV} 2^{\prime \prime} \mathrm{C}^{\# 6} 6(5 \mathrm{kV}) \oplus 1050 \mathrm{~F}$

Duct Bank 2-4"pue

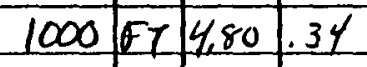

$2 E 4371-413$

1600 Fr 6.44 .75

(600 Fr
DATE $6-28-79$

TYPE OFESTIMATE: CONCGPTUAL

COST OR M/MAS

PER UNIT

MATERIAL

\begin{tabular}{|l|l|l|}
\hline MATL & M/H & LABS \\
\hline
\end{tabular}

EXPENSE

SUBCONTRACT

\begin{tabular}{|l|l|l|l|}
\hline M/HRS & DOLLARS & M/HAS & DOLLARS \\
\hline
\end{tabular}$$
+
$$ 
ESTIMATE WORKSHEET

\begin{tabular}{|c|c|c|c|c|c|c|c|c|c|c|c|c|c|c|c|c|c|c|c|}
\hline M.T.O. & BY F.j:M & & & & & & DATE & $6-1$ & $2-79$ & & & & & & EET_- & $Z$ & OF & & \\
\hline JOB NO & $\therefore 5872-1$ & & & TYPE OF & FESTIM & ATE: & & & & & & & & & KD BY & - & $\operatorname{tins}$ & & \\
\hline UNIT/A & FFP-WTF & & & & $T$ OR M/F & & & & SUBC & NTP & BACT & & & 808 & & & & & \\
\hline DESCRI & PTION UTILITTES -7000 & QUANTITY & |unit & & ER UNIT & & $\begin{array}{l}\text { MATER } \\
\text { EXPEN }\end{array}$ & & 30,0 & & HACI & & & & & & & DOLLAR: & \\
\hline CAPACI & & & & MATL & $M / H$ & LABS & & & M/HRS & & $\overline{\text { LLARS }}$ & & M/HRS & & LLAR & & & & \\
\hline ACCNT & ELECTRICAL & & & & & & & & & & & & & & & & & & \\
\hline 2202 & POWER DISTRIBUTIUN & & & & & & & & & & & & & & & & & & \\
\hline & & & & & & & & & & & & & & & & & & & \\
\hline & 2-DUCT BANKS CONC ENCASCD & & LS & 二 & - & & 33 & 010 & & & & & 1666 & & & & & & \\
\hline & $w / 8-4^{\prime \prime} \& 2-3^{\prime \prime} P V C E A C H$ & & & & & & & & & & & & & & & & & & \\
\hline & $\therefore 700^{\prime}$ RUN EACH BAVR & & & & & & & & & & & & & & & & & & \\
\hline & & & & & & & & & & & & & & & & & & & \\
\hline & TRENCHING \& BACK FICC $61400^{\circ}$ & & $\angle S$ & $=$ & $E$ & & 2 & 100 & & & & & 700 & & & & & & \\
\hline & - & & & & & & & & & & & & & & & & & & \\
\hline & CASLE $600 \mathrm{VOLT} 600 \mathrm{MCM}$ & 9000 & Ft & 4.79 & .063 & & 36 & 710 & & & & & 567 & & & & & & \\
\hline & & & & & & & & & & & & & & & & & & & \\
\hline & CABLE $5000 \mathrm{~V} \quad 500 \mathrm{MCM}$ & 2250 & F7 & 3.201 & $.06 \pi$ & & & 200 & & & & & 151 & & & & & & \\
\hline & $r$ (OKOQUACD EPR) 6 & 4800 & FT & .169 & .014 & & & 810 & & & & & 67 & & & & & & \\
\hline & CABLCE. CONTROL $19 / \mathrm{C} \# / 2$ & 45.00 & $\mathrm{Fy}$ & 2.40 & .039 & & 10 & 800 & & & & & 176 & & & & & & \\
\hline & a & & & & & & & & & & & & & & & & & & \\
\hline & $30 \mathrm{HP} C T$ FANS - $400 \mathrm{FT}$ EACM & 3 & 5 & $933-$ & $115^{-}$ & & 2 & 800 & & & & & 345 & & & & & & \\
\hline & & & & & & & & & & & & & & & & & & & \\
\hline & $1 \ldots \quad \because \because 66$ & $1 \%$ & : & & & & $\because$ & & & & & & . & & & & & & \\
\hline & $\therefore \quad i \quad \therefore \quad \therefore y$ & !! & & & & & $\because$ & & & & & & & & & & & & \\
\hline & $1 k=:$ & & & & & & & & & & & & & & & & & & \\
\hline & & & & & & & & & & & & & & & & & & & \\
\hline & MANHOLES & 8 & $E$ & 2200 & 4,00 & & & 600 & 二- & & & & 32 & & & & & & \\
\hline & PULLHOLE & 6 & $E$ & 890 & 2.00 & & & 340 & & & & & 12 & & & & & & \\
\hline & 2 - DUCT BANKS, COUC EUCA $5 \mathrm{CD}$ & & & & & & & & & & & & & & & & & & \\
\hline & $w / 2-5^{\prime \prime}$ PUC EACH $-200^{\prime} /$ BANK & 400 & FT & 6.44 & .75 & & $z$ & 580 & & & & & 300 & & & & & & \\
\hline & TR?BF & 400 & $r+r$ & 1.75 & .05 & & & 700 & & & & & 20 & & & & & & \\
\hline & CABCE $15 K V-3 / / C \# 4 \not$ & 1300 & $F 7$ & 1.895 & .063 & & & 460 & & & & & 82 & & & & & & \\
\hline & & & & & & & & & & & & & & & & & & & \\
\hline & SUBSTATION FECDERS $3 "-3^{\# 2} 15 \mathrm{KV}$ & 120 & Fy & 21.17 & 1.67 & & & 540 & & & & & 200 & & & & & & \\
\hline & $\left(4 \times 30^{\prime}\right)$ (OKOGI)AKR $\left.=E P R\right)$ & & & & & & & & & & & & & & & & & & \\
\hline & & & & & & & & & & & & & & & & & & & \\
\hline & & & & & -1 & & $=$ & + & & - & - & - & & - & 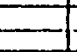 & - & & & \\
\hline & & & & & & & 124 & 650 & & & & & 4318 & & & & & & \\
\hline & & & & & & & & & & & & & & & & & & & \\
\hline
\end{tabular}


ESTIMATE WORKSHEET

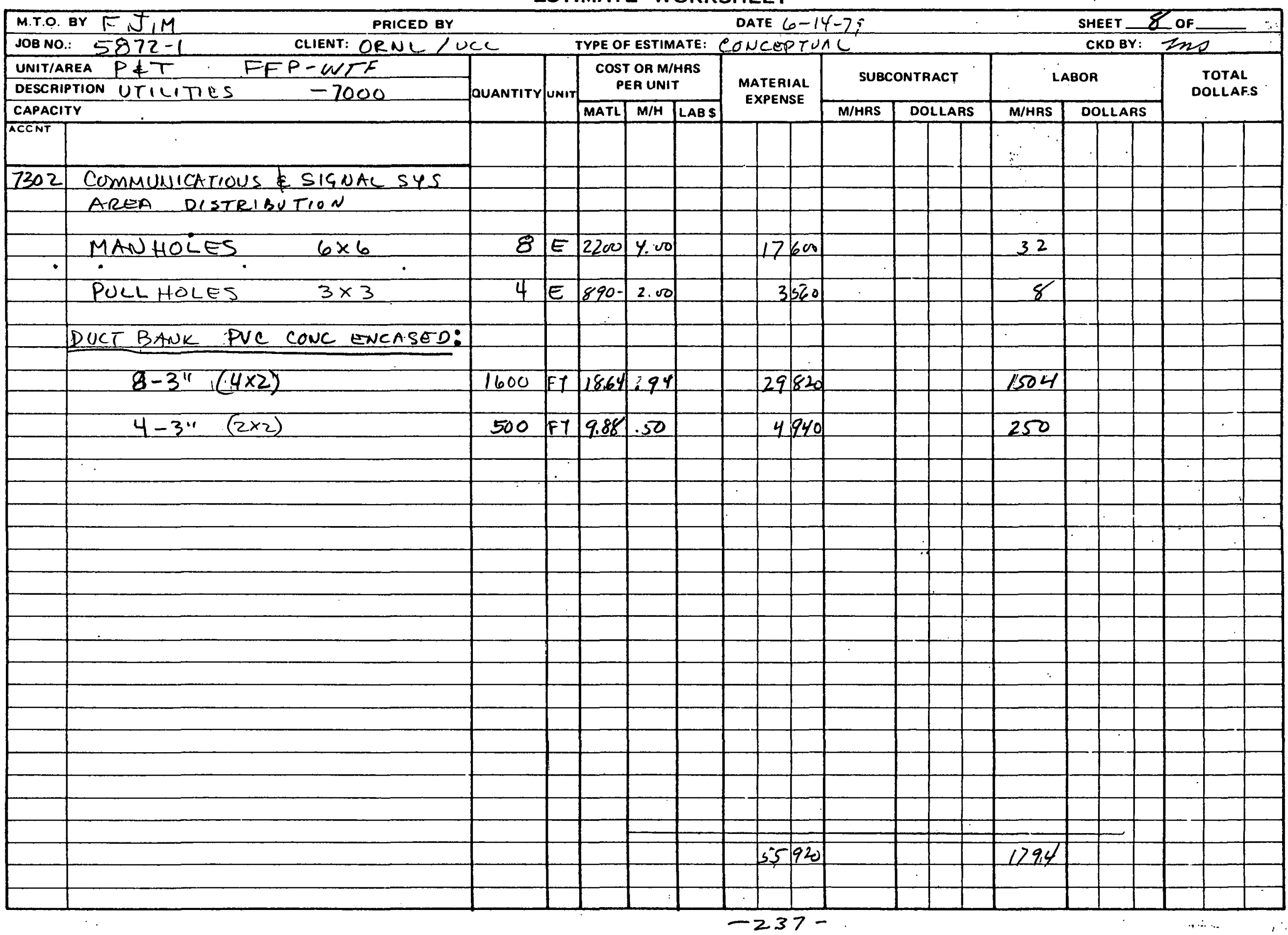


THE RALPH M. PARSONS COMPANY

ESTIMATE WORKSHEET

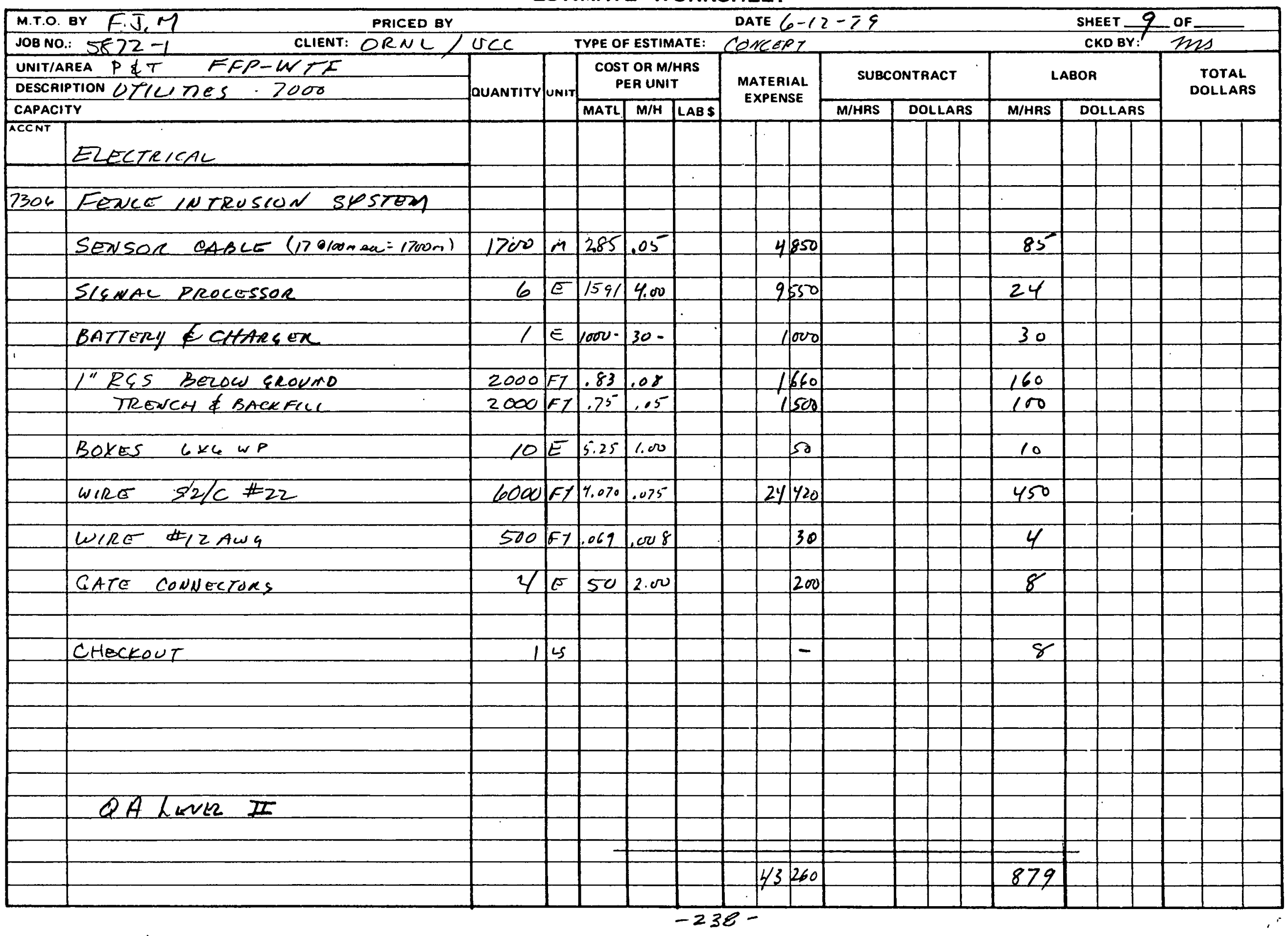


ESTIMATE WORKSHEET

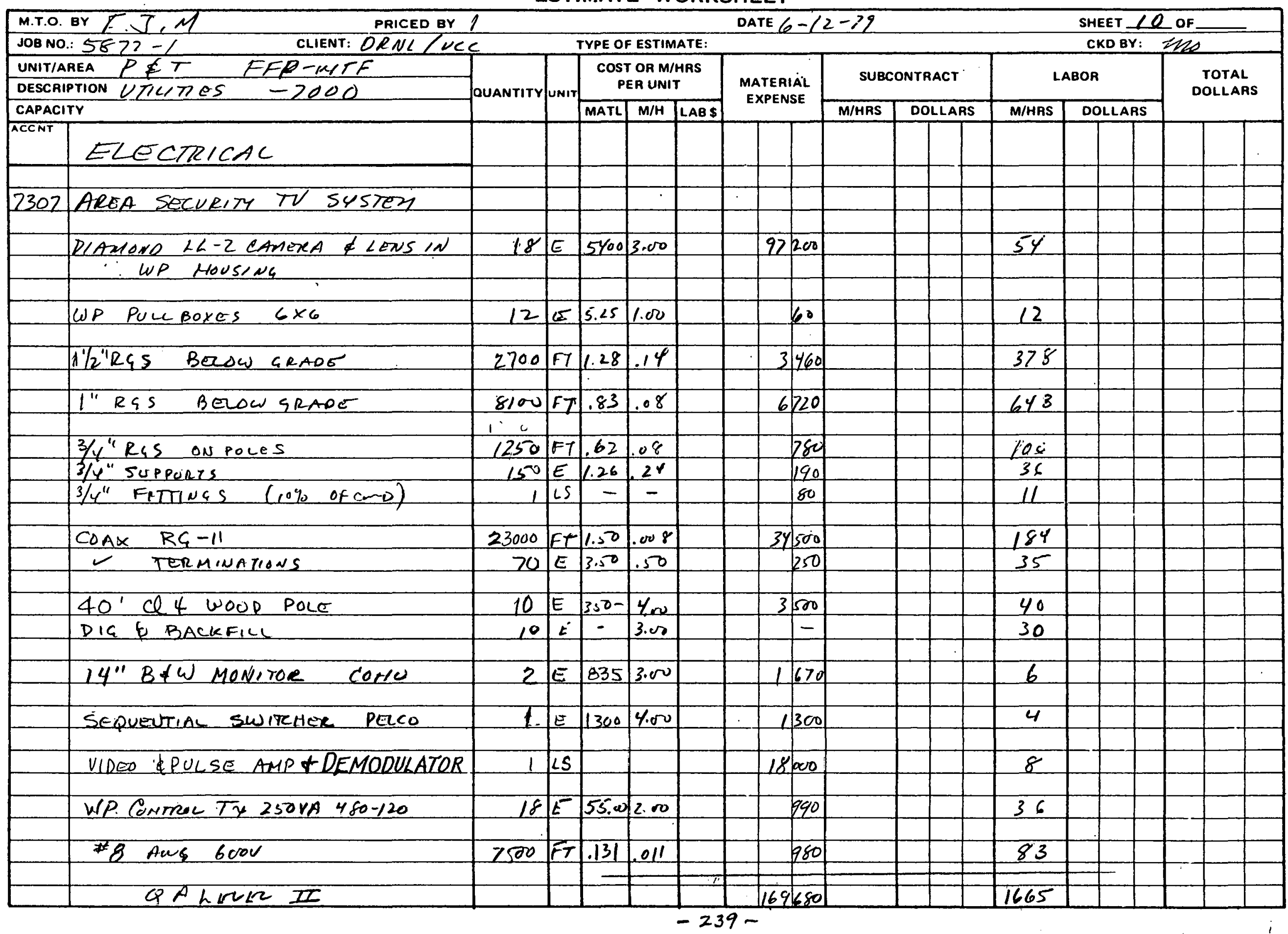




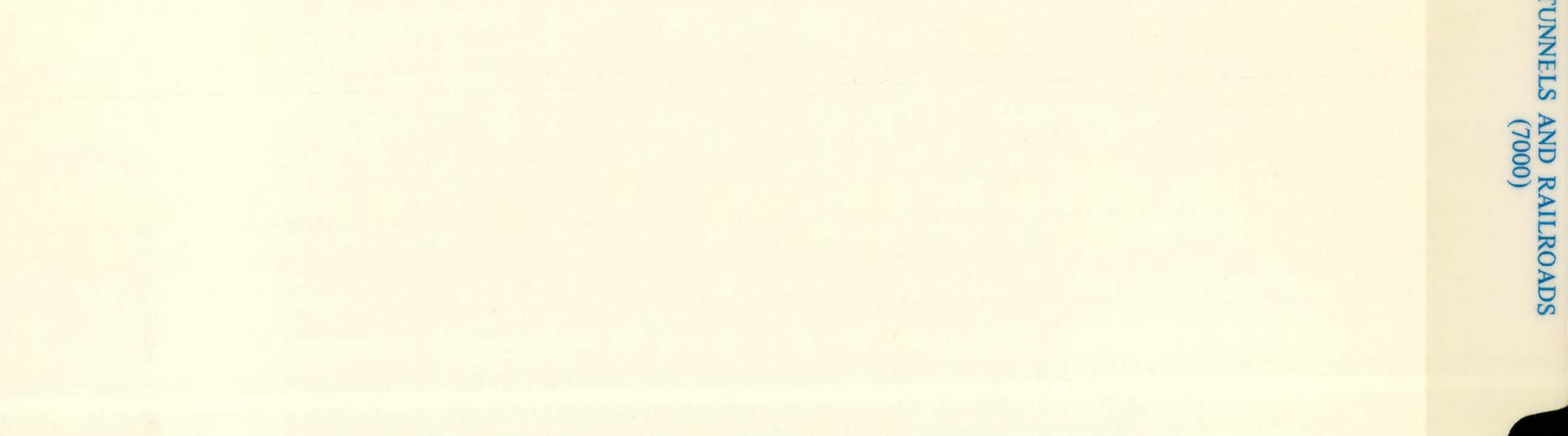

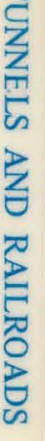




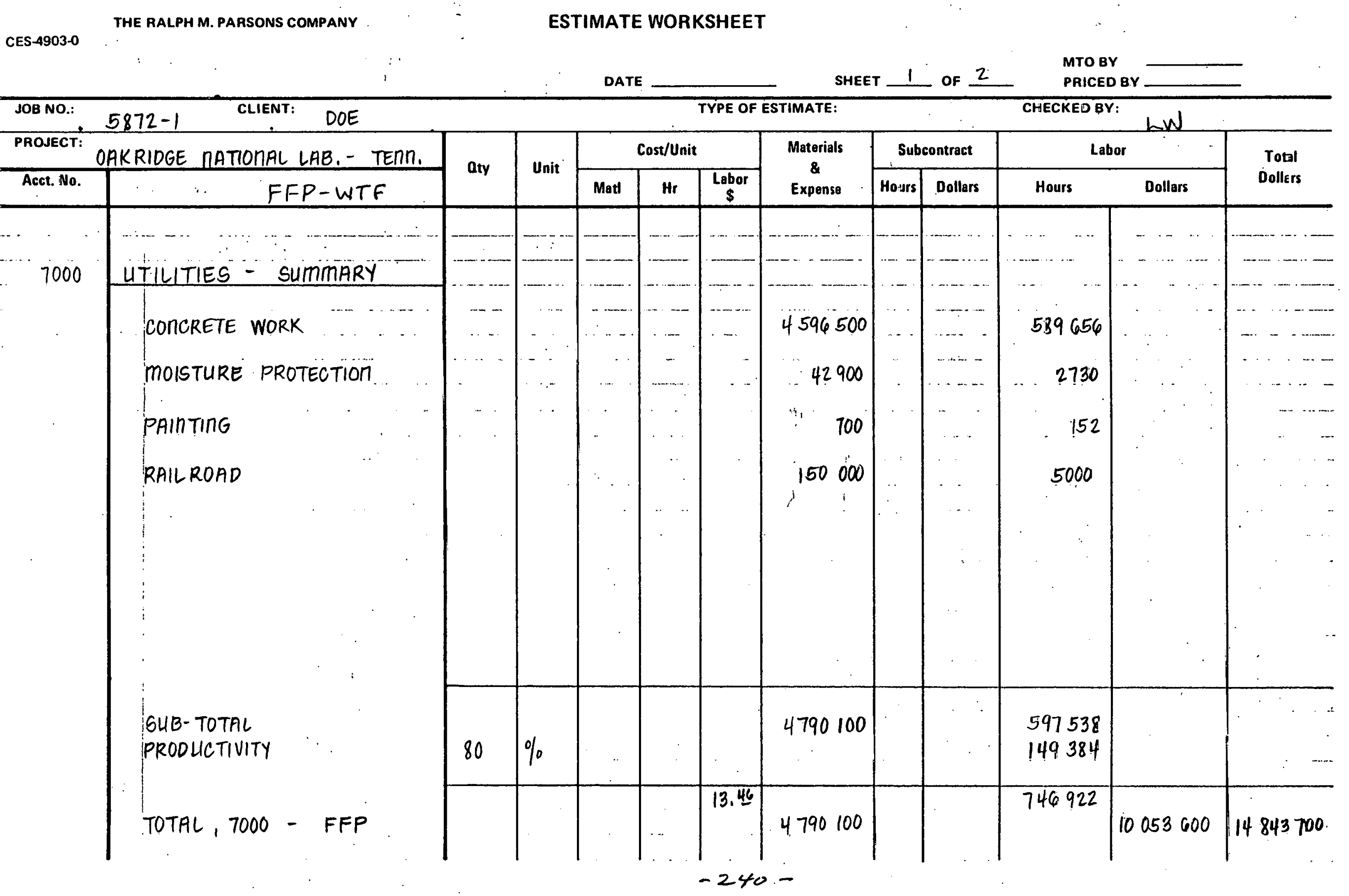


THE RALPH M. PARSONS COMPANY

ESTIMATE WORKSHEET

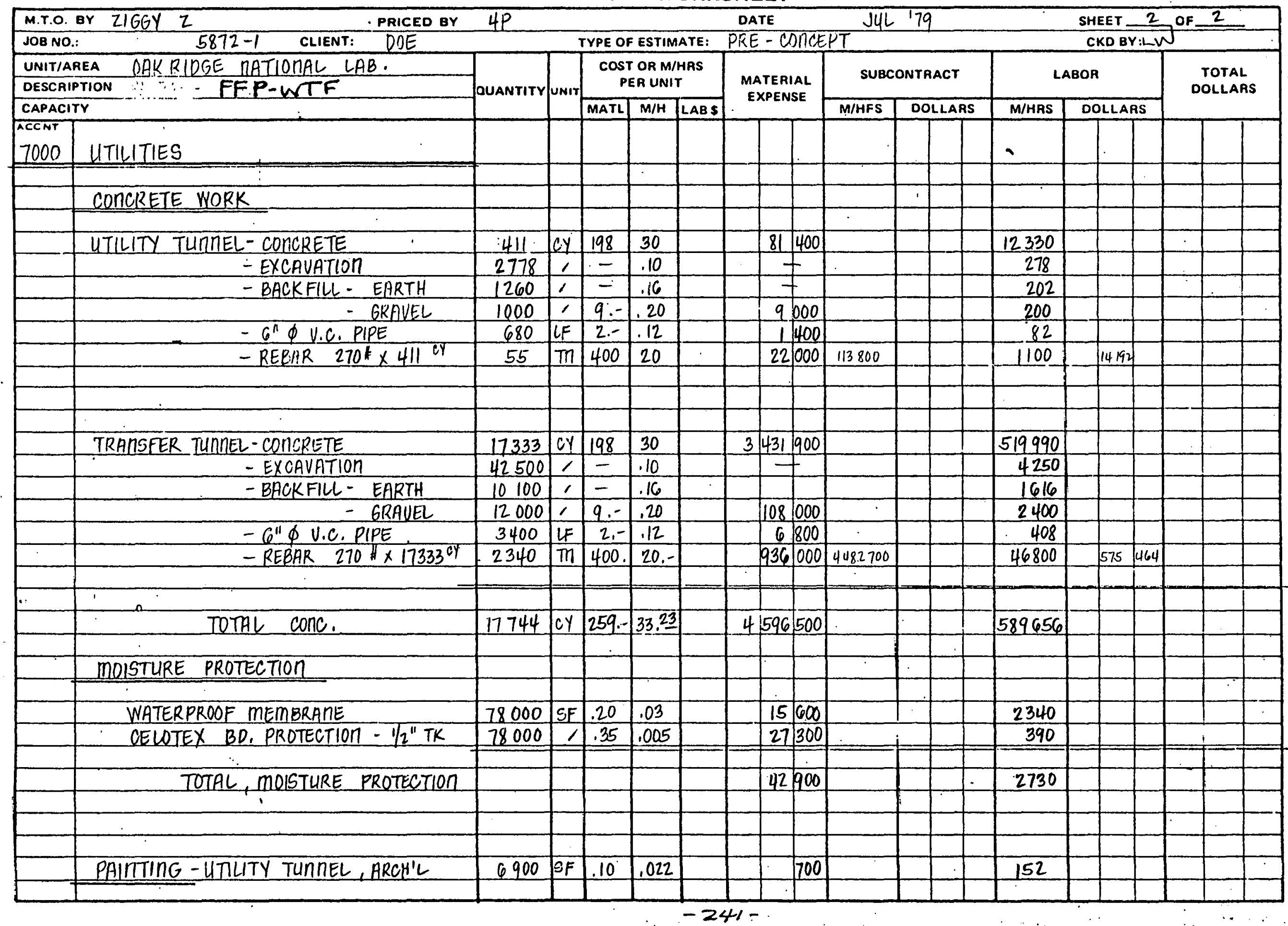


ESTIMATE WORISHEET

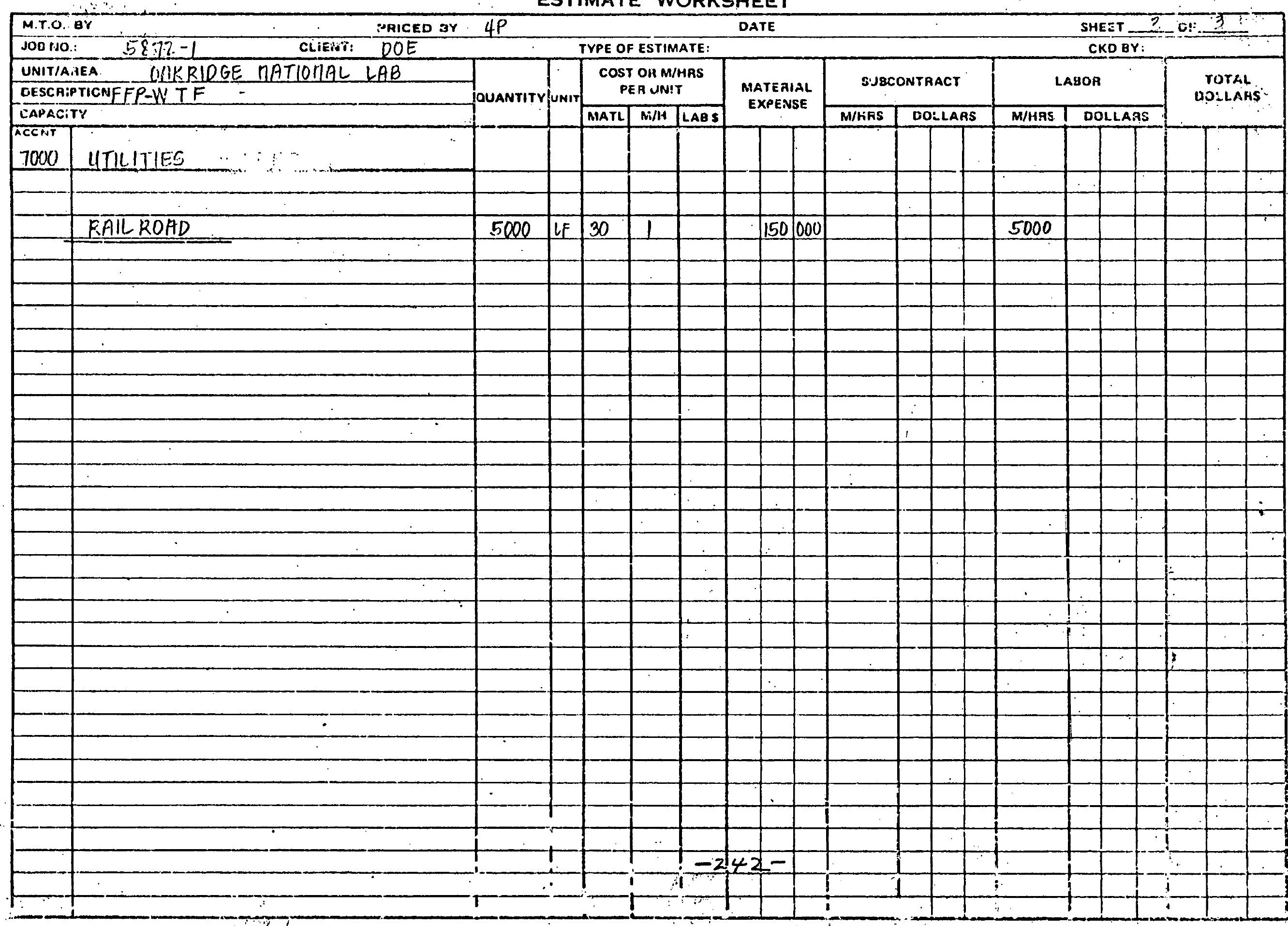


ठ으 
THE RALPH M. PARSONS COMPANY

CALCULATION SHEET

$\because$ RIP

TITLE _P

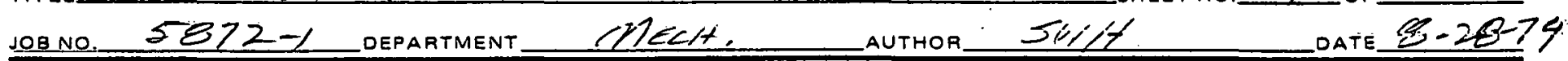

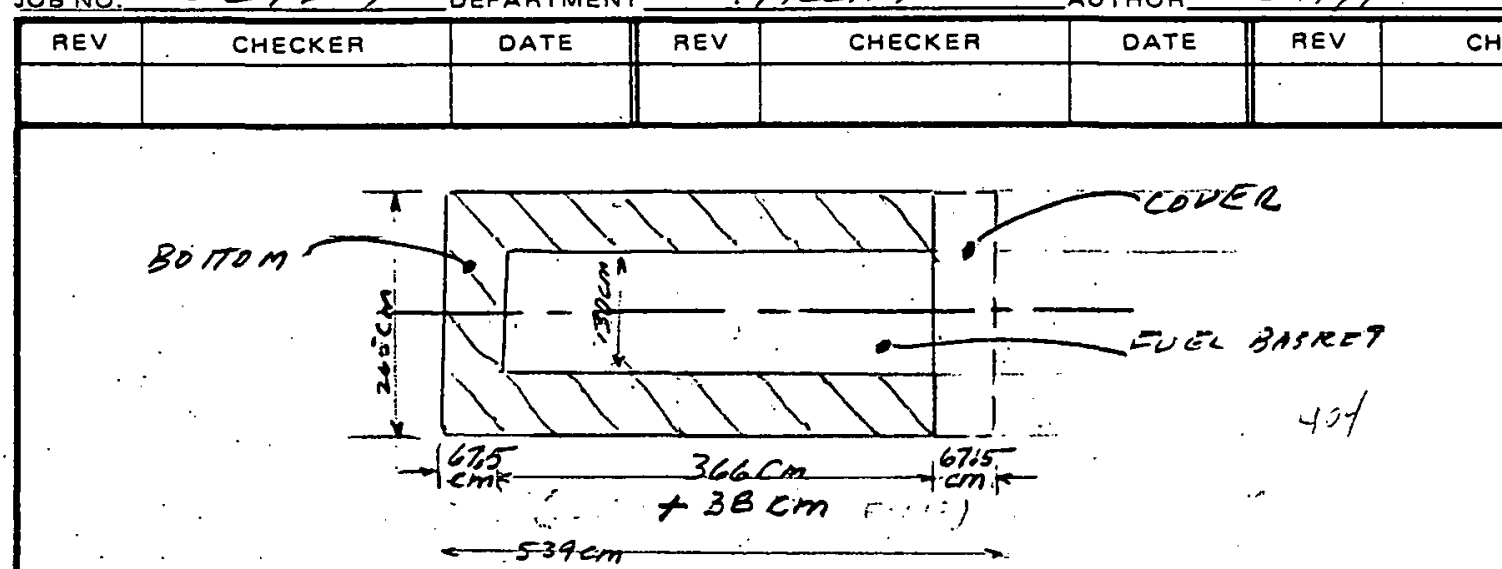

1) ADO BOTTOM A.SOECOVER WITH SAME THICKNESS AS SIDES

2) $A D D 38 \mathrm{~cm}$ TO $366 \mathrm{~cm}$ LENGTH TD PRoVIDE SPACE FOR MECH. EQUIP,

"Material Posts

A. 304 starless steel

1) $5 \mathrm{~cm} 304: 5.5 \%$ OUTER SHELL

$$
\begin{aligned}
& \text { er } C Y, L=\text { Wow }_{\text {- Dewsiry }}=\frac{\pi}{4}\left(D_{2}^{2}-D_{1}^{2}\right)^{2} \\
& l=\operatorname{leng} \text { th }=539 \mathrm{~cm} \\
& d=\text { density }=0.292 \mathrm{lb}^{\prime} \mathrm{in}^{3} \\
& D_{1}=265 \mathrm{~cm} \\
& D_{1}=251 \mathrm{~cm}
\end{aligned}
$$

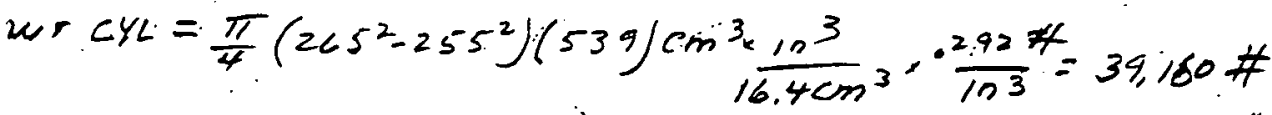
Pavis estimated wi, Finances aw o pintles 6,000 \#

Peers: Wt. End Pares $=2 \times 5 \mathrm{~cm}$ A, d $2=265 \mathrm{~cm}$

$$
=2 \times 5 \times \frac{\pi}{4} \frac{(265-10)^{2} \times 0.292}{14.4}=9,0904
$$

Torse $54,260 \#$

$$
\begin{aligned}
& \operatorname{Cos}(\text { at } 100 / 16)=54,26040-100 / 16=\$ 54,260 \\
& \text { zeus Packacinc at Swipiere } \cdots \frac{2500}{\$ 56,760}
\end{aligned}
$$

$-243-$

AMP 307 (2/78)

PAGE NUMBER 
THF RAI PH M. PARSONS COMPANY

CALCULATION SHEET

title P.T lask - Best Estimate Beculations 2 sheetino. 2 of

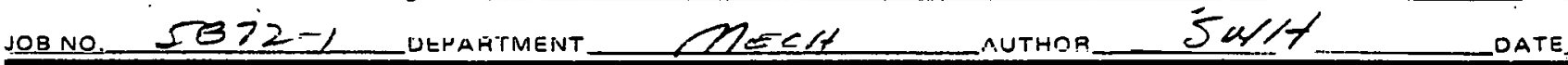



2) $2.5 \mathrm{~cm} .55$ PLATE

$$
\begin{aligned}
& \text { Wt. CYyL }=\text { Aecen } \times 42,465 \mathrm{lbs} / \mathrm{ft}^{2} \\
& =\pi-D_{14 .}+\operatorname{leng} t \pi=42,6.65 / 65 / 4 t^{2}
\end{aligned}
$$

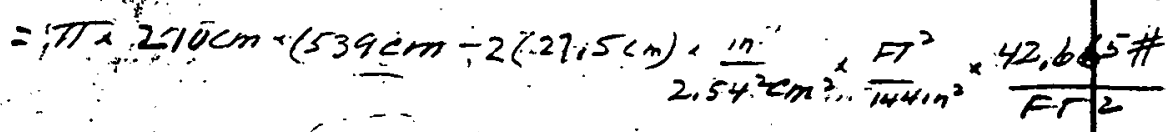

$$
\begin{aligned}
& =\frac{3,14 \times 210 \times 484 \times 42,645}{4.2,54^{2} \times 144}=14,660^{\circ}
\end{aligned}
$$

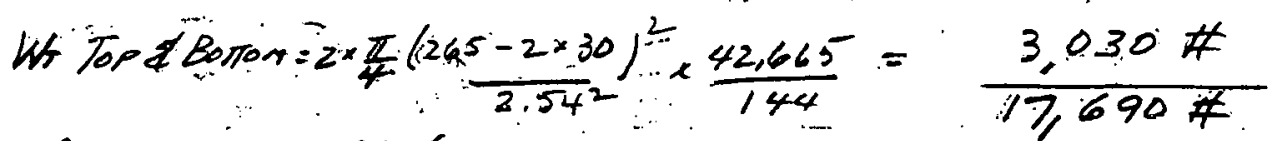

Cost@1 $1 \#-\ldots$

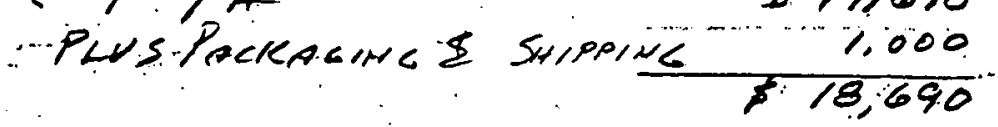

3) $5,0 \mathrm{~cm} 55$ piare

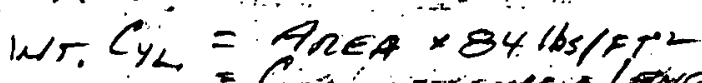

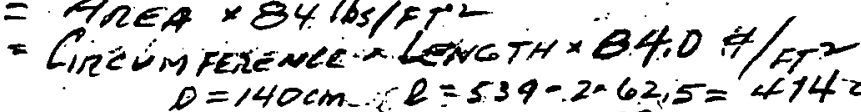

$$
\begin{aligned}
& D=140 \mathrm{~cm}, \mathrm{C}=539-2 \times 62,5=414 \mathrm{~cm} \\
& =\pi \cdot 140 \mathrm{~cm} \times 414: \frac{\mathrm{cm} \times \mathrm{in}^{2}}{2,54^{2} \mathrm{~cm}^{2}} \times \frac{\mathrm{FT}^{2}}{\mathrm{~A}^{4} \mathrm{in}^{2}} \times \frac{\theta 4 \#}{F T^{2}} \\
& =\frac{3.14 \times 140 \times 474 \times 84}{2.54^{2}+144}=16,460 \#
\end{aligned}
$$

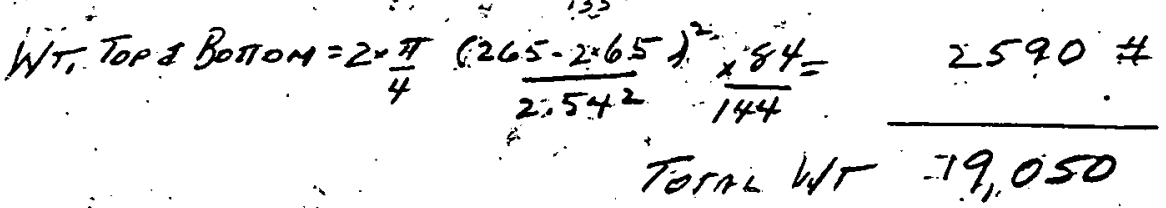

$$
\begin{aligned}
& \text { Cost } 0100 / 16 \ldots \ldots t, 050
\end{aligned}
$$

TOTAL 30455 costs:

Ourer SHeck - 56,760

$=2.5 \mathrm{~cm}$ Piate $-18,690$

$5,0 \mathrm{~cm}$ PLATE 20,050

$-244-$

USE 96000

RMP.307 (2/78)

PAGE NUMBER 
ALP

THE RALPH M. PARSONS COMPANY

GALCULATION SHEET

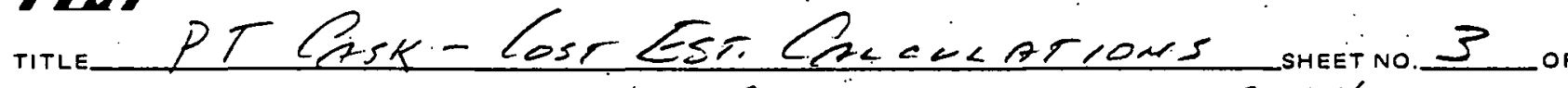

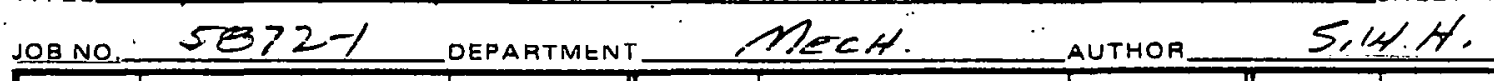

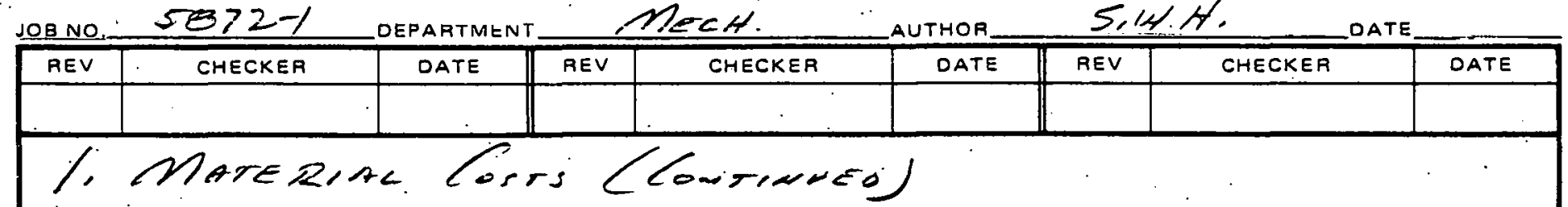

B. Aluminum Fuer Basket (6061-TL)

$$
\begin{aligned}
& H_{1}=1 / 0<.<D_{\text {exrsty }}=\frac{\pi}{4} D^{2}<d \\
& D=D_{1 A}=130 \mathrm{~cm} \\
& \angle=\angle E N G T N=404 \mathrm{~cm} \\
& d=\text { DENSITY }=0.0975 \mathrm{H} / \mathrm{mm} \\
& \text { wrr }=\frac{\pi}{4} \times \frac{130^{2}}{2.542} \times \frac{404}{2.54} \times 0.0975=31,905 \#
\end{aligned}
$$

Peus MFC Losses (15\%) $\frac{4,785}{36,6907}$

$\operatorname{Cost} 175 / 13=36,690 \times 1,75=\$ 64,210$ plus Prekncinc and Suippinc. 2,500

Ner INT $=31,905 \times .70=22,300$ TOMAL $\$ 66 \geqslant 10$.

C. Copper Coume Fims in LiH USE 67000

$$
\begin{aligned}
& \text { Estimate } 2200 \#\left[374 / 16={ }^{\$} \theta, 340\right.
\end{aligned}
$$

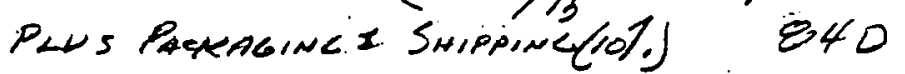

Toras 9,180

Use 10,000

2. SUB-AssembLy-losts

A, Borom Laroide

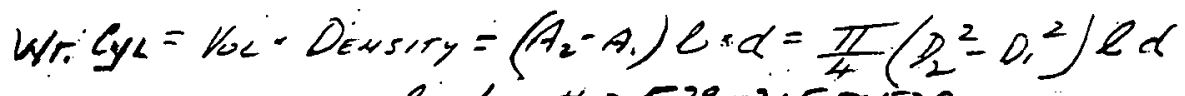

$$
\begin{aligned}
& l=\text { length }=539-2 \times 5^{4}=5-29 \ldots \ldots \\
& D_{2}=255 \mathrm{~cm} \\
& D_{1}=210 \mathrm{~cm} \quad d=0.0916 \# / \mathrm{in}^{3} \text {. } \\
& w_{T_{1}} C_{4 L}=\frac{\pi}{4}\left(255^{2}-210^{2}\right) \times 529=\frac{\mathrm{m}^{3} \times 1 \mathrm{n}^{3}}{16.4 \mathrm{~cm}^{3}} \times c 916 \mathrm{~A} / \operatorname{lin} 3=48,560 \\
& -245-
\end{aligned}
$$

PAGE NUMBER 
THE RALPH M. PARSONS COMMPANY

C.AL.C.III.ATINN SHEET

ALP

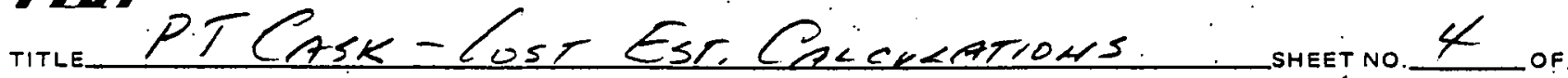
Jo8no. $5872-1$

Depattmeni Mest, айтно OATE

C.

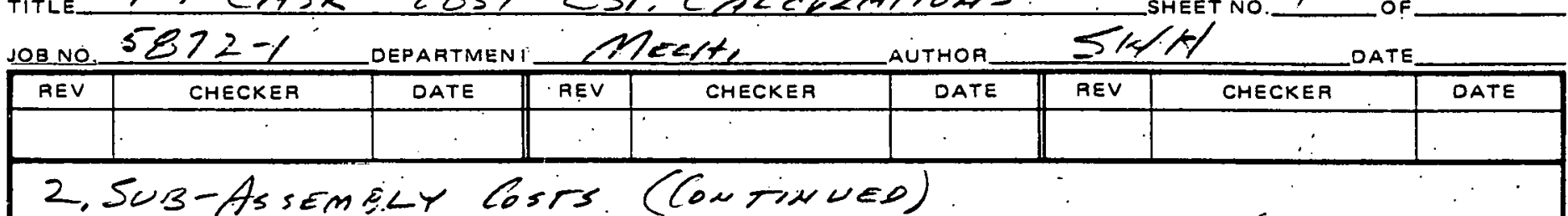

2. SuB-Assemeliy Cosirs. (Con tinued)

A.: Boroularbide (Continueo)



$$
\begin{aligned}
& =2 \times \frac{\pi}{4} \cdot 210^{2} \mathrm{~cm}^{2}(127,5=105) \mathrm{cm} \cdot \mathrm{in}^{3}=\frac{\pi}{4} a \cdot 210^{2} \\
& \frac{1 \mathrm{n}^{3}}{16.4 \mathrm{~cm}^{3}}=\frac{0.091 / \#}{103} \\
& =8,110016 \mathrm{~s}
\end{aligned}
$$

Torre Wr $B_{4}<=46,560 \#+B, 710 \quad \#=57270 \%$

PLUS Bétikaté and los.sés $(10 \%) \frac{5,730}{63000 \#}$

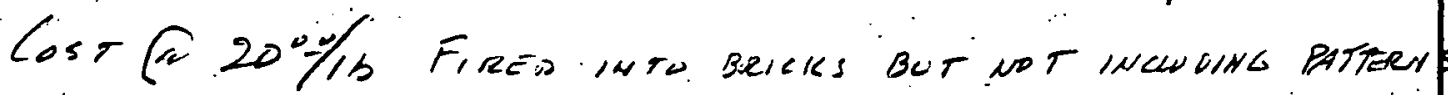

$$
\begin{aligned}
& \text { Cosr }=63,000 \text { \#.2 20\% } / 16=\cdots 1,260,000 \\
& \text { Puss Precricinc (2\%) 25,200 } \\
& \frac{2,000}{1,287,200}
\end{aligned}
$$

B. Litium Hyorioe

$$
\begin{aligned}
& \text { WT CQunderas } \frac{\pi}{4}\left(O_{2}^{2}-0,{ }^{2}\right) d \cdot d \\
& \text { USE } 1,280,000 \\
& D_{2}=205 \mathrm{~cm} \\
& D_{1}=167.6 \mathrm{~cm} \\
& l=539.2 \cdot 30^{\circ}=47.9 \mathrm{~cm} \\
& d=0.028 \mathrm{H} / \mathrm{m}^{3}
\end{aligned}
$$

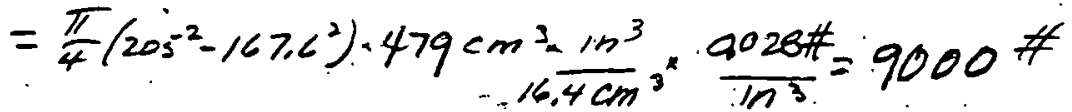

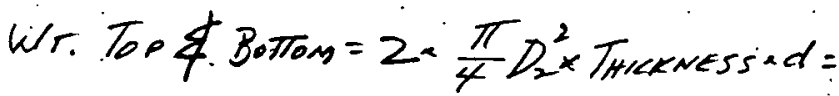



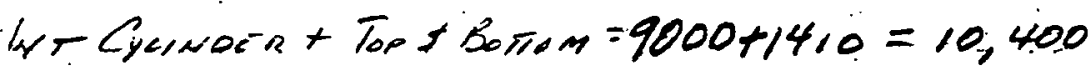

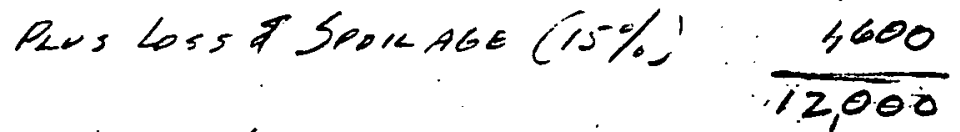

los, at $46 \mathrm{~s}=0 / 16=12,00046 \mathrm{C}=7552,000$

Pavs Packacieic

Pius Surppine

$-246-$

22,000

$\frac{1,200}{575,200}$

RMP.307 (2/78)

PAGE NUMBER 
THE RALPH M. PARSONS COMPANY

CALCULATION SHEET

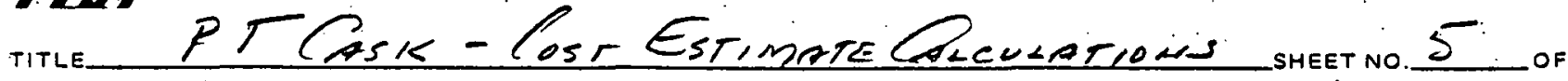

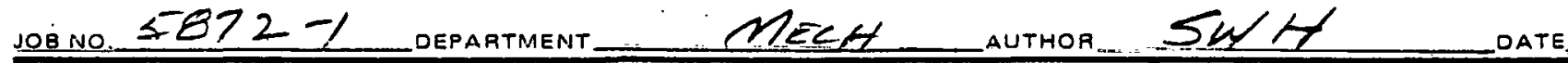

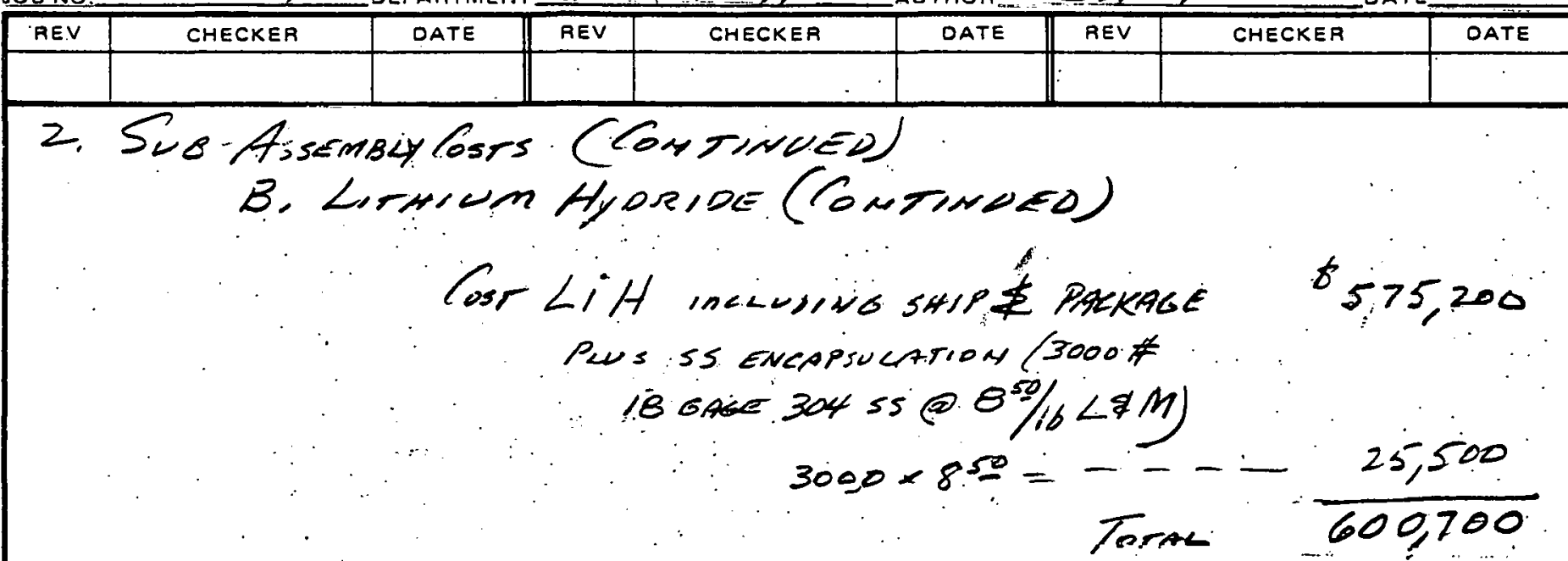

C. Depieteo Vranium

Use 610,000

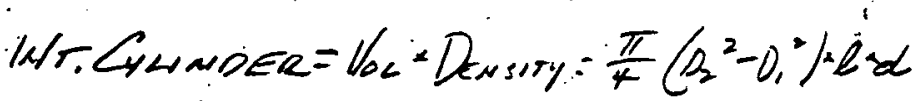

$$
\begin{aligned}
& D_{i}=167.6 \mathrm{~cm} \quad l=539 \mathrm{~cm}-2.48 .7 \mathrm{~cm}=441.6 \mathrm{~cm} \\
& D_{1}=160 \mathrm{~cm} \quad d=0,6753 \neq / 1^{3}
\end{aligned}
$$

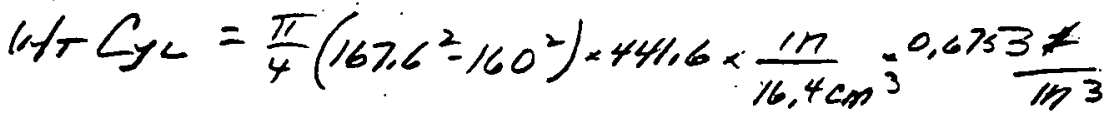

$$
\begin{aligned}
& =\frac{\pi}{4} \times 2490 \times \frac{441,6}{16.4} \times 0.6753=-35,560 \#
\end{aligned}
$$

Wit TO, \& BoTTON $0,=205-2.52,5 \times 160 \mathrm{~cm}$

$$
\begin{aligned}
& =2 \cdot \frac{\pi}{4} D_{1}^{2} \cdot T_{\text {TrCKNESS }}
\end{aligned}
$$

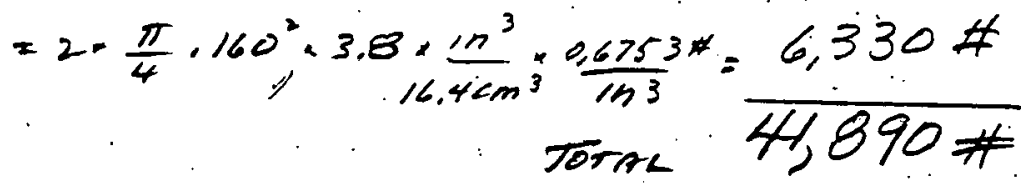

Cost $20 \% / 16$ (maCNINE)

$$
=41,890165 \times 20 \frac{01}{13}=\$ 837,800
$$

Peus Pacieasina (2\%) - 16,800

Rus suip 3:tart $\cdots \frac{1,500}{\text { ToraL }}$

USE 860,000

$-247-$

RMP.307 (2/78)

PAGE NUMBER 
ALP

THE RALPH M. PARSONS COMPANY

CALCULATION SHEET.

TitLe PT Cask - Cost Estimete Caccuentions sheEt no. 6 or



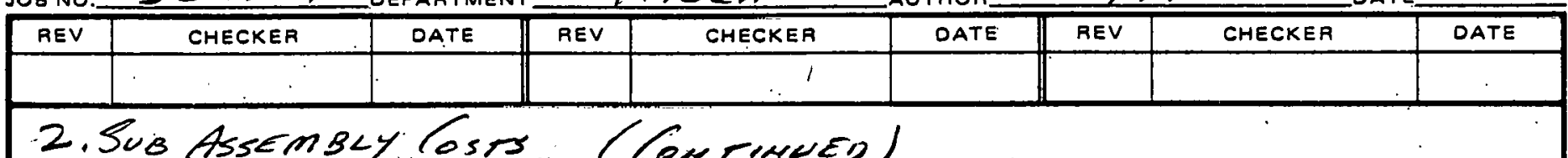

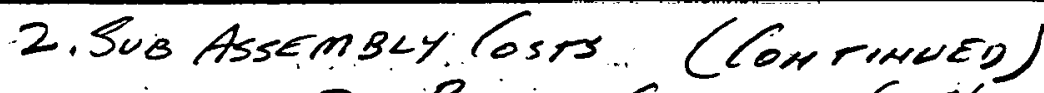

D. Borion Cinkide (70\%) wurn Gopete $(30 \%)$

$$
\begin{aligned}
& \text { wr. } \text { Cyurvoce }=V_{0<} \cdot D_{\text {ewrory }}=\frac{\pi}{4}\left(D_{2}^{2}-0_{1}^{2}\right) L \cdot d_{m} \\
& D_{2}=00=160 \mathrm{~cm} \\
& D_{1}=10=140 \mathrm{~cm} \\
& l=1 \mathrm{congth} \text { ey })=539 \mathrm{~cm} \cdot 2(132.5-80) \mathrm{km}-434 \mathrm{~cm}
\end{aligned}
$$

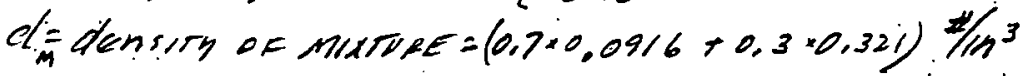

$$
\begin{aligned}
& =0.064+0.096=0.16 \mathrm{H}^{1} / \mathrm{ln}^{3} \\
& =, 7854 \times \frac{6000 \times 444 \times 0,16}{10,4}=\cdots \cdots \quad 19,9607
\end{aligned}
$$

ive Tor $\geq$ Botron

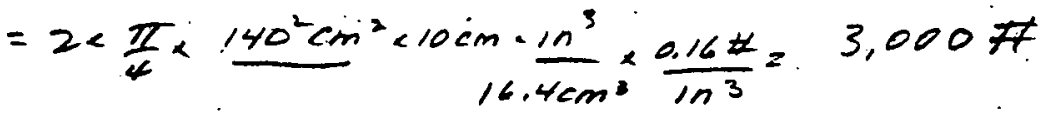

$$
\begin{aligned}
& \text { Sos TornL } \overline{22,9604} \\
& \text { Plus Spiclace (10\%) Torral } \frac{2300}{25,260 \text { H }}
\end{aligned}
$$

lost a 20\%\% Formeo into grimes but less comtainees

$$
\begin{aligned}
& \text { Cort }=25,260 \% \times 20 \% / 4=\quad \$ 505,200 \\
& \text { Purs Prokecenc (2\%) 10,100 } \\
& \text { Puis shispinc a 2 28/curt } 700 \\
& \text { Pws 3000\#.186000 304 25,500 }
\end{aligned}
$$

is conraineas

8Se/16 uasol 7 MlaTL

$$
\begin{aligned}
& \text { Torta } \$ 541,500 \\
& \text { Use } 550,000
\end{aligned}
$$

$-248-$

RMP $307(2 / 78)$

PAGE NUMBER 
ALP

THE RALPH M. PARSONS COMPANY

CALCULATION SHEET

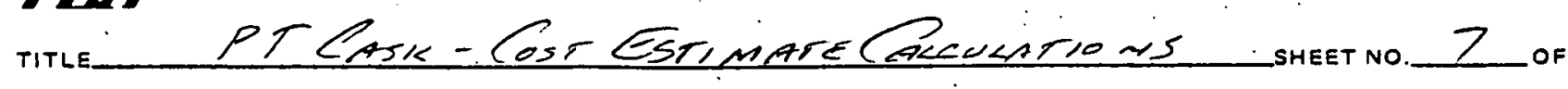
JOBNO. DEPARTMENT

$$
\text { AUTHOR }
$$

DATE

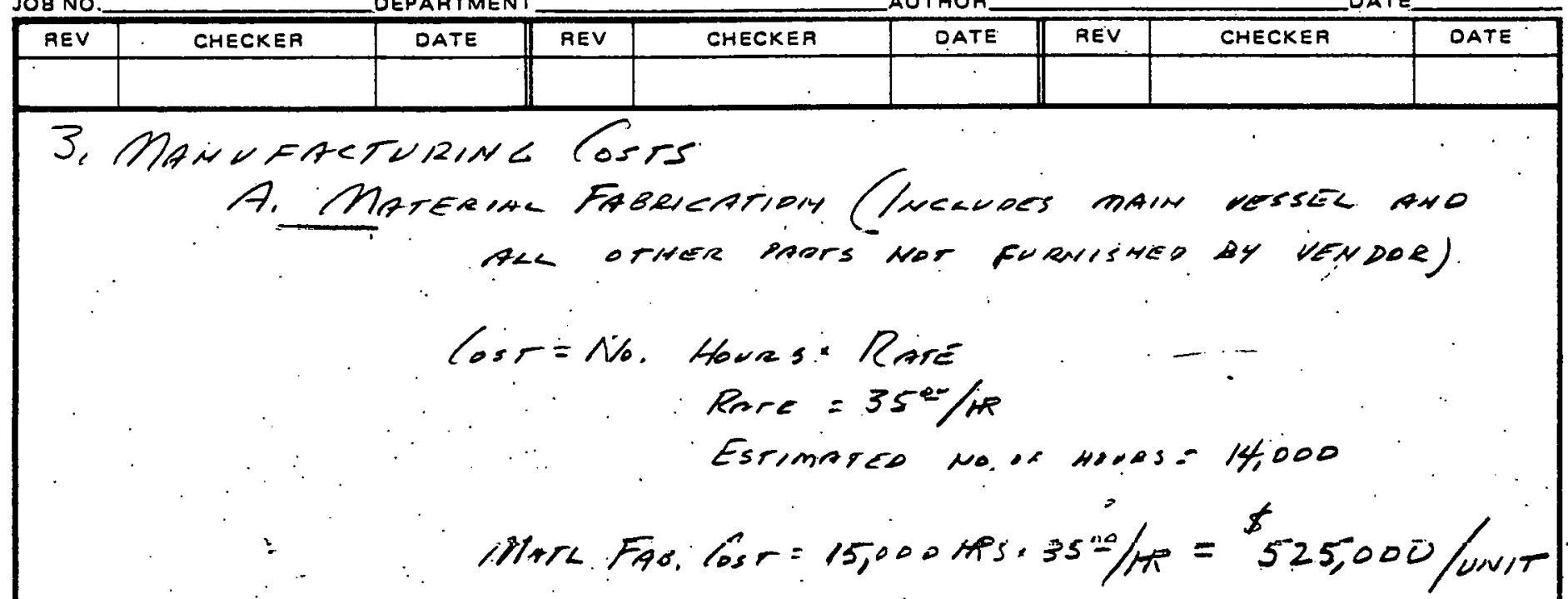

B. AssembLy (VENONR Paets assembeLED into VESSEL SHEN)

Estimarea mannous $=3000$

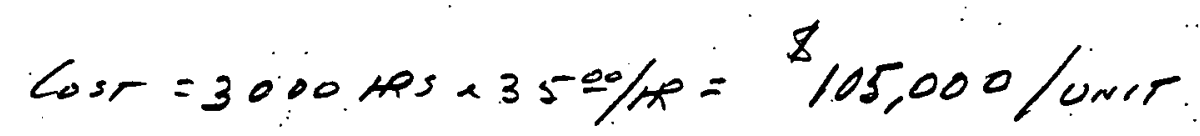
Use 100,000

4 Design, License al Test losts

A. Desion

Estimarco Mry Howns - 12,000

Cost $=12,000$ Res $235^{\circ} \mathrm{y} / \mathrm{Re}=\cdots \quad 8420,000$

Cost/unit (AMOetizero ORER 25 units)

Des: Corrfunit $=\frac{420,000}{25}=\frac{\$ 16,800 / \text { unir }}{15020,000}$

B. Test, Resesicn \& ReBuich FOR DOT License 20,000 Estinated Gor $\$ 1000,000$ (Tora)

Cost(unir(moetizes over 25 un irs) $=1,000,000=540000 /$ wir

Pus costs of Licensinc 20,000/urret

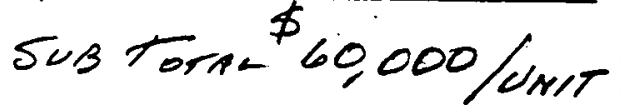

$\frac{\text { Torat Pesicu, Laenge } \$ \text { Tesr }=60 K+20 K=80,000 / \text { wint }}{.249 \cdot}$ 
THE RALPH M. PARSONS COMPANY

CALCULATIAN SHFFT

Tithe PT Cask-Cost Estimate Preculations SHEET NO. $\$$ OF JOBNO. $5872-172$ OEPARTMENT AUTHOR SW/T ATE

(



Estimater man-noves - 4000

$$
\begin{aligned}
& \text { Losir }=4000405 \times 35=1 / m 0=0 / 40,000 \text {. (Toran) }
\end{aligned}
$$

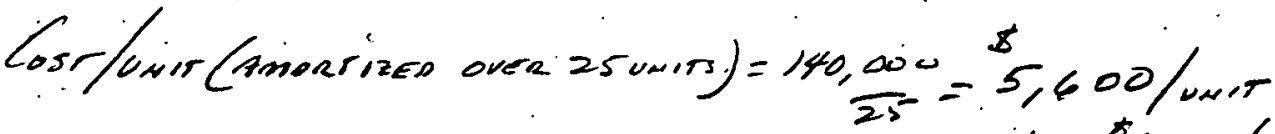

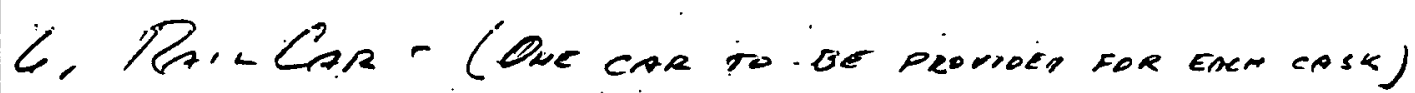
Ws=otosog/me

Estimiateo Cost - \$,75,000

COST SUMMARY

i: Materiacs

304 :STAIN LESS STEEL - - -96,000

6061-T6 ALUMINUM 67,000

Coppere Gounc Fins $\quad 10,000$

Misceilaneous 1,000

$18.4,000$

2. Sur Assembues.

Bonon Carbide

$1,290,000$

Lirhium Hypriog

$0.10,000$

Depretes VRanium 860,000

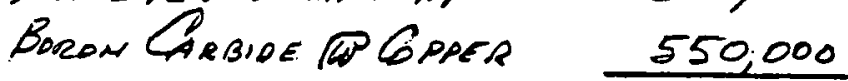

Total Materials amo Subassemblies

$\frac{3,3 \% 0,000}{3,494,000}$

3. Mamufacturing

Materna Fabelcation 525,000

AssemBLY 105,000

SUB TOTAL

4 Desicu, Lisense an, Test (1/5 of cort)

$\frac{630,000}{4,124,000}$

5. Tooung ano Setoup (1/25 of cist)

80,000

i. Rarl Che

6,000

$-250-$

Costíunt $\frac{175,000}{4,385,000}$

RMP 307 (2/78)

PAGE NUMBER 


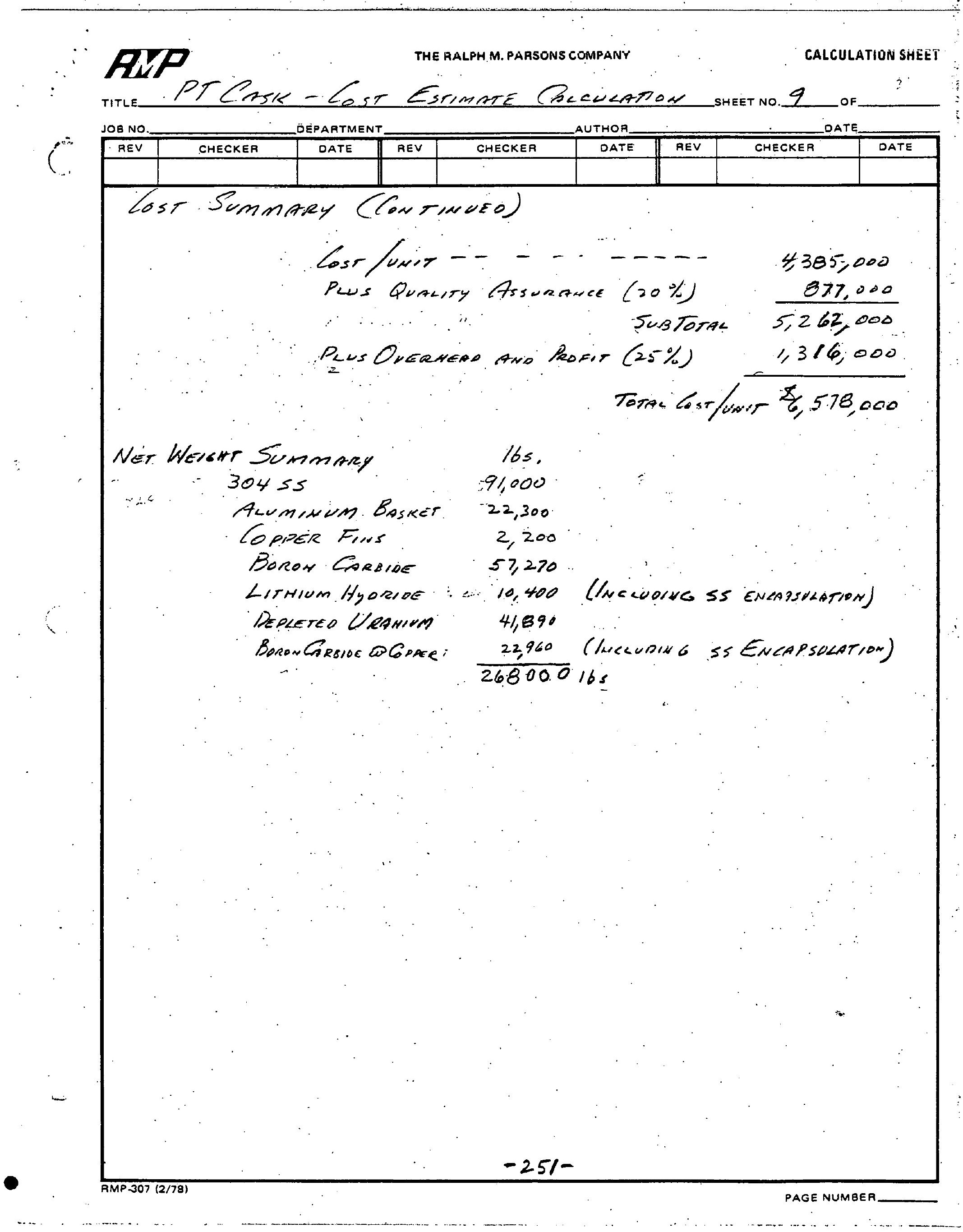




\section{INTERNAL DISTRIBUTION}

1-10. J. 0. Blomeke

11. A. G. Croff

12. B. C: Finney

13. Centràl Kesearch Library

14. ORNL Patent Section

15. Laboratory Records

16. Laboratory Records, RC

17. ORNL Y-12 Technical Library, Document Reference Section

\section{EXTERNAL DISTRIBUTION}

Science Applications, Inc., 5 Palo Alto Squaré, Suite 200, Palo Alto, CA. 94304

Los Alamos Technical Associates, P.0. Box 410, 1553 Myrtle St., Los Alamos, NM 87544

19. S. E. Logan

Ralph M. Parsons Company, Power Division, 100 Walnut St., Pasadena, CA 91124

20-24. A. E. Smith

Georgia Institute of Technology, Atlanta, GA 30332

25. D. W. Tedder

DOE-SRO, P.O. Box A, Aiken, SC 29801

26. T. B. Hindman

DOE-HQ, Washington, DC 20545

27. R. D. Walton

DOE-ORO, Oak Ridge, TN 378.30

28. Office of Asst. Manager for Energy Research and Development

29-30. Technical Information Center, Oak Ridge, TN 37830 